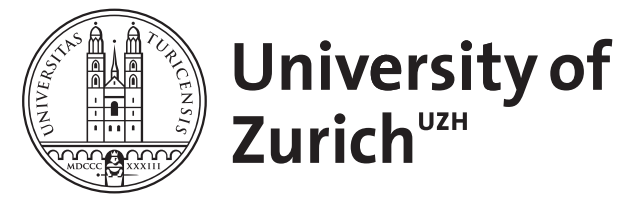

Zurich Open Repository and Archive

University of Zurich

University Library

Strickhofstrasse 39

CH-8057 Zurich

www.zora.uzh.ch

Year: 1977

\title{
La condition du croyant dans l'évangile selon Matthieu
}

\author{
Zumstein, Jean
}

Posted at the Zurich Open Repository and Archive, University of Zurich ZORA URL: https://doi.org/10.5167/uzh-150082

Monograph

Published Version

Originally published at:

Zumstein, Jean (1977). La condition du croyant dans l'évangile selon Matthieu. Fribourg, Switzerland / Göttingen, Germany: Éditions Universitaires / Vandenhoeck Ruprecht. 
ZUMSTEIN - LA CONDITION DU CROYANT 
Publié par

Othmar Keel, Bernard Trémel et Erich Zenger au nom de l'Institut Biblique de l'Université de Fribourg, Suisse et du Seminar für Biblische Zeitgeschichte de l'Université de Münster

Notice biographique:

Jean Zumstein (1944) a fait ses études de théologie aux Universités de Lausanne, Strasbourg et Göttingen. Il s'est spécialisé en Nouveau Testament à l'Institut des sciences bibliques de l'Université de Lausanne et à l'Université de Heidelberg. La présente monographie est la thèse de doctorat qu'il a soutenue en 1974 à Lausanne. Depuis 1975, il est professeur de Nouveau Testament à la Faculté de Théologie de l'Université de Neuchâtel. 


\section{JEAN ZUMSTEIN}

\section{LA CONDITION DU CROYANT DANS L'EVANGILE SELON MATTHIEU}

EDITIONS UNIVERSITAIRES FRIBOURG SUISSE VANDENHOECK \& RUPRECHT GÖTTINGEN 1977 
(c) 1977 by Editions Universitaires Fribourg Suisse Imprimerie St-Paul Fribourg

EU ISBN 2-8271-0124-6 V \& R ISBN 3-525-53319-5

Digitalisat erstellt durch Florian Lippke, Departement für Biblische Studien, Universität Freiburg Schweiz 
A PIERRE BONNARD 

AVANT - PROPOS

Première partie : LES DISCIPLES ET LES ADVERSAIRES DE JESUS

1. Survol statistique

2. Les disciples comme groupe

3. La figure des disciples dans les récits de controverses

4. La figure des disciples dans les grands discours

5. La figure des disciples dans les débats d'école et dans les récits de miracles
a) la destinée des disciples (37); b) la compréhension des disciples (41).

6. La figure des disciples dans l'histoire de la Passion et de la Résurrection

7. Conclusion

A. Les adversaires de Jésus avant la Passion

1. Les pharisiens seuls ou associés à d'autres groupes

2. Les scribes seuls ou associés à d'autres groupes

3. Conclusion

B. Les adversaires de Jésus durant la Passion

1. Les grands prêtres seuls ou associés à d'autres groupes

2. Les anciens seuls ou associés à d'autres groupes 73

3. Conclusion 
C. Les pécheurs et les païens chez Mathieu

1. Les pécheurs, les péagers et les païens, image de l'incroyance

2. Les péagers et les pécheurs, bénéficiaires de l'appel du Christ

3. Les péagers et les prostituées, image de la foi refusée par les Juifs

D. L'importance des partenaires de Jésus dans la

composition du premier évangile

Deuxième partie : LE FONDEMENT CHRISTOLOGIQUE

CHAPITRE TROIS : LE RESSUSCITE ET LE JESUS TERRESTRE (Mt 28, 16-20)

1. Analyse littéraire

a) tradition et rédaction (87); b) la tradition christologique utilisée dans les vv. 18b-20 (90);

c) l'arrière-fond ecclésial (92); d) forme littéraire et contexte (93).

2. Interprétation

3. L'identité du Ressuscité et du Jésus terrestre

CHAPITRE QUATRE : LE DIDASCALE ESCHATOLOGIQUE (Mt 5,17-20)

1. Analyse littéraire

a) tradition et rédaction (107); b) forme littéraire (110); c) l'arrière-fond ecclésial (114).

2. Interprétation

3. Le statut de la prédication du Christ $\mathrm{mt}$

CHAPITRE CINQ : LE REVELATEUR (Mt 11, 25-20)

1. Analyse littéraire

a) critique des sources (131); b) le contexte de $Q$ et le contexte $\mathrm{mt}(134)$; c) les paralleles historicoreligieux (135).

2. Interprétation 
3. Le joug du maitre doux et humble

Troisième partie: LA COMMUNAUTE MATTHEENNE

CHAPITRE SIX : LES RESPONSABLES DE LA COMMUNAUTE MA T T HEENNE

154

1. Les scribes 156

a) bref état de la question (156); b) 23, 8-12 (157);

c) 13,51-52 (159); d) résultats (162).

2. Les prophètes

163

a) bref état de la question (163); b) les prophètes au service de la communauté (164); c) les prophètes envoyés à Israël (167); d) résultats (170).

CHAPITRE SEPT : LES DIVISIONS DE LA COMMUNAUTE MA T T HEENNE

1. La terminologie de la lutte contre l'hérésie
a) $\dot{\alpha} \vee 0 \mu i \alpha$
(171); b)
b) $\pi \lambda \alpha \vee \alpha ́ \omega$ (174);

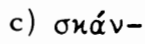

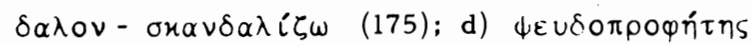

2. La parénèse du Sermon sur la Montagne (Mt 7, 15-23)

181

a) vv. 15-20 (181); b) vv. 21-23 (184)

3. L'explication de la parabole de l'ivraie (Mt 13,36-43) 187

4. Les grandes tribulations (Mt 24, 9-14) 195

5. Conclusion

198

Quatrième partie : LE CROYANT ET SON SEIGNEUR

201

CHAPITRE HUIT : LE CROYANT ET LE JESUS TERRESTRE 202

1. Comprendre 203

a) ouvı́์ (203); b) Mt 13,10-17 (206);

c) le problème de l'idéalisation (212).

2. Suivre Jésus

a) Mt 4,18-22; 9, $9(216)$; b) Mt 8, 18-22 (220);

c) Mt 10, 37-39 (225). 
CHAPITRE NEUF : LE CROYANT ET LE PRESENT DE LA FOI

1. La foi

a) bref état de la question (233); b) la foi dans les récits de miracles (234).

2. La crise de la foi

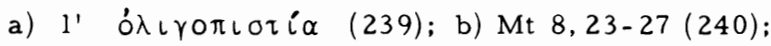

c) Mt 14,22-23 (245); d) conclusion (254).

CHAPITRE DIX : LE CROYANT FACE A L'AVENIR

256

1. La venue inopinée de la parousie (Mt 24, 37-44)

2. La parabole du serviteur (Mt 24, 45-51)

3. La parabole des dix vierges (Mt 25, 1-13)

4. Conclusion

Cinquième partie: LE CROYANT ET L'ETHIQUE

CHAPITRE ONZE : LE POINT DE DEPART DE L'ETHIQUE (Mt 5, 3-10)

1. Tradition et rédaction

2. L'accentuation de l'éthique

3 Forme littéraire et eschatologie 295

4. Le contexte de communication 300

5. Le contexte littéraire des béatitudes 303

6. Le point de départ de l'éthique 306

CHAPITRE DOUZE : LE THEME DE L'ETHIQUE (Mt 5, 43-48)

1. La Loi et le thème de l'éthique 309

2. Analyse littéraire 313

3. Le prochain et l'ennemi

4. Imiter Dieu 320

5. La perfection 
CHAPITRE TREIZE : L'ENJEU DE L'ETHIQUE

(Mt 25, 31-46)

1. Analyse littéraire

2. Le champ de l'éthique

3. L'évidence de l'éthique

4. Le retour au fini

Sixième partie : LE CROYANT ET L'EGLISE

CHAPITRE QUATORZE : LE RASSEMBLEMENT DE L'EGLISE

1. L'invitation (Mt 22, 1-14)

2. Incrédulité d'Israël, foi de l'Eglise (Mt 8, 11-12)

3. Désobéissance d'Israël, obéissance de l'Eglise (Mt 21, 33-46)

4. L'Eglise, corpus mixtum

1. La discipline fraternelle (18, 15-20)

2. La sollicitude envers les petits $(18,6-14)$

a) le scandale, vv. 6-9 (397); b) la brebis perdue, vv. 10-14 (400).

3. Le pardon $(18,21-35)$

a) l'entretien sur le pardon, vv. 21-22 (405);

b) le serviteur impitoyable. vv. 23-35 (407).

4. La véritable grandeur (18, 1-5)

1. Devenez ce que vous êtes (Mt 5, 13-16)

2. Un témoignage en paroles et en actes

a) l'envoi en mission, Mt 10.1.7-8 (429); b) la crise des charismes. Mt 17, 14-20 (435).

3. Souffrance et échec (Mt 10,17-25) 
Abréviations

454

Bibliographie

456 


\section{A V A N T - P R O P O S}

Le présent travail est la thèse de doctorat que j'ai soutenue le 23 novembre 1974 devant la Faculté de Théologie de l'Université de Lausanne. Ma nomination à la chaire de Nouveau Testament de l'Université de Neuchâtel au printemps 1975 m'a empêché de prendre en considération pour la publication les études critiques consacrées à l'évangile selon Matthieu et publiées après l'été 1974. Indépendamment de ce fait, le nombre des travaux sur le premier évangile a pris ces dernières années des proportions si considérables que le dépouillement exhaustif de la bibliographie est devenu impossible. J'ai donc été obligé d'opérer un choix que j'espère judicieux en regard des questions abordées dans mon ouvrage.

C'est pour moi un agréable devoir que d'exprimer ma gratitude à tous ceux qui, d'une manière ou d'une autre, ont soutenu mon travail de recherche. Je remercie en particulier la Faculté de Théologie de l'Université de Lausanne qui m'a permis d'élaborer et de défendre cette thèse de doctorat; le Fonds national suisse de la recherche scientifique qui, dans ce but, m'a accordé une bourse de jeune chercheur; les professeurs E. Schweizer et C. Senft qui ont fait un rapport critique sur mon travail à l'occasion de la soutenance; les professeurs O. Keel et B. Trémel qui ont accepté d'éditer cette thèse dans la collection "Orbis Biblicus et Orientalis"; l'Eglise évangélique réformée du canton de Neuchâtel qui a contribué à la publication par un don appréciable. Enfin, je mentionnerai tout spécialement mon maître de Nouveau Testament et directeur de thèse, le professeur Pierre Bonnard, à qui cette étude de théologie matthéenne est offerte en signe de reconnaissance. 



\section{INTRODUCTION}

Le point de vue que nous avons choisi pour aborder la théologie de l'évangile selon Matthieu, est la condition du croyant. Notre intention est d'essayer de montrer comment le rédacteur du premier évangile a traité le problème de l'existence chrétienne à travers les traditions qu'il a reçues, ordonnées et rédigées. En retenant ce thème, nous ne pensons pas faire violence à l'évangile ou lui imputer artificiellement une problématique qui lui est étrangère. L'attention particulière que le rédacteur voue au groupe des disciples, la fonction paradigmatique qu'il leur attribue, sont des résultats avérés de la recherche mt récente ${ }^{l}$. Notre

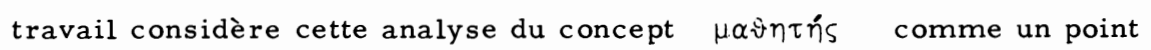
de départ qu'il s'agit de vérifier, de fonder et de développer systématiquement. La méthode que nous utilisons dans les pages qui suivent, est connue sous le nom de "Redaktionsgeschichte". Appliquée pour la pre mière fois à l'évangile selon Mt, en 1948, par G. Bornkamm, à l'occa sion d'une étude sur la tempête apaisée ${ }^{2}$, cette procédure d'analyse est si connue qu'elle n'appelle pas d'exposé particulier.

Le propos de cette introduction est plutôt d'expliquer la démarche que nous avons suivie pour traiter notre sujet. Nous avons ordonné notre recherche en six grandes parties. Dans la première partie, nous avons

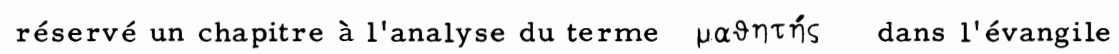
selon Mt. Cette étude qui démontre à la fois l'ambivalence du concept, sa présence constante dans le procès de l'évangile, mais aussi sa fonction variable selon la forme littéraire envisagée, justifie méthodologiquement notre recherche en indiquant de manière précise le champ

1 Voir notre chap. 1. Par ailleurs, nous n'entendons pas présenter à titre préalable une histoire de la recherche mt. Le lecteur découvrira au gré des chap. à la fois les hypothèses actuellement défendues et notre position propre.

2 BORNKAMM, Sturmstillung 48-53. 
sémantique dans lequel le rédacteur aborde notre problématique. Le deuxième chap. qui est consacré aux adversaires de Jésus, semble s'éloigner de la perspective centrale de notre travail. En réalité, son utilité est de montrer que le phénomène d'ambivalence établi pour le groupe des disciples se répète au niveau des autres partenaires du Christ mt si bien que nous butons ici sur un élément caractéristique de la rédaction $\mathrm{mt}$ : les groupes qui entourent le Christ $\mathrm{mt}$ sont non seulement les partenaires du Jésus terrestre, mais aussi des paradigmes grâce auxquels la communauté vit et interprète sa propre histoire.

La deuxième partie de notre thèse s'intitule "le fondement christologique". Son intention est de penser théologiquement la structure littéraire mise en évidence dans la première partie - et en particulier l'usage conséquent de catégories ambivalentes -. Il ne suffit pas, en effet, de constater que Mt rend transparent, pour le présent, l'histoire du Jésus terrestre et de ses compagnons, encore faut-il expliquer pourquoi une telle continuité historique est possible, pourquoi le croyant est appelé à se reconnaître et à se comprendre dans la narration évangélique. A notre avis, la réponse à cette question se trouve dans la christologie mt. L'exégèse de $28,16-20 ; 5,17-20 ; 11,25-30$ est destinée à montrer que, pour $\mathrm{Mt}$, on ne peut connaître le Ressuscité qui règne actuellement qu'en se mettant à l'écoute et à la suite du Jésus terrestre. L'unité paradoxale du terrestre et du Ressuscité confère à la destinée historique du Christ $\mathrm{mt}$ - et, en particulier, à sa prédication - un statut de normativité pour le présent de la foi. Cette concentration de la christologie mt sur le Jésus terrestre explique la fonction typologique des disciples dans l'évangile. Nous pourrons dès lors renoncer à faire, dans notre deuxième partie, une présentation exhaustive de la christologie $\mathrm{mt}$, et borner notre effort à en discerner la portée spécifique pour la détermination de la condition du croyant.

La troisième partie de notre thèse s'efforce de ressaisir quelques traits caractéristiques de la communauté mt. Cette démarche est 
méthodologiquement justifiée pour deux raisons. D'une part, si, dans notre première partie, nous avons établi que le $\mu \alpha \vartheta \eta \tau$ ńs de la narration mt est le paradigme du croyant, il s'agit de ne pas oublier que ce paradigme n'est pas conçu de façon idéale, mais en fonction de la situation concrète que vit l'église mt. Pour interpréter correctement l'argumentation de l'évangéliste, il convient de la replacer dans son contexte historique spécifique. D'autre part, si, dans notre deuxième partie, nous avons montré que l'existence chrétienne se constituait comme relation avec l'histoire - et singulièrement l'enseignement - du Jésus terres tre, il apparaît indispensable d'observer comment la communauté mt a accueilli, transmis et interprété la tradition qui lui révélait son Seigneur. A ce double objectif, nous avons tenté de répondre en étudiant deux aspects de l'église $\mathrm{mt}$ : les charges qu'elle reconnaît, les conflits qu'elle traverse. Ce choix s'imposait car, à notre avis, ces deux enquêtes nous permettent immédiatement de découvrir les deux points théologiques sur lesquels Mt s'est inlassablement battu : la christologie et la loi. Nous n'avons donc pas eu l'ambition, dans cette troisième partie, de présenter une esquisse développée de l'église $\mathrm{mt}$, mais d'éclairer le champ conflictuel dans lequel la condition du croyant a été pensée ${ }^{1}$.

Après avoir circonscrit notre problématique aussi bien à son niveau littéraire que dans ses présupposés christologiques et historiques, il nous était possible d'entreprendre la description proprement dite de la condition du croyant. Nous l'avons d'abord fait, dans notre quatrième partie, en nous concentrant sur la relation qui se noue entre le disciple et son Seigneur. Dans la mesure où le statut du croyant est le même que celui du compagnon historique de Jésus, plus précisément, dans la

1 Le conflit de l'église $m$ t avec la synagogue fait naturellement partie de ce champ conflictuel. Nous ne sommes pas revenus sur ce point dans notre troisième partie, car nous l'avons abondamment traité dans les pp. $26 \mathrm{ss}$ de notre chap. 1 et dans l'ensemble de notre chap. 2 . 
mesure où le chrétien est invité à devenir le contemporain des premiers disciples pour se mettre à l'écoute et à la suite du Jésus terrestre, il importait de montrer à travers quelles catégories théologiques une telle relation s'institue. Il nous a semblé à cet égard que le ouvı́́vaı en tant que modèle d'adhésion à l'enseignement du Jésus terrestre et

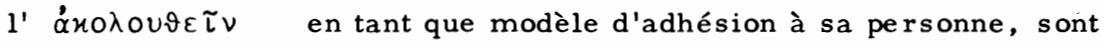
particulièrement appropriés pour rendre compte de la pensée mt sur ce point.

En devenant le contemporain du Jésus terrestre, le croyant se place sous la grâce et l'autorité du Kyrios qui règne actuellement. Cette contemporanéité n'a de sens que si elle amène le disciple à vivre le temps présent dans la fidélité à la vocation reçue. Nous pensons que cet accomplis sement de la condition chrétienne dans le présent, vécu à la fois dans la misère et la grandeur, a tout particulièrement préoccupé Mt. Il a essayé de présenter la relation sans cesse menacée, mais toujours recréée du chrétien avec son Seigneur à travers les catégories de la foi orante et du doute.

Enfin, si le Jésus terrestre est le Kyrios qui règne actuellement, il est également le Fils de l'homme qui va revenir pour le jugement. La christologie détermine non seulement la relation du croyant avec le passé et sa saisie du présent, elle décide également de son avenir. Plus que tout autre, Mt s'est attaché à replacer la vie chrétienne dans la perspective de l'eschaton. En prenant acte du retard de la parousie, mais en soulignant avec la dernière énergie la venue inéluctable du jugement, il a vu dans l'avenir le sujet d'une promesse illimitée, mais d'une promesse qui met le croyant ici et maintenant en situation de responsabilité radica le. C'est ce que nous avons tenté d'illustrer en analysant la parénèse de la vigilance.

Notre cinquième partie essaie de rendre compte de cette mise en responsabilité radicale de la vie chrétienne en s'attaquant au problème de l'éthique. Nous devons à cet égard écarter d'emblée un malentendu. 
Nous n'avons pas suivi la voie royale qui consiste à traiter de l'éthique mt en se concentrant sur la problématique de la Loi. Il nous importait de mettre en évidence, non pas l'éthique en tant que telle, mais la manière dont elle structure la condition chrétienne. A travers l'étude des béatitudes, nous avons tenté de décrire le point de départ de l'éthique, càd son enracinement dans la prédication eschatologique du Règne. Les antithèses du Sermon sur la Montagne nous ont permis de mettre en lumière le thème de l'éthique : les valeurs invoquées, la justice, l'amour, la perfection, réclament la consécration totale de l'existence humaine à Dieu. Enfin, par notre analyse de la fameuse fresque du jugement dernier $(25,31-46)$, nous avons voulu dégager l'enjeu de l'éthique. Selon nous, ce texte démontre de manière exemplaire que, pour $M t$, l'éthique est la médiation nécessaire qui permet au croyant d'inscrire sa foi dans le quotidien.

La sixième partie de notre thèse s'inter roge sur la condition du croyant dans l'Eglise. On pourrait à juste titre nous faire remarquer que les parties précédentes pourraient, elles aussi, trouver place dans "l'ecclésiologie $\mathrm{mt}^{\prime}$ : la notion de disciple est un concept ecclésiologique; la christologie décrit comment le Seigneur est présent dans sa communauté; l'éthique nourrit la catéchèse et édifie l'Eglise. Si notre présentation de la matière est différente, c'est que nous étions animé par une autre intention. Il s'agissait pour nous d'organiser l'ensemble de la théologie mt en fonction de notre thématique propre, la condition du croyant. Aussi dans notre partie consacrée à l'ecclésiologie mt, on trouvera ce qui peut être affirmé de la condition chrétienne en fonction de la réalité de l'Eglise - et en fonction de cette réalité seulement -. Dans un premier temps, nous avons dépeint le rassemblement de l'Eglise sur le fond de la crise d'Israël et nous avons mis en évidence les conditions de constitution de ce nouveau peuple - la foi et l'obéissance -. Nous avons insisté sur l'ouverture universelle qui caractérise ce rassemble ment, et qui a son envers paradoxal, mais inévitable dans l'apparition 
d'un corpus mixtum. Dans un second temps, nous nous sommes penché sur le discours communautaire (Mt 18) pour montrer comment ce statut de corpus mixtum peut être vécu et assumé par ses membres. Enfin, nous avons tenté de ressaisir le rapport que Mt établit entre l'Eglise et le monde : si, d'une part, l'Eglise est appelée à fournir un témoignage en paroles et en actes, d'autre part, le monde répond à cette prétention ecclésiale par la dérision et la haine. 
PRE MIERE PAR T I E

LES DISCIPLES ET LES ADVERSAIRES DE JESUS 


\section{Survol statistique}

La concordance nous apprend que $\mu \alpha \vartheta \eta \tau n ́ s$ comme désignation des disciples de Jésus apparaît 68 fois dans le premier évangile (Mc :

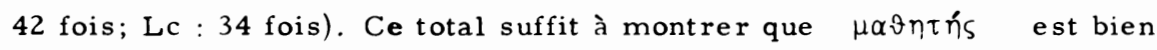
le concept usuel dont se sert Mt pour nommer les compagnons de Jésus.

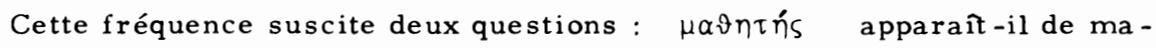
nière uniforme dans tout l'évangile ? Quelle est la part rédactionnelle dans l'emploi de ce terme?

Pour répondre à la première question, il suffit d'observer si $\mu \alpha \vartheta \eta \tau$ h́s apparaît avec une fréquence relativement constante dans les différentes formes littéraires propres à la tradition synoptique et reprises par Mt. Servons -nous à cet effet de la classification élaborée par Bultmann ${ }^{l}$ :

Evangile selon Matthieu

1 La tradition des paroles de Jésus (28 fois)

1.1 Apophtegmes (16 fois)

1.11 polémiques ou didactiques : $9,10.11 .14 ; 12,1.2 ; 13,10$; $15,2.12 .23 ; 19,10 ; 21,20$

1.12 biog raphiques : 8,$21 ; 12,49 ; 19,13 ; 24,1 ;(26,8)$

1.2 Les "dits du Seigneur" (= Herrenworte) 12 fois

1.21 les logia proprement dits ou paroles sapientielles : 9, 37; $10,24.25 ; 16,5.24 ; 19,23.25$

1.22 les dits apocalyptiques et prophétiques : 17,$10 ; 24,3$

1.23 les paroles relatives à la Loi et les règles en vue de la vie communautaire : 10,42

1.24 les paroles à la première personne du singulier $: 16,21$

1.25 les paraboles et dits du même genre : 13,36

1 Voir BULTMANN, Trad. 
Evangile selon Matthieu (suite)

2 La tradition narrative ( 34 fois)

2.1 Récits de miracles (13 fois)

2.11 récits de guérisons et d'exorcismes : 9, 19;17,16.19

2.12 actes violant 1 les lois naturelles : 8,$23 ; 14,15.19^{2} .22 .26$; $15,32.33 .36^{2}$

\subsection{Histoires et légendes (21 fois)}

2.21 du baptême à l'entrée à Jérusalem : 16,13.20;17,6;21,1.6

2.22 Passion:26,8.17.18.19.20.26.35.36.40.45.56;27,64

2.23 Pâques : 28,7.8.13.16

3 Cadre rédactionnel ( 7 fois)

5,$1 ; 10,1 ; 11,1 ; 17,3 ; 18,1 ; 23,1 ; 26,1$

La lecture du tableau ci-dessus montre que $\mu \alpha \vartheta \eta \tau$ ns apparait dans toutes les rubriques de la classification de Bultmann et que sa fréquence est relativement constante.

Notre deuxième question concerne l'activité rédactionnelle de $\mathrm{Mt}^{1}$ dans l'emploi de ce terme. La comparaison synoptique - et plus précisé ment la critique des sources - nous permet de résoudre ce problème. 24 mentions de $\mu \alpha \vartheta \eta \tau$ ńs sur 68 sont empruntées à $M c(9,10.11 .14 ; 12,1$; $14,15.19^{1} .22 ; 15,2.12 .32 .33 .36^{1} ; 16,13.24 ; 17,16.19 ; 19,13.23 ; 21,1$; $26,17.18 .19 .36 ; 28,7 ;) 1$ ressortit à $Q(10,24)$ et 3 au Smt $(27,64$; $28,13.16)$. Les 40 attestations restantes sont rédactionnelles $(5,1$; $8,21.23 ; 9,19.37 ; 10,1.25 .42 ; 11,1 ; 12,2.49 ; 13,10.36 ; 14,19^{2} .26$; $15,23.36^{2} ; 16,5.20 .21 ; 17,6.10 .13 ; 18,1 ; 19,10.25 ; 21,6.20 ; 23,1$; $24,1.3 ; 26,1.8 .20 .26 .35 .40 .45 .56 ; 28,8)$. Cette répartition fait apparaître clairement que $M t$ attache une importance particulière au concept

1 Nous parlons d'activité rédactionnelle de Mt lorsque nous détectons dans le corpus envisagé une intervention du rédacteur final de l'évangile. 


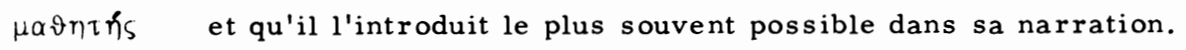
Quelle est alors la réalité décrite par ce terme auquel Mt voue une attention aussi constante ?

2. Les disciples comme groupe ${ }^{1}$

L'analyse des emplois rédactionnels montre que chez $\mathrm{Mt}$, plus que chez Mc, les disciples forment un groupe homogène et non différencié. Quatre séries de faits étayent cette constatation.

a) Sans qu'il soit question de nier la place particulière de Pierre ${ }^{2}$ parmi les disciples, il faut tout de même remarquer qu'en 21,20

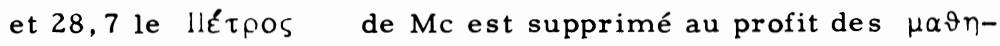
$\tau \alpha i$.

b) Nous observons la même tendance dans la place qui est accordée au "cercle des trois ou quatre intimes" de Jésus : s'il est repris de Mc en 17,1 et 26,37 il disparaît en 9,23 et 24,3. Même phénomène en 17,6 où $\mathrm{Mt}$ parle des disciples en général et en 17,14 où il évite, à la différence de Mc, d'opposer les "trois" aux autres disciples.

c) En 24,1 ce sont les disciples - et non pas l'un d'entre eux (ainsi Mc) - qui questionnent Jésus. En $26,17 \mathrm{ss}$, ce sont les disciples qui préparent la Pâque - et non pas deux d'entre eux (ainsi Mc) -.

d) Enfin en 13,10,Mt, à la différence de $\mathrm{Mc}$, refuse de distingue $\mathrm{r}$ les douze d'un groupe plus vaste de disciples.

1 Sur ce point, voir BARTH 93, note 2 .

2 Au niveau de la rédaction, Mt attribue un rôle particulier à Pierre en $14,28-29 ; 15,15 ; 16,18 ; 17,24 ; 18,21$. Dans ces passages, Pierre est à la fois le porte-parole des disciples et le garant de la tradition halachique de la communauté mt. Cf. SCHWEIZER, Gemeinde 152-153. 
A cette première tendance s'en ajoute une seconde : Mt distingue clairement le groupe des disciples de la foule. A cet égard, mentionnons trois observations :

a) En 5,1 et 23,1, foules et disciples sont clairement séparés. Voir aussi la redondance significative en 14,19 et 15,36 . Cette distinction atteint son point culminant dans le chap. 13 (cf. en particulier $13,10-17)$.

b) Ceux qui entourent Jésus et font sa volonté ne peuvent être que ses disciples et non pas un groupe indéterminé (comparer 12, 39 avec Mc 3,34).

c) Jésus adresse les exigences de la "Nachfolge" aux seuls disciples et non à la foule ${ }^{1}$ (comparer 16, 24 avec Mc 8, 34); voir aussi 8,$21 ; 10,5.37-39 ; 19,23-30$.

Concluons: en supprimant nombre de détails dans sa source Mc, Mt montre clairement qu'il n'est pas guidé par un souci d'historicisation. Il n'est pas attaché à décrire qui fut exactement le groupe des disciples historiques de Jésus. Sa tendance (pour une raison qu'il s'agira de découvrir) est à la stylisation. Il modèle le groupe des disciples en une grandeur uniforme. clairement distincte des autres protagonistes de l'évangile : pris en eux-mêmes, les disciples forment un tout homogènè et nondifférencié: par rapport aux autres groupes, ils sont clairement séparés et des adversaires de Jésus et de la foule.

Si l'on veut dépasser cette caractérisation formelle et découvrir l'image que Mt se fait de ce groupe, le rôle qu'il lui attribue, il s'agit

1 Nous utilisons le terme "Nachfolge" quil. ،'a pas d'équivalent satisfaisant en francais, pour désigner le partage de la destinée de Jésus et plus généralement la consécration à son service et à sa cause. Certes, Mt dit aussi des foules qu'elles suivent Jésus (4, 25; 8, 1; $12,15 ; 19,2 ; 20,29 ; 21,9 ;)$, mais c'est plus pour bénéficier de son secours que pour se soumettre à ses exigences. 
d'analyser la fonction des disciples dans les différentes parties de l'évangile. Or, de manière remarquable, à chaque forme littéraire correspond une fonction particulière des disciples. Nous examinerons successivement le rôle des disciples dans les récits de controverses, dans les grands discours, dans les débats d'école et les récits de miracles, dans l'histoire de la Passion et de la Résurrection.

\section{La figure des disciples dans les récits de controverses}

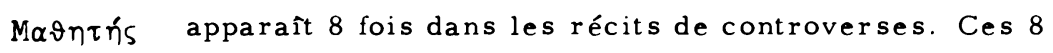
mentions se répartissent dans les 5 péricopes suivantes : 9, 9-13.14-17; $12,1-8 ; 15,1-20 ; 19,3-12$. Pour ce qui est de ces 8 usages, Mt suit 6 fois $\operatorname{Mc}(9,10.11 .14 ; 12,1 ; 15,2.12)$; à deux reprises, le terme est rédactionnel $(12,2$ : élucidation du par. mc; 19,10 : adjonction permettant d'introduire un logion tiré du Smt). A première vue, Mt semble donc assez fidèle à sa source Mc et sans originalité affirmée. Mais ce jugement formel doit être vérifié. Il convient d'examiner le rôle joué par les disciples dans les récits de controverses.

Dans 4 des 5 récits de controverses où ils apparaissent (exception : 19, 3-12), les disciples font figure d'accusés. Les pharisiens leur reprochent de violer la Loi. Formellement, le point de départ de chaque scène est stéréotypé. Les pharisiens, seuls $(9,11 ; 12,1 ; 19,3)$ ou associés à d'autres groupes $(9,14 ; 15,1)$, s'approchent de Jésus pour l'interroger. Leur question vise à mettre en cause la conduite des disciples, soit parce que ces derniers, à l'image de leur maître, mangent avec les péçheurs $(9,11)^{2}$, soit parce qu'ils ne jeûnent pas $(9,14)$, soit encore

1 Sur ce sujet, voir avant tout : HUMMEL 34-75.

2 La question des pharisiens met explicitement en cause Jésus; mais le v. 10 montre clairement que les disciples suivent la pratique de leur maître. 
parce qu'ils violent le sabbat $(12,2)$, soit enfin parce qu'ils ont renoncé à pratiquer les abblutions rituelles $(15,2)$.

Face aux accusations portées contre ses disciples, le Christ mt, à la différence de $\mathrm{Mc}$, n'invoque pas l'ordre instauré par sa venue. Acceptant la Tora comme critère de vérité, il défend la conduite de ses disciples en démontrant sa conformité avec la volonté de Dieu. En 9,9-13, la citation d'Os 6,6 (9,13a : adjonction rédactionnelle) tend à montrer que la communion avec les pécheurs est l'accomplissement de la volonté de Dieu annoncée dans l'Ecriture. En 12,1-8, l'argumentation rédactionnelle des vv. 5-7 établissant l'autorité de Jésus sur le sabbat et la priorité du commandement d'amour, est étayée par 2 passages de l'AT : $\mathrm{Nb} 28,9 \mathrm{~s}$ et Os 6,6. En 15,1-20, la halacha libérant les disciples des ablutions rituelles est légitimée par une discussion détaillée de la tradition orale et des prescriptions alimentaires. En 19,3-12, la halacha sur le mariage $(19,9)$ est le résultat d'une exégèse minutieuse de Gn 1,27; 2,24 et Dt 24,1 . Toutes les réponses de Jésus aux insinuations pharisiennes visent donc à établir que les disciples sont accusés à tort puisqu'en fait ce sont eux qui pratiquent l'authentique Tora (cf. le double $\dot{\alpha}-$ vaí ᄂos de $12,5,7)$.

Résumons : dans les récits de controverses $\mathrm{mt}$, les disciples font figure d'accusés, injustement mis en cause par les pharisiens et trouvant dans le Christ le défenseur qui démontre la conformité de leur règle de vie avec la Tora. Les compagnons de Jésus ou, plus précisément, la halacha qu'ils pratiquent, sont l'objet d'une défense assumée sur le terrain choisi par les adversaires - la pratique de la Tora -. Pour ces disciples, l'heure est à l'apologétique qui doit faire triompher leur bon droit et leur bonne foi; le Christ, interprète eschatologique de la Tora, se porte garant de leur éthique. Cette analyse appelle immédiatement une question : quelle situation historique une telle description suppose-t-elle? S'il est bien vrai que les récits de controverses $m$ t ont avant tout un but apologétique - défendre les règles de vie des disciples 
en butte à la polémique pharisienne en rappelant l'interprétation de la Loi donnée par le Christ -, alors nous nous trouvons en situation anachronique par rapport à la vie du Jésus historique. En effet, s'il est hors de doute que le Jésus historique a mené un débat polémique avec le judaîsme, s'il est possible qu'à quelques occasions, le motif de la controverse ait été éveillé par le comportement des disciples, il est à l'inverse douteux - preuve en soit Mc ! - que la confrontation ait été assumée par Jésus dans une visée apologétique, toute consacrée à éta blir la fidélité de ses adhérents à la Loi. Il est également peu vraisemblable qu'une tension ait existé entre le judaïsme et les disciples, Jésus ne jouant qu'un second rôle en prenant la défense de ses compagnons menacés. La situation des disciples dans les récits de controverses évoque bien plutôt une situation ecclésiastique : l'église $\mathrm{mt}$, figurée par les disciples, est.attaquée par le pharisaisme d'après 70 ; elle défend son enseignement et son mode de vie en se réclamant de la prédication prononcée naguère par son maître.

Notre interprétation selon laquelle les disciples deviennent l'image de l'église mt, est appuyée par trois autres arguments. Premièrement, nous montrerons dans le chapitre suivant que tant les adversaires de Jésus que les pécheurs et les païens ont une signification ambivalente : si les pharisiens figurent le judaïsme d'après 70 , les pécheurs et les païens représentent la destinée des pagano-chrétiens. Cette tendance ne peut manquer d'avoir des répercussions dans les passages où les différents groupes s'affrontent : c'est l'église mt qui est confrontée au judaïsme rabbinique : Deuxièmement, tous les récits de controverses où interviennent les disciples, se terminent par l'énoncé d'une halacha: halacha sur la communauté de vie entre Juifs et paiens convertis $(9,12$ 13), halacha sur le jeûne $(9,15 b)$, halacha sur le sabbat $(12,8,12 b)$, halacha sur les ablutions rituelles $(15,20)$, halacha sur le mariage $(19,9)$. Or les halachoth $\mathrm{mt}$ sont avant tout les règles qui régissent la vie de

1 Voir nos pp. $76-80$. 
l'église mt et qui la justifient. Troisièmement, certains récits de controverses ont subi des retouches de détail qui trahissent un point de vue ecclésial : dans la halacha sur le jeûne $(9,15)$, Mt a supprimé la restric-

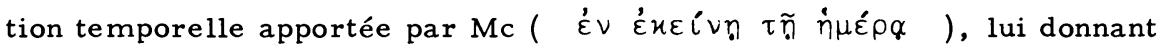
ainsi une portée générale; en $12,1-8$, il y a tension entre la halacha $\mathrm{mt}$ habilitant les oeuvres d'amour le jour du sabbat et la situation de départ qu'elle est censée légitimer (arracher des épis n'est pas une oeuvre

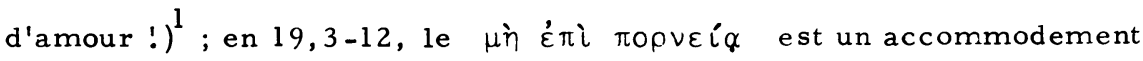
dû à la pratique ecclésiale ${ }^{2}$.

Le résultat de notre enquête est donc clair. Dans les récits de controverses mt, $\mu \alpha \vartheta \eta \tau n ́ s \quad a$ une signification ambivalente : à travers les disciples de Jésus, c'est l'église mt qui se profile. Une église violemment attaquée par le judaïsme d'après 70 et qui trouve les arguments de son apologétique dans le conflit de Jésus avec le pharisaisme. Une église qui puise dans l'enseignement passé de son Seigneur la justification de sa halacha.

\section{La figure des disciples dans les grands discours}

L'évangile selon Mt comprend cinq ou même six ${ }^{3}$ grands discours de Jésus $(5-7,10,13,18,23,24-25)$ qui font pour une bonne part son originalité. Comment les disciples sont-ils impliqués dans ces discours? D'un point de vue purement formel, il faut tout d'abord remarquer qu'ils

1 SCHWEIZER (Mt 181) défend la cohérence du récit en montrant que l'accusation portée par les pharisiens $(12,2)$ témoigne d'un manque d'amour en regard de la situation des disciples $(12,1: \dot{\varepsilon} \pi \varepsilon \dot{\imath} \nu \alpha \sigma \alpha \nu$, adjonction rédactionnelle).

2 Analyse chez HUMMEL 51.

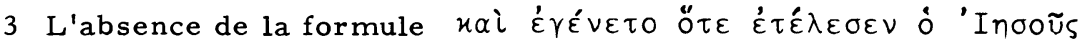

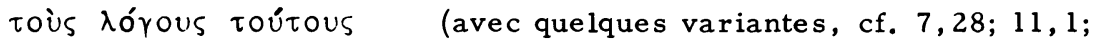
$13,53 ; 19,1 ; 26,1)$ à la fin du chap. 23 ne nous semble pas un motif suffisant pour dénier à ce passage le titre de grand discours. 
sont l'auditoire privilégié, sinon exclusif des discours du Christ mt.

L'auditoire du Sermon sur la Montagne est mentionné en 5,1-2, pas sage incontestablement rédactionnel ${ }^{l}$. Comme le confirme 7,28-29, il est composé des foules et des disciples. Les disciples sont néanmoins clairement distingués des foules : Jésus "voit" les foules, les disciples "s'approchent" de lui; ils forment le cercle des intimes qui entourent le maître. La préface $(9,35-38)$ du discours d'envoi est un sommaire rédactionnel ${ }^{2}$ qui culmine dans l'appel lancé aux disciples $(9,37: \tau \delta ́$ : $\tau \varepsilon$ $\gamma \varepsilon \iota \tau o \tau \varsigma$ $\mu \alpha \vartheta \eta \tau \alpha \tau_{\zeta} \alpha \dot{v} \tau o \tilde{v}$, rédactionnel !). L'introduction $(10,1.5)$ et la conclusion $(11,1)$ du di scours proprement dit ${ }^{3}$ confirment la ligne entrevue dans la préface : le chap. 10 est une instruction exclusivement adressée aux disciples. Le discours en paraboles semble contredire notre hypothèse, car il est adress é aux seules foules $(13,2-3)^{4}$. Pourtant, dès le v. 10 (rédactionnel), les disciples interviennent. Une courte ins truction (vv. $10-17)^{5}$ consacre leur place particulière et privilégiée par rapport aux foules. Les vv. 18-23 livrent à leur seule intention l'interpré-

1 Ainsi p. ex., BULTMANN, Trad 358; GRUNDMANN, Mt 111; KOLSTERMANN, Mt 32 pour $5,1 \mathrm{~b}$.

2 9,35-38 est une composition $\mathrm{mt}$ constituée d'un sommaire rédactionnel (v. 35), d'un emprunt à Mc 6,34 (v. 36) et à $Q(v .37)$.

310,1 , reformulation rédactionnelle de $M c 6,7$, et 11,1 , conclusion rédactionnelle stéréotypée des discours $\mathrm{mt}$, adressent le chap. 10 aux $\delta \omega ́ \delta \varepsilon \varkappa \alpha \mu \alpha \vartheta \eta \tau \alpha \iota$. 10,5 parle des seuls $\mu \alpha \vartheta \eta \tau \alpha \iota$. Par ailleurs, l'emploi $m t$ de $\delta \omega ́ \delta \varepsilon \varkappa \alpha$ n'autorise aucune conclusion (ainsi STECKER : tendance à l'historicisation !). Mt utilise $\delta \omega ́ \delta \varepsilon-$ $x \alpha$ de manière rare et hétérogène. Pour lui le cercle des douze est une réalité qui va de soi, mais à laquelle il n'attache aucune impor tance particulière (LUZ, Jünger 142-43). Preuve en soit le fait que Mt ne reproduit pas le récit de l'institution des douze qui figure dans sa source Mc (3,13-19; voir en particulier l'omission significative de Mc 3, 14a).

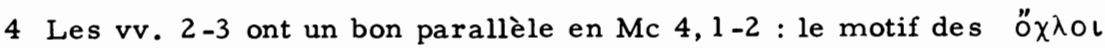
comme auditoire des paraboles est donc traditionnel.

5 13,10-17 est une composition typiquement mt élargissant et réinter prétant complètement Mc 4,10-12 par l'adjonction de Mc 4, 25 et $Q$ (cf. Lc 10,23-24). 
tation de la parabole du semeur. Les vv. 24-33 sont de nouveau adressés à la foule. Mais au v. 36 (rédactionnel !) le cadre change : Jésus se retire avec ses seuls disciples et les instruit ${ }^{1}$. Cette instruction "privée" culmine dans les vv. 51-52 où, à tit re de conclusion du discours dans son ensemble, Jésus s'assure que ses disciples ont bien compris son enseignement. Ces derniers sont donc incontestablement l'auditoire privilégié du Christ $\mathrm{mt}$ au chap. 13. Le discours communautaire s'adresse exclusivement aux disciples : les questions des disciples (v. 1, rédactionnel !) et de Pierre ( $v$. 2la, rédactionnel !) qui en constituent le cadre, en sont la preuve éloquente. Le discours contre les scribes et les pharisiens a un cadre analogue au Sermon sur la Montagne : le v. 1 (rédactionnel) nous présente les foules et les disciples comme les auditeurs du chap. 23. L'apocalypse $m t$ développe un enseignement réservé aux disciples : la petite scène introductive (inspirée de Mc 13,1-2, mais totalement remaniée) se passe entre Jésus et les disciples $(24,1.2)$; le v. $3^{2}$ qui donne le prétex-

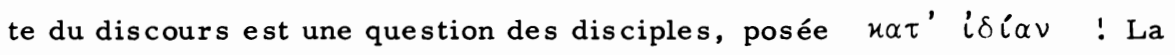
conclusion rédactionnelle et stéréotypée du discours $(26,1)$ confirme le cadre fixé en $24,1-3$.

La conclusion de ce rapide survol est claire : le cadre rédactionnel des grands discours mt présente les disciples comme l'auditoire privilégié des instructions du Christ. On sera également attentif au fait que dans quatre des six grands discours apparait la formule stéréotypée $x \alpha i$

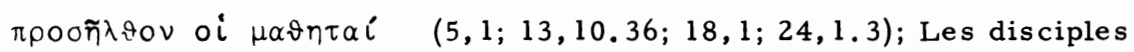
"s'approchent" de leur maître pour se faire instruire par lui ${ }^{3}$.

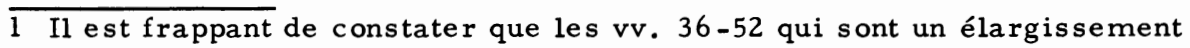
puisé dans le Smt, soient précisément adressés aux seuls disciples.

2 Conformément à sa tendance à voir les disciples comme un groupe homogène et non différencié, Mt substitue le groupe des $\mu \alpha \vartheta \eta \tau \alpha i ́$ au quatuor de Mc 13,3.

3 Sur ce point, bonne remarque de LUZ (Jünger 151): "Kurz: Während die lukanischen Apostel Zeugen alles dessen, was Jesus im Land der Juden und in Jerusalem getan hat (Ac 10,39), besonders aber der Aufer stehung (Ac 1,22) sind, also primär Augenzeugen, sind die matthäischen Jünger Menschen, die alles, was Jesus zu seinen Lebzeiten gelehrt hat, gehört und verstanden haben, also Ohrenzeugen". 
Mais comment se présentent ces auditeurs privilégiés ? Quelle image historique en est-il donné ? Le double auditoire auquel Mt a adressé le Sermon sur la Montagne, a mis bien des exégètes dans l'embarras et a souvent suscité des hypothèses artificielles ${ }^{l}$. R. Schnackenburg nous semble avoir trouvé la solution à ce problème lorsque, distinguant les différentes intentions de la rédaction $\mathrm{mt}$, il écrit : "Die an sich aufällige 'doppelte' Adresse wird sich so erklären, dass im Rahmen des Berichts vom Wirken Jesu die damals zusammengekommenen Menschen ein Beispiel der 'Lehre' Jesu 'in Vollmacht' erhalten sollen (vgl 7,29), die spätere Kirche aber einen Katechismus der sittlichen Unterweisung Jesu in die Hand bekommt ${ }^{2}$ ". Le discours public que le Christ mt adresse aux foules devient un catéchisme communautaire construit selon un schéma connu de la parénèse communautaire et analogue à Did 1-6 ${ }^{3}$. Il communique la charte d'entrée dans le Royaume et, à ce titre, il instruit le disciple du chemin qui y mène. Ce caractère catéchétique de la composition $\mathrm{mt}$ est d'une importance décisive, car il signifie que les disciples auxquels est adressé le Sermon sur la Montagne, sont aussi bien les compagnons historiques de Jésus que les croyants de l'église mt. La parénèse finale du chap. 7 confirme ce point de vue : les disciples sont mis en garde contre des ennemis qui sont ceux de la communauté postpascale - les faux prophètes $(7,15-20)$-; semblablement, l'annonce du jugement qui vient $(7,21-22)$, précédée par les malheurs des derniers temps (7,15-20) et cul minant dans l'appel à l'obéissance vigilante

1 Résumé de la discussion chez EICHHOLZ $20-25$.

2 SCHNACKENBURG 185.

3 BORNKAMM, Enderwartung 15. Dans la $\mathrm{RGG}^{3}$ 1, 1047 (art. Bergpre digt), le même auteur écrit : "Mt hat also deutlich den Rahmen der Rede aus $Q$ übernommen, aber ihn beträchtlich erweitert und das Ganze durch Aufnahme anderen Spruchgutes unter dem Thema der 'Gerechti keit, die besser ist als die der Schriftgelehrten und Pharisäer' $(5,20)$, zu einer Katechismusartigen Jünggerede (c'est nous qui soulignons) ausgestaltet. Das zugrunde liegende Schema ist in urchristlicher Parä nese auch sonst erkennbar". 
$(7,24-27)$, constitue la description mt classique du temps de l'Eglise.

Concluons : le disciple auquel Mt s'adresse dans les chap. 5-7 est, avant tout, le croyant vivant le temps de l'Eglise et recevant les instructions menant au Royaume.

Le discours d'envoi est une instruction réservée aux "douze". Mais s'agit-il d'une collection de recommandations concernant la seule mis sion galiléenne et pré-pascale des compagnons de Jésus ou le chap. 10 décrit-il également une possibilité actuelle de la communauté ${ }^{1}$ ? Si l'interprétation des vv. 1-16 est discutée, personne ne conteste la signification ecclésiale des vv. 17-42 qui traitent de la condition souffrante du croyant. Pour l'appréciation d'ensemble du discours, on se souviendra de deux faits fort significatifs à notre avis. Premièrement - et comme dans les autres grands discours -, Mt est guidé par un souci thématique et non historique; pour présenter la mission et la destinée des envoyés de Dieu, il fond en une seule composition les traditions d'origines les plus diverses (cf. notamment l'emprunt à l'a pocalypse mc !). Deuxièmement, Mt efface les circonstances concrètes liées aux traditions de l'envoi : ni récit de l'exécution de l'ordre d'envoi (au contraire de Mc 6, 12-13 par Lc 9,6), ni description du retour des envoyés (au contraire de Mc 6,30; Lc 9,$10 ; 10,17-20)$. Souci thématique et suppression du cadre vont de

1 La plupart des études sur Mt admettent que le discours d'envoi dépasse le cadre de l'activité galiléenne de Jésus et a une résonnance ecclésiale (voir BARTH 93; BORNKAMM, Enderwartung 16; BULTMANN, Trad 155-56; GRUNDMANN, Mt 284; KLOSTERMANN, Mt 84-85; RIGAUX 205-206; SCHNIEWIND, Mt 176; SCHLATTER, Mt 118). STRECKER (Weg 134.197), au nom de la tendance historicisante qu'il découvre chez $\mathrm{Mt}$, et de la stricte distinction qu'il opère entre le temps de Jésus et le temps de l'Eglise, voit dans les 16 premiers vv. du chap. - d'ailleurs en opposition avec 28,16-20 - un passage concernant exclusivement l'époque pré-pascale. Les vv. 5-6 sont le point fort de l'argumentation de STRECKER; cependant, ils ne décrivent pas d'abord la mission impartie aux disciples, mais bien la fidélité de Dieu à son peuple, manifestée dans l'itinéraire du Messie. Bonne dis cussion des études récentes sur ce point chez LUZ, Junger 143-146. 
pair : Mt entend instruire son église; la grâce et l'exigence sous les quelles furent placés les douze, deviennent le modèle de tout disciple dépositaire de la Parole ${ }^{1}$.

Dans le discours en paraboles, les foules et les disciples forment chez Mt deux fronts distincts, désormais inassimilables l'un à l'autre (vv. 10-17). Ce durcissement se conçoit avant tout durant l'époque post pascale. Les foules - entendez le judaîsme - sont sourdes à la prédica tion de Jésus; leur endurcissement est pour Mt le châtiment de leur désobéissance (cf. le ö Ł du v. 13); n'ont-elles pas persécuté Jésus, puis ses envoyés? Les disciples sont au contraire les témoins bienheureux qui comprennent ${ }^{2}$. Cet accent porté par Mt sur la compréhension dans le chap. 13 (cf. en particulier le v. 5l) est fort intéressant : il révèle le souci d'une saisie exhaustive et correcte de la prédication de Jésus, seule garantie d'une juste transmission de son enseignement. Or cette exigence ne prend-elle pas tout son sens lorsque le maitre est parti et qu'il s'agit de conserver sa parole ${ }^{3}$ ? Cette perspective post-pascale dans le chap. 13 se manifeste encore par un autre biais. La parabole de l'ivraie (vv. 24-30), mais surtout son explication (vv. 36-43) montrent que l'Eglise est un "corpus mixtum" en route vers le jugement ${ }^{4}$. Le

1 Avec BARTH, 93-94. Voir aussi la judicieuse remarque de LUZ (Jünger 146) à propos de la "validité" du chap. 10 : "Di e Gebote Jesu sind eben grundsätzlich für immer gültig".

2 BARTH 99-104 a clairement montré que Mt, dans sa narration, supprime l'incompréhension mc des disciples; le rédacteur abolit ainsi la différence que Mc établit entre le disciple pré-pascal et post-pascal, attestant que cette différence ne lui tient pas à coeur. LUZ (Jünger $148-150$ ) reprend la thèse de BARTH en lui apportant une correction pertinente : dans le chap. 13, la compréhension des disciples n'est pas sans faille; souvent, ils ne saisissent pas la prédication du maitre, mais, par des instructions privées et répétées de Jésus, ils parviennent en définitive $(13,51 !)$ à une juste intelligence de son message.

3 TRILLING, Amt 33; cf. aussi LUZ, Jünger 149.

4 BORNKAMM, Enderwartung 17. 
discours du chap. 13 - dont les disciples sont l'auditoire privilégié - est de nouveau en prise directe sur la réalité de l'Eglise.

Le discours communautaire est adressé aux disciples historiques de Jésus; en fait, il aborde une situation ecclésiale précise à laquelle il essaie de répondre ${ }^{l}$. Comme le montre l'interprétation mt de la parabole de la brebis perdue (vv. 10-14), mais aussi la règle communautaire des vv. 15-18, l'horizon de compréhension du chap. 18 est l'attitude qu'il convient de prendre vis-à-vis du petit, càd du frère égaré. Dès lors, à travers les disciples, l'enseignement du Christ mt est adressé à une communauté cherchant à conserver son unité dans la foi ${ }^{2}$.

Le discours contre les scribes et les pharisiens témoigne d'un stade avancé du conflit entre l'église et la synagogue : le judaísme, qui forme désormais un front uni, est jugé et condamné sans nuance. La manière dont le chap. 23 parle des disciples est également suggestive. La règle communautaire des vv. 8-12 met les disciples en garde contre la tentation de revendiquer le titre de $\dot{\rho} \alpha \beta \iota^{3} \quad{ }^{3}$ : cet avertissement ne peut se comprendre que sur le fond de l'organisation ministérielle d'une église déjà structurée, où certains risquent de prendre la place du Christ. Semblablement, $l^{\prime}$ annonce du jugement (vv. $33-36$ ) dresse un catalogue des persécutions qui s'abattent sur les envoyés post-pascaux de Jésus 4. A travers les disciples, c'est en vérité l'église mt qui est interpellée.

L'apocalypse $\mathrm{mt}$, dans sa partie spécifiquement apocalyptique $(24,3-36)$, décrit le temps précédant immédiatement la parousie : ce

1 L'impact communautaire du chap. 18 est unanimement reconnu, cf. en dernier lieu : BORNKAMM, Lösegewalt 37 -45; PESCH, Seelsorger 68-76; TRILLING, Is rae1 106-123.

2 BORNKAMM (Lösegewalt 41), PESCH (Seelsorger 54-55), TRILLING (Israel 106-107) admettent que les $\mu \alpha \vartheta \eta \tau \alpha i ́$ sont ici clairement les représentants de l'église mt.

3 Voir notre analyse de 23,8-12, pp. 157-159.

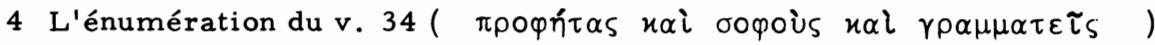
indique qu'il ne s'agit pas des compagnons historiques de Jésus, cf. nos pp. $155-156$. 
temps se caractérise par la persécution universelle des croyants $(24,9)$, par de graves crises internes à la communauté (apostasie, dénonciation réciproque, faux prophètes, faux messies, relâchement éthique, cf. $24,4-5,10-12 \cdot 23-28)^{l}$, par la prédication de l'évangile sur toute la terre $(24,14)$, par la profanation du Temple $(24,15-22)$. Pareille description reflète incontestablement la situation de la communauté ${ }^{2} \mathrm{mt}$. La parénèse qui forme la seconde partie du discours $(24,37 ; 25,46)$ indique comment l'église mt est appelée à vivre ce temps. Prenant acte du retard de la parousie, elle trace - par les paraboles eschatologiques le chemin d'une obéissance vigilante. Ces deux brèves observations suffisent à montrer que l'a pocalypse $\mathrm{mt}$ reflète une situation précise de la communauté $m$ t à laquelle elle tente d'apporter une réponse par le biais de la parénèse. Enseignement des disciples $(24,3 !)$ et enseigne ment de l'Eglise vont de pair.

En conclusion, dans les grands discours $\mathrm{mt}$, les disciples sont l'auditoire privilégié de Jésus. L'instruction qu'ils reçoivent concerne essentiellement le temps de l'Eglise. A travers le compagnon historique

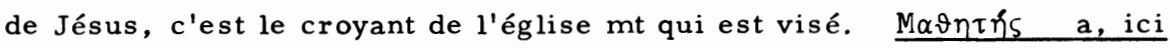
aussi, une valeur paradigmatique.

\section{La figure des disciples dans les débats d'école et les récits de}

\section{miracles.}

On pourrait s'étonner que nous abordions dans une même analyse les

1 On pourrait objecter que ce sont là des motifs apocalyptiques sans rapport nécessaire avec la situation de l'église $\mathrm{mt}$. A cela il faut répondre que la mise en garde contre les faux prophètes et le relâchement éthique occupent déjà une large place dans la parénèse finale du Sermon sur la Montagne - donc dans un contexte non apocalyptique -; il en va de même de la persécution dans Mt 10. Pour l'ensemble de cette question, voir notre chap. 7 .

2 Ainsi BORNKAMM, Enderwartung 19-20; GRUNDMANN, Mt 500-503; HAENCHEN, Weg 454. 
débats d'école et les récits de miracles. Cependant, comme l'a bien montré H.J. Held ${ }^{l}$, les récits de miracles chez Mt revêtent la forme du paradigme dont ils poursuivent par ailleurs l'intention. Il est donc justifié d'aborder simultanément ces deux formes.

$\mathrm{Si}$, dans les récits de controverses, les disciples étaient avant tout des accusés mis en demeure de justifier leur conduite, si, dans les grands discours, ils étaient les auditeurs privilégiés d'un enseignement destiné au temps de l'Eglise, dans les débats d'école et les récits de miracles, leur rôle change. C'est leur destinée comme telle qui est envisagée et interprétée.

Il nous semble judicieux de traiter cette abondante matière en deux temps : a) la destinée des disciples (le disciple comme tel est le sujet du récit), b) la compréhension du disciple (la situation du disciple apparaît de manière incidente, mais significative dans la narration).

\section{a) La destinée du disciple :}

Un premier groupe de récits est constitué par l'histoire de la tempête apaisée $\frac{(8,18-27)}{2}$ et par celle de Jésus et Pierre marchant sur les eaux $(\underline{14,22-33})^{2}$. Du point de vue de la critique littéraire, $\mu \alpha \vartheta \eta \eta \hat{n}$ en $8,21.23$ et 14,26 ressortit à la rédaction, tandis qu'en 14,22 le terme provient de Mc. Pour le fond, on remarquera que les deux récits se jouent exclusivement entre Jésus et les disciples. Leur but est d'expliciter la nature de la Nachfolge et, plus particulièrement encore, de dénon-

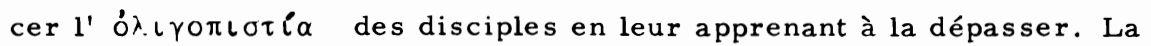
métaphore du bateau menacé figurant l'Eglise sous la croix de même que

1 HELD 229-234.

2 Notre exégèse de ces deux récits s'inspire avant tout de : BORNKAMM, Sturmstillung 48-53; HELD 189-195;253-256;259-260. Voir aussi les analyses détaillées dans : ZUMSTEIN, Maître et disciple 189-205. 


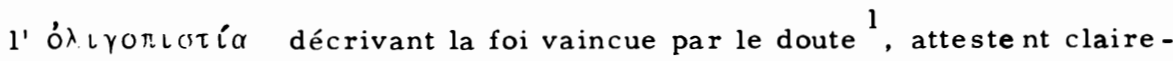
ment la signification paradigmatique des deux récits, et, par conséquent, le rôle typologique des disciples. L'aventure vécue naguère par les compagnons de Jésus devient le modèle de la Nachfolge placée sous la promesse, mais menacée par la tentation. De ces deux récits, mais surtout de $8,18-22$, on peut rapprocher $16,24-28$, péricope énonçant les exigences de la Nachfolge. A la différence de Mc, mais aussi de Lc, et en vertu d'une correction significat ive, elle n'est adressée qu'aux disciples. Seuls ces derniers se voient annoncer le sens de la Nachfolge. Mais

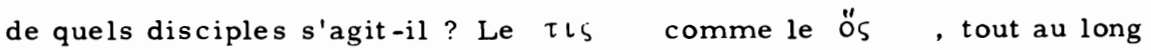
de l'instruction de Jésus, désignent le disciple comme tel, sans limitation d'aucune sorte et, donc, en définitive le croyant. Le v. $27^{2}$ atteste d'ailleurs qu'il s'agit d'un enseignement valable jusqu'à la parousie. Dans le même ordre d'idée, on évoquera 10,24-25 (pour $\mu \alpha \vartheta \eta \tau n ́ s: 10,24=$ $Q, 10,25$ = rédaction) : le contexte $(10,17-23)$, et notamment le v. 23 , montre clairement que ce texte traite de l'existence du disciple postpascal en butte à la persécution. Le singulier à valeur typologique de $\mu \alpha \vartheta \eta \tau$ ńs dans les vv. 24-25 confirme cette observation. Ce qui est dit du disciple ici concerne la similitude de sa condition avec celle du maître, similitude résidant dans leur commune destinée de souffrance (cf. v. 25b).

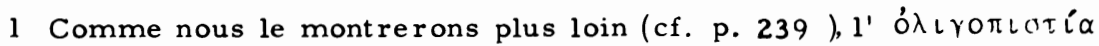
est une catégorie post-pascale. Elle pose le problème de la foi à une communauté déjà "installée" et dont le problème majeur n'est pas la conversion, mais la persévérance et la fidélité dans la vie chrétienne. Semblablement, le scandale du doute ne prend toute son ampleur que dans le cadre de l'existence croyante.

2 La validité jusqu'à la parousie des exigences liées à la Nachfolge est attestée par le fait que le Fils de l'homme revenant en gloire pour le jugement demandera compte à chacun $(16,27 \mathrm{~b}:$ : $\alpha \grave{i} \tau \delta \dot{\tau \varepsilon} \dot{\alpha} \pi 0 \delta \hat{\omega}-$

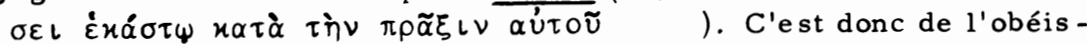
sance comme telle du croyant dans la perspective du jugement selon les oeuvres qu'il est question dans 16,24-28 (cf. BARTH 88; BORNKAMM, Enderwartung 45). 
Un deuxième groupe de textes aborde le thème de l'impuissance du disciple dans l'exercice de sa mission ${ }^{l}$. L'histoire de la guérison de l'enfant épileptique $(17,14-20)$ est devenue dans la rédaction mt un paradigme consacré à ce motif. Si les deux mentions de $\mu \alpha \vartheta \eta \tau$ ńs (vv.16. 19) sont empruntées à $\mathrm{Mc}$, on remarquera l'accent porté par Mt sur leur échec et surtout l'interprétation originale qu'il en donne : seule l'ó $\lambda \iota \gamma o-$ $\pi \iota \circlearrowleft \tau i ́ \alpha$ a empêché les disciples de remplir leur office, car à la foi, si humble soit-elle, tout est possible. On retrouve la même problématique

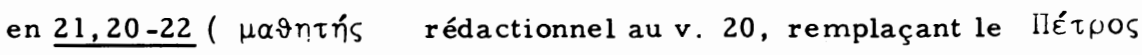

de Mc) : seule $1^{\prime} \dot{\partial} \lambda \iota \gamma \circ \pi \iota \sigma \tau i ́ \alpha$ empêche les disciples d'accomplir les actes merveilleux promis à la foi. Le point central de ces deux passages est de nouveau l'ó $\lambda \iota \gamma \circ \pi \iota \sigma \tau i \alpha$ qui envahit les disciples et dont nous avons dit qu'elle était une catégorie post-pascale. Elle se manifeste dans ce cas particulier par l'impuissance.

Un troisième groupe de textes traite des dangers et de la promesse attachée à la Nachfolge. Le débat d'école (19,23-30) qui suit l'histoire du jeune homme riche est très instructif à cet égard ( $\mu \alpha \vartheta \eta \tau$ ńs : v. 23, source Mc; v. 25 : adjonction $\mathrm{mt}$ ). Deux détails méritent d'être relevés : le sens de la scène qui vient de se dérouler, à savoir le danger que la richesse représente pour la Nachfolge, est expliqué aux seuls disciples ${ }^{2}$. Or ces derniers se sentent concernés par cet avertissement du Christ mt : cela s'accorde mal avec ce que nous savons des compagnons du Jésus historique, qui étaient pauvres, et renvoie sans doute à un auditoire plus large. Cette impression est confirmée par la forme que le Christ mt donne à la promesse récompensant la fidélité dans la Nachfolge : si, dans un premier temps, seuls les douze sont visés (v. 28), il est aussitôt

1 Il s'agit de I'impuissance du disciple à accomplir les actes de guérison dont l'a chargé le maitre. Sur ce problème particulier de l'église mt, voir: HELD 177-182; LUZ, Jünger 154-156. Nous avons donné une étude détaillée de ce thème dans : Maître et disciple 274-289.

2 Ainsi déjà $\mathrm{Mc}$ (cf. 10,23a). 


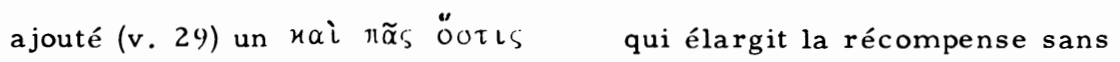
limitation d'aucune sorte et autorise à voir dans la Nachfolge des disciples une destinée exemplaire. Le logion de $\underline{10,42}$, en tant qu'il exprime la bénédiction attachée à l'accueil d'un disciple, peut être placé dans le même contexte. Il est, cependant, d'un intérêt capit al en vertu de la correction qu'il opère sur sa source Mc 9,41 : alors que Mc a év óvó-

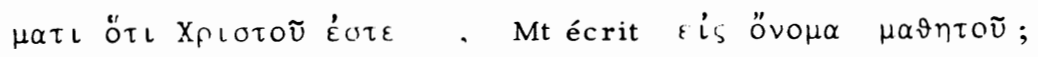
cela signifie : pour Mt, être chrétien ou être disciple est équivalent. Notre thèse sur le rôle typologique des disciples est donc confirmée.

Il faut enfin évoquer quelques textes fort importants, mais plus isolés thématiquement dans la forme littéraire que nous traitons ici. La préface du discours d'envoi (9,35-38) se termine par une parole adressée aux disciples (adjonction rédactionnelle introduisant $Q$ ) : les disciples sont les chargés de mission du Christ, comme le confirmera le chap. 10 (cf. v. $38: \delta \varepsilon \eta ́ \vartheta \eta \tau \varepsilon)$. Le paradigme de 12,46-50 est aussi fort suggestif : par la correction qu'il opère sur la source Mc au v. 49 ( Toù

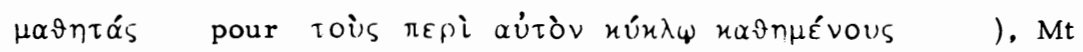
définit le disciple comme étant celui qui fait la volonté de Dieu. Cette assertion s'entend évidemment du disciple en tant que modèle du croyant. Les deux multiplications des pains, enfin (14,13-21 et 15,32-39) l' accentuent la fonction médiatrice des disciples entre la foule et Jésus $(14,15$. $19^{1}$ et $15,32.33 .36^{1}$ proviennent de $\mathrm{Mc}$, mais $14,19^{2}$ et $15,36^{2}$ sont rédactionnels :). Leur participation à la toute-puissance de Jésus au-

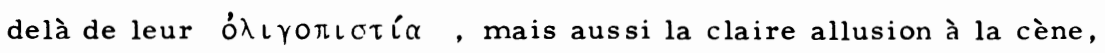
donnent à nouveau au rôle des disciples une signification exemplaire .

Concluons : le rapide survol des débats d'école et des récits de miracles où les disciples jouent un rôle constitutif, nous amène à la constatation suivante : dans ces péricopes, la destinée des disciples du Jésus terrestre est explicitée de manière exemplaire si bien qu'elle devient le paradigme de l'existence croyante.

1 Cf. HELD $171-177$. 


\section{b) La compréhension des disciples :}

Dans une deuxième grande série de débats d'école, nous passerons en revue le rôle des disciples, parfois incident, parfois constitutif, un rôle qui se rapporte moins à la Nachfolge et plus à la compréhension que les disciples ont de la destinée de leur maitre. Nous avons déjà eu l'occasion de signaler l'importante place que Mt ménage à sa réflexion sur la "compréhension" des disciples, et nous avons indiqué pourquoi, à notre avis, cette thématique était typiquement post-pascale ${ }^{l}$.

La péricope de $\underline{13,10-17}$ est capitale dans l'économie du discours en paraboles. Elle nous présente les disciples $(13,10$ : adjonction rédactionnelle) comme les bénéficiaires compréhensifs de la prédication de Jésus. La compréhension qui leur est gracieusement accordée par opposition aux autres hommes, ne se réduit pas à la fonction de témoin oculaire (voir la signification de $\beta \lambda \varepsilon \tilde{\pi} \omega$ et ákoúw dans les vv. 10-17 et la correction rédactionnelle en ö ఒ du v. 16); c'est l'intelligence spirituelle conférée à tout croyant, impliquant un bonheur immédiat (vv. 16-17) et porteuse du salut eschatologique (v. 12). De ce passage, il convient de rapprocher l'explication de la parabole de l'ivraie (13,36-43) où les disciples (v. 36 rédactionnel) reçoivent de nouveau à l'exclusion des foules le sens des propos du Christ. L'empreinte post-pascale - et par conséquent le rôle typologique que jouent les disciples - est de nouveau claire : il leur est donné de comprendre que l'Eglise, "corpus mixtum", est en route vers le jugement. Cette insistance toute $\mathrm{mt}$ sur la compréhension des disciples se retrouve dans le passage parlant "du levain des pharisiens et des sadducéens" (16,5-12). Les disciples (adjonction rédactionnelle, v. 5) sont éduqués au-delà de leur peu de foi (vv. 7 -8) à discerner le danger de l'enseignement des pharisiens (v. 12 : $\tau \delta \tau \varepsilon$ ouv $\tilde{x} \alpha \nu$ !). Nous rencontrons le même écho dans l'instruction sur le retour d'Elie $(17,10-13)$ : les disciples (les 2 mentions des vv. 10 et 13 sont rédactionnelles) reçoivent de manière compréhensive la juste

1 Cf. nos pp. 34-35. 
intelligence de l'histoire du salut.

A cette connaissance de la prédication du Christ, de la doctrine des adversaires ou de la place de Jean-Baptiste s'ajoute une connaissance plus fondamentale encore qui est la connaissance de la véritable identité du Christ mt. La confession de Pierre à Césarée de Philippe (16, 13 -20) nous montre comment les disciples (mention du v. 13 empruntée à Mc, celle du v. 20 est rédactionnelle), questionnés par Jésus à propos du Fils de l'homme, confessent par la voix de Pierre sa véritable identité ! Comme en 13,10 (cf. 16,17), cette connaissance est une connaissance reçue. Fait capital, comme le montrent les vv. 18-19, elle devient le fondement sur lequel l'Eglise s'édifie. A nouveau, la condition des disciples et la réalité de l'Eglise sont étroitement imbriquées. La première annonce de la Passion qui suit immédiatement $(\underline{16,21-23})$ reste dans la même ligne : il est donné aux disciples (la mention du v. 21 est rédactionnelle) de découvrir l'avenir vers lequel s'avance leur maître - sa passion et sa résurrection -, même s'ils se méprennent sur le rôle qu'il leur faudra jouer ( $v v .22-23$ ). Le récit de la transfiguration enfin (17,1-9) poursuit le développement amorcé avec la confession de Césarée de Philippe : les disciples (v. 6 : mention rédactionnelle) sont les témoins de l'épiphanie du Christ qu'ils accueillent positivement - au contraire de Mc (suppression de Mc 9,6-10) -.

Conclusion : Ce rapide survol des débats d'école et des récits de miracles axés sur la compréhension des disciples nous montre que, pour Mt, le disciple est fondamentalement celui qui reçoit une juste intelligence de la prédication, de la destinée et de l'identité du Christ. De

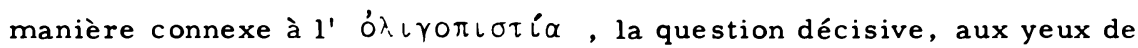
Mt, n'est pas celle de la conversion, voire de la foi, mais de la juste intelligence du Jésus terrestre. Ce souci d'une saisie correcte du Jésus terrestre prend tout son poids durant le temps de l'Eglise. Le disciple de naguère devient ainsi le modèle du croyant actuel. 


\section{La figure des disciples dans l'histoire de la Passion et de la}

\section{Résurrection.}

Dans notre étude consacrée au rôle des adversaires de Jésus dans la Passion, nous remarquerons que l'intérêt typologique de Mt fort perceptible jusque -là (inscription du conflit Eglise-judaisme dans la vie de Jésus) cède le pas devant une intention plus historicisante ${ }^{1}$. Le résultat de l'analyse de la figure des disciples dans la Passion n'est pas aussi net; il faut néanmoins reconnaître qu'une partie des passages entrant dans l'enquête n'ont plus une signification paradigmatique. Ainsi le récit de l'onction à Béthanie (26,6-13) où les disciples $(26,8$ : élucidation rédactionnelle de $\mathrm{Mc}$ ) jouent avant tout le rôle de ressort dramatique, malgré le v. 11 qui leur accorde un avenir dépassant celui de Jésus. Semblable ment, la préparation de la Pâque (26,17-20) leur attribue (les mentions des vv. 17.18.19 sont reprises de Mc alors que celle du v. 20 est rédactionnelle - texte incertain !) une fonction essentiellement anecdotique. Les deux textes apologétiques $(27,62-66$ et $28,11-15)$ font jouer aux disciples $(27,64$ et $28,13: S)$ un rôle analogue à celui qu'ils avaient dans les récits de controverses. Ils sont pris à parti par la polémique juive qui vise à les faire passer non plus pour des transgresseurs de la Loi, mais pour des imposteurs. La polémique est certes encore actuelle (cf. 28, 15b), mais elle ne s'applique pas directement aux croyants de l'église mt; elle concerne plutôt leurs prédécesseurs dans la foi. L'ins titution de la Cène (26,26-29) est un cas à part : elle a certes une actualité ecclésiale immédiate, mais c'est son caractère de "légende culturelle" ${ }^{2}$ qui lui confère sa portée exemplaire. De ce point de vue, les disciples $(26,26$ : élucidation rédactionnelle de Mc) occupent une place paradigmatique : ce que Jésus leur accorda naguère est accordé à tout croyant.

1 Cf. notre chap. 2, pp. 68-75.

2 BULTMANN, Trad 285. 
Par contre, toute une série de textes reprend la figure du disciple telle que les récits d'avant la Passion nous avaient appris à la connaître. Les deux motifs retravaillés sont la compréhension du disciple et son échec. La péricope de 26,1-5 (composition originale de Mt sur la base de Mc 14, 1-2) ouvre le récit de la Passion. Elle contient une déclaration de Jésus aux disciples $(26,1$ : rédactionnel) qui leur dévoile ce qui va arriver. Les disciples s'avancent dans la passion comme ceux qui savent $(26,2)$ : ils savent que Jésus est le Fils de l'homme et qu'il va être crucifié. Nous sommes en situation d'interprétation théologique post-pascale : les disciples sont déjà placés dans la perspective de la confession de foi communautaire. Avec l'annonce du reniement de Pierre $(26,30-35) \mathrm{com}$ mence la description de l'échec des disciples, de leur infidélité. Ce récit nous montre que Pierre et les disciples $(26,35$ élucidation rédactionnelle) sont dans la même condition (remarquer l'alternance entre le $\dot{u} \mu \varepsilon \tau_{\zeta}$

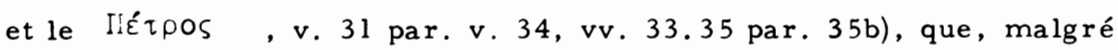
leur prétention, l'épreuve du maître est un scandale qui va briser leur fidélité. La scène de Gethsémané $(26,36-46)$ poursuit la réflexion amor cée en 26,30-35 (v. 36 : source Mc; vv. 40.45: adjonctions rédactionnelles) : les disciples sont incapables d'assister leur maître à l'heure du danger; ils succombent à la tentation. Remarquons que Mt fait alterner indifféremment $\mu \alpha \vartheta \eta \tau \alpha i$, le groupe des trois et Pierre (comparer les vv. 36.37.40 !). L'arrestation de Jésus (26,47-56) mène ce motif de l'échece à chef : les disciples $(26,56$ adjonction rédactionnelle) abandonnent Jésus à son sort, ils le renient. Le v. 56b qui décrit la fuite de tous les disciples fait pendant au reniement de Pierre (cf. 26,69-75).

Le rôle des disciples dans le cycle mt de la Résurrection est maigre. Dans le récit du tombeau vide (28,1-8), ils sont réduits (v. 7 : source $\mathrm{Mc}$; v. 8 : rédactionnel ou $S$ ) à être les auditeurs du témoignage des femmes. Par contre, le manifeste $(28,16-20)^{1}$ a une importance capitale pour notre propos. Les disciples (v. 16 rédaction ?) sont présentés dans la

1 Voir notre exégèse de $28,16-20$ au chap. 3. 
situation typique du doute et de l'adoration. L'instruction que leur donne le Ressuscité a l'accent suivant : eux, les onze, sont chargés d'appeler tout homme à la condition de disciple (v. $19: \mu \alpha \vartheta \eta \tau \varepsilon u ́ \sigma \alpha \tau \varepsilon$ ) et ceci jusqu'à la parousie. Or la condition de disciple ne consiste en rien d'autre qu'à "garder" l'enseignement normatif du Christ tel que l'évangile vient de le rappeler. Les croyants sont donc installés dans la condition même qui fut celle des compagnons de Jésus. L'usage typologique de $\mu \alpha \vartheta \eta-$ $\tau$ ńs reçoit ici son ultime confirmation et son fondement christologique.

\section{Conclusion}

Le terme $\mu \alpha \vartheta \eta \tau n ́ s$ apparaît de manière constante et uniforme tout au long de l'évangile. Cet emploi répété est dû dans la majorité des cas au rédacteur lui-même. C'est dire que nous découvrons ici un élément perceptible et cohérent de la rédaction matthéenne et qu'il convient de s'interroger sur le sens de ce procédé littéraire.

Les disciples forment un groupe homogène et indifférencié, ils sont clairement distingués de la foule. La tendance de la description est la stylisation plutôt que l'historicisation. Plus fondamentalement, à chaque forme littéraire correspond une fonction particulière des disciples. Dans les récits de controverses, ils sont accusés par la synagogue et défendus par Jésus. Dans les grands discours, ils sont les auditeurs privilégiés et compréhensifs du didascale eschatologique. Dans les débats d'école et les récits de miracles, c'est leur destinée comme telle qui est abordée. Mais au-delà de cette pluralité de perspectives, une constante se dessine. Le groupe des disciples ne désigne pas seulement les compagnons historiques du Jésus terrestre, il représente également la communauté des croyants. Le terme $\mu \alpha \vartheta \eta \tau \eta \dot{s}$ est donc une catégorie littéraire $\underline{\text { ambivalente }}$ : à la fois dénomination des adhérents du Jésus terrestre et 
concept ecclésiologique ${ }^{l}$.

De cette ambivalence du terme $\mu \alpha \vartheta \eta \tau \dot{n}$, ambivalence qui n'est sans doute pas une création matthéenne ${ }^{2}$, mais qui est élevée dans le premier évangile au rang d'une structure constitutive du récit, nous re tiendrons tout d'abord la signification littéraire. Par ce biais, la narration s'ouvre sans cesse sur l'auditoire auquel elle s'adresse. Le passé devient transparent pour le présent. L'église matthéenne est directement impliquée dans l'histoire qu'elle se remémore.

Cette structure littéraire n'est pourtant pas sans soulever un problème théologique décisif. L'ambivalence de la catégorie $\mu \alpha \vartheta \eta \tau$ ńs présuppose une imbrication mutuelle du temps de Jésus et du temps de l'Eglise. Comment cette imbrication est-elle possible ? ou, en d'autres termes, comment les facteurs d'historicité et de transparence s'articulent-ils dans le concept $\mu \alpha \vartheta \eta \tau n ́$ ? La solution à cette question qui est au coeur même de l'esquisse kérygmatique conçue par Matthieu, doit être cherchée dans la christologie du premier évangile. C'est l'objet de notre deuxième partie.

1 Bon état de la recherche chez LUZ, Jünger 159 : "Die Jünger Jesu sind für die Gegenwart transparent. Hinter ihnen erscheint die matthăische

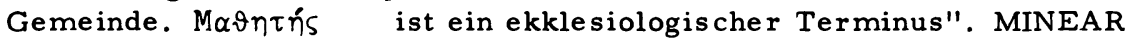
(en particulier 76-79) en limitant l'application du paradigme aux chefs de l'Eglise, ne nous semble pas tenir compte du corpus $m$ t dans son ensemble.

2 Sur la signification ambivalente de $\mu \alpha \vartheta \eta \tau \dot{n} s$ dans la tradition chrétienne primitive, voir LUZ, Jünger 165-171. 
A côté des disciples, un second groupe entoure constamment Jésus dans le premier évangile : ce sont ses adversaires. Il convient de diviser l'étude des adversaires de Jésus chez Mt en deux parties :(A) les adversaires de Jésus avant la Passion, (B) les adversaires de Jésus durant la Passion. Cette distinction est recommandée par le texte lui-même : les adversaires de Jésus avant et durant la Passion ne sont pas les mêmes. Ce phénomène se comprend d'autant mieux que le récit de la Passion était beaucoup plus élaboré dans son cadre que le reste de la tradition synoptique ${ }^{1}$. C'est dire à l'inverse que l'activité rédactionnelle de Mt se fera plus sentir dans les chap. $1-25$.

\section{A. Les adversaires de Jésus avant la Passion (chap. 1-25)}

Trois principaux groupes d'adversaires de Jésus apparaissent dans l'évangile avant la Passion : les pharisiens, les sadducéens et les scribes. Ils ne se présentent pas sous une forme monolithique, mais dans des combinaisons variables.

1) $\varphi \alpha \rho \iota \subset \alpha \tau o \iota$ apparait sous 4 formes :

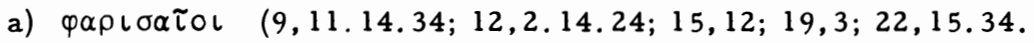
$41 ; 23,26)$, soit 12 fois.

b) $\varphi \alpha \rho \iota \sigma \alpha \tau o l \quad x \alpha i \quad \gamma \rho \alpha \mu \mu \alpha \tau \varepsilon \tau \varsigma \quad(5,20 ; 12,38 ; 15,1 ; 23,2.13$. 15.23.25.27.29), s oit 10 fois.

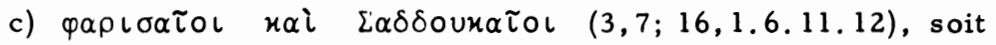
5 fois.

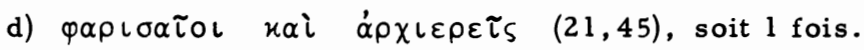

2) $\Sigma \alpha \delta \delta$ ouk $\alpha$ โol apparait sous 2 formes :

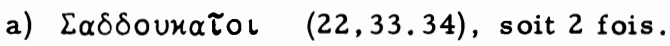

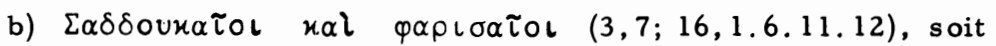
5 fois.

1 DIBELIUS, Formgeschichte 21. 
3) $\gamma \rho \alpha \mu \mu \alpha \tau \varepsilon \cup ́ s$ apparait sous 4 formes :

a) $\gamma \rho \alpha \mu \mu \alpha \tau \varepsilon \cup ́ s \quad(7,29 ; 8,19 ; 9,3 ; 13,52 ; 17,20 ; 23,34)$, soit 6 fois.

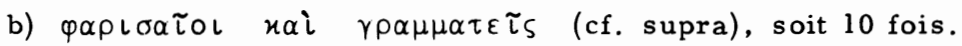

c) $\dot{\alpha} \rho \chi\llcorner\varepsilon \rho \varepsilon \tau \varsigma \quad x \alpha i \quad \gamma \rho \alpha \mu \mu \alpha \tau \varepsilon \tau \varsigma \quad(2,4 ; 20,18 ; 21,15)$, soit 3 fois.

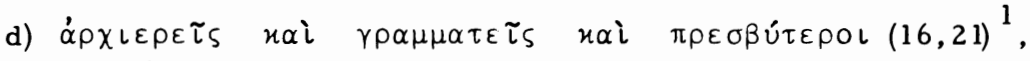
soit 1 fois.

L'inventaire des adversaires de Jésus que nous venons de dresser, nous révèle une forte prédominance des pharisiens (28 attestations) et des scribes (20 attestations). Les sadducéens ( 7 attestations) ont une place à part. Les grands-prêtres (6 attestations), mais surtout les anciens (2 attestations) n'interviennent qu'associés à d'autres groupes; c'est dire qu'ils ont un rôle mineur, voire insignifiant ${ }^{2}$.

Nous ne pouvons certes pas baser notre analyse sur la seule enquête statistique. Ce qui, pour notre sujet, nous semble d'un grand intérêt, c'est que les résultats de notre première démarche sont confirmés par la critique des sources : les groupes statistiquement prédominants sont précisément ceux qui portent une forte empreinte rédactionnelle. Neuf

1 Toutes les expressions apparaissent exclusivement dans les chap. 1-25,

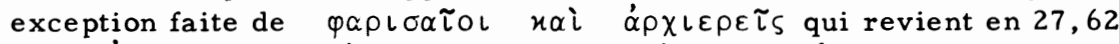

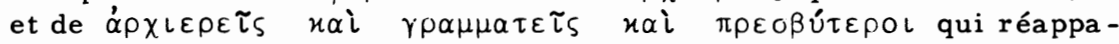
raît en 27,41 . Seule constellation non enregistrée et qui apparaît dans

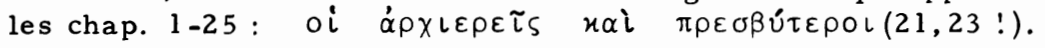

2 Cette répartition des adversaires de Jésus est bien évidemment tributaire des sources utilisées par Mt. Pourtant la seule statistique nous révèle déjà un profil différent de celui de $M c$ surtout, mais aussi de celui de Lc. Ainsi :

- $\varphi \alpha \rho\llcorner\sigma \alpha$ Los apparait 28 fois chez Mt, 12 fois chez Mc, 12 fois chez Lc;

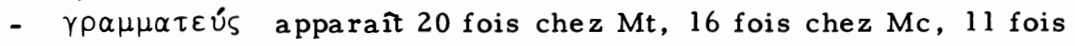
chez Lc;

- $\sum \alpha \delta \delta$ oux $\alpha$ Ios apparait 7 fois chez Mt, 1 fois chez Mc, 1 fois chez Lc;

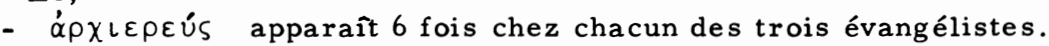

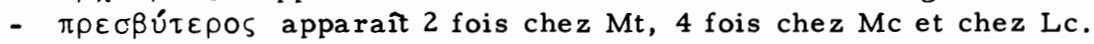


des douze emplois de $\varphi \alpha \rho \iota \sigma \alpha \tau o s$ utilisé seul sont rédactionnels, soit par correction, soit par adjonction. Les expressions $\gamma \rho \alpha \mu \mu \alpha \tau \varepsilon \tau_{\zeta} \quad x \alpha i$

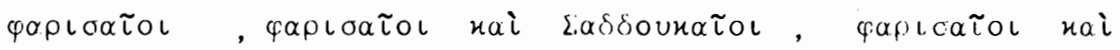
$\dot{\alpha} \rho \chi \iota \varepsilon \rho \varepsilon \tau_{\zeta}$ sont des expressions rédactionnelles; elles n'apparaissent ni chez Mc, ni chez Lc. Гم $\alpha \mu \mu \alpha \tau \varepsilon u ́ s$ et ses constellations sont moins caractéristiques : certes quatre emplois sur six de $\gamma \rho \alpha \mu \mu \alpha \tau \varepsilon u ́ S$ utilisé seul remontent soit à la rédaction, soit au Smt, mais les expres -

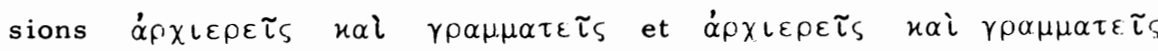

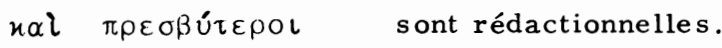

Ce rapide survol des chap. 1 -25 nous apprend que les pharisiens et, en second lieu, les scribes - sont les adversai res par excellence de Jésus. Cette répartition des antagonistes du Christ ne se trouve que chez Mt. Elle appelle la question suivante : si Matthieu compose un nouveau cadre pour son évangile, le fait-il dans une intention précise ? Que signifie cette description originale des groupes hostiles à Jésus ? Nous tenterons de résoudre ce problème en analysant les différentes constellations recensées plus haut.

\section{Les pharisiens seuls ou associés à d'autres groupes.}

Seuls ou associés à d'autres groupes, les pharisiens sont par excellence, dans l'évangile selon $\mathrm{Mt}$, les adversaires de Jésus ${ }^{1}$. Par l'analyse des constellations enregistrées, cherchons à déterminer les caractéris tiques $\mathrm{mt}$ de ce groupe.

a) oi $\varphi \alpha \rho \iota \sigma \alpha \tau \circ \iota^{2}$

La critique des sources nous permet de classer les douze emplois de oi $\varphi \alpha \rho \iota \sigma \alpha \tau o l$ entrois groupes. Dans le premier groupe, on mettra

1 WALKER 22 ne partage pas cette opinion. En cela, il s'oppose à la critique unanime (cf. GLASSON 317; HUMMEL 13; KILPATRICK 106; TRILLING, Israel 90) et surtout l'état du texte de Mt !

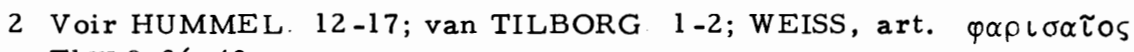
ThW 9, 36-40. 
les trois emplois traditionnels, c'est-à-dire les trois mentions que $\mathrm{Mt}$ a trouvées dans ses sources : 12,2 par. Mc 2,24; 19,3 par. Mc 10,2; 23, 26 expression empruntée à $Q m t$. Dans le deuxième groupe, on range ra les deux adjonctions rédactionnelles, plus précisément les deux mentions de oi $p \alpha \rho ı \alpha$ iol que Mt introduit dans sa source sans, par ailleurs, la modifier $(15,12 ; 22,15)$. Dans le troisième groupe - de loin le plus nombreux - on classera les sept corrections rédactionnelles. Par correction rédactionnelle, nous entendons soit que Mt supprime d'autres protagonistes pour laisser le champ libre aux seuls pharisiens (9,11.14; $12,14)$, soit que Mt substitue les pharisiens à d'autres groupes ( 9,34 ; $12,24 ; 22,34.41)$. Les acteurs qui s'effacent au profit des pharisiens sont les disciples des pharisiens $(9,14)$, les Hérodiens $(12,14)$, la foule $(22,41)$, mais surtout les scribes $(9,11.34 ; 12,24 ; 22,34$; voir aussi 15 , 12 où les seuls pharisiens succèdent aux "pharisiens et aux scribes" de 15,1). En résumé et d'un seul point de vue littéraire, Mt a voué une at tention particuli ère au groupe des pharisiens. Il les a introduits aussi souvent que possible dans son propos ${ }^{l}$ aux dépens d'autres groupes - le plus souvent les scribes -. Si nous envisageons le contexte, le groupe des seuls pharisiens n'apparaît que dans la section qui va du chap, 9 au chap. 23, c'est-à-dire précisément là où l'affrontement avec le judaîsme au sujet de la prédication et de l'oeuvre du Christ est le plus violent. Cette constatation générale est confirmée par l'analyse des formes littéraires dans lesquelles les pharisiens interviennent. Dix fois sur douze, il s'agit de récits de controverses $(9,11.14 ; 12,2.14 .24 ; 15,12 ; 19,3 ; 22,15.34$. 41); les deux derniers cas $(22,34.41)$ sont particulièrement suggestifs : du fait que Mt y a introduit les pharisiens, ces deux scènes qui étaient à l'origine des débats d'école, se sont transformées en récits de controverses. En 9,34 et en 23,26, nous n'avons certes pas affaire à des récits de controverses, mais l'attitude des pharisiens n'en est pas moins hostile : ils accusent Jésus d'être un démoniaque $(9,34)$, ils sont objet de

1 HUMMEL 13. 
malédiction $(23,26)$. Notre hypothèse de départ se trouve donc confirmée : choisis et introduits avec un soin particulier par Mt, les pharisiens le sont pour être par excellence les ennemis de Jésus ${ }^{1}$.

La place prépondérante que les pharisiens occupent comme adver saire de Jésus dans les récits de controverses, nous permet de découvrir l'image que Mt se fait d'eux. Les pharisiens apparaissent en effet comme les représentants privilégiés et les défenseurs compétents de la foi juive. Ce sont eux qui accusent Jésus de se souiller avec le am ha -aretz $(9,11)$, de violer le jeûne $(9,14)$, le sabbat $(12,2,14)$, les ablutions rituelles $(15,12)$. Ce sont eux qui débattent avec Jésus du divorce $(19,3)$, de l'impôt $(22,15)$ et du plus grand commandement $(22,34)$. Ce sont eux que Jésus interroge au sujet du Christ $(22,41)$. Aux yeux de Mt, les pharisiens occupent une place-clef dans le judaisme, ils en sont le porte-parole qualifié, ils en défendent l'orthodoxie face à Jésus. Si l'on ajoute à ce fait que Mt présente les pharisiens comme un groupe uni et homogène, il faut bien admettre que ce portrait ne correspond plus ni à la place, ni aux caractéristiques du groupe des pharisiens au temps de Jésus (les pharisiens du début du ler s. n'ont pas encore pris en main les destinées du judaìsme, de plus ils sont fort divisés entre eux). Mt ne connaît donc plus les particularités historiques des pharisiens contemporains de Jésus, il en donne une description anachronique ${ }^{2}$.

Le jugement que le Christ mt porte sur les pharisiens est accablant. Les pharisiens se prétendent les dépositaires et les défenseurs de la tradition des anciens (15,2-3); Ils défendent la conception juive de la Loi. Pourtant, de l'avis du Christ mt, ils ne connaissent justement pas la volonté de Dieu $(9,13 ; 12,3.5 .7 ; 19,4)$. Cette ignorance n'est pas accidentelle, elle est l'indice d'une profonde culpabilité, car les pharisiens sont

1 HUMMEL 14; KILPATRICK 106.121; TRILLING, Israel 90; WEISS, art. cit. 36.

2 KILPATRICK 101; TRILLING, Israel 90; WEISS, art. cit. 36-38. 
hypocrites ( inouflıń; : 15,7-9;22,18;23,26), aveugles ( $\tau \cup \varphi \lambda \delta_{S}$ :

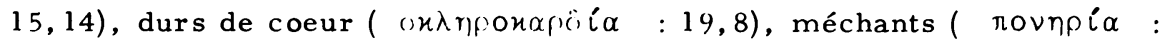
$22,18)$. C'est pourquoi ils essaient de tenter Jésus ( $\pi \varepsilon \iota \rho \alpha ́ \alpha \zeta \omega: 19,3$; $22,18 ; 22,35)$, de le prendre au piège $(22,15)$, de l'accuser ( Ka tทro$\rho \varepsilon ́ \omega: 12,10)$. Scandalisés par sa prédication $(\sigma \mathcal{K} \alpha \nu \delta \alpha \lambda i \zeta(u): 15,12)$, mais aussi par ses miracles, ils l'accusent d'être un démoniaque $(9,34 ; 12,24)$ et finalement décident de le faire périr $(12,4)$. Cet endurcissement cons tant et criminel fait qu'ils sont déjà jugés $(12,24 ; 15,12-14 ; 23,26)$. D'un point de vue historique, cette condamnation radicale et sans nuance s'accommode mal de ce que nous connaissons du ministère de Jésus (exemple : Mc 12,28-34!); elle trahit une situation ultérieure où les fronts se sont durcis.

Concluons : Mt a délibérément choisi les pharisiens pour en faire par excellence les ennemis de Jésus. Il leur attribue un rôle directeur qui ne correspond pas aux conditions historiques qui prévalaient au temps de Jésus ${ }^{l}$. Si notre description est exacte, elle pose un problème sur lequel nous devrons revenir : pourquoi Matthieu donne-t-il l'image d'un pharisaísme violemment et massivement hostile à Jésus, uni en son sein et apte à représenter, voire à défendre la foi juive?

1 Parlant de la description $m$ tes pharisiens, van TILBORG écrit : "The literary character of this name is apparent in (nearly) all the texts" (1). STRECKER va dans la même direction en écrivant "dass Matthäus den Pharisaismus schematisch, als Antitypus darstellt" (Weg 140). TRILLING, lui, conclut à une "Typisierung" dépourvue de tout relief historique (Israel 90). Si nous tombons d'accord avec ces auteurs pour discerner dans l'image mt des pharisiens une composition littéraire, témoignant de schématisme, conduisant à la typicisa tion, nous pensons que cette constatation est insuffisante. Elle rejaillit en effet dans la problématique suivante : pourquoi $\mathrm{Mt}$ a-t-il schématisé de cette manière et pas d'une autre ? quels sont les présupposés de cette composition littéraire? 


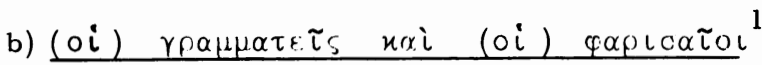

La critique des sources nous permet de constater que Mt associe avant tout les pharisiens aux scribes ( 10 fois). Pour attester cette allian-

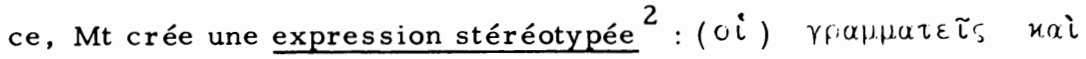

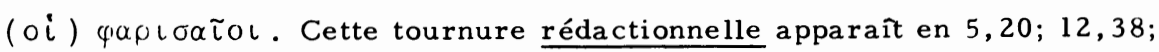
$23,2.13 .15 .23,25.27$. Seule exception : 15,1 qui est d'origine traditionnelle (voir le par. Mc 7,1), mais dont l'ordre de présentation est préci-

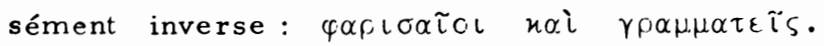

Si l'on examine les formes littéraires dans lesquelles apparaît notre expression, nous remarquons de nouveau une forte tendance à la polémi que. Excepté 5,20 qui fait partie d'une règle communautaire (mais où notre tournure joue le rôle de pôle négatif !), 12,38 et 15,1 appartiennent à des récits de controverses tandis que 23,2 13.15.23.25.27.29 ressortissent au grand discours dirigé précisément contre les scribes et les pharisiens (13.15.23.25.27.29 sont les destinataires des malédictions !). Expression rédactionnelle de Mt, les "scribes et les pharisiens" sont, à l'image des "pharisiens", intrinsèquement les ennemis de Jésus.

Pour ce qui est de l'image historique, il convient de se demander si le portrait que Mt trace des "scribes et des pharisiens" a une quelconque pertinence. De manière analogue aux "pharisiens", les "scribes et les pharisiens" sont les représentants du judaiisme dans son ensemble ${ }^{3}$ : en 5,20 , les "scribes et les pharisiens" sont représentatifs de tout Israël; semblablement le chap. 23 juge tout le judaiisme (voir surtout la conclusion du discours). Cette fonction représentative des "scribes et des pharisiens" est identique à celle des seuls pharisiens, si bien que Mt peut faire alterner l'une et l'autre expression sans difficulté (cf. 12,24.38;

1 HUMMEL 14-15; van TILBORG 3-4; WALKER 17-23; WEISS, art. cit.

2 Avec HUMMEL 14; WALKER 17.

3 Van TILBORG 4. 
$15,1.12 ; 23,25.26)^{1}$. Les "scribes et les pharisiens" semblent donc former un groupe homogène ${ }^{2}$ fort proche - sinon identique - à celui des pha risiens.

Cette image contredit les circonstances de la vie de Jésus et, à ce titre, est anachronique. Cela peut être prouvé de deux manières. Premièrement, l'histoire du judaísme au ler siècle nous apprend que les "scribes et les pharisiens" ne formaient pas un groupe homogène, les premiers appartenant à un "état", les seconds à un "parti". En fait, les scribes pouvaient appartenir et appartinrent jusqu'à l'année 70 aux tendances les plus variées du bas-judaísme ${ }^{3}$ (par exemple au parti sadducéen). Deuxièmement, l'étude des traditions utilisées par Matthieu montre que ces dernières opéraient une telle distinction : même si Mt attribue aux "scribes et aux pharisiens" le pouvoir doctoral $(23,2)$, le droit à un respect général $(23,6)$, le titre de $\dot{\alpha} \beta \beta \hat{\imath} \quad(23,7)$, à nouveau le pouvoir doctoral $(23,13)$, la tradition synoptique (notamment $Q$ ) sait que de telles prérogatives n'appartenaient qu'aux scribes; à l'inverse, les vv. 23.25.27 s'adressaient d'abord aux pharisiens (voir par. QLc) ${ }^{4}$. Cette double re marque nous montre que Mt ne distingue plus clairement les scribes des pharisiens. En cela, comme dans le cas des pharisiens, il ne connait plus la figure historique des adversaires de Jésus ${ }^{5}$.

Le jugement que Mt porte sur les "scribes et les pharisiens" est accablant. Leur justice est celle qui empêche d'accéder au Royaume des Cieux $(5,20)$. Ils constituent "une génération mauvaise et adultère" $(12$,

1 van TILBORG écrit à ce sujet (3): "From the fact that some things are ascribed to the scribes and the Pharisees which also apply to the other Jewish Leaders, it follows that one should not stress the content of the terminology".

2 WALKER 18. parle d'un "einheitliches Phänomen".

3 JEREMIAS, Jérusalem 339-343; BILL 1, 250; 4,343-352; WEISS, art. cit. 40 .

4 JEREMIAS, art. $\gamma \rho \alpha \mu \mu \alpha \tau \varepsilon \cup ́ S$, ThW 1, 742.

5 HUMMEL 14; WALKER 23. 
39) qui est déjà jugée et contre laquelle témoigneront les Ninivites et la reine du Midi (12,41-42), une génération qui - plus que ses devancières pour avoir refusé le Christ, est la proie des démons (12,43-45). Les "scribes et les pharisiens", tout en prétendant défendre la Loi, la trans gressent $(15,3)$, ce sont des hypocrites $(15,7)$. Toutes ces accusations culminent dans le chap. 23 : les "scribes et les pharisiens" sont ceux qui ne font pas ( $\pi$ olḱw v. 3). Ce sont des hypocrites (vv. 13.15.23.25. 27.29) qui sont désormais sous le coup de la malédiction du Christ (oن́$\left.\alpha i^{\prime}\right)$. Ils ferment l'accès du Royaume aux hommes et à eux-mêmes (v. 13), leur mission conduit à la géhenne (v. 15), ils dénaturent la volonté de Dieu (vv. 16-22), en oubliant l'essentiel (v. 23). Leur obéissance rituelle (vv. 25.27) n'est qu'extérieure, leur respect des ancêtres, un faux sem-

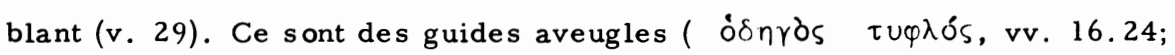
$\tau u r p \lambda \delta s$, vv. 17.19.26), des serpents, une race de vipères (v. 33), promis au jugement de la géhenne (v. 33). Le jugement va s'accomplir sur cette génération ( $\mathrm{v}$. 36) : le Messie se détourne de son peuple et Jérusalem court à sa ruine (vv. $37-39$ ). Cette radicalisation polémique présuppose évidemment une situation où les fronts se sont durcis, où la conversion des chefs du judaïsme est désormais impossible.

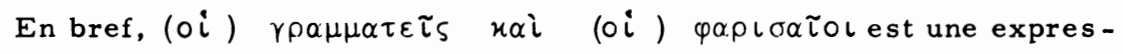
sion rédactionnelle de Mt qui décrit un groupe d'adversaires de Jésus, fort proche sinon identique aux seuls $\varphi \propto \rho \iota \sigma \alpha \tau o \iota$. Ce groupe est anachronique; il ne correspond pas aux conditions historiques qui prévalaient à l'époque de Jésus. Ce résultat nous pose une question sur laquelle nous reviendrons : pourquoi $M t n^{\prime} a-t-i l$ pas suivi ses sources $M c$ et $Q$ ? pourquoi $a-t-i l$ mis en scène un tel groupe ?

1 WALKER $17-29$ voit dans "les scribes et les pharisiens " un groupe homogène, sans enracinement historique, dont le rôle est de représenter les docteurs d'Israël dans leur affrontement avec Jésus. Ce groupe, par ailleurs, n'a aucune caractéristique particulière qui le distingue rait d'autres adversaires de Jésus. Parmi d'autres concepts équiva lents, il représente "die eine, das (damalige) Israel des Matthäus Evangeliums literarisch repräsentierende Lehrerschaft" (29). Si nous pensons que WALKER a bien cerné la démarche "littéraire" de Mt, 


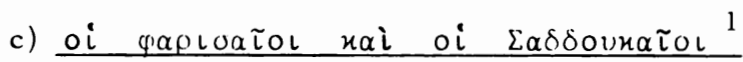

Mt fait intervenir les sadducéens plus fréquemment que les deux autres synoptiques ( 7 fois; Mc : 1 fois; Lc: 1 fois). La critique des sources nous apprend que six des sept mentions sont rédactionnelles (le seul emploi traditionnel est 22,23 par. Mc 12,18). Sur ces six emplois rédactionnels, cinq apparaissent sous la forme de l'expression stéréo-

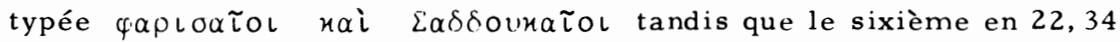

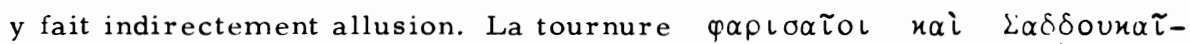
ol est donc $\underline{\text { rédactionnelle }}^{2}$ et, à ce titre, particulièrement représen-

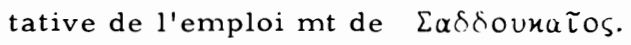

Au niveau du contexte, "les pharisiens et les sadducéens" apparaissent dans la première moitié de l'évangile, mais sont totalement absents de la Passion. Fait remarquable, en 8,7 (à la différence des foules de Lc 3,7), ils sont les interlocuteurs hostiles du Baptiste. Ils interviennent ensuite dans les récits de controverses et les débats d'école du chap. 16. Du point de vue des formes littéraires, la note polémique est patente : il y va de la prédication du Baptiste $(3,1-2)$, de la controverse évoquant la demande d'un signe (16,1-4), du débat d'école mettant en garde contre le levain des pharisiens et des sadducéens (16,5-12). Ainsi, création de Mt, le groupe "des pharisiens et des sadducéens" est, au même titre que les "pharisiens", les "scribes et les pharisiens", un ensemble destiné à montrer l'hostilité du judaĩsme face à Jésus.

nous remarquons que son analyse n'est pas menée à chef. En effet, Mt ne crée pas des fictions littéraires; il transmet et réinterprète les traditions dont il dispose. Aussi il convient de se demander pourquoi il les modifie et, dans le cas qui nous occupe, pourquoi il introduit la tournure "les scribes et les pharisiens" plutôt que de garder les protagonistes figurant dans ses sources.

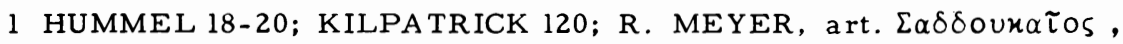
ThW 7,52; van TILBORG 2-3; WALKER 11-16; WEISS, art. cit.

2 HUMMEL 18; van TILBORG 2; WALKER 11. 
Excursus sur les Sadducéens : les seuls $\sum \alpha \delta \delta$ ouna โol comme groupe n'apparaissent qu'au chap. 22 , et à deux reprises seulement $(22,23.24)$. Comme l'indique le par. Mc 22,23, la mention de ce groupe est d'origine tradi tionnelle et son apparition est liée au récit de controverse relatif à la résurrection. La mention de 22,34 est certes rédactionnelle, mais il s'agit d'un verset de transition qui permet de passer du débat sup la résurrection à celui sur le plus grand commandement. On fera deux remarques à propos de ce double emploi de $\sum \alpha \delta \delta$ oun $\alpha$ Tos : $1^{\circ}$ ) en 22,23 - à la différence de sa source Mc 12,18 -, Mt semble ignorer que la négation de la résurrection soit constitutive de $1^{\prime}$ enseignement des sadducéens ${ }^{2} ; 2^{\circ}$ ) en 22,34 , la défaite cuisante des sadducéens provoque l'intervention des pharisiens, ce qui présuppose une sorte d'alliance entre les deux groupe ${ }^{3}$. En conclusion, Mt emprunte à la tradition la figure des sadducéens comme adversaires de Jésus. La double mention qu'il en fait, nous conduit à penser que Mt ne connait plus la doctrine sadducéenne, qu'il associe de manière étrange ce groupe à celui des pharisiens (comme le confirment les cinq

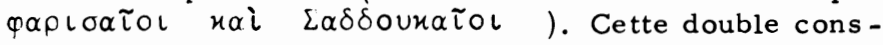
tatation pose un problème d'ordre historique.

Plus que le ròle conducteur des "pharisiens" ou l'association étroite des "scribes et des pharisiens", l'expression "pharisiens et sadducéens" s'oppose aux circonstances historiques de la vie de Jésus. Mt présente les pharisiens et les sadducéens comme un front uni alors qu'il s'agissait de partis rivaux. Il leur prête un enseignement commun (16,6-11.12) alors que leurs doctrines respectives étaient fort éloignées l'une de l'au tre. Il confond délibérément ce qui est inassimilable et ignore ici encore le profil historique des véritables adversaires de Jésus ${ }^{4}$. On peut certes admettre que Mt ne connaissait plus le parti des sadducéens, mais cette constatation n'explique rien et surtout pas que Mt ait, plus que Mc et Lc, manifesté une prédilection pour ce terme. Sans prétendre résoudre cette

1 van TILBORG 2; WALKER 11 .

2 Analyse chez HUMMEL 19; KILPATRICK 120; WALKER 11.

3 Développement chez HUMMEL 19.

4 Argumentation détaillée chez R. MEYER, art. cit., ThW 7,52. 
énigme, on peut néanmoins faire les remarques suivantes. Mt a trouvé le groupe des sadducéens dans la tradition (cf. Mc 12,18), il l'a conservé et introduit dans d'autres contextes. En jugeant nécessaire de faire une plus large place à des antagonistes qu'il sait avoir joué un rôle dans la destinée de Jésus, Mt témoigne d'un intérêt historicisant ${ }^{1}$ (même si le résultat est aberrant !). Deuxièmement - et c'est là un point caractéris tique - Mt introduit certes plusieurs fois les sadducéens dans la narration, mais en liant leur destinée à celle des pharisiens. Les pharisiens deviennent ainsi vraiment le centre autour duquel s'ordonnent tous les autres groupes, que ce soient les scribes (voir plus haut) ou les sadducéens ${ }^{2}$.

Le jugement négatif et stéréotypé ${ }^{3}$ qui s'abat sur le groupe "des pharisiens et des sadducéens", ne pouvait guère être différent de celui qui avait frappé les "pharisiens", mais aussi "les scribes et les pharisiens". Pas plus que les pharisiens, les sadducéens ne connaissent l'Ecriture $(22,29.31)$. Les "pharisiens et les sadducéens" tentent Jésus

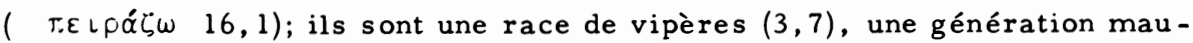
vaise et adultère $(16,4)$, mais surtout leur enseignement 4 est une doctrine

1 HUMMEL 20.

2 van TILBORG 3 remarque pertinemment à propos de l'association des pharisiens et des sadducéens : "Since the historical differences between the various groups could not be understood any longer, the groups themselves could be combined". Concernant l'usage mt de Eaôôu-

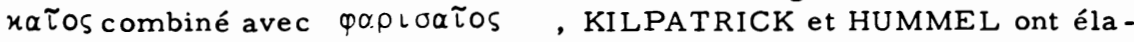
boré d'intéressantes hypothèses qui n'ont malheureusement pas d'appui décisif dans les textes. Pour KILPATRICK 120-121, "sadducéen" est un terme utilisé par Mt pour désigner tous les Juifs non-pharisiens et non-chrétiens si bien que l'expression "pharisiens et sadducéens" décrirait l'ensemble du judaísme non-chrétien. Pour HUMMEL 19-20, la combinaison "pharisiens et sadducéens" exprime un jugement dégradant de Mt sur les pharisiens - et ceci par réciprocité - dans la mesure où dans le bas-judaísme rabbinique, sadducéen est un terminus technicus appliqué aux hérétiques (voir BILL 1, 344).

3 WALKER 13. 4 WALKER 12 parle avec raison, pour résumer le jugement $\mathrm{mt}$, de 
mauvaise et vaine dont il faut se garder $(16,6,11,12)$.

En bref, le groupe "des pharisiens et des sadducéens" est une création rédactionnelle de $\mathrm{Mt}$. Il n'a pas de caractéristique particulière, mais, au même titre que d'autres et formant avec eux un front uni, il re présente les ennemis de Jésus ${ }^{1}$. Fait frappant, il est privé de toute base historique bien que, paradoxalement, il traduise un intérêt historicisant de l'évangéliste. Cette étrange association met une fois encore en évidence la place clef qu'occupent les pharisiens; il nous faudra revenir sur ce point.

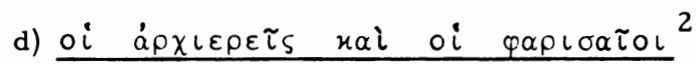

Cette expression est rare $(21,45 ; 27,62)$. Elle est rédactionnelle : ni Mc, ni Lc ne la connaissent (mais bien Jn, cf. $7,32.45 ; 11,47.57 ; 18,3$ ). Elle n'apparait que dans le cadre du séjour de Jésus à Jérusalem.

L'emploi rédactionnel de $21,45^{3}$ (avant le récit de la Passion proprement dit !) est fort suggestif. Il reprend en effet les protagonistes cités en 21,23 tout en modifiant leur composition. 21,23 parle "des grands prêtres et des anciens du peuple" alors que le par. Mc 11,27 - pour désigner le sanhédrin jérusalémite - utilise à juste titre l'expression "les grands prêtres, les scribes et les anciens". Mt 21,23 omet "les scribes", car, d'une part, il leur attribue moins d'importance que Mc, d'autre part, il préfère les couples de protagonistes. Nouveau glissement en 21,45: alors qu'apparemment l'auditoire n'a pas changé, Mt substitue "les pharisiens" aux "anciens". La tendance mt d'introduire les pharisiens le plus souvent possible comme adversaires de Jésus se vérifie.

Une fois encore, l'expression rédactionnelle introduite par Mt ne correspond pas à la réalité historique de la vie de Jésus ${ }^{4}$. Cela peut être

1 Avec WALKER 13.

2 Voir HUMMEL 15-16; van TILBORG 5; WEISS, art. cit., ThW 9,39.

3 HUMMEL 15.

4 HUMMEL 16. 
montré d'une double manière. Premièrement, cette tournure allie deux groupes de nature différente : "grand prêtre" désigne une fonction sans préjuger de l'adhésion à un parti alors que "pharisien" décrit l'apparte nance à un parti sans préjuger d'une fonction; les deux groupes peuvent donc aussi bien se mélanger que rester étrangers l'un à l'autre. Deuxièmement, en 21,45, notre expression est supposée désigner le sanhédrin jérusalémite : or l'association "grands prêtres - pharisiens" n'en donne pas une description adéquate. On se posera, dès lors, la question suivante : pourquoi $\mathrm{Mt}$, en contradiction avec sa source, a-t-il voulu associer les pharisiens comme tels à l'autorité officielle du judaissme ? qu'est-ce qui l'a poussé à leur attribuer ce rôle ?

Dans la réponse que l'on apportera à cette question, on ne perdra pas de vue que le contexte de 21,45 - la parabole des métayers révoltés est une réflexion secondaire qui pense de manière originale les rapports Israél - Jésus - Eglise et qui, à ce titre, témoigne d'une forte imbrica tion entre le passé de Jésus et le présent de la communauté.

Le jugement porté sur "les grands prêtres et les pharisiens" est aussi sévère que dans les autres cas. En 21,45, ils sont ceux qui se sentent visés par la parabole des métayers révoltés et qui, pour cette raison, tentent de s'emparer de la personne de Jésus (v. 46); ils sont en rupture avec la foule qui tient Jésus pour un prophète.

\section{Les scribes seuls ou associés à d'autres groupes}

Comme nous l'avons montré dans notre analyse statistique l, à côté des pharisiens, les scribes occupent une place privilégiée parmi les adversaires de Jésus avant la Passion. Nous avons à voir maintenant si les scribes - seuls ou associés à d'autres groupes - offrent des caractéristiques aussi remarquables que le groupe des pharisiens. En ce qui

1 Voir p. 48 
concerne l'étude de l'expression oi $\gamma \rho \alpha \mu \mu \alpha \tau \varepsilon \tau \varsigma$ n $\alpha i$ oi $\varphi \alpha \rho \iota \sigma \alpha \tau-$ ol, nous renvoyons au paragraphe précédent 1 .

a) $0 i \gamma \rho \alpha \mu \mu \alpha \tau \varepsilon \tau_{s} \quad 2$

La critique des sources nous fait découvrir que Mt biffe à 10 reprises $\gamma \rho \alpha \mu \mu \alpha \tau \varepsilon u ́ s$ dans sa source Mc. Cette importante série de suppressions ${ }^{3}$ laisse entrevoir deux tendances : d'une part, l'effacement des scribes se produit au profit des pharisiens $(9,11 ; 12,24 ; 21,45 ; 22,41)$; d'autre part, plutôt que d'utiliser l'expression historiquement correcte de Mc "les grands prêtres, les scribes et les anciens", Mt préfère celle "des grands prêtres et des anciens (du peuple)"; voir 21,23;26,47; 27, 1, mais aussi 26,3. Dans la première partie de l'évangile, les scribes cèdent donc le pas devant les pharisiens, alors que, dans la Passion, ils ne font plus massivement partie des autorités juives au mème titre que chez Mc. Comment peut-on expliquer cet effacement relatif ?

L'étude du mot $\gamma \rho \alpha \mu \mu \alpha \tau \varepsilon u ́ s$ utilisé de manière isolée contribue à fournir une réponse, car - au contraire des groupes passés en revue jus qu'ici -, les scribes comme tels ne tombent pas nécessairement sous le coup d'un jugement négatif. Le premier évangile connaît, en effet, trois acceptions dans l'utilisation du terme "scribe". Nous recensons, en premier lieu, deux emplois péjoratifs $(7,29 ; 9,3)$, tous deux d'origine traditionnelle (voir les par. Mc 1,22; 2,6). L'emploi de 7,29 est particulière ment intéressant car Mt ajoute un $\alpha \dot{u} \tau \tilde{\omega} \nu$ à $\gamma \rho \alpha \mu \mu \alpha \tau \varepsilon \tau_{\zeta}$, détail qui suggère une appartenance, l'adhésion à un parti. Ces scribes qui appartiennent au "parti des Juifs", sont jugés négativement : ils n'ont pas l'autorité de Jésus pour enseigner $(7,29)$, ils considèrent Jésus comme un blas phémateur $(9,3)$ et leur coeur est plein de méchanceté $(9,4)$. En deuxième lieu, à côté de cet emploi traditionnel et peu représentatif de

1 Voir pp.53-55.

2 HUMMEL 17-18; van TILBORG 3 ; WALKER 24-29.

3 HUMMEL 17. 
la rédaction $\mathrm{mt}$, nous avons deux emplois neutres, l'un d'origine traditionnelle $(17,10$, par. Mc 9,11) et l'autre de provenance rédactionnelle $(8,19)$. Contrairement à la majorité des exégètes ${ }^{1}$, nous ne pensons pas que le texte permette de décider en 8,19 si le scribe est un adversaire ou un disciple de Jésus - là n'est d'ailleurs pas son intention -. Sembla blement, en 17,10, l'enseignement des scribes sur Elie n'a aucune connotation négative. A ces deux emplois neutres, il faut ajouter, en troisième lieu, deux attestations positives $(13,52 ; 23,34)$ qui proviennent soit des traditions propres à Mt (Smt), soit de la rédaction. Le scribe de 13,52 est devenu le disciple du Royaume des Cieux; les scribes de 23,34 qui n'ont rien de commun avec ceux qui les précèdent dans le chap. 23, sont envoyés par Jésus !

Nous remarquons donc que le scribe comme tel, chez Mt, n'est pas une figure stéréotypée. Seul le parti auquel il appartient le qualifie in bonam ou in malam partem ${ }^{2}$. Cet élargissement de la fonction du scribe aux cercles chrétiens est indiscutablement un apport de Mt, voire de sa communauté ${ }^{3}$.

$D^{\prime}$ un point de vue historique, il est loisible d'admettre que l'emploi négatif ou neutre du terme "scribe" s'insère bien dans le cadre d'une vie de Jésus; il n'en va pas de mẽme des "scribes chrétiens". L'existence de ces derniers présuppose une Eglise constituée, dotée de fonctions, une Eglise dont les scribes s'opposent aux scribes juifs (voir chap. 23).

1 Voir, par exemple, SCHULZ, Q 434, avec état de la discussion.

2 HUMMEL 18.

3 Nous reviendrons en détail sur ce problème dans notre chap. consacré aux charges dans l'église mt (voir notre chap. 6). WALKER 24-29 s'élève avec force contre l'existence de scribes chrétiens dans la communauté $\mathrm{mt}$, mais sans produire des arguments convaincants. Van TILBORG 3 escamote la difficulté lorsqu'il écrit : "This shows that Mt does not deal with the scribes as such and that had already lost something of historical importance in his eyes". 
En résumé, Mt, à la différence de Mc, laisse moins souvent les "scribes" s'opposer directement à Jésus ou faire partie intégrante des autorités juives. Cette retenue s'explique par le fait que $\gamma \rho \alpha \mu \mu \alpha \tau \varepsilon u ́ s$, employé de manière isolée, n'est pas une figure stéréotypée, mais une fonction en vigueur aussi bien dans la synagogue que dans l'Eglise. Dès lors, seul le parti auquel le scribe appartient, le qualifie en bien ou en mal. Cet élargissement de la notion fait qu'historiquement elle ne se laisse plus enfermer dans la seule vie de Jésus, mais qu'elle garde son actualité durant le temps de l'Eglise.

b) oi $\dot{\alpha} \rho \chi \iota \varepsilon \rho \varepsilon \tau \tau_{S} \quad x \alpha i \quad \gamma \rho \alpha \mu \mu \alpha \tau \varepsilon \tau_{\zeta}{ }^{1}$

L'expression "les grands prêtres et les scribes" est relativement rare (trois attestations : 2,$4 ; 20,18 ; 21,15)$. Mt 1'a trouvée chez Mc $(20,18$ par. Mc 10,33) et l'utilise deux fois dans les traditions qui lui sont propres $(2,4 ; 21,15)$. Cette constellation désigne les autorités juives jérusalémites que les mages interrogent lors de la naissance du Christ $(2,4)$, qui condamnent Jésus à mort et le livrent aux paiens $(20,18)$, qui sont les gardiens du Temple $(21,15)$. Ignorant la Loi $(21,16)$, indignés par l'activité de Jésus dans le Temple $(21,15)$, agents de sa mort $(20,18)$, ils sont les adversaires de Jésus et tombent sous un jugement négatif. Si cette expression procède d'une tendance historicisante (raconter les cir constances de la vie de Jésus), elle reste néanmoins imprécise ${ }^{2}$.

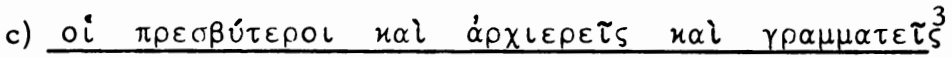

Cette expression est rare chez Mt $(16,21 ; 27,41)$ et ne survient qu'une fois dans le contexte qui nous intéresse $(16,21)$. Empruntée à Mc $(16,21$ par. Mc 8,31), elle décrit avec précision le sanhédrin jérusal émite, responsable de la mort du Christ. La note est historicisante, mais la

1 van TILBORG 5.

2 Jugement justifié de van TILBORG à propos "des grands prêtres et des scribes" : "Therefore no far-reaching consequences can be expected" (5).

3 van TILBORG 6. 
tournure reste peu représentative de la rédaction $\mathrm{mt}$.

\section{Conclusion}

L'analyse statistique et la critique des sources nous ont montré que Mt donne des adversaires de Jésus avant la Passion une image originale par rapport aux deux autres synoptiques. Cette image se caractérise par une répartition nouvelle des ennemis du Christ; elle n'est pas due à la conjonction des sources $\mathrm{Mc}, Q$ et $S$, mais à l'activité rédactionnelle de Mt. Dès lors il convient de déterminer les tendances qui ont guidé $\mathrm{Mt}$ dans cette nouvelle élaboration du cadre "de la vie de Jésus". Nous en discernons au moins trois que l'on peut décrire comme suit :

a) Les pharisiens sont le groupe prédominant autour duquel s'ordonnent les autres unités d'antagonistes. Cette nette mise en évidence des pharisiens en tant qu'adversaires par excellence de Jésus est l'oeuvre de l'auteur du premier évangile, même si elle est déjà amorcée dans la tradition. Voyons plutôt. A la différence de $\mathrm{Mc}$, chez Mt, les pharisiens sont les ennemis de Jésus qui interviennent le plus fréquemment. Cet accrois sement de leur rôle s'opère principalement aux dépens des scribes. Mais s'il explique leur prédominance, il ne prouve pas pour autant leur fonction centrale. Cette dernière se manifeste avant tout dans les relations qu'ils entretiennent avec les scribes et les sadducéens, relations qui apparaissent de manière privilégiée dans les deux expressions rédaction-

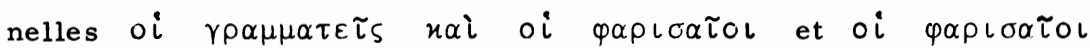

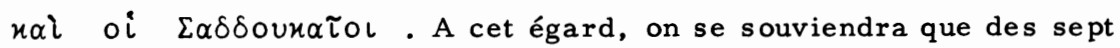
emplois de "sadducéen" chez Mt, six sont rédactionnels et que ces six mentions mettent précisément en rapport très étroit les sadducéens et les pharisiens. Cette constatation signifie que, chez Mt, les sadducéens n'interviennent qu'en compagnie des pharisiens et n'existent pas sans eux. L'analyse du terme "scribe" permet de tirer une conclusion analogue.

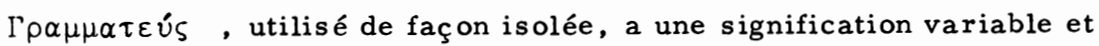


seul le parti auquel il appartient, le qualifie en bien ou en mal. Or le parti auquel Mt associe les scribes de manière stéréotypée est précisément celui des pharisiens. Le $\alpha \dot{u} \tau \tilde{\omega} \nu$ de 7,29, mais surtout l'expres sion rédactionnelle oi $\gamma \rho \alpha \mu \mu \alpha \tau \varepsilon \tau \varsigma$ $x a i$ oi $\psi \alpha \rho \iota \sigma \alpha \tau o l$ en font foi. Les scribes juifs dont parle Mt, sont ceux du parti pharisien et en dehors de ce dernier, il n'en existe pas ${ }^{1}$. Enfin, l'étrange tournure oi d $\alpha-$

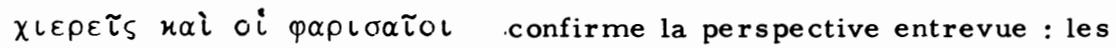
pharisiens de Mt vont jusqu'à s'implanter dans le sanhédrin dont ils de viennent un parti influent ! La conclusion s'impose d'elle-même : le judaisme auquel se heurte le Christ mt est dominé par les pharisiens.

b) A cette première tendance, on peut en ajouter une seconde : les adversaires de Jésus forment un front uni, hostile à Jésus et définitivement endurci. Bien que les ennemis du Christ mt puissent intervenir dans des combinaisons différentes, ils constituent néanmoins un front homogène. Cette unité découle évidemment de notre première remarque qui rappelait l'étroite solidarité liant, d'une part, les scribes et les pharisiens, d'autre part, les pharisiens et les sadducéens. Mais elle se manifeste aussi de manière indirecte : tous, pharisiens, scribes, sadducéens tombent sous le coup du même jugement, sans nuance et stéréotypé. A leur méchanceté et à leur incrédulité criminelles répond la même condamnation sans appel. Ce qui vaut des uns vaut des autres ${ }^{2}$. Le judais me dont parle Mt, forme donc un front uni qui est déjà sous le coup d'un verdict ir rémédiable.

1 Cette dépendance des scribes par rapport aux pharisiens est encore attestée par le fait que la signification de $\gamma \rho \alpha \mu \mu \alpha \tau \varepsilon u ́ s$ est variable et que des trois combinaisons recensées, seule l'expression "les scribes et les pharisiens" est rédactionnelle, alors que les constellations "les grands prêtres et les scribes", "les grands prêtres, les scribes et les anciens" sont empruntées à la tradition. Mt a donc moins retravaillé les expressions relatives aux scribes que celles concernant les pharisiens.

2 Sur ce point, voir en particulier van TILBORG 8-72, où l'auteur analyse la terminologie polémique utilisée par Mt contre les Juifs. 
c) Troisième tendance, enfin : tous les groupes juifs évoqués par Mt ont perdu leur profil historique initial. Nous entendons par là que la des cription mt des ennemis de Jésus ne correspond plus aux circonstances prévalant dans le bas-judaïsme palestinien du début du ler siècle. Les pharisiens forment un parti uni qui a pris en main les destinées du judaís me alors qu'il s'agissait d'un parti traversé par les courants les plus divers qui, bien qu'en expansion, n'assumait pas encore la direction de la communauté juive. Les scribes n'appartiennent plus qu'au parti pharisien auquel ils ont cédé leurs prérogatives alors qu'en fait, il s'agissait d'une fonction encore en pleine constitution et qui ne préjugeait pas de l'adhésion à un quelconque parti. Les pharisiens et les sadducéens forment un seul front alors qu'il s'agissait de partis rivaux. Les pharisiens associés aux grands prêtres représentent le sanhédrin alors qu'ils n'ont jamais siégé dans l'autorité jérusalémite à titre de parti, mais qu'ils y étaient représentés par les scribes et les grands prêtres qui partageaient leur doctrine. L'existence de scribes chrétiens $(23,34)$ enfin est impensable dans le cadre "de la vie de Jésus" qui n'était pas un rabbi ${ }^{1}$ et qui s'est entouré, avant tout, de pécheurs.

$\mathrm{Si}$, à quelques exceptions près ${ }^{2}$, la recherche récente s'accorde à reconnaître les trois tendances que nous venons de formule ${ }^{3}$, les esprits se séparent sur l'interprétation qu'il convient de donner de ces résultats. Schématiquement, deux grandes options s'offrent à l'exégèse. Soit on souligne la forte élaboration littéraire entreprise par Mt et 1 'on met l'accent sur les anachronismes qui en résultent; dans ce cas, la conclusion qui

1 Voir ZUMSTEIN, Maître et disciple 88-112.

2 Contre toute évidence, WALKER 22-23, par exemple, nie que les pharisiens soient, par excellence, les adversaires de Jésus avant la Passion chez Mt. Pour lui, les véritables ennemis de Jésus sont les docteurs d'Israel qui transparais sent à travers tous les groupes. La faiblesse de l'hypothèse consiste en ce que Mt ne connait pas ce concept.

3 Notre apport spécifique par rapport à la recherche récente est d'avoir entrepris une analyse systématique et détaillée du sujet. 
s'impose est que Mt vise à présenter le judaísme comme une unité afin de pouvoir l'utiliser comme modèle opératoire dans sa réflexion théologique. En tant qu'exemple d'incrédulité et d'iniquité, les Juifs deviennent alors l'antithèse du modèle éthique élaboré par $\mathrm{Mt}^{\mathrm{l}}$ ou encore le pôle négatif d'une histoire du salut ${ }^{2}$. Ils se transforment en une figure dépour vue de toute insertion historique et au service de l'argumentation théologique. C'est la position défendue dans leurs monographies respectives par Strecker, Walker et, plus récemment, van Tilborg ${ }^{3}$.

A cette interprétation, il faut opposer deux objections majeures. Premièrement, bien que cette théorie souligne à juste titre l'élaboration littêraire effectuée par Mt dans son portrait des adversaires de Jésus, elle n'explique pas quels sont les facteurs qui ont inspiré Mt dans cette redis tribution des rôles; compte tenu de ce que nous savons de la rédaction des évangiles, il est en effet peu probable que Mt se soit laissé guider par des critères purement fictifs et arbitraires. Deuxièmement, cette inter préta tion présuppose que le judaïsme est devenu un phénomène sans consis tance historique pour Mt. L'ensemble de l'évangile, mais surtout l'apologie menée dans les récits de controverses ${ }^{4}$, démentent cette opinion.

A cette première ligne de lecture s'en oppose une seconde essentiellement représentée par Kilpatrick et Hummel ${ }^{5}$ et à laquelle nous nous rallions. Tout en soulignant l'originalité littéraire dont Mt fait preuve

1 STRECKER écrit à ce sujet : "Der Pharisäismus reflektiert im Mat thäusevangelium nicht primär die Situation des zeitgenössischen Judentums, sondern hat die Funktion eines Topos, der im Gegenüber zur ethischen Forderung die Haltung des Unglaubens und d.h. der Ungesetzlichkeit reprăsentiert (Geschichtverständnis)".

2 WALKER 38 se prononce dans ce sens : "Die Repräsentanten Israels sind im Matthăusevangelium die Vertreter der innerhalbs des Evange liums dargestellten, uberschaubaren und zeitlich determinierten heilsgeschichtlichen Grösse Israels".

3 STRECKER, Weg 140-141; van TILBORG. passim; WALKER 11-33.

4 Voir l'analyse de HUMMEL 35-56.

5 HUMMEL 12-26; KILPATRICK 101-123; voir aussi WEISS, art. cit., ThW 9, 36-38. 
dans sa présentation des adversaires de Jésus, cette analyse fait remarquer que les nouvelles caractéristiques du judaísme mises en relief par Mt ne sont pas arbitraires, mais correspondent pour l'essentiel aux conditions qui prévalent après 70. La prédominance des pharisiens, l'autorité qu'ils exercent sur l'ensemble du judaísme, l'appartenance massive des scribes à leur parti, la perte de consistance des sadducéens, la constitution d'un front uni face aux hérétiques sont autant d'éléments propres au judaïs me rabbinique. Mt a donc retouché l'image des ennemis de Jésus, transmise par la tradition, en fonction de la réalité qu'il vivait, à savoir le judaf'sme né de la crise de 70. Ce faisant, il inscrit dans l'histoire de Jésus les conditions qui prévalent à son époque; en d'autres termes, il reformule le conflit entre Jésus et les Juifs à la lumière de l'affrontement qui se déroule entre la synagogue et l'Eglise. Fait capital, nous retrouvons ici la technique qui avait déjà présidé à l'élaboration de la figure des disciples. Nous tenterons de développer le sens de cette convergence dans la conclusion de ce chapit re.

\section{B. Les adversaires de Jésus durant la Passion}

Alors qu'avant la Passion, les adversaires de Jésus étaient avant tout les pharisiens, mais aussi les scribes et accessoirement les sadducéens, l'image change complètement durant la Passion. Pharisiens, scribes et sadducéens disparaissent presque complètement et laissent principalement le champ libre à deux groupes : les grands prêtres et les anciens. Ici, encore, nous n'avons pas affaire à des groupes monolithi ques, mais à des constellations variables organisées autour d'un concept.

(1) oi $\dot{\alpha} \rho \chi\llcorner\varepsilon \rho \varepsilon \tau \varsigma$ apparaît sous 4 formes :

a) oi $\dot{\alpha} \rho \chi\llcorner\varepsilon \rho \varepsilon \tau \varsigma \quad(26,14.59 ; 27,6 ; 28,11)$, soit 4 fois.

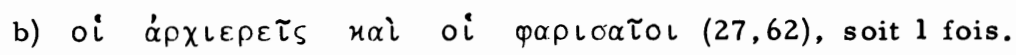

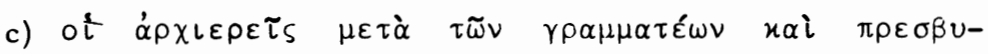
$\tau \varepsilon \rho \omega \nu(27,41)$, soit 1 fois.

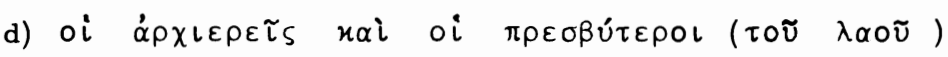
$(26,3.47 ; 27,1.3 .12 .20)$, soit 6 fois. 
(2) oi $\pi \rho \varepsilon \cup \beta u ́ \tau \varepsilon \rho o l$ apparaît sous 4 formes :

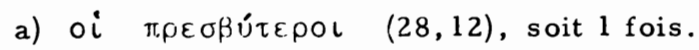

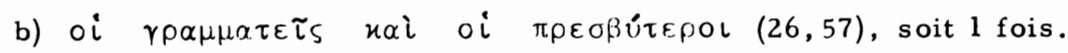

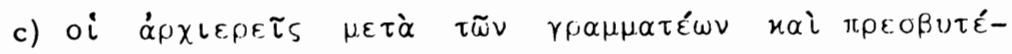
$\rho \omega \nu$. Cf. supra, soit 1 fois.

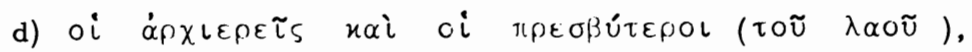
soit 1 fois.

Ce simple inventaire montre déjà que les grands prêtres jouent le premier ròle dans la Passion; ils sont immédiatement suivis et soute nus par les anciens. De plus, comme dans la première partie de l'évangile, Mt retravaille fortement les formulations traditionnelles : les quatre constellations enregistrées sont en effet de son cru.

\section{Les grands prêtres seuls ou associés à d'autres groupes}

a) oi $\alpha \rho \chi\llcorner\llcorner\rho \varepsilon \tau \varsigma$

Le groupe des grands prêtres intervient deux fois comme reprise verbale de Mc $(26,14$ par. Mc 14,40; 26,59 par. Mc 14,55) et deux fois dans le Smt $(27,6 ; 28,11)$. Son unique contexte d'apparition est la Passion. Ce groupe est, lui aussi, jugé négativement par Mt : ce sont les grands prêtres qui négocient la trahison de Judas $(26,14$, accent $\mathrm{mt}$ sur le négoce), ce sont eux ensuite qui abandonnent le traître à son sort $(27,6)$. Asso-

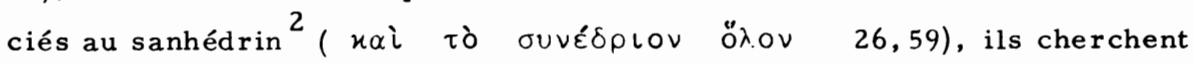
le faux témoignage qui condamnera Jésus; avec les anciens, ils tentent de polémiquer contre la réalité de la résurrection $(28,11-15)$.

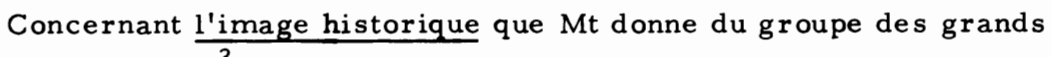
prêtres, Hummel ${ }^{3}$ a relevé que dans les trois passages où ces derniers

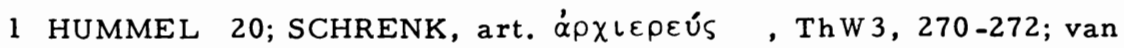
TILBORG 4; WALKER 29-30.

2 Nous sommes à la limite de la constellation, voir van TILBORG, 6.

3 HUMMEL 21. 
apparaissent seuls, il y va de leur juridiction : la police du temple $(26,14$; $28,11)$, les finances du temple $(27,6)^{1}$. Hummel en conclut que, sur ce point au moins, Mt possède des connaissances historiques exactes ${ }^{2}$. Ce jugement nous parait quelque peu rapide, car, dans les trois cas invoqués, la suite du récit fait intervenir d'autres antagonistes qui exercent les mê mes compétences ${ }^{3}$. Il est donc exagéré de discerner dans le rôle joué par les grands prêtres la preuve de la sagacité historique de $\mathrm{Mt}^{4}$, et plus prudent d'y voir le reflet de la tradition dont disposait le rédacteur et que - fait intéressant - il a transmise sans grande modification sur ce point précis.

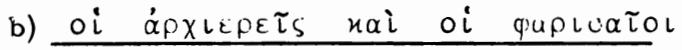

En parlant des pharisiens, nous avons déjà dit ce qu'il fallait penser de cette expression ${ }^{5}$. Il s'agit d'une tournure d'origine rédactionnelle et fortement anachronique. La mention de 27,62 appelle néanmoins les deux remarques suivantes. Premièrement, par le biais de cette expression et

1 Bon résumé historique de SCHRENK : "Die Oberpriester scheinen ein festes Kollegium mit den Funktionen der Leitung des Kultus, der Tempelverwaltung und - polizei, der Verwaltung der Tempelgelder und der priesterlichen Gerichtsbarkeit gebildet zu haben; sie hatten Sitz und Stimme im Synedrium (art. cit. 270)". Voir aussi BILL 2, 56. 626631.634-635;3, 696-697.

2 HUMMEL 21.

3 Avec WALKER 30. En 27,6, en tant que responsables des finances du Temple, ce sont certes les grands prêtres qui disposent de l'argent de la trahison, mais, en 27,3 , Judas s'adresse "aux grands prêtres et aux anciens" pour leur rendre les trente pièces. Semblablement, en 26,14 , Judas négocie la trahison avec les grands prêtres, chefs de la police du Temple, mais la troupe de 26,47 est mandatée par "les grands prêtres et les anciens du peuple". Enf in en 28,11 , les soldats informent les grands prêtres (police du Temple) de la disparition de Jésus, mais ces derniers s'associent immédiatement aux anciens $(28,12)$ pour riposter.

4 van TILBORG 4 : "In any case it cannot be said that Mt attributes a special place to the high priests as such".

5 Voir pp. 59-60. 
à la différence des deux autres synoptiques, Mt associe les pharisiens à la Passion; eux aussi, portent la responsabilité de la mort de Jésus. Ainsi se trouve également vérifiée la tendance de la première partie de l'évangile qui est de faire intervenir le plus souvent possible les phari siens en tant qu'adversaires de Jésus. Deuxièmement, la conjonction des principaux antagonistes des chap. 1-25 avec ceux de la Passion est doublement anachronique : d'une part, Mt attribue un rôle aux pharisiens durant la Passion, rôle que toute la tradition ignore ${ }^{1}$; d'autre part, associés aux grands prêtres, ils sont à nouveau supposés représenter les plus hautes autorités juives-jérusalémites. Sur ce point aussi, il s'agira d'expliquer pourquoi Mt modifie ses sources.

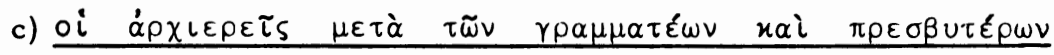

En parlant des scribes, nous avons déjà dit ce qu'il fallait penser de cette expression ${ }^{2}$ qui est une désignation correcte du sanhédrin jérusalémite. Remarquons simplement qu'en 27,41, Mt entreprend une correction de sa source (par. Mc 15,31) en introduisant le groupe des anciens. Cette adjonction peut s'expliquer d'une double manière : soit Mt tient à ce que tous les adversaires du Christ soient présents devant la Croix; soit, selon la tendance qui lui est chère dans le récit de la Passion, il réduit le rôle des scribes au profit de celui des anciens. Dans les deux cas, la correction n'a pas un motif historique. Ce groupe est, lui aussi, qualifié négativement : il insulte le Christ en croix.

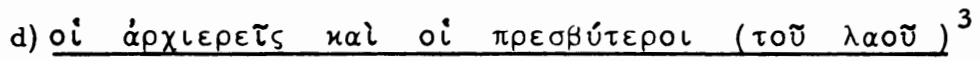

L'expression "les grands prêtres et les anciens du peuple" qui

1 Cf. HUMMEL 13. Certes en Mc 3,6, les pharisiens associés aux Herodiens complotent de faire périr Jésus. Mais ce passage n'appartient pas au cycle mc de la Passion dans lequel les pharisiens n'apparaissent jamais.

2 Voir pp. 63-64.

3 BORNKAMM, art. $\pi \rho \varepsilon \sigma \beta \cup S \quad x \tau \lambda$, ThW 6, 659; HUMMEL 18; van TILBORG 4-5; WALKER 29-33. 
n'apparaît qu'une fois dans les chap. $1-25$ (voir 21,23), est rédaction$\underline{\text { nelle }}^{1}$. Elle désigne le groupe prédominant des adversaires de Jésus dans la Passion mt. Elle apparaît soit sous la forme oi $\dot{\alpha} \rho \chi\llcorner\varepsilon \rho \varepsilon \tau \varsigma$ xai oi $\pi \rho \varepsilon \sigma \beta u ́ \tau \varepsilon \rho O L \quad(27,3.12 .20)$, soit encore avec l'adjonction

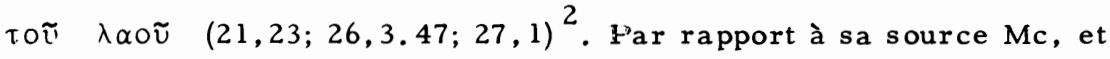
pour constituer ce groupe, Mt suit une double tendance. D'une part - et à deux reprises $(27,12.20)$-, il ajoute "les anciens" au seul groupe des grands prêtres représentés chez Mc. D'autre part, il supprime quatre fois "les scribes" présent chez Mc $(21,23 ; 26,3.47 ; 27,1)$, dont une fois pour leur substituer "les anciens" $(26,3)$. Dans la formation de cette expression rédactionnelle, Mt tend donc incontestablement à un efface ment des scribes au profit des anciens ${ }^{3}$.

$D^{\prime}$ un point de vue historique, Hummel ${ }^{4}$ à la suite de Jeremias a soutenu que cette expression désigne le sanhédrin jérusalémite et que le terme $\pi \rho \varepsilon \sigma \beta u ́ t \varepsilon \rho \circ$ englobe aussi bien l'aristocratie laique que les scribes. Si cette interprétation est vraisemblable, il faut pourtant se garder d'être trop catégorique, car, d'une part, dans le même récit, ce groupe peut être relayé par d'autres sans la moindre difficulté ${ }^{5}$, d'autre part, la fusion des scribes et de la noblesse lafque en un seul

1 van TILBORG 5.

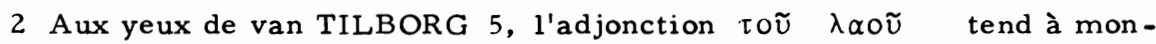
trer que, pour $\mathrm{Mt}$, les grands prêtres et les anciens représentent le peuple dans son ensemble et agissent véritablement en son nom.

3 BORNKAMM, art. cit., ThW 6, 659.

4 HUMMEL 18. Le titre IPT qui était parfois appliqué aux rabbis (cf. par exemple Mt 15,2) peut avoir facilité l'assimilation des scribes au groupe des anciens.

5 Un seul exemple suffira à éclairer notre propos. Dans le récit de la garde du tombeau, les interlocuteurs de la force romaine sont succes sivement les grands prêtres et les pharisiens $(27,62)$, les grands prêtres $(28,11)$, les grands prêtres et les anciens $(28,12)$. Pareille alternance tend à effacer les caractéristiques historiques propres à chaque groupe. Autres exemples chez van TILBORG 5. 
parti reste surprenante ${ }^{1}$.

"Les grands prêtres et les anciens du peuple" se caractérisent par leur duplicité $(21,23-27 ; 26,4-\delta o ́ \lambda \psi)$. Ce sont eux qui décident de tuer Jésus $(26,4)$, qui dirigent son arrestation $(26,47)$, qui décident une fois encore sa mort $(27,1$ : rédactionnel !), qui laissent Judas se suicider $(27,3-4)$, qui mènent l'accusation dans le procès de Jésus $(27,12-20)$. Aux yeux de Mt, ils sont donc les agents de la mort du Christ.

En bref, sans que nous puissions ici taxer Mt d'anachronisme - bien que d'un point de vue historique sa présentation reste floue -, nous cons tatons qu'en faisant intervenir ce groupe, le rédacteur a d'abord une visée théologique : établir la responsabilité d'Israél à travers ses chefs dans la mort du Messie ${ }^{2}$.

\section{Les anciens seuls ou associés à d'autres groupes}

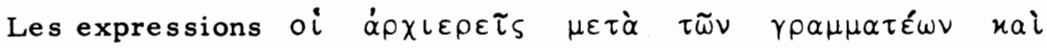

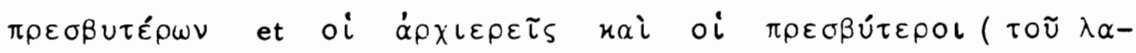
õ̃) sont étudiées aux pp. $71-73$. Nous n'y revenons donc pas.

1 Au sens strict, les "anciens" sont les représentants de l'aristocratie jérusalémite lalque au sanhédrin; ils sont généralement d'obédience sacerdotale et sadducéenne (BORNKAMM, art. cit., ThW 6, 659). Les scribes, au contraire, sont recrutés dans toutes les couches sociales et sont, dans leur majorité, ralliés au parti pharisien (JEREMIAS, Jérusalem, 315 -342).

2 Bien qu'excessive dans sa conclusion, la position de WALKER est suggestive : "Die Oberpriester und Ältesten des Volkes sind für Matthaus die Exponenten der Christus -Todfeindschaft Israels auf dem Boden Jerusalems. Erst Matthaus hat dieses Bild der Oberpriester und Åltesten als einer Aktionseinheit geschaffen und ihm seine charakteristischen Zuge sich aufgeprägt. Es handelt sich also umeinen durchaus ungeschichtlichen Personenkomplex... (32)". 
a) $\circ i \pi \rho \varepsilon \sigma \beta u ́ \tau \varepsilon \rho \circ \iota^{1}$

Les "anciens" n'ont pas d'existence indépendante chez Mt. En 28, 12 $(\mathrm{Smt})$, ils sont incontestablement en relation avec les grands prêtres $(\text { voir } 28,11)^{2}$. Le v. 15 b a pporte un complément intéressant à l'image historique que nous avons tracée jusqu'ici : nous sommes dans un contexte apologétique où le judaîsme du temps de Jésus et le judaĩsme contempo-

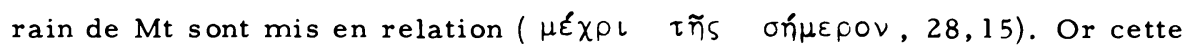
relation dénote une identité : ce qui fut dit alors est cru aujourd'hui. La polémique mt a donc un impact immédiat. Cette note sur la continuité et l'homogénéité du judaîs me doit être retenue pour l'interprétation globale.

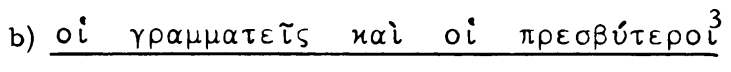

Cette expression $(26,57)$ est unique chez Mt. Rédactionnelle, elle est engendrée par la reprise du par. Mc 14,53 et la suppression des grands prêtres présentés dans la source. Cette suppression - en opposition avec la tendance $m$ t observée dans la Passion - se laisse difficilement expliquer. Elle est d'ailleurs historiquement incorrecte. Serait-elle due au goût de Mt pour les groupes de deux, ce d'autant plus que les grands prêtres réapparaissent en force au v. 59 ?

\section{Conclusion}

Nous avons déjà dit que le récit de la Passion se distinguait du reste de l'évangile (chap. 1-25) par l'entrée en scène de nouveaux adversaires de Jésus. Ce nouveau groupe est conduit par les grands prêtres, appuyés le plus souvent par les anciens. Il est désigné par une série d'expressions variables qui portent une forte empreinte rédactionnelle. Le travail de Mt obéit-il aussi dans cette section particulière de l'évangile à des tendances précises ? Nous en discernons deux. Tout d'abord, par rapport

1 HUMMEL $21-22$; WALKER $30-31$.

2 Pour l'analyse de cette association, voir pp. 71-73.

3 van TILBORG 6. 
ì Mc, Mt stylise les ennemis de Jésus. L'expression rédactionnelle oi $\alpha \rho \chi \iota \varepsilon \rho \varepsilon \tau \varsigma$ nai oi $\pi \rho \varepsilon \sigma \beta u ́ \tau \varepsilon \rho \circ \iota$ - qui est la plus fréquente est devenue un stéréotype. Les tournures oi $\alpha \rho \chi \iota \varepsilon \rho \varepsilon \tau \varsigma \quad \mu \varepsilon \tau \dot{\alpha} \quad \tau \tilde{\omega} \nu$

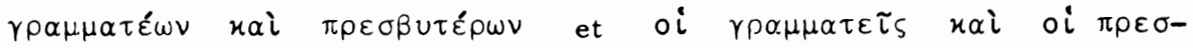
$\beta u ́ \tau \varepsilon \rho \circ$, mais aussi oi $\pi \rho \varepsilon \sigma \beta u ́ \tau \varepsilon \rho o l$ employé seul, font allusion à la même réalité que le stéréotype décrit ci-dessus. On a alors l'impression que Mt concentre son attention sur le même groupe qui revient in lassablement.

La deuxième tendance que nous détectons, est une relative historicisation. Alors que dans les chap. 1-25, Mt faisait volontiers intervenir des groupes fortement anachroniques, tous les acteurs de la Passion mt, à une exception près $(27,62 !)$, sont des personnages ou des groupes qui ont pu avoir leur place dans le procès et la mort de Jésus. Certes l'image reste floue et la description imprécise. Mais la description des compétences des grands prêtres et l'accent porté sur le rôle du sanhédrin sont, sous toute réserve, vraisemblables.

Au contraire des chap. l-25, les adversaires de Jésus durant la Passion n'ont pas une signification ambivalente (exceptions 27,$62 ; 28$, $11)^{1}$. La description semble plutôt animée par la volonté d'établir la claire responsabilité des Juifs d'alors dans la mort du Christ (voir notamment 27,15-23.24-26, le Smt, les adjonctions rédactionnelles). L'a pologétique n'est pas directe, elle passe par la médiation d'une histoire du salut qui s'esquisse ${ }^{2}$.

1 L'ambivalence en 27,62 et en 28,11 est liée à la signification apologé tique du passage : la calomnie qui fut proférée alors par les chefs d'Israel est reprise comme telle par les dirigeants du judaîsme d'après 70 .

2 La version $m$ de la Passion, en établissant la responsabilité totale des Juifs dans la mort du Christ, justifie la formation de la communauté et légitime sa prétention à être désormais la porteuse de la $\beta \alpha \sigma \iota \lambda \varepsilon \iota_{\alpha} \alpha$. A l'inverse, elle établit pourquoi Israel a été dépos sédé de son élection. 
C. Les pécheurs et les paiens chez Matthieu ${ }^{1}$

Pour désigner les pécheurs et les paiens dans son évangile, Mt re court à quatre concepts génétiques qu'il utilise seuls ou dans des combinaisons variables : ce sont $\dot{\alpha} \mu \alpha \rho \tau \omega \lambda \delta_{S} \quad(9,10.11 .13 ; 11,19 ; 26,45)$,

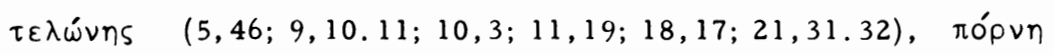
$(21,31.32)$ et $\dot{\vartheta} \vee\llcorner x \delta \varsigma \quad(5,47 ; 6,7 ; 18,17)$. Fait intéressant, ces concepts ou les combinaisons qu'ils forment entre eux, sont liés à des motifs précis et spécifiques qu'il s'agit d'analyser.

1. Les pécheurs, les collecteurs d'impôts et les paíens, image de l'incroyance

Le "pécheur" ( $\left.\alpha \dot{\alpha} \alpha \rho \tau \omega \lambda \delta_{S}\right)$, dans le bas-judaísme, désigne soit le membre du am ha - aretz, soit le paien ${ }^{2}$. Est membre du am ha - aretz le Juif qui ne vit pas en se conformant à la Tora, plus particulièrement encore celui qui se signale par une conduite immorale ou qui exerce une profession déshonorante (ainsi le $\tau \varepsilon \lambda \omega ́ v \eta n s)^{3}$. Dans l'un et l'autre cas, il est exclu du véritable Israël et assimilé à la masse des infidèles, des paíens ( $\varepsilon \& \vee\left\llcorner x_{o ́ s}^{4}\right.$ ). A la suite de la tradition synoptique, Mt reprend cette distinction typiquement juive entre fidèles et infidèles et l'applique - à l'Eglise (voir 18,17 !). A ce titre, les collecteurs d'impôts et les paiens deviennent un stéréotype utilisé comme pôle négatif dans l'argumentation. En 5,46 , le collecteur d'impôts et, en $5,47^{5}$, le pafen, sont ceux dont il

1 HUMMEL 22-26; KILPATRICK $117-118$.

2 Voir JEREMIAS, Zöllner 293 -300; RENGSTORF, art. $\dot{\alpha} \mu \alpha \rho \tau \omega \lambda \sigma_{S}$, ThW 1, 320-337 (en particulier 328-333).

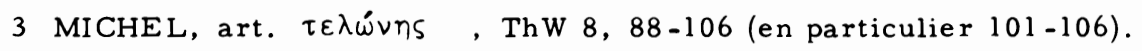

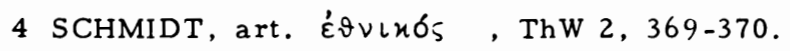

5 Les $\tau \varepsilon \lambda \tilde{\omega} \vee \alpha \iota$ et les $\varepsilon \vartheta \vee\llcorner ห o i$ de $5,46.47$ proviennent de la source $Q$, mieux conservée chez Mt que chez Lc. Etat de la discussion et référence chez SCHULZ, Q 129. 
faut se garder d'imiter la conduite minimaliste. En $6,7^{1}$, le paien apparaît comme le prototype de la prière superficie lle et verbeuse. En 18,17 (Qmt), l'excommunication qui frappe le frère endurci le réduit, pour l'Eglise, au rang du collecteur d'impôts et du paíen. Chez Mt, à la suite

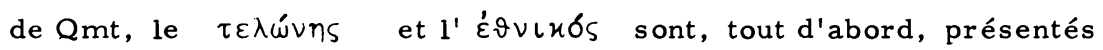
comme l'image de l'immoralité et de l'incrédulité.

Quelle est alors la relation établie par Mt entre le collecteur d'impôts et le païen ? S'il est vrai que notre auteur a trouvé ces deux termes - et probablement leur usage conjugué - dans ses sources ${ }^{2}$, il reste néanmoins le seul des synoptiques qui conserve $\varepsilon \vartheta \vee \iota x o ́ s$ dans sa narra-

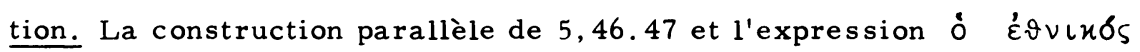

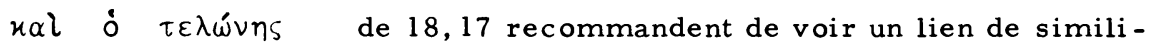
tude entre ces deux concepts; ils sont équivalents : ce qui vaut de l'un vaut

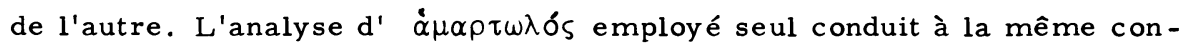
clusion : en 9,13, il a un sens généralisant par rapport à 9,10.11 où il

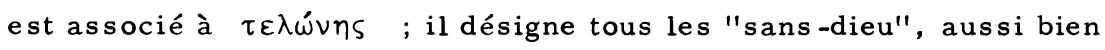
juifs que paiens. En 26,45, il fait clairement allusion aux Romains.

En bref, Mt, à la différence de Mc et de Lc, mais en continuité avec la tradition ( $\mathrm{Qmt}$ ) établit une étroite solidarité entre les pécheurs et les païens. La similitude de leur condition a tout d'abord un accent négatif : c'est leur commune incrédulité et immoralité qui les réunit.

\section{Les collecteurs d'impôts et les pécheurs, bénéficiaires de l'appel du}

\section{Christ.}

Il est fort intéressant de remarquer que les trois fois $(9,10.11$;

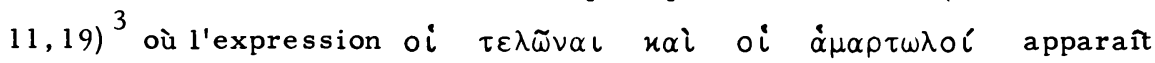

1 Il est difficile de savoir si 6,7 a relève de $\mathrm{Smt}$ ou de la rédaction.

2 HUMMEL 22 -23.

3 En 9,10.11 l'expression "les collecteurs d'impôts et les pécheurs" est tirée de $\mathrm{Mc}$, en 11,19 de $\mathrm{Q}$. 
chez Mt, elle est liée au thème de l'appel. Le Christ mt est celui qui se sent spécialement envoyé auprès des brebis perdues d'Israël $(10,6$; $15,24)$. Il appelle un collecteur d'impôts à la condition de disciple $(9,9$; 10,3); il partage le repas des péagers et des pécheurs, leur montrant par là qu'il leur accorde sa communion et sa sollicitude $(9,10.11)$; les pharisiens le savent, eux qui croient l'insulter en le traitant "d'ami des collecteurs d'impôts et des pécheurs" $(11,19)$.

Alors que l'appréciation négative de la tradition synoptique frappait indistinctement pécheurs, collecteurs d'impôts et paíens, il semble que l'appel de Jésus s'adresse au seul am ha - aretz ( oi $\dot{\alpha} \mu \alpha \rho \tau \omega \lambda \circ i$, cf. aussi 10,$6 ; 15,24)$.

Cette constatation appelle néanmoins deux remarques. Nous avons

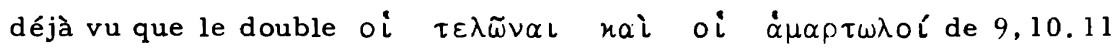
culmine dans le seul $\dot{\alpha} \mu \alpha \rho \tau \omega \lambda$ o ́ de 9,13 qui a un sens généralisant ${ }^{l}$. A cela il faut ajouter l'hypothèse suggestive de Hummel ${ }^{2}$ sur le "Sitz im Leben" $\mathrm{mt}$ de 9,13: en rappelant l'appel que Jésus adressa naguère au am ha-aretz, Mt - attaqué par la polémique juive -, essaie de justifier l'existence d'une communauté chrétienne où voisinent. Juifs et paíens convertis.

En résumé, le groupe des "collecteurs d'impôts et des pécheurs" ne tombe pas seulement sous le coup d'un jugement négat if. Reconnu dans son incrédulité et son immoralité, il est l'objet de la sollicitude du Christ qui lui offre sa communion. L'appel qui lui est adressé est l'image de l'appel qui s'adresse également aux païens. La similitude de condition entre le am ha-aretz et les palens fait que les premiers forment une figure dans laquelle les seconds peuvent se reconnaitre.

1 Mt emprunte cette explication à Mc.

2 HUMMEL 39-40; voir aussi KILPATRICK 117. 
3. Les collecteurs d'impôts et les prostituées, image de la foi refusée par les Juifs

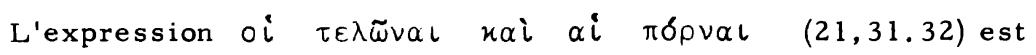
liée, elle, au motif de l'exemplarité de la foi, acceptée par les pécheurs et refusée par les Juifs pieux. La parabole des deux fils $(21,28-32)$ montre, de manière polémique, que l'appel à la repentance adressé aux parias religieux d'Is raël rencontre un accueil favorable. Leur foi (vv. $31-32)$ est l'exemple que les autorités juives auraient dû prendre en considération et imiter.

Le motif de l'exemplarité de la foi "des collecteurs d'impôts et des prostituées", suggéré aux vv. 28-31a, mais développé aux vv. $31 \mathrm{~b}-32$ l, est particulièrement intéressant. Il appartient plutôt à une couche ultérieure de la tradition, car les textes les plus anciens parlent de préférence de la justice des pharisiens et de l'appel du am ha-aretz pécheur

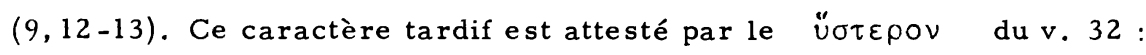
la foi "des collecteurs d'impôts et des prostituées" est déjà vue comme un événement passé et le refus initial des autorités juives est demeuré inébranlable ${ }^{2}$.

Le contexte immédiat de la parabole des deux fils nous permet d'aller plus avant. L'endurcissement patent d'Israël, déjà suggéré et condamné dans les vv. $31-32$, prend toute sa signification dans les deux péricopes qui suivent : la parabole des métayers révoltés et celle du festin nuptial $(21,33-46 ; 22,1-14)$. Les refus répétés d'Is raël sont châtiés : le peuple est dispersé, le temple est détruit, le Royaume lui est enlevé. Mais les héritiers du Royaume qui lui sont opposés ne sont plus "les collecteurs d'impôts et les prostituées"; ce sont les païens $(22,9)$ ou, plus simplement, l'Eglise composée aussi bien de Juifs que de paiens

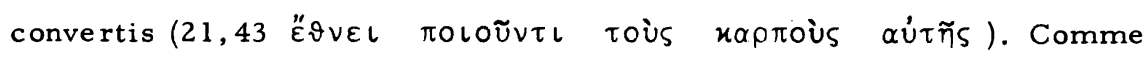

1 Avec BORNKAMM, Enderwartung 25; BULTMANN, Trad 192; HUMMEL 23; STRECKER, Weg 153; WALKER 66.

2 HUMMEL 24. 
l'écrit à juste titre Hummel ${ }^{l}$ : "Die Zusammenordnung des Gleichnisses von den ungleichen Söhnen mit den beiden folgenden Gleichnissen setzt die 'Sünder' des jüdischen Volkes und die Heiden zueinander in Bezie hung". Cette similitude de condition est encore renforcée par le fait que, dans l'évangile de Mt (fidèle sur ce point à ses sources), les seuls modèles de foi - à part "les collecteurs d'impôts et les prostituées" sont deux paíens : le centenier de Capernaüm $(8,5-13)^{2}$ et la Cananéenne $(15,21-28)$.

Concluons. Que ce soit au niveau de leur indignité, de l'appel qui leur est adressé ou de la foi exemplaire avec laquelle ils accueillent la proclamation du Christ, les pécheurs et les pafens se situent sur une même ligne, témoignent d'une condition semblable ${ }^{3}$. Il en résulte pour la communauté mt que toutes les affirmations portées sur les "pécheurs"

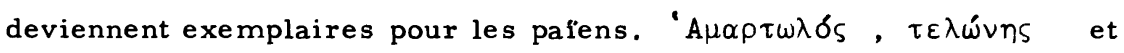
$\pi \delta \rho \vee \eta \quad$ ont également une valeur typologique; ils sont utilisés comme des catégories ambivalentes dans le récit.

\section{HUMMEL 25.}

2 L'histoire du centurion de Capernaüm s'achève par les vv. 10-12 où, comme en 21,31-32, se conjuguent les motifs de l'exemplarité de la foi, de l'appel des pécheurs (ici les paiens) et du rejet d'Israél (v. 12a).

3 Des passages tels que 10,5-6 et 15,24 (inventaire détaillé STRECKER, Weg 99-117) semblent contredire notre analyse et nier toute relation entre les pécheurs et les paléns chez Mt. Pourtant la limitation du ministère de Jésus et de ses disciples à Israël, n'est pas destinée, dans le premier évangile, à régler la stratégie missionnaire, mais à penser le problème d'Israël. Dans la personne du Christ, Dieu est resté fidèle à son peuple et seul l'endurcissement de ce dernier a provoqué son rejet, ouvrant ainsi la voie aux paíens. 10,5-6 et 15,24 n'ont pas une visée particulariste en ecclésiologie, mais apologétique en christologie (cf. SCHWEIZER, Gemeinde $31-34$; STRECKER, Weg 99-122; TRILLING, Israel 99-105). 


\section{L'importance des partenaires de Jésus dans la composition du}

premier évangile

L'enquête détaillée que nous avons menée sur les disciples et les adversaires de Jésus, se justifie par l'intérêt et l'importance de ses résultats. Sans exclure d'autres approches, elle nous permet d'observer de manière exemplaire la démarche rédactionnelle de Mt.

De manière tout élémentaire, cette démarche rédactionnelle se manifeste d'abord dans la redistribution des rôles des partenaires de Jésus. Cette redistribution qui force l'attention et dont tout le monde convient, n'est pas fortuite. Les innombrables corrections, adjonctions, suppres sions opérées dans les sources, la création d'expressions nouvelles pour désigner les différents groupes, témoignent d'une volonté bien arrêtée. Certes, cette volonté dont nous avons tenté de ressaisir les nombreuses manifestations dans le texte, n'est pas exécutée de manière parfaitement rigoureuse. Néanmoins, elle modèle le récit avec une cohérence suffisante pour que nous en découvrions le projet.

Que l'on se tourne vers les disciples, vers les pharisiens et leurs alliés ou encore vers les pécheurs, une même constatation s'impose : Mt s'attache à souligner la valeur typologique de ces différents groupes. Les compagnons de Jésus sont l'image de la communauté croyante; les adversaires du Christ ont le visage de ceux qu'affronte l'église mt; les pécheurs appelés et pardonnés préfigurent l'accueil des paiens. Cette dimension typologique dans sa description des partenaires de Jésus doit être prise au sérieux et analysée dans sa globalité. Elle nous indique notamment que, dans sa présentation, Mt est guidé par un souci de corrélation entre l'histoire qu'il vit et l'histoire qu'il raconte. Cette recherche de la corrélation se manifeste par l'apparition de catégories ambivalentes, c'est-à-dire par l'emploi d'éléments qui sont constitutifs de la trame du récit, tout en ayant leur correspondant immédiat dans la réalité vécue par la communauté mt. Nous touchons ici un point capital dans l'histoire de la rédaction du ler évangile : appliqué à établir une corrélation entre l'histoire de Jésus et le temps de l'Eglise, Mt se devait d'introdui re dans 
son oeuvre des médiations qui réalisent cette visée. Il les a trouvées dans les interlocuteurs de Jésus dont il a dégagé la dimension typologique et qui sont devenus par là-même des catégories littéraires ambivalentes.

Ce traitement rédactionnel des partenaires de Jésus qui aboutit à la mise en place de catégories ambivalentes, est plus important qu'il n'y paraît au premier abord. Il implique notamment l'apparition d'un modèle original dans le fonctionnement du récit. Ce modèle peut être défini par deux règles qui sont dans un rapport dialectique. D'une part, le récit s'applique directement à la réalité de l'église mt (ce qui est dit des dis ciples vaut des croyants, ce qui est affirmé des Juifs concerne la synagogue syrienne, etc...). Cette proposition qui admet une telle immédiateté dans la transmission du sens, présuppose une continuité historique sur laquelle il faudra revenir. D'autre part, le vécu de l'église mt ne peut s'interpréter qu'à travers le récit raconté; cette seconde règle confère à l'histoire de Jésus une dimension normative sur laquelle il faudra également s'expliquer. Le meilleur exemple du modèle que nous venons d'esquisser, est certainement le récit de controverse qui fait intervenir deux catégories ambivalentes : les disciples et les adversaires de Jésus. A titre d'illustration, nous en rappelons brièvement le fonctionnement. A travers l'affrontement entre Jésus et les Juifs - et notamment les accusations que ces derniers portent contre les disciples -, c'est le conflit entre l'Eglise et la synagogue qui est évoqué (première règle !). Mais, à l'inverse, le conflit trouve son aboutissement et le bon droit de l'Eglise est rétabli grâce au rappel de l'enseignement du Jésus terrestre (deuxième règle).

En conclusion, les partenaires de Jésus, jouent un rôle capital dans la composition du premier évangile. Conçus comme des catégories ambivalentes, ils impliquent un fonctionnement particulier du récit. Il faut pourtant bien se garder de ne voir dans ce phénomène qu'un procédé lit téraire et formel. Ce mode de présentation est indissociable d'une conception théologique qu'il s'agit d'éclaircir et de situer dans l'ensemble de la réflexion mt. Pour engager l'analyse de cette théologie implicite, 
nous rappelons en les problématisant les deux présupposés qui ont cons tamment resurgi au cours de notre étude du modèle littéraire : qu'estce qui autorise l'affirmation d'un rapport de continuité entre l'histoire de Jésus et celle de l'Eglise ? Pourquoi et comment l'histoire de Jésus peut-elle être normative pour le présent de la communauté mt ? 
DEU X I E M P A R T I E

LE FONDEMENT CHRISTOLOGIQUE 
CHAPITRE TROIS : LE RESSUSCITE ET LE JESUS TERRESTRE

(Mt 28, 16-20)

L'analyse des protagonistes du premier évangile nous a permis de mettre en évidence l'utilisation conséquente de catégories littéraires ambivalentes. Le même signifiant désigne simultanément un groupe participant à l'histoire du Jésus terrestre et son correspondant dans la situation actuelle de la communauté $\mathrm{mt}$. L'ambivalence des concepts utilisés n'implique pas une similitude de fonction pour les groupes en question. C'est le groupe de l'époque ecclésiale qui se reconnaît et s'identifie au groupe originaire dont l'historicité est maintenue. La transparence du passé pour le présent résulte donc de la possibilité de cette identification.

La mise en oeuvre par Mt de catégories ambivalentes pose deux problèmes : 1) Comment le présent de l'Eglise peut-il se reconnaître dans le passé du Jésus terrestre? 2) Pourquoi le passé du Jésus terrestre a - $t$-il une valeur normative pour l'Eglise au point que cette dernière juge bon de s'y référer sans cesse ? La solution à ces deux questions se trouve dans la conception christologique dont se réclame Mt. Pour répondre à la première qui soulève le problème de la continuité entre le passé de Jésus et le présent de l'Eglise, nous étudierons la conclusion de l'évangile $(28,16-20)$. Pour éclairer la seconde qui est axée sur la place du Jésus terrestre dans la christologie $\mathrm{mt}$, nous nous référerons à $\mathrm{Mt}$ 5,17-20 (notre chap. 4) et à Mt 11,25-30 (notre chap. 5).

\section{Analyse littéraire}

Le plan de la péricope est clair ${ }^{1}$. Il comprend deux parties : les vv. 16-18a décrivent succintement l'apparition du Ressuscité devant ses disciples en Galilée tandis que les vv. 18b-20 rapportent la déclaration que Jésus aurait faite à cette occasion. Cette déclaration comporte trois

1 Cf. BORNKAMM, Der Auferstandene 291; HAHN, Mission 53; MICHEL 19; STRECKER, Weg 210; WILCKENS 69. 
éléments : la révélation de la toute-puissance de Jésus (v. 18b), l'ordre de mission aux disciples (vv. 19-20a), la promesse d'assistance de Jésus (v. 20b). Ces trois éléments sont étroitement liés entre eux : on passe de 18 bà 19-20a par l'adverbe oũv et de 19-20a à 20b par l'expression

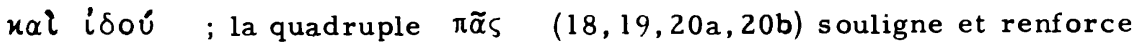
cette unité.

\section{a) Tradition et rédaction}

Mt 18,16-20 appartient au Smt. Nous ne disposons donc d'aucun parallèle, mais nous sommes fondés à croire que Mt - comme tout au long de son évangile - retravaille ici des traditions transmises par sa commu nauté. Notre connaissance des techniques rédactionnelles et du vocabulaire propres à l'évangéliste nous permet d'opérer une séparation élémentaire entre tradition et rédaction ${ }^{l}$.

Nous n'avons pas les moyens de nous faire une conviction définitive $\operatorname{sur} \underline{16 a}^{2}$, mais il semble bien que $\underline{16 \mathrm{~b}}$ soit rédactionnel. D'une part, őpos est dans tout l'évangile le lieu de la révélation (cf. 5, 1; 15,29; 17, 1); ensuite, le verbe $\tau \alpha \dot{\sigma} \sigma \sigma \varepsilon \iota v$ est $\mathrm{mt}$; enfin, le motif rédactionnel du rassemblement sur la montagne introduit une tension avec 28,7,10, passages qui annoncent le nôtre, mais où le motif de l' őpos manque.

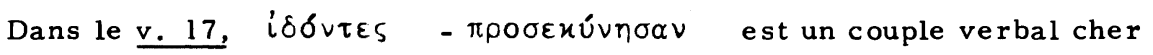
à Mt. Le verbe $\pi \rho \circ \sigma \chi u v \varepsilon \tau \nu$ apparait 13 fois chez Mt, 2 fois chez Mc

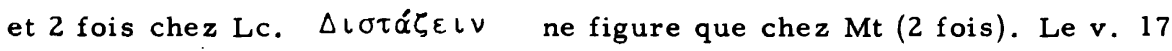
laisse entrevoir un glissement par rapport à $28,7.10$ : alors que $28,7,10$ mettent tout l'accent sur l'apparition de Jésus, 28,17 la rapporte de

1 Voir sur ce point : BARTH $122-123$; KILPATRICK 48-49; STRECKER, Weg 208-210.

2 STRECKER, Weg 208, pense pouvoir affirmer que $\check{\varepsilon} \nu \delta \varepsilon \varkappa \alpha$ et $\varepsilon i \varsigma$ iǹ $\Gamma \alpha \lambda \iota \lambda \alpha i \alpha v$ sont rédactionnels. Ce jugement est trop caté-

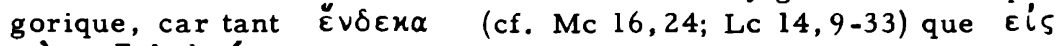

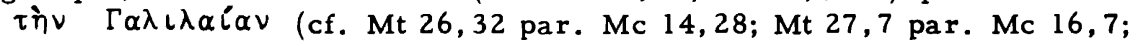
Mt 27,10$)$ sont des motifs bien connus de la tradition pascale des synoptiques. L'argumentation d'adéquation au contexte n'est pas fondée puisqu'il y a précisément tension. 
manière quasiment allusive. Le v. 18a peut être considéré comme ré-

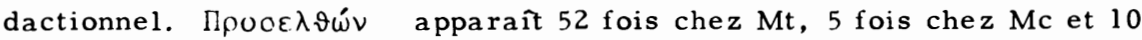

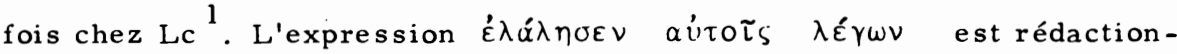
nelle en 13,$3 ; 14,27 ; 23,1$. Nous notons une nouvelle tension : en $28,7,10$ était annoncée une vision d’l Ressuscité et nous assistons à une instruction. Le v. 18b n'est pas rédactionnel. L'opposition oúpavós - $r \tilde{\eta}$, p.ex., est constamment pré-mt ${ }^{2}$. La terminologie du v. 19a est typique -

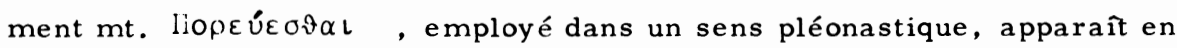

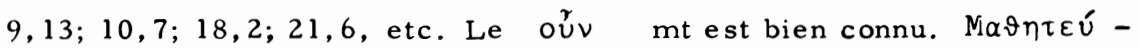
$\varepsilon \iota \downarrow$, verbe propre à Mt, se retrouve dans deux autres passages $(13,52$ et $27,57)$ de manière également rédactionnelle. Ilá $\nu \tau \alpha$ ¿à employé 4 fois dans l'évangile ${ }^{3}$ : une fois sur la base de Mc (Mt 24, 14), une fois en complétant Mc (Mt 24,9), les autres attestations ressortis sent au Smt (Mt 25,32;28,19). Le v. 19b est une tradition liturgique pré $\mathrm{mt}^{4}$. Le v. 20 contient nombre de mots typiquement $\mathrm{mt}$ : $\tau \eta \rho \varepsilon \tau \nu$

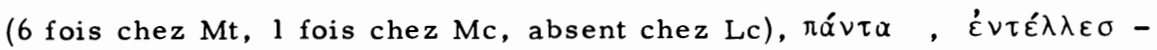

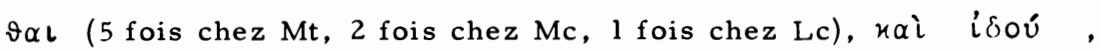

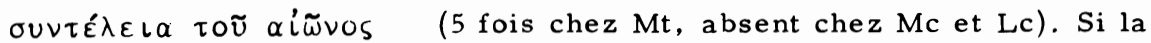
forme actuelle du logion est incontestablement rédactionnelle, ses motifs sont traditionnels (cf. 18,20).

Concluons: L'analyse de la terminologie de notre passage fait apparaître une forte imbrication entre les traditions recueillies par $\mathrm{Mt}$ et la

1 De plus, l'expression formée du participe $\pi \rho \circ \circ \varepsilon \lambda \vartheta \omega ́ \nu$ suivi d'un "verbum dicendi" est exclusivement $\mathrm{mt}$.

2 Avec STRECKER (voir le matériel rassemblé par ce dernier, Weg 209, note 8 ) et contre KILPATRICK 49.

3 TRILLING, Israel 26.

4 Certains ont vu dans le $v$. $19 \mathrm{~b}$ une interpolation tardive et lisent $\mu \alpha-$

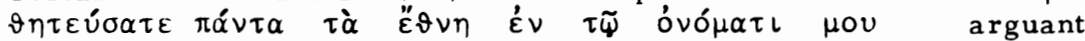
que la formule trinitaire n'apparaît qu'après le Concile de Nicée; mais c'est négliger, d'une part, la bonne tradition textuelle de notre passage, d'autre part, une attestation chronologiquement proche de Mt, celle de Did 7,1 (avec GRASS 31). 
rédaction qu'il en donne. Ainsi, s'il est vrai que les vv. 16-17 utilisent un motif bien attesté par la tradition - l'apparition du Ressuscité aux Onze en Galilée - il n'en reste pas moins que l'empreinte théologique du premier évangéliste est très forte (voir 16b.17). Semblablement, la déclaration faite par Jésus à ses disciples est composée de trois motifs traditionnels (voir plus loin), mais également fortement réinterprétés par Mt (voir en particulier 19a.20a).

Le rédacteur ne réinterprète pas seulement la tradition en la reformulant, mais également en unissant des motifs primitivement indépendants les uns des autres. D'où la question: les traditions figurant dans notre passage constituaient-elles déjà une unité sous leur forme pré-mt ? Et, en particulier, les trois éléments de la déclaration du Christ étaientils déjà liés ? Selon Strecker ${ }^{1}$, 18b.19-20a.20b se présentaient déjà comme une unité achevée dans la tradition reprise par l'évangéliste et avaient leur "Sitz im Leben" dans la liturgie de la communauté mt. Un examen détaillé de la tradition synoptique invite à une plus grande prudence. A part Ac 1 et 2,33.39, Mt 28,18b-20 est le seul passage synoptique où ces motifs sont mis en rapport les uns avec les autres. Dans tous les autres cas, chacun des motifs apparaît séparément (ainsi le motif du v. 18b en Mt 11,27, Jn 3,35; celui de 19b en Mc 16,15; celui de 20b en Mt 18,20). La tradition $n^{\prime}$ atteste que rarement une parenté entre ces trois thèmes et il est donc plus vraisemblable que ce soit l'évangéliste lui-même qui a réuni ces trois logia ${ }^{2}$.

Ce résultat a une conséquence directe. En effet, si 18b-20 sont des logia réunis par l'évangéliste, il est impossible qu'ils aient été transmis avec 16-17. D'ailleurs, la tradition de l'apparition de Jésus aux Onze en Galilée a été traitée de tout autre façon par Mc et donc pas dans le sens

\section{STRECKER, Weg 210.}

2 Avec BARTH 124; BORNKAMM, Der Auferstandene 291; HAHN, Mis sion 53; MICHEL 20.21. Sans les attribuer explicitement à Mt, GRASS 30 voit également dans les vv. 18b-20 une composition tardive. 
suggéré par les vv. $18 \mathrm{~b}-20^{\mathrm{l}}$.

Concluons. En 28,16-20, l'activité rédactionnelle de Mt se manifeste aussi bien par la collation de motifs traditionnels indépendants que par leur reformulation. Notre passage est une composition originale du premier évangéliste et, à ce titre, il est représentatif de sa théologie.

\section{b) La tradition christologique utilisée dans les vv. 18b-20}

Malgré son importance capitale pour la compréhension du Christ mt, 28,16-20 ne contie nt aucun titre permettant d'identifier d'emblée la tradi tion christologique qui lui est sous-jacente. Il convient donc d'analyser les motifs de notre passage pour décider à quel type de christologie il se rattache. La plupart des exégètes admettent que Dn $7,13.14$ est le modèle qui a inspiré Mt 28,18-20 et qu'à ce titre notre péricope est dominée par la christologie du Fils de l'homme ${ }^{2}$. Mais Dn 7,13.14 est-il vraiment un point de départ satisfaisant pour interpréter notre passage ? C'est le mérite de Vögtle ${ }^{3}$ d'avoir montré qu'il n'en est rien. Nous rappelons brièvement le point décisif de sa démonstration. Mt 28,18 ne peut être considéré comme l'accomplissement de Dn 7,13 dans la mesure où, chez Mt (cf. 24,30c; 25,31 -46;26,46b), la venue du Fils de l'homme sur les nuages appartient au cycle de la parousie et non de Pâques ${ }^{4}$; en d'autres termes, Dn 7,13 est chez Mt l'objet d'une espérance et donc inadéquat pour rendre compte de l'événement décrit en 28,18. De plus, la narration daniélique développe une série de thèmes absents chez Mt (présentation du Fils de l'homme devant Dieu, éternité et indestructibilité de son royaume, soumission politique des peuples) et ne comporte pas ceux qui

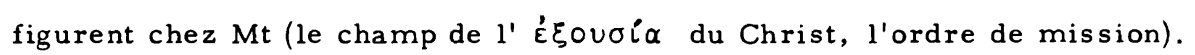

1 Sur ce point, voir plus bas notre analyse de l'arrière-fond ecclésial.

2 Ainsi, p. ex., BARTH 125; BONNARD, Mt 418; GRASS 30; LOHMEYER, Mt 425s; MICHEL 22; SCHNIEWIND, Mt 276; WILCKENS 70.

3 VOEGTLE $266-294$.

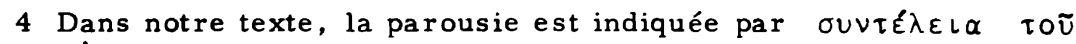
aĩ̄vos : elle est donc située dans le futur. 


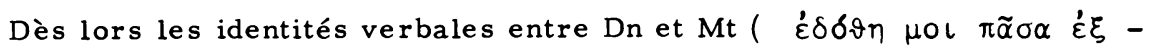

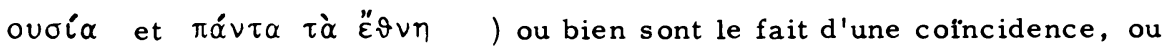
bien remontent effectivement à $\mathrm{Dn} 7,13$; dans ce cas, Dn 7,13 a été si complètement réinter prété par Mt qu'il n'est plus d'aucune utilité pour élucider notre péricope.

A notre avis, pour parvenir à une solution satisfaisante, il convient plutôt de se demander : comment le Ressuscité est-il présenté dans les vv. 18-20? Comme le signale le v. 18 et, en particulier, la notion d' $\dot{\xi} \zeta o v o i ́ \alpha$, le Ressuscité est investi de la toute-puissance sur le ciel et sur la terre, il occupe désormais la fonction de Kyrios. C'est dire que, dans notre texte, résurrection et élévation sont conçues comme un seul événement ${ }^{l}$. L'identification de ces deux motifs n'est pas propre à Mt; elle est très ancienne et se retrouve en particulier dans les traditions prépauliniennes $^{2}$. Elle signifie que, par la résurrection, le Christ a été élevé par Dieu au rang de Seigneur tout-puissant. A cette première connexion archafque s'en ajoute une seconde tout aussi intéressante : la xupıó̃ns du Ressuscité est liée à la mission universelle. Cette association n'est pas née dans le judéo-christianisme palestinien où la tâche missionnaire - d'ailleurs souvent limit ée à Israẹl - est légitimée par l'imminence de la parousie du Fils de l'homme, mais bien dans le christianisme hellénistique ${ }^{3}$. S'il est vrai qu'à l'origine, dans ce dernier, la

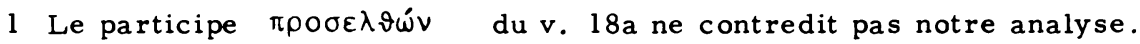
En effet, Mt l'utilise fréquemment comme un stéréotype signalant le début d'une instruction du Christ.

2 Sur ce point, voir WILCKENS 37-39.53-55.70.71. A titre de tradition prépaulinienne liant résurrection et élévation, on peut citer p.ex. Rm 1,3.4. Il convient d'ajouter que tant la notion d'élévation (HAHN, Mis sion 53.54) que la foi au Kyrios (BORNKAMM, Der Auferstandene 295) ont leur origine dans le judéo-christianisme hellénistique et, en particulier, dans le groupe des "hellénistes".

3 Dans le judéo-christianisme palestinien particulariste, la mission ne s'exerce qu'à l'égard d'Israêl; la conversion des paiens est réservée à Dieu lors de la fin. La conception d'une mission adressée d'emblée à tous les peuples et légitimée par la kupıó̃ns du Christ ne peut se développer que dans le judéo-christianisme hellénistique (HAHN, Mis sion 43-48. 57). 
xuplóns du Christ signifiait d'abord la victoire sur les puissances du monde, elle suscite rapidement une compréhension définie des charis mes et de la prophétie, c'est-à-dire de la mission des disciples ${ }^{l}$.

Concluons ces brèves remarques. L'étroite connexion que Mt établit entre résurrection et élévation, d'une part, élévation et mission univer selle, d'autre part, nous invite à penser que la tradition christologique dont il s'inspire ici est la confession de foi hellénistique au Kyrios et non le modèle daniélique. Nous verrons plus loin comment il infléchit cette tradition christologique à partir de sa compréhension de la Loi ${ }^{2}$.

c) L'arrière-fond ecclésial ${ }^{3}$

Pour déterminer l'insertion ecclésiale de notre péricope, il est capital de remarquer que Mt n'accorde quasi aucune importance à la description de l'apparition du Christ comme telle; le cadre historique a presque

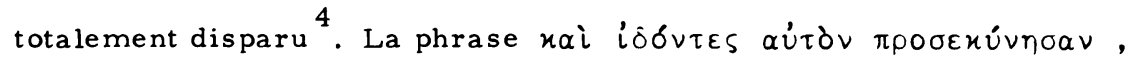
oi $\delta \varepsilon \dot{\varepsilon} \delta i \sigma \tau \alpha \sigma \alpha \nu \quad(28,17)$ présuppose l'apparition sans la décrire. Le texte ne nous dit rien sur les circonstances de l'apparition du Christ, puis de sa disparition, rien sur la joie, la frayeur ou la confession de foi des disciples alors que ces divers éléments occupent généralement une place importante dans les récits parallèles (Jn 20,16.20; Lc 24,25s s., 3 lss.). Dans ces conditions, le motif du doute $(28,17)$ prend une impor -

1 BORNKAMM (Der Auferstandene 294-299) a fait une étude détaillée de l'histoire des relations entre élévation et mission universelle à laquelle nous renvoyons. A titre d'exemples où le culte du Kyrios débouche sur une compréhension particulière de la mission, citons la conclusion se condaire de $\mathrm{Mc}(16,15 \mathrm{ss})$, la conception de l'apostolat et la description des "hellénistes" dans les Ac, l'interprétation de l'apostolat dans les deutéro-pauliniennes.

2 Avec BORNKAMM, Der Auferstandene 297.298.30l et BULTMANN, Trad 313.

3 Voir avant tout sur ce point : BORNKAMM, Der Auferstandene 289. 290 , et MICHEL $17-19$.

4 BORNKAMM, Der Auferstandene 289; DIBELIUS, Formgeschichte 285; LUZ, Jünger 162 . 
tance particulière ${ }^{l}$ bien qu'il s'agisse là encore d'un lieu commun des péricopes pascales ${ }^{2}$. L'originalité de Mt tient dans la réponse qu'il donne au doute des disciples. Le doute n'est pas dissipé par un geste matériel du Christ (Lc 24,4lss.), ou par une vérification empirique des disciples (Jn 20,24ss.), ou par une nouvelle apparition du Ressuscité (Mc 16, 14ss.), mais par la seule parole de l'Elevé. Le disciple doit surmonter son doute en renonçant à toutes preuves matérielles et en s'en remettant à la seule parole de l'Elevé ${ }^{3}$. Si, à la problématique de la ré-

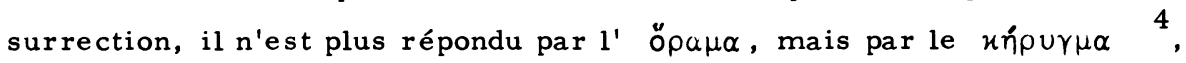
alors nous n'avons plus affaire à la couche la plus ancienne de la tradition, mais à une situation plus tardive. Cette situation est celle de la communauté $m$ t en quête d'une certitude quant à la résurrection. Mt 28 , $16-20$ répond à cette question ${ }^{5}$ : comment confesser le Ressuscité dont la manifestation historique appartient apparemment à un passé révolu ?

d) Forme littéraire et contexte

La détermination de la forme littéraire des vv. 16-20 est difficile, car nous n'avons pas affaire à un texte construit selon un schéma classique et bien connu. Notre péricope n'est assimilable ni au schéma de révé-

1 BORNKAMM, Der Auferstandene 290, et LUZ, Jünger 162.

2 Le motif du doute s'amplifie avec le développement de la tradition. Il est déjà beaucoup plus massif chez Lc $(24,36-49)$ et chez Jn $(20,19$ 23.24-29), cf. l'analyse de WILCKENS $71-74$. Il atteint son paroxysme dans les évangiles apocryphes (matériel chez GRASS 29).

3 Ainsi WILCKENS 69. Par ailleurs, on retrouve le même schéma dans la finale secondaire de Mc $(16,9-20)$.

4 MICHEL 19.

5 Le fait que Mt réponde à une question présente de sa communauté par la narration d'un événement passé est souligné par la manière dont s'achève la péricope : les paroles de l'Elevé ne sont suivies d'aucun récit de séparation ou d'ascension, mais s'adressent directement à l'église mt et ceci jusqu'à la fin des temps. 
lation gnostique ${ }^{l}$, ni à celui des premières confessions de foi chrétiennes célébrant l'intronisation du Christ ${ }^{2}$. Il ne s'agit pas non plus, à proprement parler, d'un récit d'apparition ${ }^{3}$, puisque le fait de l'apparition ne joue quasi aucun rôle (voir plus haut). Mt ne nous présente pas non plus un discours d'adieux ${ }^{4}$ puisque le thème du départ est précisément évité, ni une légende cultuelle accréditant la pratique du baptême ${ }^{5}$, car ce n'est pas là le but du texte. Il faut plutôt y voir une histoire pascale dont le centre est l'ordre de mission donné aux disciples par le Ressuscité et qui a sa source dans la tradition archaique des apparitions en Galilée ${ }^{6}$. On ajoutera que notre scène a un caractère "idéal" dans la mesure où, en elle, Mt a résumé et concentré toutes les traditions d'apparition aux disciples ${ }^{7}$.

Il est important de remarquer que Mt 28,16-20 clôt à la fois le cycle pascal et l'évangile de Mt. Mais il ne l'achève pas en tant que dernière péripétie chronologique d'une longue hist oire. Notre péricope est tout à la fois la conclusion théologique et le sommaire de l'évangile dans son

1 Contre DIBELIUS, Formgeschichte 285. Le schéma gnostique suggéré par DIBELIUS se compose de deux éléments : autorecommandation et appel. Or le v. 18 ne décrit pas la qualification du révélateur (comme 11,27 p.ex.), mais l'installation du Ressuscité dans sa fonction de Kyrios.

2 Contre HAHN, Mission 52-57 et MICHEL 22.23. Le schéma des premières confessions de foi (ex.: $\mathrm{Ph} \mathrm{2,9-11)} \mathrm{est} \mathrm{composé} \mathrm{de} \mathrm{trois} \mathrm{élé-}$ ments : intronisation-proclamation-acclamation. Or le v. 18b présuppose l'intronisation du Kyrios sans la décrire, les vv. 19.20a ne sont pas une proclamation adressée aux puissances, l'acclamation ne retentit nulle part.

3 Contre LOHMEYER, Gewalt 26.27.

4 Contre MUNCK 165.

5 Contre BULTMANN, Trad 313.314, et, proche de lui, STRECKER, Weg 210.

6 Avec BORNKAMM, Der Auferstandene 289, et BULTMANN, Trad ibid. Cf. aussi Lc 24,44-49; Ac 1,4-8; Jn 20,19-23.

7 Avec von CAMPENHAUSEN, Osterereignisse 17, et GRASS 28. 
ensemble; elle est "la clef de compréhension du livre dans sa totalité"l.

\section{Inter prétation}

Selon le v. 16, le cercle des disciples qui assistent à la scène finale de l'évangile est restreint aux Onze ( oi $\delta \varepsilon \check{\varepsilon} \nu \mathcal{E} \varepsilon \varkappa \alpha \mu \alpha \vartheta \eta \tau \alpha \iota$ n'inter -

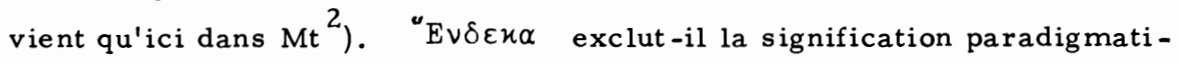
que de $\mu \alpha \vartheta \eta \tau \eta ́ s$, pourtant si fréquente dans l'évangile? Trois raisons nous invitent à voir dans les Onze les premiers disciples, mais en tant qu'ils sont représentatifs de l'Eglise dans son ensemble. Première ment, l'étude de l'arrière-fond ecclésial de notre péricope nous a montré qu'à travers l'attitude des Onze, c'est la question de la communauté mt quant à la résurrection de son Seigneur qui se fait jour. Deuxièmement, le v. 17 évoque la condition paradoxale et typique du disciple, c'est-àdire d'un disciple partagé entre la foi et le doute. Troisièmement, l'utilisation du verbe $\mu \alpha \vartheta \eta \tau \varepsilon \cup ́ \varepsilon \iota \nu$ au v. 19 démontre que la condition du disciple n'est pas limitée aux seuls témoins oculaires et croyants du Jésus terrestre.

Le voyage des disciples en Galilée est conforme à l'ordre donné par l'ange en 28,7 et par le Ressuscité en 28,10 (cf. aussi 26,32). Il n'est d'ailleurs théologiquement pas indifférent que le Ressuscité retrouve les siens en Galilée, lieu où se déroula l'essentiel de son ministère; "cela suppose une continuité entre le Christ terrestre et le Christ ressuscité, continuité que le v. $20 \mathrm{a}$ soulignera explicitement" ${ }^{3}$. L'élément introduit par Mt consiste en ce que le rendez-vous a lieu sur une montagne ${ }^{4}$. En

1 MICHEL 21.

228,7 et 28,10 qui annoncent le même événement, parlent respective -

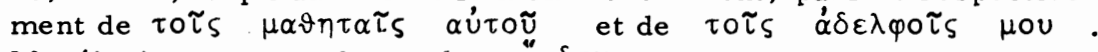

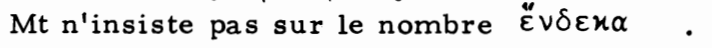

3 BONNARD, Mt 417.

4 Il en résulte une tension avec 28,7,10 qui ignore tout du motif de l'őpos. 
insérant le motif de l' őpos dans sa narration, l'évangéliste veut donner à comprendre que la rencontre qui suit a valeur de révélation, car la montagne désigne constamment dans l'évangile le lieu de la révélation ${ }^{1}$.

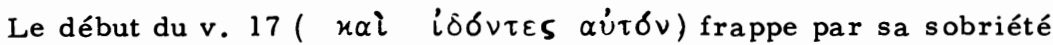
dans la description de la rencontre entre Jésus et les Onze. L'apparition du Ressuscité n'est même pas décrite, elle est simplement présupposée

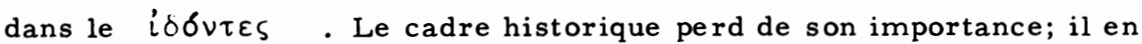
résulte une tension avec $28,7,10$ où tout le poids porte sur l'apparition en

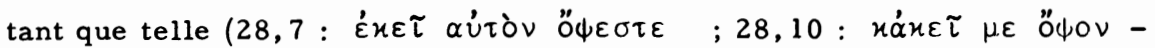
$\tau \alpha \iota)$. Ce recul de l'intérêt documentaire rend perceptible l'imbrication de la situation de la communauté $\mathrm{mt}$ dans le récit, car, pour elle, le thème de la vision du Ressuscité a perdu sa force de persuasion et de signification $^{2}$.

A l'apparition du Ressuscité répond le proskynèse des disciples. Le

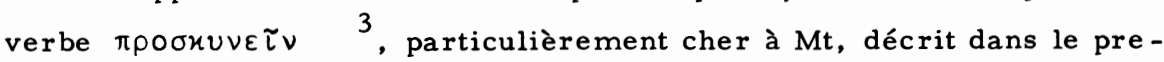
mier évangile la dépendance confiante, voire l'adoration que l'homme voue à Jésus. Cette relation de soumission résolue est l'indice de la foi. Mais confrontés au Ressuscité, les disciples ne font pas que croire, ils doutent aussi. $\triangle \iota \sigma \tau \alpha ́ \zeta \varepsilon \iota \nu{ }^{4}$ est un mot propre à Mt et qu'il applique aux seuls

1 Avec LOHMEYER, Gewalt 24.25, et SCHMAUCH 67-80 (en particulier 71). Plus hésitant : DELLING, art. őpos , ThW 5, 484-485.

2 Avec BORNKAMM, Der Auferstandene 290; GRUNDMANN, Mt 576 ; MICHEL $17-19$.

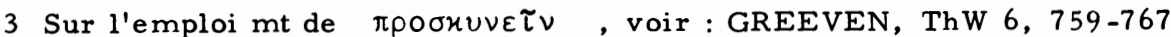
(en particulier 764); HAHN, Hoheitstitel 86 (note 1); HELD 217. Проб-

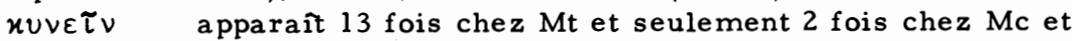
2 fois chez Lc. Il décrit toujours une attitude de confiance et de respect face à Jésus (jamais affirmé de ses adversaires !). Se prosternent devant Jésus : a) les mages $(2,2.8 .11)$, b) les disciples $(14,33$; $28,9.17$; voir aussi 18,26$)$, c) les personnes sollicitant son aide $(8,2$; $9,18 ; 15,25 ; 20,20) .4,9,10$ sont deux attestations appartenant au récit de la tentation.

4 HELD 283. 
disciples $(14,31 ; 28,17)$; ce verbe décrit, de manière analogue à $l^{\prime}$ ó $\lambda \iota$ -

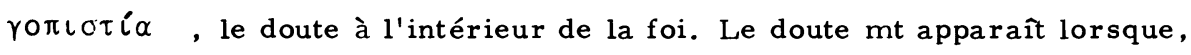
dans la vie des disciples, les évidences mondaines mettent en échec la foi alors que la foi devrait dépasser les évidences mondaines. Ici, par exemple, l'évidence de l'impossibilité de la résurrection prend le pas sur la confiance dans la promesse de Jésus $(c f .26,32)$ et sur la réalité de son apparition.

Les disciples ${ }^{1}$ qui rencontrent le Ressuscité sont partagés entre la foi et le doute. Ils sont incroyants au sein même de leur existence croyante $^{2}$. N'est-ce pas là la situation de la communauté mt en quête d'une nouvelle certitude quant à la résurrection ?

Le v. 18a est rédactionnel (voir plus haut). Il introduit directement $l^{\prime}$ instruction du Ressuscité sans que l'on sache si les disciples sont dans la joie ou la frayeur, s'ils ont reconnu leur maître ${ }^{3}$. Le cadre historique est de nouveau réduit à sa plus simple expression si bien que tout le poids du récit porte sur les paroles qui suivent. Mt utilise les vv. 16-18a pour préparer et introduire les vv. 18b-20; la mention de $1^{\prime}$ ŏp $\alpha \mu \alpha \quad(16-18 a)$ est au service du $u n ́ \rho \cup \gamma \mu \alpha(18 b-20)$. Au doute de la communauté est

1 Le oi $\delta \varepsilon ̀ ~ દ ́ \delta i ́ \sigma \tau \alpha \sigma \alpha \nu$ doit-il être traduit par "mais certains doutèrent" (ainsi BONNARD, Mt 416; LOHMEYER, Mt 412; SCHNIEWIND, Mt 275; mais aussi Bl-D par. 250), en supposant que le oi $\delta \xi$ a une valeur à la fois adversative et restrictive ? Cela nous semble contestable car le oi $\delta \varepsilon$, construit sans le oi $\mu \varepsilon ́ v$, peut égale ment avoir une valeur exclusivement adversative (cf. Bl-D par. 251). Nous traduirions alors : "mais ils doutaient" (avec GRUNDMANN, Mt 572).

2 Tant ALLEN, Mt 385, que LAGRANGE, Mt 543, méconnaissent cette situation de crise dans l'existence croyante, pourtant si typique de la rédaction $\mathrm{mt}$. Le $\dot{\delta} \delta \zeta \sigma \tau \alpha \sigma \alpha \nu$ se rapporte dès lors soit à d'autres personnes, soit à un événement antérieur.

3 Il s'agit d'un motif constant dans les récits du cycle pascal. Dans Lc 24, Jésus dissipe le doute des disciples (vv. 25-27.30.38-43), provoque leur frayeur (v. 37), leur joie (vv. 41.52). Dans Jn 20, le motif de la joie apparaît au v. 20 , celui de la dissipation du doute aux vv. $16.24-28$. 
opposée non pas l'apparition du Ressuscité comme telle, mais la parole qu'il a prononcée à cette occasion. Le Ressuscité édifie sa communauté en allant à elle par sa parole ${ }^{1}$. Mais quelle est cette parole ?

Le centre de la première partie de l'instruction du Ressuscité à ses disciples (v. 18b) est constitué par la notion d' $\mathrm{d}^{\prime} \zeta o v \sigma i ́ \alpha$. Il ne s'agit pas là d'un élément qui distingue le Ressuscité du Jésus terrestre ${ }^{2}$. Avant Pâques déjà, toutes choses lui ont été remises par le Père $(11,27)^{3}$. Que ce soit son enseignement $(7,29)$, son activité miraculeuse $(8,9)$, son action pardonnante $(9,6,8)$, tout son ministère est déjà placé sous le signe

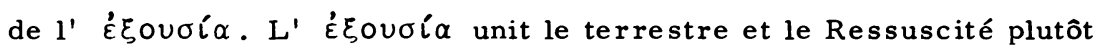
qu'elle ne les sépare. Bien plus, tant chez le terrestre $(11,25-20 ; 11,29$ : $\mu \alpha \vartheta \varepsilon \tau \nu$ ) que chez le Ressuscité $(28,18-20 ; 28,19: \mu \alpha \vartheta \eta \tau \varepsilon v ́ \varepsilon \iota \nu)$, elle se concrétise dans l'appel à revêtir la condition du disciple ${ }^{4}$.

Par rapport au reste de l'évangile la nouveauté de Mt 28,18 ne réside donc pas dans le fait que le Ressuscité est le dépositaire de l' ȩ́ovoía, elle consiste en ce que cette Éร̧ovoía s'exerce désormais partout sans

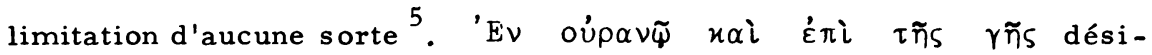
gne ici, comme d'ailleurs en 5, 18 et en 24,35, le tout, l'ensemble du monde, la création dont Dieu est le Seigneur, cf. $11,25^{6}$. Le don qui est fait au Ressuscité est donc à la fois la confirmation de l'ézovoía qu'il détenait déjà et son extension universelle.

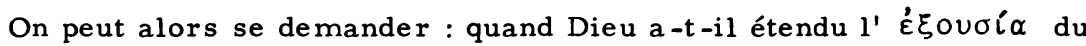
Fils à l'universalité ? Comment faut-il interpréter l'aoriste passif $\dot{\varepsilon}$ -

1 GRUNDMANN, Mt 576; MICHEL 21.22; WILCKENS 69.

2 Contre KLOSTERMANN, Mt 231.

3 Comme en témoigne le verbe $(\pi \alpha \rho \alpha) \delta i \delta \omega \mu \iota$ qui apparaît aussi bien en 11,27 qu'en $28,18 \mathrm{~b}$, le Père est la source souveraine de l'autorité de Jésus avant et après sa résurrection.

4 BORNKAMM, Der Auferstandene 293.

5 Ainsi STRECKER, Weg $211-212$.

6 SCHMID, Mt 391, et TRILLING, Israel 24. 
$\delta \delta \vartheta \eta \quad$ ? W. Grundmann ${ }^{l}$ propose de fixer ce moment au $\alpha^{\prime} \pi^{\prime \prime} \alpha \rho-$ $\tau \iota$ de 26,64 , certains le lient à l'événement de la résurrection, d'autres enfin le situent entre la résurrection et l'événement dont parle le texte ${ }^{2}$. L'absence de solution contraignante est significative, car le texte ne dit pas quand le Christ a été investi par le Père ${ }^{3}$, mais il insiste en revanche sur le fait que le Ressuscité règne universellement, maintenant et jusqu'à la fin des temps (v. 20). Résurrection et élévation constituent un seul événement chez $\mathrm{Mt}^{4}$. Les vv. 19.20 préciseront comment $\mathrm{Mt}$ comprend cette tradition très ancienne (cf. p.ex. l Co 15,20-28) qui trouve son expression achevée dans la confession du Kyrios ${ }^{5}$. L'instruction mis -

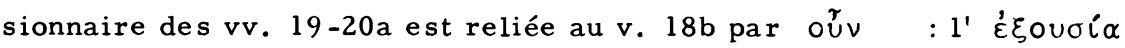
du Christ sur la création débouche concrètement sur la tâche qu'il confie à ses disciples. Quelle est la structure interne des vv. 19-20a ? On peut admettre que le v. 19a présente l'idée principale, développée ensuite par

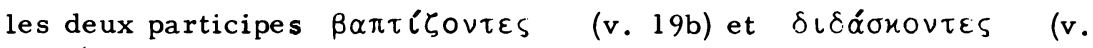
20 a) ${ }^{6}$.

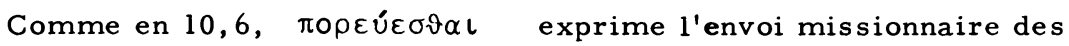
disciples ${ }^{7}$. Cette mission consiste à faire de toutes les nations les dis -

1 GRUNDMANN, Mt 577.

2 BONNARD, Mt 418 , et LOHMEYER, Mt 416.

3 Une fois encore, $18 \mathrm{~b}$ n'est pas une assertion directe sur l'acte d'intronisation, mais une parole témoignant d'un acte déjà accompli ( $\varepsilon \delta \delta \vartheta \eta$ ). Ce fait souligne la faiblesse de la thèse voyant dans notre passage $1^{\prime}$ l'emploi du schéma d'intronisation.

4 Déjà ainsi : B. WEISS, Mt 507.

5 On souligne souvent que le v. $18 \mathrm{~b}$ est proche de Dn 7,14a (LXX) :

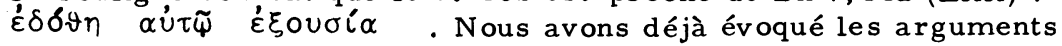
qui s'opposent à une telle filiation. Remarquons simplement que $\delta i \delta \omega \mu \iota$ et $\dot{\varepsilon} \xi o v \sigma i \alpha$ sont également mis en rapport par Mt dans d'autres contextes parfaitement étrangers à Dn 7,$14 ; \mathrm{cf} .9,8 ; 10,1$; 21,23 .

6 BAUMBACH, Mission 891; SCHLATTER, Mt 798; WEISS, Mt 508.

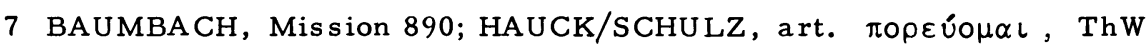
6, 574-575; WEISS, Mt 508 . 


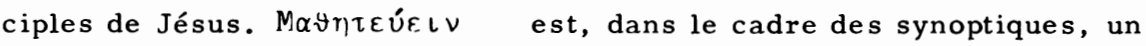
terme propre à $\mathrm{Mt}^{\mathrm{l}}$. On le retrouve en 13,52 et en 27,57 sous la forme d'un déponant signifiant "être, devenir disciple" alors qu'en 28, 19, il s'agit d'une forme transitive active se traduisant par "faire de quelqu'un un disciple" ${ }^{2}$.

L'emploi mt du verbe $\mu \alpha \vartheta \eta \tau \varepsilon u ́ \varepsilon \iota \nu$ dans un contexte missionnaire est riche de sens. Il signifie en effet que la condition du disciple telle qu'elle est décrite dans l'évangile n'est pas l'apanage des seuls compagnons historiques du Jésus terrestre, mais qu'au contraire, elle est la condition dans laquelle tout homme est désormais invité à entrer ${ }^{3}$. S'il en est ainsi, la figure du disciple telle qu'elle apparaît dans le tout de l'évangile, n'appartient pas à un passé désormais révolu, elle est, au contraire, une catégorie paradigmatique dans laquelle chaque croyant est appelé à se reconnaître. " $0 i \quad \mu \alpha \vartheta \eta \tau \alpha i ́$ est la notion ecclésiologique spécifique de l'évangéliste" 4 .

Les disciples sont placés devant l'exigence de faire de toutes les

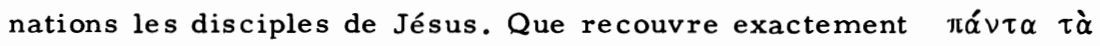

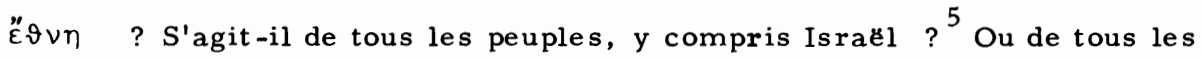
peuples à l'exclusion d'Israël, à supposer que l'opposition עמ / גוימ

1 Pour décrire l'activité missionnaire, Mc $(16,15)$ et Lc $(24,47)$ utili-

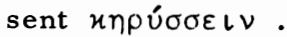

2 BAUER, WB 148; RENGSTORF, art. $\mu \alpha \vartheta \eta \tau \varepsilon \cup ́ \omega$, ThW 4, 465; TRILLING, Israel 28-31.

3 BORNKAMM (Der Auferstandene 301) écrit à ce propos : "Offensicht lich wird mit dem entsprechenden Begriff $\mu \alpha \vartheta \eta \tau \varepsilon u ́ \varepsilon \iota \nu(28,19)$, der ursprünglich von dem Verhältnis zum Irdischen hergenommen ist, eben dieses Verhältnis auch jetzt nach der Auferstehung für bleibend verbindlich erklärt". Voir aussi, dans la même direction, LOHMEYER, Mt 419 , et TRILLING, Israel 30-31.

4 BORNKAMM, Der Auferstandene 301.

5 LOHMEYER, Mt 418; SCHLAT TER, Mt 798; SCHMID, Mt 391; SCHMIDT, art. "̈ษ vos, ThW 2, 366-367; STRECKER, Weg 117; TRILLING, I'srael $31-32$. 


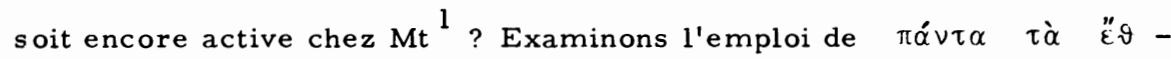
$\eta$ qui, hormis notre passage, apparait encore en $24,9.14 ; 25,32 ; 24,9$ et 25,32 désignent toutes les nations y compris Israël, alors que le sens de 24,14 est ambigu ${ }^{2}$. Si l'on ajoute à cela la visée rhétorique du texte qui parle moins d'exclusion que d'extension, on comprendra de préférence

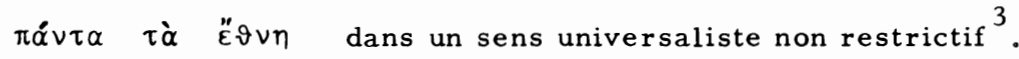

En élucidant $\mu \alpha \vartheta \eta \tau \varepsilon \cup ́ \varepsilon \iota \nu$ par le baptême d'abord, par l'enseigne ment ensuite, Mt montre que son intérêt est dirigé vers la vie de l'Eglise plutôt que sur la pratique missionnaire comme telle, qu'il cherche à définir la règle de vie d'une Egli se universaliste ${ }^{4}$.

L'homme devient d'abord le disciple du Christ en recevant le baptême

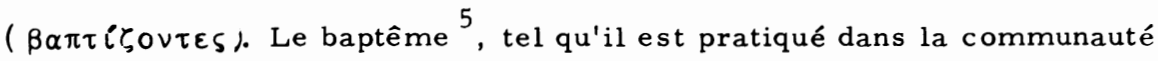
$m t$, est l'acte par lequel le croyant accepte dans l'obéissance l'appel et l'exigence que lui adresse le Christ; il fonde et scelle son appartenance au Seigneur et, du même coup, à l'Eglise. L'expression $\varepsilon$ is ővor $\alpha$ souligne l'aspect sotériologique de l'acte baptismal : en invoquant sur le prosélyte le Père, le Fils et le Saint-Esprit, la communauté l'introduit dans la réalité du salut. La formule triadique appartient à la pratique liturgique de $1^{\prime}$ église $\mathrm{mt}^{6}$.

1 WALKER $111-113$.

2 Analyse détaillée chez TRILLING, Is rael 26-28.

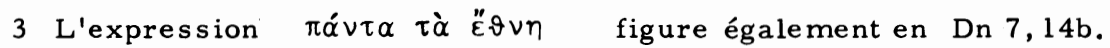
Mais si le Fils de l'homme daniélique asservit et soumet les peuples par sa force et son autorité, pour le Christ mt, ils sont objet de mis sion (GRUNDMANN, Mt 577 et LOHMEYER, Mt 418).

4 BORNKAMM, Der Auferstandene 304.

5 Voir sur ce point : BIETENHARD, art. ővora , ThW 5,274; DELLING, Taufe 94-96; GRASS 30; GRUNDMANN, Mt 578; MICHEL 24; STRECKER, Weg 216.

6 BORNKAMM, Der Auferstandene 304; STRECKER, Weg 210. 
L'homme devient ensuite le disciple du Christ en conservant l'enseignement de Jésus ${ }^{l}$. Cette dernière élucidation est plus importante que la première, car si, pour évoquer le baptême, Mt utilise une tradition liturgique, pour présenter l'enseignement de Jésus il recourt à une terminologie qui lui est propre et donc représentative de sa théologie ${ }^{2}$. Le verbe $\tau \eta \rho \varepsilon \tau \nu$, dans $M t$, désigne l'obéissance fidèle et concrète aux commandements divins (cf. Mt 19,17 et 23,3$)^{3}$. Mais quelle obéis sance les Onze doivent-ils enseigner ? Quel est le contenu de la fidélité dont ils sont les dépositaires ? La réponse tient dans l'expression ŏ $\sigma \alpha$

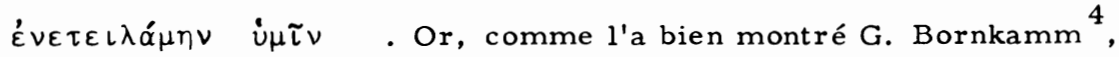
cette expression a deux particularités. Premièrement, le verbe $\varepsilon^{\nu}$ $\tau \varepsilon ́ \lambda \lambda \varepsilon \sigma \vartheta \alpha \iota$ définit et caractérise l'ensemble de la prédication de Jésus comme un commandement. C'est là un cas unique dans toute la tradition synoptique ${ }^{5}$; dans un contexte semblable, Lc présente la prédication de

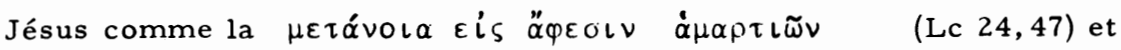

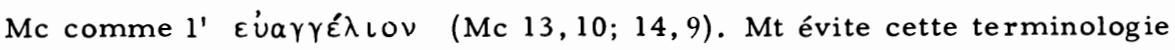

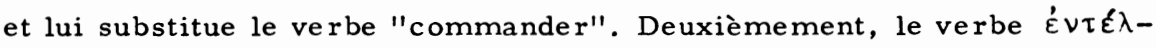
$\lambda \varepsilon \sigma \vartheta \alpha \iota$ est à l'aoriste. Ici encore, Mt fait cavalier seul, car la tradi tion préfère parler de la prédication de la parole présente du Seigneur élevé par la bouche de ses prophètes inspirés (Jn 16,13ss.; 14,25ss.;

1 L'ordre de succession baptême - enseignement ne reflète pas une pratique ecclésiale. Le point de vue adopté ici par Mt est principiel : de même que le baptême, la pratique des commandements divins est constitutive de la condition chrétienne (ainsi aussi TRILLING, Israel 39).

2 Ainsi déjà WEISS - BOUSSET, Schriften NT I 392. Ajoutons : le fait que Mt utilise une tradition liturgique pour parler du baptême suppose sa pratique régulière dans la communauté. Par contre, sa mention de l'enseignement des commandements ne reflète aucune activité ecclésia le définie.

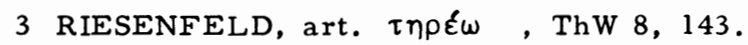

4 BORNKAMM, Der Auferstandene 304.

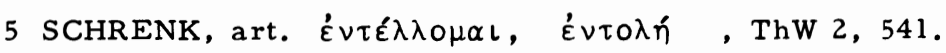


Ac 13,2; Ap. 2,7.11.17; 18,20). Mt se concentre non pas sur ce que le Seigneur élevé dit présentement à l'Eglise, mais sur ce que le Jésus

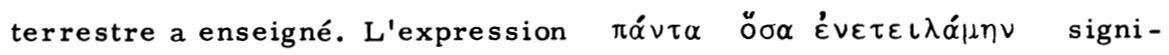
fie donc que "le Ressuscité, et l'Elevé, rend normatif pour l'Eglise... de tous les temps et jusqu'à la fin du monde, la parole du Jésus terres tre" ${ }^{1}$. Le message du Ressuscité est identique à celui du Jésus terrestre. Le Kyrios n'est personne d'autre que le didascale Jésus de Nazareth. Annoncer le Ressuscité implique le rappel de la prédication du Jésus his -

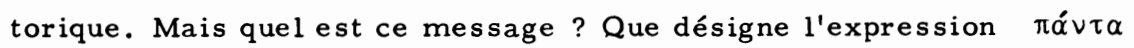

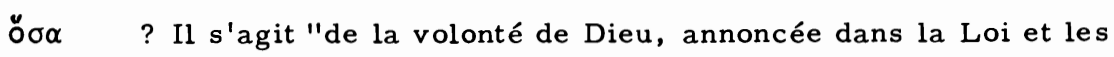
Prophètes, interprétée souverainement dans l'enseignement de Jésus, concrétisée et résumée dans le commandement d'amour" ${ }^{2}$.

Le v. $20 \mathrm{~b}$ constitue la troisième et dernière partie de la déclaration du Ressuscité. Son thème est l'assistance permanente du Ressuscité à ses disciples ${ }^{3}$. Le nai iEoú relie $20 \mathrm{~b}$ à ce qui précède. L'expres sion $\mu \varepsilon \vartheta^{\prime} \dot{u} \mu \tilde{\omega} \nu \varepsilon i \mu \iota$ est d'origine vétérotestamentaire ${ }^{4}$ : Yahvé promet son assistance à des individus particuliers ou au peuple dans son ensemble, quand il s'agit de remplir une mission ou d'affronter un danger (exemples : Jos 1,5; Es 43,1s . 4s ). De même dans les synoptiques et singulièrement chez $\mathrm{Mt}$ (cf. 1,23;18,20), Jésus promet aux disciples la

1 BORNKAMM, Der Auferstandene 305; dans le même sens : BONNARD, Mt 418, et STRECKER, Weg 212. Autrement, WEISS, Mt 508.

2 BORNKAMM, Der Auferstandene 306; dans le même sens : BONNARD, Mt 419.457, et TRILLING, Israel 38.39. Voir, p.ex. , 5,17-20 et les antithèses. L'originalité de Mt est ici absolument décisive : le contenu de la prédication missionnaire n'est ni le kérygme (annonce de la mort et de la résurrection du Christ), ni la proclamation de la $\beta \alpha \sigma \iota \lambda \varepsilon i$ $\alpha$, mais bien l'enseignement de la Loi réinterprétée par Jésus.

3 Comme le montre bien GRASS 31.32 , cette promesse d'assistance permanente souligne à nouveau combien le Ressuscité est déjà, aux yeux de Mt, le Seigneur élevé.

4 GRUNDMANN, Mt 579, et art. oúv - $\mu \varepsilon \tau \alpha \dot{\alpha}$, ThW 7,775; LOHMEYER, Mt 422; TRILLING, Is rael 40.41. 


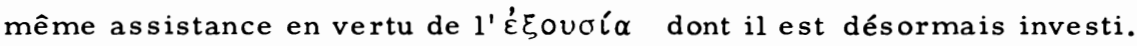
Il promet son secours à ses envoyés engagés par lui au sein même de

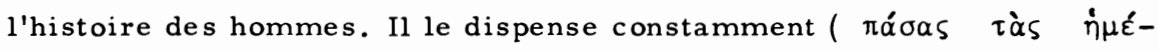

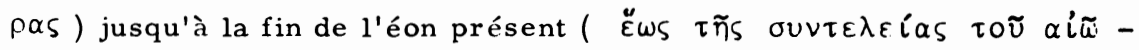
$\operatorname{vos})^{1}$.

L'évangile s'achève par cette ouverture illimitée sur l'avenir, laquelle démontre, s'il en est encore besoin, que l'instruction finale du Ressuscité s'adresse par delà les Onze, à tous les croyant s de toutes les époques. Le Christ $\mathrm{mt}$ autorise ainsi l'avenir de l'Eglise.

On se permettra enfin de souligner que si 20 a affirmait que le Ressuscité n'est personne d'autre que Jésus de Nazareth, $20 \mathrm{~b}$ amène la proposition inverse : Jésus de Nazareth n'est personne d'autre que le Kyrios qui règne avec autorité et miséricorde sur la création.

\section{L'identité du Jésus terrestre et du Ressuscité}

L'interprétation de Mt 28,16-20 nous place devant une conception christologique profilée dont il s'agit de dégager l'enjeu et les conséquences. La tradition christologique utilisée dans le manifeste est d'origine hellénistique? . En accord avec elle, Mt présente le Ressuscité comme le Kyrios tout-puissant habilitant ses envoyés à la mission universelle. Mais la similitude s'arrête là. Alors que, pour le christianisme hellénis tique, les envoyés du Kyrios sont remplis de l'Esprit, accomplissent prodiges et miracles, annoncent un évangile libéré de la Loi, pour Mt, la mission des disciples est recentrée sur l'annonce du "commandement" du Jésus terrestre (v. 20a). La confession de foi hellénistique du Kyrios est

1 STRECKER (Weg 212-213) voit dans le v. 20b l'expression de la seule présence de Jésus par sa parole et récuse toute idée d'assistance. $20 \mathrm{~b}$ serait alors le doublet de $20 \mathrm{a}$, ce qui contredit le plan, clai rement marqué, des vv. 18b-20. Voir aussi LUZ, Jünger 162-163.

2 BORNKAMM, Der Auferstandene 297-307. 
réinterprétée à partir de la tradition palestinienne de l'obéissance à la Loi (Nachfolge). Cette réinterprétation présuppose une unité essentielle entre le Ressuscité et le Jésus terrestre. Si annoncer le Ressuscité consiste à rappeler l'enseignement du terrestre, alors le Ressuscité qui règne actuellement n'est personne d'autre que le Jésus terrestre qui en-

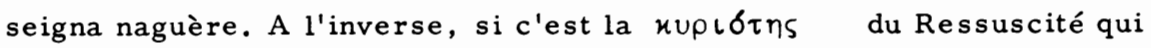
confère à l'enseignement du terrestre sa valeur normative, alors $1 \mathrm{e}$ Jésus terrestre qui enseigna naguère n'est personne d'autre que le Ressuscité qui règne actuellement. L'identité du Christ mt est donc cons tituée par l'unité paradoxale du terrestre et du Ressuscité.

Ce modèle christologique a un rôle constitutif dans la théologie $\mathrm{mt}$; à ce titre, il détermine la condition du croyant. En effet, si le Ressuscité est identique au Jésus terrestre et réciproquement, cel a signifie que le croyant qui désire rencontrer le Ressuscité ne peut le faire qu'en se mettant à l' écoute et à la suite du Jésus terrestre, mais que, ce faisant, il est confronté au Ressuscité. On se demandera alors : comment le croyant pe ut-il se mettre à l'écoute et à la suite du terrestre ? Il ne le peut qu'en devenant le contemporain des disciples historiques de Jésus, ou, si l'on préfère, en se reconnaissant dans la figure des $\mu \alpha \vartheta \eta \tau \alpha i$ qui se mirent jadis à l'écoute et à la suite de Jésus, et en s'identifiant à ceux-ci. Cette caractérisation de la condition du croyant a son corollaire - au niveau du récit - dans l'emploi de catégories littéraires ambivalentes pour désigner les partenaires du Jésus terrestre. En effet, si le Ressuscité rend normatif ce que fit et dit le Jésus terrestre, alors la condition des premiers disciples - sans rien perdre de son historicité - devient le paradigme de toute existence croyante. La similitude de condition entre les premiers disciples et les générations ultérieures - et partant, la possibilité de rendre transparents les premiers pour les seconds par le biais de catégories littéraires - a son fondement dans l'identité du terrestre et du Ressuscité.

Concluons. L'identité du terrestre et du Ressuscité est la thèse christologique fondamentale qui justifie à la fois l'emploi des catégories 
littéraires ambivalentes mises en évidence dans notre première partie, mais aussi la continuité historique essentielle que Mt établit entre l'his toire du Jésus terrestre et le temps de l'Eglise. S'il en est ainsi, le sujet de notre recherche se trouve circonscrit méthodologiquement. Pour décrire la condition du croyant dans l'évangile selon Matthieu, il s'agit d'analyser la relation qui s'institue entre le Jésus terrestre et ses dis ciples. 
CHAPITRE QUATRE : LE DIDASCALE ESCHATOLOGIQUE (Mt 5, 17 -20)

Le manifeste qui conclut l'évangile accorde une place décisive à la prédication du Jésus terrestre. Etre le disciple du Christ consiste fondamentalement à "garder tout ce qu'il a prescrit" (v. 20a). La relation entre le croyant et son Seigneur ressuscité s'édifie donc sur la base de l'enseignement du terrestre - enseignement réputé désormais normatif -.

La fonction essentielle que le manifeste attribue à la prédication de Jésus dans la christologie $\mathrm{mt}$, doit retenir toute notre attention. Elle appelle notamment un examen minutieux de la manière dont le Christ $\mathrm{mt}$ comprend le statut, le contenu et la portée de son enseignement. Une telle réflexion apparaît de façon privilégiée dans le texte programmatique de 5,17-20. Dans cette péricope, en effet, le didascale du Sermon sur la Montagne révèle non seulement comment il conçoit son office doctoral, mais encore la fonction qu'il accorde à son discours.

\section{Analyse littéraire}

Notre péricope se signale par son originalité dans le cadre des synoptiques. Elle est en effet sans parallèle à l'exception du v. 18 dont on retrouve une correspondance approximative en Lc 16,17. Pour ouvrir la voie à une interprétation pertinente du passage, il convient de distinguer dans la mesure du possible - de quelles traditions Mt s'inspire, quelles corrections il leur apporte, quel rôle il leur attribue dans l'ensemble du Sermon sur la Montagne.

\section{a) Tradition et rédaction}

Concernant le v. 17 on ne peut décider avec certitude si Mt reprend un logion en le reformulant ${ }^{l}$ ou si le $v$. est une création rédactionnel-

1 BORNKAMM (Enderwartung 21) attribue la formation des vv. $17-19$ à la communauté judéo-chrétienne, tandis que BULTMANN (Trad 147) voit l'origine du même ensemble dans la source $Q$. 


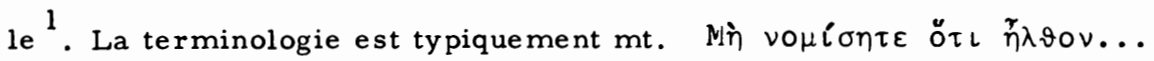

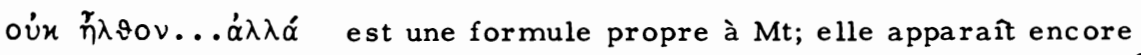
en $10,34 \mathrm{~s}$ où elle est visiblement secondaire par rapport à Lc $12,51-53^{2}$; elle correspond au souci mt de simplifier et de condenser ses sources.

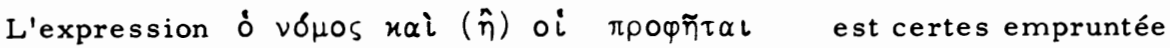
à la tradition (Mt 11,13 par. Lc 16,16), mais l'auteur du premier évangile l'a introduite de son propre chef dans d'autres passages $(7,12 ; 22,40)$. Le verbe $\pi \lambda \eta p \rho \tilde{v} \nu$ est l'un des termes importants de la rédaction $\mathrm{mt}$; il apparait 2 fois chez Mc, 9 fois chez Lc, 16 fois chez Mt; son emploi est rédactionnel en 3,$15 ; 23,32$ et dans toutes les introductions aux citations scripturaires.

La plupart des exégètes s'accorde à voir dans le $\underline{\text { v. } 18}$ la reprise d'un logion tiré de la source $Q^{3}$ et qui se trouve chez Lc dans un tout autre

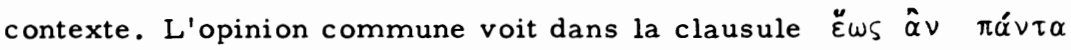
$\gamma \varepsilon \nu \eta \tau \alpha \iota$ (18d) un commentaire rédactionnel ${ }^{4}$; cette adjonction s'accorde

1 Ainsi BARTH 62; EICHHOLZ 61; HUMMEL 66; STRECKER, Weg 144. LUEHRMANN, Logienquell e 116 laisse la question en suspens tout en remarquant une forte empreinte rédactionnelle dans la terminologie.

2 BARTH 62, note 3; TRILLING, Is rael 171.

3 BRAUN, Radikalismus II, 11 (note 2); BULTMANN, Trad 147; DUPONT, Béatitudes I 134; LUEHRMANN, Logienquelle 116; STRECKER, Weg 143; TRILLING, Is rael 167. BARTH 61 et HUMMEL 67 sont plus réservés et ne parlent que de l'existence pré-mt du logion. WREGE 39-40 n'accorde pas ce logion à $Q$, attaché qu'il est à nier en bloc l'existence de cette source. Récemment, SCHWEIZER (Gemeinde 79-83) a proposé une nouvelle hypothèse : le rédacteur $\mathrm{mt}$ connaissait ce logion sous deux formes. La première était une variante de Mc 13,30 et correspondait à Mt $5,18 \mathrm{a}, \mathrm{c} . \mathrm{d}$; la se conde était la version $Q$ et équivalait à $M t 5,18 \mathrm{~b}$. c. Le rédacteur $\mathrm{mt}$ a corrigé la variante mc sur la base de $Q$ : il a introduit $18 \mathrm{~b}$ et réinterprété dans un sens éthique $18 \mathrm{~d}$ qui décrivait primitivement l'eschaton.

4 BARTH 60-61; BORNKAMM, Wandlungen 77; DUPONT, Béatitudes I 134; HUMMEL 67; SCHULZ, Q 114; STRECKER, Weg 143; TRILLING, Israel 168. 
en effet mal avec la structure du v. Le $\dot{\alpha} \mu \grave{\eta} \nu ~ \gamma \grave{\alpha} \rho \lambda \varepsilon \varepsilon_{\gamma \omega} \dot{v} \mu \tau \nu$

est

contesté; certains le considèrent comme relevant de la rédaction ${ }^{1}$; Lührmann ${ }^{2}$ a sans doute raison en attribuant l'origine de cette expression à la communauté $\mathrm{mt}$, car elle se rattache à une forme halachique typique du premier évangile (cf. plus bas), forme qui est parfois pré-rédactionnelle.

L'origine pré-mt du v. 19 n'est pas contestée. La plupart des exégètes l'attribue au Smt ${ }^{3}$. Mais ce logion était-il déjà associé au v. 18 avant la rédaction $\mathrm{mt}^{4}$ ? Si tel est le cas, il faudrait voir dans le v. 19 non une tradition indépendante s'ajoutant au v. 18 à un moment donné, mais plutôt l'application casuistique du v. 18, application formulée par la communauté $\mathrm{mt}$, dépositaire, mais aussi interprète de $\mathrm{Q}^{5}$.

Selon l'opinion commune, le v. 20 est une adjonction rédactionnelle ${ }^{6}$. La terminologie du v. ( $\lambda \varepsilon ́ \gamma \omega \gamma \grave{\alpha} \rho \dot{u} \mu \tau \nu, \delta \iota \varkappa \alpha \iota \circ \sigma u ́ v \eta, \gamma \rho \alpha \mu \mu \alpha \tau \varepsilon \tau \varsigma$

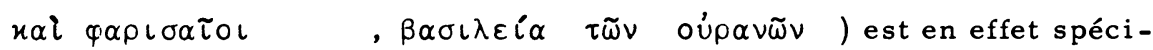
fiquement mt. Le rôle du v. 20 qui annonce le thème développé dans les antithèses, confirme cette opinion .

L'activité rédactionnelle ne se manifeste pas seulement dans la composition d'une péricope, mais aussi dans la position de celle-ci dans l'ensemble de l'évangile. A cet égard, 5, 17-20 est exemplaire. Le Sermon sur la Montagne se compose en effet de trois parties ${ }^{7}$. 5,3-20 tient

1 STRECKER, Weg 143; TRILLING, Israel 168.

2 Logienquelle 119.

3 BARTH 61; DUPONT, Béatitudes I 138; HUMMEL 67; STRECKER, Weg 145; TRILLING, Israel 182; WREGE 41. Autrement, BULTMANN, Trad 147.

4 Ainsi BARTH 61; TRILLING, Israel 182; WREGE 41.

5 LUEHRMANN, Logienquelle 117.

6 BULTMANN, Trad 147; BARTH 61; EICHHOLZ 61; KAESEMANN Anfänge 85; LUEHRMANN, Logienquelle $117-118$; STRECKER, Weg 151. Optent pour une origine traditionnelle : DUPONT, Béatitudes I $132-133$ et WREGE $42-44$.

7 BARTH 68. 
lieu d'introduction; 5,21-7,12 constitue le corps central; 7,13-27 forme la parénèse conclusive. Les exégètes ont depuis longtemps reconnu que 5, 17-20 est la transition reliant l'introduction au corps central; plus précisément, nos vv. donnent le thème qui sera traité dans $5,21-7,12$. Mais de quelle manière ?

Il ne fait guère de doute que 5,20 formule le "programme" des antithèses $(5,21-48)$ : la "meilleure justice" réclamée dans le v. 20 est développée dans les vv. $21-48^{l}$. Quant aux vv. 17-19, ils posent l'exigence de l'accomplissement de la Loi et des prophètes dont le sommaire est donné dans la conclusion, en 7,12. Autrement dit, 5,17-19 et 7,12 encadrent le corps central du Sermon sur la Montagne dont l'objet est précisément de donner l'interprétation exemplaire de la Loi ${ }^{2}$.

Concluons. 5,17-20 joue un rôle décisif dans le Sermon sur la Monta gne. En énonçant la position principielle du Christ $\mathrm{mt}$ face à la Loi, il donne le thème qui sera développé de 5,21 à 7,12. Pour le formuler, Mt reprend une tradition de la source $Q(v .18)$ déjà commentée par sa communauté (v. 19). Il l'interprète en y introduisant la clausule de $18 \mathrm{~d}$ et en l'encadrant par les vv. rédactionnels 17 (?).20. Le devoir de l'exégèse consiste à déterminer comment et dans quel sens Mt interprète, voire corrige, à l'aide de ses adjonctions la tradition reçue.

\section{b) La forme littéraire}

C'est Käsemann qui nous a rendus attentifs aux énoncés de droit sacré (Sätze heiligen Rechtes) dans le $\mathrm{NT}^{3}$. Dans ces énoncés construits selon le schéme du ius talionis eschatologique, transgression et châtiment, obéissance et récompense se correspondent exactement. La fidélité à garder ou le péché à éviter sont formulés dans une subordonnée en

1 BARTH 68; BORNKAMM, Enderwartung 14; BULTMANN, Trad 147; GRUNDMANN, Mt 151; HUMMEL 66; DESCAMPS, Les Justes 120; STRECKER, Weg 152. Voir aussi DUPONT, Béatitudes I 131.

2 BARTH 68; GRUNDMANN, Mt $141-142$.

3 Cf. KAESEMANN, Sätze 69-82. 
$\varepsilon \ddot{~} \tau \iota \varsigma$ ( $\dot{\alpha}^{\alpha} \nu \tau \iota \varsigma$ ou ôs $\delta^{\prime \prime \alpha} \alpha$ ) typique du droit casuistique, tandis que la sanction correspondante apparaît dans une principale au fu$\operatorname{tur}^{1}$. Récompense et châtiment seront prononcés par le juge eschatologique dont la venue est proche. Or c'est le schéma que nous retrouvons dans $5,19.20^{2}$. Cette découverte ne doit pourtant pas nous amener à tirer des conclusions hâtives. Käsemann a, en effet, également montré que le "Sitz im Leben" du droit sacré est la prophétie de la chrétienté primitive. Or non seulement Mt n'appartient plus à cette époque, mais il combat toute manifestation enthousiaste qui compromettrait l'obéissance à l'enseigne ment du Jésus terrestre ${ }^{3}$. Comment expliquer alors que Mt reprenne cette forme littéraire avec une ampleur inconnue des deux autres synoptiques ? En écrivant un évangile, l'intention de Mt est de doter son église

1 Ex. : Mt 19,28-29;10,41.42;10,13-14.

2 Dans le v. 19, le ius talionis eschatologique est appliqué rigoureuse ment : une obéissance qui fait l'économie des plus petits commande ments sera sanctionnée de la plus petite place dans le Royaume; à l'inverse, l'obéissance parfaite sera récompensée par la grandeur eschatologique. Le v. 20 est construit selon le même principe : seule une "meilleure justice" donne accès au Royaume. A la suite de BERGER, SCHWEIZER (Gemeinde 79-81) discerne dans le v. 18 une forme littéraire typique (parole en amen). Il s'agit d'une sentence apocalyptique

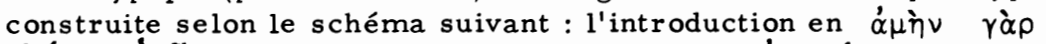

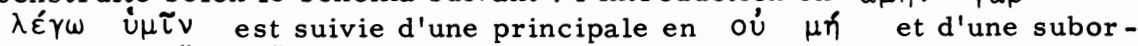

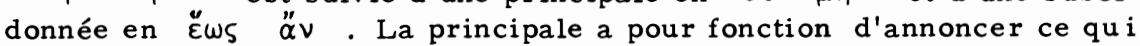
arrive jusqu'à l'eschaton mentionné dans la subordonnée. Cette analyse mérite la plus grande attention, mais elle n'éclaire guère le sens du v. 18 au niveau rédactionnel. En effet, d'une part, l'imminence de la parousie n'est plus un facteur déterminant (cf. contexte rédactionnel du v. 18 !), d'autre part, le v. 18 est lié au v. 19 que SCHWEIZER (Mt 62) considère également comme un énoncé de droit sacré issu de la prophétie chrétienne primitive. Il s'agit donc de déterminer la fonction et la forme des vv. 18-19 au niveau rédactionnel, ce que fait LUEHRMANN.

3 Voir le thème des "faux prophètes" chez Mt (cf. nos pp. 178-180). et BORNKAMM, Der Auferstandene, passim. 
d'une tradition doctrinale et disciplinaire; dans ce but, il présente la prédication de Jésus comme l'expression de la Loi divine. C'est ce qui explique sa prédilection pour les formulations inspirées du droit. L'usage du droit sacré n'est plus, chez lui, l'expression d'une conscience prophé tique, elle vise à la constitution d'une halacha. Käsemann écrit à ce propos : "Ich... bemerke..., dass ich in ihm die Uebernahme und Modifika tion des prophetischen Erbes durch den christlichen Rabbi konstatiere" 1 . Lührmann ${ }^{2}$ a voué une attention particulière à la forme de la halacha $m$ t inspirée des énoncés de droit sacré, et mis ainsi en évidence une forme littéraire particulière au premier évangile (précisant par là l'analyse plus générale de Käsemann). Grosso modo, le schéma de cette forme halachique se compose de deux éléments : a) une proposition de forme thétique de teneur eschatologique introduite par $\dot{\alpha} \mu \bar{\eta} \nu \quad \lambda \hat{\gamma} \gamma \omega$ vं$\mu \tau \nu$ b) deux propositions conditionnelles antithétiques en ôs $\dot{\kappa} \alpha \dot{\nu}$ - ôs $\delta^{\prime} \not \ddot{\nu}$. Or c'est précisément ce schéma que nous retrouvons dans les

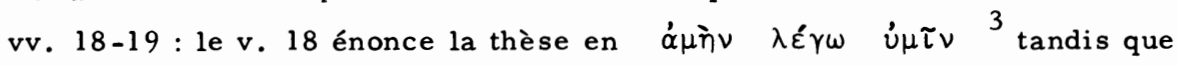
le v. 19 contient les deux conditionnelles antithétiques donnant l'application casuistique du v. 18. Le v. 20 entre également dans ce cadre : il est

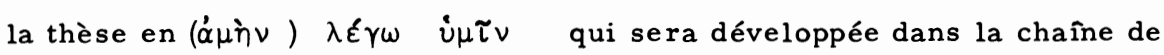
conditionnelles constituées par les antithèses. Relevons enfin que le v. 17 est lié étroitement à ce qui suit, car les instructions formulées de manière négative sont fréquentes dans les contextes où apparaît la forme halachique ${ }^{4}$.

1 KAESEMANN, Sätze 80; voir aussi Anfänge 92.

2 Voir LUEHRMANN, Logienquelle 107-121, mais aussi HUMMEL 5659.

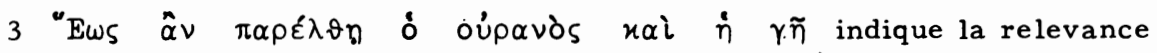
eschatologique (cf. BORNKAMM, Wandlungen 77).

4 Cf. 10,34-39: les vv. 34-36 développent la thèse principale et sont suivis dans les vv. $37-39$ de trois logia de forme conditionnelle. 
Cette forme halachique est née dans la communauté $\mathrm{mt}{ }^{1}$. Son "Sitz im Leben" est une église soucieuse d'édicter le droit destiné à régir son existence et, en particulier, son enseignement et sa pratique religieuse. Mais quelle est alors l'autorité de ce droit ? Comme le montre le v. 19, l'obéissance ou la désobéissance aux exigences proclamées par ce droit engagent la destinée eschatologique de l'homme. C'est dire que le droit de la communauté est garanti par l'autorité du juge eschatologique. Il exprime sa volonté et anticipe son verdict. L'autorité du droit de la communauté mt est donc absolue : en le respectant ou en le transgressant, l'église, corpus mixtum, gagne ou perd son salut.

Il faut encore dégager les implications christologiques de cette forme littéraire. Dans notre passage, comme d'ailleurs dans tout l'évangile de Mt, la halacha de la communauté et l'enseignement du terrestre sont identiques. La communauté place sa halacha dans la bouche de son Seigneur, et inversément, elle déclare normatif l'enseignement de celui-ci. La halacha mt est placée sous l'autorité de Jésus et, simultanément, l'enseigne ment du terrestre est énoncée en terme de droit sacré. Dès lors, c'est de la fidélité à la prédication du Christ mt que dépend l'entrée dans le Royaume. La prédication du terrestre est le critère du salut, sa mise en pratique la seule voie qui y mène ${ }^{2}$.

1 Pour LUEHRMANN (Logienquelle 107-121), cette forme halachique est constamment pré-rédactionnelle. SCHWEIZER (Mt 63) défend avec raison une opinion plus nuancée : cette forme est certes pré-rédactionnelle en 6,2-3,5-6,16-17, mais rédactionnelle en $12,31-32 ; 18,3-$ 5. 10-14.15-18.

2 Dans la même direction, KAESEMANN (Anfänge 96) écrit à propos des vv. 17-20: "Wir sind schon von Mt 5,17 aus darauf gestossen, dass es in ihnen nicht bloss darum geht, für spätere Geschlechter festzustellen, was einmal war, sondern darum, an Auftrag und Werk Jesu ein Kriterium für das zu gewinnen, was christlich heissen darf". HUMMEL 70 remarque avec plus de précision encore : "Für Matthăus kommt die Gerechtigkeit der Jünger aus dem Gehorsam gegenubber der Lehre Jesu, diese Lehre wiederum besteht in der Auslegung des Gesetzes". 


\section{c) L'arrière-fond ecclésial}

Notre passage est né dans une situation précise qui, seule, permet d'en apprécier correctement l'argumentation et d'en saisir la pointe. Pourtant il faut se garder de reporter sans autre le "Sitz im Leben" des traditions qui y sont recueillies, sur la situation vécue par l'évangéliste.

Etablissons tout d'abord le "Sitz im Leben" originel de ces traditions. La critique littéraire nous a permis de reconnaître dans les deux vv. 18 19 le noyau traditionnel le plus ancien de notre passage. Ces vv. ont toujours frappé les commentateurs par leur accent juif palestinien ${ }^{l}$. Ainsi le v. 18 reprend le dogme juif de l'éternité de la Loi et le formule à l'aide d'une image connue des rabbis ${ }^{2}$. Semblablement, le v. 19 repose sur deux présupposés typiquement juifs : d'une part, l'accomplissement des petits commandements est aussi important que celui des grands ${ }^{3}$, d'autre part, toute critique de la Loi est interdite ${ }^{4}$. Cette stricte allégeance à la Tora comprise au sens juif-rabbinique ne concorde pas avec l'attitude du Jésus historique ${ }^{5}$. Elle correspond par contre à ce que nous savons des

1 Voir en particulier sur ce point : KUEMMEL, Traditionsgedanke 33 34 , et BILL $1,240-252$.

2 KUEMMEL, Traditionsgedanke 18 (note 14) et BILL 1, 244-247 ont rassemblé le matériel dénotant la croyance à l'éternité de la Tora dans le bas-judaísme. Mentionnons cette citation d'Ex Rabba 6,2 par. 6,1 (BILL 1,244) qui exprime le dogme de l'éternité de la Tora en utilisant la même image que Mt 5,18: "Dieu dit : Salomon et mille autres, semblables à lui, passeront, mais de la Loi (= Tora), je ne laisserai pas le moindre trait passer".

3 Voir, p. ex., Ab 2,$1 ; 4,2$.

4 Sifré Nb 15,31 par. 112 (KUEMMEL, Traditionsgedanke 33, note 83) : "Si quelqu'un dit : 'j'accepte toute la Tora, cette parole mise à part', il a méprisé la parole de Dieu (Nb 15,31). Si quelqu'un dit : 'Moíse a prononcé toute la Tora au nom du Saint, mais cette parole il l'a dite en son propre nom', il a méprisé la parole de Dieu".

5 BORNKAMM, Wandlungen 78-79; BRAUN, Radikalismus II 7-14 (en particulier 7 , note 2 , et 11 , note 2 ); KUEMMEL, Traditionsgedanke $26-35$. 
positions de la communauté judéo-chrétienne palestinienne. Mais alors, dans quelles circonstances ce groupement a -t-il été amené à s'exprimer ainsi ? Les controverses bien connues sur la validité de la Loi nous semblent être à l'origine de ces paroles judaîsantes : en opposition à des cercles plus libéraux qui prônent l'abrogation partielle ou totale de la Loi - ainsi le cercle d'Etienne, par exemple -, ce groupement conservateur réaffirme, avec l'autorité du droit sacré, la validité intrinsèque de la Loi ${ }^{1}$.

S'il est exclu, en bonne méthode, de reporter sans autre cet affrontement entre nomistes et antinomistes au niveau de la rédaction $\mathrm{mt}$, il convient néanmoins de souligner deux points importants. Premièrement ${ }^{2}$, la communauté mt a véhiculé le v. 18 et l'a commenté en lui adjoignant le v. 19; cela signifie qu'el le a été un milieu où s'est posé le problème de la Loi et qu'elle l'a résolu de manière nettement conservatrice. Deuxiè mement, Mt a non seulement recueilli cette tradition, mais il l'a introduite dans un contexte-clef de son oeuvre : les vv. 17-20 sont en effet l'introduction à la partie centrale du Sermon sur la Montagne et acquiè rent ainsi une signification programmatique. C'est dire que pour le rédacteur du premier évangile aussi, l'affirmation de la validité intrinsèque de la Loi est d'une importance capitale ${ }^{3}$.

Les 2 vv. rédactionnels (17.20) nous permettent de confirmer et de préciser cette opinion. Le v. 17 montre comment Mt interprète les vv. 18-19. Il combat le malentendu selon lequel Jésus serait venu pour abolir

1 Partagent cette opinion : BULTMANN, Trad 146-147; BRAUN, Radi kalismus II 8 ; BAR TH 60-61; KAESEMANN, Anfänge 86; LUEHRMANN, Logienquelle 117 (note 2); SCHWEIZER, Mt 5,17-20, 401, et Gemeinde 81; STRECKER, Weg 145; TRILLING, Israel 181-182; WREGE 40-41.

2 Sur l'insertion $m$ t de notre passage, voir BARTH 60-66; BORNKAMM, Wandlungen 74-75; HUMMEL 66-71.

3 Lc 16,17 (le parallèle de Mt 5, 18) figure au contraire dans une collection de logia où il ne joue aucun rôle particulier. 
la Loi. Reprenant au niveau mt la problématique des vv. 18-19, il présuppose, la présence dans l'église mt de cercles - et singulièrement de maîtres - prônant l'abolition (partielle ou totale ?) de la Loi et se tourne violemment contre eux. Cette attaque n'est pas purement rhétorique, des tinée seulement à mettre en valeur les vv. 18-19 ${ }^{1}$. Elle forme l'arrièrefond de toute la parénèse du Sermon sur la Montagne $(7,12-27)^{2}$.

Notre texte nous permet-il de mieux localiser les adversaires de Mt ? Le v. 17 formule le reproche d'une manière très générale; l'évangéliste ne juge pas nécessaire d'examiner en détail la position qu'il combat; il se borne à condamner quiconque ne se rallie pas à sa conception de la volonté de Dieu ${ }^{3}$.

Mais il est clair aussi que la critique élevée par Mt contre des cercles plus 1 ibéraux dans l'appréciation de la validité de la Loi, n'implique pas une solidarité ou une bienveillante tolérance vis-à-vis du bas-judaĩsme rabbinique. Bien que la polémique de notre passage soit exclusivement dirigée contre des maîtres chrétiens, le v. 20 montre que les "scribes et les pharisiens" - représentants typiques du judaîsme d'après 70 - sont également pris à parti, mais dans un tout autre sens; leur "justice", càd leur pratique de la Loi divine, les promet à la géhenne. Cette polémique anti-pharisienne domine les antithèses et le chap. 6.

Dans les vv. 17-20, - et de manière plus générale dans tout le Ser mon sur la Montagne -, Mt s'adresse aux disciples, càd aux membres de sa communauté. Il les instruit de l'enseignement du Jésus terrestre, en se battant sur un double front. Par le biais de sa halacha, il réaffirme la validité absolue de la Loi contre les cercles plus "libéraux", mais en même temps il conteste la position juive en cette matière. Dans ce double conflit, le recours aux termes du droit sacré doit nous faire comprendre

1 Avec BORNKAMM, Wandlungen 74 (note 3) et contre TRILLING, Israel 171 .

2 Voir aussi 11,12-13;24,10-13 (et l'ensemble de notre chap. 7).

3 KAESEMANN, Anfänge 84. 
que Mt prétend, à l'exclusion de tout autre, posséder la vérité en ma tière d'enseignement chrétien.

\section{Interprétation}

Le Christ mt entend, dans le v. 17, écarter un malentendu qui touche

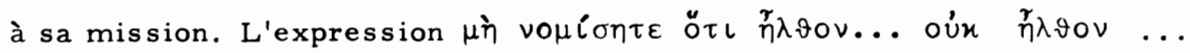
$\dot{\alpha} \lambda \lambda \alpha_{\alpha}$ est destinée à opérer cette mise au point. Elle n'est pas l'écho de débats communautaires ${ }^{1}$; elle n'est pas non plus une formule purement rhétorique, sans lien avec une situation concrète ${ }^{2}$, il s'agit d'une tournure rédactionnelle citant pour la réfuter l'opinion des cercles libéraux ${ }^{3}$. L'opinion soutenue par les "antinomistes" et jugée aberrante par Mt concerne l'interprétation du ministère de Jésus : les paroles en $\stackrel{\Upsilon}{\eta} \lambda \vartheta \circ v$ décrivent, en effet, de manière rétrospective et synthétique le sens de la mission du Christ ${ }^{4}$.

Quelle est donc la thèse christologique combattue par Mt et que ce dernier impute à ses adversaires ${ }^{5}$ ? Elle peut s'énoncer ainsi : Jésus est venu pour abolir la Loi, il est la "fin de la Loi". Dans le contexte du Sermon sur la Montagne qui présente le Christ avant tout comme ensei -

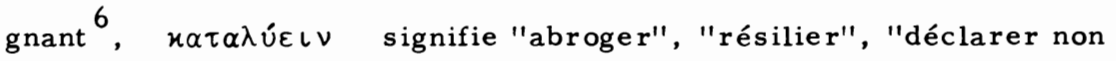

1 BULTMANN, Trad 146.

2 TRILLING, Israel 171.

3 BARTH 62; GRUNDMANN, Mt 144; HUMMEL 66.

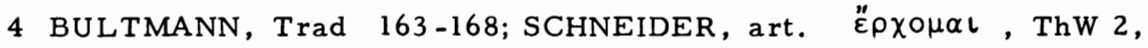
$664-665$.

5 Dire que cette thèse reflète fidèlement l'opinion des adversaires de Mt dépasse nos compétences; en tout état de cause, le rédacteur du premier évangile leur impute cette position. Le ton polémique n'exclut assurément pas un jugement excessif et simplificateur.

6 Voir le cadre du discours et en particulier le double usage du verbe $\delta \iota \delta \alpha ́ \sigma x \omega \quad(5,1 ; 7,29)$ qui fait inclusion. 


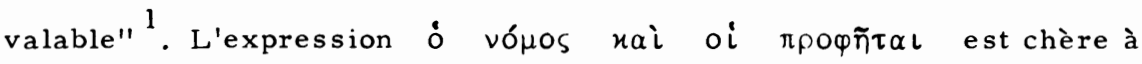
Mt (cf. 7,$12 ; 11,13 ; 22,40)$; elle désigne l'ensemble de l'AT comme exigence éthique, comme expression de la volonté de Dieu ${ }^{2}$. Ce qui est en cause, c'est l'obéissance à la Loi.

A la thèse qui lie apparition du Christ et fin de la validité de la volonté de Dieu sous sa forme vétérotestamentaire, Mt oppose sa propre conception : bien loin de supprimer la Loi, le Christ "l'accomplit". On a souvent perdu de vue que le sens premier de la conception mt est la reconnaissance et la ratification par le Christ de l'AI comme expression de la volonté de Dieu. Or quel est le sens de cette reconnaissance ? En quoi est-elle un "accomplissement" ? Que signifie ici le verbe $\pi \lambda \eta \rho \delta \omega ?$

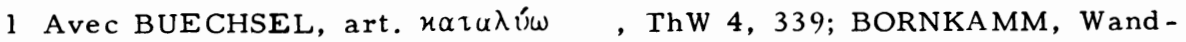
lungen 76; KLOSTERMANN, Mt 40; KUEMMEL, Traditionsgedanke 34 (là, l'histoire du mot grec et origine sémitique); voir aussi BILL 1 , 241 et GRUNDMANN, Mt 141. BONNARD, Mt 61 et SCHNIEWIND, Mt $53-54$ insistent sur la valeur plus pratique que théorique de $x \alpha \tau \alpha-$ $\lambda u ́ w \quad$ : s'il est bien évident qu'en abolissant la Loi, le Christ sous trairait l'homme à son autorité concrète, le v. 17 décrit d'abord un aspect discuté de l'enseignement du Christ.

2 Mt utilise $\ddot{\eta}$ et non $x \alpha i ́$ comme particule de liaison à cause de la forme négative du logion; il n'y a pas de différence de sens, cf.

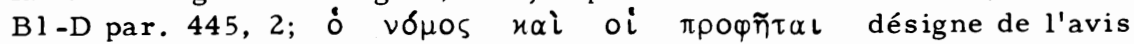
unanime l'ensemble de l'Ecriture; cette expression est rare dans la littérature juive tardive (cf. BILL 1, 240; GRUNDMANN, Mt 144;

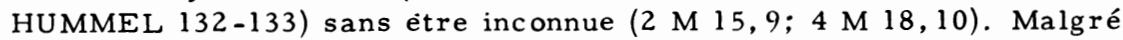
DUPONT (Béatitudes I 143, note 1) et SCHNIEWIND (Mt 53) qui pensent aux promesses divines, c'est l'Ancien Testament en tant qu'expression de la volonté de Dieu qui est visé; 7,12 et 22,40 , mais aussi l'enchaînement par vópos au v. 18 et $\dot{\varepsilon} \nu \tau o \lambda \hat{n}$ au v. 19, le montrent clairement. Les prophètes sont avant tout conçus comme les interprètes et les prédicateurs de la Tora (ainsi BARTH $86-88$;

BORNKAMM, Wandlungen 75, note 7; GRUNDMANN, Mt 144; GUTBROD, art. vófos , ThW 4, 1051 ; HUMMEL 132-133; KLOSTERMANN, Mt 40 -41; SCHLATTER, Mt 153; STRECKER, Weg 144; TRILLING, Israel $172-174)$. 
$\Pi \lambda \eta \rho \delta \omega$ est un terme-clef de la théologie $\mathrm{mt}^{1}$. Le plus souvent, il pparait sous une forme passive et dans l'introduction aux citations de Ancien Testament. L'idée directrice est alors la suivante : dans une irconstance donnée de la vie du Christ, une promesse de l'Ancien Tes ament trouve sa réalisation, passe dans les faits, acquiert son entière ralidité. Dans notre passage, il n'y va pas de la vie du Christ, mais de son enseignement ${ }^{2}$. En se souvenant du sens $\mathrm{mt}$ de $\pi \lambda \eta \rho \delta \omega$, on peut zlors donner la paraphrase suivante : dans l'enseignement du Christ, la volonté de Dieu telle qu'elle est exprimée dans l'Ancien Testament, trouve sa réalisation, atteint son accomplissement. Mais en quoi consiste cet

1 Sur les 16 mentions mt de $\pi \lambda$ no $6 \omega, 12$ parlent de l'accomplissement des Ecritures. SCHWEIZER (Mt 5,17-20,400) relève en conséquence que $\pi \lambda \eta \rho \delta \omega$ a un sens profilé chez Mt, également valable pour notre passage : porter à son accomplissement, réaliser. Dans la même direction, BARTH $62-65$ souligne que $\pi \lambda \eta n \delta \omega$ exprime plus l'idée d'une réalisation que d'une modification, mais que cet "accomplissement" a précisément lieu dans l'enseignement de Jésus. Cette double remarque préliminaire rend problématique l'interprétation de $\pi \lambda \eta p \delta \omega$ au seul sens de "dégager la véritable signification, expliquer" (ainsi KLOSTERMANN, Mt 41; KUEMMEL, Traditionsgedanke 34), car elle néglige l'idée d'accomplis sement. Semblablement, l'élucidation au seul sens de "faire la volonté de Dieu" (ainsi SCHLATTER, Mt 154; SCHNIEWIND, Mt 54) ne tient pas compte du contexte qui est celui de l'enseignement. GRUNDMANN (Mt 145-146), LUEHRMANN (Logienquelle 117) et DESCAMPS (Les Justes 130-131) refusent l'alter native et interprètent l'accomplissement de la Loi comme son enseigne ment nouveau et sa pratique; cette interprétation synthétique (bien étayée par l'évangile dans son ensemble !) ne résoud rien car elle n'explique pas pourquoi l'enseignement de la Loi prodigué par le Christ est un accomplissement. B ONNARD (Mt 61) et DELLING (art. $\pi \lambda \eta \rho \delta \omega$, ThW 6,292 -93) élargissent encore le champ d'explication en discernant dans l'accomplissement du v. 17 la réalisation de l'Ancien Testament à la fois comme exigence et comme promesse; une telle analyse repose sur une appréciation à notre avis erronée de l'expression d voros $x \alpha i$ oi $\pi \rho \circ \varphi \tilde{n} \tau \alpha \iota$. La même critique s'applique à DUPONT, Béatitudes I 138-144 et WREGE 36-37, qui, pour avoir compris "la Loi et les Prophètes" sous l'angle de la promesse, voient dans $\pi \lambda \eta p \delta \omega$ le terme technique de leur réalisation.

2 Avec BORNKAMM, Wandlungen 76. 
"accomplissement" de la volonté divine, réalisé dans l'office doctoral du Christ ? Il réside dans l'interprétation totale, définitive et décisive de la Tora, càd dans la révélation de la meilleure justice dont parle le $v$. $20^{l}$. Le contenu de cette meilleure justice est développé dans les antithèses et récapitulé en 7,12 : il s'agit du commandement d'amour (cf. aussi 22,40). L'"accomplissement" dont parle le v. 17 peut alors se définir comme suit : Jésus accomplit la Tora, la réalise en en dévoilant le sens véritable, en en dégageant l'intention directrice qui est l'exigence de meilleure justice concrétisée dans le commandement d'amour ${ }^{2}$.

Face aux "antinomistes", Mt défend donc la thèse suivante : la mis sion de Jésus consiste à établir le droit de Dieu, à mettre en vigueur sa volonté. Il la réalise par son enseignement ${ }^{3}$ - qui ne consiste dans rien d'autre que dans la juste interprétation de la Tora -.

Pour appuyer et étayer la thèse émise au v. 17, Mt introduit un logion isolé de la source $Q(\underline{v} .18)$ et commenté par sa communauté (v. 19).

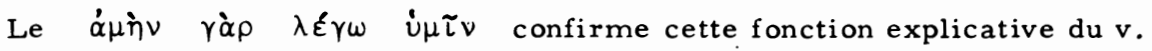
18, car, dans l'évangile selon $\mathrm{Mt}$, cette expression est toujours placée en tête d'une phrase fondant théologiquement l'affirmation précédente et ayant un rôle quasi-axiomatique dans l'argumentation du passage en

1 Cf. BORNKAMM, Wandlungen ibid., qui invoque deux preuves tirées du contexte : la conséquence tirée à l'intention de l'Eglise au v. 19 se situe au niveau de l'enseignement; le v. 20 est la suscription des antithèses.

2 Ainsi BORNKAMM, Wandlungen 79 (en particulier note 14); EICHHOLZ 65-66; SCHWEIZER, Mt 5, 17-20, 404-405; STRECKER, Weg 145. 147. Nous n'abordons pas ici la discussion entre BARTH et HUMMEL, d'une part, SCHWEIZER et STRECKER, d'autre part, sur la place du commandement d'amour dans la Loi. Chacun admet qu'il en constitue le centre et le principe critique.

3 Il va de soi que l'évangile dans son ensemble voit l'accomplissement de la Tora par le Christ non seulement dans son enseignement, mais encore dans son obéissance. Dans cette perspective globale, il faut bien admettre que 5,17 traite en priorité de l'accomplissement au niveau de l'enseignement (autrement : SCHWEIZER, Gemeinde 84). 
cause ${ }^{1}$.

La déclaration sur la Loi qui forme le corps du v. émane de cercles conservateurs et a un accent nettement judaisant. Tant la forme que le fond ont des parallèles frappants dans la littérature rabbinique. Le iod ( $i \tilde{\tau} \tau \alpha)$ est la plus petite lettre de l'écriture hébraíque carrée; le trait de lettre ( $x \varepsilon \rho \alpha i ́ \alpha)$ est le plus petit signe calligraphique de l'écriture hébraique, il sert à enjoliver les lettres. Ces deux caractères ont une fonction métaphorique dans les sentences rabbiniques et l'affirmation de leur éternité expriment en fait la croyance à l'éternité de la Loi dans son intégralité (y compris les adjonctions rabbiniques $=x \varepsilon \rho \alpha i \alpha)^{2}$. "Ews

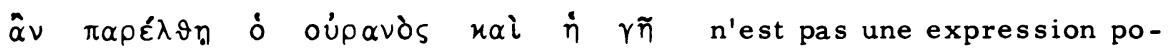
pulaire signifiant simplement "jamais" ${ }^{3}$. Comme le souligne justement Bornkamm ${ }^{4}$, cette tournure présente l'histoire du monde comme une his toire qui s'achemine vers sa fin (cf. 24,35). Cette première clausule en ह̌ws $\ddot{\alpha} \nu$ signifie alors que la Loi reste en vigueur jusqu'à l'accomplissement eschatologique.

Comment Mt comprend-il cette tradition affirmant la validité de la

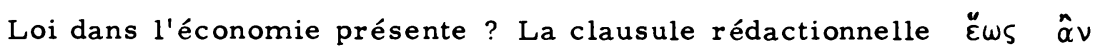

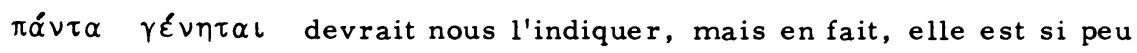

1 Cf. BERGER 29-34.71-86 (pour le v. 18, voir en particulier 33.73-74). Dans le même sens : BORNKAMM, Wandlungen 76.

2 Pour le tout, cf. BILL 1, 244-249, et KUEMMEL, Traditionsgedanke

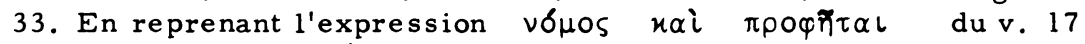
par le seul terme vópos, le v. 18 confirme la rectitude de notre analyse du v. 17, càd l'Ecriture au sens de Loi, de volonté de Dieu.

3 KLOSTERMANN, Mt 41; après lui, BARTH 61; STRECKER, Weg 144.

4 Wandlungen 77. Avant lui, LUEHRMANN, Logienquelle 116. 
claire que toute interprétation reste hypothétique ${ }^{l}$. C'est conscient de cette limite que nous tentons une explication. La conjonction temporelle

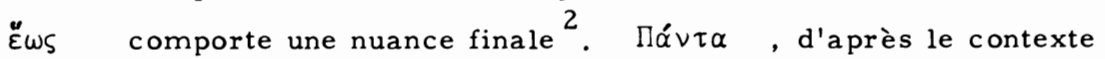
immédiat, désigne tout ce qui est exigé par la Loi ${ }^{3}$. Comme en 6, 10 et

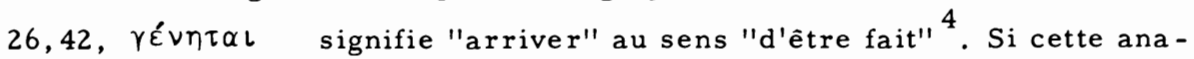
lyse des concepts est correcte, 18d exprime alors le but de la validité de la Loi. La Loi est valable afin que tout ce qu'elle exige, soit accompli par la communauté ${ }^{5}$. L'intention dernière qui justifie son autorité est l'instauration du droit de Dieu par les disciples ${ }^{6}$. Il va de soi que la volonté divine dont il est question ici est la Loi "accomplie" par le Christ au sens du v. $17^{7}$.

1 Schématiquement, trois types d'interprétation sont constamment proposés pour comprendre 18d. L'interprétation temporelle, s'appuyant sur le parallèle formel de Mt 24,34-35, voit dans 18d une redondance de $18 \mathrm{~b}$; la clausule rédactionnelle signifierait alors "jusqu'à la parousie" (ainsi BONNARD, Mt 62; KLOSTERMANN, Mt 41; SCHNIEWIND, Mt 54). La difficulté de cette exégèse tient dans le fait qu'elle aboutit à une tautologie : Mt ajouterait $18 \mathrm{~d}$ pour répéter ce qu'affirmait déjà $18 \mathrm{~b}$ ! L'interprétation historico-salutaire rapporte $18 \mathrm{~d}$ à la destinée de Jésus. Le sens est alors le suivant : Jésus accomplit les exigences de l'Ecriture (ainsi SCHLATTER, Mt 157) ou ses promesses (ainsi DUPONT, Béatitudes I 134-135) ou les deux à la fois (WREGE 38-39). Dans ce cas, $18 \mathrm{~d}$ est une redondance de 17 . Contre cette exégèse, on répétera que le contexte ne parle pas de la destinée de Jésus, mais de son office doctoral. Pour l'interprétation éthique, cf. notre analyse.

2 BARTH 61; SCHWEIZER, Gesetz und Enthusiasmus 51, et Gemeinde 83. BORNKAMM, Wandlungen 78 , maintient à juste titre la composante temporelle.

3 BARTH 61; GRUNDMANN, Mt 148; SCHLATTER, Mt 157.

4 TRILLING, Israel 169 (en particulier note 11).

5 Avec BORNKAMM, Wandlungen 77; GRUNDMANN, Mt 148; SCHWEIZER, Gemeinde 83; STRECKER, Weg 143-144; TRILLING, Israel 169-170.

6 BARTH 65.

7 Comme le souligne bien TRILLING (Israel 179), le v. 18 en tant que commentaire et développement du v. 17 perd l'accent nomiste qu'il avait dans la tradition. Il doit être compris comme une hyperbole (BORNKAMM, Wandlungen 77). 
L'argumentation des vv. 17-18 peut dès lors s'énoncer ainsi : loin de vouloir abolir la Loi, le Christ est venu pour l'établir définitivement en en dégageant l'intention directrice. Par cet accomplissement plénier, il ouvre dans l'histoire du monde qui chemine vers sa fin, une nouvelle période - la période où le droit de Dieu doit être instauré sur la terre -. En regard de l'eschaton qui s'approche, le temps vécu par les disciples est celui de la décision; placé sous l'impératif de la meilleure justice, il appelle de manière urgente les croyants à réaliser la volonté de Dieu.

Comme l'indique le oưv, le v. 19 tire l'application pratique du "dogme" qui vient d'être proclamé. La vie de la communauté doit se régler sur la thèse développée dans les vv. 17-18 ${ }^{1}$. Cette concrétisation éthique - déjà̀ anticipée en $18 \mathrm{~d}$ - est formulée en termes de droit sacré (voir plus haut). Elle peut se résumer ainsi : seule l'observation scrupuleuse de la Loi permet d'accéder à la grandeur eschatologique.

Le critère selon lequel seront attribuées les places dans le Royaume est le respect des petits commandements. On a proposé d'identifier les "plus petits commandements" soit avec la deuxième table du Décalogue ${ }^{2}$, . soit avec les antithèses qui suivent ${ }^{3}$. Si l'on se souvient que notre v. est

1 Avec BARTH 61; GRUNDMANN, Mt 148; LUEHRMANN, Logienquelle 117. Au niveau traditionnel, le v. 19 était l'application casuistique du seulv. 18. Au niveau rédactionnel, la thèse sur la validité de la Loi a été élargie par le v. 17.18d dont le v. 19 formule désormais la conséquence éthique.

2 Ainsi F. DIBELIUS, Gebote 188-190; GRUNDMANN, Mt 149; MICHEL,

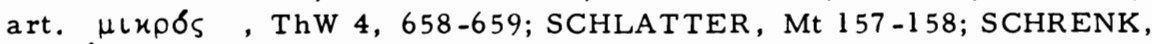
art. '่́ $\nu \tau \lambda \dot{n}$, ThW 2, 544. Contre ces auteurs, il faut souligner que jamais dans la tradition vétérotestamentaire-juive, un commandement est réputé "petit" à cause de la brièveté de sa formulation.

3 Cette thèse émise par CARLSTON et reprise par SCHWEIZER (Gesetz und Enthusiasmus 52) repose sur une surinterprétation du $\tau 0 u ́ \tau \omega \nu$ et néglige la conclusion du $v$. : il est peu probable que celui qui trans gresse les antithèses, obtienne une place dans le Royaume - si petite soit -elle ! -. 
né dans les cercles conservateurs du judéo-christianisme palestinien, on pensera de préférence à la distinction rabbinique entre petits et grands commandements ${ }^{1}$. Le $\tau o u ́ \tau \omega \nu$ ne renvoie pas au v. $18^{2}$, il n'est pas non plus l'écho d'un débat communautaire ${ }^{3}$ : il s'agit d'un sémitisme où le démonstratif a valeur générale et ne se traduit pas 4 .

L'obéissance requise est un "faire" et un "enseigner"; dans le monde juif déjà, ces deux notions forment une unité et sont les modalités de l'obéissance à la Loi ${ }^{5}$. Le comportement critiqué est exprimé par les verbes $\lambda u ́ \omega$ et $\delta\llcorner\delta \alpha ́ \sigma K \omega$ : si le sens de $\delta\llcorner\varepsilon \alpha ́ \sigma K \omega$ est évident inviter les croyants à ne plus respecter les petits commandements -,

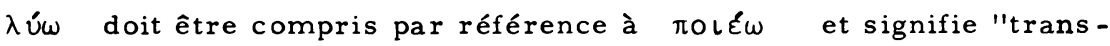
gresser volontairement", "abolir par son comportement" 6 .

Toute proposition de droit sacré est construite selon le schéma du "ius talionis" eschatologique. Elle établit une correspondance exacte entre la conduite actuelle de l'homme et sa rétribution à venir. Ainsi, dans notre v., à l'inobservation délibérée de l'un des plus petits commande-

l Avec BILL 1, 249; DUPONT, Béatitudes I 133; HUMMEL 67 (note 8); KUEMMEL, Traditionsgedanke 33; TRILLING, Israel 181. BILL 1, 249.900-905, traite cette distinction : la grandeur d'un commandement dépend de l'effort qu'il demande et de l'expiation qu'il procure. Un commandement important est "lourd" ou "grand"; un commandement moịns central est "léger" ou "petit".

2 Ainsi DUPONT, Béatitudes I 137 et HUMMEL 67. Le v. 18 n'opère pas la distinction entre petits et grands commandements. 'I $\tilde{\omega} \tau \alpha$ et uEpaía ne désignent pas les petits commandements.

3 Ainsi SCHMID, Mt 86.

4 Cf. BARTH 61 (note 3).

5 BILL 3, 85-88 traite des rapports entre l'étude et la pratique de la Tora dans le rabbinisme. Jusqu'au 2 e s., la pratique est toujours placée avant l'étude, cf. Ab $1,15.17 ; 3,9.17$. Bien qu'il faille se garder de toute surinterprétation, Mt mentionne, ici aussi, le faire avant l'étude.

6 Avec GRUNDMANN, Mt 150; SCHWEIZER, Gesetz und Enthusiasmus 52; autrement, BUECHSEL, art. $\lambda \dot{u} \omega$, ThW 4, 337. 
ments correspond la plus petite place dans le Royaume; inversement, l'observation rigoureuse de toute la Loi est sanctionnée par la grandeur eschatologique. Nous avons là un curieux mélange de motifs juifs et nonjuifs. Comme notre v., le judais me tardif interdit toute critique de la Loi ${ }^{1}$; il affirme que l'observation des petits commandements est aussi importante que celle des grands; enfin, il partage l'opinion de notre tradition selon laquelle le Royaume comprend plusieurs rangs ${ }^{2}$. Il se refuse, par contre, absolument à penser que la transgression des petits commandements conduise tout de même au Royaume ${ }^{3}$. C'est là une opinion propre à certains cercles judéo-chrétiens.

Quel est, en définitive, le rôle du v. 19 dans la composition mt ? L'évangéliste ne s'attache ni à l'idée de rang, ni à celle de petit commandement comme telle. Ce qu'il veut, c'est formuler un solennel avertissement. Le Christ est le garant eschatologique de la pratique de la Loi dans l'Eglise. Toute critique de la Tora, toute abrogation - si minime soit -elle - entraînera un "déclassement dans le Royaume".

Le lien entre les vv. 17-19 et le $\underline{\text {. } 20}$ est lâche. Tandis que les vv. 17-19 traitent le thème de la Loi, le v. 20 aborde la question de la justice. Le front, lui aussi, est différent : la polémique ne se dirige plus contre les "antinomistes", mais contre les "scribes et les pharisiens", càd contre le bas-judaîsme rabbinique d'après 70 . Pourtant, au-delà de ces

1 KUEMMEL, Traditionsgedanke 33.

2 BILL 1, 249-250.

3 BILL 1, 902. GRUNDMANN (Mt 150), STRECKER (Weg 145), DUPONT (Béatitudes I $137-138$ ), DESCAMPS (Les Justes 121), SCHNIEWIND (Mt 5I), MICHEL (art. $\mu$ lupos , ThW 4, 659) admettent que le présupposé de notre v. est l'idée de rang. BONNARD (Mt 62) et SCHWEIZER (Mt 62) pensent en revanche que le v. 19a décrit la damnation eschatologique des adversaires. Cette dernière interprétation est éga lement possible. De toute manière, pour le rédacteur mt, le v. 19 est "in globo" un avertissement parénétique. La clause d'entrée dans le Royaume est formulée au v. 20 . 
différences, une unité de réflexion subsiste, car, comme le montre la notion de justice, c'est encore de fidélité à la volonté de Dieu dont parle le v. 20 .

Le v. 20, suscription aux antithèses, présente le thème qui sera développé dans les vv. $21-48$, sous la forme d'une sentence d'entrée dans le Royaume ${ }^{1}$. Cette catégorie de logia est construite selon un schéma fixe : une conditionnelle en éá $\varepsilon^{\prime}$ formule l'exigence à remplir pour avoir part au salut décrit dans la principale. "Entrer dans le Royaume des cieux" désigne, en effet, la participation à la récompense eschatologique ${ }^{2}$. L'admission dans le Royaume est liée ici à la pratique de la justice.

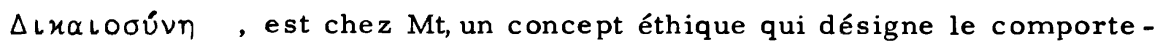
ment conforme à la volonté de Dieu ${ }^{3}$. Or, selon notre v., la justice des

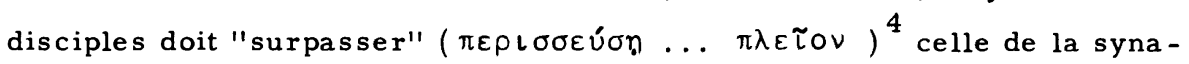
gogue. Que signifie ce dépassement ? Il a d'abord une nuance quantitative $^{5}: l^{\prime}$ obéissance de la communauté doit être plus riche, plus complète, plus abondante que celle des scribes et des pharisiens qui disent, mais ne font pas (cf. chap. 23) ${ }^{6}$.

1 Voir à ce sujet WINDISCH, Sprüche vom Eingehen $163-192$; S CHNEIDER,

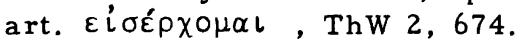

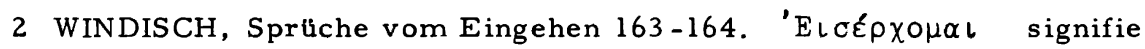
"entrer" au sens "d'être admis", il présuppose la sanction positive du

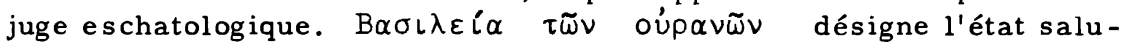
taire final promis par les prophètes et l'apocalyptique.

3 L'accord est unanime sur ce point. Cf. BARTH 130-131; BONNARD, Mt 62; BORNKAMM, Enderwartung 28; DESCAMPS, Les Justes 182; DUPONT, Béatitudes I 131; GRUNDMANN, Mt 152; SCHRENK, art.

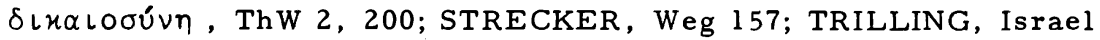
184.

4 Grammaticalement, cette expression est une gradation du comparatif (B1-D par. 246; KLOSTERMANN, Mt 41).

5 DUPONT, Béatitudes I 132; HAUCK, art. $\pi \varepsilon \rho \iota \sigma \sigma \varepsilon u ́ \omega$, ThW 6, 59; SCHLATTER, Mt 159; SCHNIEWIND, Mt 56.

6 L'hypocrisie des scribes et des pharisiens, càd leur désobéissance sous des couverts de piété, est un topos typique de la polémique mt. 
Mais ce dépassement est aussi qualitatif ${ }^{1}$ : l'obéissance des disciples doit se régler sur la volonté de Dieu telle que le Christ l'a accomplie et telle qu'elle va être illustrée par les antithèses (vv. $21-48$ ). La justice à laquelle Mt appelle sa communauté, se distingue donc d'une double manière de celle de la synagogue : elle représente une intensification de l'obéissance et une radicalisation de l'exigence.

Quel est donc le rôle du v. 20 dans le cadre de la petite composition qui nous occupe ? Le v. 20 est le corollaire sotériologique de la christologie développée dans les vv. $17-19^{2}$ : le salut réside dans la perfection éthique, laquelle est pratique de la Loi "accomplie" par le Christ.

\section{Le statut de la prédication du Christ mt}

Dans notre interprétation du manifeste $(28,16-20)$, nous avons montré que l'affirmation christologique centrale du passage accrédite une certaine conception de l'histoire et détermine corrélativement la condition du croyant. En bref, l'identité paradoxale du terrestre et du Ressuscité a boutit à rendre normative pour le présent l'histoire du Jésus terrestre, si bien que le croyant rencontre son maitre ressuscité en devenant le contemporain de l'homme de Nazareth. L'histoire passée du Jésus terrestre et le temps présent de l'Eglise ne sont pas deux époques qualitativement différentes, mais le présent se situe dans une continuité essentielle avec le passé dans la mesure où le passé reste fondamentalement transparent pour la foi.

Comment Mt 5,17-20 présente-t-il les rapports entre le passé et le présent ? entre les croyants et le Christ ? Y trouvons-nous une confirmation de la perspective entrevue dans le manifeste bien que le problème

1 GRUNDMANN, Mt 152; BONNARD, Mt 62, a bien vu que, loin de s'exclure, sens quantitatif et qualitatif se conjuguent.

2 Nous n'ignorons pas en affirmant cela que $18 \mathrm{~d}$ et 19 ouvrent déjà une perspective éthique à relevance eschatologique. 
traité soit différent ? Nous penchons pour l'affirmative et nous nous proposons de le montrer en éclairant le statut que le texte attribue à la prédication du Christ mt.

Ce statut apparait tout d'abord dans la forme de l'argumentation dont Mt use vis-à-vis de ses adversaires. Dans la polémique qu'il mène contre des cercles de son église qui ne partagent pas sa conception de la validité de la Loi, Mt n'engage pas une discussion approfondie; il se limite à rappeler ce qu'il croit être ${ }^{l}$ l'enseignement du terrestre sur ce point. C'est dire qu'à ses yeux, la solution à tout conflit de nature doctrinale trouve sa solution dans le rappel de la prédication de Jésus. Cette dernière est donc pour le présent de l'Eglise le critère de la vérité. Cette affirmation trouve une confirmation éclatante dans la forme littéraire utilisée. Nous avons montré plus haut que les vv. 18-19, mais aussi $20 \mathrm{ss}$, sont formulés de manière halachique. Cette reprise d'une forme en honneur dans la communauté de l'évangéliste est inséparable d'une certaine appréciation de la prédication de Jésus. Si les logia sont devenus des énoncés de droit sacré, cela signifie que l'enseignement du terrestre est la norme sous laquelle est placée l'Eglise et donc que cet enseignement comme tel de vient la règle de la vie ecclésiale. Enseignement de Jésus et doctrine de l'Eglise sont une seule et même réalité.

L'examen du contenu théologique des vv. $17-20$ nous permet d'avancer dans la même di rection. La notion de l'accomplissement de la Loi par le Christ mt signifie en priorité que l'enseignement de ce dernier est l'expression exhaustive, pleinement valable et décisive de la volonté de Dieu à l'égard de l'homme. Par conséquent, le Christ mt est le didascale eschatologique et sa prédication la parole ultime de Dieu qui instaure et déclare sa volonté. Le v. 18 qui fonde cette affirmation, en dégage l'enjeu historique : l'accomplissement plénier réalisé dans l'enseignement du

1 L'attitude que Mt impute au Jésus terrestre sur le problème de la Loi, ne doit pas être reportée sans autre sur le Jésus historique (voir BORNKAMM, Wandlungen $78-80$ ). 
Christ détermine tout le temps à venir comme un temps placé sous l'autorité de cet enseignement, comme le temps de la décision vis-à-vis de cet enseignement. Bornkamm ${ }^{1}$ voit d'ailleurs dans ce temps nouveau, ouvert par le Christ, la pointe de la péricope.

L'introduction aux antithèses se place donc dans une perspective théologique fondamentalement cohérente avec celle du manifeste. Cette cohérence se manifeste dans la même appréciation de l'enseignement du ter restre, du temps de l'Eglise, de la condition du croyant. L'enseignement du Christ mt, présenté comme l'accomplissement de la Loi ${ }^{2}$, est l'événement capital et décisif de son ministère. Avec lui s'ouvre un temps nouveau qui prévaudra jusqu'à la fin. La condition du croyant n'est donc qualitativement pas différente de celle des premiers disciples. Comme eux, le chrétien se trouve confronté à l'enseignement de Jésus; comme eux, il est appelé à placer son existence sous l'autorité de cette nouvelle justice. La parole qui fut adressée aux compagnons historiques de Jésus est le contenu du message de l'Eglise. Pour entendre ce message dont dépend le salut eschatologique, le croyant doit devenir l'auditeur fidèle et attentif du Jésus terrestre. Il le devient en s'identifiant au $\mu \alpha \vartheta \eta \tau \hat{n} \zeta$

de la narration $\mathrm{mt}$. Ici encore, l'ambivalence du concept $\mu \alpha \vartheta \eta \tau \hat{n} s$ trouve sa justification dans la christologie ${ }^{3}$.

1 BORNKAMM, Wandlungen 78.

2 La même famille terminologique désigne la prédication du Christ en

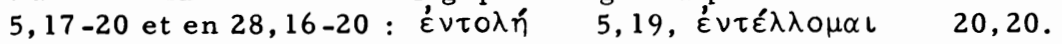

3 La même démonstration pourrait être faite à propos des adversaires de Jésus : la parole du terrestre - rendue normative par le Ressuscité condamne le judaísme d'après 70 de la même manière qu'elle avait condamné les ennemis historiques de Jésus. 
CHAPITRE CINQ : LE REVELATEUR (Mt 11,25-30)

Mt 28,16-20 et 5,17-20 nous ont fait découvrir la place absolument décisive que le Jésus terrestre - et singulièrement son enseignement occupe dans la théologie $\mathrm{mt}$. Cet axiome christologique posé par Mt, détermine la condition du croyant comme un retour au Jésus terrestre, comme une écoute de son enseignement. S'il en est ainsi, la condition du croyant n'est pas différente de celle des premiers disciples. Ces derniers deviennent dès lors dans la narration $\mathrm{mt}$ le paradigme de l'existence chrétienne.

Cette thèse, attestée dans des contextes aussi importants que le ma nifeste et le Sermon sur la Montagne, conditionne l'interprétation de l'évangile dans son ensemble. Elle mérite, par conséquent, d'être vérifiée soigneusement. Dans ce but, nous avons choisi d'analyser 11,25-30. Ce choix se justifie pour les raisons suivantes. D'une part, la péricope occupe une place-clef dans le plan de l'évangile : elle conclut la longue présentation christologique entreprise dans les chap. 5-1l. Ensuite, elle établit de manière originale les rapports qui prévalent respectivement entre le révélateur, la révélation et ses destinataires. Enfin, les vv. 28-30 qui sont un commentaire $\mathrm{mt}$ des vv. 25-27 (Q) montrent en toute clarté comment Mt comprend l'identité du révélateur et de quelle manière il définit le contenu de la révélation.

\section{Analyse littéraire}

Notre péricope se compose de trois parties ${ }^{1}$ : a) les vv. $25-26$ con-

1 Se prononcent pour ce plan : BULTMANN, Trad 171-172; DIBELIUS, Formgeschichte 280; GRUNDMANN, Mt 315; HAHN, Hoheitstitel 322; LEGASSE, Enfant 130-151; LUEHRMANN, Logienquelle 65-67; KLOS -

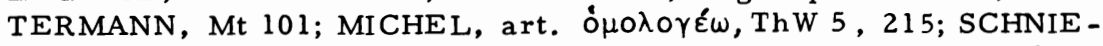
WIND, Mt 149. CERFAUX 381 rattache le v. 26 au v. 27, le $v \alpha l$ du v. 26 marquant à son avis le début d'une nouvelle unité. C'est oublier cependant que le v. 26, comme le v. 25, mais à la différence du v. 27 , est rédigé dans la terminologie de la louange vétérotestamentaire. 
tiennent une louange adressée au Père et le bénissant de s'être caché aux sages et de s'être révélé aux simples, b) le v. 27, appelé traditionnelle ment "logion johannique". est une parole de révélation sur la toute-puis sance et la connaissance du Fils; c) les vv. 28-30 sont un appel de Jésus invitant ses auditeurs "accablés" à accepter son joug.

$\mathrm{Si}$, au niveau de la rédaction $\mathrm{mt}$, les $\mathrm{vv}$. 25-30 forment un ensemble cohérent développant en trois temps un seul thème ${ }^{1}$, il est néanmoins nécessaire d'examiner la genèse de notre passage afin de découvrir les accents spécifiques mis par le rédacteur du premier évangile.

\section{a) Critique des sources}

Du point de vue de la critique littéraire, les vv. 25-27 ne soulèvent pas de grandes difficultés. Ils sont issus de la source Q. La formule de transition qui les introduit $(11,25 a)$, figurait probablement déjà dans $Q$, mais sous une forme que nous ne connaissons plus ${ }^{2}$. Quant aux deux logia proprement dits $(11,25 b-26.27)$, les versions qu'en donnent Mt et Lc, sont quasiment semblables ${ }^{3}$. Etant donné que notre enquête se limite à la rédaction mt de cette péricope, nous n'avons pas à dire si les vv. 25-27 associent deux logia à l'origine indépendants - Mt les a déjà trouvés grou pés dans sa source -, ni à statuer sur leur authenticité documentaire Mt les considérait comme des paroles de son Seigneur -. Les vv. $28-30$ posent par contre, un difficile problème, car ils sont sans parallèle canonique. Pour déterminer leur origine, on a le choi $x$ entre trois hypo-

1 Malgré de nombreuses propositions, il ne convient pas d'opérer des corrections dans le texte. Avec SCHULZ, Q 214 (note 268).

2 La version de Lc 10,21a est vraisemblablement rédactionnelle, cf. LUEHRMANN, Logienquelle 64; SCHMIDT, Rahmen 259; SCHULZ, Q 213 .

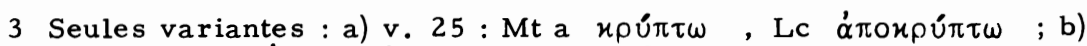
v. 27 : Mt a $\dot{\pi} \iota \gamma \iota \nu \omega ́ \sigma \varkappa \omega$, Lc $\gamma \iota \nu \omega \dot{\sigma} \varkappa \omega$; de plus, Mt construit $\dot{\varepsilon} \pi \iota \gamma \iota \nu \omega ́ \sigma \varkappa \omega$ avec un complément d'objet direct alors que Lc place une inter rogative indirecte. Ces variantes n'engendrent aucune différence de sens notable. 
thèses : soit ils faisaient partie de la source $Q$ et Lc les a omis pour une raison à déterminer; soit $M t$ les a recueillis dans une tradition qui lui est propre (Smt); soit, enfin, ils sont dus à la plume du rédacteur du premier évangile. La décision à prendre ne manque pas d'importance car, comme le montre l'histoire récente de l'exégèse ${ }^{l}$, c'est d'elle que dépend l'appréciation d'ensemble de notre péricope. Les interprétations qui discernent dans notre passage un schéma de prédication de propagande caractéristique de l'Orient ancien ${ }^{2}$ ou celles qui croient reconnaître tout au long de nos vv. la voix du Jésus historique ${ }^{3}$, présupposent l'unité prérédactionnelle de notre passage et donc l'appartenance des vv. 28-30 à la source $Q$.

Montrer, au contraire, que les vv. 28-30 sont une adjonction $\mathrm{mt}$, ébranle gravement les hypothèses que nous venons d'évoquer et accrédite l'idée selon laquelle les vv. 25-30 sont plutôt une composition originale de Mt. Or - il faut bien l'admettre -, si la documentation dont nous dis posons ne permet pas de trancher de manière définitive, elle milite néanmoins nettement pour la dernière hypothèse. A cet égard, mentionnons trois observations. D'une part, on s'explique mal pourquoi Lc aurait supprimé la troisième strophe du passage. Arguer de son caractère juif, voire anti-pharisien ${ }^{4}$, est peu convaincant, car le v. 25 est déjà une

1 LEGASSE, Enfant 125-129 et MERTENS 21-33, présentent chacun un court tableau de l'histoire de l'interprétation de notre péricope depuis le début du siècle.

2 La thèse du schéma de prédication de propagande caractéristique de l'Orient ancien a été élaborée par NORDEN 277-308, puis reprise avec quelques aménagements par DIBELIUS, Formgeschichte 279-284. FEUILLET, Sagesse 169, y adhère également.

3 Parmi les défenseurs les plus résolus de l'authenticité documentaire de notre passage dans son intégralité, citons : FEUILLET, Sagesse 170. 196; HUNTER 241-249; MERTENS qui y consacre le plus clair de sa monographie (cf. notamment 50 ).

4 Ainsi, FEUILLET, Sagesse 170. 
polémique anti-juive et, d'ailleurs, Lc ne supprime pas les logia trop "juifs" à son goût, mais les réinterprète ${ }^{1}$. Montrer qu'un tel appel n'a pas de sens à l'occasion du retour des $70^{2}$, n'explique rien: Lc 10,17-20 est une composition rédactionnelle qui crée librement le contexte préva lant pour Lc 10,21-24 ${ }^{3}$. Si l'on s'explique mal les motifs d'une éventuelle suppression lucanienne de "l'appel du Sauveur", on distingue bien au contraire pourquoi $\mathrm{Mt}$ introduit précisément à cet endroit une tradition qui lui est propre : il place en complément de $Q(11,25-27)$ et juste avant de reprendre le fil de la narration de $M c(12,1)$ une tradition qui joue, à ses yeux, le rôle de commentaire. Cette tendance à procéder à des adjonctions aux sutures du récit, est caractéristique de la rédaction mt. Mais un autre fait doit retenir notre attention : l'évangile de Thomas cite la troisième strophe de notre péricope comme un logion indépendant 4 . Or, si l'évangile de Thomas ne présuppose pas celui de Mt, mais s'ins pire d'une tradition pré-mt ${ }^{5}$, il est prouvé que le logion des vv. 28-30 était dans le trésor traditionnel une unité autonome que $\mathrm{Mt}$ (ou sa communauté) est le premier à avoir mis en rapport avec l'hymne de jubilation emprunté à $Q$. Un dernier point, enfin, mérite d'être mentionné. Si les vv. 25-27 ne trahissent aucune retouche ou correction mt, les vv. 28-30 portent une forte empreinte rédactionnelle. Selon Légasse ${ }^{6}$, les expres -

1 Voir à cet égard, le matériel suggestif rassemblé par LEGASSE, Enfant 132 .

2 DIBELIUS, Formgeschichte 280.

3 On s'expliquerait mal que Lc introduise ce passage de la source $Q$ précisément dans un contexte qui nécessite son amputation.

4 Evangile de Thomas, Logion 90 : "Venez à moi, parce que mon joug est commode et que ma domination est douce, et vous trouverez le repos pour vous". Rétroversion en grec de BAUER, Joch 103 : $\lambda \varepsilon \hat{\varepsilon} \iota^{\prime}$ 'I

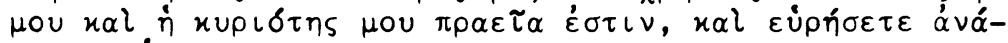
$\pi \alpha \cup \sigma \iota v \dot{u} \mu \tau \nu$.

5 Sur ce point, voir BAUER, Joch 103-106;' LEGASSE, Enfant 132-135; STRECKER, Weg 172 .

6 LEGASSE, Enfant 134. 


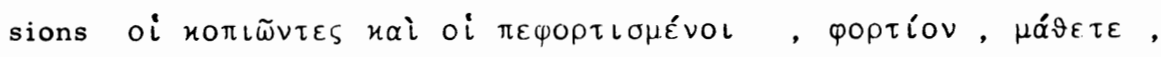
$\pi \rho \alpha u ̈ s, \tau \tilde{n} \operatorname{u} \operatorname{\rho } \rho \delta i_{\alpha}$, sont probablement des adjonctions ou des corrections dues à $\mathrm{Mt}$ ou à sa communauté. Une pareille précision reste hypothétique, car les termes que Légasse attribue à la rédaction $\mathrm{mt}$, appar tiennent au vocabulaire de la Sagesse. Néanmoins, cette terminologie -

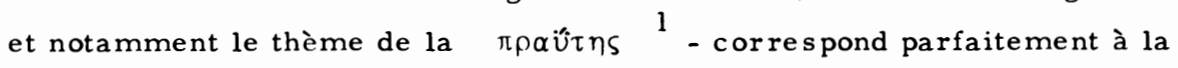
conception christologique de $\mathrm{Mt}$.

Concluons : les vv. 28-30 sont vraisemblablement un logion isolé que Mt a trouvé dans le trésor traditionnel de sa communauté ${ }^{2}$ et qu'il a placé a près l'hymne de jubilation pour en donner l'interprétation qui lui semblait adéquate ${ }^{3}$. C'est dire que ce commentaire rédactionnel devra particulièrement retenir notre attention lors de l'exégèse.

b) Le contexte de $Q$ et le contexte $\mathrm{mt}$

Dans la minutieuse étude qu'il a consacrée à la rédaction de la source $Q$, Luhrmann a montré que, dans cette collection de logia, l'hymne de jubilation (Mt 11,25-27 par. Lc 10,21-22) concluait un ensemble formé par les traditions d'envoi (Mt 9,37-10,15 par. Lc 10,2-12) et les malédictions sur les villes galiléennes (Mt 11,21-23 par. Lc 10,13-15) 4 . Il avait alors pour rôle de fonder la prétention des disciples en mission : si les disciples sont les authentiques dépositaires de la révélation, alors la décision que chacun prend à l'égard de leur prédication, engage sa des tinée ultime. La pointe du texte est plutôt sotériologique que christolo-

\section{BARTH 96 (note 1).}

2 La présence de ce logion dans l'évangile de Thomas, mais aussi les six hapaxlegomena qu'il contient par rapport à $M t$, empêchent d'y voir une formation rédactionnelle de l'évangéliste (avec STRECKER, Weg 172).

3 Partagent cette conclusion : BONNARD, Mt 169; BULTMANN, Trad 171 -172; LUEHRMANN, Logienquelle 67; STRECKER WEG 172-173; GRUNDMANN, Mt 315; SCHNIEWIND, Mt 149; SUGGS $77-83$.

4 LUEHRMANN, Logienquelle $60-63$. 
gique $^{1}$.

Chez Mt - et c'est là aussi un moyen d'interprétation - le contexte est entièrement différent ${ }^{2}$. Le chap. 11 dans lequel figure notre péricope, se présente comme une charnière dans l'économie de la pensée $\mathrm{mt}$ : il mène à chef la pensée développée dans les chap. 5-10 et prépare la pers pective abordée dans les chap. 12, voire 13. Qu'est-ce à dire ? Dans les chap. 5-10, Mt a présenté le Christ comme le Messie de la Parole (5-7) et de l'action (8-9), mais aus si comme le Seigneur tout-puissant qui associe sa communauté à son oeuvre (10). Cette ample présentation de la prédication et de l'oeuvre du Messie trouve son aboutissement naturel dans le chap. 11 qui pose la question christologique : "Es-tu Celui qui doit ve nir ou devons-nous en attendre un autre ?" $(11,3)$. Le chap. 11 dévoile sans équivoque l'identité de celui qui se prévaut d'une révélation unique $(11,25-30)$ et qui accomplit des oeuvres extraordinaires $(11,2-6)^{3}$.

Mais il montre également que le Christ va diviser irréductiblement les hommes $(11,16-25)$ et prépare ainsi le dur affrontement de Jésus avec le judaïsme au chap. 12; le chap. 13 développe cette perspective en établissant une stricte distinction entre les disciples et les foules. Notre péricope est, à cet égard, le point culminant du chap. 11 : elle caractérise le Christ comme l'authentique révélateur (axe christologique) tout en désignant polémiquement les dépositaires fidèles de son message.

c) Les parallèles historico-religieux

L'origine des traditions religieuses qui affleurent dans notre péricope est très controversée ${ }^{4}$. Néanmoins, à notre avis, les travaux de Suggs

1 LUEHRMANN, Logienquelle 68.100.

2 Cf. sur ce point, HELD $237-240$.

3 Le rôle récapitulatif du chap. 11 apparaît avec une netteté particulière

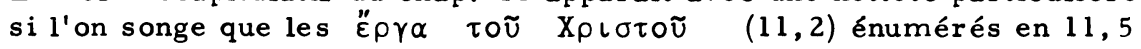
ne font que reprendre les miracles accomplis dans les chap. 8-9.

4 DINKLER 82; WILCKENS, art. $\sigma 0 \varphi i \alpha$, ThW 7,517. 
marquent un tournant dans l'histoire de la recherche. Cet exégèse propose de voir dans 11,25-30 la reprise de motifs sapientiaux juifs véhiculés par un milieu apocalyptique ${ }^{l}$. Nous nous proposons de vérifier cette hypothèse en examinant la provenance des différents motifs qui appa raissent dans notre passage.

Concernant les vv. 25-26, la quasi unanimité des exégètes s'accorde sur le caractère sémitique, voire la provenance araméenne, de notre lo-

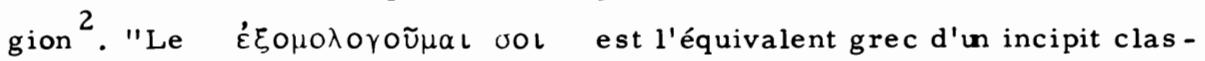
sique des formules d'action de grâces israélites" ${ }^{3}$. Le vaí (v. 26) qui reprend la louange du v. 25 est également caractéristique de ce genre littéraire ${ }^{4}$. Mais quel est donc l'objet de cette eulogie ? Dieu s'est révélé aux uns et caché aux autres. Le motif de la révélation divine de ce qui est caché, est caractéristique de l'apocalyptique ${ }^{5}$, sans pourtant être

1 SUGGS 83. DAVIES (Setting 207) a donc raison de prétendre qu'il n'est pas nécessaire de dépasser les limites du judaís me et de recourir à la mystique hellénistique paf́enne pour expliquer l'arrière-fond de notre passage (contre BOUSSET 50; BULTMANN, Trad 171-172; DIBELIUS, Formgeschichte 281-284; NORDEN 277-308. voir avant tout 290-293. 303).

2 BULTMANN, Trad 172; BRAUN, Radikalismus II 18 (note 3); CONZELMANN, art. oUvinul, ThW 7, 891; DIBELIUS, Formgeschichte 280; HAHN, Hoheitstitel 322; LEGASSE, Enfant 148; LUEHRMANN, Logienquelle 65; KLOSTERMANN, Mt 102.

3 LEGASSE, Enfant 148. L'action de grâces est commune à la tradition vétérotestamentaire-juive : Ancien Testament: Ps 75,2;111,1; 138,1-2; Si 51,1, Dn 2,23; judal's me rabbinique : voir la prière d'après le repas (BONSIRVEN, Textes 4) et la 17e bénédiction des semonè esré; Qumrân : voir l'incipit des Hodayot (BRAUN, Qumran I 23-24), ex : 1 QH 7,26-27. Cf. aussi SCHULZ, Q 217 .

4 KLOSTERMANN, Mt 102-103; LUEHRMANN, Logienquelle 65; NORDEN $50-51$.

5 Voir l'excursus de LUEHRMANN (Offenbarungsverständnis 98-104) sur la notion de révélation dans l'apocalyptique. 
ignoré de la sagesse ${ }^{1}$. Cette dernière sait que les bénéficiaires de la

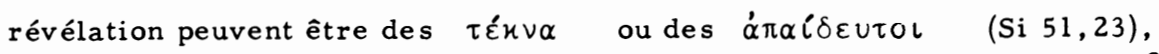
elle se propose même d'arracher les sots et les petits à leur ignorance ${ }^{2}$. La sagesse, dans son stade tardif, est donc probablement le milieu préformateur de l'opposition savant-simple du v. 25. Toutefois, elle ignore la formulation particulière que $Q$ a donnée à cette opposition, formulation qui doit être considérée comme une nouveauté dans le cadre de la tradi tion vétérotestamentaire-juive ${ }^{3}$. L'élection des petits au détriment des sages est l'expression de l' $\varepsilon \dot{U} \delta 0 x i ́ \alpha$ divine. Nous retrouvons ici un motif sapiential, car c'est dans le Si que cette notion fait l'objet d'une réflexion conséquente dans le cadre de l'AT ${ }^{4}$.

La question dominante posée par le v. 27 peut s'énoncer comme suit : dans quelle tradition la christologie du Fils s'enracine-t-elle? Пá $\nu \tau \alpha$ Hol $\pi \alpha \rho \varepsilon \delta \delta \vartheta \eta \quad$ évoque, à première vue, Dn 7, 14. Pourtant la suite du v. , nous montre qu'il n'y va pas d'une christologie du Fils de l'homme, mais de la relation réciproque du Père et du Fils. D'ailleurs, le titre viós, en tant qu'il désigne le médiateur de la révélation, n'est pas attesté dans la littérature apocalyptique juive ${ }^{5}$. Le modèle sous-jacent à

1 P.ex. Si 4,18. CHRIST 83 (note 302) a rassemblé tout le matériel concernant la révélation cachée dans la sagesse.

2 Sur le rôle des petits dans l'histoire de la sagesse et sur leur valorisation progressive dans ce milieu, voir CHRIST 83-85; sur l'histoire de l'adjectif $v n ́ \pi l o s$ dans l'Ancien Testament et le bas-judaisme, voir LEGASSE, Enfant 168-175. Cf. aussi BERTRAM, art. vท́ ThW 4, 917-918; BILL 1, 606-607; GRUNDMANN, Die $\nu \hat{n} \pi \iota 0 l$ in der urchristlichen Paränese, NTS 5, 1958-59, 180-205.

3 CHRIST 83, LUEHRMANN (Logienquelle 15), SCHULZ (Q 219), WIL CKENS (art. Coqí , ThW 7,517-518) soulignent l'originalité frappante de la formulation, tandis que HAHN (Hoheitstitel 322) et SUGGS 83-87 défendent son enracinement dans la tradition sapientiale tardive.

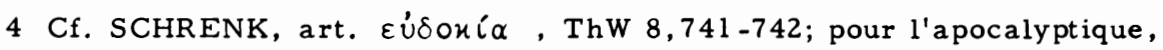
voir SCHULZ, Q 220; pour Qumrân, voir l QS 11,15-18; 1 QH 10,27ss; 14,12-15.25 (SCHWEIZER, Mt 175).

5 LOHSE, art. viós $x \tau \lambda$, ThW 8,361-363. 
notre $v$. se rapproche bien plutôt de la représentation philonienne du גóros 1 - ou, plus encore, de l'hypostase de la Sagesse ${ }^{2}$. Dans la tradition sapientiale, Dieu et la Sagesse sont dans une relation exclusive de connaissance et d'amour; cette situation privilégiée de la Sagesse 1'autorise à révéler Dieu ${ }^{3}$. Dans ce même univers sapiential tardif, Suggs nous a rendu attentif à la figure du "fils" (ou du juste) dans la Sagesse de Salomon. Cette figure est particulièrement intéressante, car en elle, comme en 11,25-27, se trouvent réunis les motifs de l'élection, de la connaissance eschatologique, de la relation intime avec le Père et enfin de l'hostilité des hommes ${ }^{4}$. Que l'on opte pour l'une ou l'autre hypothèse, on est de nouveau renvoyé à un arrière-fond sapiential.

Si notre analyse est exacte, alors le verbe $\dot{\pi} \pi \curlyvee \iota \nu \omega ́ o x \in \iota \nu$ doit être compris dans son sens vétérotestamentaire-juif ${ }^{5}$. Il signifie élire, reconnaître. Dans cette acception, il est déjà attesté chez Osée ${ }^{6}$ et connaît une large diffusion à Qumrân où $y_{7}$, désigne à la fois élection, connaissance de Dieu et révélation à d'autres ${ }^{7}$.

La quasi totalité des exégètes ${ }^{8}$ voit, à juste titre, dans "l'appel du

1 SCHWEIZER, art. viós $x \tau \lambda$, ThW 8,357.375. Voir aussi LUEHR MANN, Logienquelle 66 et l'analyse détaillée de SCHULZ, Q 224-225.

2 FEUILLET, Sagesse 179-181 et CHRIST 87-91.

3 La littérature sapientiale a été dépouillée dans cette perspective par FEUILLET, Sagesse 179-181, et CHRIST 89.

4 SUGGS 89-95.

5 Contre Norden 287 et BOUSSET 50 qui interprètent la connaissance réciproque du Père et du Fils à partir de la mystique hellénistique orientale.

6 HUNTER 245.

7 SCHWEIZER, art. viós, ThW 8,374-375 (en particulier la note 281).

8 Ainsi BONNARD, Mt 169.438; BULTMANN, Trad 171-172; FEUILLET,

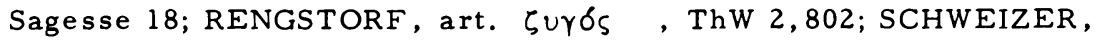
Mt 177; SUGGS 99-106. Dans la même ligne : CHRIST 103; HAHN, Hoheitstitel 322; KLOSTERMANN, Mt 103-104; WILCKENS, art. бoфía, ThW 7,517. Autrement CERFAUX 336-342 et PERCY 109110. 
sauveur" (vv. 28-30) l'écho de traditions sapientiales. Les parallèles les plus souvent évoqués sont $\operatorname{Pr} 8, \mathrm{Si} 6.24 .51^{1}$. Les vv. 28-30 ne sont certes pas une citation textuelle de $\mathrm{Si} 6$ ou $51^{2}$, mais ils reprennent incontestablement la tradition classique de l'invitation lancée par la Sagesse. A l'image de la Sagesse, Jésus, après s'être recommandé (vv. 25-27), invite les hommes à se joindre à lui, à se mettre sous son joug; comme elle, il promet le repos à ceux qui acceptent son exigence.

En invitant les hommes à se mettre sous son joug, la Sagesse les appelle en fait à s'instruire dans la Tora ${ }^{3}$. Cette connexion entre joug de la Sagesse et apprentissage de la Tora est à l'origine de la tradition rabbinique qui identifie ces deux termes et crée l'expression caractéristique "joug de la Tora". Cette tournure typique qui désigne la fidélité à la volonté de Dieu, est sans doute le parallèle le plus proche du v. $29 a^{4}$.

Concluons : ces quelques indications nous laissent pressentir combien composites sont les motifs qui se trouvent à l'arrière-fond de notre pas sage. Les représentations venant de la sagesse sont dominantes, mais elles n'excluent pas l'influence apocalyptique (vv. 25 et 27 ); les motifs émanant du judaîsme palestinien et hellénistique sont mêlés.

Il convient alors de se demander quel est le milieu qui a pu véhiculer

1. Ici encore FEUILLET, Sagesse 180-183, mais surtout CHRIST 103 117, ont dépouillé la littérature sapientiale sous cet angle.

2 Preuves chez CHRIST 112. Les seules identités verbales avec Si 6

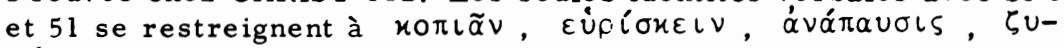
rós.

3 Voir SUGGS 101-107.

4 DINKLER 84. L'interprétation rabbinique de کưós est recommandée, d'une part, par l'opposition - voire le parallélisme paradoxal : du v. 29 avec le v. 28, d'autre part, par le ton anti-pharisien de l'ensemble du passage. Pour l'histoire de la notion de joug dans l'Orient ancien et, en particulier, dans la tradition vétérotestamentaire juive, voir : BAUER, Joch 99-106; CHRIST 108-109; RENGSTORF,

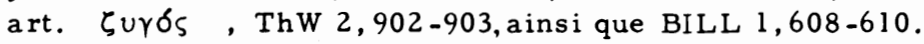


tant de traditions diverses et notamment combiner la sagesse et l'apocalyptique. A notre connaissance, seul le judaísme hellénistique du premier siècle (également bien vivant à Jérusalem !) comprenait une telle multitude de courants et les laissait librement coexister en son sein. Or, c'est précisément dans ce milieu que la foi chrétienne en pleine expansion s'est alimentée et s'est développée. Dès lors, notre péricope a probablement été façonnée dans le milieu du judéo-christianisme hellénistique. C'est ce que nous avions déjà constaté pour Mt 28,16-20 ${ }^{1}$.

\section{Interprétation}

Dans notre péricope, seuls les vv. 25-26, construits sur le modèle de l'action de grâces vétérotestamentaire-juive, sont à proprement parler un "hymne de jubilation". Cette caractéristique littéraire des vv. 25b26 ne doit pourtant pas conduire à une interprétation excessive du v. 25a :

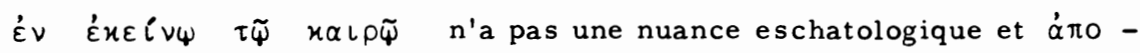

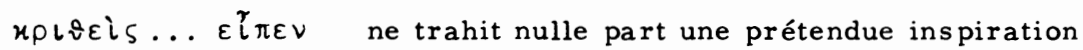
prophétique de Jésus (à la différence de Lc 10,21) ${ }^{2}$. Le v. 25 a est une formule de transition stéréotypée dont le seul rôle est d'introduire les logia qui suivent ${ }^{3}$.

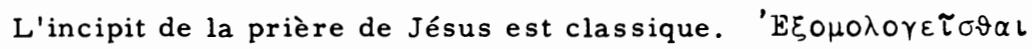

1 BORNKMANN pour Mt 28,16-20 (Der Auferstandene 298-299) et LUEHRMANN pour notre passage (Logienquelle 66) ont montré que la genèse de $\mathrm{Mt}$ est à rechercher dans le judéo-christianisme hellénis tique. Cf. aussi SCHULZ, Q 228.

2 Contre p.ex. GRUNDMANN, Mt 316, et SCHNIEWIND, Mt 149.

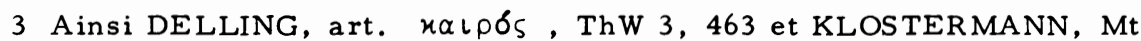

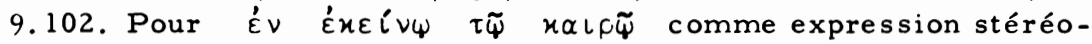
typée, voir 12,$1 ; 14,1 ; 24,15$. Dans le contexte actuel, on pourrait éventuellement la mettre en relation avec la malédiction des villes galiléennes (CHRIST 94). L'association $\dot{\alpha} \pi$ oxp $\diamond \varepsilon i s \ldots \varepsilon \imath \pi \varepsilon \nu$ en tant qu'elle introduit un discours apparait plus de 40 fois chez Mt ! 
signifie "reconnaître publiquement", "confesser" ${ }^{1}$. La littérat ure juive attribue également à Dieu le titre de "Seigneur du ciel et de la terre" ${ }^{2}$; les deux interpellations "Père" s'expliquent par la christologie du v. 27 (voir interprétation plus bas). Mais cette forme traditionnelle ne doit pas faire perdre de vue l'essentiel. Comme l'a bien montré Dibelius, cette prière solennelle et publique que le Christ mt adresse à son Père, est en réalité une prédication ${ }^{3}$. Elle montre quels sont les véritables destinataires de la révélation; elle dévoile le fondement de la communauté.

Qu'est-ce à dire?

Le v. 25 présente la révélation comme une action souveraine et paradoxale de Dieu parmi les hommes ${ }^{4}$. Dieu, de manière gratuite, dévoile "quelque chose" aux "simples" et le cache aux "sages". L'interprétation du $\tau \alpha \tilde{u} \tau \alpha$ a donné lieu à une discussion nourrie. Cependant si l'on se souvient du contexte $\mathrm{mt} d \mathrm{v}$., on ne s'étonnera pas que le $\tau \alpha \tilde{v} \tau \alpha$ f asse al lusion aux ceuvres du Christ évoquées aux vv. 2.20-24: ce que Dieu refuse aux uns et accorde aux autres, c'est de comprendre le sens de la prédication et de l'oeuvre de son Fils en tant que Sagesse incarnée ${ }^{5}$.

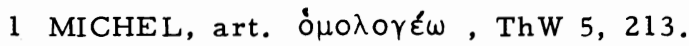

2 Matériel rassemblé par LEGASSE, Enfant 148-149.

3 Formgeschichte 282. L'action de grâces vétérotestamentaire laisse déjà présager ce fait, car la louange adressée à Dieu par le croyant, est

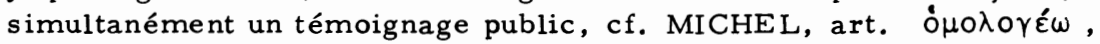
ThW 5, 213.

4 On remarquera que, dans son évangile, Mt ne parle jamais de la révélation comme telle, la racine $\dot{\alpha} \pi 0 \varkappa \alpha \lambda \cup \pi \tau$-est rare $(10,26 ; 16,17$ à part notre v.). Il préfère témoigner de l'action révélatrice de Dieu; il emploie à cet effet deux verbes dont Dieu est le sujet : हैน $\dot{\alpha} \pi \varepsilon \varkappa \alpha \dot{\alpha} \lambda \cup \phi \alpha \varsigma$.

5 Avec DAVIES, Setting 207 et SUGGS 95. Optent pour une interprétation

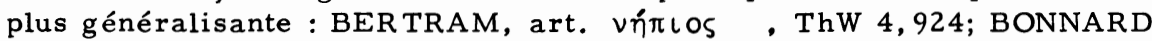

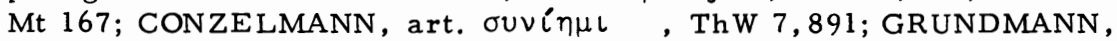
Lc 215; LUEHRMANN, Logienquelle 65; SCHNIEWIND, Mt 149. CER FAUX 743 e t LEGASSE, Enfant 151-168, rapprochent notre v. de Mt 13,11 et discernent dans le $\tau \alpha \tilde{v} \tau \alpha$ les "secrets du Royaume". Proche d'eux, CHRIST 94 voit dans le $\tau \alpha \tilde{v} \tau \alpha$ le mystère de l'élection 
Révélation et élection vont de pair. Ceux qui sont exclus de l'oeuvre salu-

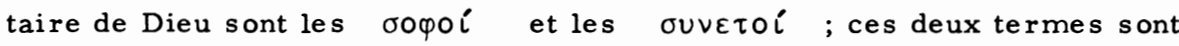
synonymiques et désignent l'élite religieuse d'Israél, càd les rabbis et les pharisiens ${ }^{1}$. Les bénéficiaires de la révélation sont les vñ Ce mot est d'une interprétation difficile, car, hormis notre passage, il n'apparaît qu'une fois chez $\mathrm{Mt}$ - et encore au sens propre -. Il est dès lors hasardeux d'inférer dans notre $\mathbf{v}$. tout le contenu idéologique que vñ $\operatorname{los}$ a acquis dans sa longue histoire vétérotestamentaire-juive ${ }^{2}$. On préférera interpréter ce concept d'après son contexte immédiat. Son

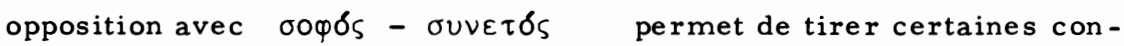

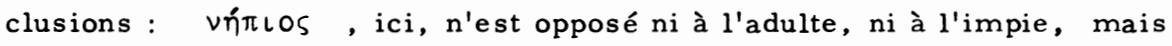
au sage. Il ne désigne donc ni l'enfant en tant que modèle de foi, ni le pauvre en tant qu'exemple de la vie religieuse, mais bien l'ignorant. Les Vท́ঁı๐ sont donc des hommes incultes, sans compétence religieuse ${ }^{3}$. A l'origine, ils s'identifient avec le am ha-aretz galiléen, méprisé par

et de l'endurcissement. DIBELIUS, Formgeschichte 280-281, éclaire le $\tau \alpha \tilde{u} \tau \alpha$ par le v. 27, càd la connaissance réciproque du Père et du Fils.

1 L'accord est général sur ce point; voir : BONNARD, Mt 167; CONZELMANN, art. cit., ibid. ; GRUNDMANN, Mt 316; KLOSTERMANN, Mt 102; OEPKE, art. xpú $\pi \omega$, ThW 3, 974. La subtile distinction de

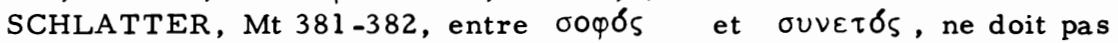
être retenue. Ces deux termes ne sont pas des désignations techniques de fonctions particulières, mais renvoient globalement aux élites religieuses. GRUNDMANN, Lc 215 , a relevé que cette association apparaît déjà dans la littérature juive $(1 Q H 1,35$, Syr. Bar 46,5$)$ pour désigner les scribes.

2 Nńđlos n'est pas un terme caractéristique de la théologie mt. Il n'apparait que 2 fois dans le premier évangile : en 11,25 au sens figuré, en 21,16 au sens propre; de plus, les 2 emplois sont dictés par l'utilisation de sources. Il est donc erroné de projeter dans notre texte tout le contenu idéologique que ce terme a dans la littérature vétérotestamentaire-juive.

3 Voir les remarques pertinentes de LEGASSE, Enfant 176-178, 183-185, mais aussi CHRIST 83 (note 305). 
l'élite pharisienne-rabbinique, et vers lequel Jésus s'est tourné avec prédilection. Dans l'évangile selon Mt, ils prennent place parmi les pauvres des béatitudes $(5,3-6)$, les pécheurs et les malades $(9,12-13)$, les petits $(10,42)$, les brebis sans berger $(9,36)^{1}$. Ce sont ceux-là que Dieu choisit pour accueillir sa révélation, c'est parmi les humbles et les ignorants qu'il recrute ses disciples.

Le v. 26, introduit par vai qui reprend la louange du v. 25, confirme avec force ce qui précède. Si la révélation est accueillie par les humbles et les ignorants, mais méprisée par les puissants et les sages, cela n'est pas dû à un échec de Jésus auprès des élites ou à son prétendu goût pour le petit peuple, mais à $l^{\prime} \varepsilon \dot{u} \delta o x i ́ \alpha$ divine. $L^{\prime} \varepsilon \dot{u} \delta o x i ́ \alpha 2$ décrit la volonté souveraine et l'amour gratuit de Dieu. Dans l'élection des petits et le rejet des grands, c'est le plan de Dieu qui se réalise.

On pourrait alors se demander comment l'église mt a compris une pareille affirmation. A n'en pas douter, elle y a vu l'élucidation théologique de son dramatique conflit avec Is raël ${ }^{3}$. Le peuple juif, personnifié dans ses élites, s'est refusé à écouter Jésus et persécute ses envoyés; à l'inverse, la communauté, se reconnaissant dans les vท́đıol de naguère, le confesse ${ }^{4}$. A l'endurcissement d'Is raèl répond l'élection de la communauté, désormais dépositaire de la révélation. La volonté divine paradoxale qui se fait jour dans les vv. 25-26, fonde la légitimité et la mission de l'Eglise face aux hommes et, en particulier, face aux Juifs. Mais quelle est cette révélation revendiquée par la communauté ?

1 Avec GRUNDMANN, Mt 316; KLOSTERMANN, Mt 102. Voir aussi BERTRAM, art. vímıOS, ThW 4, $922-924$.

2 Cf. SCHRENK, art. $\varepsilon \dot{U} \delta o x i ́ \alpha$, ThW 2, $741-748$ (en particulier 745); LEGASSE, Enfant 180-183; CHRIST 85.

3 Bien remarqué par LUEHRMANN, Logienquelle 65.

4 Nous n'avons pas à revenir sur la figure du judaísme dans l'évangile selon Mt. Notons que l'Israël endurci et représenté par ses chefs - et, en particulier, ses rabbis - reflète la situation prévalant après 70. D'autre part, comme l'a bien vu SCHLATTER (Mt 382), dans notre pas sage, les $v$ ńtıl désignent en définitive le cercle des disciples. 
Si les vv. 25-26 nous parlaient des destinataires de la révélation, le v. 27 décrit son médiateur. Le v. 27 est composé de quatre affirmations : $27 \mathrm{a}$, rédigé à la première personne, proclame l'investiture de Jésus; $27 \mathrm{bc}$ expriment la connaissance réciproque du Père et du Fils; $27 \mathrm{~d}$ revient, dans une perspective christologique, sur les destinataires de la révélation.

La difficulté principale du v. 27 a consiste à discerner le sens de $\pi \alpha \rho \alpha \delta \iota \delta \delta \vee \alpha \iota$. Ce verbe désigne-t-il ici l'investiture dans une autorité ou la transmission d'une connaissance ? ${ }^{1}$ Tant le sens d' $\varepsilon \dot{u} \delta o x i ́ \alpha$ (v. 26) exprimant l'initiative et l'intervention de Dieu dans l'histoire, que la

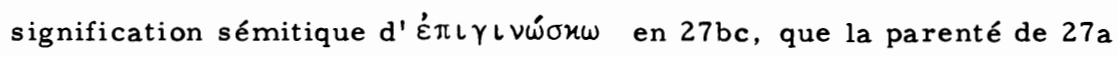
avec 28,18 militent en faveur de la première hypothèse : le Christ mt se déclare investi de toute autorité par le Père. En revanche, le contexte immédiat - et notamment la terminologie de la révélation au v. 25 et celle de l'instruction dans les vv. 28-30 - recommande la seconde hypothèse : le Christ $\mathrm{mt}$ est déclaré dépositaire de la révélation divine. En fait, il ne s'agit pas d'une alternative : l'autorité qui est conférée au Christ mt par le Père est celle du révélateur.

27 bc explicite 27 a dans un style didactique ${ }^{2}$. 'É $\pi$

1 Optent pour l'investiture dans une autorité : BONNARD, Mt 168; BUECH SEL, art. $\pi \alpha \rho \alpha \delta i \delta \omega \mu l$, ThW 2, 173; CERFAUX 333 -334; FEUILLET, Sagesse 188; HAHN, Hoheitstitel 323; LEGASSE, Enfant $137-138$; SCHLATTER, Mt 383-384; SCHNIEWIND, Mt 151; SCHRENK, art. $\pi \alpha \tau$, ThW 5, 993; WEISS-BOUSSET, Schriften NT I 310; d'autres exégètes préfèrent voir dans $\pi \alpha \rho \alpha \delta i \delta \omega \mu \iota$ la transmission d'une connaissance : GRUNDMANN, Lc 217; HUNTER 246; KLOS TERMANN, Mt 102; NORDEN 288-290; SCHMID, Mt 199; WELLHAUSEN, Mt 57. CHRIST 86 pense qu'il ne s'agit pas d'une alternative et que les deux nuances sont combinées. SCHWEIZER, art. viós, ThW 8,374-375. 382 , discerne dans le v. 27 le motif apocalyptique de l'élection et de la toute-puissance du Fils, mais ajoute que dans $Q$ et chez Mt, l'accent prédominant porte sur la fonction de révélateur.

2 Ainsi HAHN, Hoheitstitel 322.326; KLOSTERMANN, Mt 102. Cet accent de commentaire didactique se fait sentir dans l'alternance des personnes des verbes : on passe d'une déclaration de la lère personne à une explication à la 3 e personne. 
le sens vétérotestamentaire-juif de choisir, reconnaître. La connaissance exclusive du Fils par le Père (v. 27b) signifie dès lors que le Père choisit le Fils - celui que le monde ignore -, qu'il en fait son représentant uni que et exclusif ${ }^{l}$. A l'inverse, la connaissance du Père par le Fils signifie que seul le Fils confesse vraiment le Père, vit en communion avec lui. Comme le laisse présager 27a, mais comme l'atteste également la succession de $27 \mathrm{bc}$, c'est l'initiative élective du Père qui fait du Fils le médiateur de la révélation dans le monde.

Notre interprétation vétérotestamentaire de la connaissance réciproque du Père et du Fils ouvre la voie à une compréhension cohérente de 27d : si le Fils est le représentant autorisé et exclusif du Père, alors la connaissance du Père est liée à la prédication du Fils, elle n'est trans mise que par lui. L'élection du Fils est inséparable de sa tâche de révélateur.

L'intention de notre $v$. est dès lors claire : l'habilitation du Fils par le Père n'a d'autre but que de faire du Fils le représentant exclusif du Père dans le monde. Si la communauté est le destinataire de la révélation, le Fils en est le seul médiateur ${ }^{2}$. 27 fonde 25-26 christologique ment.

La structure des vv. 28-30 est dominée par le parallélisme qui existe entre les vv. 28 et $29^{3}$. A l'impératif de 28 a répond celui de $29 a$, à la

1 SCHLATTER, Mt 382 -383; SCHNIEWIND, Mt 151; WEISS-BOUSSET, Schriften NT I 311 , interprètent négativement la connaissance du Fils par le Père : Dieu reconnaît pleinement celui-là même que les hommes rejettent. Ce motif, déjà attesté dans la sagesse tardive, explique l'ordre de succession surprenant de 27 bc; de plus, il montre bien que "l'inconnu" qui est au centre du propos, n'est pas Dieu, mais bien le Christ.

2 Cette pointe est bien mise en relief par BONNARD, Mt 168; DIBELIUS, Formgeschichte 281, note 1 (mais à partir d'autres présupposés); HAHN, Hoheitstitel 326; LUEHRMANN, Logienquelle 65; SCHWEIZER, art. viós, ThW 8,375; SCHNIEWIND, Mt 152.

3 Pour le plan de $11,28-30$, voir les judicieuses remarques de HAERING $3-4$; dans le même sens, SCHMID, Mt 165. 
promesse de $28 \mathrm{~b}$ correspond celle de $29 \mathrm{c}$. Ce double parallélisme est d'importance, car il signifie que 28a.29a, respectivement 28 b. 29c, s'élucident mutuellement. Cependant, à la différence du v. 28, le v. 29 comprend une double explicative : l'appel de 29a est fondé en $29 \mathrm{~b}$ et la promesse de 29 c dans le v. 30.

Considérons tout d'abord le double appel des vv. 28a.29a. En reprenant à son compte l'antique invitation de la Sagesse, le Christ mt prétend être la Sagesse incarnée; et dans la mesure même où, pour le courant sapiential tardif (Si 24,23) et le bas-judaisme rabbinique, Sagesse et Tora ne font qu'un, le Christ $\mathrm{mt}$ prétend être la manifestation de la Tora divine. C'est en tant que présence achevée et définitive de la volonté de Dieu dans le monde que le Christ $\mathrm{mt}$ appelle les hommes à lui. ${ }^{1}$

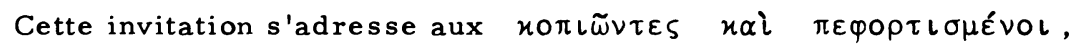
càd à ceux qui peinent sous les lourdes exigences de la conception pharisienne de la Loi et qui sont oppressés par la compréhension rabbinique de la Tora ${ }^{2}$. L'accent anti-rabbinique et anti-pharisien est violent; l'intention du texte est plus polémique que missionnaire ${ }^{3}$. Si, à l'origine, les $о \pi\llcorner\tilde{\omega} \nu \tau \varepsilon \varsigma \quad \varkappa \tau \lambda$ désignaient le am ha-aretz palestinien, en eux c'est maintenant la communauté $\mathrm{mt}$ - en lutte ouverte avec le judaísme - qui se

1 Avec SCHWEIZER, Gemeinde 54-57, et SUGGS 106-108.

2 Cette interprétation est aujourd'hui unanimement acceptée. A son actif, on notera l'accent anti-pharisien prononcé de tout le contexte $(11,12,16,20-24,25 ; 12,1-14$, etc...) et l'emploi suggestif de $\varphi \circ \rho$ $\tau \ell \alpha \quad \beta \alpha \rho \varepsilon \alpha$ en 23,4. Voir notamment: BARTH 139 (note 1); BON NARD, Mt 169; GRUNDMANN, Mt 317; KLOSTERMANN, Mt 103;

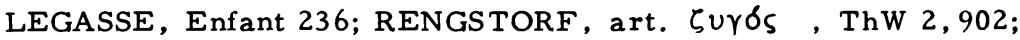
SCHNIEWIND, Mt 153-154; STRECKER, Weg 173; WEISS, art. 甲op$\tau \hat{\imath} \zeta \omega$, ThW 9,89. Autrement : CERFAUX 338 et PERCY 110. L'appréciation négative portée par Mt sur l'obéissance légale est polémique et unilatérale; le judaísme rabbinique voit, au contraire, dans le joug de la Loi un motif de joie (cf. BONNARD, Mt 170, note 1).

3 Contre LEGASSE, Enfant 237, qui voit dans notre texte un appel a Israel dans son ensemble. 
reconnaît.

Le v. 29a élucide 28a. Abandonner les maîtres du judaísme pour re joindre Jésus, ne signifie pas renier la Tora. Le Christ mt presse les hommes de le suivre, mais pour les mettre sous un nouveau joug et leur dispenser un nouvel enseignement. Le joug que Jésus recommande, désigne, de manière métaphorique, l'obéissance absolue à la volonté de Dieu ${ }^{1}$. Comme l'indique le $\mu$ ou qualifiant Cuross, Jésus oppose sa conception de l'obéissance à la Tora à celle du rabbinisme pharisien. Le

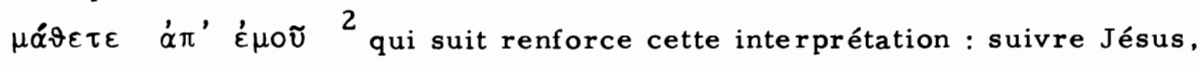
prendre le joug qu'il propose, c'est se laisser instruire par lui, c'est apprendre à son école quelle est la volonté de Dieu ${ }^{3}$. Le double appel des vv. 28a.29a signifie donc que Jésus invite les hommes à se soumettre à la volonté de Dieu, mais que cette obéissance ne peut être apprise et réa lisée qu'à sa suite. La progression de la pensée par rapport aux vv. 25-27 est dès lors claire : si les vv. 25-26 nous indiquaient quels étaient les

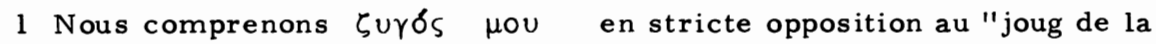
Tora" rabbinique, càd que dans le joug proposé par Jésus, nous voyons avant tout son "commandement" par opposition à la législation rabbi nique. Ce commandement est la volonté de Dieu telle qu'elle apparait, p.ex., dans le Sermon sur la Montagne (avec nous : BONNARD, Mt 170; LEGASSE, Enfant 237 -238; SCHNIEWIND, Mt 154; STRECKER, Weg

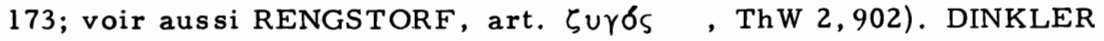
34 voit plutôt dans l'opposition des deux jougs la manifestation de l'ir ruption du Royaume; le joug de Jésus est celui des temps messianiques et provoque la disparition du joug de la Loi. Contre DINKLER, il faut relever que notre passage ne dit rien de la disparition de la Tora ou de l'avènement des temps messianiques. La christologie de 29b traite d'ailleurs des rapports entre Jésus et la Loi (voir plus bas).

2 L'emploi absolu du verbe $\mu \alpha \nu \vartheta \alpha ́ \alpha \omega$ construit avec la préposition $\dot{\alpha} \pi \delta$ est unique chez Mt. Il s'agit d'un sémitisme; on lira $\alpha \pi \delta$ au sens de $\pi \alpha \rho \alpha ́$ + génitif (voir Bl-D par. 210) et l'on traduira l'ensemble de l'expression par "se laisser instruire par quelqu'un".

3 La volonté de Dieu reste dans notre passage une catégorie formelle qui ne peut être précisée que sur la base de l'évangile tout entier. 
destinataires de la révélation, si le v. 27 nous faisait découvrir son unique médiateur, les vv. 28-30 nous rendent attentifs à son objet - la volonté de Dieu souverainement interprétée et manifestée par le Fils -.

Le v. 29b fonde la prétention du Christ à offrir désormais le seul joug authentique; le ǒ $\iota$ n'a pas une valeur complétive, mais causale ${ }^{1}$. Pour justifier son appel, le Christ $\mathrm{mt}$ - reprenant une formule vétérotes tamentaire $(\mathrm{Za} 9,9)$ - se déclare doux et humble de coeur ${ }^{2}$. Tant $\tau \alpha-$ $\pi \varepsilon \iota \nu \delta_{S}$ que $\pi \rho \alpha u ̛ ́ s$ sont deux concepts éthiques qui décrivent l'obéis sance du Christ mt. L'humilité de Jésus consiste en ce qu'il est complè tement dévoué et soumis à Dieu; l'adjonction $\tau$ ñ $x \alpha \rho o ́ l q$ indique que cette soumission est volontaire, librement consentie. Si l'humilité exprime la fidélité du Christ face à Dieu, sa douceur manifeste son attitude envers les hommes ${ }^{3}$; cette douceur qui trouve sa forme achevée dans la communion que le Christ accorde aux pécheurs et aux méprisés (cf. v. 28 !), est également de manière fondamentale obéissance, concrétisation de la volonté de Dieu. L'accent anti-pharisien est de nouveau perceptible : l'obéissance parfaite de Jésus le recommande par opposition aux rabbis hypocrites et durs de coeur. Jésus accomplit exemplairement la volonté de Dieu dont il est le prédicateur; il s'habilite comme le juste. Cette unité

1 Contre STRECKER, Weg 174, et GRUNDMANN, Mt 318; avec GAECH TER, Mt 385-386; KLOSTERMANN, Mt 104; LEGASSE, Enfant 238; SCHMID, Mt 165-166. 29b n'invite pas les hommes à découvrir dans Jésus le contenu de leur obéissance, il montre pourquoi il faut se mettre à son école.

2 Sur le sens de cette expression, voir : GRUNDMANN, art. $\tau \alpha \pi \varepsilon \iota-$ vos $x \tau \lambda$, ThW 8,20-21; HAUCK/SCHULZ, art. $\pi \rho \alpha \hat{u} S-\pi \rho \alpha-$

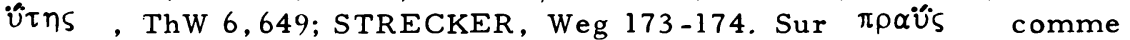
prédicat christologique chez Mt, voir BARTH 117-128. CHRIST 114 117 interprète notre expression à partir de $\mathrm{Za} \mathrm{9,9;} \mathrm{il} \mathrm{discerne} \mathrm{dans}$ notre passage la première rencontre entre la tradition du Messie davidique et celle du Serviteur de Yahvé. Ce résultat n'a d'intérêt que s'il joue un rôle au niveau de la rédaction $\mathrm{mt}$, ce que CHRIST ne vérifie pas.

3 Cf. 5,$5 ; 21,5$, mais aussi 9,$13 ; 12,7$. 
jamais démentie entre le dire et le faire, cette "justice" sans égal l'autorise à appeler les hommes à le suivre, à les instruire ${ }^{l}$.

A l'image de la Sagesse encore (cf. Sg 8,16; Si 6,19-31), Jésus lie son appel à une promesse : celui qui le suit et obéit à la Loi, trouvera le repos. Comme pour l'appel, la promesse apparaît sous une double forme (28b. 29c); les deux versions en sont si semblables qu'elles peuvent être jugées équivalentes ${ }^{2}$. Quelle est la nature du repos offert par Jésus ? Dans la tradition prophétique-apocalyptique vétérotestamentaire-juive, le repos désigne la félicité messianique, le bonheur eschatologique accordé aux justes ${ }^{3}$. Chez Mt, il conserve son sens sotériologique et décrit simplement le salut. La seule question qui se pose, est de savoir si le repos promis par le Christ mt est actuel ou eschatologique? A vrai dire, il ne s'agit pas d'une véritable alternative. Comme l'indique le présent du v. 30 , les futurs du v. 28b. 29c ont un sens conditionnel : si quelqu'un répond à l'appel de Jésus, accepte son joug, alors il éprouve immédiatement le repos. Mais dans la mesure où il obéit ainsi à la volonté authentique de Dieu, il est promis au salut eschatologique ${ }^{4}$. La déclaration du Christ

1 Cf. BORNKAMM, Enderwartung 33-34; STRECKER, Weg 177. En interprétant $29 \mathrm{~b}$ dans un sens éthico-christologique, nous refusons d'une part, une exégèse psychologico-philantropique (ainsi WEISS, Mt, ad loc; KLOSTERMANN, Mt 104), d'autre part, une analyse paulinisante (BARTH 139, note 1; SCHLATTER, Mt 387 -388; SCHNIEWIND, Mt 154).

2 En 28b, c'est explicitement J ésus qui dispense le repos; en 29c, ceux qui viennent à Jésus "trouvent le repos", mais ils le trouvent précisément parce qu'ils viennent à Jésus. Le v. 29c appelle trois remarques : a) il a été formulé sur le modèle de $\mathrm{Jr} 6,16$; b) le verbe $\varepsilon \dot{p} \hat{\text { í } \sigma n \omega}$ signifie ici "trouver" au sens de recevoir, d'obtenir un don divin, cf.

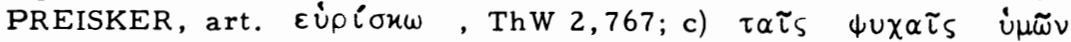
en vertu de son origine vétérotestamentaire, ne désigne pas la vie int érieure ou la personne spirituelle, mais la personne dans son ensemble (GRUNDMANN, Mt 318).

3 Sur la notion de repos dans l'Ancien Testament et le bas-judaisme, voir CHRIST 103-106; LEGASSE, Enfant 239-242.

4 Avec STRECKER, Weg 174. LEGASSE, Enfant 241-242, opte pour une interprétation présentéiste et psychologisante; GRUNDMANN, Mt 318 , ne retient que le sens eschatologique. 
est donc profondément paradoxale. Elle unit indissolublement impératif et indicatif : c'est en acceptant ce joug de Jésus que l'on accède au repos ou, comme le dit fort bien Bonnard, "il n'y a de repos que...dans... une nouvelle fidélité à la Loi" 1 .

De même que l'impératif, la promesse du repos salutaire est fondée dans une proposition explicative, le v. 30 ( ró $\rho)$. L'homme trouve le re -

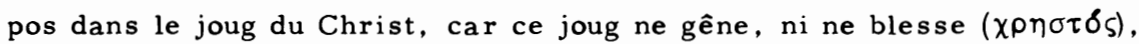
car la charge qu'il impose, est légère à porter $(\dot{\varepsilon} \lambda \alpha \varphi \rho \delta \varsigma)^{2}$. L'image doit être comprise dans son intention polémique : au contraire des rabbis qui lient des fardeaux pesants sur les épaules de leurs fidèles $(23,4)$, le Christ propose une obéissance non-oppressive et libératrice. Non pas qu'il soit moins exigeant, ni qu'il respecte mieux la psychologie de ceux qui se tournent vers lui, mais il annonce et pratique l'authentique volonté de Dieu. C'est pour cela qu'il procure le salut à ceux qui l'écoutent. De même que le joug, le repos qui lui est lié, est fondé christologiquement ${ }^{3}$.

\section{Le joug du maitre doux et humble}

En reprenant l'hymne de jubilation figurant dans sa source $Q$ (vv. $25-$ 27), Mt exprime sa christologie dans des termes que l'on ne retrouve nulle part ailleurs dans 1'évangile. Aussi est-il intéressant d'observer comment il a compris cette tradition. Nous pensons que cette élucidation est donnée

1 BONNARD, Mt 170. STRECKER, Weg 174-175, nous semble avoir jus tement cerné l'originalité théologique de notre texte lorsqu'il affirme que, pour Mt, impératif et indicatif sont identiques, càd que l'impéra tif a une signification salutaire (au contraire de Paul!). Le salut se trouve en effet dans l'exigence éthique prêchée par le Kyrios.

2 KLOSTERMANN, Mt 104, et LEGASSE, Enfant 242 (note 3).

3 Contre STRECKER, Weg 174, qui ignore la valeur explicative du v. 30 en prétendant que le joug est léger parce qu'il apporte le repos alors que l'ordre des propositions est précisément inverse. 
par le rédacteur lui-même qui développe le propos des vv. 25-27 par l'adjonction des vv. 28-30.

Or ce qui retient l'attention dans les vv. 28-30, c'est l'apparition massive du thème de la volonté de Dieu qui entraîne un réajustement de toutes les notions-clefs du passage. Ainsi, p.ex., le Fils que le v. 27 déclare représentant exclusif de Dieu parmi les hommes, est présenté au v. 28 comme la Sagesse incarnée, comme la manifestation décisive de la Tora divine dans le monde. Le v. 29 va dans le même sens en plaçant l'activité révélatrice du Fils dans la perspective de la parfaite obéissance au Père et de l'accomplissement exemplaire de sa volonté. Ce qui vaut de la destinée du Christ, s'applique également à la révélation proprement dite. Celle-ci est présentée comme un joug; cette métaphore fait allusion à l'enseignement du Jésus terrestre et, plus particulièrement, à son accomplissement plénier de la Tora. La christologie du Fils, proclamée dans les vv. 25-27, est donc spécifiée dans les vv. 28-30 à partir du thème de la Loi. Dans l'activité révélatrice du Fils parmi les hommes, il s'agit de reconnaître le Jésus terrestre qui accomplit parfaitement la volonté de Dieu aussi bien dans sa vie que dans son enseignement.

Le même réajustement se produit pour les destinataires de la révéla tion. Alors que le v. 25 saluait l'élection merveilleuse et paradoxale des vñıol, les vv. 28-29 nous montrent clairement que le don de la révélation aux simples, ne s'opère pas sur le mode d'une illumination pneu-

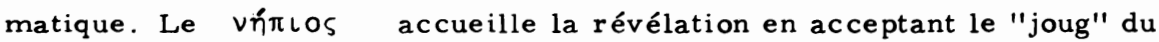
maître doux et humble, càd en se mettant à l'écoute du Jésus terrestre. La révélation est donc l'objet d'un enseignement ( $v$. 29), mais d'un enseignement qui est inséparable du maître qui le dispense : le Jésus terrestre (v. 28a). Participe à la révélation celui qui se met à la suite du Christ et qui pratique son commandement. C'est précisément dans cette relation obéissante à la prédication du terrestre que se trouve le salut eschatologique.

Concluons. Au-delà de leurs divergences de forme, de contexte, de tradition, les trois grands textes christologiques que nous avons analysés 
$(28,16-20 ; 5,17-20 ; 11,25-30)$ sont animés par la même conception. Ils recentrent l'ensemble de la proclamation chrétienne sur la personne - et singulièrement l'enseignement - du Jésus terrestre. Même le Ressuscité n'introduit pas une discontinuité essentielle dans cette perspective : il confère une normativité décisive à ce que le Jésus terrestre a dit et fait, si bien qu'il se situe dans son prolongement.

La condition du croyant est déterminée par ce modèle christologique. Elle se définit comme relation au Jésus terrestre - élevé aujourd'hui au rang de Kyrios.

La médiation qui ouvre la possibilité de cette relation est la relation même du maître et du disciple telle qu'elle est décrite dans le premier évangile et dans laquelle chaque croyant est appelé à se reconnaître (catégories ambivalentes !). La narration $\mathrm{mt}$ prend ainsi une importance insoupçonnée : en racontant l'histoire de Jésus et de ses premiers compagnons, elle révèle comment peut et doit être vécue la foi dans l'aujourd' hui de la communauté, elle est la médiation indépassable qui s'institue entre le croyant et son Seigneur. 
TROISIEME PARTIE

LA COMMUNAUTE MATTHEENNE 
MATTHEENNE

Dans nos deux premières parties, nous avons montré que l'existence chrétienne est au centre de la problématique mt et que, pour se mettre à l'écoute et à la suite de son Seigneur, le croyant de la fin du premier siècle est invité à se reconnaître dans la figure des disciples du Jésus terrestre. Dans notre troisième partie, nous voudrions mettre en évidence le contexte historique et ecclésial dans lequel ce projet théologique s'ef fectue. Certes, pour Mt, la tradition du Jésus terrestre est la seule médiation qui est donnée au croyant pour rencontrer son Seigneur, mais cette tradition est transmise et interprétée dans une église qui vit une situation précise. Nous nous proposons d'éclairer cette situation en examinant deux éléments caractéristiques de l'église mt : les charges qu'elle reconnaît et les conflits qu'elle traverse ${ }^{l}$.

Pour pénétrer avec précision dans la vie de l'église $\mathrm{mt}$, il est néces saire tout d'abord de s'interroger sur ses structures et notamment sur les ministères ${ }^{2}$ qu'elle reconnaît. Une enquête sur ce thème peut sembler illusoire, car la tradition synoptique recueillie par $\mathrm{Mt}$, ne décrit apparemment pas l'organisation ministérielle des communautés de la 2 e moitié du premier siècle. Pourtant, il est de fait que la réalité ecclésiale imprègne de part en part la rédaction matthéenne, si bien qu'il n'est pas erroné de rechercher des allusions sur ce point, même si elles doivent

1 Nous ne revenons pas sur le conflit de l'église mt avec le judaísme, qui a été traité dans le chap. 2. Nous nous limitons à deux problèmes qui permettent de découvrir le débat théologique dans lequel est engagé Mt.

2 Par pure commodité, nous choisissons le terme ministère pour désigner les diverses fonctions existant dans la communauté mt et reconnue par elle. Dans le présent chapitre, ministère a donc une signification purement formelle; il n'autorise aucune conclusion d'ordre historique ou ecclésiologique. 
rester vagues et fragmentaires.

Une lecture attentive de l'évangile nous fait découvrir deux grands groupes de ministres, disciples de Jésus et à l'oeuvre durant l'époque post-pascale : ce sont les scribes et les prophètes ${ }^{1}$. Evoquant l'intense activité des missionnaires chrétiens auprès de la Synagogue jusqu'à la chute de Jérusalem, le Christ mt déclare: "C'est pourquoi, voici que moi, j'envoie vers vous des prophètes, des sages et des scribes (23, $34 a)^{2}$ ". La présence de prophètes et de maîtres dans les communautés locales de Syrie est également attestée par une ancienne tradition figurant dans les Actes des Apôtres $(13,1)$ : "Il y avait à Antioche, dans l'église

1 L'existence de ces deux ministères dans l'église mt est largement admise dans la critique, voir : BORNKAMM, Enderwartung 22.36; DAVIES, Setting 94.106; HUMMEL, 27; KILLPATRICK 111.126; SCHWEIZER, Gemeinde 140-151; STECK 313 (note 2); STRECKER, Weg 37-38; TRILLING, Israel $81-82$ et Amt.

2 STECK a consacré une analyse approfondie à 23, 34 (cf. 26-33, 289297). La thèse classique concernant ce $v$. (voir en particulier BULT MANN, Trad 119-120 et HAENCHEN, Matthăus 23, 53-54) peut se résumer ainsi : Mt corrige sa source $Q$, bien conservée chez Lc

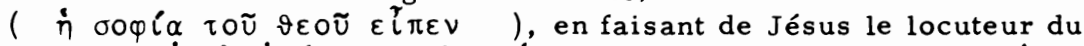

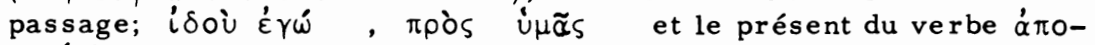
$\sigma \tau \varepsilon \dot{\lambda} \lambda \omega$ sont dès lors des corrections rédactionnelles. Deuxième point de cette hypothèse : Mt serait fidèle à $Q$ en énumérant la triade

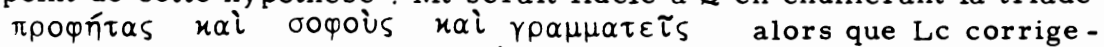
rait la source en introduisant $\alpha \pi 0 \sigma \tau \delta$ lous. Si STECK tombe d'accord sur la première partie de l'analyse, il s'en sépare sur la seconde. Pourquoi ? Les exégètes admettent généralement la priorité de la triade $m t$ en vertu de son caractère typiquement juif. Or, remarque STECK, l'ordre de succession de la triade est inconcevable pour le judaisme qui place constamment les oọoi après les $\gamma \rho \alpha \mu \mu \alpha-$ $\tau \varepsilon \tau \varsigma$ (les $\gamma \rho \alpha \mu \mu \alpha \tau \varepsilon \tau \varsigma$ sont les maitres de l'époque pré-chrétienne, les oopoi ceux de l'ère chrétienne, cf. WILCKENS, art. oo$\varphi i \alpha x \tau \lambda$, ThW 7, 505-506). Dès lors, il est plus vraisemblable de voir dans oopoùs $x \alpha i$ $\gamma \rho \alpha \mu \mu \alpha \tau \varepsilon i s$ une correction rédactionnelle du árootónous de QLC. Si STECK a raison, force est de constater que Mt a apporté un soin tout particulier à l'énumération des envoyés chrétiens auprès de la Synagogue et que le critère de cette énumération est la réalité ecclésiale. 
du lieu, des prophètes et des hommes chargés de l'enseignement ( rpo-

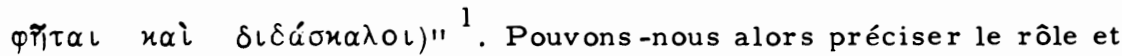
les responsabilités respectives des scribes et des prophètes dans l'église mt?

\section{Les scribes}

a) Bref état de la question :

Hummel $^{2}$ a souligné, à juste titre, que l'originalité de la communauté $m$ t n'est pas tant de compter des maîtres parmi ses membres, mais

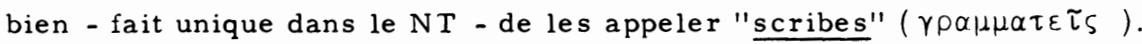
Ce titre est symptomatique du rôle que le maître chrétien assume dans l'"univers" matthéen.

Dans notre chapitre consacré "aux adversaires de Jésus" ${ }^{3}$, nous a-

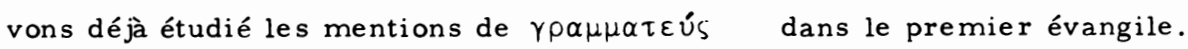

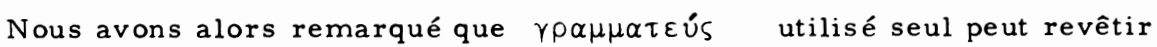
aussi bien une connotation négative $(7,29 ; 9,4)$ que positive $(13,52 ; 23,34)$. Parfois même, son emploi est neutre $(8,19 ; 17,10)$. Le scribe est donc qualifié par le parti auquel il appartient. Quels sont sur cette base les textes auxquels il convient de recourir pour poursuivre notre enquête ? Le bref maschal de 13,52 décrit de manière exemplaire l'office du scribe chrétien tandis que 23,34 évoque sa responsabilité missionnaire face à la

1 CONZELMANN, Apostelgeschichte 73, suppose que la liste citée en 13,1 repose sur une source antiochienne. HAENCHEN, Apostelgeschichte 336, admet également l'origine traditionnelle du v.; il montre que ces deux ministères archaïques s'éteignirent progressivement dans les communautés hellénistiques (337 note 2 ). Les communautés pauliniennes connaissent également l'existence de maîtres et de prophètes : 1 Co 12,28-29, voir aussi 1 Co 14,26.29, Rm 12,6-8. La Did fournit, elle aussi, des attestations : 11,$3 ; 13,2 ; 15,1-2$.

2 Auseinandersetzung 28.

3 Voir pp. 61-63. 
Synagogue. A ces deux mentions explicites, Jeremias, suivi par Bon nard ${ }^{l}$, propose d'ajouter 5,$19 ; 16,19 ; 18,18$ et $23,8-12$, passages qui, sans nommer les $\gamma \rho \alpha \mu \mu \alpha \tau \varepsilon \tau$ in verbis, n'en font pas moins allusion à la fonction de l'enseignant dans l'Eglise ${ }^{2}$.

Parmi ces différentes péricopes, deux passages nous semblent particulièrement clairs et féconds : il s'agit de 13,52 et de 23,8-12. Nous allons donc les analyser avec précision.

b) 23,8-12:

La règle de 23, 8-12 nous permet de percevoir le danger que représente le désir d'autonomie et de suprématie des maîtres dans la communauté. Elle s'oppose de façon tranchante à la formation d'un "rabbinat chrétien" ${ }^{3}$. "P $\alpha \beta \beta i \quad$ (v. 8) et $\pi \alpha \tau \dot{n} \rho \quad(v .9)$ font en effet partie de la terminologie rabbinique s'appliquant au maitre, tandis que $x \alpha \vartheta \eta \gamma \eta-$ $\tau$ ńs (v. 10) traduit cette titulature pour un auditoire hellénistique et pe u familier des usages juifs ${ }^{4}$. Les études récentes consacrées à cette péricope ${ }^{5}$ ont montré que les vv. $8-9$ constituent le noyau primitif et pré-mt de la règle $(=\mathrm{Smt})$. Le v. 10 qui élucide les vv. 8-9 à l'intention de $1 \mathrm{a}$ communauté hellénistique, est un élargissement secondaire. Mt, enfin,

1 JEREMLAS, art. $\gamma \rho \alpha \mu \mu \alpha \tau \varepsilon u ́ s$, ThW 1,742; BONNARD, Mt 210.

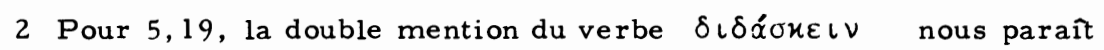
bel et bien présupposer l'activité d'enseignants. Pour 16,19 et 18, 18, tout dépend de l'interprétation du pouvoir des clefs : s'agit-il d'une activité disciplinaire ou doctrinale ? S'il s'agit d'une activité doctrinale, qui est habilité à l'exercer ? Sur ce problème voir plus bas. Pour 23,8-12, voir l'exégèse qui suit immédiatement.

3 Cf. KAESEMANN, Anfänge 85.

4 STRECKER, Weg 216-17, en particulier 217 (note 2).

5 HUMMEL 27 -28; HAENCHEN, Matthäus 23,42 -45; TRILLING, Amt 30 32; WALKER 24-25; ZUMSTEIN, Maitre et disciples 165-171. 
a commenté les vv. 8-10 par l'adjonction des vv. $11-12^{1}$, adjonction dans laquelle transparaît son interprétation de la règle.

Cette brève analyse "formiste" montre l'importance que Mt accorde à cette règle. Non seulement il juge bon de la commenter (vv. 11-12), mais encore - et surtout - il lui accorde dans l'économie du chapitre 23 une place de choix qui rompt d'ailleurs la progression logique de la polémique contre les pharisiens ${ }^{2}$. Quelle est alors l'intention du rédacteur ? Mt s'en prend à un usage abusif des titres dans sa communauté. Il ne condamne donc pas l'existence comme telle des scribes chrétiens, au contraire, il la présuppose ${ }^{3}$. Il entend fondamentalement mettre une li-

1 La preuve définitive sur ce point a été apportée par TRILLING, Amt 31-32, qui présente les arguments suivants : a) les vv. $11-12$ ont des parallèles dans la tradition synoptique alors que les vv. 8-10 ressortissen au Smt; b) dans les autres textes synoptiques, les vv. $11-12$ apparais sent toujours séparément si bien que notre passage est secondaire; c) les vv. 8-10 sont étroitement liés au contexte par les mots-crochets $\dot{\rho} \alpha \beta \beta i$ et $x \alpha \lambda \varepsilon \tau \sigma \vartheta \alpha \iota$ ce qui n'est pas le cas des vv. $11-12$; d) les vv. 8-10 ont un style cohérent - celui de la règle - qu'on ne retrouve pas dans les vv. $11-12$. Concernant l'interprétation rédactionnelle des vv. $11-12$, TRILLING formule deux remarques qui méritent d'être évoquées : a) les logia des vv. $11-12$,d'instruction des disciples qu'ils étaient à l'origine, deviennent des ordonnances ecclésiales ("Kirchengebote"); b) la règle communautaire des vv. 8-10 est examinée de manière critique à la lumière des logia de Jésus (vv. 11-12), si bien que, chez Mt, ces derniers revêtent une fonction herméneutique.

2 Placée après la courte description des ennemis (vv. 2 -7) à laquellı elle est rattachée par la brève transition rédactionnelle du v. $7 \mathrm{~b}$ (mots crochets : $x \alpha \lambda \varepsilon \tau \sigma \vartheta \alpha \iota, \dot{\rho} \alpha \beta \beta i$ ) notre règle précède immédiatement les sept malédictions (vv. 13-31) qu'elle n'introduit en aucune manière. Les liaisons avec le contexte sont donc extrêmement lâches. Cet état de fait montre bien que les vv. 8-12 n'appartenaient pas à cet ensemble traditionnel et que le rédacteur les a introduits volontairement ici. C'est dire leur importance pour lui.

3 SCHLATTER, Mt 670, appuyé aujourd'hui par WALKER 24-25, voit dans une telle règle l'attestation de l'absence de toute organisation mi nistérielle structurée dans la communauté $\mathrm{mt}$. S'il en est ainsi, il faut admettre que Mt introduit dans le chap. 23 une règle qui n'a aucun impact sur la vie de son église et qu'il y adjoint un commentaire parénéti que pareillement inutile. Cet te manière de faire s'oppose à tout ce que nous avons observé dans le reste de l'évangile et s'avère bien improbable 
mite aux privilèges et aux honneurs que s'arrogent les maitres dans son église. C'est ainsi qu'il reprend la critique christologique des vv. 8-10 et qu'il la valorise dans un sens parénétique (vv. 11-12) : l'autorité incon-

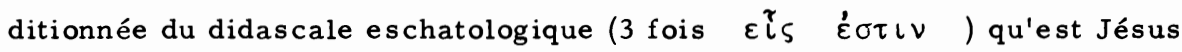
place tout ministère sous le signe de l'humilité et du service.

En conclusion, pour ce qui est de notre problématique, les vv. 8-12 nous laissent entrevoir la vie d'une église où les scribes prennent une place de plus en plus importante. Face à ce développement, le rédacteur du premier évangile juge bon à la fois de rappeler les droits du Christ sur sa communauté et d'en tirer les conséquences éthiques pour la vie ecclésiale - notamment celles qui concernent les rapports entre ministres et fidèles -.

c) $13,51-52$ :

$13,51-52$ constitue la conclusion du discours en paraboles ${ }^{1}$. Le v. 51 est vraisemblablement rédactionnel de même que les premiers mots du

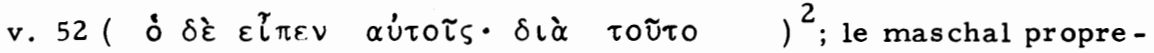
ment dit appartient au Smt. La première grande question qu'il convient de poser à propos des vv. 5l-52, concerne leur place dans l'économie du chap. 13. Pourquoi Mt a-t-il estimé judicieux de conclure son grand enseignement sur le Royaume en évoquant la compréhension nécessaire des disciples (v. 51) et la figure du scribe (v. 52) ?

1 La formule classique qui clôt les grands discours matthéens (cf. 7,28;

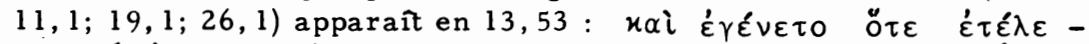

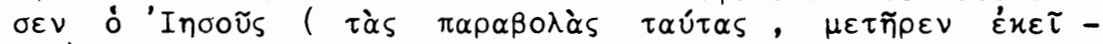
$\vartheta \varepsilon \nu)$.

2 Le v. 51 est sans parallèle. Son objet est de souligner la compréhen-

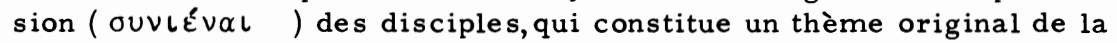
théologie matthéenne; d'où son origine rédactionnelle. La formule introductive du v. 52 est également de la main de Mt (remarquer le vocabulaire typiquement $\mathrm{mt}$ !); elle est destinée à assurer la liaison entre le v. 51 et le maschal qui suit. 
Les études récentes ${ }^{l}$ consacrées au premier évangile ont abondamment souligné l'importance accordée par le rédacteur au thème du ouvLÉval : les disciples sont par excellence ceux qui comprennent l'enseignement de Jésus, ceux qui sont ouverts à la révélation. Mais que doivent-ils comprendre ici ? Le style catéchétique des vv. $51-52^{2}$ (question-réponse-conclusion) montre qu'il y va d'un enseignement. Le chap. 13 est un enseignement exemplaire sur le Royaume; aussi faut-il que les disciples, appelés à en être les prédicateurs ${ }^{3}$, saisissent droitement la prédication de leur maître. Ils doivent éviter toute erreur d'interpréta -

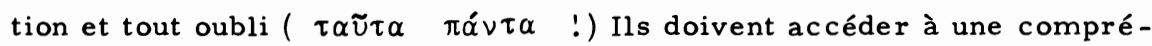
hension plénière et exhaustive de l'enseignement du Christ. Le "oui" $(v \alpha \zeta)$ qu'ils prononcent, les habilite en tant que témoins fidèles e $\mathrm{t}$ di gnes de confiance. On ne peut dès lors s'empêcher de voir dans le v. 51 une réflexion critique de Mt sur la tradition : un juste enseignement est inséparable d'une tradition vraie et exhaustive 4 .

La relation entre les vv. 51 et 52 constitue un difficile problème

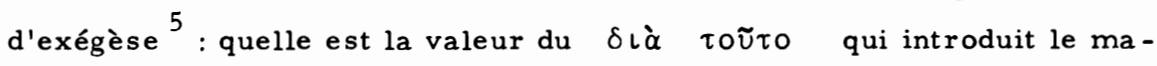
schal ? quel rapport y a-t-il entre les $\mu \alpha \vartheta \eta \tau \alpha i$ interpellés au v. 51 et le $\pi \tilde{\alpha} \varsigma$ $\gamma \rho \alpha \mu \mu \alpha \tau \varepsilon u ́ s$ évoqué auv. 52 ? Nous pensons qu'il est vain de chercher une solution "logique" à ces difficultés, car, à notre avis,

1 Sur le thème de la compréhension des disciples, voir : BARTH 99-104; HELD 279-280; LUZ, Junger 148-151; STRECKER, Weg 228-230; ZUMSTEIN, Maître et disciple 173-188. Sur notre péricope (13,51-52), voir : DUPONT, Nova 55-63; TRILLING, Amt 32-34, et Israel 14546; WALKER 27 -29; plus anciennement : HOH $256-269$.

2 TRILLING, Amt 33.

3 Cf. 10,7;28,19-20a.

4 TRILLING, Amt ibid., écrit : "Es scheint schon die Sorge um die Vollständigkeit und um das rechte Verständnis des 'Paradosis', die Sorge also um die 'wahre Lehre' zu geben."

5 Cf. HOH $257-59$; KLOSTERMANN, Mt 125. 
ici, Mt n'argumente pas avec rigueur, mais par association d'idées ${ }^{1}$. Qu'est-ce à dire ? Le v. 51 en évoquant la compréhension plénière et exhaustive de l'enseignement de Jésus par les disciples, a posé le problème de la tradition. Au v. 52, Mt conserve la même thématique, mais il l'applique à la réalité de son église; or, dans sa communauté, le scribe est celui qui recueille l'enseignement de Jésus et le transmet. La "compréhension" est donc le fil conducteur de notre passage et le maschal du v. 52 est destiné à illustrer quelle est l'exacte vocation du scribe, comment il est appelé à "comprendre" l'enseignement du Christ en vérité.

Le scribe instruit du Royaume ${ }^{2}$ est comparé à un maître de maison qui puise dans son trésor. Comme l'a bien souligné Dupont ${ }^{3}$, l'élément original du maschal et sa pointe - réside dans le fait que le maître de maison possède aussi bien du neuf que du vieux et que l'ordre de succes -

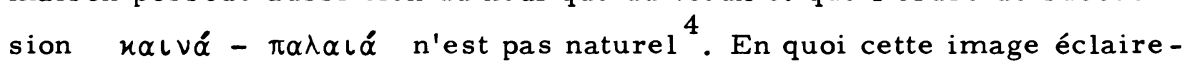
t-elle le ministère du scribe ? Si la tradition fidèle de l'enseignement de Jésus est bien la question fondamentale, l'idée est alors la suivante : le scribe est bien appelé à transmettre les instructions données jadis par le maître ( $\pi \alpha \lambda \alpha \iota \dot{\alpha})$, mais il doit le faire en les interprétant toujours à

1 Il est dès lors hasardeux de vouloir établir un rapport précis et organique entre les disciples du v. 51 et les scribes du v. 52, non qu'il n'existe pas, mais parce que Mt ne l'a pas explicité. Semblablement,

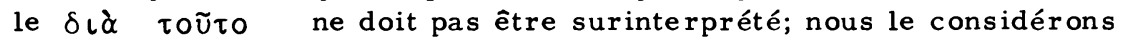
comme une simple particule de liaison.

2 B $\alpha \sigma \iota \lambda \varepsilon i q$ est un datif de relation (= quant au Royaume); $\mu \alpha \vartheta \eta$ $\tau \varepsilon \cup ́ \varepsilon \iota \nu$ a ici la nuance particulière d'instruire; voir STRECKER, Weg 192 et TRILLING, Israel 145. Le Royaume est donc ici encore matière d'enseignement (HOH $261-63)$.

3 DUPONT, Nova 56-57; WALKER 28, refuse de prendre cet élément en considération pour ne pas céder à l'allégorisation et met l'accent sur le fait que le propriétaire puise sans retenue dans son trésor. Ques tion : pourquoi le maschal culmine-t-il alors dans la formule $x \alpha \iota-$

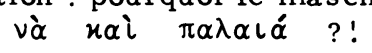

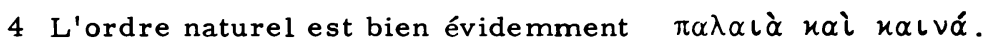


nouveau ( $x \alpha \iota \vee \alpha ́$ ) en fonction de la situation qui est la sienne ${ }^{l}$. La tradition n'est "comprise" par le scribe que si elle reste vivante, que si elle est sans cesse interprétée dans la fidélité à ses origines, que si elle est à la fois nouvelle et ancienne.

Nous avons écrit plus haut que l'une des originalités de l'église $\mathrm{mt}$ était d'avoir accordé à ses maîtres le titre de "scribe". Si notre exégèse est exacte, cette appellation n'est pas usurpée : le travail du scribe chrétien consiste précisément dans l'étude attentive, dans la transmission fidèle et dans l'actualisation sans cesse reprise de l'enseignement du mấtre. Le premier évangile est d'ailleurs le meilleur exemple de ce labeur de scribe chrétien dans la mesure où il recueille avec minutie ce que Jésus a dit et fait, tout en l'adaptant à la situation de son église.

d) Résultats :

Les scribes occupent une place assurément importante dans l'église de Mt. Ils sont appelés à transmettre l'enseignement de Jésus en l'inter prétant toujours à nouveau en fonction de la diversité des situations his toriques que vit la communauté $(13,52)$. A cette fonction ecclésiale clef s'ajoute une responsabilité missionnaire : le scribe est envoyé auprès de ses frères juifs pour les appeler à la repentance et à la conversion (23, 34).

Le rôle directeur que jouent ces maîtres n'est pas sans danger : ils risquent constamment d'usurper un rang et une autorité qui n'appartiennent qu'à leur Seigneur. La petite règle du chap. 23 apporte à cet égard le correctif nécessaire : même le ministère le plus prestigieux est un service qu'il convient d'exercer dans l'humilité.

1 L'interprétation classique indentifie le "vieux" avec l'Ancienne Alliance et le "neuf" avec l'accomplissement surgi en Jésus-Christ; cf. : DUPONT, Nova 62; SCHMID, Mt 229; SCHNIEWIND, Mt 173-74. Le point faible de cette exégèse réside dans le fait qu'elle n'est pas appuyée par le contexte (ni le chap. 13 dans son ensemble, ni le v. 51). L'hypothèse que nous défendons, figure déjà chez $\mathrm{PESCH}$, Seelsorger 76; SCHLAT TER, Mt 450-51; TRILLING, Amt 33-34. 
Semblablement, le devoir d'interprète n'est pas livré à la libre ins piration de chacun : le juge eschatologique saura reconnaitre l'enseignant fidèle de celui qui a égaré ses frères $(5,19)$.

\section{Les prophètes}

a) Bref état de la question :

Le second ministère qui a laissé quelques traces dans l'évangile se lon Mt est celui du prophète. Ici encore, il ne s'agit pas d'un trait original de la communauté $\mathrm{mt}$, mais d'un phénomène bien connu dans l'Eglise du premier siècle ${ }^{1}$. Ce ministère revêt pourtant des expressions différentes selon les régions dans lesquelles il apparaît : le prophète des communautés "pauliniennes" n'est pas semblable à celui que présente la tradition synoptique, ni à celui que révèle le livre de l'Apocalypse. Q'en est-il alors dans la Syrie du premier siècle, patrie de notre évangile ? La Didachè (chap. 11-13) - comme l'a montré Kretschmar ${ }^{2}$ - atteste l'existence des prophètes charismatiques et itinérants qui vont de village en village visiter les chrétiens qui y sont établis, à une époque où la rupture entre l'Eglise et la Synagogue n'est pas encore consommée. Ils prêchent la Parole et prononcent la prière eucharistique. Cette description est-elle également valable pour $\mathrm{Mt}$, et, dans l'affirmative, quelle position prend-il face à ce phénomène ?

La documentation qui permet de répondre à cette question, est relativement mince. Elle se compose de deux séries de textes. D'une part, nous disposons de quatre passages $(5,12 ; 10,41 ; 23,34.37)$ qui font allusion à l'existence de prophètes chrétiens. D'autre part, deux péricopes sont consacrées à la polémique contre les faux prophètes $(7,15-23 ; 24$, $9-14)$. Nous nous limitons à 1 'étude de ces passages qui traitent explicite-

1 Cf. VIElHaUer, Prophetie $422-427$.

2 Cf. KRETSCHMAR $37.47-49.65$. 
ment du ministère prophétique, sans oublier que ce phénomène nous est attesté par un tout autre biais : de larges couches de la tradition synoptique sur laquelle repose le premier évangile, sont l'oeuvre de ces inspirés qui prétendaient parler au nom du Seigneur élevé et qui prirent ainsi une part importante à la constitution de la paradosis évangélique ${ }^{l}$.

\section{b) Les prophètes au service de la communauté :}

Une attestation du ministère prophétique se trouve en $10,40-42$, petite péricope concluant le discours d'envoi en mission. Le v. 40 appartient à la source $Q$; étant donné que, dans l'évangile selon Lc (cf. 10,16), il forme également la conclusion de l'envoi en mission des soixante-douze dis ciples, il est raisonnable d'admettre que la source $Q$ l'utilisait déjà à la fin de son instruction missionnaire ${ }^{2}$. Le v. 41 fait partie du Smt; sans pouvoir le prouver absolument, nous pensons que cet élargissement du v. 40 n'est pas l'oeuvre du rédacteur de l'évangile, mais bien du milieu pré-mt, traditeur de $Q^{3}$. Ce développement de la tradition est fort instructif : il montre comment la communauté pré-matthéenne a actualisé l'instruction du v. 40. Pour elle, les envoyés qu'il convient d'accueillir, ce sont les "prophètes" et les "justes"; le fait même qu'il faille leur accor der l'hospitalité, suppose qu'il s'agit d'envoyés itinérants ${ }^{4}$. Nous nous

1 Cet élément est une preuve supplémentaire de l'existence des prophètes chrétiens. Leur influence apparaît surtout à travers les dits a pocalyptiques et prophétiques, les paroles relatives à la Loi et les règles en vue de la vie communautaire (BULTMANN, Trad 113-161). Les énoncés de droit sacré mis en évidence par KAESEMANN (Exegetische Versuche und Besinnungen II, 69-82,82-104) ont également leur place ici.

2 TRILLING, Amt 38. Remarquer que Mt supprime la partie négative du logion.

3 Avec LUEHRMANN, Logienquelle 111 et STECK 214.

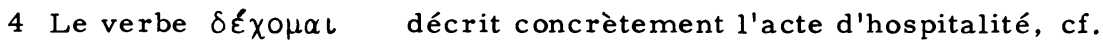
GRUNDMANN, ThW 2,52. Par ailleurs, le fait même que les croyants soient placés devant leur devoir d'hospitalité, montre bien qu'il s'agit de prophètes au service de l'Eglise. 
trouvons donc en face d'une règle communautaire qui nous fait découvrir une structure ecclésiale extrêmement ancienne, appliquée dans les petites communautés de Syro-Palestine ${ }^{1}$, et attestant l'existence de prophètes itinérants.

Or, fait absolument décisif pour notre problématique, Matthieu n'a pas repris cette règle telle quelle. Il l'a complètement réinter rétée par l'adjonction du v. $42^{2}$. Désormais, ce qui est au centre du propos de l'évangéliste, ce n'est plus l'accueil qu'il convient de réserver aux missionnaires en chemin, mais la sollicitude et le respect auxquels a droit le membre le plus humble de la communauté, le kiนpós 3 . "Il suffit que quelqu'un soit un $\mu \alpha \vartheta \eta \tau n ́ s$ pour que la communauté le respecte et le traite avec autant d'égard que d'autres membres importants ${ }^{4}$. D'une règle codifiant un ordre communautaire, nous passons à un plaidoyer en faveur des petits. Comment cela est-il possible? Il nous semble que la

1 MEYER, le premier (Ursprung and Anfange des Christentums I, 143, note 1), a émis l'hypothèse selon laquelle les vv. 40-42 reflétaient un ordre communautaire fort ancien; il est suivi aujourd'hui notamment par KAESEMANN, Anfänge 90. Si l'identification des prophètes ne pose pas trop de problèmes - on leur attribue une fonction directrice dans la communauté -, il n'en va pas de même des $\delta$ íx $\alpha \iota \iota$ : s'agit-il de chrétiens éprouvés ou de ministres (= maitres) ?

2 Le v. 42 est emprunté à Mc 9,41; le rédacteur du premier évangile apporte deux modifications à sa source : il élucide le í $\mu \tilde{\alpha} s$ de Mc

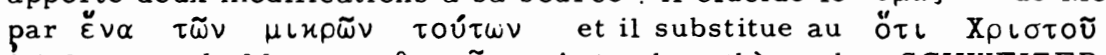

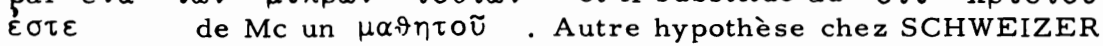
(Mt 164) : Mt a conservé la forme traditionnelle et pré-mc du logion alors que la version de Mc 9,4l (notamment la deuxième personne du pluriel) est rédactionnelle.

3 Le changement de perspective est caractéristique : l'enchaînement apôtre (v. 40) - prophète (v. 41) - juste (v. 41) dont le principe est l'envoi en mission, est brisé au profit de l'évocation de la condition du

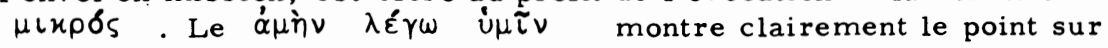
lequel le rédacteur entend s'arrêter.

4 TRILLING, Amt 39. 
réinterprétation $\mathrm{mt}$ s'explique dès l'instant où l'on admet que la structure ecclésiale suggérée au v. 41 a perdu son actualité pour le rédacteur et que le maintien conme tel de la règle risque d'accréditer le malentendu selon lequel les ministres auraient droit à des égards particuliers. Mais cela signifie alors que le prophète itinérant est en voie de disparition au moment de la composition de l'évangile ou du moins qu'il a perdu de son influence et de son importance.

Ce déclin du prophétisme dans l'église mt est confirmé par la polémique contre les faux prophètes ${ }^{1}$. Dans notre recherche sur les disputes confessionnelles, nous analyserons $7,15-20.21-23^{2}$. Nous nous limite rons donc ici à résumer nos résultats. Dans la communauté de l'évangéliste, le prophétisme est devenu un phénomène ambigu face auquel il s'agit de faire preuve de discernement ( $\dot{\pi} \iota \gamma \iota \nu \omega ́ \sigma K \varepsilon \iota \nu, 7,16-20$ ). Ses représentants semblent être des membres du peuple de Dieu $(\pi \rho \delta \beta \alpha \tau \alpha \quad 7,15)$ alors qu'en réalité ils en provoquent la perte $(\lambda u ́ x \circ \iota, 7,15)$. Leur influence néfaste tient à leur infidélité fondamentale à la volonté de Dieu, infidélité qui se manifeste aussi bien dans leur prédication que dans leur comportement concret (vv. 16-20). Pour eux, la Loi a probablement per du sa valeur normative. Certes, ce sont des charismatiques : ils excellent dans la prophétie, l'exorcisme et la guérison miraculeuse (v. 22). Il n'est pas question pour Matthieu de leur dénier ces dons, ni même de les critiquer. Le Christ $\mathrm{mt}$ a conféré à ses disciples l'égovoía néces saire à l'exercice de leur mission $(10,1.8)$ et la foi bien comprise est une foi toute-puissante $(17,20)$. Mais il ne saurait y avoir d'authentique adoration du Kyrios (v. 21) là où les charismes débouchent sur l' $\alpha$ vo $\mu l \alpha \quad$ (v. 21), c'est-à-dire sur la transgression de la volonté du maitre.

Si 10,41 nous fait découvrir un prophète itinérant, la parénèse finale du Sermon sur la Montagne nous met en contact avec sa dimension charismatique. Or, précisément, cette activité charismatique du prophète est

1 Voir notre analyse du terme $\psi \varepsilon v \delta o \pi \rho \circ \varphi$ iñ

2 Voir notre chap. 7. 
sujette à discussion. Au lieu d'être un fruit de l'obéissance, elle camoufle l'infidélité. Sans doute issue d'une piété hellénistique vulgaire qui met l'accent sur l'invocation du Kyrios glorifié et sur les dons de l'Esprit, elle est détachée de la prédication du Jésus terrestre et notamment de l'exigence d'obéissance à la volonté de Dieu. Privée de ses racines, la piété "enthousiaste" des prophètes mt ignore sa responsabilité éthique; elle s'expose ainsi au laxisme ou, pour employer un mot cher à Matthieu, au reproche "d'hypocrisie". Certes la situation n'est pas sans issue et le sens de la critique mt est bièn de ramener les égarés à la fidélité. Mais historiquement, le constat est clair : le ministère du prophète a perdu son prestige, il est désormais exposé au soupçon. Ce fait va accélérer son déclin.

c) Les prophètes envoyés à Is raël :

Steck $^{1}$ a fait remarquer à juste titre que lorsque Mt évoque la condition des prophètes chrétiens, il ne fait pas d'abord allusion à un minis tère intra-ecclésial, mais à une mission auprès d'Israël. Mt 5,12b est significatif à cet égard. Le rédacteur a trouvé ce logion dans la source $Q^{2}$. Sa recension se distingue du parallèle lc par la continuité qu'elle

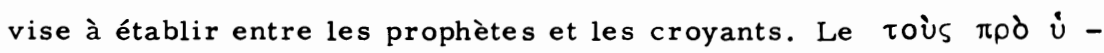
$\mu \tilde{\omega} \nu$ doit en effet être compris comme une apposition signifiant : ceux qui étaient avant vous, càd vos prédécesseurs ${ }^{3}$. Les prophètes de l'Ancien

1 STECK 308.313.

2 STECK 25-27 a fait une étude détaillée de l'histoire de la tradition de $5,12 b$. Ses résultats - convaincants à nos yeux - sont les suivants. La

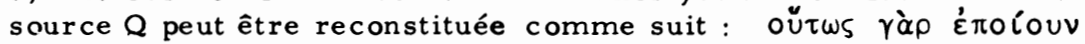

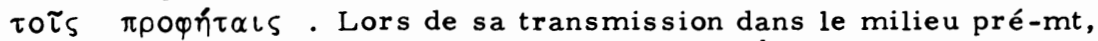
deux modifications interviennent : le verbe $\delta \iota \omega \kappa \omega$ remplace $\pi 0 \iota-$

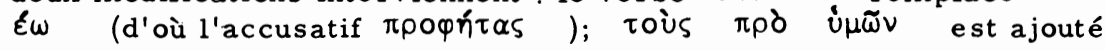
à la source. Ces deux corrections donnent au logion un sens fort original et bien dans la ligne de la prédication deutéronomiste.

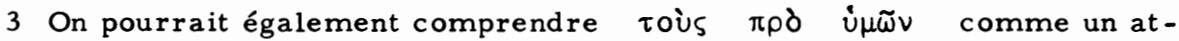
tribut avec répétition de l'article et y voir une simple détermination temporelle. On serait alors en présence d'un truisme dont on ne dis cerne pas l'intérêt. 
Testament et les personnes qui sont visées à travers le $\dot{v} \mu \varepsilon \tau_{\zeta}$ ont la même mission et endurent le même sort. De même que les prophètes de l'Ancien Testament qui appelaient Is raël à la repentance et à l'obéissance ont été violemment rejetés, ainsi en est-il des missionnaires envoyés auprès du peuple saint. Le thème de l'échec répété des envoyés de Dieu auprès d'Israël - concrétisé ici dans l'échec de la mission judéo-chrétienne en Palestine - est emprunté à la compréhension deutéronomiste de l'his toire ${ }^{1}$.

La conception du ministère prophétique sous-jacente à 5,12 b est-elle encore actuelle pour le rédacteur du premier évangile ou reflète-t-elle plutôt une situation antérieure de la communauté $\mathrm{mt}$ ? Le contexte général des béatitudes recommande la dernière hypothèse : 12b n'y figure pas en tant que consolation à l'usage des prophètes persécutés, mais à titre de motif parénétique concernant tous les croyants.

Le prophète chrétien rejeté par Israël réapparaît au chapitre 23 et particulièrement au v. 34. Nous avons analysé plus haut l'histoire de la rédaction du v. $34 \mathrm{a}^{2}$. Pour ce qui est de $34 \mathrm{~b}$, le parallèle $1 \mathrm{c} 11,49 \mathrm{~b}$

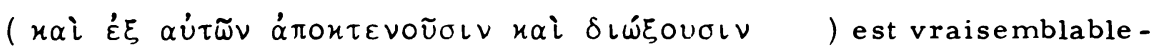
ment le reflet de $Q$. Matthieu, ici aussi, retouche profondément sa sour ce : il conserve la 2 e personne du pluriel qu'il a introduite en $34 a$ ( $\pi \rho \delta \varsigma$ $\dot{v} \mu \tilde{\alpha} \varsigma$ ) et il élargit le thème de la persécution en adjoignant les motifs de la crucifixion, de la flagellation dans les synagogues et le $\alpha \dot{\alpha}$

l STECK a consacré sa thèse à l'étude de l'image deutéronomiste de l'histoire dans l'Ancien Testament, le judaîsme et le Nouveau Testament (cf. Geschick ); pour plus de détails, nous renvoyons à ce travail. Rappelons simplement la structure fondamentale de cette vision historique : l'histoire d'Israèl se présente comme la consécration in fatigable de Dieu à son peuple et comme le refus et l'endurcissement non moins constant de ce peuple. Cette consécration de Dieu et ce refus du peuple apparaissent de manière exemplaire dans le destin des prophètes : envoyés sans relâche par Yahvé pour appeler Is raél à la repentance et à l'obéissance, ils sont rejetés avec violence.

2 Voir p. 155. 


\section{$\pi \delta \lambda \varepsilon \omega \varsigma \varepsilon i \varsigma \pi \delta \lambda_{\iota} \quad 1$.}

L'exégèse que nous avons défendue en 5,12, reçoit en 23,34 une confirmation décisive. Les prophètes chrétiens sont bien les envoyés postpascaux de l'Eglise auprès d'Israël (cf. l'ordre d'envoi de Jésus en 34a). A l'exemple des prophètes qui les ont précédés (vv. 29-31.35), ils sont rejetés et deviennent les victimes d'une haine criminelle (v. 34b). Mais ce dernier refus de la part d'Israël est d'une gravité insoupçonnée : la mesure est comble $(\mathrm{v} .32)^{2}$, la condamnation est inéluctable ( $\mathrm{vv} .33 .35-$ $36)^{3}$, le temps du repentir est passé. Comme le montre la lamentation sur Jérusalem (vv. 37-39), le dernier mot du Messie à son peuple rebelle est celui de l'abandon qui va être consommé dans la catastrophe de $70^{4}$.

En résumé ${ }^{5}$, le prophète chrétien qui apparaît en 5,12 et 23,34 . (37), est une figure post-pascale dont la mission et le destin sont compris à partir de la vision deutéronomiste de l'histoire. Sa tâche consiste à ga gner Israël au Royaume qui s'est approché en Jésus. Son activité est limitée à Israël et s'achève avec le terrible jugement de 70 qui met fin à la

1 HAENCHEN, Matthäus 23, 54; STECK 34.

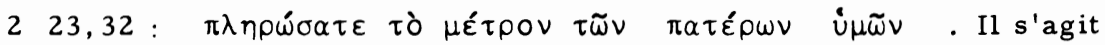
d'une représentation vétérotestamentaire-juive selon laquelle Dieu donne toujours à nouveau à l'homme la possibilité du repentir et ne juge pas avant que la mesure des péchés soit pleine (BILL 1, 939-40). Mais lorsque cette derni ère est remplie, le jugement survient inéluc tablement. La "goutte fatale" qui appelle la colère de Dieu est précisément la persécution des prophètes chrétiens (voir HUMMEL 87 -88).

3 Le v. 33 reprend la terminologie dont se sert le Baptiste pour condamner sa génération (cf. 3,7), mais - fait significatif - sans y ajouter l'appel à la conversion (voir 3,8 !).

4 Sur ce point, voir HUMMEL 85-90; STRECKER, Weg 113-115; TRILLING, Is rael 86-87. Citation significative chez HUMMEL 85 : "La destruction de Jérusalem et du temple est le jugement punitif pour la longue histoire du refus de l'appel divin, qui a culminé dans la persécution de la communauté post-pascale".

5 Pour l'ensemble du paragraphe, voir STECK $306-309$. 
période de l'appel et du repentir. Historiquement, ce prophète s'apparente au prédicateur synagogal itinérant, et la persécution qu'il subit consiste surtout dans le fait qu'il est chassé de lieu en lieu. On le remarque donc, ce ministère - qu'il ne faut pas confondre avec l'activité intra ecclésiale du prophète - n'a plus sa place dans la communauté $m t$ : il appartient à un passé certes récent, mais définitivement clos. Désormais, Israél a perdu tous ses privilèges et les Juifs sont l'objet de la mission universelle au même titre que les païens ${ }^{l}$.

\section{d) Résultats :}

Dans l'église $\mathrm{mt}$, le prophète a un double visage. Tourné vers Israël, il lance un vibrant appel à la repentance et à l'obéissance en vue d'accepter le Messie crucifié. Tourné vers la communauté, il est fort proche de la description donnée par la Didachè : son caractère charismatique est prononcé et son rôle de visiteur des croyants disséminés dans les villages est bien connu.

Pourtant que l'on envisage l'un ou l'autre champ d'action, le minis tère est sur le déclin malgré son passé prestigieux et douloureux : la catastrophe de $70 \mathrm{a}$ mis un point final à sa mission spécifique auprès d'Israèl et l'itinérance est en voie de disparition. De plus, la conduite - voire la prédication - de certains inspirés jettent le discrédit sur la "corporation". Comme dans l'ensemble de l'Eglise de la fin du premier siècle, la figure du prophète devient problématique et s'efface devant le "scribe" mieux adapté pour remplir les tâches qui incombent désormais à la communauté (transmission et interprétation de la tradition, catéchèse, etc...).

1 STECK $313-316$. 
L'église matthéenne n'est pas seulement menacée de l'extérieur. Elle est divisée contre elle-même ${ }^{l}$. Elle connaît, selon l'heureuse expression d'Ernst Käsemann, des "disputes confessionnelles" ${ }^{2}$. Ces conflits ont laissé des traces dans l'évangile. C'est ainsi que son rédacteur recourt à la terminologie de la lutte contre l'hérésie. L'examen de cette terminologie nous permet de retrouver quelques indications fragmentaires sur ces luttes "intra muros". Parmi les termes caractéristiques utilisés par

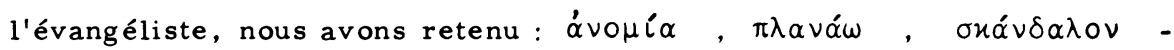

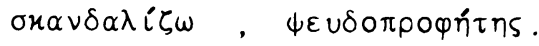

\section{La terminologie de la lutte contre l'hérésie}

\section{a) $\dot{\alpha} v o \mu i \alpha$}

'Avopía est un terme propre à Mt dans le cadre des synoptiques. Il apparait quatre fois : 7,$23 ; 13,41 ; 23,28 ; 24,12$. Nous avons de bonnes raisons de penser que les quatre attestations sont rédactionnelles, car elles figurent dans des contextes où l'activité de l'évangéliste est parti culièrement perceptible : $7,21-23^{3}$ est composé à partir de deux logia de la source $Q(\operatorname{Lc} 6,46$ et 13,25-27) et adapté à la thématique du passage

1 La thèse classique du double front a été formulée et défendue de maniè re conséquente par BORNKAMM. Elle peut s'énoncer ainsi : l'évangile selon Mt s'oppose à la fois au judaîsme d'après 70 et à un mouvement pagano-chrétien enthousiaste (cf. Enderwartung 19.20.36.46-47; Der Auferstandene 299.306-307).

2 Voir le bel article de KAESEMANN, Les débuts de la théologie chrétienne, dans : Essais exégétiques 174-198. A la p. 175, nous lisons : "... Je vais maintenant montrer à l'aide d'un petit nombre d'exemples, que l'Histoire qui précède nos évangiles a été remplie de tensions théologiques très violentes; elle a vraiment connu quelque chose comme une dispute confessionnelle (c'est nous qui soulignons)".

3 Voir notre exégèse de 7,21-23, pp. 184-187. 
qui précède; 13,36-43 - l'explication de la parabole de l'ivraie - est considéré par l'exégèse actuelle comme une création de Matthieu l'; dans le grand discours du chap. $23^{2}$, le v. 28 est un commentaire rédactionnel; enfin, 24,9-14 est une composition de Mt dans laquelle les vv. 10-12 émanent indubitablement de sa plume ${ }^{3}$. Si notre critique des sources est exacte, $\dot{\alpha} v o \mu i \alpha$ est un concept spécifique de la théologie matthéenne, probablement emprunté à la LXX ${ }^{4}$. Nous devons dès lors nous demander : pourquoi $\mathrm{Mt}$ a-t-il introduit ce terme dans son évangile et que veut-il exprimer en l'utilisant ?

L'examen du contexte nous permet de constater ce qui suit : dans trois cas, $(7,23 ; 13,41 ; 24,12), \dot{\alpha} \nu 0 \mu i ́ a \quad$ s'applique de manière critique à des membres de la communauté alors qu'en 23,28 , il vise le judaìsme à travers ses chefs. Dans les trois cas où $\dot{\alpha} v o \mu i ́ \alpha$ stigmatise le comporte ment des croyants, le ton est à la parénèse : le sort réservé aux fauteurs d' $\dot{\alpha} \vee O \mu l \propto \alpha$ doit inviter les disciples à l'obéissance; dans le cas où $\dot{\alpha} v o \mu l \alpha \quad$ s'applique aux Juifs, le ton est à la malédiction : l'iniquité séculaire du peuple d'Israèl est sanctionnée par une condamnation sans appel. Fait intéressant, tous les passages où figure $\dot{\alpha} v o \mu l \alpha$, ont un accent eschatologique : ils parlent du jugement qui s'approche et qui rétribuera chacun selon ses oeuvres.

Le concept d' $\mathrm{d}^{\prime} v o \mu i \alpha$ décrit donc une crise d'une extrême gravité; mais de quelle crise s'agit-il ? Les fauteurs d' $d^{\prime} \nu 0 \mu l \alpha$ de 7,23 sont opposés à "quiconque fait la volonté de mon Père qui est dans les cieux"

1 Voir notre exégèse de 13,36-43, pp. 187-195.

2 Cf. BULTMANN, Trad 118.352; HAENCHEN, Matthäus 23, 39.

3 Voir notre exégèse de 24,9-14, pp. 195-198.

4 Nous avons en 7,23 une réminiscence du Ps 6,9 (LXX) : $\alpha \dot{\alpha} \sigma \sigma \tau \eta \tau \varepsilon$

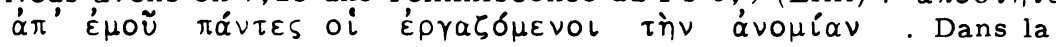
LXX, $\dot{\alpha} \vee o \mu i \alpha$ intervient comme traduction de différents termes hébrafques désignant le péché, mais d'un péché compris comme transgression de la Tora (BAUMBACH, Bösen 103; GUTBROD, art. ávoHi $\alpha$ ThW 4, 1078). 
$(7,21)$. L' $\dot{\alpha} \vee O \mu \iota^{\prime} \alpha$ est en conséquence le refus de reconnaître la volonté de Dieu et de lui obéir, étant entendu qu'il s'agit de la Loi divine telle que le Christ $\mathrm{mt}$ vient de la réinterpréter dans le $\mathrm{SM}^{1}$ et non de la Loi juive. En 13,4l, le thème de $1^{\prime} \alpha_{\alpha} v o \mu i ́ \alpha$ est mis en relation avec celui du scandale : les auteurs de scandales sont un groupe de personnes qui séduit la communauté et menace de la mener à l'apostasie; or ce groupe risque de mener la communauté à l'apostasie précisément dans la mesure où il transgresse la Loi du Fils de l'homme et invite ses frères dans la foi à faire de même ${ }^{2}$. En 23,28 , ce n'est plus une partie de la communauté qui est accusée, mais les chefs juifs : leur $\dot{\alpha} v o \mu i ́ \alpha$ consiste dans leur hypocrisie et s'oppose à la justice. En 24,12, enfin, l'accrois sement de $1^{\prime} \dot{\alpha} \vee \mu \mu \imath_{\alpha}$ est lié au dépérissement de $1^{\prime} \dot{\alpha} \gamma \alpha \dot{\pi} \eta \eta$ qui est précisément, chez Mt, le sommaire de la Loi réinterprétée par le Jésus terrestre, cf. $22,34-40$.

On ne saurait émettre de conclusions définitives sur le sens mt de 1' $\alpha$ $v o \mu i ́ \alpha$ avant d'avoir procédé à l'exégèse détaillée des péricopes en question. Pourtant certaines lignes de force se dégagent déjà. L' ávo $\mu l \alpha$ est le refus de reconnaître et de pratiquer la Loi de Dieu telle qu'elle est enseignée par le Christ mt. Ce refus ne résulte pas d'abord d'une tiédeur éthique générale, il est le fait de certaines fractions ecclésiales : les faux prophètes et les fauteurs de scandales. Cette attitude - intoléra ble aux yeux de Mt - menace gravement la vie de la communauté et mène à l'apostasie. Autrement dit, $l^{\prime} \dot{\alpha} \vee o \mu i ́ \alpha$ est l'indice d'un conflit doctrinal et par l'accusation d' $d^{\prime} \vee o \mu \iota ́ \alpha$, Matthieu s'attaque à une fraction de la communauté qui ne partage pas sa conception de la Loi et en conséquence ne la met pas en pratique.

1 Voir p.ex. DAVIES, Setting 205.

2 Le vocabulaire de ce passage typiquement mt est axé sur la probléma-

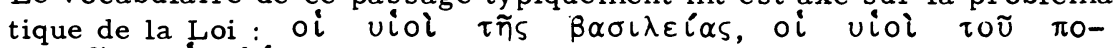
vทpou, oi síxalol. 
b) $\pi \lambda \alpha v \alpha ́ \omega$

Le verbe $\pi \lambda \alpha \nu \alpha ́ \omega$ n'est pas propre à Mt, mais, parmi les synoptiques, c'est lui qui l'utilise le plus fréquemment ( 8 fois; Mc : 4 fois; Lc : 1 fois). Les mentions de $\pi \lambda \alpha \nu \alpha ́ \omega$ en 22,$29 ; 24,4.5 .24$ provien nent de la source Mc alors que les 4 autres emplois $(18,12.12 .13 ; 24,11)$ sont rédactionnels. Sans solliciter abusivement la statistique, nous pouvons admettre que le rédacteur du premier évangile voue un intérêt cer tain à ce terme.

L'analyse du contexte nous apprend qu'à l'exception de 22,29 , les emplois de $\pi \lambda \alpha \nu \alpha ́ \omega$ sont groupés dans deux des grands discours $\mathrm{mt}$ : le discours communautaire $(18,12,12.13)$ et l'apocalypse $\mathrm{mt}(24,4.5,11$. 24). Chaque fois, dans ces deux discours, $\pi \lambda \alpha \nu \alpha ́ \omega$ s'applique à la condition du croyant et désigne de manière générale l'égarement qui me nace les membres de la communauté. Peut-on préciser la nature de cet égarement ?

Dans le cadre du chap. 18, $\pi \lambda \alpha \nu \alpha ́ \omega$ apparaît exclusivement dans la parabole de la brebis perdue (vv. 10-14) où il a été introduit par Mt (Lc utilise $\dot{\alpha} \pi \delta \lambda_{\lambda} u \mu \iota \quad$ ). Or cette originalité terminologique s'explique par l'interprétation que $\mathrm{Mt}$ donne de cette parabole ${ }^{l}$. Chez lui, il ne s'agit plus comme chez Lc, de dire la joie liée à la conversion du pécheur (Lc 15,7); l'attention se porte sur l'égarement qui menace les "petits". Cet égarement est provoqué par le scandale (voir la péricope qui précède) et il mène à l'apostasie. L'égarement semble donc désigner l'erreur doctrinale plutôt que l'infidélité éthique.

Dans le dialogue entre Jésus et les sadducéens sur la résurrection, le $\pi \lambda \alpha \nu \tilde{\alpha} \sigma \vartheta \varepsilon$ accusateur adressé aux adversaires du Christ $(22,29)$

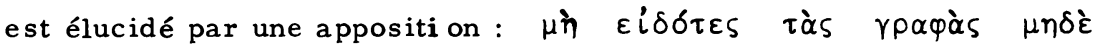

1 Voir BORNKAMM, Lösegewalt 41 : "Die Parabel dient darum hier nicht mehr primär dazu, die göttliche Gnade gegenüber den Verlore nen zu verkündigen, sondern der Gemeinde die Pflicht der Fürsorge für die Irrenden einzuprägen". Voir aussi JEREMIAS, Gleichnisse $35-37$. 


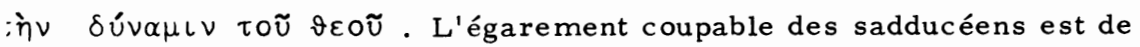
lature doctrinale : il y va d'une interprétation fausse des textes et d'une néprise quant à la personne de Dieu.

En 24,3-8, les disciples sont mis en danger par l'apparition de faux prophètes (thème non explicite !) qui prêcheront le retour d'un faux Christ. Même note en 24,23-28; des faux prophètes et des faux Christ, membres de la communauté et porteurs de dons charismatiques étonnants, vont jusqu'à mettre en péril les "élus" en les trompant sur la personne de Jésus. Dans ces deux passages de l'apocalypse $\mathrm{mt}$, le sens de $\pi \lambda \alpha \nu \alpha ́$ $\omega$ est bien profilé : une fraction de la communauté - les faux prophètes va introduire l'erreur dans l'Eglise - une erreur fondamentale puisqu'elle conduit à se méprendre sur la personne du Christ; l'égarement consiste à prêter foi à cette erreur.

Un dernier passage de l'apocalypse mt nous permet de franchir encore un pas : 24,9-14 décrit à nouveau l'apparition des faux prophètes qui égarent les croyants, mais il montre de manière intéressante que l'erreur ainsi introduite dans l'église culmine dans la recrudescence de 1 ' $\alpha$ vo lía et le dépérissement de l'amour. L'égarement, dans ce passage typiquement rédactionnel, est donc lié à la thématique de la Loi.

Concluons : le verbe $\pi \lambda \alpha \nu \alpha ́ \omega$ stigmatise une perversion des affir mations fondamentales de la foi: Dieu, l'Ecriture, le Christ, la Loi. Cette erreur est répandue dans l'Eglise par les faux prophètes et les ouvriers du scandale. Elle menace tous les croyants, même les élus, mais ce sont les $\mu \iota x \rho o i$ qui sont le plus vulnérables. Le chap. 18 et surtout 24,9-14 nous indiquent clairement que cette mise en garde contre l'égarement, loin de n'être qu'un topos apocalyptique dans le premier évangile, atteste que la communauté $m$ t est en butte à un conflit doctrinal dans lequel Mt prend violemment parti.

c) $\sigma x \alpha ́ v \delta \alpha \lambda o v$ - $\sigma x \alpha \nu \delta \alpha \lambda i \zeta \omega$

La terminologie du scandale est un élément original du vocabulaire matthéen. La statistique nous apprend en effet que Matthieu utilise 5 fois 


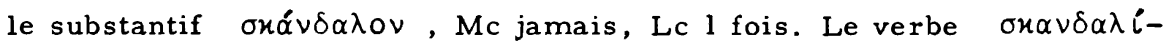
$\zeta \omega$ figure 14 fois chez Mt, 8 fois chez Mc, 2 fois chez Lc. La critiqu des sources confirme l'analyse statistique : des 5 mentions de ơó $v \delta \alpha-$ $\lambda$ ov, 4 sont rédactionnelles $(13,41 ; 16,23 ; 18,7.7)$ tandis que la seconde attestation de ce terme en 18,7 est probablement empruntée à la source $\mathrm{Q}$ (cf. Lc 17, 1). Des 14 utilisations du verbe $\sigma x \alpha \nu \delta \alpha \lambda \iota \zeta \omega, 6$ provien. nent de la source Mc $(5,29.30 ; 13,21.57 ; 26,31.33), \quad 1$ de la source $Q$ $(11,6), 1$ du Smt $(17,27)$ tandis que les 6 dernières sont rédactionnelles $(15,12 ; 24,10 ; 26,33 ; 18,6.8 .9$ sont employés en doublet). L'emploi de la terminologie n'est pas homogène. Nous nous concentrerons donc sur les passages où cette terminologie est appliquée à la vie de l'église $\mathrm{mt}$ ou permet de l'éclairer.

a) $\sigma x \alpha \dot{v} \delta \alpha \lambda$ ov

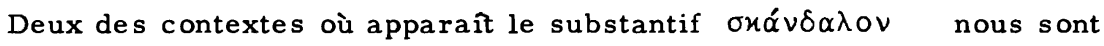
déjà connus : il s'agit de l'explication de la parabole de l'ivraie $(13,41)$ et du discours communautaire du chap. 18, plus précisément du plaidoyer en faveur des petits qui commence au v. 6. En 13,41, l'emploi de ơóv$\delta \alpha \lambda$ ov est fort singulier ${ }^{l}$ : il désigne un groupe de personnes assimilé à ceux qui pratiquent l' $\mathrm{\alpha}^{\prime} \vee 0 \mu l \alpha$ ( $\alpha \alpha \hat{\imath}$ explicatif) : les fauteurs de scandale sont alors ceux qui mènent leurs frères à l'apostasie en les invi tant à transgresser la Loi. En 18,7, la célèbre malédiction sur les au teurs de scandale est insérée dans le plaidoyer en faveur des petits : l'auteur de scandale est alors celui qui égare le petit, qui l'amène à re nier sa foi en le séduisant. Le passage de 16,23 est d'un haut intérêt, car, même s'il n'est pas en relation avec la vie de l'église mt, il nous offre une définition du scandale tel que le conçoit $\mathrm{Mt}$. La mention rédac-

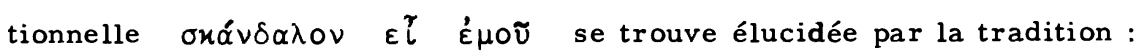

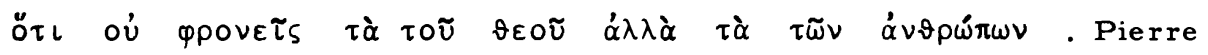
est un scandale pour Jésus en ce qu'il essaie de le détourner du cheminde

1 Voir notre exégèse de l'explication de la parabole de l'ivraie, pp. $187 \mathrm{ss}$. 
la Passion. Et il essaie de le détourner de la Passion parce qu'il s'attache

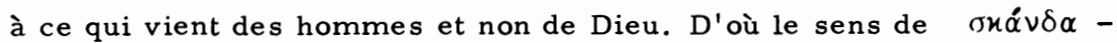

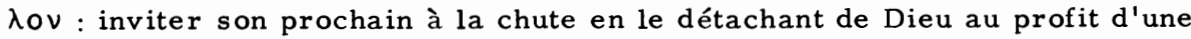
compréhension mondaine.

Un mot de conclusion : il existe dans la communauté mt des hommes qui sont une occasion de chute pour leurs frères (cf. 13,41;18,7), qui les détournent de Dieu en les séduisant et notamment en les invitant à transgresser la Loi telle que Mt la comprend.

\section{B) $\sigma x \alpha \nu \delta \alpha \lambda i \zeta \omega$}

Nous repérons chez Mt trois emplois principaux du verbe $\sigma x \alpha \nu \delta \alpha-$ $\lambda i \zeta \omega$. En premier lieu, ơ $\alpha \nu \delta \alpha \lambda i \zeta \omega$ apparait dans les sentences paradoxales dans lesquelles une partie du corps est objet de scandale si bien qu'il vaut mieux la supprimer $(5,29.30 ; 18,8.9)^{l}$. En second lieu, notre verbe décrit le scandale qui est attaché à la personne de Jésus : il est une occasion de chute pour l'homme naturel et, en particulier, pour le Juif $(11,6 ; 13,57 ; 15,12 ; 17,27)$. En troisième lieu, enfin, $\sigma \mathfrak{x} \alpha \nu \delta \alpha-$ $\lambda i \zeta \omega$ décrit une crise qui survient dans la vie du disciple par le fait des circonstances ou même de ses frères $(13,21 ; 18,6 ; 24,10 ; 26,31.33$. 33). C'est le troisième emploi - le plus fréquent - qu'il convient d'approfondir, car c'est lui qui a la plus nette connotation ecclésiale.

La mention du verbe $\sigma x \alpha \nu \delta \alpha \lambda i \zeta \omega$ en 13,21 (explication de la parabole du semeur) est - bien que traditionnelle - extrêmement significa tive. Un homme qui a reçu l'évangile avec joie, le renie aussitôt sous l'empire de la tribulation ou de la persécution. Le danger qui est attaché à la foi, devient une occasion d'apostasie. Le sens est le même en 24,10

1 Th. ZAHN, J. WEISS et W. BOUSSET, appuyés aujourd'hui par PESCH, Seelsorger 27, militent pour une interprétation allégorique de Mt 18,6. 9 : les membres scandaleux sont des membres hérétiques de la communauté qu'il convient de retrancher, càd d'excommunier. Cette interprétation qui conviendrait bien au contexte (question de discipline ecclé siale) fait pourtant intervenir une conception ecclésiologique paulinienne (L'Eglise comme corps) par ailleurs inconnue de Mt. 
où c'est également la persécution venant de l'extérieur, mais aussi les divisions internes causées par la prédication des faux prophètes, qui provoquent l'apostasie. Dans l'annonce du reniement de Pierre $(26,31.33$. 33), c'est la passion du Christ qui amène la chute des disciples; ơ $\alpha \nu \delta \alpha-$

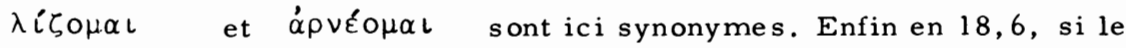

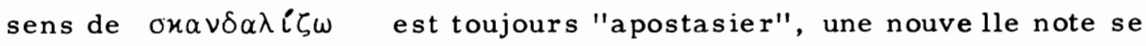
profile : le disciple peut être pour son frère, en l'occurence le "petit", l'occasion de la chute fatale.

Résumons-nous. Les quelques mentions du verbe $\sigma x \alpha \nu \delta \alpha \lambda i \zeta \omega$ que nous avons examinées, nous montrent clairement que le risque d'apostasie est réel dans la communauté $m$. Le scandale survient lorsque de graves difficultés accablent l'Eglise. Ces difficultés peuvent être externes (persécutions) aussi bien qu'internes (divisions, faux prophètes). C'est le danger de l'hérésie qui, dans ce dernier cas, provoque la réaction de Mt.

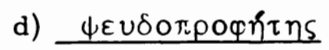

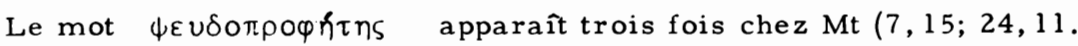
24), une fois chez Mc et une fois chez Lc (Mc 13,22; Lc 6, 26). Si en 24, 24, $\psi \varepsilon \cup \delta \circ \pi \rho \circ \varphi \eta \tau n s$ est d'origine traditionnelle (reprise verbale de Mc 13,22), les mentions de 7,15 et de 24,11 sont rédactionnelles. 7,15 , en effet, est un verset d'introduction composé par Mt, 24,11 appartient à un passage de l'apocalypse $\mathrm{mt}$, librement rédigé par le premier évangéliste ${ }^{l}$.

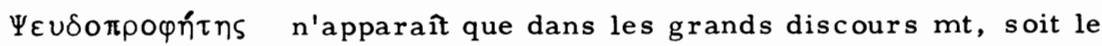
$\mathrm{SM}(7,15)$ et l'apocalypse $\mathrm{mt}(24,11,24)$. Dans les trois cas, le contexte est parénétique ${ }^{2}: 7,15$ se trouve dans la parénèse finale du SM tandis

1 Sur 1'origine rédactionnelle de 7,$15 ; 24,11$, voir nos exégèses de 7,15$20 ; 24,9-13$, pp. 181.195-196.

2 Le SM et l'apocalypse $\mathrm{mt}$ sont des instructions adressées aux disciples (voir notre analyse sur la place des disciples dans les grands discours $\mathrm{mt}$, chap. 1). L'intention parénétique de la conclusion du SM $(7,12-27)$ est soulignée par l'ensemble des exégètes. Concernant 24,9-14, on soulignera la portée parénétique du v. 13 et pour ce qui est des vv. $23-28$, on relèvera la fonction parénétique du v. 25 . 
que 24,11 appartient à une péricope mettant en garde contre les dangers liés à la persécution; 24,24 prévient les croyants contre les faux prophètes.

A quelle réalité $\mathrm{Mt} \mathrm{s}^{\prime}$ attaque-t-il en polémiquant contre les faux prophètes ? On remarquera tout d'abord qu'en 7,15 , il s'agit d'une réalité présente, alors qu'en 24,11.24, il est fait état d'une réalité future, bien évidemment en accord avec l'accent apocalyptique du contexte. Cette simple remarque suggère déjà que les faux prophètes constituent un problème qui concerne l'existence des disciples, mais non celle du Jésus terrestre ${ }^{1}$. L'analyse plus précise des trois passages permet de confirmer ce point de vue.

Le $\log$ ion de 7,15b montre clairement qu'il ne peut s'agir d'adversaires juifs. Les faux prophètes se présentent comme des "brebis" alors qu'ils sont en fait des "loups". Or "brebis" désigne, dans son sens imagé, le membre du peuple de Dieu ${ }^{2}$. Les faux prophètes sont donc des membres de la communauté chrétienne. En effet, ces mêmes faux prophètes sont présentés en 7,21 comme ceux qui invoquent constamment le Kyrios Jésus et en 7,23 comme ceux qui prétendent agir en son nom.

C'est la même note qui apparaît dans le chap. 24. 24,9-14 décrit la destinée de souffrance de la communauté chrétienne aux prises principalement avec les paiens $(v .9 b)^{3}$. Les $\pi 0 \lambda \lambda \circ i$ des v. 10 et 12 sont des chrétiens qui succombent au scandale, qui se laissent aller à la haine et à la délation, qui se laissent abuser. Or, comme en 7,15 , ceux qui les abusent sont avant tout leurs frères dans la foi.

1 Le Christ mt met en garde les disciples contre l'intrusion des faux prophètes dans la communauté (période post-pascale !); lui-même ne les affronte jamais tout au long de l'évangile. Ses adversaires sont les représentants du peuple juif qui ne sont jamais assimilés à de faux prophètes (voir p.ex. chap. 23).

2 Sur ce point, voir notre exégèse de 7, 15, pp. 181-183.

3 La période visée est donc incontestablement post-pascale. 
La mention de 24,24 est encore plus claire. Les faux prophètes sont rapprochés des faux Christ; or, où peut-il y avoir de faux Christ, sinon dans l'Eglise ? Semblablement le v. 25 parle de l'égarement possible des "élus"; il est bien improbable que les "élus" se laissent séduire par des personnes étrangères à la communauté et, en particulier, par les Juifs; on connaît les sentiments de la communauté mt à leur égard. Notre conclusion est claire : les faux prophètes annoncés et dénoncés par le Christ mt, sont des chrétiens, membres de la communauté post-pascale. L'intérêt appuyé que Mt leur voue est l'indice d'un problème actuel dans sa communauté.

Comment doit-on alors se représenter ces chrétiens dangereux que sont les faux prophètes ? Ou plutôt : comment Mt les qualifie-t-il ? Les faux prophètes ont tout d'abord une apparence d'agneaux, càd qu'extérieu rement ils sont identiques aux croyants. Ils sont même des fidèles à la vie spirituelle très riche, probablement des charismatiques: ils confes sent publiquement le Christ $(7,21)$. Ils ont le don de prophétie $(7,22$; $24,23.26)$, ils accomplissent des exorcismes et des miracles $(7,22$; 24,24). Matthieu - notons -le bien - ne condamne pas cette richesse charismatique en elle-même. Mais il prétend qu'elle est trompeuse ( $\downarrow \varepsilon \cup-$ So ...), qu'elle égare ceux qui lui font confiance $(24,11.24 \pi \lambda \alpha \nu \alpha ́ \omega)$ ). Les faux prophètes sont en réalité des loups $(7,15)$. Leur inauthenticité se manifeste dans leur $\dot{\alpha} \vee o \mu l \alpha \quad(7,23 ; 24,12)$ : ils ne portent pas les fruits que produit toute obéissance véritable $(7,16-20)$. La question qui se pose à ce point de l'analyse est la suivante : Mt incrimine -t -il la tiédeur éthique des charismatiques de son église, ou bien le conflit est-il plus profond? Dans ce dernier cas, les faux prophètes égareraient les croyants moins en transgressant la volonté de Dieu, qu'en enseignant que la Loi est abolie depuis la venue du Christ ${ }^{l}$. Seule une exégèse approfondie peut apporter une réponse à cette question.

1 C'est la thèse antinomiste établie par BARTH et défendue par BORNKAMM et HUMMEL. 


\section{La parénèse du Sermon sur la Montagne (Mt 7,15-23)}

Les vv. 15-20 sont une composition de l'évangéliste ${ }^{1}$. Le v. 15 est rédactionnel; le v. 16a est une transition, également de la main du rédacteur, qui introduit ainsi le logion traditionnel de $16 \mathrm{~b}$ (voir Q Lc 6,44). Le v. 17 est un développement pédant ajouté par l'évangéliste, le v. 18 est emprunté à $Q \operatorname{Lc} 6,43$, tandis que le v. 19, déjà présent en 3,10, est utilisé à nouveau ici pour introduire le motif de jugement. La conclusion, v. 20, qui reprend textuellement le v. 16, est également rédactionnelle. On le constate d'emblée : l'activité rédactionnelle est déterminante dans notre passage. Pour l'essentiel, elle consiste à relier de manière fort originale le thème des faux prophètes avec le motif de l'arbre et du fruit.

Mais comment le problème des faux prophètes est-il abordé ? Il est abordé dans la perspective du discernement des esprits. Matthieu entend donner à sa communauté un critère qui lui permette de distinguer les es prits bons des esprits pernicieux ( $\dot{\pi} \pi \curlyvee \iota \vee \omega ́ \sigma \varkappa \omega, 7,16-20)$, il entend doter son église d'une règle qui l'autorise à préserver son orthodoxie en éliminant les hérétiques. On notera, à cet égard, que l'auditoire visé par notre passage n'est pas le groupe des hérétiques, mais la communauté qui doit être avertie et apprendre à se défendre. Pourquoi cet apprentis sage dudiscernement des esprits semble-t-il si urgent au rédacteur ? pourquoi se presse-t-il d'enseigner son église sur ce point particulier dans un contexte aussi important que le $S M$ ? Le v. 15b nous suggère une réponse : les faux prophètes ne sont pas immédiatement reconnaissables, ils sont déguisés en brebis, ce qui signifie : ils ont l'apparence des membres du peuple de Dieu ${ }^{2}$. Les vv. $21-23$ nous apprendront même que ces faux prophètes sont les dépositaires de charismes étonnants. L'hérésie

1 Pour la critique des sources, voir surtout BARTH 68 et BULTMANN, Trad 99.131.

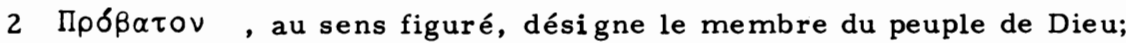
hormis les commentaires, voir PREISKER - SCHULZ, art. $\pi \rho \delta ́-$ Barov, ThW 6,690. 
- ou du moins l'erreur - n'est donc pas évidente, elle se cache sous une vie religieuse apparemment intense et fidèle. Il importe de la démasquer, car les faux prophètes sont en réalité des loups rapaces (v. 15b), càd qu'ils sont par excellence les ennemis du peuple de Dieu et menacent de le détrui re ${ }^{l}$. Quel est alors le critère qui permet de rendre manifeste l'erreur, de dénoncer l'hérésie, et de la retrancher de la communauté ? Ce critère est présenté sous la forme d'une image : un bon arbre donne de bons fruits, un mauvais arbre de mauvais fruits; un faux prophète ne peut donc avoir qu'un comportement à la mesure de son erreur. Mais qu'entend-on exactement par fruit, par porter du fruit, par bon arbre, etc... ? Schweizer ${ }^{2}$, analysant en détail la reformulation $m$ de cette tradition provenant de $Q$, a bien montré que l'accent dominant est éthique. L'homme qui ne porte pas de fruits ou en porte de mauvais, est un homme qui transgresse la volonté de Dieu. Il ne faut pourtant pas comprendre ce critère éthique de manière limitative et introduire dans le texte des distinctions que l'anthropologie biblique ignore. Comme le rappelle pertinemment Bornkamm ${ }^{3}$, de mème que la nature du fruit est indissociable de la qualité de l'arbre, de même le comportement éthique d'un homme exprime ce qu'il est globalement et fondamentalement. Dès lors, dire d'un faux prophète qu'il porte de mauvais fruits, ce n'est pas seulement affirmer qu'il se conduit mal, mais c'est le condamner massivement,

1 Le loup est, dans l'imagerie biblique, l'ennemi par excellence du troupeau des brebis (= le peuple de Dieu). Voir GRUNDMANN, Mt 232; KLOSTERMANN, Mt 69; SCHMID, Mt 150; SCHWEIZER, Gesetz und Enthusiasmus 63 (note 52).

2 SCHWEIZER (Gesetz und Enthusiasmus 64, en particulier note 59) relève que le véritable parallèle $m t$ à Lc 6,43-45 ne se trouve pas ici, mais en 12,33-35. Mt introduit ici une variante du motif qu'il applique unilatéralement aux faux prophètes (cf. l6a et le aútoús du v. 20) et qu'il accentue de manière éthique : remarquer le pluriel de $x \alpha \rho-$ $\pi \delta s$, le remplacement de $x \alpha \lambda \delta \delta_{\nu}$ par $\alpha \gamma \alpha \vartheta \delta \nu$, de $\sigma \alpha \pi \rho \delta \nu$ par tounpoús par opposition à Mt 12,33.

3 Voir BORNKAMM, art. גúnos, ThW 4,311. 
aussi bien au niveau de son enseignement que de ses actes ${ }^{1}$. En dernière analyse, le faux prophète est aux yeux de Mt en opposition irréductible avec la volonté de Dieu telle qu'elle vient d'être proclamée dans le SM.

Résumons-nous : pour prémunir la communauté du danger à la fois insidieux et mortel que représentent les faux prophètes, Mt énonce le critère qui permet d'opérer le discernement des esprits : ce critère, c'est l'obéissance à la volonté de Dieu telle qu'elle est définie dans le SM.

Ceci posé, une question demeure : pourquoi Mt choisit-il ce terrain pour s'attaquer à ses adlversaires ? A -t-il une raison majeure pour procéder de la sorte ? Nous avons le choix entre trois réponses. Première possibilité : pour éliminer des adversaires, Mt recourt à un lieu commun de la polémique chrétienne primitive contre l'hérésie qui consiste à les discréditer sur le plan éthique ${ }^{2}$. Nous jugeons cette solution peu probable, car le motif de l'obéissance à la volonté de Dieu n'est pas seulement un topos polémique chez Mt, c'est un thème constitutif de sa théologie ${ }^{3}$. Deuxième possibilité : Mt entend ramener à la fidélité éthique les prophètes de sa communauté qui font preuve d'un laxisme moral. Cette solution

1 Nous refusons ainsi l'exclusive posée p.ex. par SCHWEIZER, Gesetz und Enthusiasmus $64:$ "... vor allem nicht mehr auf die Verkündigung, sondern auf das Handeln der Pseudopropheten...". Nous préférons la vision plus globalisante de BARTH 69: "Dass sie keine Frucht bringen, d.h. den Willen Gottes nicht tun, soll hier die Gegner doch nicht nur ethisch diskreditieren, sondern ihre Lehre treffen". Cf. aussi BAUMBACH, Bösen 83 .

2 Voir à ce sujet l'excursus de DIBELIUS sur les hérétiques dans Pastoralbriefe $52-54$.

3 Il n'est pas nécessaire d'argumenter sur ce point tant c'est évident. Remarquons simplement qu'au niveau de la parénèse du $\mathrm{SM}$, le thème de la volonté de Dieu est introduit par la règle d'or $(7,12)$ qui fait charnière entre le corps du SM et sa conclusion. Il est ensuite développé avant notre péricope par le fameux passage sur les deux voies. 
est possible, mais on ne comprend plus alors pourquoi les prophètes sont fondamentalement des faux prophètes, ni pourquoi ils font courir à la communauté un risque si grave qu'il nécessite leur exclusion. La tiédeur éthique est, en effet, une caractéristique de l'église mt dans son ensemble. Troisième possibilité : les faux prophètes sont des hérétiques et $\mathrm{Mt}$ les attaque en choisissant le thème de l'obéissance à la Loi parce que c'est précisément sur ce point que porte le litige. Dans ce cas, les faux prophètes transgressent la volonté de Dieu dans la mesure où ils n'en reconnaissent plus l'autorité. Le point central du débat est alors la validité de la Loi comme telle. La querelle n'a pas pour objet une question de ferveur, elle est un conflit théologique dans lequel Mt prend nettement et passionément parti.

b) Vraie et fausse piété (vv. $21-23$ )

Les vv. 21-23 proviennent de la source $Q$. L'activité rédactionnelle de Mt - pour autant que nous puissions encore la discerner - apparait à un double niveau. D'une part, Mt a rassemblé en une seule péricope deux traditions $(7,21$ et $7,22-23)$ que Lc utilise dans des contextes différents $(L c 6,46 ; 13,25-27)^{1}$. D'autre part, Mt a procédé à des réinterprétations perceptibles au niveau de la terminologie. C'est ainsi qu'au v. 21 , les

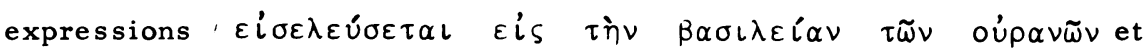

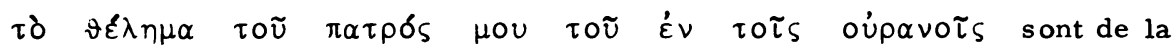
main de l'auteur; le logion est ainsi transformé par Mt en une sentence d'entrée dans le Royaume ${ }^{2}$. Il est impossible d'établir la forme que les vv. $22-23$ avaient dans la source $Q$. Néanmoins l'effort d'actualisation de

1 Trois arguments militent en faveur de cette hypothèse : a) nous remar quons l'apparition de la technique du mot-crochet, chère à $\mathrm{Mt}$, pour

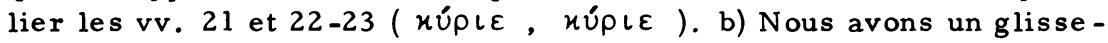
ment de sens entre le $v$. 21 qui condamne le verbalisme religieux et les vv. 22-23 qui s'attaquent à des charismatiques. c) Les vv. $21-23$ dans leur ensemble reflètent la situation de la communauté.

2 BARTH 69; GRUNDMANN, Mt 229; STRECKER, Weg 160; TRILLING, Israel 189. 
Mt est perceptible : le discours de Jésus n'est plus adressé aux Juifs (cf. Lc 13,26), mais appliqué à sa communauté ${ }^{1}$. Le $\mu \varepsilon \nu$ est sans doute de sa main. La citation exacte de la fin du v. 9 du LXX Ps 6 lui permet d'introduire le concept d' $d^{\prime} \vee o \mu \iota \alpha{ }^{2}$. En résumé, dans cette péricope, l'empreinte rédactionnelle est particulièrement forte.

La question la plus difficile et sans doute décisive pour notre problématique consiste à savoir si les vv. 21 -23 parlent encore des faux prophè tes ou si Mt aborde un autre point de sa parénèse. Les exégètes sont hésitants et certains nient même tout rapport entre $15-20$ et $21-23^{3}$. Pour notre part, nous discernons une continuité entre 15-20 et 21-23: dans les deux péricopes, ce sont les faux prophètes qui sont en accusation. Nous appuyons ce choix par deux arguments distincts. Premièrement, Mt re court au procédé du mot-crochet pour lier les deux péricopes : le $\psi \varepsilon v-$

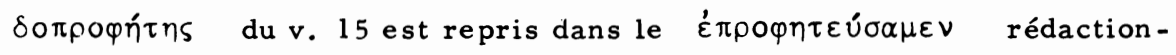
nel du v. 22; le verbe $\pi 0\llcorner\varepsilon \tau \nu$ apparaît en 7,17.17.19.21.22. Deuxièmement, l'homogénéité thématique est indubitable : au thème du "fruit" répond celui de l'accomplissement de la volonté de Dieu et dans les deux cas, c'est la transgression de cette norme qui est sanctionnée; enfin, le thème du jugement, introduit par le rédacteur au v. 19, annonce la grande scène eschatologique des vv. $21-23$.

Si notre analyse est exacte, qu'apprenons -nous sur les faux prophètes ? Le v. 21 nous les décrit animés d'une piété intense où l'invocation du Kyrios tient une place centrale - détail qui n'est pas sans importance si nous entendons les situer dans l'histoire du christianisme primitif -.

1 Voir le thème de l'invocation du xúplos et celui des charismes !

2 Dans la réminiscence du Ps 6,9a LXX, Lc restitue fidèlement la première partie, Mt la seconde.

3 SCHMID, Mt 151-152, p.ex. hésite. KLOSTERMANN, Mt 70, et TRILLING, Amt 35, nient tout rapport. BARTH 68-69, BORNKAMM, Wandlungen 75, SCHWEIZER, Gesetz und Enthusiasmus $63-64$, au contraire l'admettent. 
Le v. 22 évoque les charismes dont les accusés se réclament en vain de vant le juge eschatologique : il s'agit de la prophétie, de l'exorcisme et de la guérison miraculeuse. Ces trois dons ont pour dénominateur commun d'être attribués à l'Esprit par le christianisme primitif. Insistons : les faux prophètes ne se réclament pas de leur connaissance, nous ne sommes donc pas en contexte gnostique; ils ne se réclament pas de leur foi - le conflit évoqué par Mt n'a donc rien à faire avec celui mentionné dans l'épitre de Jc - l. Les faux prophètes se réclament de leurs charismes. Nous sommes dès lors vraisemblablement en présence d'une forme vulgaire du christianisme hellénistique que nous pourrions caractériser comme suit : les faux prophètes sont les tenants d'une piété enthousiaste; ils se savent comblés par l'Esprit du Kyrios élevé et présent si bien que pour eux, la prédication du Jésus terrestre perd de son importance ${ }^{2}$.

A ce point de l'analyse, il importe de préciser la critique que $\mathrm{Mt}$ adresse à ses adversaires. Il ne critique aucunement la valeur des charismes revendiqués par les faux prophètes : pas une remarque sur l'invocation du Kyrios, sur le don de prophétie comme tel, l'exorcisme ou la guérison miraculeuse. Ces manifestations pneumatiques vont de soi pour l'église mt et leur cessation possible provoque même des problè mes $^{3}$. Le thème dominant de la critique $m t$ est à nouveau l'obéissance à la volonté de Dieu : seul entrera dans le Royaume celui qui fait la volonté de Dieu telle que le Christ mt l'annonce (v. 21); les faux prophètes seront rejetés lors du jugement à cause de leur $\alpha$ áouía (v. 23). Plus encore que dans les autres passages étudiés, il est difficile d'imaginer que 1' 'áojía reprochée aux faux prophètes n'est qu'un manque de con-

1 Ce double point a été bien développé par BARTH 149-154.

2 Thèse déjà développée par BORNKAMM, Der Auferstandene 299 et Wandlungen 75 .

3 Cf. SCHWEIZER, Gesetz und Enthusiasmus 56-57; TRILLING, Amt 37. Cette problématique apparait avec acuité dans l'évangile en 10,1.8; $17,14-20$. 
sécration religieuse, une indifférence coupable face aux exigences de la. vie chrétienne. Les charismes reconnus par Mt à ses adversaires s'accordent mal avec une telle explication. Il est plus vraisemblable de penser que la piété enthousiaste des prophètes, l'accent mis sur la présence actuelle du Kyrios attestée par les manifestations de l'Esprit, se double d'une relativisation, voire d'une abolition de la valeur normative de la Loi. La parole du Seigneur élevé transmise par ses prophètes inspirés prend le pas sur la prédication du Jésus terrestre et en particulier sur sa réinterprétation souveraine de la Loi. On le voit, le conflit ne porte plus seulement sur la validité de la Loi comme telle, il a des racines christologiques 1 .

Concluons : l'analyse de 7,15-23 nous semble accréditer l'hypothèse selon laquelle l'église mt a connu des conflits confessionnels. Si notre exégèse est exacte, nous nous trouvons face à deux fractions qui défendent véritablement deux compréhensions différentes de la foi chrétienne, notamment en ce qui concerne la validité de la Loi et la christologie.

\section{L'explication de la parabole de l'ivraie (Mt 13,36-43)}

Dans la septième édition de son livre sur les paraboles, Jeremias a fait le point de la recherche sur notre péricope ${ }^{2}$. Ses conclusions nous semblent convaincantes, aussi les résumons -nous brièvement. L'explication de la parabole de l'ivraie est composée de deux parties bien diffé$\underline{\text { rentes }}^{3}$ : dans les vv. $37-39$, les sept éléments les plus importants de la

1 Nous voulons faire allusion au conflit christologique opposant les tenants d'une piété axée sur le Kyrios glorifié et présent et ceux qui soulignent l'indissoluble unité entre le Jésus terrestre et le Ressuscité. Sur cette question, voir en priorité BORNKAMM, Der Auferstandene.

2 GLEICHNISSE 79-83.

3 TRILLING, Israel $124-126$. 
parabole sont interprétés allégoriquement si bien que nous sommes en face d'une espèce de "petit dictionnaire"; le ton change complètement dans les vv. 40-43 qui présentent le dest in de l'ivraie et du blé comme le destin des pécheurs et des justes lors du jugement - nous sommes alors confrontés à une petite apocalypse -. On s'aperçoit immédiatement que l'explication de la parabole modifie la pointe primitive : la parabole de l'ivraie était un appel à la patience ${ }^{l}$; son explication - partie intégrante de la parénèse du jugement - devient un appel à l'obéissance ${ }^{2}$. Qui donc est l'auteur de cette explication qui gauchit le sens de la parabole ? Toute une série de tournures et d'éléments théologiques empêchent de l'attribuer à Jésus ${ }^{3}$. A l'inverse, pas moins de 37 termes appartiennent au vocabulaire spécifiquement matthéen si bien que nous pouvons admettre que les vv. $36-43$ sont l'oeuvre du rédacteur de l'évangile 4 . S'il en est ainsi, nous sommes en droit de nous demander dans le cadre de notre problématique quelles sont les raisons qui ont amené $M t$ à composer cette explication et si, à travers elle, il n'évoque pas des difficultés et des soucis inhérents à sa communauté. En d'autres termes, la transcription allégorique de la parabole sert-elle à l'établissement d'une vérité ét hique générale ou est-elle conçue dans le cadre d'une polémique intra-ecclésiale ?

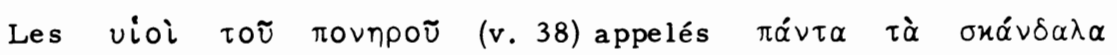

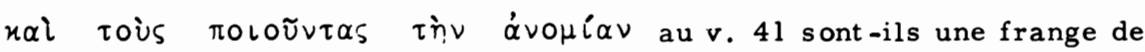
l'Eglise, qui serait alors un corpus mixtum, ou bien s'agit-il des hommes en général - chrétiens ou palens - qui ont une condui te déplorable ? La réponse à cette question nous semble dépendre de l'interprétation de

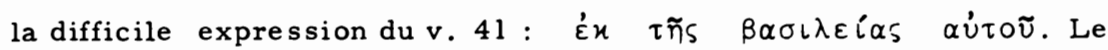

1 Voir p.ex. BULTMANN, Trad 203; JEREMIAS, Gleichnisse 79.

2 GRUNDMANN, Mt 349.

3 JEREMIAS, Gleichnisse 79-80.

4 JEREMIAS, Gleichnisse $81-83$. Il n'est cependant pas exclu que pour les vv. $37-39$, le rédacteur s'inspire d'un catalogue traditionnel (SCHWEIZER, Mt 201). 
Royaume du Fils de l'homme désigne-t-il l'Eglise - nous serions alors en situation de lutte ecclésiale - ou le monde - nous serions alors en contexte purement éthique et universaliste - ? L'analyse de l'emploi mt de

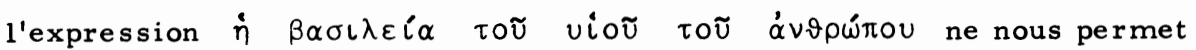
malheureusement pas de résoudre le problème, car, en dehors de notre passage, elle n'apparaît qu'une seule fois en 16,28 où elle désigne le Royaume à venir ${ }^{1}$. Or, dans notre passage, il ne s'agit pas d'un Royaume survenant lors du Jugement ${ }^{2}$, mais d'un Royaume qui précède la fin. Il nous faut en conséquence tenter de trancher la question à la fois à partir du contexte immédiat et de l'évangile dans son ensemble.

L'interprétation éthico-universaliste a été défendue de manière brillante et approfondie par Vögtle ${ }^{3}$; elle est reprise aujourd'hui notamment par Dupont ${ }^{4}$. Elle s'appuie sur cinq arguments principaux qu'il convient d'examiner. Elle fait valoir en premier lieu que la cohérence des vv. 36 43 suppose qu'il y a équivalence entre le monde du v. 38 et le Royaume du Fils de l'homme du v. 41; les deux expressions correspondent à la même image du champ. En fait, cet argument est en contradiction avec la forme littéraire du passage qui est formé de deux unités distinctes : le lexique allégorique des vv. 37-39 et la petite apocalypse des vv. 40-43. Ce qui lie ces deux unités et en fait un tout cohérent, ce n'est pas d'abord un parallélisme terme à terme des expressions utilisées, mais le souci de décrire le destin des justes et des méchants lors du jugement.

Le deuxième argument peut se résumer ainsi : l'action prêtée au diable ne se comprend bien que s'il s'agit du monde, car il n'appartient pas au diable d'introduire des méchants dans l'Eglise. A ceci, on répondra que, dans la parabole, l'action du diable sert à expliquer pourquoi bons

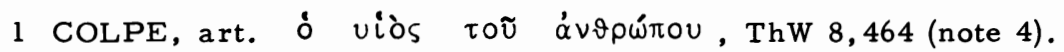

2 Contre BULTMANN, Trad 203 (note 1).

3 Anliegen 286-293.

4 Paraboles 223-229. 
et méchants sont mêlés. Ce ne sont pas les domaines respectifs du Fils de l'homme et du diable qui sont au centre du propos, mais l'énigmatique réalité de leur coexistence. Or cette coexistence n'est vraiment problématique que dans l'Eglise; hors d'elle, elle est triviale :

Le troisième argument s'appuie sur le glissement de sens entre la parabole et son explication. On souligne alors que le rédacteur n'est plus tellement préoccupé, comme dans la parabole, par la coexistence des bons et des méchants dans l'état actuel des choses, mais par le sort qui est réservé aux uns et aux autres lors du jugement. En soi, la remarque est exacte, mais elle nous semble mal exploitée. En effet, si l'auteur juge nécessaire d'appeler les croyants de son église à l'obéissance, d'adresser la parénèse du jugement à sa communauté, c'est précisément parce que l'église mt est un corpus mixtum.

Le quatrième argument relève de la christologie : le Fils de l'homme mentionné dans les vv. 36-43 est le Kyrios glorifié, càd le Kyrios qui règne sur le kosmos dans son ensemble et non pas seulement sur l'Eglise. Sans nier du tout cette dimension de la christologie mt, nous remarquons pourtant que cette interprétation dépasse ce qui est dit dans notre périco-

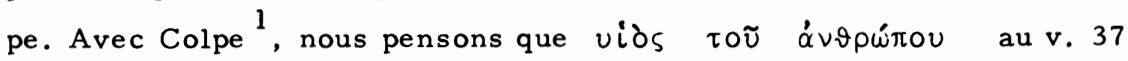
désigne tout simplement Jésus sans autre spécification - ce qui est carac téristique de la rédaction $m t$ - et qu'au v. 4l, il s'agit du juge daniélique. Par ailleurs, on sait qu'au niveau rédactionnel, chez Mt, le titre viòs

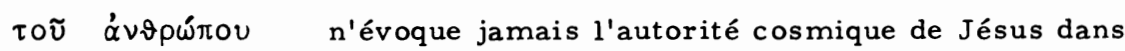
l'éc onomie présente ${ }^{2}$.

1 COLPE, art. cit. ibid.

2 COLPE, art. cit. 465 : "Matthäus bietet damit eine Synthese aller bis zu ihm und durch ihn enstandenen Bedeutungen : der Menschensohn, immer in statischer Identifikation mit Jesus, ist der auf Erden Predigende, der sühnend Leidende, der zum Herrn der Kirche Erhöhte, der als Richter und Anwalt zum Endgericht Wiederkommende und der in seinem Reich, dem Gottesreich, über den neuen fon Herrschende". 
Le cinquième argument découle des précédents : le champ où se font les semailles est identifié au monde ( $v$. 38) et, en même temps, il est présenté comme le domaine sur lequel le Fils de l'homme exercera ses

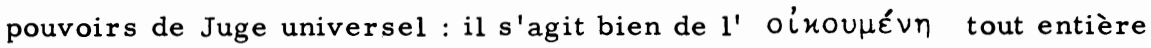
à laquelle s'étend la mission chrétienne, mais où Satan suscite également les mauvais. Nous nous bornerons à remarquer que cette thèse est ad-

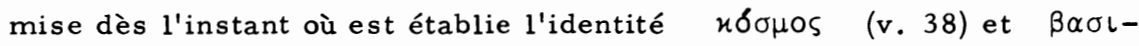

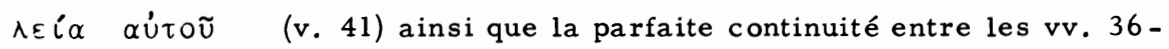
39 et les vv. $40-43^{1}$.

Malgré la qualité des arguments invoqués par Vögtle et que nous sommes conscient de n'avoir pas entièrement réfutés, il est important et peut-être décisif de remarquer que l'interprétation éthico-universaliste débouche dans une aporie. Dupont, en effet, tirant les conséquences théologiques de l'argumentation de Vögtle écrit : "le domaine dans lequel 'Fils du Royaume' et 'Fils du Mauvais' se trouvent mêlés les uns aux autres est aussi celui où le jugement universel doit intervenir : il s'agit du monde entier et pas seulement de l'Eglise... La question essentielle de l'évangéliste n'est pas de savoir si l'on est chrétien ou pas, si l'on appartient à l'Eglise ou non, mais plus largement si l'on accomplit la volonté du Père ou si l'on pratique $l^{\prime} \dot{\alpha} v o \mu i \alpha$. La perspective n'est pas proprement ecclésiologique, mais morale" ${ }^{2}$. La visée universaliste en arrive donc à lier exclusivement la thématique de la Loi et celle du Juge ment. Dupont écrit encore : "Seul l'accomplissement de la justice peut faire d'un homme un Fils du Royaume" ${ }^{3}$.

Or il est évident que l'évangile selon Mt dans son ensemble, s'oppose à une telle perspective. Seul le Maitre restitue la volonté véritable de Dieu; seule la Nachfolge permet l'accomplissement de la volonté du Père;

1 Ajoutons, à titre d'exemple, que la figure du Fils de l'homme en tant que juge est absente des vv. 37-39.

2 DUPONT, Paraboles 229.

3 ibid. 
or ceux qui s'engagent dans la Nachfolge constituent précisément l' $l^{\prime} \chi-$ xhnoía. Si, comme le souligne notre passage, Loi et jugement vont de pair, Loi et Eglise, Eglise et jugement ${ }^{1}$ sont tout aussi inséparables. La pointe - particulièrement manifeste dans notre péricope - de la parénèse du jugement est dès lors la suivante : certes tous sont en route vers le jugement, mais en premier lieu l'Eglise. L'appartenance à l'Eglise ne confère aucune sécurité, les fauteurs de scandale et d'iniquité seront jetés dans la géhenne. A cette première remarque de théologie matthéenne, on ajoutera ce qui suit : si notre péricope décrivait simplement la rétribution nécessaire et générale de l'obéissance à une loi naturelle, elle aurait un accent trivial et non-évangélique. Le scandale de la coexis tence des bons et des mauvais, décrit par la parabole, est précisément un scandale parce qu'il est intra ecclesiam. Semblablement la parénèse du jugement $n$ 'acquiert sa véritable pertinence évangélique que si elle s'applique à l'Eglise mise en demeure d'obéir.

A près avoir critiqué l'interprétat ion éthico-universaliste de notre passage, abordons les arguments qui soutiennent la perspective ecclésiale. Le premier argument se rapporte au cadre de la péricope qui ne doit pas être négligé : le v. 36 montre clairement que l'explication de la parabole est une instruction adressée aux disciples seulement et sollicitée par eux. Or les disciples sont, dans le premier évangile, l'image de la communauté. C'est donc la communauté qui veut connaître la signification de la parole du maître à son égard.

Deuxième argument : l'ensemble de la péricope ne peut être comprise indépendamment de la problématique de la Loi. Le vocabulaire déjà en

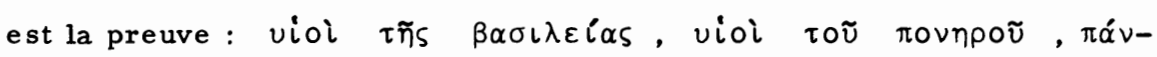

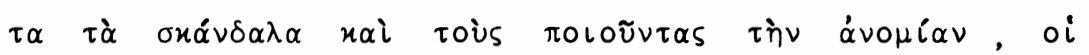
ó́xaしol. L'admission ou l'exclusion du Royaume dépendent de l'obéis sance à la volonté du Père. Or volonté du Père et Nachfolge sont indis -

1 BORNKAMM, Enderwartung 13-21. 
sociables.

Troisième argument : il convient d'être sensible au fait que ơ⿱x́ $\nu$ $\delta \alpha \lambda o v$ et $\alpha \operatorname{vou} \iota_{\alpha}$ appartiennent à la terminologie de la lutte contre

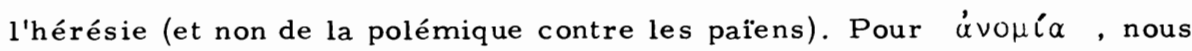
renvoyons à 7,23 et 24,12 , pour la racine $\circlearrowleft \mathcal{H} \alpha \nu \delta \alpha \lambda-$, au chap. 18 et à 24,10. Or si le rédacteur recourt à pareille terminologie, c'est que nous sommes en perspective ecclésiale.

Quatrième argument : la liaison entre Eglise et attente du jugement apparaît dans tout le chap. $13^{1}$ et se manifeste particulièrement en 13 , $24-30.36-43 \cdot 47-50^{2}$. La terminologie de la "séparation" qui a pparaît aussi bien en 13,36-43 qu'en 13,46-50 nous amène à nous poser la ques tion du "Sitz im Leben" de la péricope. Sommes-nous, comme le pense Käsemann, confrontés à une question de discipline ecclésiastique ? A l'Eglise qui se demande comment agir contre l'hérésie, Matthieu répondrait par un appel à la patience et à l'obéissance en soulignant que le jugement appartient au seul Fils de l'homme.

Cinquième argument : une étude attentive des groupes de protagonistes opposés dans le chap. 13 nous conduirait sans doute à une conclusion similaire ${ }^{3}$.

$\mathrm{Si}$, sur la base des arguments invoqués, nous préférons l'interprétation ecclésiale, qui sont alors les personnes dont parle le v. 41 ? que

1 BORNKAMM, Enderwartung 16-17.

2 La parenté thématique de l'explication de la parabole de l'ivraie et de la parabole du filet est frappante. De nouveau, l'Eglise comme corpus mixtum et marchant vers le jugement est au centre du propos. On

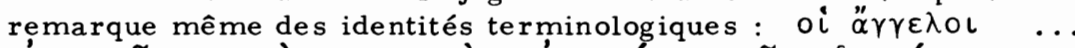

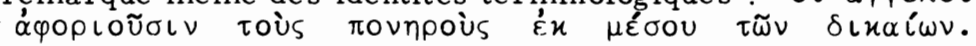

3 Nous ne voulons pas ici entrer dans l'étude détaillée de ce thème. Nous pensons à l'opposition introduite par Mt dans le chap. 13 entre la foule et les disciples. La foule est endurcie et ne comprend pas ce que dit Jésus (voir en particulier 13,10-16). Le discours en paraboles vi se donc et édifie exclusivement les disciples. 


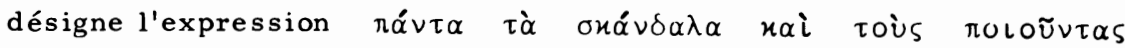

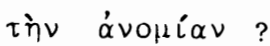

$D^{\prime}$ un point de vue terminologique ${ }^{1}$, Mt 13,41 rapelle So 1,3, texte qui décrit l'anéantissement eschatologique des méchants. L'équivalence

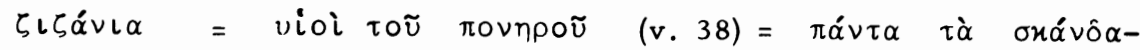

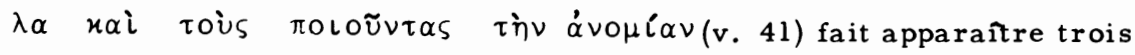
choses. Premièrement, ici - et ici seulement dans le NT - ơó́ $\delta \delta \alpha-$ $\lambda o \nu$ désigne un groupe de personnes. Deuxièmement, le terme d' ávo$\mu i ́ \alpha$, au v. 41, introduit la thématique de la Loi comme horizon de compréhension. Si le $x \alpha i$ est explicatif, alors les $\sigma \mathcal{L} \alpha \dot{\nu} \delta \alpha \lambda \alpha$ sont ceux qui séduisent leurs frères et les induisent à transgresser la Loi. Ce faisant, ils les précipitent dans le péché. La Loi refusée et transgressée par les $\sigma \mathcal{K} \alpha \delta \delta \lambda \alpha$ est celle duFils de l'homme, en l'occurence celle du Christ $m$ t telle qu'elle est enseignée dans l'évangile. A l'opposé, les fils du Royaume, les justes sont ceux qui écoutent la volonté de Dieu et

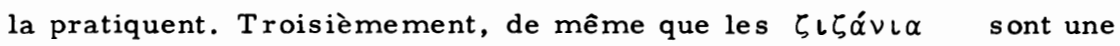
moisson du diable qui pousse mêlée au blé, de même les oxáv $\delta \alpha \lambda \alpha$ sont les enfants du diable qui, au milieu du Royaume du Fils de l'homme, càd l'Eglise, agissent contre Dieu et tentent de perdre le plus grand nombre possible de croyants en les éloignant de l'obéissance à la Loi.

Peut-on risquer une hypothèse pour identifier historiquement ce groupe dans l'église $\mathrm{mt}$ ? Bultmann et Fridrichsen ${ }^{2}$ pensent aux faux prophè-

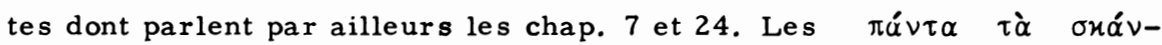

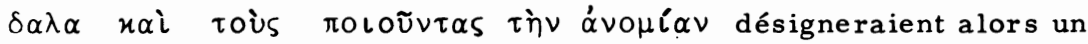
groupe réuni autour de (faux) prophètes qui ne partagent pas la compréhension mt de la Loi, càd qui ne la mettent pas en pratique. Nous ne saurions dire sur la base de notre texte s'il s'agit d'un refus comme tel de

1 BAUER, WB col. 1492; GRUNDMANN, Mt 351; STAEHLIN, art.

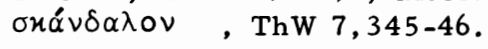

2 BAUMBACH, Bösen 62-64; BULTMANN, Trad 203 qui cite FRIDRICHSEN. 
la Loi (sous prétexte que le Christest la fin de la Loi, cf. 5, 17) ou d'une compréhension plus libérale et moins juive. En tout état de cause, si notre hypothèse est défendable et s'il ne s'agit pas simplement d'une polémique éthique de valeur générale, alors nous sommes en situation de lutte confessionnelle. L'église mt est divisée en des groupes antagonis tes qui se réclament de conceptions divergentes de la Loi.

\section{Les grandes tribulations (Mt 24,9-14)}

Dans la première partie du dernier grand discours de Jésus, à savoir Mt 24,1-36, Mt suit assez fidèlement la petite apocalypse de Mc 13. Il omet certes la conclusion de l'apocalypse mc (13,33-37), mais c'est pour mieux la reprendre dans une vaste parénèse de la vigilance (Mt 24,37; 25,46). Pour le reste, les petites modifications et adjonctions sont certes nombreuses, mais une seule péricope est récrite de bout en bout avec beaucoup d'originalité, c'est 24,9-14. La question qui se pose alors est la suivante : pourquoi cette modification, quelle est son ampleur et sa signification ? Mt, dans son dernier grand discours, saute Mc 13,9-12. Pourquoi ? La réponse est bien connue. Mt a déjà utilisé cette tradition dans son discours d'envoi $(10,17-22)$ et ne juge pas néces saire de la répéter. L'argument est exact, mais insuffisant. Il n'expli que notamment pas pourquoi Mt - qui n'évite pas les doublets ${ }^{1}$ - évite celui ci, ni surt out pourquoi il juge nécessaire de le remplacer par une composition de son crû. Avant de résoudre cette question, voyons comment se présente notre péricope du point de vue de la critique des sources.

$\begin{array}{ll}9 a & \text { rédaction } \\ 9 \mathrm{~b} & \text { Mc 13,13a } \\ 10 & \text { rédaction cf. Mc 13,12 } \\ 11 & \text { rédaction cf. Mc 13,22 } \\ 12 & \text { rédaction } \\ 13 & \text { Mc 13,13b } \\ 14 & \text { profonde reformulation de Mc 13, 9-10 }\end{array}$

1 Voir KUEMMEL, Einleitung 34. 
Grosso modo, la péricope composée par Mt reprend donc, dans son introduction et sa conclusion, des éléments de la source Mc, mais son centre qui nous intéresse particulièrement est entièrement rédactionnel (vv. $10-12)^{l}$. Il est pourtant utile de souligner que si les vv. 10-12 sont rédactionnels, ils restent en harmonie avec la terminologie et les représentations du chap. 24 : chaque v. contient des topoi apocalyptiques attestés aussi bien dans la littérature apocalyptique juive que dans la tradition chrétienne primitive ${ }^{2}$.

Quel est alors le but poursuivi par Mt en composant ce texte ? Pour le découvrir, il importe de constater que cette petite péricope juxtapose deux éléments. D'une part, elle est rédigée à l'aide de topoi a pocalyptiques - ce qui est normal -. D'autre part, elle se caractérise par une forte accentuation de la problématique ecclésiale : v. 9 les persécutions, v. 10 l'apostasie et ses tragiques conséquences, v. 11 les faux prophètes, v. 12 l'effondrement de l'obéišsance, v. 13 parénèse, v. 14 mission. La question est alors la suivante : Mt parle-t-il de la destinée de l'Eglise comme telle dans les derniers temps ou interprète-t-il à l'aide de notions traditionnelles la situation de sa communauté ?

A notre avis, c'est la seconde solution qui semble la plus probable pour les raisons suivantes:

1) Il serait étonnant que Mt brise l'ordonnance de la narration de Mc qu'il suit par ailleurs fidèlement dans le chap. 24 pour n'énoncer que des idées générales. Cette composition est l'indice d'un souci aigu, d'une question

1 Les vv. 10-12 utilisent une terminologie typiquement mt. Pour le v.

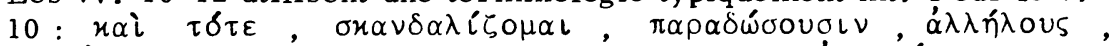

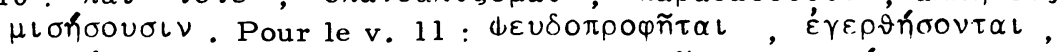

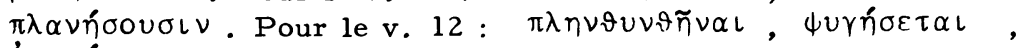
$\dot{\alpha} \vee \circ \mu \hat{\imath} \alpha$.

2 BILL 1,951 a relevé toutes les références utiles concernant le judaîs me. Ajoutons pour le v. 12 : 4e Es 4,2; Hen 91,7. Concernant la tradition chrétienne primitive, nous relevons pour le v. 10 : Mc 13,12; pour le v. 11: Mc 13,22; 1 Jn 2, 18; $2 \mathrm{Th} \mathrm{2,9-12;} \mathrm{Ap} \mathrm{13,3.8.13-17,}$ pour le tout Did 16,3-5. 
qu'il est urgent et important d'aborder.

2) Ce n'est pas sans surprise que nous retrouvons dans les seuls vv. 10 12 la terminologie classique de la lutte contre l'hérésie que Mt utilise dans d'autres contextes, et toujours dans une perspective de polémique

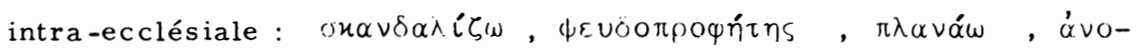
$\mu \imath \alpha, \dot{\alpha} \gamma \alpha ́ \pi \eta$.

3) Les topoi utilisés sont adaptés à une situation concrète :

- La persécution (v. 10) est désormais le fait des seuls paiens (adjonction de $\tau \tilde{\omega} \nu$ $\dot{\vartheta} \vee \tilde{\omega} \nu$, comparer avec 10,17-18) ce qui est l'indice d'une église vivant en terre païenne.

- Chez Mt, ơ $\alpha \delta \alpha \lambda i \zeta($ ) (v. 11) appliqué aux croyants décrit le drame de l'apostasie, ici de l'apostasie résultant d'une situation difficile de la communauté.

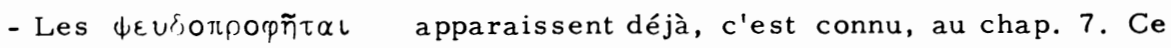
qui me semble important dans l'économie du chap. 24, c'est qu'ils sont nettement distincts des pseudo-messies empruntés à Mc et cités en $24,3-8.23-28$. Le v. 12 qui explicite l'action catastrophique des faux prophètes parle d' $\mathrm{d}^{\prime} v o \mu i \alpha$ et non de confusion christoiogique.

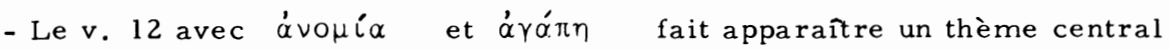
de la théologie et de la lutte de Mt : la juste interprétation et l'obéis sance à la Loi d'amour.

- Le v. 14 expose une conception purement $\mathrm{mt}$ de la mission.

On en conclura que, dans notre passage, les représentations apocalyptiques sont utilisées pour évoquer des événements concrets de la vie ecclésiale, événements qui ne sont pas considérés comme des défaillances humaines, mais comme des signes des derniers temps.

Peut-on alors décrire sur la base de notre passage, la situation des auditeurs de l'évangile ? Une sourde menace plane sur la communauté. L'église mt est mal tolérée par le monde paien qui réagit en la persécutant ( $\pi \alpha \rho \alpha \delta i \delta \omega \mu \iota$ appartient au vocabulaire de la Passion, $\vartheta \lambda \tau \psi \iota \varsigma$ décrit la souffrance résultant de la captivité et des mauvais traitements). 
Cette menace a des effets désastreux sur l'église : elle provoque la division et l'apostasie ( ơ $\left.\alpha \nu \delta \alpha \lambda i \zeta_{\omega}+\mu \iota \sigma \varepsilon \omega\right)$. Cet éclatement de la

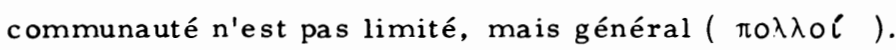

Pourtant cette crise n'a pas qu'une cause extérieure - la menace de persécution -. Elle s'explique avant tout par l'apparition au sein de la communauté, de faux prophètes qui égarent les croyants (v. 11). L'égarement causé par ces faux prophètes est élucidé auv. $12: 1^{\prime} \alpha \dot{\alpha} v o \mu i ́ \alpha$ augmente tandis que l'amour diminue. La transgression de la Loi telle que Jésus l'a enseignée ( $\left.\alpha_{\alpha} v o \mu i \alpha\right)$ provoque une dégénérescence éthique :

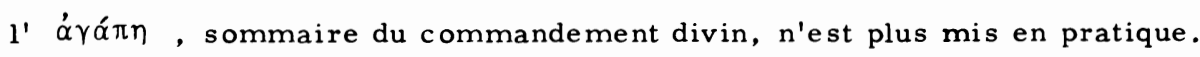
Les faux prophètes éloignent les croyants de la véritable obéissance et provoquent ipso facto la destruction de la communauté. Le remède à cette crise se trouve dans un retour à l'enseignement du Jésus terrestre comme

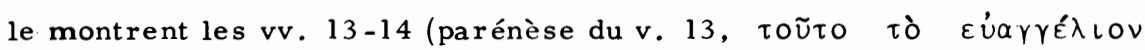
$\tau \tilde{n} \varsigma \quad \beta \alpha \sigma \iota \lambda \varepsilon(\alpha \varsigma$ v. 14 l).

L'originalité de Mt tient dans le fait qu'il combine dans notre péricope la problématique de la destinée de souffrance des croyants avec celle de la dispute confessionnelle. Si le point de départ de l'argumentation est la question de la persécution, le point culminant est la question de la juste obéissance (par opposition à l' $1^{\prime} \nu o \mu i ́ \alpha$ ) et de sa propagation universelle.

\section{Conclusion}

L'étude de la terminologie de la lutte contre l'hérésie et l'analyse de

1 STUHLMACHER 241-42 (voir aussi SCHWEIZER, Gesetz und Enthu-

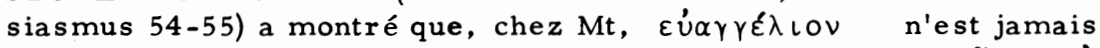
employé de manière absolue, mais toujours déterminé : Toũ o Tठ

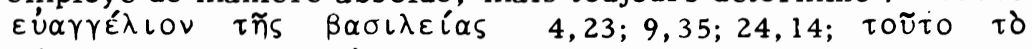

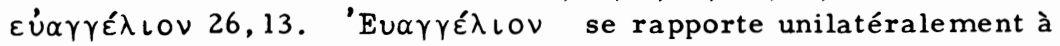
l'enseignement de Jésus, à sa prédication dans son ensemble. 
trois passages particulièrement représentatifs ${ }^{1}$ confirment notre hypothèse de départ. L'église mt connaît un grave conflit confessionnel. Les chrétiens ne sont pas d'accord sur la foi qu'ils confessent. Le conflit porte sur la validité de la Loi. Une partie de la communauté, regroupée autour des prophètes enthousiastes, ne respecte ${ }^{2}$ pas la volonté de Dieu telle que Mt la comprend. La piété de ce groupe est axée sur le culte de la présence du Kyrios. Elle attache un prix tout particulier aux manifes tations de l'Esprit et en particulier aux charismes. Cet attachement spiritualiste à la présence de l'Elevé dans l'Eglise l'amène à se détacher de la prédication du Jésus terrestre et à en ignorer la valeur salutaire. Le différend sur la Loi dévoile en fait un désaccord sur la christologie. A lors que Mt souligne avec insistance l'indissoluble unité entre le Jésus terrestre et le Ressuscité, les charismatiques de sa communauté - sans doute représentatifs d'un certain christianisme hellénistique ${ }^{3}$ - concentrent leur foi dans l'adoration du Kyrios présent.

Nous n'ignorons pas que cette hypothèse sur l'église mt, déjà défendue notamment par Barth, Bornkamm et Hummel, est gravée de tous les aléas inhérents à une reconstruction historique. Son mérite pourtant est de jeter une nouvelle lumière sur l'évangile et de rendre compte de nombreux points du texte, qui autrement resteraient obscurs. Elle ouvre

1 Il va de soi qu'on aurait pu en ajouter d'autres, notamment 5, $17-20$ (voir notre chap. 4), le chap. 18 (voir notre chap. 15) et 28, 16-20 (voir notre chap. 3) qui tous, à leur manière, soulignent de manière polémique la valeur normative de la prédication du Jésus terrestre.

2 On ne saurait qualifier ce groupe de libertiniste ou d'antinomiste, car le seul constat irréfutable que $l^{\prime}$ on peut faire, est négatif : une fraction de la communauté ne partage et n'applique pas la conception $\mathrm{mt}$ de la Loi. Son point de vue propre sur le litige reste dans l'ombre.

3 Ici aussi, toute précision est difficile. BORNKAMM (Wandlungen 75) parle d'un christianisme hellénistique vulgaire qui, après avoir é té le terrain de prédilection du paulinisme, en est devenu le champ de combat. 
en particulier deux voies fort fructueuses pour une meilleure compréhension de Mt. D'une part, elle permet de mieux situer l'enracinement his torique de l'évangile. Ce dernier n'est pas aussi juif-palestinien qu'on voudrait le croire parfois. Il a été élaboré dans le sein du christianisme hellénistique et il constitue le point de rencontre difficile et passionné du judéo-christianisme et du christianisme hellénistique ${ }^{l}$. D'autre part, l'hypothèse que nous défendons, permet de mieux saisir l'argumentation du rédacteur à propos de la Loi et singulièrement sa perspective éthique. Il ne fait pas de doute que la conception $m$ te la Loi ${ }^{2}$ prend un sens différent selon qu'on y voit un appel polémique destiné à regagner les croyants à une fidélité essentielle ou un durcissement résultant d'un attachement trop profond à l'héritage juif. L'enjeu de l'hypothèse proposée est donc considérable; à sa façon, il rappelle que le premier évangile doit être compris dans sa dynamique historique particulière.

1 Thèse historique développée par BORNKAMM dans ses différents articles sur Mt (Der Auferstandene, Lösegewalt, Wandlungen).

2 L'argumentation de Mt sur la Loi a un double visage : dans les récits de controverses, elle est tournée contre le judaïsme rabbinique; dans les grands discours notamment (exception chap. 23), elle est axée sur les problèmes communautaires. C'est de ce dernier aspect que nous nous occupons ici. 
QUATRIEME PARTIE

LE CROYANT ET SON SEIGNEUR 
L'étude de la christologie $\mathrm{mt}^{\mathrm{l}}$ nous a montré le lien très étroit que l'évangéliste établit entre le Ressuscité et le Jésus terrestre. Le Res suscité rend normatif ce que fit et dit le Jésus terrestre si bien que la prédication de ce dernier occupe désormais une place décisive dans la théologie mt. De ce point de vue, la condition du croyant consiste à deve nir le contemporain des premiers disciples afin de se mettre comme eux à l'école du didascale de naguère. Aussi n'est-il pas étonnant de constater que les $\mu \alpha \vartheta \eta \tau \alpha i$ sont présentés avant tout comme ceux qui écoutent l'enseignement du Maître. Plus que les foules et les adversaires de Jésus, ils sont les témoins constamment présents de sa prédication. Cette volonté $m t$ de faire des disciples l'auditoire privilégié du Christ enseignant se manifeste particulièrement dans les grands discours ${ }^{2}$ et son application systématique ne manque pas de créer quelques situations piquantes. Ainsi, au chap. 10, les disciples envoyés en mission ne partent, ni ne reviennent, mais restent les auditeurs attentifs de Jésus. Ainsi le chap. 13 est adressé aux foules $(13,2)$, mais ce sont les disciples qui sont déclarés heureux de ce qu'ils entendent $(13,16)$. Ainsi encore le chap. 23 est dirigé contre les scribes et les pharisiens, mais ił compte également les disciples dans le cercle de ses destinataires $(23,1)$.

La relation du croyant avec son Seigneur se constitue donc dans l'écoute de l'enseignement du Christ mt. Cet accent porté sur le thème de l'écoute soulève le problème de la compréhension : une catéchèse fidèle et une vie chrétienne authentique présupposent une saisie correcte de la prédication du Jésus terrestre. Aussi n'est-il pas étonnant que Mt ait traité le problème de l'écoute du Christ à l'aide de la catégorie critique de la compréhension.

1 Voir nos chap. $3-5$.

2 Voir notre chap. 1, pp. 29-36. 


\section{Comprendre}

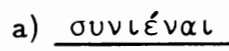

Dans les excellentes pages qu'il consacre à ouvléval dans l'évangile selon $\mathrm{Mt}$, Barth constate que "Mt a supprimé ou réinterprété tous les passages de l'évangile de Mc qui parlent de l'incompréhension des

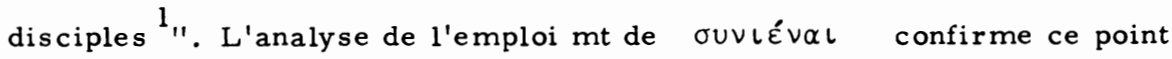

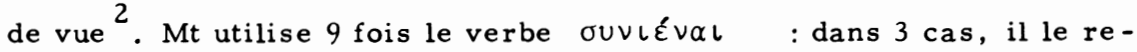
prend de sa source Mc (Mt 13,13 par. Mc 4,12; Mt 15,10 par. Mc 7, 14; Mt 16,12 par. Mc 8,21$)^{3}$ alors qu'à 6 reprises, il s'agit d'adjonctions rédactionnelles $(13,14.15 .19 .23 .51 ; 17,13)$. De plus, sur les 5 emplois mc de ouvléval $(4,12 ; 6,52 ; 7,14 ; 8,17.21)$, Mt en récuse deux (Mc $6,52$ et 8,17$)$. Or ces deux passages de Mc décrivent l'incompréhension des disciples. A Mc 6,52, Mt substitue une confession de foi (Mt 14,33) tandis qu'il supprime purement et simplement Mc 8,17b. Adjonctions et suppressions s'inscrivent dans un seul et même projet rédactionnel : il

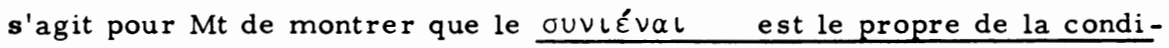
tion du disciple et qu'en conséquence il est refusé aux foules.

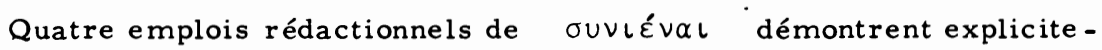
ment que le disciple est celui qui comprend. En 13,51, à Jésus qui leur demande s'ils ont compris le discours qui précède, les disciples répondent affirmativement. En 16,12 et 17,13 apparaît la même formule :

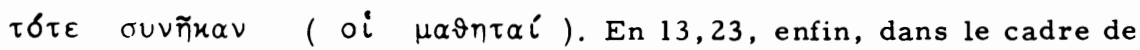

1 Gesetzesverständnis 99.

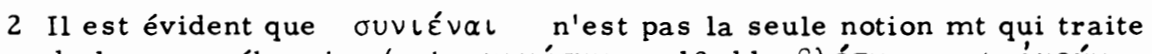

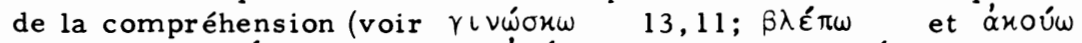

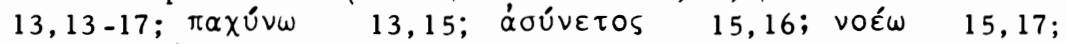

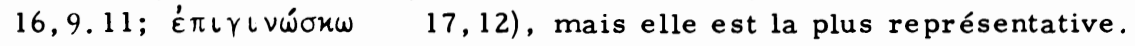
Pour l'analyse qui suit, voir BARTH 99-102, et DUPONT, Paraboles 245-249.

3 Bien que repris de Mc 8,21, Mt 16,12 utilise ouvıદ์ $\alpha \iota$ dans un autre sens : alors que l'usage mc est négatif ( oü $\pi \omega)$, celui de $\mathrm{Mt}$ est positif ( $\tau \delta \tau \varepsilon)$. 
l'interprétation de la parabole du semeur, le disciple est celui qui "entend

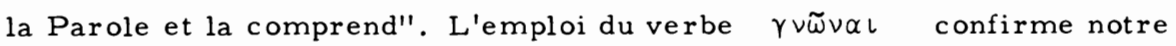
analyse : en 13,11 , les disciples sont ceux à qui est accordée la connais sance des mystères du Royaume.

La tendance mt qui se manifeste dans l'emploi explicite de ouvLÉval et de ses dérivés est confirmée par l'utilisation que Mt fait de sa source Mc: non seulement, il introduit le thème de la compréhension des disciples dans sa narration, mais encore il supprime les passages de Mc qui affirment, directement ou indirectement, leur incompréhension. A cet égard sept cas méritent d'être évoqués. En Mt 13,10.18, au contraire de Mc 4,10.13, les disciples n'interrogent pas Jésus sur la signification des paraboles puisqu'ils sont ceux qui comprennent (cf. 13,11). Pour la même raison, en 13,34, Mt supprime Mc 4,34b. Dans le récit de la trans figuration, Mt 17,14 évite la leçon de Mc 9,6, car tant Pierre que les autres disciples ne sont pas hermétiques à la révélation de Jésus. En 17 , 9, Mt saute Mc 9,10, car, si les disciples ont l'intelligence de l'enseignement de Jésus, il ne convient pas qu'ils se querellent quant à sa résurrection. En 17,23, à l'affirmation de Mc 9,32 ( $0 i$ $\delta \varepsilon$ - les disciples -

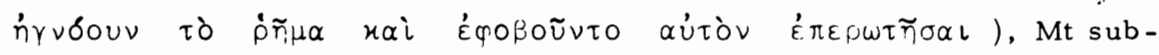
stitue l'expression $\dot{\varepsilon} \lambda \cup \pi \dot{\eta} \vartheta \eta \sigma \alpha \nu \sigma \varphi \delta \varepsilon \rho \alpha$. En 20,17, Mt ne retient pas

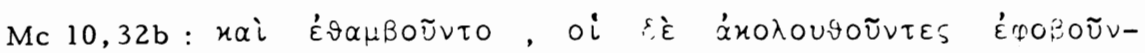
To. A ces sept corrections, il convient d'ajouter les deux cas que nous avons signalés plus haut où Mt supprime l'incompréhension des disciples (Mt 14,33 par. Mc 6,52, suppression de Mc 8,17b).

Il faut pourtant constater que si, pour Mt, les disciples sont par excellence ceux qui comprennent, le rédacteur du premier évangile apporte une nuance significative à sa thèse fondamentale. En effet, dans quatre cas au moins $(13,36 ; 15,16 ; 16,9 ; 17,13)$, il maintient l'incompréhension des disciples. Mais à la différence de Mc, cette incompréhension n'est pas durable, elle ne se dénoue pas seulement avec la résurrection ${ }^{l}$.

1 BARTH 102. Cf. Mc 9,9. 
Elle est momentanée et une nouvelle instruction du Jésus terrestre per met de la dépasser. En 13,36, les disciples demandent une explication, ce qui présuppose une incompréhension; mais comme le montre 13,51, cette crise est tout aussitôt résorbée par l'enseignement du Christ. En 15,

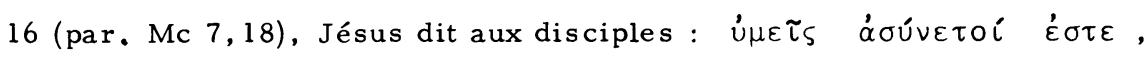
mais cette critique est radoucie par $\alpha \varkappa \mu n ́ v$ qui montre le caractère anormal de la situation et qui annonce son dépassement par une nouvelle instruction. En 16,9, Jésus met à nouveau en cause ses disciples ( oü-

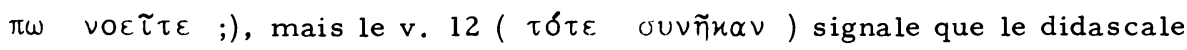
a permis aux siens de surmonter leur crise. Enfin, en 17, 13, l'expres -

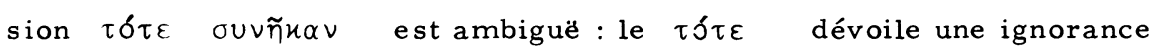
antérieure maintenant dissipée ( $\sigma u \vee \tilde{\eta} \varkappa \alpha \nu)$ grâce à la $\delta \iota \delta \alpha \chi n_{n}$ du Maître. On ne saurait donc affirmer que les disciples mt comprennent d'emblée et par eux-mêmes Jésus; ils parviennent à la compréhension par le biais de son enseignement sans cesse repris ${ }^{1}$. Le Christ enseignant est celui qui fait passer le disciple de l'incompréhension à la compréhension, lui ouvrant ainsi la possibilité de l'obéissance et du témoignage.

La compréhension est, en revanche, refusée aux foules. Ce thème

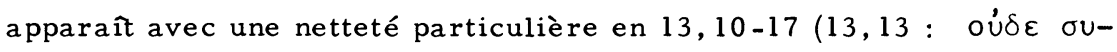

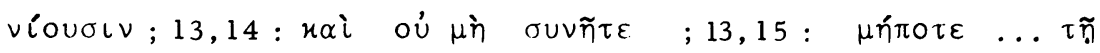

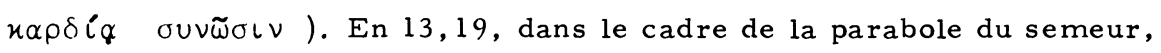

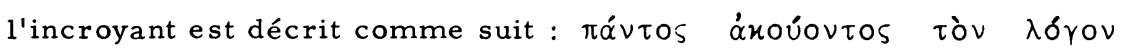

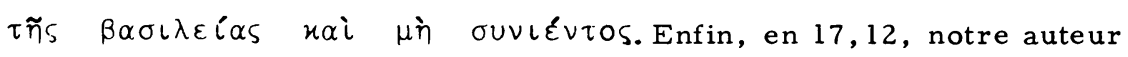
parlant des scribes - les chefs des foules -, ajoute à leur intention dans

1 LUZ (Jünger 149, en particulier note 40) a relevé à juste titre que le premier évangile est celui, parmi les synoptiques, qui contient le plus d'instructions destinées spécialement aux disciples. Il dresse l'inventaire suivant : 9,37 à 11,1 (adressé par Lc aux 70); 13,10-23 (élargissement de Mc); 16,24-28;17,10-13;17,19-20 (élargis sement de Mc); le chap. $18 ; 19,23$ à 20,$19 ; 21,21-22 ; 24,1-2 ; 24,3$ à 25,46 . 


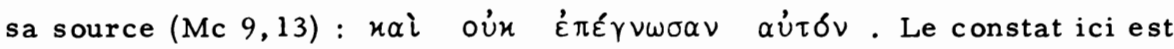
clair et ne souffre pas d'exception. Les disciples comprennent et les fou-

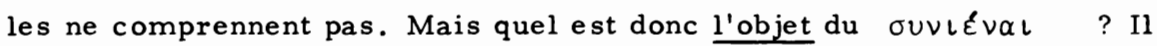
est en effet intéressant de constater que le rédacteur attribue un contenu précis à la compréhension des disciples. 13,19 donne comme objet direct

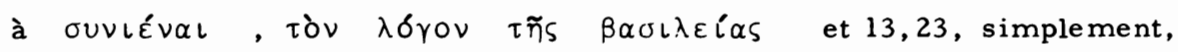

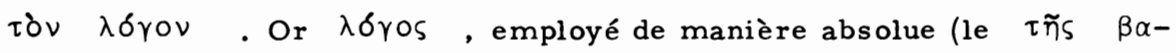
$\sigma \iota \lambda \varepsilon i \alpha s$ de 13,19 est $\mathrm{mt}$ ), est un terme technique de l'église primitive désignant l'évangile ${ }^{l}$. Ce qu'il s'agit de comprendre, c'est donc la prédication de Jésus, son enseignement. Ainsi, au chap. 13, l'objet du ou-

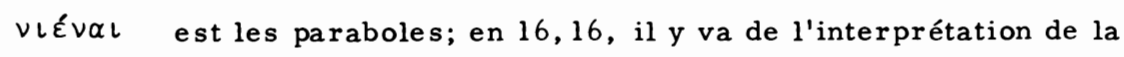
volonté de Dieu; en 16,9, des actes de puissance de Jésus; en 16,12, de l'enseignement des pharisiens; en 17,13, du Baptiste; en 17,23, de la mort et de la résurrection du Christ ${ }^{2}$. En définitive, le disciple comprend la proclamation chrétienne, càd la prédication et la destinée de Jésus, a lors que la foule ne la comprend pas.

Résumons : La figure du disciple chez Mt se caractérise par sa compréhension de la prédication de Jésus. Cette compréhension ne résulte pas d'une qualité particulière du disciple, elle est donnée par le Christ enseignant avec sollicitude les siens. Cette problématique est traitée avec une acuité particulière en 13,10-17. Nous nous tournons donc vers cette péricope pour approfondir notre thématique.

b) $\mathrm{Mt} 13,10-17$

Mt 13,10-17 est une composition originale de l'évangéliste dont le noyau se trouve en Mc 4,10-12. Mt a interprété sa source en l'élargissant : il utilise au v. 12 un logion qui apparait ultérieurement dans la narration de Mc (4,25); il introduit une citation exhaustive d'Es 6, 9-10

1 BARTH 102, note 3; KITTEL, art. $\lambda \varepsilon \gamma \omega$, ThW 4,115-120.

2 Pour suggestive qu'elle soit, l'hypothèse de LUZ qui limite le ouV८Éval à l'enseignement du terrestre compris comme instruction éthique nous semble trop limitée (cf. Jünger 150). 
(vv. 14-15) qui lui a été suggérée par Mc 4,12; enfin, il emploie aux vv. 16-17 un macarisme de la source $Q$ que $L c$ fait intervenirdans un tout autre contexte (Lc 10,23-24).

Dans son introduction (v. 10), Mt modifie totalement Mc 4,10. Le v. 10a est une formule stéréotypée dont se sert le rédacteur pour introduire un débat d'école ${ }^{1}$. Les disciples, image de la communauté, inter rogent le Christ pour recevoir son enseignement. La question qu'ils posent ( $\mathrm{v}$. 10b) est toute différente de celle présente chez Mc : ils ne sont plus en quête du sens des paraboles, mais ils demandent au Maitre pourquoi il s'adresse aux foules dans un langage qu'elles ne saisissent pas ${ }^{2}$. Le problème évoqué est donc celui de la compréhension de la prédication de Jésus et, d'emblée, un net clivage s'établit entre les disciples qui comprennent

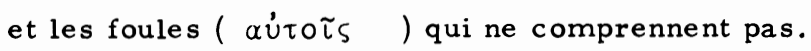

La réponse de Jésus ( $v$. 11) est formulée en fonction de la question posée : ainsile ő $\iota$ rédactionnel est la suite logique du $\delta \iota \grave{a} \tau \grave{\imath}$

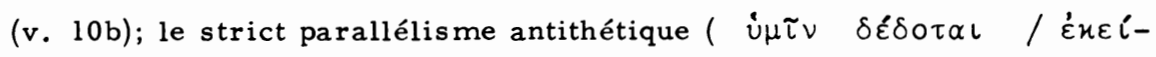
voıs oú $\delta \varepsilon ́ \delta \circ \tau \alpha \iota)$ dépend également de l'opposition mise en place dans le v. 10. La compréhension dont bénéficient les disciples est présentée comme une connaissance ( $\gamma \nu \tilde{\omega} \vee \alpha \iota !)$. Cette connaissance est définie d'un double point de vue. D'une part, Mt précise son objet : il s'agit des mystères du Royaume des Cieux. Muơńpıov est un terme emprunté à l'apocalyptique juive tardive, milieu dans lequel il désigne "les dispositions de la divinité pour la fin des temps, dispositions tenues cachées,

1 Remarquer le couple stéréotypé $\pi \rho \circ v \varepsilon \rho \chi \varepsilon \sigma \vartheta \alpha \iota-\lambda \varepsilon \gamma \varepsilon \iota \nu$ (HELD 215); par ailleurs, les $\mu \alpha \vartheta \eta \tau \alpha i$ se substituent aux oi $\pi \varepsilon \rho i$

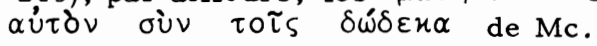

2 Le v. 10b semble présupposer que l'enseignement de Jésus a été ésotérique. Cela n'est pas conforme à ce que nous pouvons savoir par ailleurs du Jésus historique (BRAUN, Radikalismus II 18-23). Aussi sommes-nous en présence d'une affirmation émanant de la communauté (BORNKAMM, art. Huotńplov , ThW 4,824, note 133), caractérisée probablement par le conflit avec le judaîsme. Les disciples sont donc bien aussi les porte-parole de l'église $\mathrm{mt}$. 


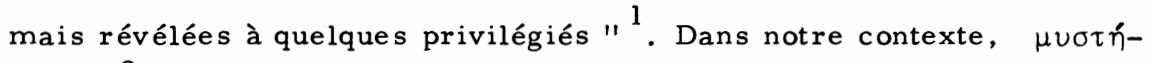
plov ${ }^{2}$ est mis en rapport avec $\beta \alpha \sigma \iota \lambda \varepsilon i \alpha$ et, de ce fait, conserve sa connotation eschatologique. De plus, ces mystères du Royaume apparaissent de manière voilée dans les paraboles. Si l'on tient compte du contenu du chap. 13 qui est un enseignement du Christ $\mathrm{mt}$, on pensera aux thèmes qui sont exposés dans les paraboles. D'abord à la manifesta tion paradoxale du Royaume dans le monde, ensuite à l'engagement radi cal qu'il requiert, enfin et surtout à la venue du Jugement dernier ${ }^{3}$.

En deuxième lieu, cette connaissance des mystères du Royaume dont bénéficient les disciples, est un don $(\delta \varepsilon \delta \circ \tau \alpha \iota$ : forme passive dont Dieu est l'agent). Elle n'est pas le fruit d'une perspicacité intellectuelle ou la récompense de bonnes oeuvres, elle est la grâce eschatologique de Dieu qui ne peut être ni méritée, ni revendiquée, mais seulement acceptée ${ }^{4}$. On comprend alors que la compréhension des mystères du Royaume présuppose la foi, car seule la foi accepte que, dans la prédication de Jésus, le Royaume devienne réalité. La compréhension est donc accordée aux disciples parce qu'ils croient, elle est refusée aux foules parce qu'elles ne croient pas ${ }^{5}$. C'est ce que confirme le ő $\tau$ du v. 13 et la longue citation du v. 14.

Par l'adjonction du logion du v. $12^{6}$, Mt dégage la portée eschatolo-

1 BONNARD, Mt 193, à la suite de BORNKAMM, art. cit. 825.

2 Pour ce qui suit, voir BORNKAMM, art. cit. 824-25.

3 Avec SCHWEIZER, Mt 194. BORNKAMM, art. cit. 825, voit dans les mystères du Royaume révélés aux disciples Jésus lui-même en tant que Messie. Cette concentration christologique, sans doute présente chez Mc, disparait chez Mt; pour ce dernier, les mystères (pluriel !) recouvrent les thèmes de l'enseignement parabolique du Christ.

4 LOHMEYER, Mt 201.

5 Cf. STRECKER, Weg 229.

6 La seule retouche rédactionnelle à signaler est l'introduction du verbe $\pi \varepsilon \rho \iota \sigma \sigma \varepsilon \cup \varepsilon \iota \nu$ dont la fonction est pléophorique (WILKENS 313). 
gique du v. 11 : la compréhension liée à la foi ou l'incompréhension liée à l'incroyance décident de la destinée de l'homme lors du jugement der nier ${ }^{l}$. Le disciple qui a reçu de Dieu la connaissance subsistera lors du jugement et accédera à la plénitude du salut. A l'inverse, celui qui n'a pas, càd celui qui ne reçoit pas l'enseignement du Christ mt, perd tout.

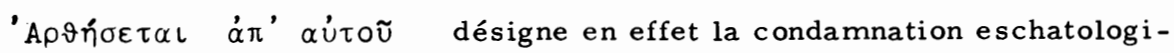
que $^{2}$. La compréhension est donc constitutive du salut.

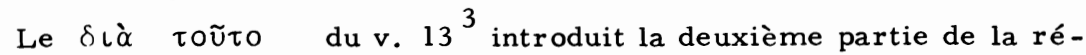
ponse de Jésus. A la constatation des vv. $11-12$ succède une explication ${ }^{4}$ : les "mystères" ne sont pas "donnés" aux foules parce qu'elles sont en durcies. Aussi le jugement annoncé au v. 12 est-il en train de s'accomplir dans la prédication de Jésus, reçue sans être comprise ${ }^{5}$. Cette interprétation est confirmée par la correction que $\mathrm{Mt}$ apporte au texte de

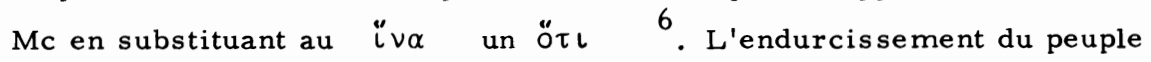
n'est pas le but du discours de Jésus en paraboles, mais il est révélé par lui dans son ultime profondeur. L'endurcissement du peuple est décrit dans une terminologie typique. Le double emploi de $\beta \lambda \varepsilon ́ \tau t \omega$ oppose la vue extérieure à la vue authentique, càd croyante. Cette vue authentique est elle-même une sorte d'écoute, elle se rapporte à l'oeuvre et à la prédication de Jésus ${ }^{7}$. L'usage contrasté d' $\mathrm{d}^{\prime}$ oúw oppose écoute extérieure et écoute authentique. Les marques de l'écoute authentique sont la

1 Les trois futurs passifs (agent : Dieu) décrivent la comparution eschatologique.

2 JEREMIAS, art. $\alpha$ i $\rho \omega$, ThW 1, 185.

3 Le v. 13a est rédactionnel.

4 TRILLING, Israel 77.

5 BONNARD, Mt 194.

6 Cette correction du $\iota_{v \alpha}$ en ŏ $\iota$ a pour conséquence logique la suppression de Mc 4,12c.

7 MiCHAELIS, art. ó $\rho \alpha ́ \omega$, ThW 5, 348. 


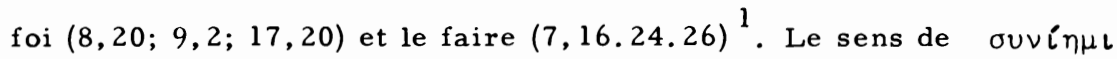
découle de ce qui précède. Il n'est fait allusion ni à la raison naturelle de l'homme, ni à une quelconque perspicacité intellectuelle. Le ouvıḱ$v \alpha \iota$ décrit l'ouverture de la compréhension pour la révélation ${ }^{2}$. En résumé, l'attitude du peuple est celle de l'incompréhension coupable, de l'ignorance causée par l'endurcissement. Cette incompréhension, cette ignorance sont la marque du jugement qui s'accomplit. Mt commente et développe cette idée en ajoutant une citation exhaustive d'Es 6, 9-10 ${ }^{3}$.

Mt conclut notre péricope non pas en reprenant le reproche d'incompréhension que Mc 4,13 adresse aux disciples (après l'avoir appliqué à la foule ${ }^{4}$ ), mais en introduisant un macarisme tiré de la source $Q$ (par. Lc 10,23-24). Formellement, ce macarisme est relié à ce qui précède par

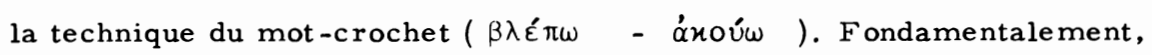
il reprend le premier terme de l'antithèse formulée au v. 1l. Car, si les vv. 13-15 ont traité du jugement qui s'abat sur le peuple endurci ${ }^{5}$, les vv. 16-17 décrivent le bonheur accordé aux disciples. A ce titre, le

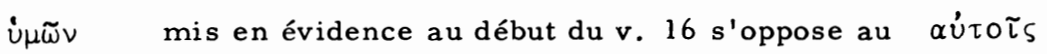
(vv. 10.13), Éxદívols (v. 11).

1 KITTEL, art. $\dot{\alpha}$ Koúw, ThW 1,220-21.

2 Voir BARTH 102-103. BONNARD, dans son article "Matthieu, éducateur du peuple chrétien" $1-7$, va dans le même sens que BARTH en proposant de traduire ouvléval par "bien comprendre", car, cette expression "implique l'idée d'une victoire sur un malentendu et celle d'une décision personnelle" (art. cit. 3).

3 Cette citation est étonnante à plus d'un titre. Son introduction n'est pas conforme à la formulation habituelle de l'évangéliste ( $\dot{\alpha} \vee \alpha \pi \lambda \eta p \circ \tilde{v} \nu$ et $\pi \rho \circ \emptyset \eta \varepsilon i \alpha$ sont des hapax dans les synoptiques). Elle est donnée dans le texte de la LXX - ce qui est un cas unique chez Mt (STRECKER, Weg 82) -. Enfin, fait sans précédent, elle est partie intégrante d'un discours du Christ mt.

4 KLOSTERMANN, Mt 119.

5 Le peuple endurci dont parlent les vv. 13-15, figure en fait le judalsme d'après 70 , car ce n'est qu'à cette date qu'une séparation si claire intervient entre le "peuple" et les disciples. Point bien développé par SCHWEIZER, Mt 195. 
Mt a opéré différentes retouches sur la source $Q^{1}$, reconnaissable encore chez Lc ${ }^{2}$. A part la mise en évidence du $i \mu \tilde{\omega} \nu$, on relèvera

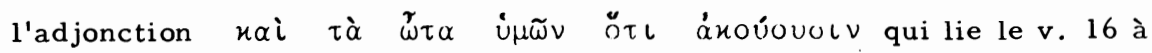
ce qui précède, mais qui, surtout, permet un strict parallélisme avec le

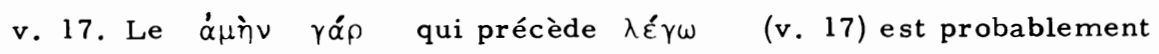

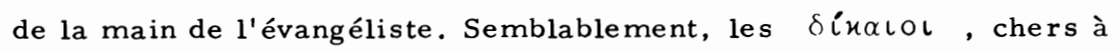
Mt, prennent la place des $\sigma \alpha \sigma \lambda \tau_{-} \tau_{S}$ de Lc. Mais la retouche la plus importante est incontestablement l'introduction des deux ö $\iota$ du v. 16 : les disciples mt sont réputés heureux non à cause de ce qu'ils voient et entendent (ainsi $Q$ et $L c$ ), mais parce qu'ils voient et entendent; leur compréhension, et non le privilège d'être des témoins oculaires, est la cause de leur bonheur. Contrairement à ce que pense Strecker ${ }^{3}$, ce ma carisme (dans sa version $\mathrm{mt}$ ) n'historicise en aucune manière la figure du disciple car, comme le montre le contexte, il n'y a pas opposition entre les compagnons historiques du Christ et les croyants ultérieurs, mais entre ceux qui voient Fésus sans le voir et ceux qui le voient authentiquement. Comme l'écrit justement Bonnard ${ }^{4}$, pour $\mathrm{Mt}$, "le bonheur

1 Voir l'analyse détaillée de SCHULZ, Q 419-420, ainsi que BARTH 100 et STRECKER, Weg 197.

2 Voir les auteurs cités à la note précédente ainsi que BULTMANN, Trad 114. Le macarisme qui célèbre le bonheur des témoins des temps messianiques est d'ailleurs déjà connu du bas-judaìsme, cf. Ps Sal 17,44; $18,6-7$.

3 STRECKER, Weg 197-198, admet que le v. 16 a une signification idéale, mais soutient que le v. 17 à cause du $\alpha$, souligne la position unique et fondatrice pour la vie de l'Eglise des disciples/témoins oculaires. C'est introduire un clivage entre premiers disciples et croyants ulté rieurs qui ne figure pas dans le texte (les disciples sont opposés à la foule et aux grandes figures de $1^{\prime} \mathrm{AT}$ ), c'est postuler entre le v. 16 et

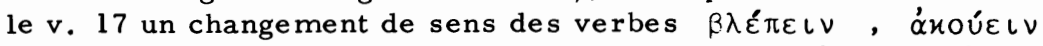
que rien ne justifie. Il s'agit pour Mt, dans cette péricope, de mettre en évidence la compréhension donnée aux disciples et non de souligner la vocation de témoin des premiers disciples pour l'Eglise.

4 BONNARD, Mt 196. 
n'est pas tant de voir Jésus, mais de le voir en le comprenant". La compréhension à laquelle est liée la déclaration de bonheur, n'est pas le privilège de la première génération, mais une catégorie de la vie chrétienne. A cet égard, il convient de faire deux remarques. Alors que dans les macarismes du chap. $5^{l}$, placés sous le signe de l'à-venir de Dieu, les verbes sont au futur, tous les verbes des vv. 16-17 sont au présent. Le bonheur est une réalité présente, immédiatement accessible. Plus encore, par opposition aux béatitudes, notre macarisme ne célèbre pas un bonheur paradoxal au sein de la détresse; l'accueil authentique de la révélation rend le croyant heureux hic et nunc.

La parole en $\alpha \dot{\mu} \tilde{\eta} v$ du v. 17 fait ressortir la grâce qui est accor dée à tous les disciples du Christ en évoquant par contraste l'histoire vétérotestamentaire. Les grandes figures de $\mathrm{l}^{\prime} \mathrm{AT}^{2} \mathrm{n}^{\prime}$ ont pas eu part, malgré leur désir, au bonheur qui est offert aux disciples. Le $\ddot{\alpha}$ ne désigne sans doute plus, comme à l'origine, l'apparition du seul Royau$\mathrm{me}^{3}$. Le Christ et le Royaume sont devenus une seule réalité. Pour $\mathrm{Mt}$, le bonheur est de comprendre la prédication et la destinée du Christ of frant le Royaume en plénitude ${ }^{4}$.

c) Le problème de l'idéalisation

Se fondant sur la suppression par Mt de l'incompréhension des dis ciples et sur l'affirmation corrélative de leur compréhension, certains exégètes ${ }^{5}$ ont accrédité la thèse selon laquelle le rédacteur du premier

1 Voir notre chap. 11.

2 En 10,$41 ; 23,29.35$, les prophètes et les justes sont les grandes figures de l'AT.

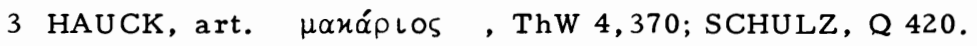

4 SCHWEIZER, Mt 195 : "Jetzt aber handelt es sich um das Hören des Gotteswortes".

5 La thèse de l'idéalisation des disciples, déjà défendue par ALLEN (Mt XXXIIIs), KLOSTERMANN (Mt 19-21), LAGRANGE (Mt LXVIIss), est représentée aujourd'hui par STRECKER, Weg 193-194, mais combattue par BARTH 110-113, et LUZ, Jünger $147-148$. 
évangile aurait idéalisé la figure des disciples ${ }^{1}$. D'autres arguments sont encore invoqués à l'appui de cette thèse. Ainsi, Mt à la différence de Mc, n'applique pas aux disciples les verbes $\vartheta \alpha \mu \beta \varepsilon \tau \sigma \vartheta \alpha \iota 2$, $\xi_{\zeta}$ เ $\sigma-$ $\tau \alpha^{\prime} \nu \alpha \iota{ }^{3}$, $\varepsilon \mu \beta \rho \iota \mu \tilde{\alpha} \sigma \vartheta \alpha \iota{ }^{4}$, $\pi \varepsilon \rho \iota \beta \lambda \varepsilon \pi \varepsilon \sigma \vartheta \alpha \iota{ }^{5}$. Il supprime certains passages dépeignant leur peur ${ }^{6}$ ainsi que certaines de leurs réactions qui les amoindrissent ${ }^{7}$. Les disciples sont au contraire ceux qui comprennent Jésus ${ }^{8}$, qui le suivent avec fidélité ${ }^{9}$, qui font la volonté de Dieu ${ }^{10}$, qui confessent le Fils de Dieu ${ }^{l} l$, qui accompagnent lucidement le maître dans la passion ${ }^{12}$. Toutes ces retouches rédactionnelles semblent être les indices d'une tendance hagiographique situant les disciples dans un

1 Pour défendre cette thèse, KLOSTERMANN s'appuie sur les passages suivants $: 8,26 ; 17,23 ; 20,20 ; 14,33 ; 16,9 ; 17,4.9 ; 18,1 ; 19,23 ; 20,17$; 26,$43 ; 13,16-17 ; 16,12.17-19$. Parmi ces références, 13,16-17;14, $33 ; 16,9 ; 17,4.9 .23$ ressortissent au thème de la compréhension.

219,23 pour Mc 10,24; 20,17 pour Mc 10,32; Mc 1,27 est absent chez Mt.

$314,32-33$ pour Mc 6,51.

426,9 pour Mc 14,5 .

$5 \quad 17,8$ pour Mc 9,8.

6 Le $\varphi \delta \beta 05$ / 17,4 pour Mc 9,6; 17,23 pour Mc 9,32; mais il subsiste en 14,26-27. 30 et 17,6 .

78,25 pour Mc 4,$38 ; 14,16-17$ pour Mc 6,$37 ; 15,33$ pour Mc 8, 4; 20, 20 pour Mc 10,35.

8 LUZ (Junger 150) remarque justement que si les disciples mt comprennent au contraire des disciples mc, l'objet de la compréhension a changé : il n'y va plus spécifiquement de la souffrance du Fils de Dieu, mais de l'enseignement du Christ.

9 8,23 pour Mc 4,35; 9, 19 pour Mc 5,24; corrections mt en 9,9-10 et en 19,28 .

$1012,49-50$ pour Mc 3,34-35.

1114,33 pour Mc 6,51 -52, cf. aussi 16,17.

1224,$3 ; 26,2$. 
passé saint et définitivement révolu '. Ce serait vrai si Mt s'en tenait là dans la description des disciples. Or ce n'est pas le cas. Car si les disciples comprennent la mission et la prédication de Jésus, ce n'est pas en vertu de leurs qualités propres, mais grâce à un don de Jésus (cf. 13,1!). Et encore, cette compréhension n'est pas une possession, elle n'est jamais parfaite, ni achevée. Sans cesse redonnée par le maître, elle tient à une décision de foi (cf. 13,11!). De plus, Mt est précisément l'évangéliste qui insiste sur le manque de foi des disciples, qui montre leur incrédulité au sein même de la Nachfolge ${ }^{2}$. Il n'en va pas autrement dans la manière dont Mt présente l'obéissance des disciples. Car si ces derniers sont ceux qui font la volonté de Dieu, parfois ils succombent à la désobéissance $^{3}$. Leur infidélité est même soulignée par le rédacte ur du premier évangile ${ }^{4}$.

Dès lors l'image que $\mathrm{Mt}$ donne des disciples n'est pas idéalisée et, par là-même, historicisée ${ }^{5}$, mais dialectique et, par là-même, paradig matique. Le disciple est celui qui comprend, mais qui parfois n'en est pas moins frappé d'incompréhension. Il croit, mais il n'échappe pas au doute. Il obéit, mais la tentation de l'infidélité ne lui est pas épargnée. Sa "Nachfolge" est tout à la fois grandeur et misère. Mais n'est-ce pas là précisément la description typique de la condition du croyant ${ }^{6}$ ?

1 Ainsi STRECKER, Weg 194.

2 Mt introduit 5 fois le reproche de manque de foi à l'égard des disciples:

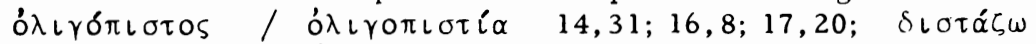
14,$31 ; 28,17 ; \vartheta \vee u \mu \alpha ́ \zeta \omega \quad 21,20$.

3 Cf. $16,22-23 ; 24,12 ; 26,70$, vv. qui montrent l'accentuation de la désóbéissance des disciples par rapport à Mc.

4 Avec BARTH 112. Voir, p. ex., comment Mt a retravaillé dans un sens négatif la figure de Pierre (matériel chez STRECKER, Weg 204-205).

5 Contre STRECKER, Weg 193-194.

6 Même STRECKER (Weg ibid.) qui défend la thèse de l'idéalisation des disciples et l'interprète dans le sens de l'historicisation, constate que Mt applique aux disciples le thème de la peur, du manque de foi, du doute et que, ce faisant, "in dem Bild der Jünger auch das Verständnis des einzelnen Christen sich abzeichnet" (Weg 194). 


\section{Suivre Jésus (ánoגougẽ̃v)}

L'accent mis par la christologie mt sur l'enseignement du Jésus terrestre a son corrélat anthropologique dans la thématique de la compréhension. Le ouvıéval est le présupposé obligé à la fois de la vie chrétienne et de l'enseignement ecclésial. Mais il convient de préciser. Même si le rédacteur n'a pas réfléchi de manière systématique sur les relations entre la compréhension et les autres catégories de la vie chrétienne (p.ex. la $\pi i \sigma \tau \iota s$ ) ${ }^{1}$, le chap. 13 - mais aussi l'enseignement sans cesse repris par le Christ - montre que le ouvikval n'est pas simplement un acte noétique. Il est une possibilité ouverte gracieusement par le Christ, acceptée dans la foi et qui décide de l'avenir eschatologique de l'homme (Mt 13,10-17). C'est dire que le ouvı́́val implique une transformation de l'existence.

Cette transformation de la vie du disci ple, liée à la compréhension, Mt a pu la décrire en termes de relation entre le Jésus terrestre et ses disciples. Il a, en effet, trouvé dans la tradition synoptique, un modèle

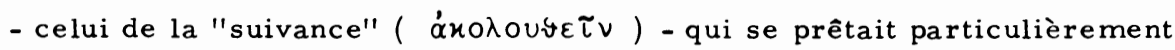
bien à ce projet. Le disciple qui comprend Jésus, le suit. Cette "suivance" est par excellence le thème qui illustre la transformation existentiel le impliquée par l'adhésion au Jésus terrestre.

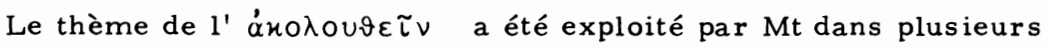
directions. Il peut tout aussi bien illustrer l'obéissance due à la Loi $(11,28-30 \text { et } 19,16-22)^{2}$ que dépeindre la grandeur et la misère de l'existence croyante ${ }^{3}$. Dans le cadre du présent chapitre, nous retiendrons

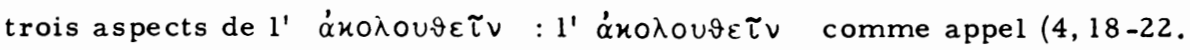
9,9), comme communauté de destin $(8,18-22)$, comme rupture avec le monde $(10,37-39)$.

1 LUZ, Junger 151 .

2 Voir notre chap. 5 et notre partie consacrée à l'éthique.

3 Voir notre chap. 9. 
a) Mt 4,18-22; 9,9

Le premier évangile compte trois récits d'appel à la suivance $(4,18$ $20.21-22 ; 9,9)$. Dans les trois cas, il s'agit d'apophtegmes biographiques 1 et, plus particulièrement de scènes idéales, càd de scènes "exprimant une vérité qui dépasse le cadre de la situation et leur conférant un caractère symbolique" ${ }^{2}$. L'intérêt documentaire ou biographique est absent, les données chronologiques ou topographiques vagues, la description psychologique des personnages inexistante ${ }^{3}$. Ces péricopes ont une signification paradigmatique; elles veulent montrer de manière exemplaire ce que signifie l'appel à la condition du disciple ${ }^{4}$. Cela est particulièrement vrai de la vocation de Matthieu $(9,9)$ qui est commentée dans les vv. 10 13 : ce récit de controverse entend en effet montrer que l'appel d'un pécheur à la Nachfolge est l'appel de tous les pécheurs ${ }^{5}$.

Dans les deux péricopes $(4,18-22 ; 9,9)$, Mt s'est inspiré de sa source Mc $(1,16-20 ; 2,14)$. A chaque fois, la recension $m$ se signale par des améliorations de style conférant une plus grande clarté formelle au récit. Seules trois modifications méritent d'être notées : en 4,22, le rédacteur déplace un $\varepsilon \dot{\imath} \varepsilon \dot{\omega} \omega s$ qui souligne dès lors la promptitude de l'obéissance

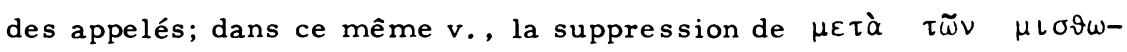
$\tau \tilde{\omega} \nu$ concentre l'attention sur la rupture radicale avec le milieu familial;

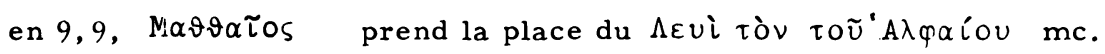

Le contexte choisi par Mt pour ces deux péricopes a une signification théologique évidente. L'appel de Pierre et André, Jacques et Jean ne suit pas le Sermon sur la Montagne, il le précède; l'enseignement de Jésus

- écouté et reçu - n'est donc pas le motif de leur adhésion. Semblablement,

1 BULTMANN, Trad $26-27$.

2 BULTMANN, Trad 59.

3 BULTMANN, Trad 27; DIBELIUS, Formgeschichte 109; SCHMIDT, Rahmen 43.

4 BORNKAMM, Jesus 134.

5 SCHWEIZER, Erniedrigung 10. 
la rencontre entre le Christ et Matthieu est fortuite. Dans les trois scènes, l'appel à la suivance est un événement complètement inattendu et imprévisible.

Nos trois scènes d'appel sont construites selon un schéma utilisé une fois dans l'AT, à savoir dans le récit de la vocation d'Elisée (1R $19,19-21=\operatorname{LXX} 3 \mathrm{R} 19,19-21)^{1}$. Ce schéma comprend quatre éléments. En premier lieu la rencontre de celui qui appelle et du futur élu : on indique la situation, on évoque la rencontre proprement dite et on précise le métier de l'élu. En second lieu l'appel. En troisième lieu intervient un motif retardateur ${ }^{2}$. Enfin, on mentionne l'abandon de l'ancienne vie et l'obéissance au nouveau maître. La tradition synoptique a repris ce schéma, mais en supprimant le motif retardateur. La Nachfolge évangélique acquiert de ce fait une radicalité incomparable.

Nous entreprenons l'analyse des trois scènes mentionnées selon le schéma que nous venons de dégager. On évoque d'abord la rencontre de Jésus et des futurs disciples. Les indications topographiques stéréotypées $(4,18 a .21 a ; 9,9 a)$ ne nécessitent pas de commentaire. La description de la rencontre, elle, mérite qu'on s'y arrête. Dans les trois cas, l'usage de $\varepsilon \hat{l} \delta \varepsilon v$ montre que c'est Jésus qui a l'initiative de la rencontre; ce terme illustre son projet d'élection ${ }^{3}$. Les hommes auxquels il va s'adres ser, ne sont ni préparés, ni prévenus; ils vaquent à leurs occupations quotidiennes. Le contexte ne permet ni de leur attribuer un intérêt mar qué pour Jésus, ni une connaissance préalable de son message - ce qui

1 BULTMANN, Trad 27 et, surtout, SCHULZ, Nachfolgen 100-103.

2 L'unanimité ne règne pas sur l'interprétation de 3R 19,20. Si les uns y voient un motif retardateur - Elisée a la permission de prendre congé de son père -, d'autres y voient une clause aggravante - il est in terdit à Elisée de saluer son père -. En tout état de cause, à l'époque de Jésus, ce $v$. était interprété comme un motif retardateur (HENGEL, Nachfolge 19).

3 SCHWEIZER, Erniedrigung 25. 
faciliterait l'explication psychologique du récit - ${ }^{1}$.

La condition des futurs disciples est significative. Pierre et André, Jacques et Jean, appartiennent au petit peuple de Galilée ${ }^{2}$, méprisé des élites religieuses. Plus intéressante encore est la profession de Matthieu. Il est collecteur d'impôts ${ }^{3}$, càd un paria aux yeux du Juif pieux. Suspect de se constituer une fortune à bon compte, impur de par ses contacts inévitables avec les païens, collaborateur de la puissance occupante, le péager représente tout ce qu'exècre la société juive qui le tient d'ailleurs à l'écart ${ }^{4}$. La personne des appelés nous montre donc que, pour le Christ, les capacités, la situation ou la piété de ceux qu'il rassemble, ne jouent aucun rôle. Il s'adresse à Israël dans son ensemble sans se sentir lié par la tradition légale. Dans cette disponibilité pour des hommes humbles et méprisés se manifeste la grâce attachée à la Nachfolge.

Le deuxième point du schéma consiste dans l'appel proprement dit. A chaque fois, il se présente comme un ordre exigeant l'obéissance in-

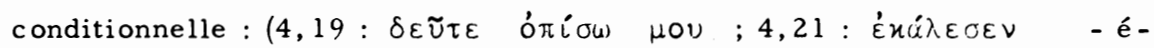

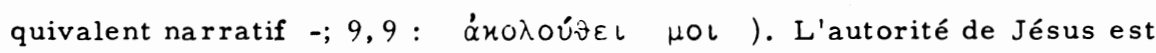
ici celle du Kyrios et le seul parallèle satisfaisant est l'appel des prophètes par Yahvé dans l'AT ${ }^{5}$ : Jésus dispose souverainement de l'homme. Corrélativement, cela signifie : la seule parole souveraine de Jésus introduit l'homme dans la condition de disciple sans égard aux capacités de

1 Avec HAENCHEN, Weg 26.

2 Le fait que Jacques et Jean soient plus riches que Pierre et André puisqu'ils disposent d'un bateau leur permettant de pêcher au large, alors que ces derniers jettent le filet depuis la rive (LOHMEYER, Mc 32-33; GRUNDMANN, Mc 4), ne change rien au problème.

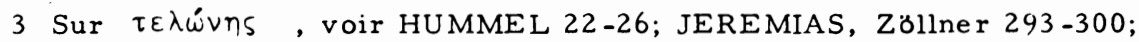
MICHEL, art. TEגívns , ThW 8, 88-106.

4 De iure, l'exclusion de la société civile et religieuse est sanctionnée par la perte des droits civils (JEREMIAS, Zöllner).

5 SCHNIEWIND, Mt 30. 


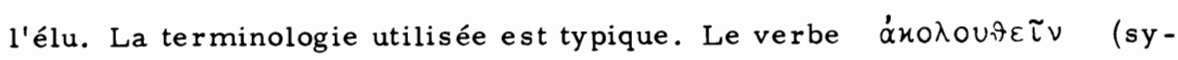
nonyme : $\delta \varepsilon \tilde{u} \tau \varepsilon$ óríow, décrit une relation exclusive entre Jésus et l'appelé. Suivre Jésus signifie s'attacher à lui totalement, lui vouer une obéissance sans limite, l'écouter et le servir, partager sa destinée. La Nachfolge est donc déterminée d'une double manière par Jésus : c'est lui qui en ouvre l'accès par son appel; elle est relation à sa personne.

La première scène d'appel $(4,18-20)$ contient un élément de plus que les deux suivantes. La nouvelle conditior créée par Jésus ( no ıńow) débouche dans un service : être parmi les hommes les témoins du Royaume instauré par Jésus(cf. chap. 10). Signalons brièvement qu'à notre avis, l'expression $\dot{\alpha} \lambda \varepsilon \varepsilon \tau \varsigma \quad \dot{\alpha} \nu \vartheta \rho \omega ́ \pi \omega \nu$ est amenée par le contexte (v. 18: $\dot{\alpha} \lambda \varepsilon \varepsilon \tau_{\varsigma}$ ) et qu'il s'agit d'un jeu de mots qu'il ne convient pas de surinterp̣réter ${ }^{l}$. Sa pointe consiste à montrer le chargeinent de condition intervenu.

Troisième point du schéma : la réponse des appelés. Dans les trois cas, elle se caractérise par sa promptitude. Pierre et André abandonnent leur filet ( $\varepsilon \dot{U} \vartheta \varepsilon ́ \omega \varsigma \quad !)$; Jacques et Jean quittent Jeur bateau et leur père

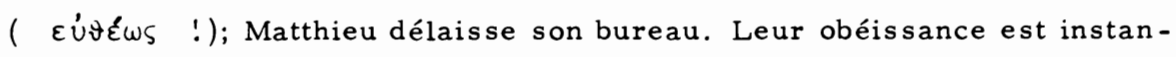
tanée et absolue. D'une part, elle se présente comme une rupture radicale avec les conditions de vie antérieures (double emploi de á $\varphi \varepsilon \varepsilon \tau \varepsilon \varsigma$

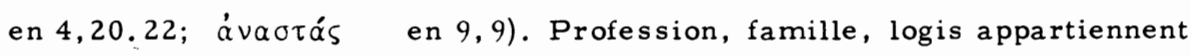
à un passé révolu. D'autre part, cette même obéissance introduit l'appelé

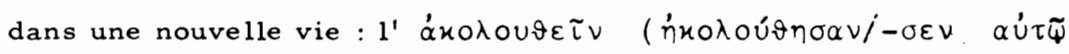
est chaque fois le dernier mot de la scène), caractérisé par l'adhésion inconditionnelle et exclusive à la personne de Jésus, le partage de sa destinée historique.

En conclusion, ces trois scènes d'appel à la suivance attestent un

1 L'image dans l'AT évoque le fait de tomber aux mains de l'ennemi; dans le bas-judaf'sme, elle décrit la tromperie (matériel chez HENGEL, Nachfolge 86 ). L'accent serait alors provocant : en proclamant le Royaume, les disciples arracheraient les hommes au Méchant. 
propos théologique et non psychologique. On n'explique pas en effet les motifs de l'adhésion des élus à Jésus. Ces a pophtegmes illustrent avec vigueur l'autorité de la parole du Christ mt qui prend possession de l'exis tence humaine, mais aussi sa grâce - il s'agit à chaque fois d'hommes sé parés de Dieu -. La Nachfolge, dont la cause est la seule initiative du Christ provoque un changement de vie radicale, une rupture complète de l'homme avec son passé. Comment se présente alors cette nouvelle vie

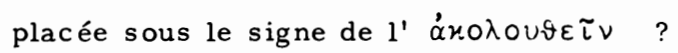

b) Mt 8,18-22

Les vv. 19-22 sont un apophtegme constitué de deux scènes construites de manière parallèle $(19-20.21-22)^{1}$. Mt a constitué d'une manière fort originale le contexte de cette unité tirée de la source $Q$. Il l'a mise en relation avec le récit de la tempête apaisée $(8,23-27)$ dont elle devient le principe d'interprétation ${ }^{2}$.

D'une part, Mt introduit les vv. 19-22 provenant de $Q$ dans le cadre $m$ de la tempête apaisée. En effet la narration mc de ce miracle débute en 4,35 (par. Mt 8,18!); or nous retrouvons Mc 4,36 en Mt 8,23. Cela signifie que le rédacteur mt place cet apophtegme non avant le récit de la tempête apaisée, mais à l'intérieur même de ce récit. A ce choix contextuel s'ajoute l'emploi de mots-crochets qui unissent étroitement 8,18-22 et 8,23-27:8,18 $\dot{\alpha} \pi \varepsilon \lambda \vartheta \varepsilon \tau \nu \quad, 8,19 \quad \dot{\alpha} \pi \varepsilon \varepsilon_{\nu} \chi \varepsilon \sigma \vartheta \alpha \iota \quad, 8,21$

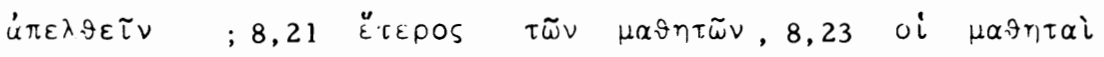

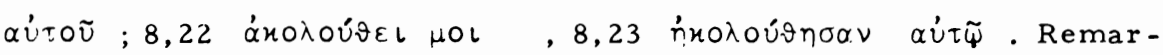

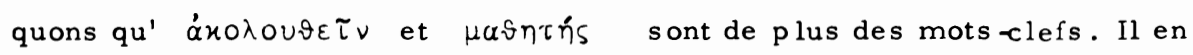
résulte une unité de thème entre les vv. 18-22 et 23-27.

Si nous examinons la rédaction $\mathrm{mt}$ de cet apophtegme, nous remarquons qu'il est intégré à un cadre (v. 18) emprunté à Mc 4,36. Mt apporte trois

1 BULTMANN, Trad 27-28; DIBELIUS, Formges chichte 34ss; SCHULZ, Q 435 .

2 HELD 190. 


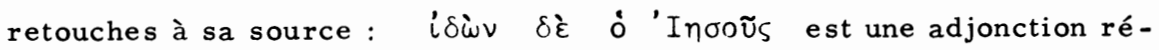
dactionnelle ${ }^{1}$; le verbe $\chi \varepsilon \lambda \varepsilon u ́ \omega$ est un élément caractéristique du vocabulaire $\mathrm{mt}^{2}$; $\alpha \dot{\alpha} \kappa \lambda \vartheta \varepsilon \tau \nu$ remplace le $\delta\llcorner\varepsilon ́ \lambda \vartheta \omega \mu \varepsilon \nu$ mc et constitue ainsi le premier maillon de la chaîne des mots-crochets signalés plus haut. La première scène (vv. 19-20) est textuellement identique avec

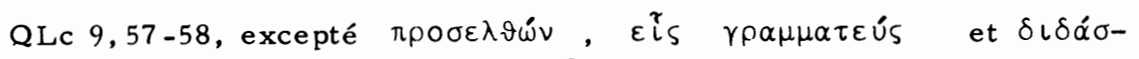
$x \alpha \lambda \varepsilon$ qui sont des adjonctions $\mathrm{mt}^{3}$. Dans la seconde scène (vv. $21-22$ ),

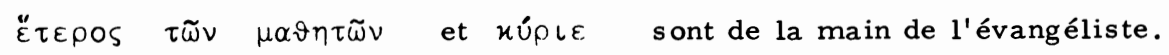
Pour le reste, l'identité verbale avec le par. lc est frappante. La différence essentielle tient dans l'ordre de présentation. Chez $\mathrm{Mt}$, la demande du disciple précède l'appel à la suivance de Jésus, alors que Lc procède de manière inverse. Il en résulte une intéressante divergence de sens : chez Mt, nous avons une parénèse - il y va de l'obéissance toujours re nouvelée dans la Nachfolge -, tandis que chez Lc, nous avons une scène d'appel - il y va de la décision pour la Nachfolge - 4 .

Le v. 18 est, pour l'essentiel, une formule de transition permettant de passer des miracles qui précèdent aux deux petites scènes idéales qui suivent ${ }^{5}$. Le verbe $\chi \varepsilon \lambda \varepsilon \cup ́ \omega$ décrit le rapport entre le maître et ses disciples (cf. v. 23) d'une manière toute mt. L'ordre de Jésus est en fait un ordre invitant à la suivance (cf. v. 23) et dont les conditions vont être exposées dans les vv. $19-22^{6}$.

1 BULTMANN, Trad 376.

27 fois chez Mt, jamais chez Mc, 1 fois chez Lc.

3 Ainsi aussi SCHULZ, Q 434.

4 Nous ne saurions dire qui de $M t$ ou de Lc a gardé l'ordre de $Q$ (état de la question: SCHULZ, Q 434-435). Par ailleurs, le thème de la proclamation du Royaume (Lc 9,60c) ressortit à la rédaction lc (HENGEL, Nachfolge 4).

5 BULTMANN, Trad 376.

6 HELD 190. 


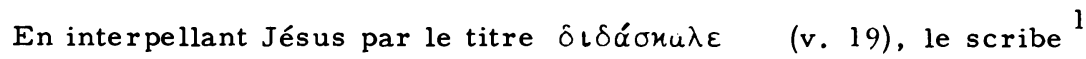
lui reconnaît la dignité d'un maître et, qui plus est, d'un maître qu'il

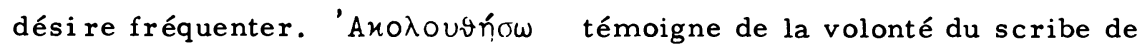

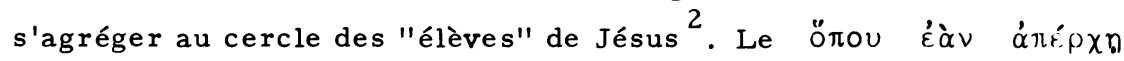

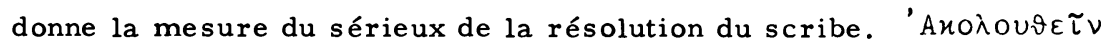
signifie dans ce cas : "suivre concrètement (Jésus) dans son itinérance, partager avec lui son destin menacé et exposé, et seulement dans un sens dérivé, devenir son élève" ${ }^{3}$.

Dans sa réponse (v. 20), Jésus reprend un topos de la sagesse populaire qui oppose l'existence menacée de l'homme à la vie des animaux ${ }^{4}$. Il décrit ainsi la nature de sa destinée qui est celle d'un rejeté, d'un sans-patrie; il veut montrer comment sa destinée de souffrance s'oppose irréductiblement à l'existence humaine normale ${ }^{5}$. Mais en quoi sa vie est-elle vouée à l'insécurité et à l'hostilité ${ }^{6}$ ? Elle ne l'est pas dans un sens naturel, du fait que Jésus n'avait ni maison, ni nourriture assurée. Elle l'est dans un sens historique : son itinérance dramatique de prophète

1 Le texte ne permet pas de décider si le scribe est un adhérent ou un adversaire de Jésus.

2 L'élève doit s'attacher à son maître, le "suivre", car il apprend non seulement par l'enseignement, mais aussi par la communauté de vie (cf. BILL 1, 187).

3 HENGEL, Nachfolge 60.

4 Ce topos est connu de l'ensemble de la sagesse antique. On le trouve aussi bien dans l'AT que chez Homère ou Plutarque (voir BULTMANN, Trad 102; HAHN, Hoheitstitel 44, note 5; SCHWEIZER, Erniedrigung 13, note 33; ZUMSTEIN, Maître et disciple 224).

5 Il est important de remarquer que J ésus applique ce topos à sa seule personne; le topos cesse donc d'être une vérité générale. Mais il l'applique à sa seule existence en tant qu'homme. " 0 vìds tov

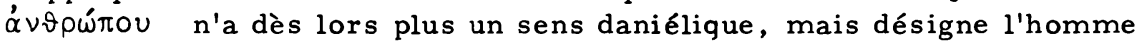

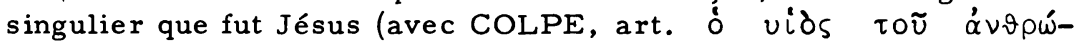
$\pi$ ov, ThW 8,435). Voir aussi HAHN, Hoheitstitel 44-45.

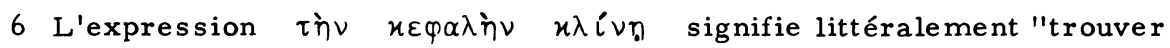
un lieu de repos après une journée de travail". 
charismatique -eschatologique va soulever la haine, provoquer des menaces, susciter le rejet et, finalement, le conduire à la croix.

Jésus veut ainsi montrer à son interlocuteur ce que signifie un par tage de vie avec lui ${ }^{l}$ : en s'engageant aux côtés de Jésus, en le suivant (cf. v. 19), l'homme renonce à toute patrie, à toute sécurité, il s'expose à l'hostilité générale ${ }^{2}$. Dès lors, dans la rédaction $\mathrm{mt}$, cette parole de Jésus prend l'accent d'un avertissement parénétique : elle indique ce qu'il en coûte de suivre l'appel du maître, tout en incitant à le faire.

Le second interlocuteur de Jésus (v. 21) ${ }^{3}$ est un disciple qui l'inter pelle par le titre $\chi u ́ p \iota \varepsilon$. Il s'agit donc d'un adhérent qui "suit" déjà Jésus et qui reconnaît en lui le Seigneur eschatologique. Mis en demeure d'accompagner Jésus (cf. v. 18), mais ayant appris la mort de son père, le disciple demande à son maître la permission d'ensevelir son père avant de le rejoindre. Apparemment, il s'agit d'une demande qui va de soi, car les devoirs funèbres sont, dans le bas-judaísme, une obligation absolue fondée sur la Tora ${ }^{4}$. Quelle est alors la valeur exacte du $\pi \rho \tilde{\omega}-$ tov? A -t-il un sens purement temporel ${ }^{5}$ ou implique -t-il une hiérarchie de valeurs ${ }^{6}$ ? L'un n'exclut pas l'autre. Le disciple désire d'abord enterrer son père, puis ensuite rejoindre Jésus (sens temporel). Mais cela implique que les devoirs filiaux précèdent à ses yeux la Nachfolge (sens axiologique).

1 Ainsi SCHLATTER, Mt 287.

2 SCHWEIZER, Erniedrigung 14.

3 Pour les vv. 21-22, on consultera en priorité l'ouvrage de HENGEL, Nachfolge, qui est spécialement consacré à ces 2 vv.

4 Les devoirs funèbres priment toutes les autres obligations religieuses. Ils dispensent de la récitation du Schema, de l'étude de la Tora; ils suspendent les règles de la pureté sacerdotale et le naziréat. Voir le matériel rassemblé dans notre travail Maître et disciple 225, mais aussi : BILL 1, 487-489; 4,578-579; HENGEL, Nachfolge 9-11; SCHULZ, Nachfolgen 106 , note 9 .

5 HENGEL, Nachfolge 7.

6 SCHULZ, Nachfolgen 107. 
La première partie de la réponse de Jésus (v. 22a) est une invitation à la Nachfolge. Fait intéressant, elle s'adresse à un croyant qui est déjà en situation de Nachfolge. Ce qui signifie : cet ordre cherche à vaincre les obstacles qui s'opposent à une adhésion inconditionnelle. Le thème est alors non pas la décision pour la Nachfolge, mais la décision toujours à renouveler de l'obéissance dans la Nachfolge. La fidélité exclusive à Jésus doit être concrétisée toujours à nouveau, elle ne l'est jamais une fois pour toutes ${ }^{1}$. Le verbe $\dot{\alpha} \varkappa 0 \lambda \circ \vartheta \tau_{\nu}$ a ici une nuance particulière; il signifie "rester fidèle à sa condition de disciple". De ce fait, il vise le problème de l'existence chrétienne. Dans cet épisode, Mt parle en vérité à sa communauté ${ }^{2}$.

La deuxième partie de la réponse de Jésus (v. 22b) tient dans le fa meux "laisse les morts enterrer leurs morts" ${ }^{3}$. Ce logion est impensable dans le milieu juif tardif. Mais il n'est pas non plus l'expression de la foi chrétienne primitive. Il appartient donc à la couche la plus ancienne de la tradition, probablement au Jésus historique ${ }^{4}$. Comment faut-il le comprendre?

Le problème exégétique principal consiste à savoir qui est désigné par le premier vexpós. Pour les uns ${ }^{5}$, il s'agit de l'homme mort au sens spirituel; Jésus ordonne alors à son disciple de laisser aux hommes qui n'ont pas été atteints par l'évangile ou qui l'ont refusé le soin d'ense velir les morts. Pour les autres ${ }^{6}$, le premier vexpós est identique au second et désigne l'homme en tant que cadavre; Jésus veut donc dire

1 Avec GRUNDMANN, Mt 259, et HELD 191-192.

2 Avec GRUNDMANN, Mt. ibid., et HELD ibid., mais aussi BULTMANN, Trad 28.

3 Pour l'histoire de l'int erprétation de ce logion, voir KLEMM 60-75.

4 Cf. HENGEL, Nachfolge 7-8, et KLEMM 74.

5 Ainsi BONNARD, Mt 119; BULTMANN, art. venoós , ThW 4,896898; HENGEL, Nachfolge 8; SCHLATTER, Mt 289.

6 KLEMM 73; SCHWEIZER, Erniedrigung 14, et Mt 142-143. 
au disciple : "Cette affaire doit prendre soin d'elle-même, tu as un travail plus important à faire " 1 . Que l'on opte pour la première ou la seconde interprétation, dans les deux cas, Jésus exige une rupture ${ }^{2}$ avec la religion et les coutumes de son milieu au nom d'une urgence plus grande. Cette urgence qui prime les devoirs familiaux les plus sacrés, est l'imminence du Royaume que Jésus, tout à la fois, annonce et inscrit dans les faits. Mais comment l'homme participerait-il à ce Royaume si ce n'est en suivant Jésus ? L'appel à la Nachfolge (v. 22a) est donc lié à une exigence (v. 22b) incommensurable et inconditionnelle, qui relati vise, voire exclut toute autre relation, tout autre devoir, tout autre égard ${ }^{3}$. L'obéissance à Jésus n'admet ni la moindre hésitation, ni la moindre exception. Cette adhésion inconditionnelle que Jésus exige du disciple apparaît de manière incomparable en Mt 10,37-39.

\section{c) $\mathrm{Mt} 10,37-39$}

Les vv. 37-39 se composent de trois logia puisés dans la source $Q$ et fort connus de la tradition synoptique. La rédaction $\mathrm{mt}$ intèg re ce matériel dans une perspective plus large qui se fait jour dès 10,17. Dans la section 10,17-42, Mt expose la condition chrétienne comme telle et, notamment, les exigences qui l'accompagnent. En conséquence, 10,37-39 deviennent une instruction de Jésus qui a valeur de règle communautaire.

\section{MANSON cité par KLEMM 73.}

2 La rupture que Jésus exige ici est d'une gravité exceptionnelle. Ainsi SCHLATTER (Mt 288) écrit : "Dass an dieser Stelle die Pietät ver leugnet und die Sohnespflicht gebrochen werden könnte, war für das jüdische Empfinden völlig undenkbar, ein schlechthin schändender Frevel". HENGEL (Nachfolge 16) ajoute: "Es gibt kaum ein Jesus Logion, das in schärferer Weise gegen Gesetz, Frömmigkeit und Sitte in einem verstösst als Mt 8,22 = Lc 9,60a...".

3 SCHLATTER (Mt 288) commente le passage en ces termes : "Gerade deshalb ist dieser Vorgang ausgewählt und in das Christusbild hineingestellt, weil er feststellt, dass Jesus jede den Jünger bindende Sitte sprengt, keine andere Verpflichtung zulässt und eine Hingabe von ihm verlangt, die den Jünger vollständig und allein an Jesus bindet". 
Ce point de vue est confirmé par le choix de l'auditoire. Dans le doublet de notre péricope, en Mt 16,24-25, le Christ $\mathrm{mt}$, à la différence des deux autres synoptiques, s'adresse aux seuls disciples (cf. v. 24). Il n'en va pas autrement du chap. 10 qui est adressé aux seuls douze, étant entendu que les douze ont une valeur paradigmatique comme en témoigne le ò / ós par lequel débute chacun des vv. 37-39 ${ }^{\mathrm{l}}$. Mt veut ainsi faire comprendre que c'est de la condition de tout croyant qu'il s'agit ici.

Comme en témoigne le par. Lc 14,26, la source de Mt 10,37 est $Q^{2}$. On admet généralement que Lc a conservé une version plus archaíque du $\operatorname{logion}{ }^{3}$. D'une part le oú $\mu \iota \sigma \varepsilon \tau$ lc est plus ancien que le نं $\varepsilon \rho \quad m t, \operatorname{car} \varphi \iota \lambda \varepsilon \tau \nu$ semble un adoucissement par rapport au oú $\mu \iota \sigma \varepsilon \tau$ et le únép renforce la relation explicite avec le Christ ${ }^{4}$.

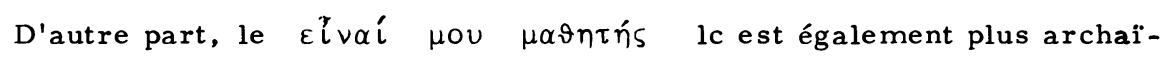

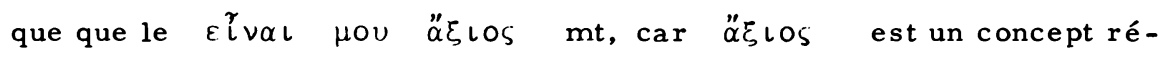
dactionnel $\mathrm{mt}$ qui apparait sept fois dans le seul chap. 10. La tradition synoptique a attaché une importance certaine au logion que nous trouvons au v. 38, car elle ne le cite pas moins de cinq fois (Mc 8,34, Mt 16,24, Lc 9,23 - source Mc -; Mt 10,38, Lc 14,27 - source Q -). Mt l'utilise deux fois, la première en s'inspirant de $Q$ (Mt 10,38), la seconde sur la base de Mc $($ Mt 16,24$)$. L'opinion qui prévaut ${ }^{5}$ est que la version de $Q$

1 Le ò / ős qui structure les vv. $37-39$, désigne évidemment tout homme interpellé par le Christ, sans limitation à la génération des douze.

2 Nous retrouvons un motif parent, mais non identique en Mc 10, 29-30 (par. Mt 19,29 et Lc 18,29-30). Ce passage traite en effet de la récompense de la Nachfolge et non de ses exigences comme en 10,37.

3 Voir BULTMANN, Trad 172-173; A. SCHULZ, Nachfolgen 80-81; S. SCHULZ, Q 446-447; SCHWEIZER, Erniedrigung 16.

4 L'explicitation croissante de la relation avec le Christ, dans la tradition synoptique, est un trait de la réflexion chrétienne post-pascale.

5 BRAUN, Radikalismus II 104, note 4; BULTMANN, Trad 173; SCHNEI-

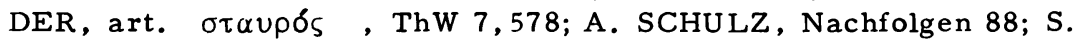
SCHULZ, Q 430-431; SCHWEIZER, Erniedrigung 15. 
est plus ancienne que celle de Mc parce qu'elle présente une formulation négative et plus brève. Enfin la forme que $Q$ revêt chez Lc, est plus ar -

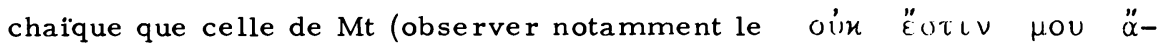
EloS rédactionnel de $\mathrm{Mt})^{\mathrm{l}}{ }^{\mathrm{l}}$. Comme le précédent, le logion du v. 39 est richement attesté (Mt 10,39, Lc 17,33 - source Q -; Mc 8,35, Mt 16,25, Lc 14,23 - source Mc -). La source $Q$ semble, ici aussi, plus a rchaïque parce que plus sobre, mais il est difficile de déterminer qui de $\mathrm{Mt}$ ou de Lc a le mieux conservé la version primitive de cette parole. La terminologie mt paraît moins retouchée que celle de Lc, encore que le $\ddot{\varepsilon} \nu \varepsilon x \varepsilon v$ Ép.ou soit une adjonction secondaire ${ }^{2}$.

Dans le temps de crise instauré par Jésus (vv. 34-36), il convient de prendre des décisions claires à son égard. Aussi le v. 37 dénonce-t-il les attachements familiaux dans la mesure où ces derniers empêchent une adhésion sans réserve au Christ ${ }^{3}$.

Le ò qui introduit aussi bien $37 \mathrm{a}$ que $37 \mathrm{~b}$, est indéfini; il désigne le croyant comme tel, sans limitation temporelle; à ce titre, il a un sens paradigmatique. Le verbe $\varphi \iota \lambda \varepsilon \tau \nu$ qui qualifie le $\dot{o}$, est rare chez Mt qui lui donne généralement un sens péjoratif : "s'attacher à", "s'af fectionner pour", "mettre son plaisir en "4". La préposition útrép

1 SCHULZ, Q ibid., ne se rallie qu'avec réserve à cette opinion. Pour

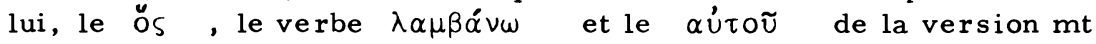
sont plus primitifs que leurs équivalents lc.

2 Démonstration par le biais de la statistique terminologique chez SCHULZ, Q 444-445. Pourtant cet auteur (au contraire de BULTMANN, Trad 97. 162 , p.ex.) pense que le Ĕ. supprimé par Lc.

3 Du point de vue de l'histoire comparée des religions, le logion rapporté par Mt contient un topos fort connu dans le monde antique : le sacrifice obligé de la famille pour servir des valeurs plus grandes. Dans le bas judaís me rabbinique, l'obéissance due à Dieu, à la Tora, au rabbi, surpasse celle rendue aux parents (BILL 1, 587). Le membre de la secte de Qumrân doit abandonner sa famille (voir p.ex. 4 Q Test 16 ss, 1 QS 6,2-3.19-20; l QH 4,8-9). Dans le stoïcisme, la quête du bien est préférable à tout lien de parenté (cf. p. ex. EPICTETE, Entretiens III, 3, 5-6).

4 BONNARD, Mt 156. 


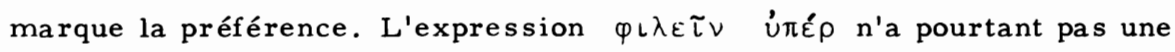
connotation affective : Jésus ne revendique pas une affection supérieure à celle que le disciple témoignerait à sa famille. L'accent est mis sur le comportement concret, sur "l'engagement". Est critiqué celui qui, en situation de crise nécessitant une décision, préférerait rester avec sa famille plutôt que suivre Jésus.

Le croyant qui opte pour sa famille plutôt que pour Jésus, n'est pas digne de Jésus ( $\stackrel{\not}{\alpha} \xi$ los ). La dignité dont il est parlé ici, n'est ni préalable à l'appel de Jésus, ni conférée par une initiation religieuse. Est digne du Christ celui qui accepte de le suivre et justement parce qu'il accepte ${ }^{l}$. Est indigne du Christ celui qui préfère ses liens familiaux et précisément parce qu'il les préfère à Jésus. La dignité ou l'indignité du disciple dépendent de la relation qu'il entretient avec le maître. La pointe du logion est dès lors la suivante : le Christ mt revendique son disciple d'une manière si totale et si exclusive qu'en situation de crise, ce dernier doit être résolu à abandonner ses affections naturelles pour suivre son maftre; les liens familiaux deviennent des obstacles à la foi s'ils limitent la fidélité.

Le v. 39, comme le précédent, s'applique au croyant comme tel sans restriction d'aucune sorte ( $\dot{o}$ paradigmatique). Il critique le disciple qui ne "se charge pas de sa croix". Nous n'avons pas à évoquer ici la discussion nourrie qui s'est engagée à la fois sur l'origine du logion et sur son sens primitif ${ }^{2}$. Dans la rédaction $m t, l^{\prime}$ expression $\lambda \alpha \mu \beta \alpha^{\prime}-$ $\nu \varepsilon \iota \nu \tau \delta \nu \sigma \tau \alpha u \rho \delta \delta$ es $\mathrm{t}$ indiscutablement mise en relation avec la passion du Christ ${ }^{3}$. Mais dans quel sens la passion du Christ intervient-elle dans

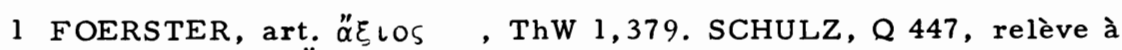
juste titre que ä́los est une catégorie de la vie chrétienne.

2 Etat de la question, SCHULZ, Q 431-433; voir aussi notre travail Maître et disciple 234-235.

3 10,21-25 établit l'identité de condition entre le maitre et le disciple; 16,24 suit immédiatement la pre mière annonce de la passion. 
notre contexte ? Dans son commentaire, P. Bonnard ${ }^{1}$ rappelle avec pertinence que "le crucifiement, supplice atroce et ignominieux, avait pour caractère principal de livrer le condamné à la furie et à la risée des foules, pour faire exemple". S'il en est ainsi, l'expression $\lambda \alpha \mu \beta \alpha ́$ $\nu \varepsilon \iota \nu$

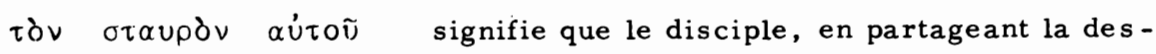
tinée de son maître et en se soumettant publiquement à sa seule autorité, encourt la même violence criminelle et la même dérision que son Seigneur ${ }^{2}$. Le disciple qui ne "se charge pas de sa croix" est, au contraire, celui qui, tout en prétendant se ranger aux côtés de Jésus, refuse d'accepter la solitude et la souffrance inhérente à sa condition.

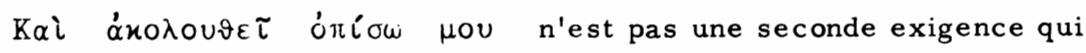

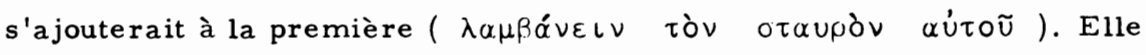
l'explique. C'est parce que le disciple suit Jésus, partage son itinérance dramatique, qu'il est exposé à la souffrance et à la moquerie. A l'inverse, la souffrance qui l'accable et la moquerie qu'il subit, n'ont de sens que parce qu'il suit Jésus. La Nachfolge est une Nachfolge dans la souffrance, souffrance qui peut culminer dans le martyre.

Celui qui n'accepte pas que la Nachfolge soit une Nachfolge dans la souffrance, n'est pas digne de Jésus parce qu'il préfère sa propre sécurité à Jésus (pour l'analyse de $\alpha^{\prime} \xi$ los, cf. v. précédent). L'adhésion à Jésus n'exige donc pas seulement une décision claire qui peut provoquer la rupture des liens familiaux (v. 37), elle implique également une des tinée douloureuse (v. 38). Mais cette double exigence est légitime, car, en s'y soumettant, l'homme gagne son salut; c'est l'objet du v. 39.

1 BONNARD, Mt 357.

2 Dans son commentaire sur Mc (175), GRUNDMANN rappelle cette réflexion de PERCY à propos de notre logion : "Und was diese Wanderung so bitter macht, ist... das Gefül, aus der Gesellschaft aus gestossen zu sein, zusammen mit dem Erlebnis, dass man Gegenstand des Spottes und der Verachtung der Menge ist". SCHULZ, Q 433, voit la pointe de notre logion chez Mt dans "die Bereitschaft zum Leiden bis ins Kreuzesmartyrium". 
Le v. 39 se présente comme une sentence paradoxale, en deux membres parallèles. On remarquera que $Q$ (cf. Mt 10,38-39) et Mc (cf. Mc $8,34-35)$ ont placé tous deux cette sentence immédiatement après le logion invitant le disciple à porter sa croix. Il est dès lors possible que dans la tradition déjà, le v. 39 était le commentaire du v. $38^{\mathrm{l}}$. Le propos de la sentence est de montrer comment l'homme gagne ou perd sa $\dot{\psi} U-$ $\chi \mathfrak{n}^{2}$. La $\psi u \chi \eta^{\prime}$ désigne la personne concrète, le "moi" dans la mesure où l'âme est la porteuse de la vie et la manifestation du "moi" ${ }^{3}$. Il faut ajouter que, dans notre v., il est question de la personne concrète dans sa vie actuelle aussi bien que dans sa vie à venir ${ }^{4}$. Plus précisément, la manière dont la personne vit sa vie actuelle, engage sa destinée future ${ }^{5}$.

Celui qui "trouve" sa vie est, par analogie au v. précédent, celui qui refuse de "porter sa croix". Il s'agit donc de l'homme qui n'accepte pas la destinée de souffrance et de dérision inhérente à la Nachfolge, qui ne renonce pas à sa volonté d'indépendance et de sécurité, qui entend être le maître de sa vie et en disposer selon ses intérêts ${ }^{6}$. Cet homme qui croit

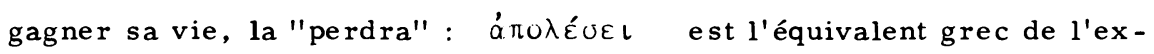
pression sémitique אבד נפס et signifie "perdre sa vie par sa propre faute ${ }^{7}$ ". Il encourra le rejet eschatologique à cause de sa culpabilité ac-

1 Cf. SCHWEIZER, Erniedrigung 16. Ce groupement des vv. 38-39 est de la plus haute importance, car il nous indique comment la tradition a interprété le v. 38 .

2 Ce motif est également bien connu du bas-judaissme rabbinique : voir BILL 1, $587-588$. 774 .

3 OEPKE, art. $\dot{\alpha} \pi \delta \lambda \lambda \cup \mu \iota$, ThW 1,394.

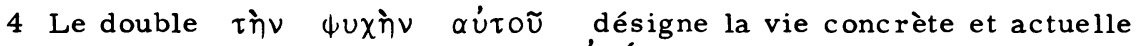

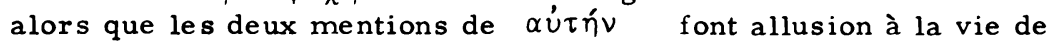
l'homme placée sous la sanction eschatologique.

5 BONNARD, Mt 251.

6 Le verbe Eúpíonw a ici le sens de "se procurer", "disposer" (cf. PREISKER, art. Eúpíonw , ThW 2,767). BONNARD, Mt 155, va dans le même sens et traduit par "posséder".

7 OEPKE, art. $\alpha \dot{\alpha} \delta \lambda \nu u \mu$, ThW 1, 393-394. 
tive. Il sera jugé pour s'être désolidarisé du Christ souffrant et pour avoir construit sa vie selon des sécurités apparentes.

Le v. 39b présente l'attitude inverse. Celui qui "perd sa vie" est celui qui l'abandonne, qui renonce à lui conférer la moindre valeur ou la mettre en sécurité, au risque même de la mort ${ }^{l}$. Cet abandon et cette renonciation $n^{\prime}$ ont pas de valeur en soi, ils sont assumés au nom de Jésus ( consistent dans rien d'autre que dans la Nachfolge décrite au v. précédent $^{2}$ (cf. aussi $8,19-22 ; 16,24$ ). Celui qui décide résolument de suivre le Christ, quelles qu'en soient les conséquences immédiates, celui-là "trouvera la vie". Le verbe Eúpíoxw a alors, un sens eschatologique prononcé et signifie "recevoir sa vie de Dieu" ${ }^{3}$, càd participer au Royaume eschatologique.

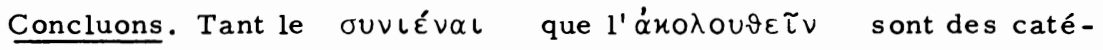
gories théologiques qui décrivent l'existence chrétienne sur le mode de la relation qui se noue entre le croyant et le Jésus terrestre. Ces deux thèmes exposent de manière conséquente - et au niveau anthropologique la perspective spécifique de la christologie mt, qui insiste sur la place

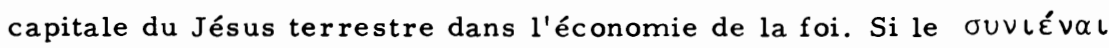
montre que la condition chrétienne s'édifie dans l'écoute fidèle et compréhensive de l'enseignement du didascale de naguère, que toute foi se cons titue à travers la Parole, 13,10-17 laisse déjà entrevoir que cette compréhension de la $\delta \iota \delta \alpha \chi n^{\prime} \quad$ n'est pas un simple acte noétique, mais un don reçu dans la foi qui procure le bonheur et engage la destinée ultime

1 OEPKE, art. cit. ibid.

2 BONNARD, Mt 158, interprète l'expression "perdre sa vie" comme signifiant "mourir de mort violente". Nous pensons que cette inter prétation est trop restrictive, car si perdre sa vie, c'est partager la des tinée souffrante du Christ (cf. 10,38), le martyre est certes une possibilité réelle, mais non inéluctable.

3 PREISKER, art. Eúpíonw, ThW 2,767. 
de l'homme. Le modèle de la suivance révèle en toute clarté que l'adhésion à l'enseignement du Jésus terrestre est inséparable d'une transformation existentielle radicale. L'appel libérateur du Christ arrache le disciple à tout ce qui faisait sa vie jusque-là. La rupture qui intervient est d'une radicalité absolue : toutes les relations qui formaient la vie du croyant - qu'elles soient d'ordre familial, professionnel, social, culturel, religieux - sont réajustées en fonction de l'adhésion à la personne de Jésus. La fidélité exigée ne souffre pas la moindre restriction et doit être concrétisée toujours à nouveau. Elle signifie un partage de destinée avec le Christ, qui peut aller jusqu'à la mort. Mais cette insécurité et cette souffrance, constitutives de l'existence croyante, ont leur justification, car, en rencontrant le Jésus terrestre et en le suivant, le disciple trouve la vie. Insistons sur ce paradoxe inlassablement souligné par $\mathrm{Mt}$ : c'est dans ce compagnonnage exclusif et aventureux, dépourvu d'apparence, exposé à la dérision et à la haine des hommes, que se trouve la vie offer te en plénitude par Dieu à chacun. 


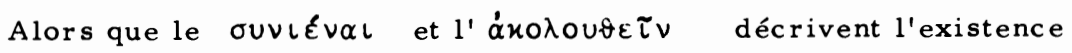
chrétienne en tant que relation entre le Jésus terrestre et le croyant, la notion $m$ de foi exprime la réalité présente de cette relation. Le croyant, en s'abandonnant avec confiance à son Maître, peut recevoir son aidé secourable et libératrice pour affronter les vicissitudes du temps vécu. Nous développerons cette thématique tout d'abord en examinant la compréhension mt de la $\pi i ́ \sigma \iota \varsigma$, notamment dans les récits de miracles. Puis, dans un deuxième temps, nous nous arrềterons plus longuement sur son contre-modèle : l' ó $\lambda \iota \gamma \circ \pi \iota \sigma \tau i \alpha . C^{\prime}$ est, en effet, une particularité du premier évangile que d'avoir longuement réfléchi sur la crise de la foi au sein même de la vie chrétienne. Le récit de la tempête apaisée ( $8,23-27)$, et celui de Jésus et de Pierre marchant sur les eaux $(14,22-33)$ en sont la démonstration.

\section{La foi}

\section{a) Bref état de la question :}

En continuité avec la tradition chrétienne - et notamment celle du christianisme hellénistique -, Mt peut entendre par foi l'existence chrétienne comme telle $(18,6 ; 27,42)$. Pourtant, l'aspect particulier qu'il retient et sur lequel il insiste est la confiance. La foi est confiance dans le Père qui veille sur ses créatures $(6,30)$, qui exauce leurs prières $(17,20 ; 21,20-22)$; elle est confiance dans $1^{\prime} \dot{\varepsilon} \xi$ ouoí $\alpha$ de Jésus $(8,10.13$; 9, 2.22.28-29). En tant que confiance, la foi comporte un accent volontariste : les verbes $\pi \iota 0 \tau \varepsilon \cup ́ \varepsilon \iota \nu$ et $\vartheta \varepsilon \hat{\varepsilon} \varepsilon \iota \nu$ peuvent devenir synony$\operatorname{mes}\left(15,28\right.$; cf. aussi 8,$\left.13 ; 9,29^{1}\right)$.

1 BARTH 107. 
Aussi n'est-il pas étonnant que cette confiance, inséparable de la volonté, devienne parfois obéissance ${ }^{1}$ : en $8,5-13 ; 21,23$, la foi se définit alors comme l'attitude de vie qui engage la destinée eschatologique de l'homme, comme la fidélité à la volonté de Dieu.

Ainsi que Barth ${ }^{2}$ le souligne à juste titre, ce bref inventaire fait apparaître que la foi, simultanément comme confiance, volonté, obéis sance, s'inscrit de manière irrécusable dans l'héritage vétérotestamentaire où la foi est la fidélité de l'Alliance, à la Tora et à la promesse. On le remarque d'emblée, pour Mt, la foi n'est pas une conviction théorique, ni seulement l'acceptation de la prédication missionnaire. Délestée de sa dimension noétique qui est développée dans la catégorie du ouvléval ${ }^{3}$, la foi décrit l'abandon confiant et résolu de l'homme à Jésus. Cet aspect est développé de manière cohérente dans les récits de miracles.

b) La foi dans les récits de miracles: ${ }^{4}$

Un bref survol de la tradition synoptique permet de constater que

1 BORNKAMM, Enderwartung 25-26, voit dans cette mise en relation de la foi avec l'attente du Jugement et la Loi, la spécificité de la réinterprétation $\mathrm{mt}$.

2 Gesetzesverständnis 108. 152.

3 BARTH 105-108 distingue avec raison le ouvléval de la $\pi i ́ \sigma \tau \iota \varsigma$. Il montre que, chez Mt, le ouvıÉval recouvre le moment noétique de la vie du croyant alors que la $\pi i ́ \sigma \tau \iota s$ désigne plutôt la confiance du disciple, sa volonté d' sbéir à Dieu. Plus discutable est la relation qu'il établit entre la foi et la compréhension lorsqu'il sc'ıtient que le

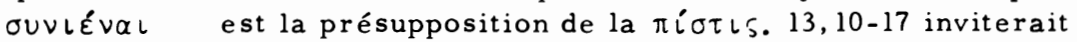
plutôt à comprendre cette relation sur le mode de la présupposition réciproque. Pourtant, au niveau de l'évangile dans son ensemble, il semble bien que Mt n'ait pas réfléchi ce problème de manière systématique et qu'il traite ces deux thèmes de manière séparée (avec LUZ, Jünger 150-151).

4 Pour l'essentiel, nous suivons l'analyse de HELD 264-276. 
pour l'essentiel, la problématique de la foi est abordée dans les récits de miracles 1 . Mt n'échappe pas à cette règle; sur ce point, il reprend l'héritage de ses prédécesseurs ( $M c$ et $Q$ ) en le profilant. Quelle est la conception de la foi qui apparaît dans les récits de miracles de la tradition synoptique?

La foi n'est pas suscitée par le miracle, elle le précède. Sans foi, il ne saurait y avoir de miracle ${ }^{2}$. La foi du demandeur est le préalable à tout acte miraculeux. Quelle est alors la nature de cette foi ? La plupart des auteurs y voient la confiance du demandeur dans le pouvoir miraculeux du Christ ${ }^{3}$. Sans être fausse, cette définition est imprécise. Comme le relève à juste titre Held ${ }^{4}$, la foi se présente fondamentalement comme une activité de la personne : des hommes et des femmes mettent tout en oeuvre pour rencontrer Jésus et pour obtenir son secours. L'exemple le plus frappant de cette "foi active" est donné dans l'histoire de la guérison du paralysé (Mc 2,1-12) : les amis de l'infirme ne s'épargnent aucune peine pour l'amener à Jésus (v. 4!) et ce dernier voit dans leurs efforts l'expression de la foi (v. 5: hai isìv ó 'Inooũs tìv $\pi i \sigma \tau ı v \quad \alpha \dot{u} \tau \tilde{\omega} \nu \quad{ }^{5}$ ).

La confiance témoignée par le demandeur à Jésus se traduit non seulement par une activité, elle suppose également une volonté sans faille. Outre l'histoire de la guérison du paralysé (Mc 2,1-12), de la femme

1 HELD 263, et LUZ, Jünger 150.

2 Cf. Mc 6,5.6; 8, 11-12.

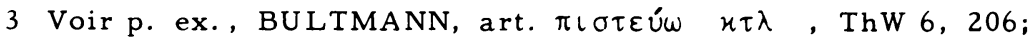
DBELIUS, Formgeschichte 75; KLOSTERMANN, Mt 75; LOHMEYER, Mc 103.

4 Wundergeschichten 266-267.

5 Voir aussi l'histoire de la femme souffrant d'hémorragies (Mc 5, 25-34, en particulier les vv. 27.28.34) et la guérison de l'aveugle Bartimée (Mc 10, 46-52, en particulier les vv. 47.48.50). 
victime d'hémorragies (Mc 5, 25-34), des deux aveugles (Mt 9, 27-31), l'exemple de la Cananéenne (Mt 15, 21-38) est particulièrement suggestif ${ }^{1}$.

Cette conception volontariste de la foi est vérifiée par la constatation terminologique suivante : la tradition fait alterner sans difficulté les

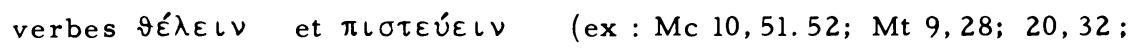
Mt 8,$13 ; 15,28)$.

Le troisième point qui mérite d'être souligné dans cette description

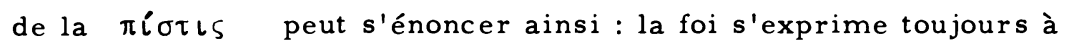
travers une demande ${ }^{2}$ - soit expresse, soit implicite - . Valable pour tous les récits de guérisons, cette constatation vaut également pour une grande partie des miracles opérés sur la nature ${ }^{3}$. Aussi est-ce à juste titre que Bultmann peut caractériser cette $\pi i \sigma \tau \iota \varsigma$, propre aux récits de miracles, comme une foi-prière (Gebetsglaube) ${ }^{4}$. Ce dernier élément est capital, car il permet de définir la relation qui existe entre la foi et le miracle. La foi est fondamentalement une prière adressée à Jésus, de même que le miracle est la réponse que Jésus donne à cette prière. Comme le formule avec bonheur Held :"Der Glaube verhält sich zu dem Wunder wie die Bitte zu ihrer Erhöhung " 5 .

1 Voir aussi la résurrection de la fille d'un notable (Mt 9, 18-26), la guérison de l'enfant épileptique (Mc 9,14-29 par. Mt 17,14-20) : même la mort (Mt 9,18), même la gravité de la maladie (Mt 17,15), même l'incrédulité personnelle $\left(M_{c} 9,24\right)$ ne viennent pas à bout de la volonté du demandeur.

2 Le miracle se présente constamment comme l'exaucement d'une demande : voir $\mathrm{Mc} 2,5$ et 2,3.4; 5,34 et 5,25-28; 5, 36 et 5,22-23; 10,52 et $10,47-48 ; 4,40$ et 4,$38 ; 7,23-24$ et $9,17-18.22 \mathrm{~b} ; \mathrm{Mt} 8,13$ et 8,6-9 et 9,27-28; 15, 28 et 15, 22-27 (inventaire établi par HELD 268, note 2).

3 Cf. Mc 4, 38; 6, 36; 14, 28.

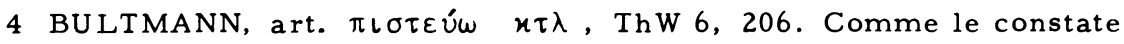
justement HELD 269, cette définition de la $\pi i \sigma \tau \iota s$ comme foi prière signifie notamment que dans les récits où une demande est adressée à Jésus, mais où la notion de foi n'apparaît pas explicitement l'appel à l'aide est l'expression de la foi (ex: Mt 8, 2; 20, 29-34; Mc 7, 26-28).

5 Wundergeschichten 269. 
Cette structure théologique rappelle la piété des Ps (ex. Ps 107) où l'orant invoque sans relâche Yahvé pour obtenir secours et délivrance. Pourtant,dans les récits de miracles, un fait nouveau surgit : cette foi orante ne connaît ni l'attente, ni l'espérance; le Christ de la tradition synoptique ne confond pas celui qui l'invoque, il l'exauce - attestant ainsi la proximité du Royaume (cf. Mt 11,4-6 !). Mt a non seulement repris

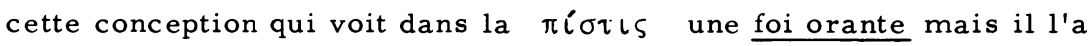
accentuée. Cette hypothèse est étayée par trois séries d'arguments. $\mathrm{D}^{\prime}$ une part, on sait que $\mathrm{Mt}$ a donné une place prépondérante au dialogue dans sa recension des récits de miracles ${ }^{1}$. Cette particularité lui permet à chaque fois de présenter la foi sous la forme d'une demande explicite, mais aussi d'établir une correspondance entre la prière adressée à Jésus et la réponse qu'il donne. D'autre part, il convient de rappeler que le rédacteur du premier évangile ne formule pas la réponse de Jésus dans les termes d'une parole souveraine et toute-puissante, destinée à opérer le miracle ${ }^{2}$; elle tient en général dans une parole sur la foi de l'homme qui l'invoque ${ }^{3}$. A cet égard, le meilleur exemple est fourni par l'histoire du centurion de Capernaüm. A la demande de l'officier (v. 8 : '́a $\lambda \lambda \grave{\alpha} \mu \delta-$

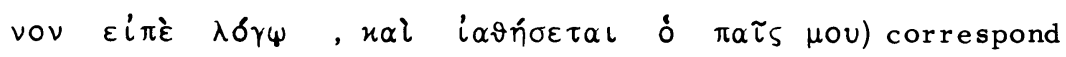

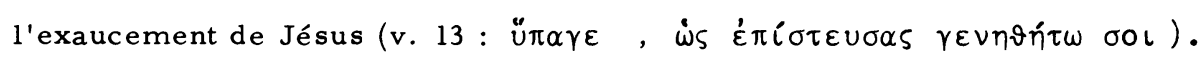
Enfin, la parole de Jésus sur la foi de l'orant est non seulement le point

1 HELD 229 a montré que la recension mt des récits de miracles est élaborée à partir d'un schéma comportant quatre points : a) une introduction formelle présentant le demandeur et évoquant un geste de prière; b) la demande proprement dite à travers laquelle s'exprime la foi, et qui peut donner lieu à un dialogue; c) la réponse de Jésus en parfaite correspondance avec la demande (soit sous la forme d'une parole, soit par un geste, soit par les deux); d) courte notice constatant l'effectuation du miracle.

2 Voir dans ce sens les suppressions par Mt de Mc 5, 41; 9, 25.

3 Cf. Mt 8, 13; 9, 22. 29; 15, 28. 
culminant de la péricope, mais l'expression adéquate de son contenu théologique ${ }^{1}$. A ce propos, il est bon de remarquer que Mt a donné à cette parole une forme nouvelle et originale : $\dot{\omega} s \quad \dot{\varepsilon} \operatorname{l} \sigma \tau \varepsilon \cup \sigma \alpha \varsigma$ $\gamma \varepsilon-$

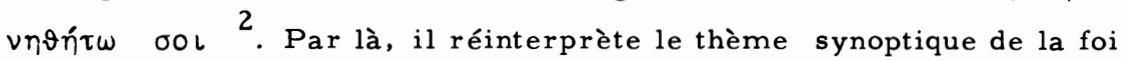
qui sauve ${ }^{3}$ par celui de la foi qui prie. Il confère ainsi une clarté nouvelle à ce que la tradition affirmait déjà : la relation entre la foi et le miracle est analogue à celle qui existe entre la prière et son exaucement.

Quel est alors l'intérêt théologique de cette description de la $\pi i ́ c-$ $\tau \iota \varsigma$ ? La présentation $\mathrm{mt}$ autorise la répétition des récits de miracles à l'intention de l'Eglise et trahit ainsi un souci à la fois catéchétique et parénétique ${ }^{4}$. En appliquant de manière conséquente la catégorie de la foi orante comme principe d'interprétation des récits de miracles, Mt veut montrer que la relation qui s'est nouée naguère entre le Jésus terrestre et des hommes venus l'implorer, reste une possibilité actuelle pour sa communauté. De même que le Christ $\mathrm{mt}$ a jadis exaucé les prières qui lui étaient adressées, il répond aujourd'hui au croyant qui l'invoque dans la foi. Les récits deviennent ainsi pour le croyant, engagé dans le temps de l'Eglise, promesse et exhortation. Le Kyrios ne confond, ni n'abandonne celui qui s'adresse à lui; il le délivre, le soutient et le console. En définitive, la foi, dans le premier évangile, est le modèle de la relation qui s'institue entre le croyant et son Seigneur durant l'époque post-pascale. Cette relation - subsumée dans la $\pi i \sigma \tau \iota \varsigma$ - se caractérise par sa dimension sotériologique : le croyant est présenté comme un quémandeur qui vit de l'appui actif et gracieux du Christ ${ }^{5}$.

1 Voir HELD 227-228.

2 Cf. Mt 8,13; 9, 29; 15, 28.

3 Mt 9,22= Mc 5, 34= Lc 8,48; Mc 10,52 par. Lc 18,42; Lc 7, 50; 17,9.

4 Cf. HELD 275.

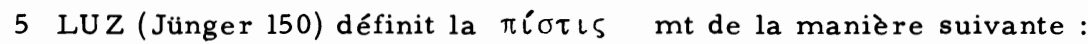
"Glaube ist für Matthäus weitgehend die der göttlichen Heilsmacht entsprechende Haltung". 
2. La crise de la foi

a) $\underline{1^{\prime}} \dot{\partial} \lambda \iota \gamma o \pi \iota \sigma \tau i \alpha \underline{\alpha}$

Pour décrire la crise de la foi qui peut affecter la vie du disciple,

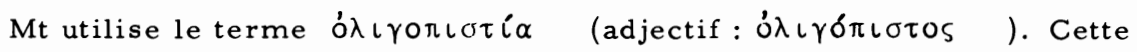
notion provient du bas-judaïsme rabbinique. Elle caractérise des croyants qui appartiennent au peuple de Dieu, qui sont justes et qui ont déjà fait preuve de foi, mais qui, dans une circonstance donnée, manquent de confiance en Dieu et singulièrement en sa bonté. "Rabbi Eliezer l'Ancien (90) disait : celui qui a du pain dans sa corbeille et qui dit : 'Que mangerai-je demain ?', celui-là appartient aux gens de peu de foi ". Le "peu de foi" se définit donc comme un moment d'incrédulité au sein même de l'existence croyante.

Cette signification correspond exactement au sens que $Q$, puis $\mathrm{Mt}$,

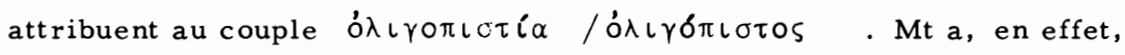
trouvé cette terminologie dans $Q$ (Mt 6, 30 par. Lc 12, 28), puis selon une technique littéraire qui lui est propre, il l'a introduite dans quatre autres passages : en 8,26 pour $\pi \tilde{\omega})$ oú que 14,$31 ; 16,8$ et 17,20 sont des adjonctions rédactionnelles. Mt n'appli-

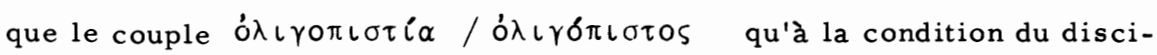

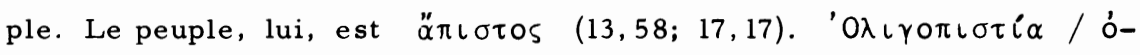

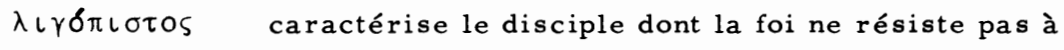
l'épreuve. Le disciple se décourage devant la menace de la tempête $(8,26 ; 14,31)$, il succombe au souci $(6,30 ; 16,8)$, il ne ré siste pas aux

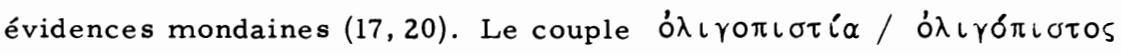
décrit paradoxalement l'incrédulité dans la suivance même de Jésus. L'alternative n'est plus celle de la foi ou de l'incrédulité (ainsi Mc); le problème est celui de la crise de la foi, de l'incrédulité dans la foi. Nous découvrons ici une situation typique de la condition du croyant et singulièrement de l'église mt.

1 BILL 1, 439. 
b) Mt 8,23-27

Le récit de la tempête apaisée se situe dans les deux grands chap. que Mt a consacrés à la narration des miracles de Jésus (8-9). Dans cet ensemble, 8, 18-9,17 forme un cycle particulier qui traite le thème de la relation entre Jésus et les disciples, càd en définitive la question de la Nachfolge ${ }^{1}$. L'analyse du contexte immédiat confirme ce découpage. En effet, notre histoire est immédiatement précédée de deux logia sur la Nachfolge $(8,19-22)$ que seul Mt a introduits à cet endroit ${ }^{2}$. Les modifications opérées par Mt sur sa source Mc vont dans le même sens (voir infra). Aussi Held ${ }^{3}$ peut-il à juste titre écrire : "So stellen nicht mehr Jesus und die Elemente das Thema der Erzählung, sondern Jesus und seine in Not geratenen Jünger". On peut donc supposer que, chez Mt, l'histoire de la tempête apaisée est "le kérygme de la misère et de la grandeur de la Nachfolge" 4 . Nous nous proposons de vérifier cette hypothèse d'interprétation en examinant les éléments caractéristiques de la rédaction $\mathrm{mt}$ dans ce récit de miracle.

Mt a stylisé l'introduction ( $v$. 23) : les disciples n'emmènent plus Jésus, ils le suivent. Cette modification trahit une intention théologique précise. Le verbe $\dot{\alpha} \varkappa \circ \lambda \circ \cup \vartheta \varepsilon \tau \nu$, ici, signifie certes d'abord "suivre" dans un sens immédiat et concret. Mais on se souviendra que les vv. 18-22 - que Mt a introduits dans notre contexte - se composent de deux logia traitant des exigences de la Nachfolge. Ce faisant, ils utilisent

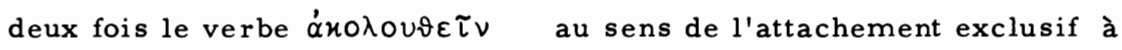

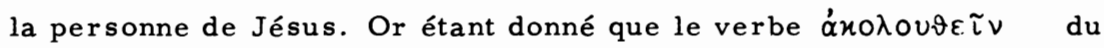
v. 23 est le mot-crochet qui relie notre récit aux vv. 18-22, il a forcé-

1 HELD 236; LEON-DUFOUR, Tempête 165.

2 Voir notre analyse de détail pp. 220-225.

3 Wundergeschichten 192.

4 BORNKAMM, Sturmstillung 53. 
ment aussi le sens imagé ${ }^{1}$. L'événement historique relaté devient une situation typique et le $\mu \alpha \vartheta \eta \tau$ ńs figure le croyant suivant son Seigneur ${ }^{2}$. Pourquoi, pour décrire la tempête (v. 24), Mt a-t-il préféré oદ lchòs

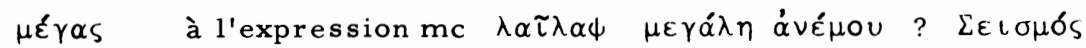
décrit généralement les tourments apocalyptiques ${ }^{3}$ et non pas la tempête au sens météorologique. Il semble bien que $\mathrm{Mt}$ ait choisi ce terme en tenant compte de sa signification apocalyptique et qu'il donne ainsi un sens symbolique à la tempête. Cette dernière est alors l'image des tribulations qu'affronte le disciple 4 .

Remarquons pourtant que le texte parle moins de la détresse des disciples que du danger qui menace le bateau (ce dernier est d'ailleurs vu comme une unité de l'extérieur) ${ }^{5}$.

Cette menace qui plane sur l'embarcation est décrite avec une sobriété remarquable à la différence de Mc. Cette stylisation s'accorde bien avec le fait que, dès les temps les plus anciens, l'Eglise ${ }^{6}$ a vu dans le

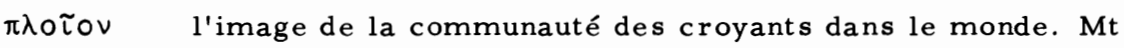
lui-même, en 14,33, n'hésite pas à désigner les disciples comme oi

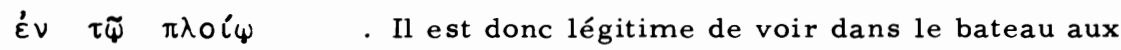
prises avec la tempête le symbole de l'Eglise sous la croix ${ }^{7}$.

1 La terminologie renforce cette interprétation : Jésus est "suivi", au contraire de Mc 4,36, uniquement par ses disciples. Le $\alpha \dot{\tau} \tau \tilde{\psi}$, puis le $\alpha \dot{\tau} \tau o \tilde{u}$ de la proposition principale soulignent ce rapport d'appartenance.

2 BONNARD, Mt 120; BORNKAMM, Sturmstillung 51; GRUNDMANN, Mt 260; HELD 191.

3 Cf. Mc 13,8; Mt 24, 7; 27,54; 28,2; Lc 21, 11; Ap 6, 5.8.12; 11,13.19; 16,8 .

4 BORNKAMM, Sturmstillung 52 et art. $\sigma \varepsilon \iota \sigma \mu \delta \varsigma$, ThW 7, 197-198. GRUNDMANN, Mt 260.

5 LEON-DUFOUR, Tempête 167, et LOHMEYER, Mt 163.

6 Matériel rassemblé par GRUNDMANN, Mt 260.

7 Ainsi BORNKAMM, Sturmstillung 51, et LEON-DUFOUR, Tempête 168. 
Alors même que la communauté des croyants est débordée par l'épreuve, son Seigneur dort (v. 24b). Comment faut-il interpréter ce sommeil ? Il signifie que Jésus n'est pas atteint, voire menacé, par la catastrophe qui frappe ses disciples (figure post-pascale). Il exprime sa confiance, le fait qu'il reste maître de la situation ${ }^{1}$.

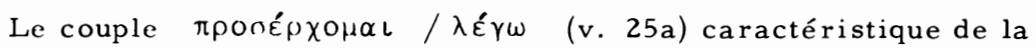
rédaction $\mathrm{mt}$, nous signale que nous entrons en contexte d'instruction ${ }^{2}$. L'appel à l'aide des disciples (v. 25b) qui l'amorce, est formulé dans la terminologie et sous la forme d'une prière. Pour l'introduire, alors que Mc utilise un titre traduisant le respect dû à un maître de type rabbinique

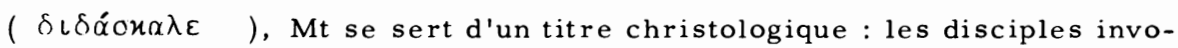
quent le xúpıos. Dans notre évangile, xúpıos n'est appliqué à Jésus que par ceux qui sollicitent son aide en toute confiance ou par les disciples ${ }^{3}$. Il présuppose donc une relation particulière entre Jésus et son interlocuteur, il a valeur de confession. ¿డ̃øov exprime un appel au secours dans un danger aigu où la vie de l'orant est menacée ${ }^{4}$. Il est tout à la fois le cri que les disciples adressent à Jésus au sein de la tempête et l'appel que l'Eglise dans l'épreuve lance à son Seigneur.'A$\pi \circ \lambda \lambda u ́ \mu \varepsilon \vartheta \alpha$ décrit simultanément la détresse concrète des disciples et la misère fondamentale du croyant en butte à la tentation ${ }^{5}$.

En donnant à l'appel des disciples la forme d'une prière, càd en utilisant une terminologie qui s'applique aussi bien à la situation des compagnons historiques de Jésus qu'à celle de sa communauté actuelle,

1 BONNARD, Mt 120; GRUNDMANN, Mt 261; OEPKE, art. $\chi \alpha \vartheta \varepsilon u ́ \delta \omega$, ThW 3, 439.

2 Sur l'utilisation $m$ t de ce couple, voir HELD 214-216.

3 Voir, p. ex., BOR NKAMM, Sturmistillung 51, et LEON-DUFOUR, Tempête 168 .

4 FOERSTER, art. $\sigma \xi \zeta \omega$, ThW 3, 989.

5 GRUNDMANN, Mt 261, et LOHMEYER, Mt 163. 
Mt décrit simultanément la relation entre Jésus et ses adhérents, et la possibilité actuclle pour la communauté ecclésiale de rencontrer son Seigneur ${ }^{l}$.

Chez Mt, à la différence de Mc, la réprimande de Jésus (v. 26a) précède le miracle. Il ne s'agit pas d'une inversion purement formelle, mais d'un moyen d'interprétation théologique utilisé par l'auteur. Du fait de ce renversement de l'économie du récit, ce n'est plus le miracle comme tel qui en constitue le centre, mais la parole de Jésus sur la foi ${ }^{2}$. En modifiant la structure du récit, en déplaçant son centre de gravité, Mt crée une situation typique - le dialogue sur la foi - et cela donne à sa narration une portée kérygmatique immédiate. Le Christ mt ne blâme pas l'incrédulité des disciples une fois la situation rétablie, mais au sein même de l'épreuve et du danger. Et l'église mt vit précisément le problème de l'incrédulité alors qu'elle est sous la croix ${ }^{3}$.

La réprimande de Jésus a la forme d'une question accusatrice. La

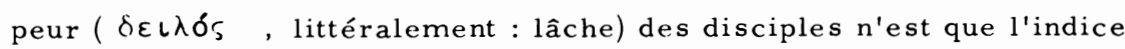

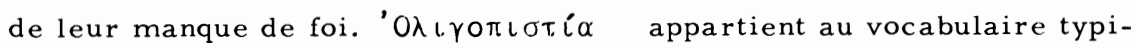
quement mt.

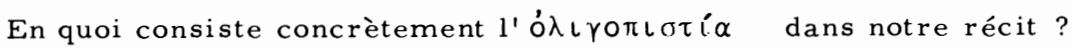
Alors même qu'ils prient ${ }^{4}$, les disciples accordent une importance plus décisive à la menace de la tempête qu'à l'autorité de leur Seigneur. Leur crainte dans l'épreuve est plus forte que leur confiance en Jésus alors que la foi authentique postule précisément la hiérarchie inverse. La question accusatrice de Jésus dénonce donc la compréhension aberrante que

\section{HELD 253.}

2 BONNARD, Mt 120, et LEON-DUFOUR, Tempête 168.

3 BONNARD, Mt ibid.

4 Le fait même que c'est la prière des disciples qui manifeste leur "peu de foi", montre bien que l' ó $\lambda \iota \gamma o \pi \iota \sigma \tau \iota \alpha$ est incrédulité dans la vie de la foi. 
les disciples ont de leur situation ${ }^{1}$ et, par là, la tentation que le croyant encourt dans l'épreuve. Voilà le centre de notre récit.

Après cet appel critique à la foi - et après cet appel seulement - ,

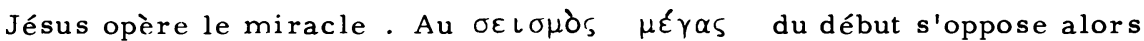

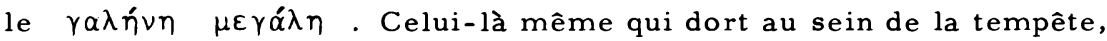
s'en révèle le maître. A celui qui lui donne toute sa confiance, Jésus révèle que l'épreuve qu'il subit, n'est pas une fatalité inéluctable et destructrice. Parce qu'il en est le maître, il permet à son disciple de l'affronter sereinement.

A la différence de Mc, le "choeur final" du récit (v. 27) n'est pas le fait des disciples, mais des $\ddot{\alpha} \nu \vartheta \rho \omega \pi \circ \iota$. Comment faut-il comprendre cela et qui sont les $\ddot{\alpha} \nu \vartheta p \omega \pi$ o ? Il convient de se souvenir que les "choeurs finaux" apparaissent dans les récits à caractère paradigmatique et qu'ils sont l'élément autorisant directement la prédication du récit ${ }^{2}$. Ainsi la conclusion de notre histoire pose la question de l'identité du

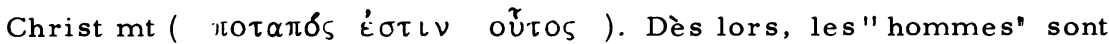
probablement les auditeurs auxquels est destinée cette histoire. Notre péricope n'est plus alors seulement la description de la grandeur et de la misère de la Nachfolge, elle devient également un appel à la Nachfolge ${ }^{3}$.

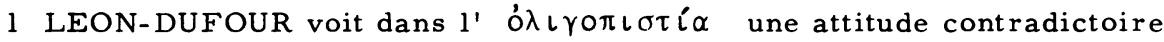
où l'adhésion de principe à Jésus jure avec le comportement réel. "Le disciple de 'peu de foi' ne vit pas selon la lumière que lui donne sa foi" (Tempête 170). Face à cette situation, dans le récit de la tempête apaisée, "Mt montre comment Jésus éduque une foi initiale pour la rendre pleinement vivante" (ibid). Quant à nous, nous ne pensons pas

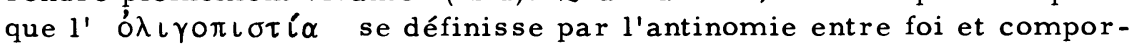
tement et que Jésus poursuive une pédagogie du progrès dans la foi à l'égard de ses disciples. L' ó $\lambda \iota \gamma \delta \pi \iota \sigma \tau$ os est un croyant devenu incrédule, il ne se comprend plus à partir de la foi. Il doit donc non pas être amené à une foi meilleure, mais simplement à la foi.

2 DIBELIUS, Formgeschichte 54-55.

3 BOR NKAMM, Enderwartung 52. Autrement SCHWEIZER, Mt 143 : la question du v. 27 traduit l'étonnement des hommes, vivant en dehors de la foi, devant les actes de Jésus. 
c) Mt 14,22-33

La source de notre récit est Mc 6,45-52. La comparaison entre les deux versions fait apparaftre une foule de différences. Abstraction faite des modifications mineures, la recension mt se distingue par trois changements importants qui modifient le sens du texte et témoignent de la théologie globale de Mt. D'une part, au v. 24a, $\beta \alpha \sigma \alpha v i \zeta \omega$ change de sujet : c'est le bateau qui est agité par les vagues et non les disciples (ainsi Mc). Ensuite, les vv. 28-31 sont une adjonction rédactionnelle. Enfin, Mt remplace Mc 6,51b-52 par son v. 33. Nous reprenons ces trois transformations pour les analyser.

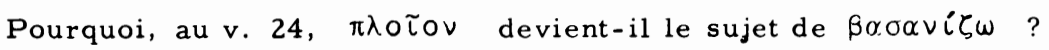
Cela provient de la conception $\mathrm{mt} d u \quad \pi \lambda \circ \tau_{0}{ }^{1}$ : le "bateau"des disciples est, en effet, pour le rédacteur du premier évangile, la figure de l'Eglise sous la croix (cf. 8, 24; 14,24.33). Cette signification autorise une interprétation symbolique du récit.

Les vv. 28-31 sont une adjonction par rapport au texte de Mc. S'agitil d'une tradition connue du seul $\mathrm{Mt}(\mathrm{Smt})^{2}$ ou d'une création rédactionnelle ${ }^{3}$ ? L'analyse terminologique livre à cet égard d'intéressants renseignements. Tant que les versions de Mc et de Mt sont parallèles,

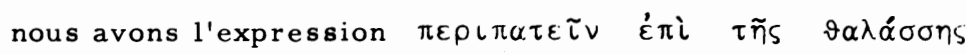

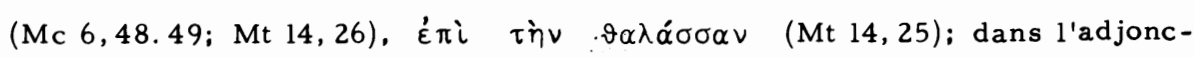
tion, au contraire, pour exprimer la même réalité, Mt utilise $\varepsilon \dot{\lambda}-$

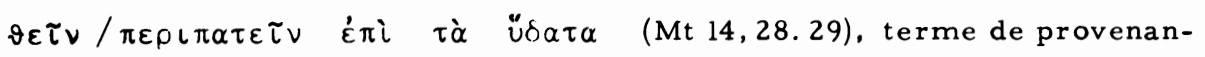
ce rédactionnelle en 8,32 . La prière $x u ́ p \iota \varepsilon$ ow̃oov $\mu \varepsilon \quad(14,30)$ se retrouve en 8,25 où elle est rédactionnelle. L'interpellation $\chi u ́ p \iota \varepsilon$

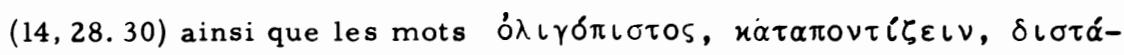

1 HELD 253-254.

2 KLOSTERMANN, Mt 130-131; STRECKER, Weg 198-199.

3 HELD 193-194. 
$\zeta \varepsilon ı \nu, \quad \varkappa \varepsilon \lambda \varepsilon \cup ์ \varepsilon \iota$ appartiennent au vocabulaire typiquement $\mathrm{mt}$. De plus, Mt relie les vv. 28-31 à l'ensemble de la péricope par la technique

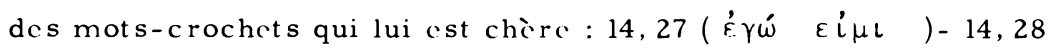

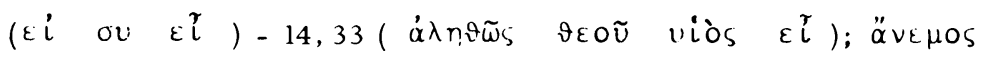

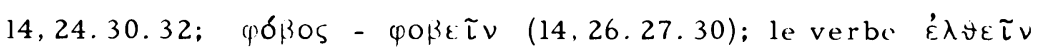
assure la cohérence des vv. 28-31 (vv. 28. 29a.29b).

Ces différentes observations attestent clairement l'important travail du rédactcur. Dès lors, avec Braumann ${ }^{1}$, on conclura ainsi : sans que l'on puisse décider de manière contraignante si Mt a utilisé ou non une tradition (Smt), on relèvera l'accent rédactionnel dominant. Mais pourquoi cette adjonction? En racontant la marche de Pierre sur les eaux à la rencontre de son Seigneur, puis sa chute, Mt introduit la thématique théologique de la foi et du doute ou, si l'on veut, de la grandeur et de la misère de la Nachfolge (réminiscerce de 8, 23-27).

Enfin, Mt remplace Mc 6,5lb-52 qui décrit l'incompréhension des disciples par une confession de foi (v. 33). Pourquoi ? Notre auteur n'insiste pas sur l'incompréhension des disciples, mais sur leur peur et leur manque de foi; plus précisément, il interprète leur. incompréhension

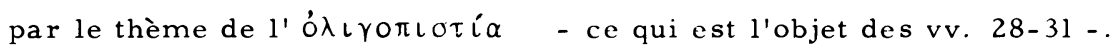

Bultmann ${ }^{2}$ voit dans notre péricope un récit de miracle violant les lois naturelles (Naturwunder). Cette classification, si elle convient bien au récit de $\mathrm{Mc}$, ne rend pas compte des modifications apportées par Mt. Il sied, à cet effet, de formuler trois remarques. D'une part, cette histoire s'adresse aux seuls disciples : 14, 22 introduit une nette séparation entre la foule et les $\mu \alpha \vartheta \eta \tau \alpha i ́$. D'autre part, le centre de la péricope n'est plus le miracle évoqué en 14,25, mais le dialogue entre Jésus et Pierre (vv. 28-31) ${ }^{3}$. Enfin, le thème central n'est plus la puissance du thaumaturge comme telle, mais bien la grandeur et la misère de la

1 BRAUMANN, Petrus 407. Voir aussi SCHWEIZER, Mt 209.

2 BULTMANN, Trad 231.

3 HELD 233. 
Nachfolge. On remarquera à l'appui de cette thèse que les catégories fondamentales du récit explicitent la situation de la communauté croyante

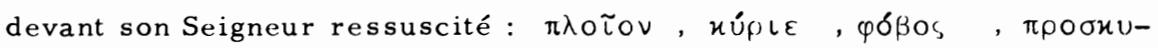

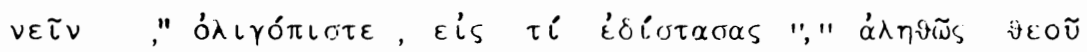
viós $\varepsilon \tilde{l}$ ". Dès lors, sans oublier qu'il s'agit d'une histoire de miracle - comme en 8,23-27 -, on notera qu'elle est devenuc un paradigme de Ia Nachfolge.

L'introduction de notre récit (v. 22) opc̀re une nette distinction entre la foule et les disciples ${ }^{1}$. Il n'y a pas de commune mesure entre ceux qui rencontrent occasionnellement le maître et ceux qui partagent sa destinée. L'événement qui va suivre concerne les seuls disciples. Pourtant l'ordre de Jésus est étonnant : c'est la seule fois dans l'évangile - passion excep-

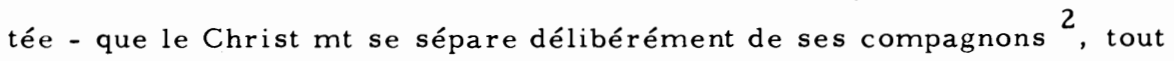
en promettant de les rejoindre. Doit-on, avec Schlatter ${ }^{3}$, voir dans cette indication une évocation indirecte de la situation post-pascale ? Cette interprétation n'est pas exclue car Mt imbrique constamment la situation de l'Eglise dans l'histoire de Jésus. Si elle se vérifiait, $\mu \alpha \vartheta \eta \tau n ́ s$ aurait d'entrée le double sens que nous lui connaissons.

La prière solitaire de Jésus (v. 23) est un motif qui suit ou précède les actes importants de son ministère (cf. $26,36 \mathrm{ss})^{4}$; dans notre cas,

1 Mt supprime le $\pi \rho \grave{s} \quad \beta \eta \vartheta \sigma \alpha i \delta_{\alpha} \nu$ de Mc parce que Jésus n'a pas traversé le lac.

2 Cf. LOHMEYER, Mt 238-239. Selon GRUNDMANN (Mt 367), le renvoi des disciples est motivé par la crainte de Jésus de les voir provoquer de fausses expériences messianiques. Rien dans le v. 22 n'appuie une telle hypothèse d'ailleurs peu conforme à la conception mt du disciple

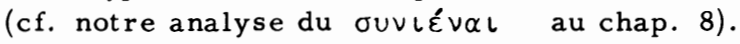

3 SCHLATTER (Mt 468) écrit sur ce point : "Die Gemeinschaft, die er durch das Mahl die Menge mit sich stellte, wird enden ... Wird auch seine Gemeinschaft mit den Jüngern enden ? Darauf gibt das neue Zeichen die Antwort. Er bleibt ihnen auch, wenn die Trennung von ihnen stattgefunden hat, nahe; er kommt zu ihnen".

4 BONNARD, Mt 222. 
elle prépare l'épiphanie du v. $25^{1}$. Il faut toutefois souligner que la mise en relation de la prière de Jésus et de sa solitude a un sens christologique $^{2}$ : Jésus n'a pas la même relation que ses disciples avec le Père; il ne saurait se confondre avec eux dans l'exercice de la prière.

Dans le v. 24, Mt fait preuve d'une grande originalité par rapport à Mc : il fait du $\pi \lambda o$ iov le centre d'intérêt de la scène et il introduit le thème des vagues. La proximité avec 8,24 est indubitable. Le thème du "bateau menacé par les vagues", motif propre à Mt, est l'image de la communauté du Ressuscité, vivant dans l'abaissement, menacée par l'impuissance et le doute ${ }^{3}$, (cf. notre exégèse de 8,24 ).

A propos du miracle relaté au v. 25, il convient de faire deux remarques. La marche de Jésus sur les eaux a tout d'abord un sens christologique : en marchant sur les eaux, le Christ $\mathrm{mt}$ agit avec l'autorité de la puissance du Dieu de l'A T (Ps 46, 2ss; 65, 7ss; 77, 20; 89, 10; 93, 38: Jb 9, 8; Es 43,16). Mais, d'autre part, comme dans l'AT, cette démonstration de puissance a un sens sotériologique : c'est pour s'approcher et venir en aide à sa communauté menacée que Jésus marche sur les eaux ${ }^{4}$.

L'apparition de Jésus (v. 26) qui devrait aider les disciples en difficulté, provoque paradoxalement leur effroi ${ }^{5}$. Ils croient, en effet, se trou-

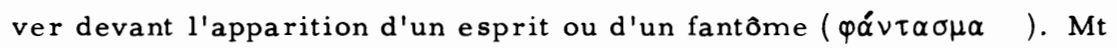
interprète la terreur des disciples par une glose explicative : $\alpha$ đò tou

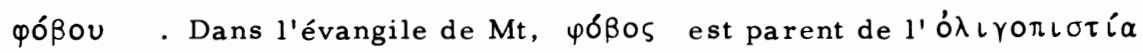
$(8,26 ; 14,30-31$; cf. aussi 10,28. 31); ce terme désigne le désarroi du croyant face à l'épreuve, la foi qui succombe dans la difficulté. Alors même que les disciples devraient être réconfortés par la rencontre de

1 LOHMEYER, Mt 239.

2 GREEVEN, art. $\varepsilon \ddot{u} \chi O \mu \alpha \iota$, ThW 2, 803.

3 BONNARD, Mt 222; GRUNDMANN, Mt 366; HELD 254.

4 GOPPELT, art. Ű $\omega \rho$, ThW 3, 322; LOEVESTAM 133-135.

5 GRUNDMANN, Mt 367. 
Jésus, ils sont ébranlés ${ }^{1}$. Ici déjà, à la différence de Mc, Mt interprète l'effroi des disciples comme défaillance de leur foi et non comme signe de leur incompréhension.

Jésus s'approche de ses disciples, càd de sa communauté, de manière secourable (v. 27). Par sa présence, il veut les libérer de la peur qui paralyse leur foi ${ }^{2}$. A cet effet, il se donne à connaître : $\vartheta \alpha \rho \sigma \varepsilon \tau \tau \varepsilon$, $\dot{\varepsilon} \gamma \dot{\omega} \varepsilon \dot{\imath} \mu \iota$ - $\mu \grave{\eta}$ $\phi о \beta \varepsilon \tau \sigma \vartheta \varepsilon$. Le verbe $\vartheta \alpha \rho \sigma \varepsilon \tau \nu$ apparaît souvent dans les évangiles comme un appel lancé par Jésus ${ }^{3}$. L'homme est invité au $\vartheta \alpha \rho \circ \varepsilon \tau \nu$ en raison de ce que Jésus est pour lui et lui donne. C'est dire que cet appel dévoile la prétention du Christ : en rencontrant l'homme, il prétend le libérer de la misère et de l'angoisse, lui offrir la joie et la confiance; il affirme implicitement être le porteur et le donateur d'une nouvelle possibilité d'existence. Le $\mu \grave{\eta} \varphi \circ \beta \varepsilon \tau \sigma \vartheta \varepsilon$ ne signifie pas autre chose et doit être considéré comme une redondance. Si notre interpréta-

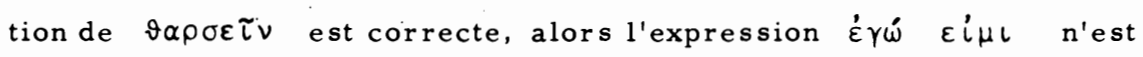
pas employée dans un sens banal, càd en vue d'établir l'identité du locuteur ${ }^{4}$. Si Jésus marche sur les eaux à la manière du Dieu (voir de la Sagesse) de l'AT et invite ses disciples ébranlés à la confiance, દ́rú $\varepsilon i \mu \iota$ a la valeur d'une formule de révélation divine, inséparable d'une prétention absolue ${ }^{5}$.

La déclaration secourable de Jésus est suivie d'un dialogue proprea Mt, qui forme le centre du récit (vv. 28-31). C'est Pierre qui prend tout d'abord la parole (v. 28) : mais, ce faisant, il n'est que l'interprète des

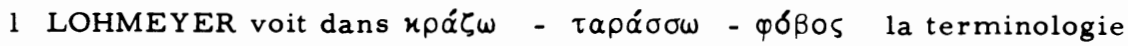
classique de l'épiphanie, décrivant la réaction de l'homme devant la manifestation du divin. Cette hypothèse est douteuse, car $x \rho \alpha ́ \zeta \omega$ et $о \beta \varepsilon \varepsilon \omega$ sont repris en 14,30 , càd dans le même contexte, et précisément dans le sens que nous avons adopté.

2 GRUNDMANN, Mt 368; SCHWEIZER, Mt 210.

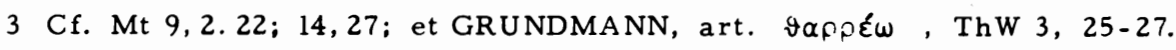

4 Ainsi STAUFFER, art. غ́ $\gamma \dot{\omega}$, ThW 2, 350.

5 LOEVESTAM 125-126. 
disciples ${ }^{1}$, car, comme le confirme la suite de la narration, son intervention ne lui confêre aucune dignité particulière. Pierre demande à Jésus l'autorisation de le rejoindre sur les eaux. Cette prière est-elle l'expression du doute (cf. en particulier le $\varepsilon \dot{i}$ où $\varepsilon \hat{l}$ ) qui désire une preuve matérielle, ou le reflet de la confiance retrouvée ? La deuxième hypothèse nous semble mieux fondée pour quatre raisons $: 1^{\circ}$ ) Jésus a dissipé la crainte des disciples par sa déclaration du v. $27 ; 2^{\circ}$ ) le titre $\chi \dot{u} p \iota \varepsilon$ qui ouvre la prière de Pierre, implique généralement chez Mt une confession de foi de la part de celui qui le prononce; $3^{\circ}$ ) la demande de Pierre est l'expression correcte de la foi, car en sollicitant de Jésus l'autorisation de venir à lui ( $x \varepsilon ́ \lambda \varepsilon v \sigma o ́ v ~ \mu \varepsilon$ ), Pierre reconnañt son impuissance personnelle à effectuer un tel acte et le pouvoir que Jésus a, de lui en donner la force; $4^{\circ}$ ) le $v$. qui suit montre que Pierre, une fois l'ordre donné, n'hésite pas à s'engager sur les eaux et cela sans que Jésus ait démontré son identité. La prière de Pierre est donc une prière faite dans la foi ${ }^{2}$, elle est la prière du disciple qui désire rejoindre son maître.

A la prière faite dans la foi ${ }^{3}$ répond l'exaucement : દ́خ.ఫદ́ (v. 29). Jésus habilite son disciple à faire ce que lui-même est en train de réaliser : marcher sur les eaux. Il lui donne de partager sa toute-puissance 4 . Ce n'est donc pas la capacité ou la volonté du disciple qui lui permet de marcher sur les eaux, mais l'ordre de Jésus, sa parole ${ }^{5}$. Par sa parole

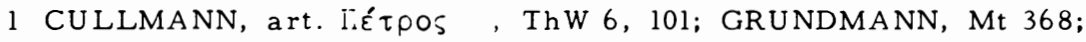
LOHMEYER, Mt 240; SCHWEIZER, Mt 209.

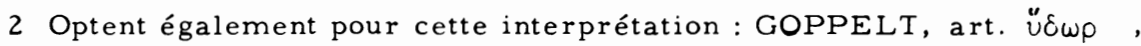
ThW 3, 322; HELD 194; LOEVESTAM 126; SCHLATTER, Mt 470.

3 On remarque que dans le dialogue entre Pierre et Jésus, toutes les répliques de Pierre (vv. 28.30) ont la forme de la prière, càd précisément du langage dans lequel le disciple de la communauté mt peut se reconnaitre et vivre sa relation avec son Seigneur.

4 GRUNDMANN, Mt 368; HELD 277-278; SCHWEIZER, Mt 210.

5 BRAUMANN, Petrus 409; SCHWEIZER, Mt ibid. 
toute-puissante, Jésus prend l'initiative de faire du disciple croyant son égal : Pierre marche sur les eaux et vient à la rencontre de Jésus. Voilà l'illustration de la grandeur de la Nachfolge.

C'est par la seule vertu de sa foi ou, si l'on préfere, parce qu'il s'en remet totalement à Jésus que Pierre peut marcher sur les eaux. Il suffira donc que cette relation soit perturbée pour que la toute-puissance du

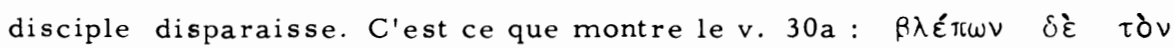

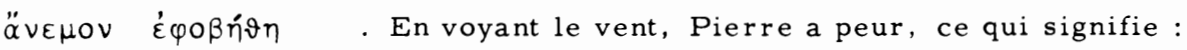
l'évidence des cataclysmes naturels est, pour lui, plus forte que l'évidence de sa foi. Sa crainte devant les fatalités de ce monde l'emporte sur sa confiance dans la puissance que Jésus lui accorde, et dans la mesure même où il cesse de s'en remettre à Jésus et se soumet aux réalités naturelles, la toute-puissance qu'il tenait du Christ s'évanouit : il coule. Alors que la marche de Pierre sur les eaux attestait sa foi, son naufrage révèle son incroyance (cf. v. 31). Mais il s'agit d'une incroyance paradoxale, car, alors même qu'il s'enfonce dans les eaux pour avoir cessé de s'en remettre à Jésus, c'est le Christ que Pierre appelle à son secours : นúple , oũoóv $\mu \varepsilon$. Nous avons fait l'exégèse d'une prière quasiment semblable en 8, 25 ; nous nous bornons donc à rappeler l'essentiel : au moment où sa vie est en danger, Pierre invoque le Kyrios en des termes liturgiques susceptibles d'être répétés par des croyants de l'église mt.

A cette seconde prière de Pierre, Jésus répond par un geste et une parole, les deux éléments formant un tout indissociable. Pour secourir Pierre, Jésus étend la main. Comme le montre l'histoire comparée des religions ${ }^{1}$, il ne s'agit pas là d'un geste banal ou réclamé par les seules circonstances. Lorsque la divinité tend la main, c'est toujours une action sotériologique. En sauvant Pierre d'un naufrage sans retour, Jésus lui montre l'inanité de son doute et lui apprend que la relation du maître

1 Matériel rassemblé par GRUNDMANN, Mt 369. 
et du disciple n'a pas son fondement solide dans la foi du disciple, mais dans la fidélité du maître ${ }^{1}$. Si Jésus sauve Pierre par le geste de la main tendue, il lui dévoile, par sa parole, la raison de son échec:

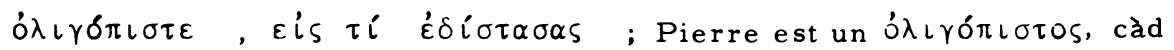
un incrédule au sein de la foi. Son incrédulité consiste dans le doute 2

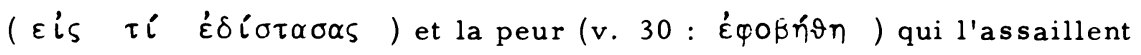
lorsque l'épreuve survient. C'est là sa "petite foi, une foi qui est trop petite comparée à la menace qui l'atteint, et face à laquelle il sous-estime la toute-puissance du maître" ${ }^{3}$. Concrètement, Pierre est un

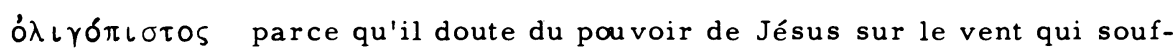
fle sur le lac. La scène entre Jésus et Pierre (vv. 28-31) "illustre donc la grandeur de la promesse accordée à la foi" - Pierre marche sur les eaux -, "mais elle ne dissimule pas l'incapacité du disciple à s'en tenir à cette promesse dans la tentation" 4 - Pierre coule -. Elle montre la grandeur et la misère de la condition du disciple ou - si l'on préfére - de la Nachfolge.

Avec lev. 32, nous reprenons le fil de la narration mc: Jésus, mais aussi Pierre chez Mt, regagnent le bateau et le vent tombe. Le motif de la tempête est ainsi mené à chef. Comme en 8,26 , ce n'est qu'après la réprimande adressée au disciple que la cause de l'épreuve disparaît ${ }^{5}$.

Mt conclut son récit (v. 33) d'une manière opposée à celle de Mc : loin de rester fermés et endurcis (ainsi Mc 6,51b-52), les disciples mt

1 SCHLATTER, Mt 472-473.

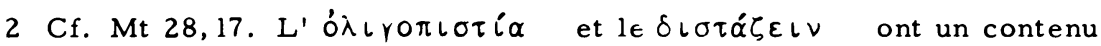
semblable; tous deux décrivent la réalité paradoxale du disciple incroyant.

3 GRUNDMANN, Mt 369.

4 HELD 195.

5 Nouvelle amorce de la parénèse ecclésiale: Pierre est appelé à une foi authentique par le geste et la parole critique de Jésus alors que le vent souffle encore; de même, l'Eglise est exhortée à croire au sein de la tentation et non pas une fois la tentation disparue. 
confessent leur foi ${ }^{1}$. Ils sont d'ailleurs désignés par l'expression signifi-

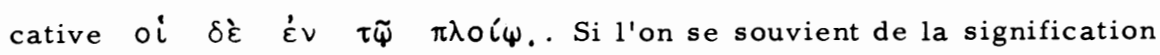
symbolique du $\pi \lambda \circ \tilde{\imath} 0 \nu$ chez Mt et si l'on voit que l'expression oi... É $\tau \tilde{\Psi} \pi \lambda \circ i \varphi$ est équivalente à oi $\mu \alpha \vartheta \eta \tau \alpha i$, on comprendra que le groupe des disciples n'est rien d'autre que l'image de la communauté post-pascale. Le $\pi \rho \circ \sigma \varepsilon \varkappa u ́ v \eta \sigma \alpha \nu$ qui suit, confirme cette interprétation, car, comme nous l'avons déjà montré ${ }^{2}$, la proskynèse mt est une catégorie ecclésiale qui décrit l'adoration du Christ élevé,par le fidèle. La

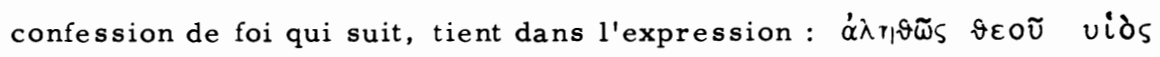
$\varepsilon \hat{l}$. Le titre viós $\vartheta \varepsilon o \tilde{u}$ n'a pas ici sa consonance mt classique qui voit, avant tout, dans le Fils de Dieu le juste souffrant ${ }^{3}$. L'apparition de

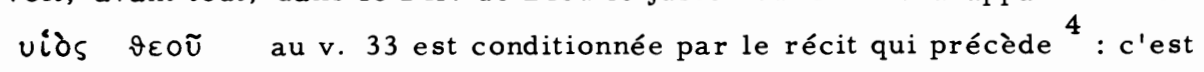
parce que Jésus s'est manifesté comme le Seigneur eschatologique en marchant sur les eaux, c'est parce qu'il a fait partager à Pierre sa toute-puis-

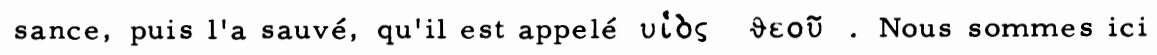
dans la ligne de 8,29 et 27,54 , passages où le Fils de Dieu est l'envoyé des derniers temps qui accomplit le miracle de la nouvelle création. Et

1 L'originalité de Mt dans la conclusion de notre récit ne doit pas nous étonner. Nous avons vu au chap. précédent que $\mathrm{Mt}$, à quelques exceptions près, voit dans les disciples des hommes qui ont reçu la compréhension de la prédication et de la destinée du Christ. Il ne peut donc dire d'eux

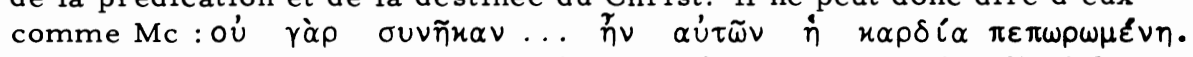
Cela ne signifie pas pour autant qu'il les idéalise, car, si les disciples mt de notre récit "comprennent", ils ne restent pourtant pas sans reproche : ils n'échappent pas à la crainte (v. $26:$ : ßßos) et Pierre succombe à $1^{\prime}$ ó $\lambda \iota \gamma o \pi \iota \sigma \tau i \alpha$ (vv. 30-31). Mt réinterprète donc les thèmes de l'effroi et de l'incompréhension propres à Mc par ceux de la peur et du manque de foi (cf. HELD 125).

2 Voir notre exégèse de $28,17, \mathrm{pp} .96 \mathrm{~s}$. Voir aussi HAHN, Hoheitstitel 86, note 1; SCHLIER, art. Yóvu , ThW 1, 738-740; GREEVEN, art.

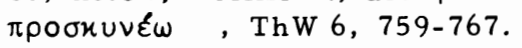

3 SCHWEIZER, art. viós, ThW 3, 381-382; cf. p.ex. 27,40.

4 SCHWEIZER, art. cit., 382, et HAHN, Hoheitstitel 313-314. 
c'est justement parce que Jésus est le Fils de Dieu que les hommes lui doivent la Nachfolge illustrée dans les vv. qui précèdent.

\section{d) Conclusion}

Les catégories $\mathrm{mt}$ de la foi et du doute décrivent l'existence chrétienne dans sa réalité présente. Elles montrent que la relation du croyant avec son Seigneur est vécue hic et nunc à la fois comme misère et comme grandeur. La condition chrétienne est dès lors paradoxale.

La misère de la condition croyante est, tout d'abord, une misère né cessaire. Le disciple vit dans un monde menaçant et tentateur. L'épreuve est inévitable et souvent elle surgit en l'absence apparente du Kyrios. La figure du bateau balloté par les éléments est celle de l'Eglise qui n'est pas triomphante, mais vit sous la croix. Le disciple ne saurait attendre autre chose que la destinée subie jadis par son maître. Mais la misère nécessaire risque toujours de devenir une misère coupable. C'est ce qui arrive lorsque le disciple se laisse aller à l'incrédulité (ó $\lambda\llcorner\gamma o \pi \iota \sigma \tau i ́ \alpha$ ) ou au doute ( $\delta\llcorner\sigma \tau \alpha \dot{\zeta} \omega)$. Cette incrédulité et ce doute ne dénotent ni une crise intellectuelle de la foi, ni un abandon résolu ou déclaré du maître. Le disciple incroyant continue à suivre le Christ. L'incrédulité du disciple est due à $1^{\prime}$ importance décisive qu'il attribue à la réalité des épreuves qui l'accablent et à son manque de confiance en l'appui efficace du maître. Le disciple incroyant transforme en fatalité l'épreuve tentatrice dont Jésus est pourtant le maître.

Pourtant à cette misère toujours nécessaire et parfois coupable s'oppose la grandeur de la Nachfolge. Cette grandeur ne tient pas à la persévérance héroïque du disciple, voire à sa sagacité ou à sa perfection, elle tient à l'appui secourable, efficace et constant que le maf́tre prête aux siens. Le Christ $m t$ exauce les prières des siens. Il vient en aide, par sa parole et sa puissance, aux disciples qui ont failli. Non seulement il permet aux croyants fidèles de surmonter la tentation, mais il arrache encore les incroyants à leurs errements coupables. Il associe les uns et les autres à sa toute-puissance, leur donnant ainsi la force de vivre 
dans un monde hostile.

Dans la thématique de la foi et du doute, l'existence chrétienne prend donc un double visage. Le Christ jette certes ses disciples dans un monde dangereux et tentateur où il exige leur complète obéissance, mais il leur donne cela-même qu'il leur commande. La réalité ultime de la condition présente du croyant n'est dès lors pas la misère qu'il endure, mais la sollicitude active et libératrice de son Seigneur. Et ce don, le croyant est appelé à le recevoir dans la foi et la fidélité. 
CHAPITRE DIX : LE CROYANT FACE A L'AVENIR

Nous avons essayé d'éclairer la condition du croyant selon Mt en décrivant tout d'abord la relation qui s'institue entre le disciple et le Jésus terrestre, sous le signe de la compréhension et de la suivance. Nous avons tenté ensuite de discerner comment le devenir chrétien était vécu dans le présent et nous avons découvert une condition paradoxale, simultanément riche de la promesse attachée à la foi et menacée par le doute. Ii nous faut maintenant nous poser la question de l'avenir du croyant.

Pour Mt - comme d'ailleurs pour l'ensemble du NT -, l'avenir se définit comme l'à-venir de Dieu. L'à-venir de Dieu est un thème, sinon le thème fondamental de l'évangile. Il donne sa substance à la proclamation du Regne aussi bien qu'à la prédication de la volonté de Dieu. Pourtant, à la suite de $M c$ et de $Q$, Mt lui réserve une place particulière en lui consacrant la grande instruction des chap. 24-25. En examinant cet ultime discours du Christ mt sous l'angle de la comparaison synoptique, le lecteur est immédiatement frappé par l'élargissement considérable que le rédacteur a apporté à l'a pocalypse mc. Jusqu'au v. 36, Mt 24 suit plus ou moins fidèlement Mc 13, mais ensuite l'auteur du premier évangile développe énormément le bref appel à la vigilance qui retentit en Mc 13 , 33-37, remplaçant les $5 \mathrm{vv}$. conclusifs de $\mathrm{Mc}$ par une vaste parénèse ne comprenant pas moins de sept (!) paraboles. Aux trois brèves paraboles des vv. 37-44 (Q), centrées sur la venue inattendue du Fils de l'homme, succèdent trois paraboles plus développées qui insistent sur l'attitude à adopter en l'absence du Kyrios : la parabole du serviteur (24,45-51, Q), celle des dix vierges $(25,1-13$, Smt), celle des talents $(25,14-30, Q)$. Le discours s'achève par l'impressionnante fresque du jugement dernier $(25,31-46, \mathrm{Smt})$. Cette composition originale doit être interprétée de manière à faire apparaître la cohérence de la conception $\mathrm{mt}$.

Comme le remarque pertinemment Grässer ${ }^{1}$, ce rééquilibrage des

1 Parusieverzögerung 94. 
traditions apocalyptiques par la parénèse de la vigilance traduit une nouve lle conception de l'avenir. L'apocalypse $(24,3-36)$ n'est plus simple ment la description de la fin imminente, le discours parénétique, qui la relaie, suppose le retard de la parousie et prend acte de son ajournement ${ }^{l}$. Certes, l'apocalypse $\mathrm{mt}(24,3-36)$ aborde le thème de la parousie $^{2}$ et traite déjà de manière conséquente les rapports entre l'eschatologie et l'éthique ${ }^{3}$; néanmoins, c'est dans la parénèse de la vigilance avant tout que Mt expose sa compréhension de l'existence chrétienne face à l'à venir de son Seigneur, et c'est sur elle en bonne méthode qu'il convient de concentrer l'attention.

A strictement parler - et comme en témoigne d'ailleurs la technique des mots-crochets ${ }^{4}$-, la parénèse $m t$ de la vigilance n'occupe pas toute la deuxième partie du discours; elle est constituée par la séquence qui va de 24,37 à $25,13^{5}$. C'e st donc sur cette séquence que nous devons concentrer notre intérêt.

\section{La venue inopinée de la parousie (Mt 24,37-44)}

Mt ouvre sa parénèse de la vigilance par trois brèves paraboles : la parabole du déluge ( $v v$. $37-39$ ), la double image de deux hommes travail-

1 Il est extrêmement significatif que Mt achève sa recension de l'apocalypse mc par l'affirmation de l'ignorance dans laquelle est tout homme - et même le Fils ! - quant à la venue de la parousie (24,36 par. Mc $13,22)$. C'est sur ce constat d'ignorance que s'engrène la parénèse. La communauté vit donc dans la conscience du retard de la parousie puisque, loin d'affirmer son imminence, elle se refuse à fixer un délai.

2 STRECKER, Weg 236-242.

3 PESCH, Eschatologie und Ethik $223-238$.

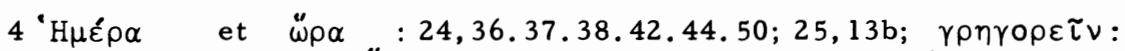

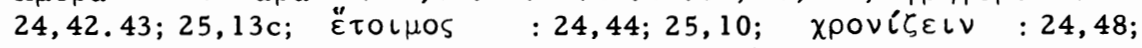
25, 10 (inventaire de GROLLINGER 239, note 1).

5 GROLLINGER 239; TRILLING, Israel 150. 
lant au champ et de deux femmes occupées à moudre (vv. 40-42) et enfin la parabole du voleur venant la nuit (vv. 43-44).

La parabole du déluge est empruntée à la petite apocalypse qui concluait la source $Q^{l}$. Comme en témoigne la double utilisation du schéma

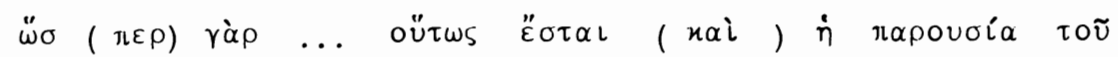

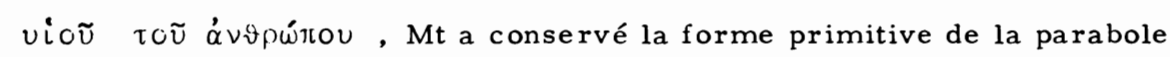
alors que Lc l'a récrite en l'élargissant (Lc 17,28-30) ${ }^{2}$. Deux modifications sont toutefois à porter au crédit de $M t:$ d'une part, aux vv. 37.39, il substitue le terme hellénistique rapovoía qu'il est seul à utiliser,

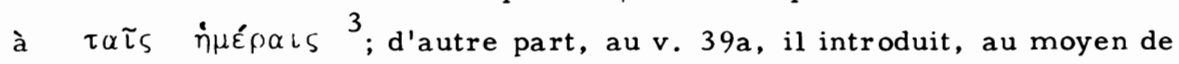

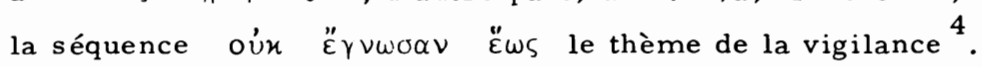

Cette parabole se présente comme un midrash de l'histoire de Noé ${ }^{5}$.

Elle établit une correspondance entre les premiers temps du monde et les derniers temps si bien que la tradition du déluge acquiert une valeur ty-

1 LUEHRMANN, Logienquelle $71-75$, suivi par SCHULZ, Q 277-287, a montré que la tradition figurant en Mt 24,27-28.37-41 par. Lc 17, 24. 26-30.34-35.37 formait une petite apocalypse qui concluait la source Q. La similitude entre $\mathrm{Mt}$ et $\mathrm{Lc}$, aussi bien dans la formulation des logia que dans leur enchaînement, le fait que Mt distribue ce matériel dans son chap. 24 alors que Lc choisit le chap. 17 (distinct de l'apocalypse $\mathrm{mc}$ ) appuient cette hypothèse.

2 La plus grande ancienneté de la recension $m$ t peut être démontrée par le fait suivant. Le premier logion, emprunté à l'apocalypse $Q$ (Mt

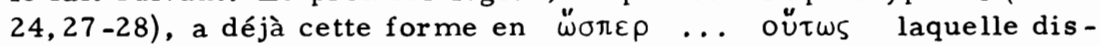
paraît tant que $M t$ suit $M c$ pour réapparaître aussitôt que $M t$ reprend le fil de l'apocalypse $Q$ (avec LUEHRMANN, Logienquelle 73; SCHULZ, Q 279).

3 Avec COLPE, art. ò viòs toũ ávîpúitov, ThW 8,436, note 255; GROLLINGER 240; GRUNDMANN, Mt 511 ; LUEHRMANN, Lo-

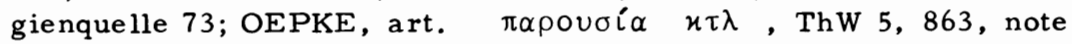
42; SCHULZ, Q 279; SCHWEIZER, Mt 300. Sur l'origine hellénistique et l'emploi mt de $\pi \alpha \rho o v o i ́ \alpha$, cf. STRECKER, Weg 237.

4 Avec LUEHRMANN, Logienquelle 74; SCHULZ, Q 279. Cf. aussi 24, 42. 44. 50; 25, 13.

5 SCHULZ, Q 282; STENDHAL 94. Le midrash est ici le support d'une parénèse. 
pologique. La mise en relation quelque peu abrupte ${ }^{l}$ entre la venue du déluge et celle de la parousie a un double accent. D'une part, elle souligne le caractère inopiné et subit de la parousie : de même que le déluge surprit les hommes dans leurs occupations quotidiennes, ainsi le Fils de l'homme viendra alors que personne ne l'attend. D'autre part, le motif du déluge, dans la tradition vétérotestamentaire-juive est inséparable du thème du jugement universel : la parousie sera le lieu du grand tri eschatologique ${ }^{2}$ auquel personne - pas même l'Eglise - ne pourra se soustraire.

Le corrélat anthropologique de cette affirmation christologique est formulé de manière originale. Si la tradition juive voit dans la génération du déluge le paradigme de la corruption et de l'impiété ${ }^{3}, Q$ - et, à sa suite, Mt - valorise l'image dans un autre sens. Les verbes $\pi i ́ v \omega$, $\tau \rho \omega ́ \gamma \omega, \gamma \alpha \mu \varepsilon \omega$, $\gamma \alpha \mu \imath \zeta \omega$ n'évoquent pas des comportements mora lement répréhensibles ${ }^{4}$. L'erreur coupable des contemporains de Noé consiste à conduire leur vie sans tenir compte de la réalité de Dieu, et singulièrement de sa venue. Noé, au contraire, comprend son existence dans la perspective de l'à-venir de Dieu et il en tire les conséquences : il monte dans l'arche. Le oúx "̌r $v \omega \sigma \alpha \nu$ rédactionnel souligne ce trait : la faute fatale de la génération du déluge est qu'elle oublie la seigneurie divine et la venue imprévisible du jugement.

La pointe parénétique de la parabole est claire : la parousie du Fils de l'homme surviendra de manière inopinée et provoquera le jugement de

1 Une comparaison correcte impliquerait la présence de $\dot{\eta} \mu \varepsilon \varepsilon_{\rho} \iota$ dans le second membre de la comparaison (aux jours de la parousie...). Ce raccourci s'explique par la volonté de Mt d'attirer l'attention du lecteur sur la parousie comme telle (GROLLINGER 240-41).

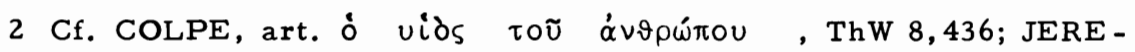
MIAS, Gleichnisse 87; LUEHRMANN, Logienquelle 73.

3 Matériel chez BILL 1,961-964.

4 Glissement bien souligné par GRUNDMANN, Mt 511 ; SCHNIEWIND, Mt 244-45; SCHULZ, Q 284-85; SCHWEIZER, Mt 301. 
tous - non seulement des pécheurs, mais aussi de l'Eglise - ${ }^{1}$. Seul celui qui a vécu - tel Noé - en prenant acte de la réalité divine et de son à-venir, sera sauvé. Celui, au contraire, qui vit dans l'oubli de son Seigneur, court à sa perte. On le voit, la vigilance, comme elle est comprise ici, ne tient donc pas dans un quelconque calcul des temps, mais dans la soumission hic et nunc de sa vie à Dieu.

Les deux images qui suivent - celle de deux hommes travaillant au champ (v. 40) et celle de deux femmes occupées à moudre (v. 4l) - proviennent également de la petite apocalypse $Q$ dans laquelle elles succèdent à la parabole du déluge. Les re touches rédactionnelles sont minimes. Outre le $\tau \delta \tau \varepsilon$ de transition (v. 40), il est possible que le $\varepsilon \dot{\nu} \tau \tilde{\Psi}$ $\alpha \gamma \rho \tilde{\varphi} \quad$ soit de la main de l'évangéliste qui créerait ainsi un strict parallélisme avec le v. 4l, en parlant à chaque fois de personnes solidaires dans leur travail ${ }^{2}$. Par contre l'exhortation du v. 42 est certainement une adjonction $\mathrm{mt}$, probablement inspirée de Mc 13,35, mais profondément retouchée par le rédacteur ${ }^{3}$.

Les deux images désignent des personnes solidai res l'une de l'autre

1 L'analyse de GROLLINGER 239-241 qui voit la pointe de la recension mt des vv. 37-39 dans le caractère inopiné de la parousie à l'exclusion de tout corrélat anthropologique est unilatérale (ainsi aussi SCHMID, Mt 340-4l). Dans un autre ordre d'idée, GRUNDMANN (Mt 541) décrit la chrétienté comme étant à l'abri de ce jugement imprévisible. La conception $m t$ du jugement - mais surtout le fait que le discours soit adressé aux seuls disciples $(24,3)$ - s'oppose à cette hypothèse.

2 Ainsi BULTMANN, Trad 123; GRUNDMANN, Mt 511 (hésitant); SCHULZ, Q 280.

3 L'exhortation parénétique du v. 42 est empruntée à $\mathrm{Mc}$; elle est même le seul élément que $M t$ a retenu de la parabole du portier (Mc 13,33-37). A l'énumération de Mc 13,35 (le soir, ou au milieu de la nuit, au chant du coq ou le matin), Mt a substitué ì $\mu \varepsilon ́ p \alpha$ qui devient un terminus technicus eschatologique. De plus, il a transformé le ó xúplos

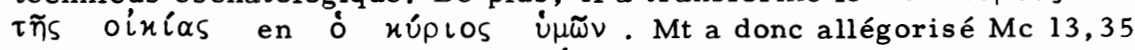
dans un sens christologique : le $\pi \delta ́ \tau \varepsilon$ devient le jour du jugement, le maître le Christ dans sa dignité judiciaire et eschatologique (cf. GROLLINGER 242; JEREMIAS, Gleichnisse 51-52; SCHMID, Mt 341). 
et apparemment semblables. La pointe ${ }^{l}$ est que la parousie va surprendre ces personnes dans leurs occupations quotidiennes et briser leur communauté de destin. Le jugement va séparer ceux qui semblaient unis et solidaires; il va détruire de manière imprévisible les liens humains les plus étroits. La parousie n'est plus l'heure de la grande joie eschatologique; elle apparaît comme une menace qui détruit toute sécurité et fait fi des apparences. Le salut n'est plus une assurance que l'on pourrait posséder ${ }^{2}$. La question qui surgit alors peut se formuler ainsi : pourquoi l'un est-il sauvé et l'autre rejeté ${ }^{3}$ ? La réponse implicite se trouve dans le v. 42 : l'un était prêt, mais non l'autre.

Comme nous l'avons signalé, le v. 42 est une adjonction rédactionnelle. A ce titre, il présente un intérêt certain, car il montre la perspective dans laquelle $M t$ a interprété la petite apocalypse qu'il tenait de $Q$. Cette appréciation est corroborée par le fait que l'exhortation du v. 42 revient de manière stéréotypée dans la séquence que nous étudions (cf. $24,44.50 ; 25,13)$. Quelle est alors pour Mt la leçon à tirer de la venue subite du Fils de 1'homme? L'incertitude du jour de la parousie place le présent vécu et l'avenir qui s'ouvre sous le signe de la vigilance (rppro$\rho \varepsilon \tau \tau \varepsilon)$. Cette vigilance ne consiste pas à supputer le temps du retour

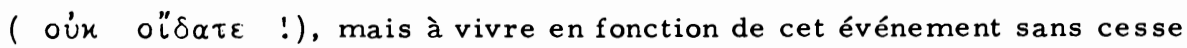

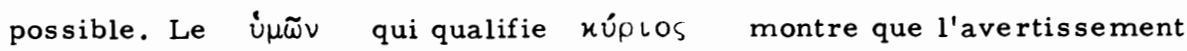
s'adresse aux croyants eux-mêmes plutôt qu'à ceux qui vivent en dehors de la foi.

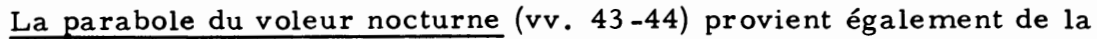

1 Voir par ex., BULTMANN, Trad 123; GROLLINGER 241; LUEHR MANN, Logienquelle 74; SCHULZ, Q 240.

2 GROLLINGER 242; SCHWEIZER, Mt 301.

3 L'agent du passif des verbes $\pi \alpha \rho \alpha \lambda \alpha \mu \beta \alpha \dot{v} v \omega$ et $\dot{\alpha} \varphi \imath \eta \mu \iota$ est le

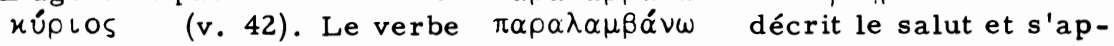
puie sur le motif du rassemblement eschatologique des élus par les anges (cf. 24,31). Voir GROLLINGER 241; GRUNDMANN, Mt 241; KLOSTERMANN, Mt 197; SCHULZ, Q 285; contre SCHMID, Mt 341. 
source $Q$ (cf. Lc 12,39-40). Dans cette dernière, elle n'est pas intégrée à l'apocalypse finale (cf. notamment Mt 24,37-41), mais elle est associée à la parabole suivante, celle du serviteur (vv. 45-51 par. Lc 12,41-46) ${ }^{1}$. L'exhortation du v. 42 permet donc de souder deux blocs primitivement indépendants en les interprétant à partir du thème commun de la vigilan$c^{2}$. Ici encore (comme en témoigne le par. Lc), Mt est resté très proche de la version $Q$ de la parabole. Deux retouches peuvent pourtant lui

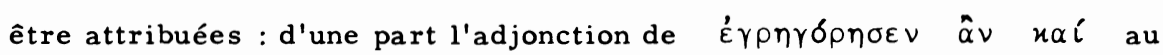
v. 43 qui fait fonction de mot-crochet et accentue l'unité thématique du

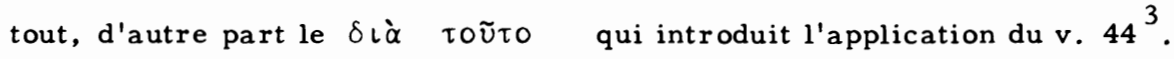

Nous n'avons pas à statuer sur l'origine de la parabole; Mt l'a trouvée dans $Q$ où elle a déjà une pointe christologique : le voleur qui survient à l'improviste durant la nuit est la figure du Fils de l'homme lors de la parousie ${ }^{4}$. La comparaison peut sembler choquante ${ }^{5}$, car qu'y a-t-il de commun entre la venue du Christ et l'activité délictueuse d'un cambrioleur ? A cette question, il est possible de donner une double réponse. D'une part, la métaphore du voleur nocturne pour décrire l'irruption de

1 Voir p.ex., GROLLINGER 245; LUEHRMANN, Logienquelle 69; SCHMID, Mt 342.

2 Cf. GROLLINGER 242 -43; KLOSTERMANN, Mt 197; SCHMID, Mt 342.

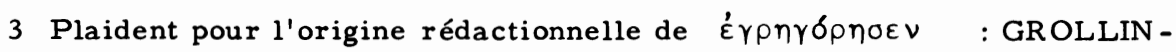
GER 243; SCHNIEWIND, Mt 300; SCHULZ, Q 268. Avec GROLLINGER ibid. et JEREMIAS, Gleichnisse 45 , nous pensons que le $\delta$ ¿à

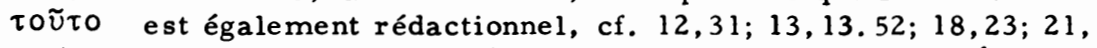

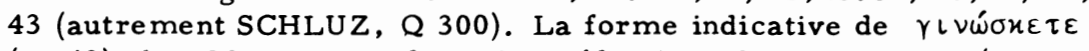
(v. 43) chez Mt est une adaptation rédactionnelle au contexte (avec (GRAESSER 93, note 1, et SCHULZ, Q 268).

4 JEREMIAS (Gleichnisse 47) et VIELHAUER (Gottes reich 73, note 79) bien que défendant une position opposée sur l'origine de la parabole, s'accordent sur son sens pré-mt.

5 SCHNIEWIND, Mt 245 : "bewusst abstossend"; cf. aussi SCHULZ, Q 269. 
la fin est connue de l'ensemble du christianisme primitif ${ }^{l}$. D'autre part, il ne faut pas presser l'image, mais en retenir la pointe : le voleur symbolise l'événement imprévisible par excellence, événement qui tourne à la confusion de celui qui n'a pas pris les dispositions nécessaires ${ }^{2}$.

La difficulté essentielle dans l'interprétation des vv. $43-44$ réside dans le passage abrupt entre l'image et l'exhortation qu'elle fonde ${ }^{3}$. En effet, le propriétaire ne sait ni si un voleur viendra, ni, s'il vient, quand aura lieu l'effraction; dans ces conditions, toute vigilance peut sembler illusoire, voire grotesque. A l'inverse, le disciple ne sait pas quand son maitre viendra, mais il est assuré de sa venue; sa vigilance est donc jus tifiée. Cette inadéquation entre l'image et son application peut être dépassée ${ }^{4}$ dès l'instant où l'on admet que la parabole évoque une règle générale ${ }^{5}$ et qu'une partie du raisonnement est implicite. Qu'est-ce à dire ? Tout propriétaire veillerait s'il savait quand vient le cambrioleur; mais justement, il ne le sait pas. Le point de comparaison entre le propriétaire et le croyant consiste dans leur inévitable ignorance ${ }^{6}$. Dès lors, l'argumentation implicite permettant de passer de l'image à l'exhortation peut s'énoncer ainsi : si le disciple connaissait l'heure de la parousie du Fils de l'homme, elle ne le surprendrait pas; mais comme le propriétaire,

1 En 1 Th 5,2.4, 2 P 3, 10, la métaphore du voleur décrit l'arrivée inopinée du "dernier jour"; en Ap 3,3; 16,15, elle s'applique à la parousie du Christ. Voir aussi Did 16,1 et Evangile de Thomas, logion 21. En tant qu'elle décrit l'arrivée subite du Messie, elle est inconnue du bas-judal's me (cf. GRAESSER 93, note 5; JEREMIAS, Gleichnisse 47; SCHNIEWIND, Mt 245; SCHULZ, Q 270).

2 Avec GRAESSER 93; SCHULZ, Q 269; VIELHAUER, Gottes reich 73.

3 Difficulté bien vue par GROLLINGER $243-244$.

4 Voir l'analyse de STRECKER, Weg $241-242$.

5 Contre JEREMIAS, Gleichnisse 45-46 qui discerne dans la parabole l'écho d'un événement réel.

6 LINNEMANN 140 : "Das Gleichnis hat seinen Vergleichspunkt in der Unmöglichkeit Vorsorge zu treffen". 
il ne la connaît pas et la venue de son Seigneur reste un événement imprévisible. L'exhortation "soyez prêts" tire alors la conséquence de cette ignorance. La vigilance requise consiste non pas à éviter d'être surpris puisque, de toute manière, la parousie sera inopinée, mais à prendre les dispositions nécessaires pour que cette surprise ne devienne pas un sujet de confusion. Ce point sera développé dans les deux paraboles qui suivent ${ }^{l}$.

Les trois brèves paraboles (vv. $37-39.40-42.43-44$ ) que nous avons analysées défendent un même point de vue ${ }^{2}$. Elles représentent une intensification de la parénèse de la vigilance et décrivent la parousie comme un événement inopiné et subit qui surprendra chacun. Semblable perspective présuppose le retard de la parousie et une église qui prend conscience de la durée à laquelle elle est appelée. En effet, s'il est incontestable que Jésus a appelé ses contemporains à la vigilance, c'est dans la cons cience aiguë de l'imminence de la fin. Tout autre est la conception de $Q$ - et partant de Mt -. L'appel à la vigilance n'est plus motivé par la proximité de la fin, mais par l'incertitude de son échéance. Le glissement est significatif : le croyant ne se comprend plus comme vivant la fin ellemême, mais dans l'attente de la fin. Le cadre temporel est maintenu, mais la question du délai est rendue caduque. La vigilance requise ne consiste plus dès lors dans un discernement des temps, mais dans une vie placée sans cesse sous l'autorité du Seigneur. Lors de la parousie qui prendra chacun au dépourvu, seul le croyant fidèle ne sera pas confondu.

1 Avec SCHULZ, Q 270-271; SCHWEIZER, Mt 302; STRECKER, Weg 242; contre GROLLINGER 244 et JEREMIAS, Gleichnisse 47. La vigilance ne consiste pas dans une attente jamais prise en défaut, mais

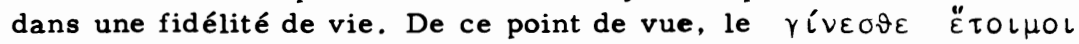
(v. 44) reprend le $\gamma \rho \eta \gamma о \rho \varepsilon \tau \tau \varepsilon$ du v. 42, mais ces deux expressions

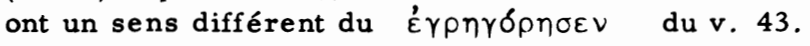

2 Pour ce qui suit, voir GRAESSER 93-94. 


\section{La parabole du se rviteur (Mt 24,45-51)}

La parabole du serviteur provient de la source $Q$ (cf. Lc 12,42-46). $\mathrm{Au}$ contraire du texte de Lc qui a été substantiellement retravaillé et élargi (cf. vv. 41.47-48), la recension mt a conservé pour l'essentiel la version $Q$ de la parabole ${ }^{l}$. On peut cependant imputer à Mt les retouches suivantes. Auv. 51, Mt substitue $\dot{u} \pi 0 x \rho \iota \tau \tilde{\omega} \nu$ à $\dot{\alpha} \pi i \sigma \tau \omega \nu$ et il ajoute la séquence finale (v. 5lc) qui est caractéristique de sa rédaction $^{2}$. Au demeurant, il est possible que le $\varkappa \alpha \varkappa \delta \varsigma$ du v. 48 soit une adjonction rédactionnelle faisant pendant au couple $\pi \iota \sigma \tau \delta$ / / ppóvi-

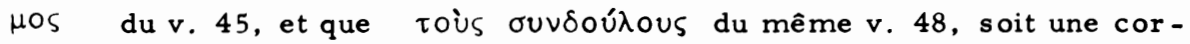
rection de caractère allégorique et inspirée de la réalité ecclésiale ${ }^{3}$.

Pour apprécier correctement la recension mt, il est nécessaire de remarquer que, dans $Q$ déjà, notre péricope présente des traits allégoriques et aborde la problématique de la parousie ${ }^{4}$. En effet, comme le montre Mt 24,43-44.45-51 par. Lc 12,39-40.42-46, la source $Q$ avait groupé l'image du voleur venant la nuit et notre parabole. Or l'application parénétique du v. 44, en traitant explicitement de la venue du Fils de l'homme pour le jugement, indique clairement que le thème du discours est l'événement de la parousie. En outre, le châtiment décrit dans le v. 5 lb sort du cadre de la parabole en évoquant le rejet eschatologique. Dès. lors, le xúplos des vv. 45-50 n'est pas un mañtre ordinaire, mais le

1 Cf. SCHULZ, Q 272, et WEISER 179.

2 Avec, p. ex., GROLLINGER 245-246; GRUNDMANN, Mt 154; JEREMIAS, Gleichnisse 54; SCHULZ, Q 272; SCHWEIZER, Mt 301; WEISER

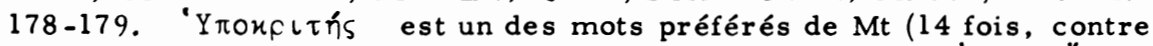

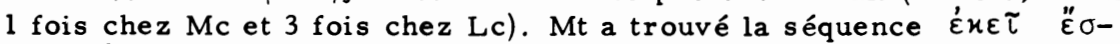
$\tau \alpha \iota$ ò $x \lambda \alpha \cup \vartheta \mu o ̀ s$ $x \tau \lambda$ dans la source $Q$ (Mt 8,12b par. Lc 13, 28a) et il l'introduit à de nombreux autres endroits : 13,42b. 50b; 22,13c; $25,30 \mathrm{~b}$.

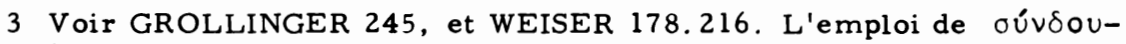
$\lambda$ os signalerait alors que la méchanceté consiste à maltraiter des frères dans la foi (cf. 18,21-35).

4 GRAESSER 91 -92; LUEHRMANN, Logienquelle 70; SCHULZ, Q 274; WEISER 180 . 
Christ régnant sur ses créatures et exerçant son pouvoir judiciaire. Sem-

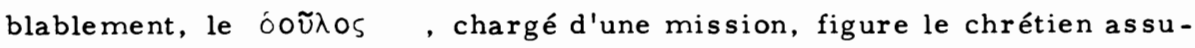
mant sa responsabilité de croyant et répondant de sa vocation devant son Seigneur. Mt a non seulement repris cette interprétation de la parabole, mais il l'a accentuée : le contexte rédactionnel est donné par la parénèse de la vigilance $(24,37 ; 25,13)$ et l'adjonction du $v$. $51 \mathrm{c}$ souligne sans équivoque 1 'enjeu eschatologique du comportement du serviteur 1 .

Ces arguments tirés du contexte et du contenu, qui plaident pour une lecture allégorique de la parabole, sont soutenus par une analyse de la forme littéraire. La question introductive en $\tau i \varsigma_{s} \ddot{\alpha} \rho \alpha$ (v. 45) n'appelle pas le lecteur à approuver la pointe du récit, mais à s'identifier avec le serviteur ${ }^{2}$. De plus, fait caractéristique, la parabole dans son développement contient deux paroles prophétiques-apocalyptiques ${ }^{3}$ : un macarisme au v. 46 et une déclaration en $\alpha \mu \eta ́ v$ au v. 47. S'il est évident que ces deux logia ont perdu leur substance prophétique, ils témoignent d'une tendance à l'allégorisation. Le macarisme du v. 46 argumente à partir du fond et non de l'image : l'esclave de l'Antiquité n'est pas récompensé pour l'accomplissement fidèle de son travail, mais bien le chrétien pour sa persévérance dans la foi. Le $\dot{u} \mu \tau \nu$ du v. 47 établit tacitement une correspondance entre le serviteur de la parabole et le lecteur. Nous n'avons donc pas affaire à une parabole au sens strict, mais à un ensemble complexe où le fond et l'image se combinent subtile ment ${ }^{4}$. Ce modèle est caractéristique des paraboles de la parousie qui,

1 Voir sur ce point, WEISER 215-216, et, avant lui, JEREMIAS, Gleichnisse 53 .

2 BULTMANN, Trad 185. WEISER 180-182 a fait une étude détaillée de cette question introductive, unique dans les paraboles synoptiques : à la suite de BEYER, il y reconnait une construction de phrase sémitique.

3 Sur ce point, voir SCHULZ, Q 273.

4 SCHULZ, Q 274. Cette complexité de forme ne met pas en cause l'unité de composition. 
de manière constante, opposent deux comportements concrets sur la base du schéma bénédiction/malédiction (cf. $25,1-13.14-30.31-46)^{1}$.

Notre parabole expose de manière antithét ique les deux comporte ments possibles pour un serviteur ${ }^{2}$ chargé d'une mission par son maitre qui s'absente. La transcription allégorique nous signale immédiatement que le thème majeur n'est pas la vigilance au sens de l'attente de la parousie, mais la conduite à adopter en l'absence du Kyrios ${ }^{3}$. De ce point de vue, la parabole du serviteur développe de manière cohérente la réflexion amorcée dans l'image du voleur nocturne qui invitait le croyant à tirer les conséquences du caractère imprévisible de la parousie 4

La première partie de la parabole (vv. 45-47) décrit le serviteur fidèle ( $\pi \iota \sigma \tau o ́ \varsigma)$ et avisé ( $\varphi \rho \delta ́ v \iota \mu \circ \varsigma)$. Sa fidélité et son intelligence consistent en ce qu'il remplit correctement la mission qui lui est impartie. Il est conscient d'être dépositaire d'un mandat ${ }^{5}$ et il agit en consé-

1 LUEHRMANN, Logienquelle 70.

2 JEREMIAS (Gleichnisse 53) a établi que la parabole parle d'un, et non de deux serviteurs. Le દ́r. $\tau$ vos du v. 48 , textuellement bien attes té, vérifie cette hypothèse.

3 Ce glissement est bien souligné par BONNARD, Mt 356; SCHMID, Mt 342; SCHWEIZER, Mt 302; WEISER 179.

4 LUEHRMANN (Logienquelle 70) rappelle à ce propos la réflexion de

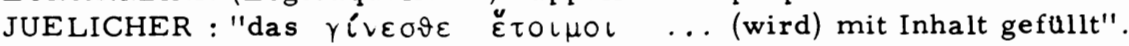

5 Plusieurs auteurs (GRUNDMANN, Mt 513; JEREMIAS, Gleichnisse 55; KLOSTERMANN, Mt 198; SCHULZ, Q 274-276) voient dans le serviteur l'image du responsable de la communauté, étant donné l'autorité dont il jouit sur la maison de son maître. Si cette interprétation est vrai semblable pour la version lc de la parabole (cf. Lc 12,41 et l'analyse

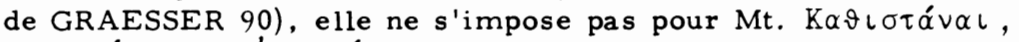

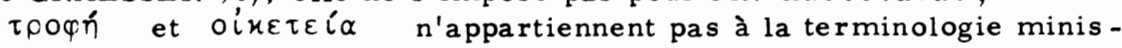
térielle typique du NT. De plus, il n'y a pas lieu de supputer une rupture avec le contexte, car le discours dans son ensemble, et singulièrement la parénèse de la vigilance, s'adresse à tous les disciples (avec BON NARD, Mt 356; BULTMANN, Trad 125; LUEHRMANN, Logienquelle 70, note 9; SCHWEIZER, Mt 301; WEISER 184-186). Par ailleurs, la responsabilité impartie à l'esclave de la parabole est courante dans le monde antique. 
quence. Aussi le retour inopiné de son maître ne tourne-t-il pas à sa confusion; la parousie est pour lui l'heure de la récompense eschatologi$q^{1}$. Le serviteur fidèle se caractérise donc par son évaluation "réalis te" de sa situation: il ne s'arroge pas une autonomie illusoire, mais il sait qu'il est responsable devant son maitre et qu'il devra rendre des comptes. Semblablement, le croyant avisé est celui qui est conscient de la réalité de Dieu et de son à-venir, et qui saisit son existence en conséquence $^{2}$.

La deuxième partie de la parabole (vv. 48-51) nous décrit le serviteur en tant qu'il choisit un comportement insensé et condamnable ( $x \alpha-$ nós). Son égarement est illustré par les motifs de la brutalité et de l'in-

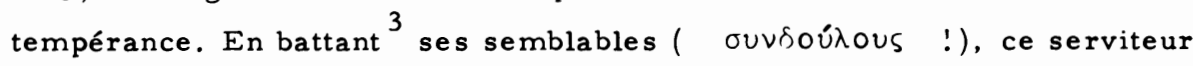
commet un abus de pouvoir incompatible avec le mandat qui lui est confié (v. 49a). Au lieu de respecter sa condition, il s'arroge un droit que seul un maître avait vis -à-vis de ses esclaves. Le motif de l'ivresse (v. $49 \mathrm{~b})^{4}$ va dans le même sens : le mauvais serviteur exploite sa position privilé-

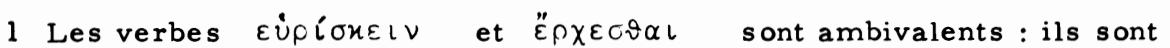
à la fois appropriés pour décrire un événement purement profane et la venue du Christ pour le jugement. Matériel néotestamentaire rassemblé par WEISER $186-187$.

2 Ainsi BERTRAM, art. $\varphi \rho \hat{\nu} \nu \quad x \tau \lambda$, ThW 9,230; GROLLINGER 246; SCHWEIZER, Mt 302 -303; WEISER 204.216.

3 Le fait pour un esclave de l'Antiquité d'être battu par son maître est constitutif de sa vie quotidienne (cf. STAEHLIN, art. $\tau u ́ \pi \tau \omega$, ThW 8,263-264; WEISER 193-195 - là matériel -). BONNARD (Mt 453) re marque à juste titre qu'en s'arrogeant les droits de son maíre, l'es clave de la parabole "a perdu jusqu'à la conscience de sa condition". Faut-il allégoriser ce trait et voir dans le v. 49 des comportements ayant cours dans la communauté $\mathrm{mt}$ ? Sans être exclue (voir, p.ex., SCHWEIZER, Mt 303), cette interprétation ne semble pas s'imposer. L'allégorie porte sur la relation entre le croyant et son Seigneur plutôt que sur les relations intracommunautaires, et les motifs du v. 49 illustrent le dommage irréparable intervenu dans le lien qui unissait le disciple à son maître.

4 PREISKER, art. $\mu \varepsilon \vartheta \eta \pi \tau \lambda$, ThW 4,552-553. Voir aussi SCHWEI ZER, Mt 302 ; l'ivresse est le symbole d'une vie dissolue qui ne tient plus compte de sa responsabilité devant Dieu (1 Th 5,6-8; Lc 21,34; 1 Co 15,$34 ; 1 \mathrm{P} 1,13 ; 5,8)$. 
giée, non pour accomplir sa mission, mais pour mener une vie déréglée et fuyant la réalité. Que ce soit par ses abus de pouvoir ou ses déborde ments, il trahit la confiance de son maitre et récuse de facto son autorité. Semblablement, le croyant égaré est celui qui, en l'absence apparente de son Seigneur, se soustrait à la responsabilité dont il est investi. Il fuit sa situation réelle placée sous le signe de Dieu et de son à-venir pour s'abandonner aveuglément à sa volonté et à ses plaisirs.

L'originalité de la deuxième partie de la parabole tient dans le fait que le comportement coupable du mauvais serviteur est expliqué : $\chi \rho \circ-$ Vį̌ hou ò xúplos (v. 48b). Le trait allégorique est flagrant ${ }^{1}$. Car s'il est douteux que, dans l'Antiquité, un esclave doué de bon sens ait pu prendre prétexte du retard de son maitre pour commettre des abus qui auraient de toute façon causé sa perte, il est à l'inverse parfaitement explicable que le retard de la parousie ait eu pareil effet ${ }^{2}$. La tentation du croyant, déçu dans son attente, est d'imaginer que désormais il n'a plus de compte à rendre - sinon à lui-même -, et d'organiser sa vie, pensant en être le maître. Le retour inopiné du Kyrios - rédigé dans la ter minologie du jugement eschatologique ${ }^{3}$ - montre l'inanité du raisonnement de l'esclave fautif et ses conséquences catastrophiques. Si le châtiment,

1 Si les exégètes sont divisés sur le sens, voire la présence, de $\chi \rho 0-$

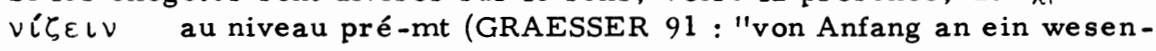
tlicher Zug des Gleichnisses";JEREMIAS, Gleichnisse 54 : "in der Geschichte liegt auf der Verzögerung der Ruckkehr des Hausherrn kein Ton"), l'unanimité règne quant à la signification de ce verbe au niveau rédactionnel : il désigne de manière allégorique le retard de la parousie. Cette interprétation est commandée à la fois par le contexte et la conclusion de la parabole. Voir, p.ex., BONNARD, Mt 357; BORN KAMM, Enderwartung 39, note 2; BULTMANN, Trad 125; GRUNDMANN, Mt 513; GRAESSER 91; LUEHRMANN, Logienquelle 70; SCHULZ, Q 276; STRECKER, Weg 44. Etat de la discussion chez WEISER, 188-193.

2 GRAESSER 91 à la suite de JUELICHER. Cf. 2 P 3,2 -4.

3 Comme en témoigne le contexte rédactionnel (cf. $24,36.42 .44 ; 25,13$ ), $\dot{\eta} \mu \varepsilon ́ \rho \alpha$ et $\ddot{\omega} \rho \alpha$ sont des termes techniques qui désignent la venue du Fils de l'homme pour le jugement. Etat de la discussion chez WEISER $196-197$. 
décrit dans le v. 5la, tout en étant d'une rigueur impitoyable, reste dans le cadre de l'image ${ }^{l}$, le v. 51 b argumente à partir du contenu théologique et désigne la condamnation eschatologique ${ }^{2}$. L'adjonction rédactionnelle du v. 5lc, en évoquant le séjour des damnés, renforce ce dernier trait.

La problématique de la parousie, dans notre parabole, est traitée de la même manière que dans les vv. $37-44$. D'une part, la communauté est invitée à prendre acte de son retard : la réflexion du serviteur au v. 48 ne saurait s'expliquer autrement; le retour inopiné du maître n'accrédite pas la croyance à une parousie proche, car il falsifie précisément le calcul de l'intéressé ${ }^{3}$. D'autre part, l'enseignement de notre péricope sur la manière de réagir devant ce retard est analogue à l'argumentation de l'apocalypse $Q$ : l'affirmation de la venue imprévisible du Kyrios (cf. v. 50) met le croyant en demeure, non pas de spéculer sur le retard de la parousie, mais au contraire d'organiser et de mener sa vie comme si le Fils de l'homme pouvait revenir à chaque instant. L'incertitude qui caractérise l'échéance de la fin aboutit à une intensification de la parénèse ${ }^{4}$.

1 Le verbe $\delta \iota \chi 0 \tau O \mu \varepsilon \tau \nu$ fait sans doute allusion à un supplice d'origine perse, répandu dans l'Antiquité, et qui consiste dans le démembre ment du coupable. Voir : BILL 1, 969; GRUNDMANN, Mt 514;

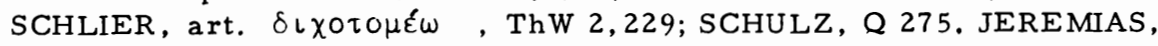
Gleichnisse 54 (note 6)pense à une mauvaise traduction de l'original araméen et suppose qu'à l'origine le sens était: "les coups seront son partage". Dans ce cas, il y aurait équival ence entre la faute et le châtiment. Etat de la question chez WEISER 198-200.

2 Avec BULTMANN, Trad 185; GRUNDMANIN, Mt 514; JEREMIAS, Gleichnisse 54; SCHNEIDER, art. HépOS , ThW 4,601; SCHULZ, Q 276; WEISEK 200-201. Si, dans la source $Q$, la cordamnatıon eschatologique du v. 5lb vise les Juifs incrédules, dans la rédactior nt, elle s'adresse aux membres infidèles de la communauté.

3 CONZELMANN (Mitte der Zeit 99, note 2) écrit à propos du comportement du serviteur : "Aber wie kommt er zu dieser Haltung ? Weil der Herr lange ausblieb". Mème analyse chez GRAESSER 91-92. Autrément GROLLINGER 246.

4 GRAESSER 95; GROLLINGER 246; STRECKER, Weg 242; TRILLING, Israel 150 . 
Dans ces conditions comment le problème de l'existence croyante se pose-t-il ? La parabole est à la fois promesse et exhortation ${ }^{1}$. Dans le temps qui précède le retour du Kyrios, càd durant son absence, le chrétien doit toujours à nouveau choisir entre deux possibilités de vie : soit celle du serviteur avisé qui place toute son existence sous l'autorité de Dieu et dans la perspective de son à -venir, soit celle du serviteur coupable qui, prenant prétexte de l'absence du Kyrios, organise sa vie au gré de ses préférences. Le serviteur qui vit dans la fidélité la responsabilité qui lui a été confiée, n'a rien à craindre de la parousie; pour lui, l'avenir est ouvert, il est le sujet d'une promesse. A l'inverse, le croyant infidele se condamne lui-même; alors qu'il s'imagine profiter de la vie, il perd son avenir. Sa désobéissance qui l'aveugle et lui masque la réalité de Dieu, l'assimile au pharisien hypocrite contre lequel le Christ s'est élevé avec véhémence (chap. 23) et qu'il a rejeté sans appel ${ }^{2}$. Le retard de la parousie et la réponse qui lui est apportée posent en vérité l'alterna tive propre à tout devenir chrétien ${ }^{3}$.

3. La parabole des dix vierges (Mt 25,1-13)

La parabole des dix vierges appartient au Smt. Les retouches rédactionnelles - pour autant que nous puissions encore les discerner - concernent en priorité le "cadre" de la parabole. Auv. la, le $\tau o ́ \tau \varepsilon$ typique ment $\mathrm{mt}$, relie notre péricope avec celles qui précèdent ${ }^{4}$. De plus, la

1 SCHWEIZER, Mt 303.

2 Sur ce point, voir l'analyse détaillée de SCHWEIZER, Gemeinde 119 120. $123-124$.

3 BONNARD, Mt 357; GRUNDMANN, Mt 514; SCHWEIZER, Mt 303.

4 BULTMANN, Trad 190-191; JEREMIAS, Gleichnisse 48; KLOSTER MANN, Mt 200; LINNEMANN 133; MAISCH 255; SCHWEIZER, Mt 304. 


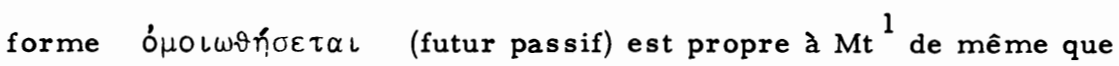

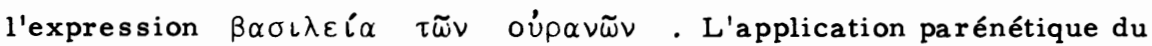
v. 13 est sans doute de la main de l'évangéliste, car, d'une part, elle reprend pour l'essentiel l'exhortation rédactionnelle du v. 42 (cf. vv. 44. 50), d'autre part, elle ne retranscrit qu'imparfaitement le sens de la parabole. Les vie rges avisées ne se caractérisent pas par leur vigilance - elles dorment comme les vierges insensées (v. 5) -, mais par leur prévoyance.

Le contexte rédactionnel $\mathrm{mt}^{3}$ de cette parabole est particulièrement approprié, non seulement parce qu'il s'agit d'une parabole eschatologique et qu'il l'intègre dans les unités thématiquement semblables de la source $Q(24,37-51 ; 25,14-30)$, mais surtout parce que l'histoire des dix vierges contient une série de motifs analogues à ceux de l'histoire qui précède. Dans les deux paraboles, il est question du retard de celui qu'on attend, d'un comportement avisé ou insensé, d'un jugement irrémédiable qui provoque la joie des uns, la confusion des autres.

La quasi totalité des exégètes s'accorde à voir dans la version mt de la parabole des dix vierges une allégorie de la venue du Fils de l'homme ${ }^{4}$.

1 BULTMANN, Trad 190-191; KLOSTERMANN, Mt 200; MAISCH, 255 256. Analyse de la formule d'introduction chez JEREMIAS, Gleichnisse 100; LINNEMANN 26-27. 134; STRECKER, Weg 214. La présence de cette formule d'introduction typiquement $\mathrm{mt}$, mais pas nécessairement rédactionnelle, n'exclut pas que, dès l'origine, la parabole ait été conçue comme une parabole du Royaume.

2 BORNKAMM, Verzøgerung 49; BULTMANN, Trad 190-191; JEREMIAS, Gleichnisse 48; KLOSTERMANN, Mt 201; LINNEMANN 133.192-193; SCHWEIZER, Mt 304.

3 Le contexte est rédactionnel dès l'instant où l'on a montré que le cadre de la parabole l'est également - ce qui est le cas - (cf. vv. la.13).

4 Voir p.ex., BONNARD, Mt 359; BORNKAMM, Verzogerung 54-55; BULTMANN, Trad 190-191; GRAESSER 119; HAHN, Hoheitstitel 98-99; JEREMIAS, Gleichnisse 48; KLOSTERMANN, Mt 199; MAISCH 248 249. 254.258; SCHWEIZER, Mt 304-305. Seule LINNEMANN 133.187quoique concédant une correspondance entre certains éléments de l'ima ge et du contenu - nie qu'il y ait allégorie, même au niveau mt. 
L'époux désigne le Christ, les dix vierges sont la figure de la communauté attendant son Seigneur, le retard de l'époux traduit celui de la parousie, sa brusque arrivée l'événement de la fin, le sort réservé aux vierges folles le jugement dernier.

Si cette analyse ne saurait être remise en cause - ne serait-ce qu'à cause du contexte choisi par Mt et de l'application parénétique qu'il ajou te -, la question difficile soulevée par le texte consiste à savoir s'il s'agit d'une parabole du Royaume secondairement allégorisée ${ }^{1}$ ou bien si, dès l'origine, nous avons affaire à une allégorie construite pour répondre au problème posé par le retard de la parousie ${ }^{2}$. Dans ce dernier cas, nous posséderions un témoignage capital sur la manière dont l'Eglise primitive a repensé sa foi dans une situation où la réalité faisait échec à son espé rance eschatologique ${ }^{3}$.

Trois arguments nous font préférer la dernière hypothèse. Tout d'abord, il convient d'être attentif au rôle constitutif que le motif du retard joue dans l'économie du récit. Il ne s'agit pas d'un trait originellement accessoire, puis secondairement mis en relief lors d'une hypothétique allégorisation ${ }^{4}$. Le retard de l'époux décide du sort des vierges, plus précisément il est le ressort dramatique qui permet de révéler la sages se des unes et la folie des autres. Sans le retard de l'époux, la parabole perd sa substance et ne répond plus à la problématique centrale du récit :

1 Ainsi JEREMIAS, Gleichnisse 48-50, à la suite de DODD. Dans la même ligne, voir, p. ex., GRUNDMANN, Mt 515-516, et MAISCH $255-259$.

2 Voir notamment BORNKAMM, Verzögerung 53 -55; GRAESSER, 126 127; HAHN, Hoheitstitel 98-99. BULTMANN, Trad 125.190-191 va dans le même sens, sans exclure absolument une parabole à l'origine.

3 Point bien souligné par BORNKAMM, Verzŏgerung 48-50. 54-55; GRAESSER 119-121. 126-127; LINNEMANN 132-133.

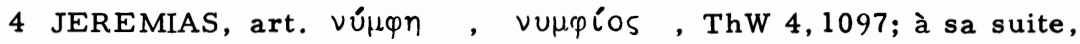
MAISCH 257. Etat de la question, GRUNDMANN, Mt 515-516. 
qui est digne de participer aux noces ${ }^{1}$ ? Le second argument qui nous semble d'importance tient à la distinction entre parabole et allégorie ${ }^{2}$. Une parabole conte un événement qui, certes, peut être exceptionnel, mais qui reste dans les limites du possible; de plus, elle contient en ellemême les conditions de son intelligibilité. L'allégorie, au contraire, est construite en fonction du problème abordé et le développement de l'image ne s'éclaire que par une référence constante au contenu. Or qu'avons-nous sous les yeux ? L'objectivité oblige à un constat surprenant. En effet, la tradition nous dépeint l'histoire d'un mariage plutôt étrange : l'épouse en est absente ${ }^{3}$, l'époux est en retard, les acteurs de la cérémonie s'endor ment, puis, quand enfin la fête peut commencer, le principal responsable du contretemps chasse la moitié des amies de l'épouse. Si l'on ne peut pas niex à priori qu'un tel événement soit possible, on est néanmoins en droit de se demander si l'auteur ne crée pas un pareil enchaînement de circons tances pour illustrer une idée préalable. Le troisième argument qui plaide en faveur d'une composition d'emblée allégorique tient dans l'inexactitude historique du récit. Comme l'a bien montré Bornkamm ${ }^{4}$, ce que nous

1 Ainsi BORNKAMM, Verzögerung 49-5l et, à sa suite, GRAESSER 120. JEREMIAS (Gleichnisse 49-50) voit dans la figure primitive du récit une parabole de crise - crise liée à l'arrivée inopinée de l'époux -. A ceci, il faut répondre qu'à strictement parler, les dix vierges ne sont pas surprises : elles ont tout le temps de se préparer. Ce qui rend leur situation problématique, c'est le retard de l'époux. Par ailleurs, l'image des noces ne suggère pas l'arrivée d'une catastrophe, mais plutôt la joie (GRUNDMANN, Mt 517; MAISCH 257). La proposition de MAISCH (ibid.) qui discerne dans la parabole primitive un appel à être prêt et à accueillir le Royaume comme un enfant ne convainc guère.

2 Voir, p. ex., LINNEMANN 13-18; MAISCH 249-250.

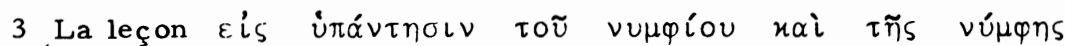
( $D \vartheta \lambda$ it vg sy) est secondaire. Démonstration par JEREMIAS, art.

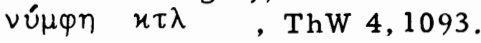

4 Verzögerung 50-51. Cf. aussi BULTMANN, Trad 191 (note 1), GRAES SER 120-121; KLOSTERMANN, Mt 199; matériel rassemblé par BILL 1,504-517, et JEREMIAS, Gleichnisse 171-174. L'état lacunaire de nos sources (JEREMIAS, Gleichnisse 172) n'accrédite ni la crédibilité, ni l'impéritie de la parabole. Mais il demeure que notre parabole 
savons des cérémonies nuptiales dans le bas -judaîsme palestinien s'accorde mal avec les faits évoqués dans la parabole. La coutume n'exige pas que le cortège nuptial aille à la rencontre de l'époux, mais bien que l'époux aille chercher son épouse (cf. vv. 1.6); la cérémonie ne commence pas au milieu de la nuit (ainsi v. 10), mais atteint son point culminant à ce moment; enfin, le retard du futur conjoint n'est pas un élément cons titutif du rite nuptial. La conclusion qui s'impose est claire : dès son origine, notre péricope est une allégorie qui traite du retard de la parousie. Elle se sert de l'image du rite nuptial mais elle l'adapte à son argumentation.

Pour vérifier notre hypothèse, il convient maintenant de montrer que les traits qui s'intègrent mal à la parabole ont leur place dans l'allégorie et qu'ils sont issus de l'attente de la parousie dans le christianisme primitif. Ainsi l'image des noces qui fixe le champ sémantique dans lequel se développe l'histoire, résulte de la façon dont les premières communautés ont conçu la parousie : le Seigneur eschatologique est l'époux qui fêtera ses noces avec sa bien-aimée - l'Eglise - ${ }^{l}$. A l'appui de cette thè -

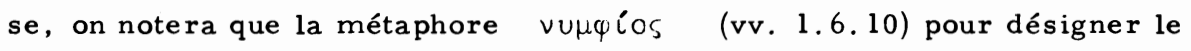
Messie est inconnue de l'AT et du bas-judaísme, mais attestée dans la tradition chrétienne primitive ${ }^{2}$. Avec l'image des noces était donnée celle

raconte un événement peu clair et difficilement comparable avec ce que rapportent les sources juives rabbiniques. Le nouveau matériel publié récemment par JEREMIAS (Gleichnisse 172-173) ne résoud pas la difficulté, car l'élément critique de la parabole - le début de la noce au milieu de la nuit - reste sans parallèle.

1 Cf. Ap 19,7.9; 2 Co 11,2. Avec LINNEMANN 191, il est juste de souligner que le bas-judaĩsme n'utilise pas fréquemment l'image des noces pour décrire les temps messianiques, mais lui préfère celle du festin (BILL 1, 517). Cette constatation ne suffit pourtant pas à mettre en dou te la métaphore des noces chez Mt pour décrire l'accomplissement eschatologique, cf. p.ex., Mt 22,1-14 (en particulier v. 2).

2 Cf. GRAESSER 121; JEREMIAS, art. $\nu$ Ú $\varphi \eta \quad x \tau \lambda$ 1094-1095.10971099, et Gleichnis se 49; LINNEMANN 191. Matériel:2 Co 11,2-3, Ep 5,23 -32; Ap 19,7.9; 21,2.9; pour la tradition synoptique, voir Mc 2, 19b-20 (sur ce point, cf. HAHN, Hoheitstitel 99). 
des vierges, amies de l'épouse. L'effacement surprenant de l'épouse et l'attention portée sur le comportement des vierges s'expliquent dès l'ins tant où l'on saisit que l'allégorie ne vise pas à illustrer les noces elles mêmes, mais à montrer quelles sont les personnes dignes d'y participer ${ }^{1}$. Les vierges symbolisent dès lors les invités et, partant, la communauté. Leur comportement contradictoire souligne l'ambiguité de la réalité ecclésiale ou, en d'autres termes, son statut de corpus mixtum ${ }^{2}$. Le retard de l'époux qui est l'événement crucial dont dépend toute la trame du récit, traduit le retard de la parousie. Seule une interprétation allégorique de

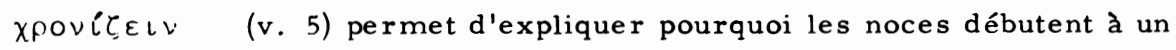
moment inattendu : a milieu de la nuit ( $v$. 6). La tradition chrétienne primitive situe en effet, la venue du Fils de l'homme précisément durant la nuit $^{3}$. Semblablement, le motif de la lumière pour décrire la fidélité des croyants est utilisé dans la parénèse eschatologique; or, dans notre récit, l'accès des vierges à la fête dépend de leur capacité à "faire briller leur lumière" ${ }^{4}$. Il est par ailleurs possible - mais indémontrable - que le cri

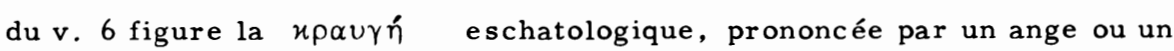
prophète, et qui signale l'irruption de la parousie ${ }^{5}$. Enfin, le trait qui a

1 C'est donc la problématique abordée qui guide l'organisation de l'image (allégorie !). Avec BORNKAMM, Verzögerung 51; GRAESSER 122.

2 Sur ce point, voir notre chap. 14, pp. 381-385.

3 Comme le remarque ironiquement BORNKAMM (Verzögerung 52), l'élément étonnant de la parabole n'est pas tellement que les magasins soient ouverts la nuit, mais qu'une noce débute à ce moment-là. Le retard en lui-même n'implique pas ipso facto pareille conséquence. Ce choix est donc lié au fait que la parousie surviendra la nuit (matériel synoptique : Mc 13,33-37; Lc 12,35-40; Mt 24,42-44; voir aussi $\operatorname{Rm~13,11,1~Th~5,1-6,~Ep~5,14,~Ap~3,3;16,15).~Dans~le~même~sens,~}$ GRAESSER 122; autrement, LINNEMANN 190.

4 Cf. Lc 12,35, l Th 5,4-5 (voir BORNKAMM, Verzögerung 52; GRAES SER 122).

5 Hypothèse émise par BORNKAMM, Verzögerung 53, et développée par GRAESSER 123-125. Cf. Ap 14,15;18,2;19,17. 
constamment intrigué les exégètes, à savoir le cortège des vierges allant au-devant de l'époux, correspond peut-être à une coutume antique reprise de manière imagée dans la tradition paulinienne (1 Th 4,17): de même que les habitants sortaient en cortège de leur ville pour aller à la rencontre de leur maître et faire ainsi acte de soumission, semblablement les croyants à l'heure de la parousie iront-ils accueillir leur Seigneur ${ }^{1}$. Pareille représentation, même si elle est unique chez Mt, expliquerait du moins pourquoi l'époux du début de la parabole devient dans les vv. 11 12 le Kyrios eschatologique exerçant son pouvoir judiciaire ${ }^{2}$. L'inventaire que nous venons de dresser montre, nous semble-t-il, que l'allégorie des dix vierges est nourrie de la réflexion et, partant, des représentations que la communauté post-pascale a utilisée pour témoigner de l'attente de la parousie. L'originalité de notre texte est d'avoir ordonné ces divers motifs dans un procès narratif dont l'unité de composition est donrée par l'image des noces ${ }^{3}$.

1 A la suite de PETERSON 682-702, BORNKAMM (Verzögerung 52 -53),

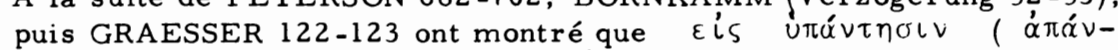

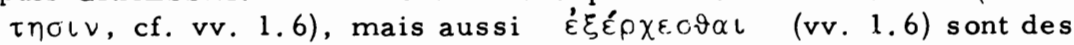
termes techniques qui, dans l'Antiquité, désignent la soumission juridique d'une ville à son nouveau maître ou à ses représentants; cette soumission est illustrée par le geste du cortège d'accueil. L'usage symbolique de cette coutume expliquerait l'impéritie de notre récit : le cortège des vierges à la rencontre de l'époux serait la figure du cortège des croyants allant à la rencontre du xúplos lors de la parousie. L'objection de LINNEMANN 190-191 qui relève que ce cortège est terrestre alors que le festin messianique est céleste (d'où incohérence !), ne porte pas. Dans 1 Th 4,17 , p.ex., les croyants sont enlevés sur des nuées.

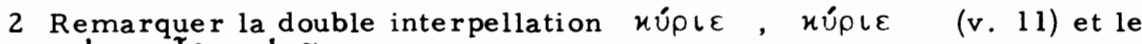

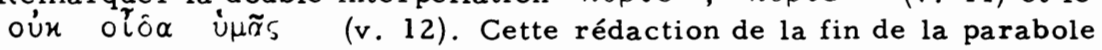
rappelle en particulier $7,21-23$ où la comparution eschatologique des faux prophètes est décrite dans des termes semblables $(7,21 \mathrm{~s}: x u ́-$

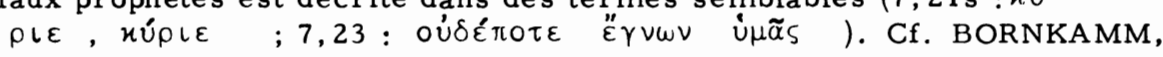
Verzögerung 53; GRAESSER 125; MAISCH 248. 258.

3 L'essai de STROBEL qui tente de rapporter tous les motifs de la parabole à la théologie de la Pâque n'emporte pas l'adhésion. Réfutation détaillée chez LINNEMANN 192. 
La formule introductive ( $v$. la) ne compare pas le Royaume des cieux avec les dix vierges, mais avec l'ensemble de l'action qui suit ${ }^{l}$. Or la trame de l'histoire vise à montrer que toutes ne participeront pas aux noces eschatologiques, mais que, lors de la rencontre décisive avec le Seigneur, les unes révéleront leur intelligence, les autres leur folie (v. 2 : couple antithétique próvluos - uupós ). En quoi consiste alors l'intelligence des cinq vierges et la folie des cinq autres ? Toutes en effet sont unies par un même dessein - aller à la rencontre de l'épouxet toutes prennent des lampes ${ }^{2}$ afin d'accueillir comme il convient ( $v$. $1 \mathrm{~b}$ ) celui qu'elles attendent. La différence qui les sépare et qui va prendre une importance capitale tient dans le fait que les unes se munissent de fioles d'huile ${ }^{3}$ en prévision d'un éventuel retard alors que les autres omettent de le faire (vv. 3-4). En d'autres termes, les unes envisagent leur condition d'invitées et la mission qui en dépend, dans la durée, alors que les autres vivent dans l'insouciance de l'instant, sans envisager l'ave nir et sans se préparer en conséquence. Le retard imprévisible de l'époux provoque la crise qui va perdre les unes et tourner à l'avantage des autres (v. 5a). Le motif du sommeil ( $v$. 5b) souligne l'ampleur du retard, mais aussi le caractère irréversible des dispositions prises. Car lorsqu' enfin retentit le cri annonçant l'arrivée de l'époux et que chaque vierge est mise en demeure de former le cortège d'accueil ( $v .6)$, toutes - bien que le désirant - ne sont plus en mesure de le faire. Le dialogue des vv. 8 -9 n'a aucun accent moralisant ou ironique ${ }^{4}$; il illustre simplement qu'en

1 Voir JEREMIAS, Gleichnisse 100.174, suivi par GRUNDMANN, Mt 516 -517; LINNEMANN 133; MAISCH 255-256; SCHWEIZER, Mt 305.

2 Il est difficile de savoir exactement ce que signifie $\lambda \alpha \mu \pi \alpha ́ s$ dans notre texte (JEREMIAS, art. $v u ́ \mu \varphi \eta x \tau \lambda$, ThW 4,1093). S'agit-il de torches alimentées à l'huile (JEREMIAS, Gleichnisse 174) ou de lampes à huile montées sur un bâton (GRUNDMANN, Mt 517; là état de la question)?

3 'Arrẽ̃ov (v. 4) désigne une petite cruche à col étroit (JEREMIAS, Gleichnisse 174).

4 Avec KLOSTERMANN, Mt 201; LINNEMANN 131; MAISCH 256; SCHMID Mt 344; SCHWEIZER, Mt 305. 
cet instant capital, il est trop tard pour changer quoi que ce soit. Dans la surprise du réveil, chaque vierge se révèle telle qu'elle est en vérité ${ }^{l}$. Les unes sont en mesure d'accomplir leur mission, elles sont dignes de l'invitation reçue et participent à la fête. Les autres ne sont pas prêtes, elles font défaut et se révèlent indignes de l'honneur qui leur avait été fait. Les vv. 11-12 montrent que cette défection est irréparable.

Le thème du retard de l'époux qui domine le récit, ainsi que celui de la prévoyance des vierges avisées, attestent que la parousie est toujours au centre du propos; mais la perspective est différente de celle des paraboles précédentes ${ }^{2}$. Si 24,37-44 insistait sur la venue inopinée du Fils de l'homme et sur la nécessité de comprendre son existence en fonction de cet événement constamment possible, si la parabole du serviteur (24, 45-51) dénonçait comme une erreur coupable le fait de spéculer sur l'absence du maître, la parabole des dix vierges souligne la nécessité vitale de prendre acte de ce retard et d'organiser sa vie en conséquence. La communauté dans laquelle cette parabole a été conçue, non seulement constate le retard de la parousie, mais elle repense sa foi en fonction de cette situation nouvelle. Sur ce point, la parabole des dix vierges se dis tingue de la fin du chap. 24 ( $\mathrm{vv} .37-5 \mathrm{l}$ ) qui, tout en admettant le retard du Seigneur dans 1 'accomplissement de sa promesse, invitait les croyants à vivre comme si ce retard n'avait pas lieu. La parabole des dix vierges s'oppose à cette conception : vivre comme si la parousie était imminente est le signe de la folie; c'est le fait de gens qui emportent des lampes, mais oublient l'huile; c'est négliger le temps qui est imparti aux croyants, $c^{\prime}$ est se réfugier dans une agitation trompeuse ${ }^{3}$.

La communauté dont les membres doivent abandonner l'illusion d'une parousie imminente, ne doute pourtant ni de la promesse de son Seigneur,

1 Point bien souligné par BONNARD, Mt 359-360; GRAESSER 127.

2 BORNKAMM, Verzogerung 49-50; GRAESSER 119-120.

3 Même situation en $2 \operatorname{Th} 2,2 ; 3,11-12$. Voir BORNKAMM, Verzögerung 55; LINNEMANN 133; SCHWEIZER, Mt 306. 
ni de l'à-venir de Dieu, Au contraire, parce qu'elle entend s'ouvrir lucidement à cet à-venir, parce qu'elle y discerne une promesse - mais aussi une menace -, parce qu'elle sait que, malgré ce retard, il faut redouter un irréparable "trop tard", elle invite les chrétiens à aborder l'avenir dans la vigilance (v, 13). Etre vigilant ne signifie pas guetter sans relâche les signes de la fin, mais accepter de vivre dans la durée. Etre vigilant signifie saisir l'existence chrétienne dans son devenir et la placer toujours à nouveau sous le signe de la vocation reçue, mais aussi de la res ponsabilité qu'elle implique.

\section{Conclusion}

La parénèse de la vigilance $(24,37 ; 25,13)$ atteste de manière irrécusable que le rédacteur du premier évangile s'est posé la question de l'avenir. Le recours au genre parénétique indique que la réflexion $\mathrm{mt}$ est développée à l'intention de l'existence croyante. Ce qui conditionne toute l'argumentation, c'est le retard de la parousie. Si la fin du chap. 24 convient de ce retard en affirmant que la date de l'eschaton est inconnue, le début du chap. 25 admet explicitement l'ajournement intervenu. Cette différence d'a'ppréciation donne lieu à des exhortations distinctes. Si le complexe traditionnel que $M t$ a emprunté à $Q$, recommande de vivre comme si le Fils de l'homme pouvait revenir à chaque instant, la parabole des dix vierges ( $\mathrm{Smt}$ ) tient compte de l'histoire qui se prolonge. Cependant, que l'on se place dans l'une ou l'autre optique, la question du délai - voire du calcul des temps - est désormais caduque ${ }^{l}$. On peut même affirmer que le retard de la parousie en tant que tel n'est pas ressenti comme un démenti apporté à la foi; les difficultés qu'il soulève, tiennent plutôt dans

1 STRECKER, Weg 242, écrit à ce propos : "Sowohl die Nah-als auch die Fernerwartung haben in der matthaischen Konzeption Platz. Uebergeordnet ist, dass die Gemeinde auf ihrem Weg stets mit der Möglichkeit des Endes rechnet...". 
les conséquences aberrantes que certains croyants peuvent en tirer : soit qu'ils négligent d'inscrire leur foi dans le quotidien en arguant d'un re tour imminent du Christ, soit qu'ils prennent prétexte du retard survenu pour se soustraire à leur responsabilité. L'exhortation à la vigilance dénonce l'une et l'autre erreur et invite à s'en dégager.

Si Mt prend acte du retard de la parousie, il n'élimine en aucune façon l'attente de la fin. Le cadre temporel est maintenu. L'accomplissement eschatologique reste un thème central de sa théologie. On pourrait même dire - cum grano salis - que l'ajournement de la venue du Fils de l'homme est le modèle qui lui permet de proclamer l'à-venir de Dieu dans toute sa radicalité. A cet égard, l'importance de plus en plus grande que la thématique du jugement prend au fil des chap. 24-25 est significative. Toute spéculation a pocalyptique étant désormais exclue, cet à-venir de Dieu pour le jugement renvoie le croyant à lui-même, et singulière ment, au présent vécu. Il l'invite à placer hic et nunc son existence sous l'autorité de son Seigneur. Sur ce point, on remarquera que, malgré une perspective théologique différenciée, 24,37-51 et 25,1-13 se rejoignent dans leur compréhension de l'existence chrétienne : c'est ici et maintenant, dans sa fidélité ou son infidélité à son Seigneur que le croyant joue son avenir; c'est dans la foi vécue qu'il le gagne, c'est dans la foi reniée qu'il le perd. La vigilance consiste, en définitive, à vivre dans la pers pective jamais oubliée de l'à-venir du Kyrios. Mais comment le croyant peut-il accueillir et assumer toujours à nouveau l'à-venir de Dieu dans le devenir de son existence? Cette question dépasse la problématique de l'avenir comme tel et nous place devant le problème de l'éthique. C'est l'objet de notre cinquième partie. 
C I N Q I E M E P A R T I E

LE CROYANT ET L'ETHIQUE 
La question éthique est au coeur du premier évangile. En effet, Mt présente le Christ comme l'interprète eschatologique de la volonté de Dieu. Il insiste sur le fait que la seule réponse possible à l'enseignement de Jésus est l'obéissance. L'existence du disciple est donc placée sous une exigence absolue.

Dans les trois chap. qui suivent, nous n'entendons pas écrire une "éthique matthéenne"; notre ambition est plus limitée : Montrer comment la question éthique se pose pour le croyant. Dans ce but, il nous paraît important de préciser tout d'abord les rapports établis par Mt entre l'eschatologie et l'éthique; les béatitudes (Mt 5,3-10) nous permettront de décrire cette articulation théologique qui constitue le point de départ de l'éthique. Nous aborderons ensuite le contenu de l'obéissance exigée du disciple; ce contenu, qui constitue le thème de l'éthique, sera explicité par l'exégèse de la sixième antithèse (Mt 5,43-48). Enfin, nous nous demanderons quel est le rôle spécifique de l'éthique dans l'économie globale de l'existence croyante; Mt 25,31-46 nous fera découvrir en quoi l'éthique est la vérification critique de la foi vécue dans la profanité du monde.

\section{Tradition et rédaction:}

La version matthéenne des béatitudes $(5,3-12)$ est fort différente de celle offerte par $\mathrm{Lc}(6,20 \mathrm{~b}-23)$. Alors que le troisième évangile rapporte quatre béatitudes suivies de quatre malédictions, Mt nous présente une série de neuf béatitudes. Les quatre béatitudes connues de Lc figurent aussi chez Mt, mais dans un ordre différent. 'Le premier évangile situe la béatitude des affligés avant celle des affamés; Lc procède de manière 
inverse.

A ces différences tout à fait fondamentales s'ajoutent des variations dans la formulation des quatre macarismes communs. Si, dans la béatitude des pauvres, Lc parle des $\pi \tau \omega \chi 0 i \quad(6,20 \mathrm{~b})$, Mt fait état des $\pi \tau \omega \chi \circ i \tilde{\Psi} \pi v \varepsilon u ́ \mu \alpha \tau \iota \quad 2$. Dans la béatitude des affamés, les $\pi \varepsilon \iota \nu \tilde{\omega} \nu \tau \varepsilon S$ de Lc $(6,21 a)$ deviennent chez Mt $(5,6)$ les $\pi \varepsilon \iota \nu \tilde{\omega} \nu \tau \varepsilon S$ Kai

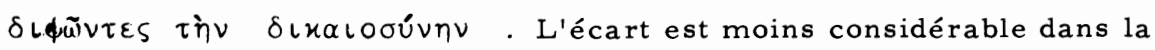
béatitude des affligés : si Lc utilise le couple $x \lambda \alpha i \varepsilon \iota \nu-\gamma \varepsilon \lambda \tilde{\alpha} \nu(6,21 b)$, Mt emploie $\pi \varepsilon \nu \vartheta \varepsilon \tau \nu-\pi \alpha \rho \alpha \varkappa \alpha \lambda \varepsilon \tau \nu \quad(5,4)$. Par contre la différence est si grande entre les versions $m$ t et lc de la béatitude des persécutés (Mt 5,11-12 par. Lc 6, 22-23) que la comparaison est malaisée. Signalons enfin que les béatitudes lc sont à la 2e personne du pluriel tandis que Mt utilise la 3 e personne (exception : 5,11-12); que le vũv lc $(6,21 a .21 b)$ manque chez $\mathrm{Mt}$.

Les versions $m t$ et $1 c$ des béatitudes dépendent de la source $Q^{3}$. Quel est sur ce point précis le contenu de $Q$ et quelle réinterprétation les béatitudes ont-elles subie chez Mt et chez Lc?

1 Il est aléatoire de conjecturer qui de Mt ou de Lc a conservé l'ordre primitif des béatitudes dans Q. SCHULZ, Q 76 opte pour Lc; DUPONT, Béatitudes I 271-272 se décide pour Mt. En tout état de cause, le texte $\mathrm{mt}$ semble plus retravaillé et l'ordre des vv. 3-4 est une réminiscence d'Es 61,1-3 alors que Lc ne trahit aucun principe de construction.

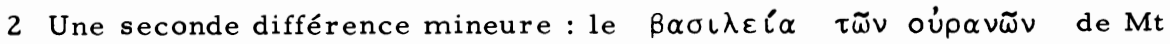

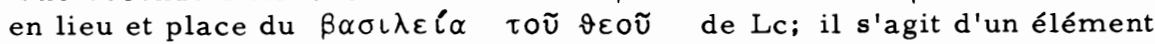
caractéristique de la terminologie du ler év. souvent remarqué, mais sans importance théologique particulière (voir, p. ex., DUPONT, Béatitudes I, 207-210).

3 Ainsi la quasi totalité des exégètes. Remarquons simplement à l'appui de cette hypothèse que tant chez Mt que chez Lc, les béatitudes constituent l'exorde d'un même discours fondamental qui se conclut chez les deux par la parabole des deux maisons. 
Envisageons tout d'abord les quatre macarismes communs à Mt et à Lc. Dans la béatitude des pauvres, le $\tau \tilde{\psi} \pi \nu \varepsilon u ́ \mu \alpha \tau \iota$ mt est de l'avis unanime une élucidation didactique du rédacteur ${ }^{1}$; semblablement le

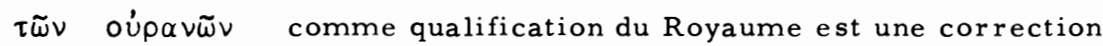
typique à mettre au compte de la communauté $m t(Q m t)$. Dans l'ensemble la version de Lc est donc vraisemblablement plus proche de Q. Il n'est par contre pas aisé d'établir qui de $\mathrm{Mt}$ ou de Lc a le mieux conservé la formulation primitive de la béatitude des affligés; l'analyse de la terminologie inclinerait sous toute réserve à opter pour $\mathrm{Lc}^{2}$. Le cas de la

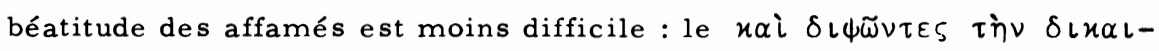
ooúvnv mt est vraisemblablement rédactionnel, car, d'une part,

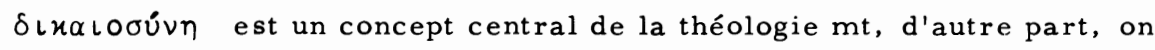
s'expliquerait mal pourquoi Lc aurait supprimé cette séquence ${ }^{3}$. Ici encore, le troisième évangile est plus proche de $Q$. La béatitude des

1 Ici aussi le consensus est réalisé (références détaillées chez DUPONT, Béatitudes I 216-17 et SCHULZ, Q 77). L'argument décisif dans la discussion est fort simple : si une adjonction de la part de Mt - d'ailleurs conforme à son souci pédagogique - se conçoit fort bien, une suppression de la part de Luc qui refuserait ainsi le sens premier de la béatitude pour l'infléchir dans une toute autre direction, est peu vraisemblable.

2 Les avis sont partagés et aucun argument vraiment décisif ne peut être produit. DUPONT, Béatitudes I 266-271, et STRECKER, Macarismes 195-96 accordent la priorité à Mt, tandis que SCHULZ, Q 77-78 et SCHWEIZER, Mt 46-47 voient dans Lc le texte primitif. Constatons néanmoins que ni $\gamma \varepsilon \lambda \tilde{\alpha} \nu$, nı $x \lambda \alpha i \varepsilon \iota \nu$ n'appartiennent à la terminologie typiquement lc alors que Mt utilise $\pi \varepsilon \nu \vartheta \varepsilon \tau \nu$ de manière rédactionnelle en 9,15 . Peut-être le rédacteur ou Qmt ont-ils introduit le couple $\pi \varepsilon \nu \vartheta \varepsilon \tilde{\tau} \nu / \pi \alpha \rho \alpha \varkappa \alpha \lambda \varepsilon \tau \nu$ sous l'influence d'Es 61,2 .

3 Ici aussi le consensus nous semble réalisé. Voir parmi les études récentes: BARTH 116; DUPONT, Béatitudes I 218-223; SCHULZ, Q 77; STRECKER, Macarismes 197; WALTER 249. Certes DUPONT et STRECKER se montrent sceptiques quant à la provenance rédactionnel-

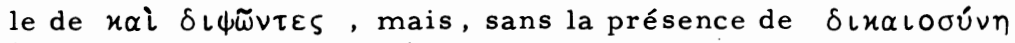
(assurément rédactionnel), cette séquence n'a aucune justification. 
persécutés a été redoublée dans le premier évangile. Mt, en effet, nous en présente deux : celle du v. 10 et celle des vv. 11-12. La béatitude du v. 10 est probablement rédactionnelle, car, à part oi $\delta \varepsilon \delta \iota \omega \gamma \mu \varepsilon ́ v o \iota$

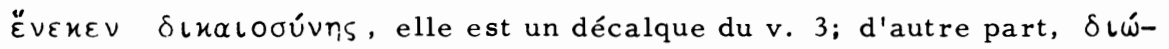
$x \omega$ et $\delta\left\llcorner x \alpha \iota\right.$ เớun sont des termes-clefs de la théologie mt ${ }^{1}$. Par contre, la béatitude des persécutés provenant de $Q$ n'est pas de la même veine que celles qui précèdent : elle est beaucoup plus longue, elle présuppose une situation historique fort différente (la persécution de la communauté) et, pour la première fois, Mt utilise la deuxième personne ${ }^{2}$. Bien que le rédacteur l'ait trouvé dans la liste $Q$ des béatitudes, ce macarisme appartient à une autre couche traditionnelle que les vv. 3-10, il dénote une autre vision théologique. Nous le mettons provisoirement entre parenthèses et concentrons notre attention sur les vv. 3-10. Le procédé d'inclusion, utilisé par Mt, pour encadrer les vv. 3-10 ( ŏ $\iota \alpha u \dot{\tau} \tau \tilde{\omega}$

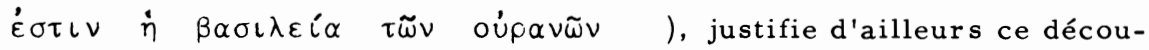
page.

Quant à l'origine des quatre béatitudes propres à Mt (vv. 5.7.8.9), trois hypothèses sont possibles. Ou bien elles figuraient dans la source $Q$ et Lc les a omises; ou bien elles ont été ajoutées à la liste initiale dans le milieu mt qui transmettait $Q^{3}$; ou encore elles sont l'oeuvre du

1 Ici encore l'accord est large. Parmi les études récentes, optent pour la composition rédactionnelle : BARTH 95; EICHHOLZ 31; KOCH 47; STRECKER, Macarismes 192.200-201; WALTER 247; DUPONT, Béatitudes I 223-227, tout en étant favorable à l'idée, laisse la question ouverte.

2 Voir à ce sujet : BULTMANN, Trad 115; KOCH 48-49; EICHHOLZ 31; SCHULZ, Q 452-54; STRECKER, Macarismes 202-203.

3 Cette hypothèse est défendue par BARTH 115; EICHCHOLZ 32; KOCH 46-49 (qui réserve néanmoins l'origine du v. 7); STRECKER, Macarismes 190-191. Voir aussi les commentaires de GRUNDMANN, SCHNIEWIND et SCHWEIZER. Dans son art. "Zum traditionsgeschichtlichen Problem der Seligpreisungen", BRAUMANN tente d'éclairer l'origine de cette tradition particulière; il montre que la terminologie des vv. 5.7.8. 9 est fort proche de celle utilisée en $1 \mathrm{P}$ et ressortit en priorité à la prédication chrétienne primitive. Son "Sitz im Leben" en serait la théologie baptismale. 
rédacteur du ler évangile ${ }^{1}$. La première hypothèse est bien improbable, car on voit mal ce qui aurait conduit Lc à biffer ces quatre béatitudes dans sa source. Par contre, le choix entre la deuxième et la troisième hypothèse est difficile; la terminologie et la méthode de composition du rédacteur inciteraient plutôt à préférer la seconde ${ }^{2}$.

Reste le problème des malédictions qui suivent les béatitudes chez Lc. S'agit-il d'une omission mt ou d'une adjonction lc ? Comme le fait remarquer Dupont ${ }^{3}$, les sutures des v. 24 ( $\left.\pi \lambda \dot{\eta} \nu\right)$ et v. 27 ( $\dot{\alpha} \lambda \lambda \dot{\alpha} \dot{u} \mu \tau \nu$

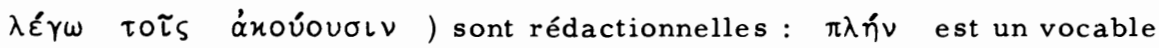
typiquement lc qui permet de relier les béatitudes et les malédictions; la séquence introduite par $\dot{\alpha} \lambda \lambda \alpha^{\prime}$ au v. 27 rétablit l'auditoire indiqué au v. 20a, auditoire qui ne convient pas pour les malédictions. Cette double suture nous incline à penser que c'est Lc qui a inséré les malédictions des vv. 24-26 pour faire pendant aux béatitudes ${ }^{4}$.

Concluons : la source $Q$ connaissait quatre béatitudes (celles des pauvres, des affligés, des affamés, des persécutés, soit Mt 5, 3.4.6.11-12); le milieu $\mathrm{mt}$ en a ajouté quatre autres (Mt 5,5.7.8.9). Le rédacteur, quant

1 Cette provenance rédactionnelle des vv. 5.7.8.9 est soutenue par DUPONT, Béatitudes I 251-263; FRANKEMOELLE 73-74; WALTER 247-249.

2 Avec STRECKER, Macarismes 190-191, il faut relever que les vv. 7-9 utilisent une terminologie et des représentations fort originales dans le cadre du premier évangile et que, par ailleurs, le rédacteur "rédige et compose plutôt que de créer des traditions". (191)

3 DUPONT, Béatitudes I 299-342.

4 Partagent ce point de vue : LUEHRMANN, Logienquelle 54; WALTER 249-250; WREGE 5-27. Autrement, FRANKEMOELLE 64-66. On peut ajouter l'argument suivant : la liste primitive des béatitudes lc $(6,20-21)$ est séparée des malédictions $(6,24-26)$ par la béatitude des persécutés $(6,22-23)$ qui n'appartient pas à la liste primitive et qui est venue s'y greffer ultérieurement. 
à lui, a composé la neuvième béatitude (v. 10), et il a retouché les vv. 3

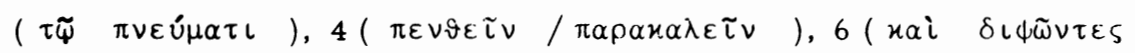

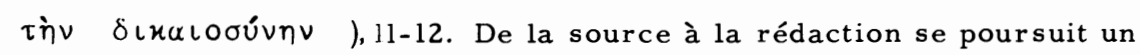
important développement - voire une profonde réinterprétation -. Peuton discerner une cohérence dans ce processus?

\section{L'accentuation de l'éthique:}

Au stade de la source $Q$, les béatitudes s'adressent aux pauvres, aux affligés, aux affamés. Les destinataires sont donc des hommes qui vivent concrètement dans la détresse 1 . A ces gens tombés dans le malheur, les béatitudes annoncent un bonheur totalement inattendu : la bonne nouvelle du Royaume; elles proclament le bonheur eschatologique.

Dans la couche suivante de la tradition ( $\mathrm{Qmt}$ ), les béatitudes s'adressent aux doux $(5,5)$, aux miséricordieux $(5,7)$, aux coeurs purs $(5,8)$ et à ceux qui font oeuvre de paix $(5,9)$. L'accent change : ce n'est plus la détresse comme telle qui caractérise les destinataires des béatitudes, mais un certain comportement éthique ${ }^{2}$.

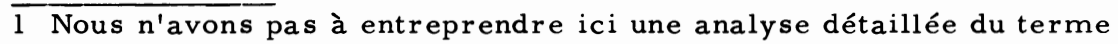
$\pi \tau \omega \chi \delta_{S}$, ni à retracer son histoire dans le monde vétérotestamentaire-juif. A l'appui de notre interprétation, nous invoquons deux arguments. D'une part, en référence au contexte, $\pi \tau \omega \chi \sigma_{\varsigma}$ est élucidé par les verbes $x \lambda \alpha i ́ \omega$ et $\pi \varepsilon \iota v \alpha ́ \omega$ qui, tous deux, font allusion à une détresse immédiate et matérielle. D'autre part, la tradition synoptique la plus ancienne nous dépeint un Jésus se tournant vers les membres les plus défavorisés de son peuple et non vers une élite religieuse (p. ex. les anawim); cette sollicitude pour le am ha-aretz apparaît très clairement, $p$. ex. en $\mathrm{Mt} 9,9-13 ; 11,18-19$. Cette exégè se "sociologique" de $\pi \tau \omega \chi \sigma \varsigma$ n'exclut nullement une implication religieuse, à savoir que seuls les plus défavorisés sont disposés à tout attendre de Dieu; elle soutient uniquement que cette dernière composante est secondaire et nullement constitutive. Partagent notre point de vue : BILL 1, 190; BRAUN, Radikalismus II 73; GRUNDMANN, Mt 122; SCHULZ, Q 81-82; SCHWEIZER, Mt 46-50; STRECKER, Macarismes 193-194; WALTER 254.

2 Cette opinion est largement partagée. Parmi les études récentes, voir : BARTH 116; DUPONT, Béatitudes I 260; EICHHOLZ 44; FRANKEMOELLE 55; STRECKER, Macarismes 199; WALTER 256. 


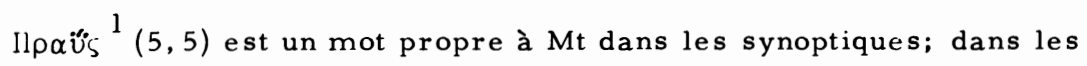
deux autres passages où il apparaît, il s'applique au Christ (11, 29; 21,5). La douceur du Christ consiste dans son abaissement et sa faiblesse, qui sont l'expression de son obéissance à la volonté de Dieu ${ }^{2}$. Cet abaissement assumé par le Maître jusqu'à la Croix - et non subi - devient un paradigme proposé au croyant pour lui indiquer la voie qui mène au bon-

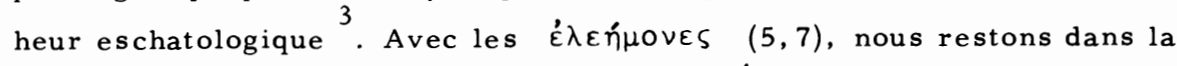
même perspective. Chez Mt, la miséricorde ${ }^{4}$ est au centre même de la Loi, elle est ce qu'il y a de plus essentiel dans la volonté de Dieu. Notre béatitude loue donc ici celui qui accomplit l'essentiel de l'exigence divine ${ }^{5}$. Les coeurs purs ${ }^{6}(5,8)$ pourraient, comme dans l'AT, désigner simple-

1 La majorité des commentateurs rapprochent la béatitude des doux du

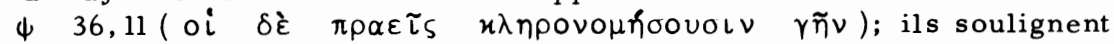
également sa quasi synonymie avec la béatitude des pauvres (p. ex. DUPONT, Béatitudes I 251-257). Est "doux" le faible, l'opprimé celui qui n'a aucun moyen de faire triompher son droit (voir les commentaires de BONNARD, EICHHOLZ, SCHMID, SCHNIEWIND, mais aussi

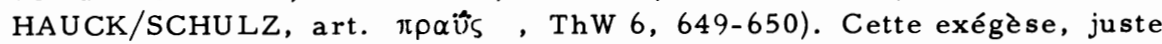
en elle-même, néglige cependant la dimension éthique et volontariste du concept : non seulement le doux est privé de son droit, mais il renonce à le faire prévaloir et ceci par obéissance à la volonté de Dieu.

2 Cf. BARTH 122.

3 Ainsi GRUNDMANN, Mt 126, mais surtout STRECKER, Macarismes 197 qui assimile $\pi \rho \alpha \hat{u} s$ à $\tau \alpha \pi \varepsilon \iota \nu \delta \zeta s$. SCHWEIZER, Mt 52, souligne le fort accent catéchétique de la béatitude.

4 Cette place centrale de la miséricorde dans la Loi est attestée par les deux citations rédactionnelles d'Os $6,6(9,13 ; 12,7)$ et par 23,23 ( $\tau \grave{\alpha}$

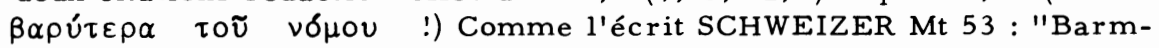
herzigkeit ist für Matthaüs die Mitte der Verkündigung Jesu, die zeigt, was Erfüllung des Gesetzes heisst". Voir aussi GRUNDMANN, Mt 128.

4 Remarquons que le v. 7 est la seule béatitude qui soit présentée sous la forme d'une proposition de droit sacré ! Cette reprise du schéma du ius talionis eschatologique souligne bien l'urgence de l'obéissance.

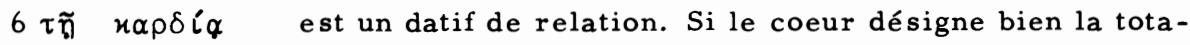
lité de la personne en tant qu'elle se décide et qu'elle agit, le sens de l'expression est alors le suivant : est un "coeur pur" celui qui agit de manière non partagée et sans arrière-pensée, celui qui est avec autrui dans un rapport de transparence et de simplicité (voir les commentaires de BONNARD, GRUNDMANN, SCHNIEWIND, SCHWEIZER). 
ment les hommes pieux ( Ps 24,$4 ; 51,12 ; 73,1)$. Cependant cette explication néglige le grand débat sur la pureté qui culmine dans la cinquième malédiction du chap. 23. La pureté extérieure, rituelle et cultuelle, est critiquée au nom de la pureté intérieure, qui est consécration résolue et sans partage à Dieu. Pour Mt, la pureté est donc une attitude de vie globale ${ }^{l}$. Dans la septième béatitude $(5,9)$, l'exigence éthique est évidente : la réconciliation est une tâche de la vie chrétienne ${ }^{2}$.

Nous constatons dans la source Q - et son développement en Qmt - une perspective cohérente, perceptible notamment au niveau des destinataires des béatitudes. En va-t-il de même à l'échelon rédactionnel ? Pour répondre à cette question, il convient d'examiner les corrections opérées aux vv. 3.6 et la composition du v. 10. Nous avons déjà remarqué qu'au v. 3 , le rédacteur précise le sens de $\pi \tau \omega \chi \sigma_{\zeta}$ en y adjoignant l'expression $\tau \tilde{\Psi} \pi \nu \varepsilon u ́ \mu \alpha \tau \iota^{3}$. Il supprime ainsi la plurivocité potentielle du concept et ne conserve que sa dimension spirituelle au détriment de sa composante sociale ${ }^{4}$; seule la pauvreté intérieure, l'humilité, permet d'accéder au

1 Cet accent éthique est souligné notamment par HAUCK, art. $x \alpha \vartheta \alpha \rho \delta \varsigma$, ThW 3, 428 et STRECKER, Macarismes 199-200. Notons par ailleurs, avec WREGE 26 que notre expression appartient à la parénèse chrétienne primitive (cf. $1 \operatorname{Tm~} 1,5 ; 2 \operatorname{Tm} 2,22 ; 1 \mathrm{P} 1,22)$.

2 Sur l'enracinement juif-rabbinique de l'expression, voir FOERSTER,

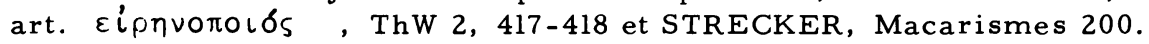

$3 \tau \tilde{\Psi} \pi \nu \varepsilon \cup ́ \mu \alpha \tau$ est un datif de relation si bien que la pauvreté dont il est parlé au v. 3 se rapporte au $\pi \nu \varepsilon \tilde{u} \mu \alpha$ (et non aux richesses terrestres!). L'esprit ne désigne ni le Saint-Esprit, ni l'intelligence, mais le tout et le centre de la personne (voir TOB $52 \mathrm{~d}$ ); il a donc un sens anthropologique (cf SCHWEIZER, art. $\pi \nu \varepsilon \tilde{u} \mu \alpha$, ThW 6, 398-399, et STRECKER, Macarismes 194-195). Est pauvre en son esprit celui qui se découvre et se reconnaît faible et démuni.

4 La plupart des commentaires (BILL 1, BONNARD, GRUNDMANN, RADEMAKERS II, SCHLATTER, SCHMID, SCHNIEWIND) définissent la pauvreté (en esprit) sans distinguer les différents auditoires auxquels la béatitude a été adressée au cours de sa transmission. La correction $\tau \widetilde{\varphi} \pi \nu \varepsilon u ́ \mu \alpha \tau \iota$ n'est dès lors pas interprétée en fonction d'un auditoire ecclésial, mais de manière théorique; semblablement, les diffé-

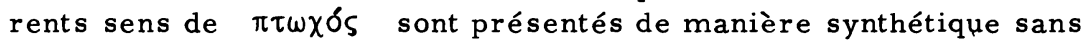


Royaume. Par pauvreté intérieure, il faut entendre l'attitude de l'homme qui découvre sa faiblesse, son incapacité et son dépouillement devant Dieu et qui, partant, attend tout de Lui ${ }^{l}$. L'adjonction $x \alpha i \quad \delta\llcorner\psi \tilde{\omega} \nu \tau \varepsilon \varsigma$ Tìv $\delta \iota x \alpha \iota$ ooúviv (v. 6) va dans la même direction : d'une détresse tout-àfait concrète - la faim -, Mt fait une exigence spirituelle. L'homme qui "a faim et soif de justice" est celui qui désire passionnément un bien spirituel sans lequel il ne saurait vivre ${ }^{2}$. Mais quelle est cette justice tant recherchée ? Comme le montre le contexte du Sermon sur la Monta gne $(5,10.20 ; 6,1.33)$, il s'agit de l'établissement du droit de Dieu sur la terre. Même si cette instauration de la volonté divine se réalise par l'enseignement et l'oeuvre du Christ, il n'en reste pas moins qu'elle est proposée aux disciples comme une tâche urgente et impérative. Dès lors, au niveau de la rédaction $\mathrm{mt}$, l'homme qui a faim et soif de justice n'est pas tellement celui qui aspire à la recevoir de Dieu comme un don, mais bien celui qui s'applique de manière active et efficace à la réaliser ${ }^{3}$.

égard à une éventuelle variation de sens dans l'histoire de la tradition mt. Pour notre part, nous pensons que l'adjonction $\tau \tilde{\varphi} \pi \nu \varepsilon \dot{u} \mu \alpha \tau \iota$ trahit un glissement de sens dans l'application du thème de la pauvreté : ce n'est plus une situation de détresse économique, sociale et religieuse qui est d'abord visée, mais bien la personne intérieure.

1 Avec BARTH 115 où le lien avec l'ensemble de la théologie mt est bien marqué. Voir aussi dans la même direction : EICHHOLZ 33-35 ("... So enthält der Makarismus...das Ja des Menschen zu seiner Lazarusexistenz als seiner wahren Existenz vor Gott", 34); SCHNIEWIND, Mt 41.

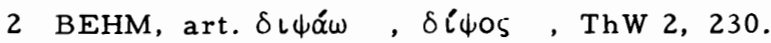

3 Pour des raisons à la fois théologiques et dogmatiques, l'interprétation éthique que nous donnons de la $\delta \iota$ h looúvn $\mathrm{mt}$ est fortement contestée. Nombre d'auteurs (BARTH 116; BILL 1, 201; EICHHOLZ 39-44; SCHLATTER, Mt 137; SCHNIEWIND, Mt 44; SCHRENK, art. Sıxa ८Ooúvn, ThW 2, 200) y discernent une réduction légaliste et moralisante de l'évangile; pour eux, la $\delta\llcorner$ L เooúvn du v. 6 est avant tout la justice de Dieu, initiative souveraine et gracieuse, qui justifie gratuitement le croyant et dont la victoire sera définitivement consacrée lors de la consommation eschatologique. Cette manifestation de l'initiative et de la grâce divine, coeur de l'évangile, est certes présente chez $\mathrm{Mt}$, mais ne se cristallise pas dans le concept de justice comme le montre son emploi constamment rédactionnel (cf. 3,15; 5,10.20;6,1.33; 21,32). 
Le v. 10 qui est une création de Mt, confirme indiscutablement notre interprétation éthique de la $\delta \iota x \alpha \iota$ coúv : les persécutés sont déclarés heureux en raison d'une conduite déterminée et, en définitive, à cause de leur fidélité concrète de la Loi de Dieu. Le travail rédactionnel témoigne donc, lui aussi, d'une cohérence remarquable : l'accentuation éthique apparaît à la fois comme le principe d'interprétation (vv. 3.6) et de composition (v. 10).

L'analyse que nous avons effectuée nous permet de retracer succintement l'histoire de la tradition des béatitudes et de formuler le problème qu'elle pose. Au stade de la source $Q$, les béatitudes annoncent le bonheur eschatologique aux hommes qui vivent dans la détresse. Dans le milieu mt pré-rédactionnel ( $\mathrm{Qmt}$ ), nous constatons un développement substantiel, mais aussi un décalage par rapport aux origines. Ce n'est plus sur la proclamation du salut comme telle que la communauté insiste, mais sur le comportement concret que réclame la venue du Royaume. La promesse devient le motif d'une exigence. L'eschatologie se développe dans un sens éthique. Le rédacteur de l'évangile, quant à lui, a donné son as sentiment à cette réinterprétation éthique en corrigeant les béatitudes les plus anciennes à la lumière des plus récentes. L'ensemble des macarismes dans les vv. 3-10 est ainsi gouverné par une conception théologique assez différente de la conception primitive, mais elle aussi cohérente.

Ce glissement de sens mérite d'être soigneusement interprété. Il ne doit pas aboutir à une alternative simplificatrice, les uns s'efforçant de maintenir le contenu essentiellement kérygmatique des béatitudes en sousestimant leur portée éthique ${ }^{1}$, les autres insistant sur la dimension impé-

Plus équilibrées - quoiqu'avec des nuances diverses - sont les analyses de BONNARD, Mt 57; GRUNDMANN, Mt 126-127; SCHWEIZER, Mt 53 qui soulignent l'implication éthique de la justice désirée. Optent nettement pour l'interprétation éthique : SCHMID, Mt 80 ; STRECKER, Macarismes 197-199; ТОВ.

1 Ainsi, p. ex., très nettement BONHOEFFER 68-77; EICHHOLZ 26-54; SCHNIEWIND, Mt 40-51. 
rative de la réinterprétation $m$ t en oubliant son point de départ indicatif ${ }^{l}$. S'enfermer dans pareil dilemme nous semble provoquer une dangereuse réduction de la réflexion $\mathrm{mt}$.

Certes l'accentuation éthique des béatitudes dans le premier évangile est nette; mais deux faits nous paraissent devoir être rappelés. D'abord qu'il est impossible d'ignorer le fondement christologique des béatitudes et de les séparer de celui qui les prononce ${ }^{2}$. Le rédacteur est clair sur ce point : celui qui parle est revêtu de l'autorité eschatologique $(7,29)$; son enseignement a valeur de révélation $(5,1)^{3}$. Dès lors, l'adhésion à son discours est inséparable de l'adhésion à sa personne. En d'autres termes, les béatitudes ne peuvent être acceptées que par celui qui donne sa foi à celui qui parle; sinon, elles ne sont guère qu'un paradoxe mystificateur. Elles ne sauraient, donc, être définies comme un simple catalogue des vertus chrétiennes nécessaires au salut; pour être admises, elles présupposent la confession de celui qui inaugure gracieusement $1 \mathrm{e}$ Règne en les proclamant. La confession du Christ est première par rapport à l'éthique; l'obéissance s'enracine dans la foi. En second lieu, pour

1 Ainsi p.ex., DUPONT (Béatitudes I 260; L'interprétation des Béatitudes 21), qui écrit : "Les béatitudes matthéennes se présentent en somme comme une liste des conditions spirituelles à remplir pour être admis dans le Royaume". Voir aussi Buch et Bücher NT 40 qui, après DIBELIUS (Bergpredigt 93), voit dans les béatitudes $\mathrm{mt}$ 'bine tafelartige Zusammenstellung christlicher Eigenschaften und Verhaltensweisen"; de même STRECKER, Macarismes 201.205-206, et WALTER 254-257.

2 Ainsi déjà BONNARD, Mt 55; SCHNIEWIND, Mt 45; SCHWEIZER, Mt 50; STRECKER, Macarismes 208.

3 7,29 souligne que le didascale du Sermon sur la Montagne est revêtu,

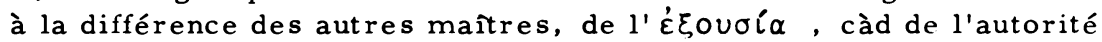
toute-puissante conférée par Dieu à travers laquelle se dévoile le sens

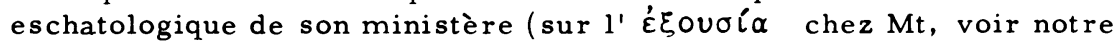
travail Maître et disciple 279-280 et STRECKER, Weg 127). Par ailleurs, en 5,1 , le verbe $x \alpha \vartheta i \zeta \omega$, affirmé de Jésus, décrit la position de

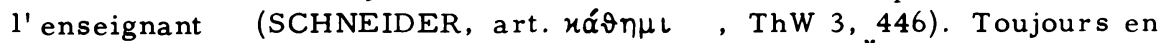
5,1 , le lieu de l'enseignement, à savoir la montagne (öpos) a une signification théologique : dans $\mathrm{Mt}$, en effet, la montagne (voir particulièrement 17,$1 ; 28,16)$ désigne par excellence le lieu de la révélation (SCHMAUCH 67-68). 
apprécier correctement l'accentuation éthique des béatitudes, il convient de souligner qu'elle se développe dans un contexte précis et absolument fondamental : la proclamation souveraine du salut désormais offert gratuitement à tous. Dans les béatitudes, la déclaration du bonheur eschatologique manifeste la venue du Règne à la fois comme une promesse et comme une invitation. Que Mt ait porté l'accent sur l'exigence liée à cette invitation, qu'il ait insisté sur "le prix de la grâce" vécu dans la suivance de Jésus, ne change rien au fait que la proclamation gracieuse du Règne est première; l'implication éthique en découle, mais ne la fonde pas.

Cette double mise au point nous autorise à reposer avec acuité la grosse question de la version mt des béatitudes : pourquoi le rédacteur, en accord avec son église, a-t-il jugé bon d'infléchir la tradition dans un sens éthique?

\section{Forme littéraire et eschatologie}

Les béatitudes synoptiques ${ }^{1}$ - et plus particulièrement mt - s'inscrivent dans la tradition vétérotestamentaire-juive des macarismes. Cette appartenance n'est pas seulement littéraire, mais également idéologique. Il apparaît, en effet, que l'héritage de l'apocalyptique juive tardive est présent dans les béatitudes 2 .

D'un point de vue historique - nous ne faisons ici que rappeler les résultats essentiels de la recherche -, le macarisme est d'abord attesté dans la tradition sapientiale. Il est alors une bénédiction adressée à l'homme

1 Sur l'origine et l'histoire du macarisme, voir p. ex. : EICHHOLZ 26-

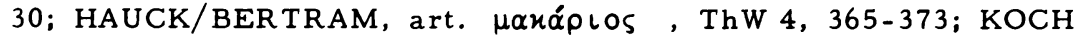
7-9. 20-22; SCHULZ, Q 78-81; SCHWEIZER Gemeinde 69-77 et Mt 45-46; TRILLING, Christusverkündigung 73-78.

2 Thèse bien développée par KOCH 21; SCHULZ, Q 80. L'existence de macarismes dans le monde grec (cf. BERTRAM, art. cit., ThW 4, 366-67) ne fait pas avancer notre analyse : la question décisive des béatitudes synoptiques - celle du salut eschatologique - n'y est pas posée. 
qui vit selon l'ordre du monde créé par Dieu et révélé par les sages ${ }^{1}$. Dans le bas-judaisme pré-chrétien, le courant apocalyptique récupère la forme littéraire du macarisme sapiential tout en la réinterprétant. A la différence de la tradition sapientiale, le macarisme apocalyptique s'adresse à des personnes qui seront sauvées lors du jugement dernier en vertu de leur fidélité à la foi ${ }^{2}$. Le macarisme chrétien primitif s'inscrit dans le prolongement de l'apocalyptique juive; cependant, il n'est plus comme dans cette dernière une fiction, un souhait, un voeu; il devient un porteur de la prédication prophétique, un véhicule de l'annonce du Royaume, c'est-à-dire d'une réalité qui s'instaure ${ }^{3}$.

S'il n'est guère contestable que la béatitude synoptique et l'annonce du Royaume vont de pair, encore faut-il se demander de quelle manière. Ici se situe la difficulté majeure dans l'interprétation des béatitudes. En effet, le schéma du macarisme est caractérisé par une polarité temporelle présent-futur. La principale qui s'ouvre par $\mu \alpha \varkappa \alpha ́ \rho \iota$ s se rapporte au présent tandis que la subordonnée en ő $\iota$ concerne le futur ${ }^{4}$. Le Royaume de Dieu - ou plus précisément le bonheur eschatologique qui lui est associé - est une réalité à la fois présente et future. Comment faut-il comprendre pareille tension ? Pour répondre à cette question, une distinction, certes schématique et sommaire, entre les différentes couches de la tradition s'impose.

$1 \mathrm{KOCH} 8$; voir p. ex., $\operatorname{Pr} 3,13$ et 1 'introduction du Ps 1 .

2 KOCH ibid., voir p. ex., Dn 12,12, Tb 13,14, Ps Sal 4, 23; 17, 44; 18, 6.

3 SCHULZ, Q 80; TRILLING, Christusverkündigung 77.

4 Difficulté bien vue par EICHHOLZ 27-28; ROBINSON, Le kérygme de

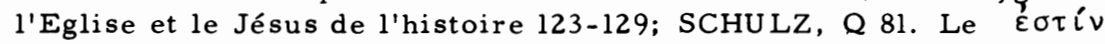
qui apparaît dans les subordonnées des vv. 3.10 ne met pas en cause la polarité temporelle que nous avons dégagée; ce présent, comme le montre d'ailleurs les futurs des subordonnées aux vv. 4.5.6.7.8.9, a valeur de futur, mais d'un futur immédiat, imminent qui conditionne déjà le présent vécu (voir p. ex. EICHHOLZ 28; SCHNIEWIND Mt 40; SCHULZ Q 81; STRECKER, Macarismes 193). 
Au niveau le plus ancien (Jésus ?), la problématique nous paraît se présenter ainsi : le Royaume est si proche, l'éon à venir si immédiatement perceptible qu'il détermine entièrement le présent, que le présent devient par excellence la temps du salut ${ }^{1}$. Parce que Dieu va établir son règne, parce qu'il commence déjà à le faire dans la personne de Jésus, l'heure est à la joie.

Dans la source $Q$, du moins dans ses composantes les plus archaĩques, un glissement de sens s'est déjà produit : l'imminence du Royaume qui donne toute sa densité au présent, est maintenue tout en subissant une réinterprétation christologique; la venue du Royaume s'identifie à l'avènement du Fils de l'homme pour le Jugement ${ }^{2}$.

Au niveau de la rédaction $\mathrm{mt}$, l'horizon de compréhension des béatitudes change complètement. La venue du Royaume n'est plus conçue comme un renouvellement cosmique imminent; le retard de la parousie est une difficulté ressentie, mais déjà surmontée ${ }^{3}$. Dès lors, dans une histoire qui se prolonge plutôt que d'être en voie d'achèvement, comment le rédacteur peut-il assumer la proclamation d'un Règne à la fois présent et à venir ? La théologie du premier évangile suggère une double réponse, ce qui permet de placer les béatitudes dans une perspective spécifiquement $\mathrm{mt}$.

1 HAUCK (art. cit., ThW 4, 372) peut ainsi écrire à propos des béatitudes: "Sie fassen die Gegenwart im Licht der Zukunft"i voir, dans la même direction, EICHHOLZ 27; SCHNIEWIND, Mt 40; SCHWEIZER, Mt 50. Sur le problème de la structuration temporelle de l'annonce du Royaume, orientation générale chez BORNKAMM, Jesus 58-87 (en particulier 82-87).

2 Concernant les différents problèmes posés par la source $Q$, bon état de la recherche récente chez LUZ, Logienquelle 527-533. Cette étude critique souligne la place centrale de la christologie du Fils de l'homme dans $Q$ ainsi que l'affirmation de l'imminence de la parousie dans la couche archaïque de la source. Par ailleurs, tant LUEHRMANN (Logienquelle 94) que SCHULZ, ( $83-84$ ) montrent clairement que la prédication du Jésus terrestre quant à l'imminence du Règne est réinterprétée par le thème apocalyptique de l'attente du jugement.

3 Sur ce point, voir notre chap. 10. 
D'une part, le Règne est présent : des logia sur la $\beta \alpha \sigma \iota \lambda \varepsilon i ́ \alpha$ tels que 11,$12 ; 12,28$ et 21,43 en sont la preuve évidente ${ }^{l}$. Tant la difficile parole de 11,12 que la fameuse affirmation de 21,43 évoquent un Règne contesté et en définitive refusé par les Juifs. Plus concrètement, ce Règne rejeté par Israël se manifeste dans la prédication de Jésus ${ }^{2}$. Aux yeux de Mt, la présence du Règne se concentre en effet dans l'enseignement du Christ. Cette conception du Règne rendu présent et manifesté dans l'enseignement de Jésus est confirmée par une série de faits. En 6, 33, la recherche du Règne est élucidée par la clausule rédactionnelle $x \alpha i$

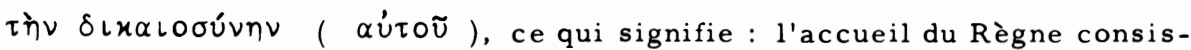
te dans l'obéissance à la volonté de Dieu telle que le Christ l'enseigne dans le Sermon sur la Montagne. En 13,52, dans la description de l'office du scribe, il est fait allusion à un $\gamma \rho \alpha \mu \mu \alpha \tau \varepsilon u ́ s$ instruit ( $\mu \alpha \vartheta \eta \tau \varepsilon \cup \vartheta \varepsilon i \varsigma$ ) du Royaume des Cieux, ce qui présuppose que la $\beta \alpha \sigma \iota \lambda \varepsilon i \alpha$ est objet d'étude et d'enseignement. Mentionnons enfin deux expressions propres

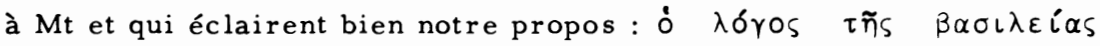

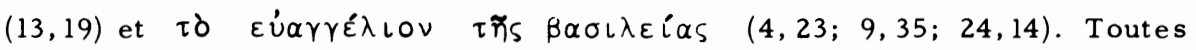
deux désignent de manière globale la prédication de Jésus désormais transmise et proclamée par l'Eglise (cf. 24,14 !). En définitive, le Règne est présent, pour la communauté $\mathrm{mt}$, étant donné que la prédication du Jésus terrestre est intégralement valable et actuelle. Cette affirmation est fondamentalement la même que celle du manifeste $(28,16-20)$ où se trouve affirmée l'identité du terrestre et du Ressuscité; cette identité suppose la valeur normative de ce que le Jésus terrestre a dit et fait - mais d'abord sous la forme d'un enseignement qui doit être gardé et accompli par la communauté.

D'autre part, il est tout aussi évident que, pour Mt, le Règne vient. A cet égard, il est révélateur que, dans le premier évangile, la difficile

1 Passages rassemblés et analysés par STRECKER, Weg 166-171.

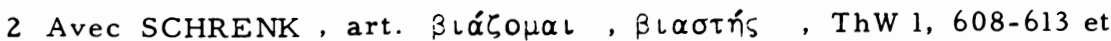
STRECKER, Weg 167-168. 


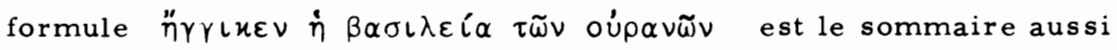
bien de la prédication du Baptiste $(3,2)$ que de celle de Jésus $(4,17)$ que de celle des disciples $(10,7) !{ }^{1}$. Deux autres traits bien connus de la théologie mt vont dans le même sens. D'abord, Mt associe volontiers l'annonce du Royaume à celle du banquet eschatologique $(8,11 ; 22,2-14$; $25,10 ; 26,29)$; ensuite il a un goat particulier pour les sentences d'entrées dans le Royaume $(5,20 ; 7,21 ; 18,3 ; 19,23-24 ; 21,31 ; 23,13)$. Que ce soit dans la polémique ou la parénèse, la participation à la venue du Royaume, représentée par l'image du festin, désigne constamment l'issue favorable du jugement. Semblablement, les sentences d'entrée dans le Royaume énoncent les conditions à remplir pour bénéficier d'une sanction eschatologique favorable. Venue du Royaume et venue du jugement vont donc de pair pour Mt. Plus précisément, le Royaume s'approche de l'homme sous la forme du jugement; de ce fait, l'heure présente devient le temps de la décision et de l'obéissance.

Cette brève analyse de la conception mt du Règne permet de comprendre plus exactement les béatitudes dans le cadre du premier évangile, et notamment de mieux saisir la structure paradoxale de ce bonheur déjà déclaré présent et pourtant appelé à un épanouissement eschatologique. Aux yeux de $\mathrm{Mt}$, le bonheur proclamé par le Christ est présent dans la mesure où l'Eglise répète fidèlement la prédication du Maftre et se l'approprie; il est futur puisque l'Eglise est en marche vers le jugement, que le bonheur qu'elle annonce ne lui appartient pas, mais reste un don sans cesse à recevoir et dont le Christ reste le seul garant. Que l'on insiste sur la pérennité de la prédication du Jésus terrestre pour souligner la présence du Règne - et donc du bonheur des béatitudes -, ou que l'on mette l'accent sur l'attente du jugement pour maintenir l'à-venir du

14,17 est emprunté à Mc 1,15 tandis que 3,2 et 10,7 sont vraisemblablement des adjonctions rédactionnelles. Prétendre que le chap. 10 et 10,7 en particulier se rapportent exclusivement à la mission pré-pascale est un raccourcissement peu convaincant du texte; la prédication des disciples en 10,7 concerne également l'époque post-pascale (voir notre chap. 16). 
Règne - et donc du bonheur des béatitudes -, dans les deux cas, la question critique est celle de l'appropriation. Dans la conception mt, aussi bien la présence du Règne que son à-venir détermine le présent vécu par l'Eglise comme le temps de la fidélité et de l'obéis sance.

L'accentuation éthique des béatitudes - càd la tendance à doubler la promesse d'une exigence -, n'est donc pas un acte arbitraire de la communauté $m t$ - ou du rédacteur -, témoignant d'un goât immodéré pour le moralisme; elle a sa cause et son sens dans la révaluation de l'eschatologie. Elle témoigne de la manière dont l'église mt a compris la situation qualitativement nouvelle dans laquelle elle se trouvait et comment elle a assumé les problèmes qui en résultaient. Séparée de son Seigneur, mais témoin de sa Parole, en route vers le jugement, mais désormais engagée dans l'histoire, familiarisée avec l'Evangile, mais mise en demeure de le vivre, l'Eglise affronte le problème de la vie chrétienne dans sa durée. Pour conserver au bonheur offert son caractère de grâce libératrice et transformatrice, elle l'infléchit éthiquement. L'éthique est alors la catégorie qui exprime la vie chrétienne dans son devenir.

Pour que cette hypothèse d'interprétation soit fondée, il faut maintenant prouver que le contexte de communication des béatitudes a changé dans la rédaction $m t$ et que les destinataires en sont désormais l'auditoire ecclésial.

\section{Le contexte de communication}

La précédente analyse révèle un fait fort intéressant : à chaque variation de sens correspondait un changement de contexte de communication. Afin de consolider cette hypothèse, répertorions les différents auditoires que nous pouvons discerner aux différents stades de la tradition.

Au niveau le plus ancien (prédication de Jésus ?), la béatitude est une proclamation qui s'adresse en particulier aux pauvres d'Israël, c'est-àdire au petit peuple exploité et méprisé et, d'une manière plus large, 
au am ha-aretz, c'est-à-dire aux parias religieux ${ }^{1}$. Sur ces hommes condamnés par le "non" de la société, Jésus, à travers les béatitudes, prononce le "oui" de Dieu. La béatitude est dès lors le sommaire de la bonne nouvelle du Royaume, sommaire qui culmine dans un appel à la conversion $(4,17)$. Au niveau de la source $Q$, la béatitude s'intègre à la prédication prophétique des inspirés post-pascaux et s'adresse à une communauté dont nous discernons mal les contours. S'agit-il d'une prédication missionnaire adressée à Is raël ? ou les termes qui désignent les destinataires des béatitudes sont-ils déjà des prédicats ecclésiologiques disant la promesse et l'exigence sous lesquelles est placée la vie dans la foi ${ }^{2}$ ?

Au niveau de la rédaction $\mathrm{mt}$, la béatitude n'est plus d'abord une proclamation prophétique; elle est un enseignement. Son intégration dans un grand discours "catéchétique", l'utilisation de la 3e personne, mais aussi l'emploi du verbe $\delta\llcorner\delta \alpha ́ \sigma \varkappa \omega$ en 5,2 l'attestent clairement. De plus, il semble bien que le Sermon sur la Montagne - et donc les béatitudes qui en constituent la préface - s'adresse non plus aux hommes en général (prédication missionnaire!), mais à l'église mt ${ }^{3}$. Dès lors, l'accentuation éthique ne doit pas être expliquée seulement par la révaluation de l'eschatologie, mais aussi par un changement d'auditoire. Ce changement que nous présupposons est-il perceptible dans le texte-même des béatitudes ? End'autres termes, pouvons-nous prouver que le prologue du Sermon sur la Montagne est une instruction ecclésiale ${ }^{4}$ ?

L'adresse du Sermon sur la Montagne nous fournit une première indication : les disciples sont présentés comme les auditeurs privilégiés

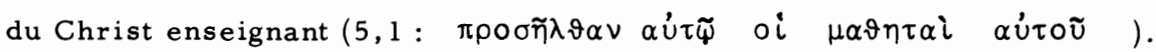

1 Voir nos pp. 289. 293.

2 Une analyse détaillée de la communauté ou des communautés qui ont transmis $Q$, nous mènerait au-delà de notre propos. A l'appui de notre argumentation, voir LUZ, Logienquelle 528.533, et SCHULZ Q 80-84.

3 Voir nos pp. 32-33.

4 Cf. Das Buch der Bücher, NT 40. 
Or, comme nous l'avons abondamment montré, ils sont à ce titre la figure de l'Eglise instruite par son Seigneur ${ }^{1}$. Dans les béatitudes elles-mêmes, ensuite, nous repérons des concepts à connotation incontestablement ecclésiale; nous entendons par là des notions qui ne sauraient être vécues indépendamment de la communauté des croyants, le lieu où le Christ est proclamé et confessé. Les doux du v. 5, par exemple, le sont à l'image de leur maître : ils accomplissent dans l'humilité la volonté de Dieu, suivant en cela le chemin ouvert par le Christ. Semblablement, les miséricordieux du v. 7 et les coeurs purs du v. 8 sont les ouvriers fidèles de la Loi recentrée sur son contenu essentiel par le didascale eschatologique. La justice qui donne son empreinte spécifiquement $m t$ aux vv. 6 et 10, ne saurait être connue en dehors de celui qui la proclame, nouvelle et radicale. Les vv. 10-12, en liant la pratique de la justice à l'épreuve de la persécution, nous font mesurer le fossé qui existe désormais entre la communauté et le monde. Les similitudes du sel et de la lumière des vv. 13-16, enfin, rappellent clairement la vocation et la responsabilité des croyants appelés à témoigner devant les hommes ${ }^{2}$.

Ces différents éléments confirment notre hypothè se : l'auditoire mt des béatitudes est l'Eglise. Mais quelle est alors la fonction des béatitudes ? que font-elles découvrir ou redécouvrir à l'Eglise ? Elles nous semblent remplir un double rôle. En tant que déclaration d'un bonheur déjà présent et pourtant à-venir, elles sont tout d'abord_paraclèse. A travers elles, le croyant s'entend rappeler la vocation reçue qui est une vocation au bonheur; il se remémore la grâce du Règne dont il est le bénéficiaire, mais aussi la consolation essentielle qui le soutient dans l'épreuve. A l'inauguration souveraine du Règne en Jésus correspond la proclamation gracieuse d'un bonheur contre lequel rien ne prévaut ni ne prévaudra. Mais l'invitation au bonheur est une invitation exigeante qui met toute l'existence en question et la transforme. C'est dire que les béatitudes sont simul-

1 Voir nos pp. 45-46.

2 Cf. nos pp. 422-428. 
tanément exigence et, à ce titre, parénèse. Leur perspective eschatologique rappelle que 1 'invitation reçue est une invitation qui engage et qui ne peut être accueillie que dans la fidélité et l'obéissance. La grâce ne supprime pas la responsabilité, elle la crée.

Pour illustrer cette réciprocité entre paraclèse et parénèse au niveau $\mathrm{mt}$, nous reprenons la première béatitude. La promesse du Règne est accordée aux pauvres en leur esprit. En tant que paraclèse, la béatitude dit que Dieu s'approche de ceux qui sont faibles et démunis, de ceux qui n'ont rien à lui offrir, aucune prétention à émettre; c'est la bonne nouvelle par excellence, en termes pauliniens, selle de la justification de l'impie. En tant que parénèse, la béatitude affirme certes que Dieu se donne, mais justement aux pauvres; cette prétention divine, le croyant doit l'accepter, la rendre présente dans son projet de vie. Dans le premier évangile, la béatitude n'est donc plus d'abord une parole missionnaire qui dévoile de manière inattendue et provocatrice la grâce du Règne, elle n'est plus un appel à la conversion. Inscrite dans le temps de l'Eglise, elle est la parole qui anime et éclaire l'existence chrétienne à la lumière de ce que le Christ donne et exige; elle est tout à la fois consolation et exhortation. La béatitude $\mathrm{mt}$ semble donc être le lieu par excellence où s'articulent indicatif et impératif, eschatologie et éthique. Nous nous proposons de vérifier cette hypothèse en examinant brièvement le contexte littéraire de notre péricope.

\section{Le contexte littéraire des béatitudes}

Avant de tirer les conclusions de notre enquête sur les relations entre l'eschatologie et l'éthique au niveau des béatitudes - et, en définitive, sur le point de départ mt de l'éthique -, il convient d'examiner le contexte littéraire dans lequel est imbriqué notre texte. Les béatitudes, nous l'avons dit, constituent le prologue du Sermon sur la Montagne. Elles sont précédées d'une série de trois péricopes qui illustrent de manière programmatique le ministère de Jésus en Galilée et elles sont suivies du 
Sermon sur la Montagne proprement dit. Pouvons-nous dégager une dominante théologique dans chacune de ces deux sections qui encadrent les béatitudes ?

Considérons tout d'abord 4,12-25. La première péricope (vv. 12-17), fortement tributaire de la vision de l'évangéliste ${ }^{1}$, a une valeur programmatique. L'énumération géographique du v. 15 qui culmine dans l'étonnan-

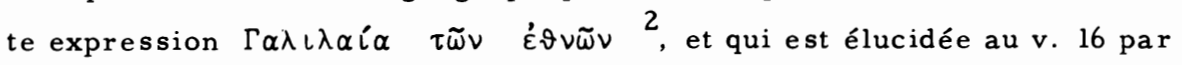
les thèmes des ténèbres et de la mort, montre clairement que le Christ mt s'adresse en priorité à des hommes qui vivent séparés de Dieu et de sa volonté formulée dans la Loi. A ces hommes perdus, Jésus est présenté comme la lumière ${ }^{3}$, c'est-à-dire comme l'accomplissement eschatologique du dessein salutaire de Dieu. Cette affirmation à la fois christologique et sotériologique est reprise et concrétisée au v. 17 par l'énoncé "classique" de la prédication du Royaume. La péricope suivante (4,18-22) relate l'appel des disciples; la pointe du récit consiste en ce que Jésus met souverainement, par pure grâce et sans aucun préalable, des membres du am ha-aretz à son service ${ }^{4}$. Le sommaire rédactionnel $(4,23-25)^{5}$ qui conclut le chap. 4, anticipe déjà le propos développé dans les chap. $5-7,8-9$ : à la parole du Messie répond son action; la bonne nouvelle du

1 Mt 4,12-17 est une composition rédactionnelle. Le point de départ de la péricope est donné par $\mathrm{Mc} 1,14$; ce v. est profondément remanié par $\mathrm{Mt}$ et scindé en deux parties : à Mc 1,14a correspond Mt 4, 12 et à Mc 1, 14b$16 \mathrm{Mt} 4,17$. Entre ces deux éléments, Mt introduit une citation d'Es $8,23-9,1$, précédée d'un $v$. de transition et de la formule d'introduction. Pour l'étude de la citation, voir STENDAHL 104-106 et STRECKER, Weg 63-66.

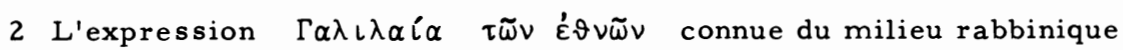
(voir BILL 1, 153-159) fait allusion à la population mêlée de Galilée où Juifs et pä̈ens cohabitent et où, en conséquence, la Loi est observée avec moins de rigueur qu'en Judée.

3 Le motif de la lumière est une désignation messianique; voir BILL 1, 161-162, GRUNDMANN, Mt 106.

4 Voir nos pp. 216-220.

5 Il s'agit d'une composition rédactionnelle faite à partir de Mc 1, 39. 28; 3, 7-8. 
Règne dresse à travers l'activité thaumaturgique de Jésus les signes qui attestent son contenu libérateur. Ainsi, malgré l'importance centrale des chap. 5-10 dans le projet global de l'évangile, cette présentation inaugurale du ministère de Jésus, que le rédacteur a soigneusement élaborée, doit être prise au sérieux et saisie dans son unité. Elle est tout entière centrée sur l'irruption du Règne qui, par delà le am ha-aretz, s'en va à la rencontre des païens; irruption du Règne qui libère, appelle, rassemble les humbles réputés indignes; irruption du Règne qui sauve en faisant échec "à toutes sortes de maladies et de tourments". La dominante théologique est claire, c'est tout l'aspect eschatologique du ministère de Jésus qui est mis en évidence, le bouleversement libérateur et décisif qu'il introduit. Cette thématique est bien évidemment reprise dans les béatitudes; bien plus, elle y culmine dans la mesure où ces dernières sont la proclamation paradoxale et immédiate du bonheur eschatologique.

Si nous envisageons maintenant le corps du Sermon sur la Montagne, le changement d'horizon est indubitable. Avec 5,17-20, le thème de la Loi accomplie par le Christ et proposée à l'obéissance du disciple, prend le devant de la scène ${ }^{1}$. Les antithèses $(5,21-48)$ annoncent souverainement $1^{\prime}$ authentique volonté de Dieu ${ }^{2}$. Quant aux bonnes oeuvres de la tradition juive $(6,1-18)$ reprises de manière critique et novatrice par le maître, elles dévoilent le comportement désormais exigé par Dieu. Le Sermon sur la Montagne se concentre donc, dans sa partie centrale, sur la dimension que nous avons également repérée dans les béatitudes, plus précisément dans ce que nous avons appelé l'accentuation éthique de la tradition. D'un point de vue littéraire et théologique, nous constatons donc que les béatitudes $\mathrm{mt}$ sont un des lieux par excellence de l'évangile où les deux composantes de la prédication de Jésus - à savoir l'eschatologie et l'éthique - se rejoignent et s'articulent. Malgré un avis aujourd'-

1 Voir notre chap. 4.

2 Voir notre chap. 12. 
hui fort répandu ${ }^{1}$, nous ne pensons pas que l'accentuation éthique due à Mt implique un affaiblissement de la ligne eschatologique avec son corrélat indicatif.

\section{Le point de départ de l'éthique}

Dans ce paragraphe conclusif, nous nous proposons de reprendre le problème de l'accentuation éthique des béatitudes $\mathrm{mt}$ en fonction de notre sujet particulier, à savoir : la condition du croyant. Comment se pose, pour le disciple $\mathrm{mt}$, le problème éthique ? quel est le contexte théologique et ecclésial dans lequel s'inscrit l'impératif sous lequel le croyant est placé ? Dibelius ${ }^{2}$, approuvé par de nombreux exégètes ${ }^{3}$, a dit des béatitudes mt qu'elles étaient une description "des vertus que le chrétien doit pratiquer "; Windisch ${ }^{4}$ y a vu les "conditions d'admission" dans le Royaume; dans la même ligne, Strecker ${ }^{5}$ prétend qu'elles "décrivent, à la manière d'exigences indirectes, la conduite à adopter comme condition préalable à l'entrée dans la future $\beta \alpha \sigma \iota \lambda \varepsilon \iota_{\alpha} \alpha$ "; Trilling ${ }^{6}$, enfin, y discerne "le portrait du parfait disciple de Jésus". Toutes ces appréciations, qui relèvent unilatéralement l'accentuation éthique des béatitudes $\mathrm{mt}$, réduisent leur message à la question: "que dois-je faire pour être sauvé ?". Dans cette perspective, l'éthique révèle au disciple le chemin du salut, plus précisément, elle lui indique les conditions à remplir pour surmonter victorieusement l'épreuve du jugement qui vient.

1 Ainsi p. ex. DIBELIUS, Bergpredigt 93; DUPONT, Béatitudes I 260; STRECKER, Macarismes 201.205-206; WALTER 254-257.

2 DIBELIUS, Bergpredigt 93.

3 BARTH 56; BORNKAMM, Enderwartung 14.

4 WINDISCH, Der Sinn der Bergpredigt 63 (note 1).

5 STRECKER, Macarismes 205-206.

6 TRILLING, Mt I 101. 
Une telle exégèse nous semble mal situer le problème éthique dans la mesure où elle repose sur une appréciation discutable du contexte christologique et eschatologique des béatitudes mt. En effet, celui qui appelle à la pauvreté, à la douleur, à la justice, à la miséricorde, à la pureté ou encore à la paix, n'est pas un maître ordinaire; à travers son discours, il prétend représenter totalement Dieu, arrềter et instituer une fois pour toutes son dessein salutaire à l'égard de l'homme. Dès lors, il n'y a pas d'acceptation possible du propos des béatitudes en dehors de la confession de celui qui les prononce. Il s'ensuit que l'impératif des béatitudes s'inscrit dans la relation première de la foi. C'est pour avoir reconnu en Jésus le didascale eschatologique que le disciple se soumet à son exigence.

Cependant, même cette référence à la christologie des béatitudes reste insuffisante si on néglige leur composante eschatologique. Nous avons, en effet, constaté que - même chez Mt - la structure des béatitudes repose sur l'affirmation d'un Règne à la fois présent et à-venir. Dans la parole du Maître, le bonheur proclamé est déjà une réalité présente, gracieusement offerte à tous en plénitude et le bonheur eschatologique n'est pas d'abord un but à atteindre en remplissant certaines conditions, mais une invitation à saisir. L'accentuation éthique ne supprime pas le caractère indicatif, elle souligne le sérieux inéluctable de l'appropriation. La problématique éthique des béatitudes mt ne consiste donc pas dans la question: "que dois-je faire pour être sauvé ?", mais bien : "par quelle décision de vie vais-je répondre à l'offre gracieuse du bonheur eschatologique ?".

L'éthique est donc la médiation que Mt a choisie pour décrire à l'intention du disciple le sérieux de l'appropriation résultant de la proclamation du Règne. Le modèle d'appropriation qui se manifeste par l'accentuation éthique des béatitudes, ne doit pourtant pas être détaché de son contexte historique - et plus particulièrement ecclésial -. L'auditoire auquel s'adresse Mt et qu'il se propose d'instruire est composé de croyants. En conséquence, le problème majeur de sa communauté n'est 
pas la conversion, mais la fidélité vécue à une libération déjà reçue. Les béatitudes $\mathrm{mt}$ ne sont pas destinées à provoquer une rupture de vie chez l'auditeur; elles se proposent de l'amener à considérer sa vie dans la durée à la fois comme objet de paraclèse et de parénèse. De ce point de vue - et c'est là certainement un paint fondamental de la théologie mt -, l'accentuation éthique vise à dire l'appropriation du Règne non pas de manière générale, mais pour l'existence chrétienne considérée dans son devenir. Elle rappelle au croyant, engagé dans le temps de l'Eglise et toujours tenté de soustraire son vécu à la prétention de la parole du Maître, le sérieux de sa condition. 


\section{La Loi et le thème de l'éthique}

Par l'étude des béatitudes, nous avons essayé de montrer comment se posait pour le disciple mt le problème de l'éthique ou, plus précisément, quel était l'horizon théologique et ecclésial sur le fond duquel devait être pensée la question de l'obéissance. Nous en venons maintenant au contenu de cette obéissance réclamée du disciple ou, en d'autres ter mes, au thème de l'éthique. Nous touchons de ce fait même une des notions fondamentales de la théologie $\mathrm{mt}$, à savoir sa compréhension de la Loi. Pourtant, étant donné le sujet particulier de notre étude - la condition du croyant - nous n'avons pas à refaire une analyse complète de la conception mt de la Loi, mais à préciser en quoi cet aspect de la pensée mt concerne particulièrement notre problématique.

Comme nous l'avons montré sur la base de travaux récents ${ }^{1}$, la Loi est au centre des préoccupations de Mt. Il élabore sa position particulière en se battant sur un double front. Contre le judais me rabbinique d'obédience pharisienne, il prétend non certes posséder une nouvelle Tora, mais la Tora restituée dans son authenticité par le Messie ${ }^{2}$. Contre les "antinomistes" de son église qui contestent la validité de la Tora, il en réaffirme l'autorité normative pour la foi ${ }^{3}$. A ses yeux, la Loi est donc une partie constitutive et inaliénable de la prédication chrétienne (cf. 5 , $17-20)$, le lieu par excellence où se trouve manifestée la volonté de Dieu. Pourtant, si Mt maintient fermement la validité de la Tora, s'il est dis posé à reconnaitre le bien-fondé de certains as pects de la tradition rabbi-

1 Voir sur ce point nos chap. 4 et 7 . Concernant l'esquisse qui suit, nous renvoyons à : BARTH 58-98; BORNKAMM, Enderwartung $21-29$, et Wandlungen 73-80; HUMMEL 66-75; SCHWEIZER, Gesetz und Enthu siasmus 50-53.

2 HUMMEL 75.

3 BARTH 68-70. 149-151; BORNKAMM Wandlungen 75; HUMMEL 64-65. 
nique $(23,2)^{1}$, si même il ne condamne pas principiellement la Loi céré monielle $(23,23 ; 24,20)^{2}$, il n'en reste pas moins que la Loi dont il se fait le défenseur "est la Loi réinterprétée par l'enseignement et le comportement de Jésus" ${ }^{3}$.

La Loi ne saurait donc être pensée sans la christologie. Mais s'il en est ainsi, comment se présente la réinterprétation décisive proposée par le Christ mt ? Nous l'avons vu, le Jésus mt n'apporte pas une nouvelle Tora. Cependant, pour lui et contrairement à la conception rabbinique, le critère de vérité de la Tora ne réside pas dans sa conformité à la tradition mosalque, mais dans son aptitude à traduire la volonté vivante du Créateur (cf. p.ex. 15,3-5; 19,4.8b). En conséquence, pour conserver sa légitimité, la Tora doit sans cesse être mesurée à la volonté originaire du Dieu vivant dont le Christ $\mathrm{mt}$ prétend être le révélateur autorisé ${ }^{4}$. Quelle est alors l'intention fondamentale de la volonté du Dieu vivant ou, en d'autres termes, quel est pour le Christ mt le principe d'interpréta tion de la Tora ? La réponse de Mt ne diffère pas de celle de l'ensemble de la tradition chrétienne primitive, elle est bien connue : il s'agit de l'exigence d'amour.

Pourtant - aussi surprenant que cela puisse paraître -, Mt est le seul parmi les synoptiques à avoir vraiment insisté sur ce thème; il lui confère une place particulière et lui accorde une réflexion dont il n'est pas

\section{BARTH $80-83$.}

2 BARTH 83 -86; BORNKAMM, Enderwartung 28-29; SCHWEIZER, Ge setz und Enthusiasmus 52-53. Dans la mesure où ils ne détournent pas le croyant d'une obéissance véritable, le sacrifice $(5,23-24)$, l'impôt du Temple $(17,24-27)$, le sabbat $(24,20)$, l'aumône, la prière et le jeûne $(6,1-8,16-18)$, le serment $(23,16-22)$, la dîme $(23,23)$ gardent leur validité.

3 SCHWEIZER, Gesetz und Enthusiasmus 51 à la suite de BORNKAMM.

4 Le thème $\mathrm{mt}$ de la restitutio principii est développé par BORNKAMM, Enderwartung 23, et TRILLING, Israel 205-206. 
inutile de rappeler les principaux éléments ${ }^{1}$. L'apocalypse mt est la seule à signaler le refroidissement de l'amour $(24,12)$ comme un des signes de la fin. Semblablement, la parabole du serviteur impitoyable $(18,23-35)$ et la scène du jugement dernier $(25,31-46)$ qui sont des traditions propres à $\mathrm{Mt}$, insistent sur l'amour dû à autrui. Dans l'histoire du jeune homme riche, la mention récapitulative du commandement d'amour $(19,19)$ est une adjonction rédactionnelle. Même note dans la parabole de la brebis égarée $(18,12-14)$ que Mt a infléchie dans le sens de la sollicitude que le disciple doit aux "petits". A cela s'ajoutent les textes classiques où Mt, de manière originale, affirme l'importance décisive du commandement d'amour par rapport à la Loi. En effet, si Mt emprunte la règle d'or à $Q$ (cf. Lc 6,31) ${ }^{2}$, s'il tient l'apophtegme sur le plus grand commandement de Mc (cf. Mc 12,28-34), il est le seul à voir dans ces logia le sommaire de la Loi : (7,12b et 22,40 sont des adjonctions rédactionnelles !). On notera enfin la même insistance à dégager le principe herméneutique de la Loi dans les deux citations rédactionnelles d'Os $6,6(9,13 ; 12,7)$ et en 23,23 où la justice, la miséricorde et la fidélité sont présentées comme

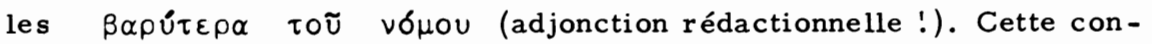
ception $\mathrm{mt}$ de la Loi dont nous venons de rappeler sommairement les traits essentiels, apparaît de manière exemplaire dans les antithèses du Sermon sur la Montagne (5,21-48). Paradoxalement, Mt ne comprend pas ces der nières comme l'abrogation de la Tora, mais comme son accomplissement

1 BORNKAMM, Enderwartung 23 et Wandlungen 96-97; BARTH $70-80$; SCHWEIZER, Gesetz und Enthusiasmus 53; TRILLING, Is rael 196198.206-207.

2 Comme le montre la comparaison entre le discours dans la plaine (Lc 6) et le SM, Mt 7,12 est le seul logion du tronc commun que le rédacteur a déplacé. Ce choix d'un nouveau contexte résulte du rôle essentiel que Mt attribue à la thématique de l'amour. La règle d'or n'est plus seulement un logion invitant à l'amour de l'ennemi, elle devient la conclusion de la partie centrale du SM, elle résume et conclut l'interprétation mt de la Loi (cf.pp. 109-110). 
par le Christ $\mathrm{mt}^{\mathrm{l}}$. La dénonciation de la compréhension rabbinique de la Tora $^{2}$ ne vise pas à annuler la Loi; elle sert de repoussoir pour la proclamation de la volonté originaire et radicale de Dieu, proclamation qui met un terme à toute obéissance juridique et légaliste ${ }^{3}$. De plus - et là nous retrouvons le principe $m$ t d'interprétation de la Loi -, les antithèses se rapportent soit à la deuxième table du Décalogue, soit à des textes analogues ${ }^{4}$; elles posent donc le problème de l'obéissance au niveau des relations interpersonnelles et culminent de manière significative dans l'appel à l'amour de l'ennemi.

Par ailleurs, les antithèses, malgré leur dénonciation de la tradition rabbinique, n'ont pas pour intention première de polémiquer contre le judaïsme ${ }^{5}$. A l'image de l'ensemble du Sermon sur la Montagne, elles sont une instruction ecclésiale qui montre aux disciples quelle est la conception spécifiquement chrétienne de la Loi par opposition au judarsme; à ce titre, elles se distinguent clairement des récits de controverse dont l'intention

1 Cette position est parfaitement cohérente avec le programme énoncé en 5,17-20. Cf. BARTH 87; BORNKAMM, Enderwartung 22-23; TRILLING, Israel $208-210$.

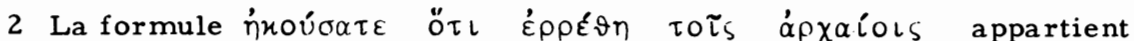
à la terminologie rabbinique (voir BILL 1,253; cf. aussi BARTH 87; TRILLING, Israel 208-209). Les "anciens" désignent le premier maillon de la chaîne traditionnelle qui a reçu la Loi de Moise et de Josué (cf. Abat 1,la). 'Huoúoate signifie "vous avez reçu comme tradi-

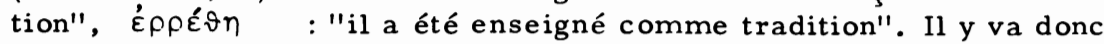
de la conception de la Loi dans la tradition rabbinique-pharisienne.

3 La lère, $2 e$ et $4 e$ antithèse radicalisent la Loi; la $3 e$, $5 e$ et 6e l'annu lent de facto en la dépassant. BORNKAMM (Enderwartung 22) dégage bien la perspective d'ensemble : "Ihr durchgangiges Motiv ist der Durchstoss durch ein in formale Rechtssätze verkehrtes Gesetz, hinter dessen Ordnung das ungehorsame Herz des Menschen sich in Ordnung wahnt, und damit das Dringen auf den ursprünglichen radikalen Willen Gottes, der zu Vollkommenheit ruft".

4 BORNKAMM, Wandlungen 96.

5 Point bien relevé par HUMMEL 74. 
est a polog étique.

Les quelques remarques qui précèdent nous permettent de mieux situer notre problématique. Nous la formulerons ainsi : les antithèses énoncent de manière exemplaire la volonté de Dieu édictée par le Christ à l'intention de l'Eglise, et elles nous permettent de découvrir le type d'obéissance exigé du croyant ou, en d'autres termes, le thème de l'éthique; elles donnent un contenu au programme de 5,20 en précisant quelle est "cette meilleure justice" à laquelle est appelé le disciple.

Nous ne traiterons pas les six antithèses, mais nous concentrerons notre attention sur la dernière $(5,43-48)$ qui est à la fois le point culmi nant et la conclusion de la liste.

\section{Analyse littéraire}

Comme l'atteste Lc 6,27-28.32-36, Mt a trouvé la matière de la sixième antithèse dans la source $Q$. Dans son ensemble, la version $\mathrm{mt}$ semble plus proche de $Q$ que celle de Lc, car elle est plus brève et moins élaborée ${ }^{l}$; par ailleurs, le vocabulaire lc convient à un auditoire de pagano-chrétiens hellénistiques tandis que Mt est encore proche du monde juif-palestinien ${ }^{2}$.

1 Lc a, en effet, écrit une véritable petite composition sur le thème de l'amour des ennemis en combinant des logia (voir l'insertion des vv. 29-31), en procédant à des élargissements rédactionnels (27c. $28 \mathrm{a}$. 34.35a.b ). Cf. BULTMANN, Trad 100, FUCHS 131 et l'analyse détaillée de SCHULZ, Q 127-131.

2 Lc reformule sa source dans un vocabulaire plus accessible à des

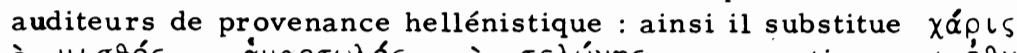

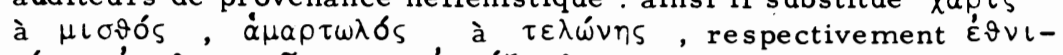
นós, $\dot{\alpha} \gamma \alpha \vartheta \circ \pi 0 \iota \varepsilon \tau \nu$ à $\dot{\alpha} \sigma \pi \alpha \zeta \varepsilon \sigma \vartheta \alpha \iota$. Voir : BRAUN, Radikalismus II 115, note 1; BULTMANN, Trad 83; position inverse chez SCHWEIZER, Mt 68 . 
Pourtant il apparait que sur quatre points au moins ${ }^{1}$, Mt a retouché sa source. Tout d'abord - et ici l'unanimité des exégètes est quasiment réalisée ${ }^{2}$-, c'est Mt qui a coulé les sentences sur l'amour des ennemis dans la forme de l'antithèse : d'une part, en effet, Mt est le seul évangile à connaître la forme de l'antithèse; d'autre part, le parallèle lc est cons titué par un simple enchaînement de sentences. Il en résulte que les vv. 43. 44a sont rédactionnels. La deuxième modification notable se situe au

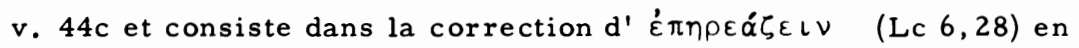
$\delta\llcorner\omega ́ x \varepsilon \iota \nu$. Mt a, en effet, une prédilection particulière pour ce verbe (emploi rédactionnel en 5,10.11.12) et Lc ne l'évite pas quand il le trouve dans ses sources ${ }^{3}$. La troisième modification est fort typique, bien que sans grande importance théologique : c'est l'application du prédicat

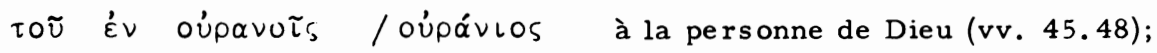
le fait est si connu qu'il n'appelle pas une démonstration. En revanche - et c'est la quatrième modification notable -, la réinterprétation $\mathrm{mt}$ du v. 48 est lourde de conséquences. Il semble bien en effet que Mt ait cor-

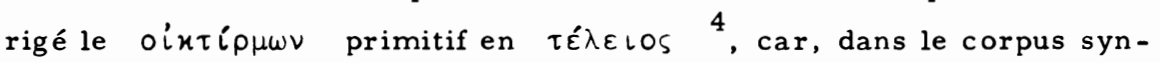
optique, cet adjectif n'apparaît que dans le premier évangile et sa seule attestation en dehors de notre contexte (cf. 19,21) est incontestablement rédactionnelle. Enfin, si le v. 48 est bien la conclusion des antithèses, destinées elles-mêmes à illustrer le programme de 5,17-20, l'idée de perfection s'accorde bien au contexte alors que celle de miséricorde n'au-

1 Dans le cas de la formulation en antithèse et dans l'utilisation du prédi cat divin oúnávlos, on ne saurait dire si l'auteur de ces transformations est le rédacteur proprement dit ou $Q m$ t.

2 Voir p.ex., BRAUN, Radikalismus II 7, note 2; BULTMANN, Trad 143-144; EICHHOLZ 70; FUCHS 131; GRUNDMANN, Mt 175; KLOSTER MANN, Mt 42; LUEHRMANN, Logienquelle 118; SCHULZ, Q 127; SCHWEIZER, Mt 66-67.

3 SCHULZ, Q 128; STRECKER, Weg 151, note 4.

4 Position partagée par BARTH 90 (voir en particulier note 5); FUCHS 131; SCHULZ, Q 130; SCHWEIZER, Mt 68; STRECKER, Weg 141; TRILLING, Israel 193. 
rait guère convenu.

Les corrections rédactionnelles que nous venons de relever, ne sont pas de simples gloses; elles infléchissent la tradition dans un sens nouveau qu'il convient de préciser. L'analyse de l'argumentation du passage nous permet de découvrir l'originalité de la réinterprétation $\mathrm{mt}$. Ainsi, l'introduction de la forme littéraire de l'antithèse donne naissance au schéma de pensée suivant : le v. 43 énonce la thèse communément admise par l'auditoire fictif; le v. 44 expose une nouvelle thèse, niant la précédente e t destinée à la remplacer; le v. 45 fonde cette nouvelle thèse tandis que la double question rhétorique des vv, 46-47 réfute la position récusée par le Christ; le v. 48 conclut le débat sous la forme d'un impératif.

La réinterprétation du v. 48 à partir de l'idée de perfection nous fait progresser dans l'analyse de l'argumentation mt. En effet, si chez Lc, l'invitation à la miséricorde $(6,36)$ est un logion que l'on peut rattacher aussi bien à ce qui précède (vv. $27-35)$ qu'à ce qui suit (vv. $37-42)^{l}$, chez Mt, l'appel à la perfection reprend clairement le propos du v. 45 et, à ce titre, conclut la sixième antithèse dans son ensemble. Pourtant son rôle rhétorique ne s'arrête pas là ${ }^{2}$ : la perfection réclamée du disciple ne se limite pas à l'amour de l'ennemi, elle correspond à la description de l'obéissance telle qu'elle est donnée dans les six antithèses (21 -47). Dès lors, le v. 48 non seulement clôt la dernière antithèse, mais il résume également le propos des vv. $21-47$ et, de ce point de vue, définit le contenu de la meilleure justice proposée programmatiquement au v. $20^{3}$.

Un dernier point de la réinterprétation mt mérite notre attention : c'est l'enracinement historique donné à l'argumentation par la correction rédactionnelle opérée en $44 \mathrm{c}$. Parlant à sa communauté, Mt pose la problématique de l'ennemi dans des termes ecclésiaux : l'ennemi est le per-

1 BULTMANN, Trad 92.

2 Sur ce point, voir : BONNARD, Mt 74; BRAUN, Radikalismus II 43, note 1; FUCHS 132; GRUNDMANN, Mt 179-180; KLOSTERMANN, Mt 51.

3 BORNKAMM, Jesus 95. 
sécuteur de l'Eglise (explicitation d' $\dot{\chi} \vartheta \rho o ́ s$, par $\delta \iota(u ́ x \omega$ ) auquel le disciple est constamment exposé. L'amour dont le croyant doit faire preuve, concerne concrètement les ennemis de la foi - que ce soit des Juifs ou des païens (cf. Mt 10,17-25) -. La sixième antithèse est donc pensée dans une situation de persécution ${ }^{1}$.

\section{Le prochain et l'ennemi}

Le Christ $\mathrm{mt}$ attribue à la Loi mosalque telle qu'elle est comprise par la tradition rabbinique pharisienne, l'ordre d'aimer son prochain et de hair son ennemi (v. 43). Rend - il ainsi justice à la position qu'il atta que ou s'agit-il d'un raccourci polémique et simplificateur ? En ce qui concerne l'Ancien Testament, le reproche semble de prime abord injustifié : si le v. 43a est une citation explicite de Lv 19,18, par contre le v. $43 \mathrm{~b}$ n'apparait jamais sous cette forme dans l'Ancien Testament. Pourtant, le problème rebondit immédiatement dans la mesure où la notion de prochain dont parle Lv 19,18, désigne le compatriote à l'exclusion de l'étranger $^{2}$; le commandement d'amour est donc bien partie intégrante de la Loi, mais il régit en priorité les rapports à l'intérieur de la communauté politique et religieuse. Cette limitation laisse ouverte l'attitude à observer face à ceux qui ne sont pas membres de l'Alliance et n'habitent pas le pays. Et, de fait, sur ce point particulier, l'Ancien Testament s'est ex-

1 EICHHOLZ 99-100; GRUNDMANN, Mt 177; SCHWEIZER, Mt 81 .

2 Cette limitation apparaît d'une double manière. D'une part, l'interdic tion de se venger qui précède immédiatement ( $\mathrm{Lv} 19,18 \mathrm{a})$ et qui est formulée de manière parallèle s'applique aux "fils de ton peuple" (:). D'autre part, Lv 19,34 étend explicitement le commandement d'amour à l'étranger en séjour ạ Is raël : cet élargissement présuppose la limitation préalable du concept de prochain. Partagent notre point de vue : BILL 1,353-54; BRAUN, Radikalismus II 92; FICHTNER, art. $\pi \lambda \eta-$ cíov, ThW 6,312-313; GRUNDMANN, Mt 175-176; KLOSTERMANN, Mt 50; SCHMID, Mt 41; SCHWEIZER, Mt 80-81. 
primé de manière très diversifiée : il peut recommander aussi bien l'a mour que l'extermination du non-Israélite ${ }^{l}$.

Le bas-judaiisme adopte la même attitude que l'Ancien Testament à cette différence près que la problématique du prochain ne se pose plus au niveau de l'Etat, mais du groupe religieux ${ }^{2}$ qui prétend incarner le véritable Israël. Si l'amour est la règle de la confrérie, le devoir vis -à -vis de l'étranger varie entre la bienveillance et la haine. La tradition rabbinique peut aussi bien déclarer : "poursuis la paix, aime les créatures et amène-les à la Tora" ${ }^{3}$ que : "un disciple qui ne hait pas n'est pas un disciple" ${ }^{4}$. L'affirmation la plus proche de Mt 5,43 se trouve cependant dans la règle de Qumrân où il est dit : "(leur but est) d'aimer tous les fils de Lumière, chacun selon son sort dans le dessein de Dieu et de haïr tous les fils de Ténèbres, chacun selon sa culpabilité dans la vengeance de Dieu" ${ }^{5}$. La conclusion de cette brève enquête semble s'imposer d'ellemême : si le Christ mt ne cite littéralement ni l'Ancien Testament, ni la tradition rabbinique d'obédience pharisienne, il restitue néanmoins assez fidèlement le sentiment qui habitait l'Israélite moyen face à son prochain

1 Outre Lv 19,34, Dt 10,18 recommande la bienveillance vis-à-vis de l'étranger. Des textes tels que $E_{x} 23,4-5,1 S 24,20, \operatorname{Pr} 24,17 ; 25,21$ appellent à l'amour face à l'ennemi. A l'inverse, les psaumes de vengeance (Ps 137,7-9 p.ex.) ou les prescriptions contre les Cananéens (Dt 20,13-18 p.ex.) prônent explicitement la ruine et l'extermination des ennemis de Yahvé et donc du peuple.

2 Nous pensons à des clivages du genre : Judée/Samarie, pharisiens/ am ha-aretz, Qumrân ${ }_{i}^{\prime}$ monde extérieur. Par rapport à l'Ancien Testa ment, il s'ensuit même une limitation plus stricte du concept : la synagogue n'accueille favorablement que l'étranger qui se convertit dans l'année. Pour l'ensemble de la question, voir : BILL 1,353-368; BRAUN, Radikalismus II $57-58$; FICHTNER, art. $\pi \lambda$ noiov, ThW 6 ,

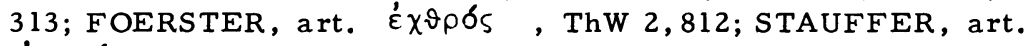

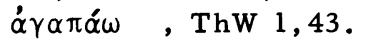

3 A bot 1,12 .

4 b Joma 22b cité par BILL 1, 365; cf. aussi SEITZ 43.

5 l QS 1,9-11 (traduction de Carmignac); cf. aussi SEITZ 49-51. 
proche ou lointain ${ }^{l}$.

A cette conception qu'il réprouve, le Christ $\mathrm{mt}$ oppose son souverain $\dot{\varepsilon} \gamma \grave{\omega} \delta \grave{\varepsilon} \quad \lambda \varepsilon \hat{\gamma} \omega \quad \dot{u} \mu \tau \nu$. Il met en balance son autorité avec celle de Molse. Il rompt avec la tradition ou plutôt il prétend l'accomplir en révélant la volonté originaire de Dieu. L'auditeur est placé devant une alternative : ou bien s'en remettre à la tradition des pères, ou bien accorder sa foi à celui qui prétend révéler le Père. La question christologique est inséparable du débat.

Mais quel est l'objet du litige ? L'amour du prochain n'est pas en cause, il est admis par tous (cf. vv. 46-47!). Les esprits se séparent sur la portée de cet amour. En revendiquant de manière provocante l'a mour de l'ennemi - et pas seulement du frère -, le Christ mt récuse toute discrimination face au prochain. En faisant de la situation la plus extrême le critère de l'amour, il scandalise l'homme naturel (vv. 46-47). L'enne mi n'est-il pas celui qui n'aime personne et que personne n'aime ? et, pour l'église $\mathrm{mt}^{2}$, l'ennemi n'est-il pas l'adversaire de Dieu, celui qui persécute le disciple précisément parce qu'il est disciple? Mais, justement, l'amour ne choisit pas le prochain. Il ne s'en tient pas aux solidarités religieuses, sociales, politiques. Il ne connaît pas d'a priori restrictif. Il consiste à être là pour l'autre, dépréoccupé de soi et n'exigeant rien de lui ${ }^{3}$. Il accepte le prochain tel qu'il est, sans l'asservir à des fins personnelles. Mais si l'amour est cela, il ne fait pas acception de personne et il n'exclut même pas celui-là par qui je suis douloureusement contesté et menacé : l'ennemi. L'amour inclut l'ennemi ou il n'est pas l'amour. L'amour de l'ennemi est le gage du sérieux et de l'authenticité

1 Ainsi également : BILL 1,353; BONNARD, Mt 74; GRUNDMANN, Mt 176; SEITZ 51.

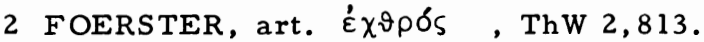

3 BULTMANN, Jesus 79 : "... Die Feindesliebe ist nicht der Höhepunkt der allgemeinen Menschenliebe, sondern der Höhepunkt der Selbst überwindung, des Verzichts auf den eigenen Anspruch". 
de l'amour du prochain : il est sa vérification critique. Encore faut-il s'entendre sur le terme amour : il ne s'agit ni d'un sentiment, ni d'une neutralité bienveillante. Mt concrétise l'amour par le thème de l'inter -

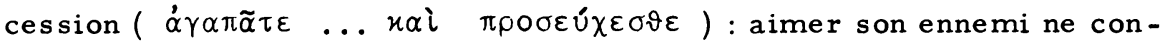
siste pas à ignorer son hostilité, à ne pas répondre à ses provocations, mais à agir en sa faveur au point de prendre parti pour lui devant Dieu ${ }^{1}$.

Si nous essayons d'élaborer la problématique éthique à partir des quelques remarques qui précèdent, nous constatons ceci : l'argumentation du Christ mt ne vise pas à instaurer une nouvelle valeur morale - l'a mour -; l'Ancien Testament et le judal'sme connaissent déjà cette exigence. L'argumentation porte sur la manière dont l'amour doit être vécu par le disciple ${ }^{2}$. En supprimant toute restriction, en faisant d'une "situationlimite" la règle, le Christ mt donne à comprendre que l'amour n'est pas une tâche limitée, circonscrite, occasionnelle. L'amour est une tâche infinie, illimitée qui n'est jamais achevée. Cette destruction de la limite, cette radicalisation de l'exigence est d'ailleurs le fil conducteur des antithèses construites selon le schéma "non seulement... mais même". Bornkamm écrit à ce sujet : "Même la colère, même le regard impur, même le divorce légal, même le simple serment..., même la vengeance qui reste dans les limites permises par la Loi, même l'amour qui exclut l'ennemi, tout cela est contraire à la volonté de Dieu" ${ }^{3}$.

Cette contestation de toute limite, de tout point d'arrêt nous permet à la fois de découvrir la pointe de la sixième antithèse et de mieux dégager le thème de l'éthique. Il ne s'agit pas d'abord pour le Christ mt de décrire des modèles de comportement et, p. ex., la règle normative à adopter

1 La prière pour l'ennemi est une nouveauté absolue par rapport à la tradition vétérotestamentaire-juive. Cf. SCHWEIZER, Mt 81 .

2 BORNKAMM, Jesus 97; BULTMANN, Jesus 63, écrit à ce propos : "er (= Jesus) weist den Frager immer auf sich selbst zurück".

3 BORNKAMM, Jesus 95 (cité ici d'après la traduction française de BARTH et de DE BUSSY 120). 
face à l'ennemi, mais bien d'interroger le disciple sur la qualité de son obéissance, c'est-à-dire de lui montrer que toute obéissance qui ne saisit pas la totalité de son être et de son vouloir est, en définitive, une fausse obéissance ${ }^{l}$. La fidélité exigée, p.ex., dans l'amour est une fidélité illimitée qui ne fait même pas la restriction de l'ennemi, une fidélité qui mobilise l'existence dans sa totalité. Car tel est le thème de l'éthique, telle est la consécration requise par le Dieu du Sermon sur la Montagne. Mais qui est ce Dieu ? comment se révèle-t-il ? C'est l'objet du v. 45.

\section{Imiter Dieu}

Le v. 45 fonde les vv. $43-44$ en en révélant la finalité (öđws ). La réfutation de la tradition des pères (v. 43) et la formulation par Jésus d'un nouvel impératif ( $\mathrm{v}$. 44) sont ainsi situés dans la seule réalité qui permette de les comprendre : la volonté et l'action de Dieu. L'éthique mt ne vise pas à défendre une certaine échelle des valeurs, elle n'est pas régie par une image idéale de l'homme ${ }^{2}$; son propos est de confronter l'homme à Dieu, de montrer comment se constitue l'existence placée en présence de Dieu et de son à-venir ${ }^{3}$. La terminologie fort originale du v. 45a le souligne d'emblée : l'amour de l'ennemi pose en réalité la question de la relation entre le Père et ses fils ${ }^{4}$.

1 BORNKAMM, Jesus 97; BULTMANN, Jesus 56, écrit à ce sujet :

"Radikaler Gehorsam ist nur dort vorhanden,... wo der Mensch ganz in dem ist, was er tut, d.h. wo er nicht gehorsam etwas tut, sondern in seinem Sein gehorsam ist".

2 Cf. BULtMANN, Jesus 53.57.61.

3 Cf. BORNKAMM, Jesus 99.

4 La terminologie du v. 45a est fort originale dans le cadre du corpus synoptique à un double point de vue : d'une part, le couple $\pi \alpha \tau \hat{n} \rho$ viós (affirmé du croyant) n'apparaît jamais ailleurs; d'autre part, c'est la seule fois où la filialité est comprise comme une condition déjà présente (voir SCHWEIZER, art. ví́s, ThW 8,392-393, et Mt 55). Il est probable que $M t$ reprend ici une idée vétérotestamentaire-juive. 
Comment le disciple peut-il devenir le fils du Père céleste ? La filialité divine n'est pas une qualité naturelle de l'homme ${ }^{1}$. Le croyant est appelé à réaliser un "être" qualitativement différent de celui de l'homme naturel (cf. vv. 46-47). Devenir le fils du Père, c'est précisément cesser de vivre comme les collecteurs d'impôts et les paiens, c'est faire quelque chose d'extraordinaire $(\pi \varepsilon \rho \iota \sigma \sigma \delta v)$, c'est accepter la possibilité de vie créée par le Père. Or cette adhésion à la condition de fils ne se réalise que là où le disciple découvre dans son Père le paradigme de son action ou, en d'autres termes, que là où le disciple aime son prochain de l'amour même du Père ${ }^{2}$.

$\underline{\text { L'exigence }}^{3}$ sous laquelle est placée la filialité, peut sembler incommensurable. Pourtant le v. 45b, en dévoilant qui est le Père, montre la grâce qui est inhérente à cette condition. Il recourt à un motif sapiential $^{4}$ : la création est la parabole de l'action divine. Plus concrètement, le soleil et la pluie qui font croître le blé dans les champs et assurent à l'homme sa nourriture, sont l'expression de la bonté divine ${ }^{5}$ qui - c'est

Dans l'Ancien Testament, en effet, la relation père -fils décrit le rapport entre Yahvé et Israël dans une double perspective : d'une part, pour souligner la distance qui sépare les deux partenaires, donc corrélativement l'autorité de Yahvé et la soumission du peuple; d'autre part, pour exprimer la bonté et l'amour de Yahvé (voir FOHRER, art. cit., ThW 8,352-53). Dans le judai'sme palestinien, la catégorie de la filialité, au sens imagé, décrit avant tout l'appartenance, étant entendu que l'homme se définit précisément par son appartenance à telle ou telle grandeur. L'appartenance d'Israël à Dieu repose sur un acte d'élection, concrétisé dans le don de la Loi et lié à la pratique de cette dernière (voir LOHSE, art. cit., ThW 8,359-361).

1 SCHLATTER, Mt 193; SCHWEIZER, art. cit., ThW 8,393.

2 GRUNDMANN, Mt 177-78; SCHWEIZER, Mt 55.

3 Le v. 45a décrit moins une possibilité qu'une nécessité : l'accent impératif est incontestable (cf. BONNARD, Mt 429; KLOSTERMANN, Mt 50).

4 BORNKAMM, Jesus 110; SCHULZ, Q 135-136.

5 EICHHOLZ 100. Le matériel rassemblé par BILL 1, 374-377 montre que le judaìsme rabbinique a également compris la présence du soleil et de la pluie comme le témoignage de la bonté de Dieu. 
l'élément capital - agit sans discrimination. L'amour de Dieu n'est pas réservé aux croyants : il s'adresse aussi bien aux méchants qu'aux bons, aux justes qu'aux injustes ${ }^{1}$. Dieu ne fait pas acception de personne; dans sa liberté souveraine et sans dresser aucun préalable, il s'approche de chaque homme pour lui offrir son amour et, en définitive, le salut. Le Dieu de la sixième antithèse est le Dieu de la prédication du Règne, le Dieu de l'évangile au sens fort de ce terme.

Nous voyons aussitôt comment rebondit la question de la filialité ! Certes le disciple est placé sous une exigence infinie, parce que, comme tout homme, il est l'objet d'un amour infini. Il n'est pas invité à imiter Dieu pour être sauvé, mais parce qu'il l'est. Comme le dit bien Schlatter ${ }^{2}$, il ne se nomme pas lui-même fils, mais il entre dans une condition qui lui est ouverte par Dieu, il ratifie par son adhésion résolue l'initiative dont il a été l'objet. Comme dans la conception vétérotestamentaire-juive de la filialité, élection gracieuse et exigence vont de pair.

Notre interprétation du v. 45 nous permet de progresser dans l'élaboration du thème de l'éthique. L'obéissance totale signifiée par l'ordre d'aimer son ennemi n'est pas une maxime morale qui possède sa légitimité en elle-même. Elle n'a de sens que dans la mesure où elle est intégrée à son contexte théologique, à savoir la rencontre entre Dieu et l'homme. En s'approchant gracieusement de tout homme, en se laissant invoquer comme le Père, en offrant à chacun la condition de fils, Dieu prend l'initiative d'une relation, il crée la situation même dans laquelle surgit la question éthique, à savoir : comment le disciple doit-il ordonner son existence face à la réalité de Dieu manifestée dans les antithèses ? com-

1 Le chiasme du v. 45b renforce l'aspect scandaleux de l'amour de Dieu.

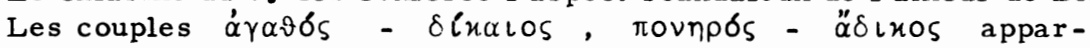
tiennent à la terminologie mt classique pour décrire les fidèles, res pectivement les adversaires de Dieu.

2 SCHLATTER, Mt 193 : "Der Sohn ernennt sich nicht selbst zum Sohn; Kindschaft Gottes gibt es nur durch göttliches Wirken". 
ment va-t-il vivre son acquiescement? Le thème de l'éthique consiste précisément à décrire cet acquiescement, à montrer comment le disciple, en choisissant la condition filiale, s'abandonne totalement à la volonté du Père au point de devenir son imitateur, au point d'aimer de l'amour même dont il est aimé.

En résumé, si les vv. $43-44$ dépeignent, à travers l'exigence de l'a mour de l'ennemi, l'obéissance radicale qui est demandée au croyant et qui est précisément le thème de l'éthique, le v. 45 affirme que cet impé ratif n'a de sens que dans la relation qui existe entre Dieu et le croyant et dont Dieu a pris l'initiative.

\section{La perfection}

Le v. 48 est à la fois la conclusion et la récapitulation des antithèses - et singulièrement de la dernière -. La difficulté majeure réside dans l'interprétation du concept $\underline{\tau \varepsilon ́ \lambda \varepsilon l o S}$. Néanmoins, il semble que l'exé-

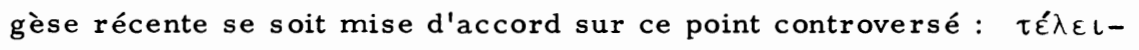
os doit être compris sur la base de la tradition vétérotestamentaire de la perfection. Cette tradition s'exprime avant tout par deux termes :

תמימ qui se dit d'un homme qui accomplit toute la volonté de Dieu et של qui décrit une obéissance non partagée à l'égard de Dieu ${ }^{1}$. La note dominante porte donc sur la totalité, sur l'intégralité d'un engagement. Il y va non pas de la pureté morale, ni d'un idéal à réaliser progressivement, ni d'une intensification quantitative de l'obéissance, mais d'un coeur non partagé, d'une consécration totale dans l'accomplissement

1 Sur la signification vétérotestamentaire-juive, puis mt de $\tau \varepsilon ́ \lambda \varepsilon\left\llcorner O S_{\text {, }}\right.$

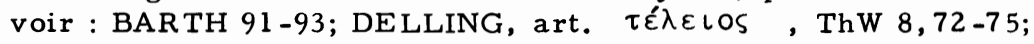
TRILLING, Israel $192-196$. 
d'un projet ${ }^{l}$.

Comment convient-il d'appliquer cette définition dans notre v. ? De quelle manière la perfection du Père est-elle le paradigme de la perfec tion du disciple ? Le $\dot{\omega} s$ fait de l'attitude du Père un exemple norma tif. La perfection du Père doit être comprise en continuité avec le v. 45 : elle réside dans sa bonté sans partage envers ses créatures. Le phénomène observé au v. 45 se répète : la perfection divine est non seulement le paradigme de la perfection du disciple, mais aussi son fondement ${ }^{2}$. L'engagement illimité de Dieu pour ses créatures ${ }^{3}$ est non seulement un modèle proposé au croyant, mais aussi et surtout sa condition de possibilité dans la mesure où le disciple est précisément au bénéfice de cette sollicitude divine. Dans ces conditions, la perfection du disciple consiste dans son engagement total en réponse à l'engagement total du Père. De

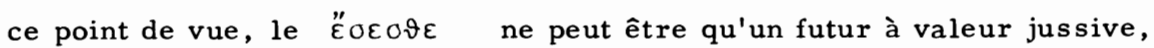
car il décrit la décision qui est exigée de l'homme face à l'action de Dieu ${ }^{4}$.

En conclusion, après cette analyse de la sixième antithèse, nous pouvons revenir à la question centrale : quel est le thème de l'éthique ? Le

1 Dans la même ligne : BONNARD, Mt 76.429; BORNKAMM, Jesus 98 99; BRAUN, Radikalismus II 43, note 1; BULTMANN, Jesus 83-84;

GRUNDMANN, Mt 180-181; SCHLATTER, Mt 197; SCHNIEWIND, Mt 73; SCHWEIZER, Mt 83 et les auteurs cités dans la note précédente. STRECKER, Weg 141-142, voit dans la perfection une intensification quantitative de l'obéissance.

2 Aspect bien souligné par BRAUN, Radikalismus II 43, note 1; TRILLING, Israel 196 et GRUNDMANN, Mt 181: "Gott ist in vollem und ganzem Sinne 'euer Vater', d.h. in vollem und ganzem Sinne für euch da. Aus dieser Botschaft aber, die den Menschen seines Gottes gewiss macht, ergibt sich für ihn die Möglichkeit, von sich selbst gelöst und doch seiner gewiss, weil er seines Gottes gewiss geworden ist, voll und ganz sich in der Liebe hinzugeben". Point longuement développé par FUCHS $134-136$.

3 La proclamation du "Christ des antithèses" est assurément le témoigna ge inaliénable, mais trop peu souligné, de l'engagement illimité de Dieu pour ses créatures.

4 Le futur jussif est un élément bien connu du langage de la Loi dans 1'Ancien Testament, cf. Bl-D par. 362 et DELLING, art. cit., ThW 8, 75 (note 36 ). 
premier évangile ne livre pas un catalogue des vertus et des devoirs qui régiraient l'existence chrétienne; il ne dresse pas une liste des valeurs qu'il conviendrait d'instaurer au niveau individuel ou collectif; il n'a pas en vue l'épanouissement de la personne ou l'amour du genre humain. Le thème exclusif et suffisant de l'éthique mt est la prétention radicale que Dieu, à travers la prédication du Christ, émet sur l'existence humaine. En ce sens, l'éthique $\mathrm{mt}$ est une éthique de l'obéissance; elle veut décrire de manière adéquate la nature de cette obéissance.

L'idée de perfection exprime de manière exemplaire ce projet ${ }^{1}$. En parfaite cohérence avec les antithèses, elle souligne que la question éthi que décisive ne porte pas sur la rectitude de l'action prise en elle-même, mais sur l'intégralité de l'obéissance. Le disciple n'est pas appelé à faire ceci ou cela, mais à se consacrer dans la totalité de son être et de son vouloir à Dieu. Quel est alors le rôle de la Loi ? A-t-elle encore un sens? Selon Mt, la Loi réinterprétée et accomplie par le Christ exprime sans équivoque la revendication radicale de Dieu sur les siens; elle dévoile comment le disciple appartient totalement à Dieu. Ainsi la sixième antithèse qui montre à propos de l'amour ce que signifie l'accomplissement de la Tora, n'a pas pour but d'ajouter une exigence supplémentaire à la pratique habituelle, mais d'indiquer à quelles conditions l'amour est abandon de toute prétention et acceptation de l'exigence divine ${ }^{2}$.

La perfection me se résume cependant pas à la radicalisation de l'obéissance requise; à ce titre, elle ne serait guère qu'une aggravation de la Tora rabbinique. Elle prétend être qualitativement différente et mettre fin à tout autre comportement éthique. Ni la justice des pères qui codifie l'obéissance ( $v$. 43), ni celle de l'homme naturel qui s'élabore à partir de la sagesse du monde (vv. 46-47), ne sont reçues par Dieu. La perfection proclamée dans les antithèses met fin aux possibilités comme

1 BULTMANN, Jesus 84 .

2 BARTH 93. 
aux impossibilités des hommes, à leur moralité comme à leur immoralité. Elle est cette justice qui surpasse toutes les autres $(5,20)$, elle est cette possibilité extraordinaire $(5,47)$ qui échappe au collecteur d'impôts et au palen ${ }^{l}$. Mais en quoi est-elle extraordinaire ? et pourquoi est-elle l'apanage des seuls disciples ${ }^{2}$ ?

A ce point de l'analyse, il convient de reprendre le contexte théologique dans lequel Mt situe l'impératif. La perfection du Sermon sur la Montagne est indissociable de celui qui la révèle et de celui qu'elle révèle. Pour la découvrir il faut faire totalement confiance à celui qui déclare, contre toute évidence et toute tradition : "Mais moi je vous dis..." (v. 44). Pour la revêtir, il faut dire non à soi-même et oui au Dieu qui s'approche pour établir sa seigneurie sur toute créature (v. 45). La perfection $\mathrm{mt}$ s'annonce comme une possibilité de vie pour celui qui a fait le deuil de lui-même et du monde, qui est prêt à vivre de la présence et de l'à-venir de Dieu, qui s'appuie désormais sur ce Père miséricordieux dont le Christ est le révélateur. L'exigence de perfection n'est pas une implication secondaire et périphérique de l'évangi le; elle est l'indice de la relation qui se noue entre le croyant et le Dieu du Sermon sur la Montagne; elle est, au niveau de l'existence chrétienne, la réduplication de l'amour du Père.

1 Le $\pi \varepsilon \rho\llcorner\sigma \sigma o ́ v$ du v. 47 ne décrit pas une obéissance qui s'élèverait au-dessus de la normale, car le comportement du païen n'est justement pas le modèle d'une obéissance normale, mais de la désobéissance face à Dieu. Le $\pi \varepsilon \rho \iota \sigma \sigma \delta$ met donc le païen en question non pas en vue d'une amélioration de sa moralité, mais au nom d'une possibilité de vie entièrement nouvelle (cf. DELLING, art. cit., ThW 8,75, note 37).

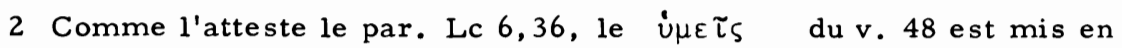
évidence par Mt (cf. BARTH 91; KLOSTERMANN, Mt 51). Il désigne la communauté des disciples par opposition à tous les autres hommes : d'abord les collecteurs et les paiens qui tombent sous le coup du $\pi \varepsilon \rho \iota \sigma o \delta v$ ( $v v .46-47$ ), mais aussiles scribes et les pharisiens condamnés par le $\pi \varepsilon \rho \iota \sigma \sigma \varepsilon u ́ o n$ du v. 20; ni les uns, ni les autres n'ont part à la perfection qui conduit au Royaume. 


\section{CHAPITRE TREIZE : L'ENJEU DE L'ETHIQUE (Mt 25, 31 -46)}

\section{Analyse littéraire}

Mt 25,31-46 est, en analyse littéraire, l'une des péricopes les plus difficiles et les plus discutées du premier évangile. Parmi les auteurs qui acceptent de se prononce $r^{l}$, d'aucuns y voient l'écho fidèle de la prédication du Jésus historique ${ }^{2}$; d'autres soutiennent la même hypothèse, mais en relevant de nombreuses retouches rédactionnelles ${ }^{3}$; d'autres encore optent pour une composition du rédacteur liant habilement deux traditions - la parabole du berger et les antithèses - ${ }^{4}$, d'autres enfin n'hésitent pas à attribuer l'ensemble du morceau à la plume de $\mathrm{Mt}{ }^{5}$. La difficulté majeure réside dans le fait que 25,31-46 est un passage propre à $\mathrm{Mt}$; on ne peut donc recourir à la comparaison pour déterminer avec sûre té l'étendue de la source et les apports rédactionnels. On peut toutefois parer dans une certaine mesure à cette lacune par la statistique terminologique et l'analyse des motifs ${ }^{6}$.

En insistant sur l'aspect glorieux de la parousie et en mettant cette dernière en étroite relation avec le jugement, le v. 31 évoque d'emblée des perspectives spécifiquement $\mathrm{mt}$ (cf. 16,$27 ; 19,28)$. Deux motifs, caractéristiques du premier évangile, méritent d'être soulignés. D'une part,

1 INGELAERE 24 refuse de se prononcer. Cette prudence, de prime abord recommandable, a ses inconvénients : elle risque de dissimuler à l'exégète la manière dont le rédacteur se situe par rapport à la tradition reçue.

2 Ainsi MANSON, cité par JEREMIAS, Gleichnisse 206.

3 BROER 276-288 et JEREMIAS, Gleichnisse 204-206.

4 LEGASSE, Enfant 86-93 et ROBINSON, Parable. Par parabole du ber ger, il faut entendre les vv. 32-33, par antithèses, les vv. 35-40.4245.

5 COPE $36-43$.

6 Bref exposé de la méthode par BROER $282-283$. 
le Fils de l'homme est accompagné par des anges. Cette représentation, étrangère à la littérature juive, apparaît en revanche à la fois dans la tradition paulinienne ( $1 \mathrm{Th} 3,13,2 \mathrm{Th} 1,7)$ et dans le corpus synoptique $($ Mc 8,$38 ; 13,27)$. L'apport spécifique de Mt est d'avoir accentué ce motif et surtout de décrire les anges comme appartenant au Fils de l'homme ${ }^{l}$. D'autre part, le v. 31 fait allusion à la session du Fils de l'homme sur le trône de gloire. Rare dans l'apocalyptique juive, ce motif est en principe réservé à Dieu dans la littérature biblique. C'est une caractéristique de Mt que d'attribuer cette prérogative au Fils de l'homme ${ }^{2}$. Si donc le v. 31 reprend des motifs traditionnels, il les présente de manière spécifiquement $\mathrm{mt}$ : désormais, le jugement, la gloire, les anges, le trône appartiennent au Fils de l'homme. Cette constatation donne à penser que le v. 31 est rédactionnel ${ }^{3}$. Cette hypothèse est appuyée par le fait que le v. 31 fait figure de corps étranger dans l'ensemble du récit; il s'accorde mal notamment avec la titulature royale qui intervient dès le v. 34 .

Le v. 32a semble également émaner de l'évangéliste, car il assure la transition entre le v. 31 qui est un en-tête rédactionnel et les vv. $32 \mathrm{bc}-33$ qui relèvent du genre parabolique. Cet argument fonctionnel est renforcé par des considérations de syntaxe et de terminologie : la cons truction de la phrase est semblable à celle de 13,2a qui est rédactionnel

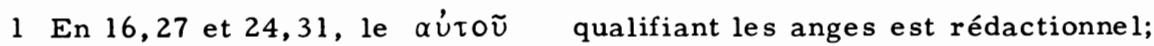
l'explication de la parabole de l'ivraie qui est de la main de Mt (voir notre chap. 7) va dans le même sens : 13,4l parle des anges du Fils

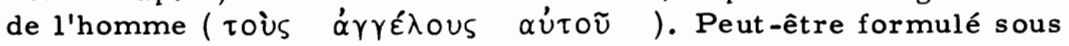

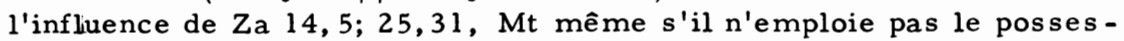
sif, met explicitement en relation les anges avec le Fils de l'homme ( $\mu \varepsilon \tau$ ' $\alpha \dot{U} \tau o \tilde{v})$. Voir : BROER 276-277; COPE 86; INGELAERE 27; ROBINSON, Parable $227-228$.

2 Cf. 19,28. Signalent la coloration $\mathrm{mt}$ du motif : BROER 277; COPE 36; INGELAERE 27; ROBINSON, Parable 227 -228.

3 C'est un avis largement partagé parmi les exégètes : BROER 277;

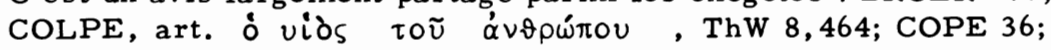
JEREMIAS, Gleichnisse 204 (avec réserve); LEGASSE, Enfant 87-88; ROBINSON, Parable 229; TOEDT 71; VIELHAUER, Gottes reich $62-63$. 
(cf. Mc 4,1 !); par ailleurs, Mt emploie volontiers $\ddot{\varepsilon} \mu \pi \rho \circ \sigma \vartheta \varepsilon \nu$, le

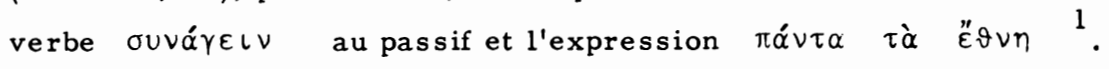

Les vv. $32 \mathrm{bc}-33$ présentent une brève séquence parabolique décrivant une activité typique du berger palestinien. On ne peut tirer de la statistique terminologique aucune conclusion sur l'origine de ces vv. ${ }^{2}$. Cependant, notre connaissance de la technique rédactionnelle de Mt nous permet de faire certaines constatations. D'une part, le rédacteur n'a pas coutume d'exprimer sa position personnelle en composant des paraboles. D'autre part, lorsqu'il dépeint la parousie, Mt n'utilise généralement pas l'image du berger séparant le bétail. Enfin, dans les descriptions rédactionnelles du jugement, ce sont les anges - et non le Fils de l'homme qui effectuent le tri ${ }^{3}$ alors que notre texte confie cette prérogative au berger. Ces différents indices nous amènent à penser que Mt restitue ici une tradition conservée dans sa communauté ${ }^{4}$. Cette hypothèse est confirmée par le décalage qui existe entre $32 \mathrm{a}$ et $32 \mathrm{~b}$ : en effet, le v. $32 \mathrm{a}$ parle du rassemblement des nations ( $\pi \dot{\alpha} \nu \tau \alpha$ tà $\left.\varepsilon^{\prime} \vartheta \vee \eta\right)$ alors qu'au v. $32 b$, ce sont les individus ( $\alpha \dot{\tau} \tau o u ́ s$ ) qui sont en cause.

Qu'en est-il du v. 34 ? Certaines retouches rédactionnelles sont évi -

1 Mt utilise $\ddot{\varepsilon} \mu \pi \rho 0 \circ \vartheta \varepsilon \nu 17$ fois pour 2 fois chez Mc et 10 fois chez Lc. Le verbe $\sigma u \vee \alpha ́ \gamma \varepsilon \iota \nu$ au passif est fréquent dans la couche ré-

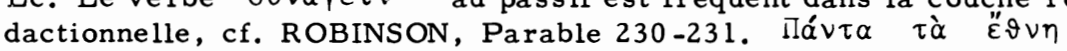
est emprunté à Mc 13,10 en 24,14, mais rédactionnel en 24, 9 et 28, 19. Pour l'ensemble, voir BROER $277-278$.

$2 \mathrm{Si} \alpha \dot{\alpha} \mathrm{S}(\zeta \varepsilon \iota \nu$ est utilisé rédactionnellement en 13,49 (JEREMIAS, Gleichnisse 83), si $\pi \rho \delta \beta \alpha \tau o v$ est un mot cher au premier évangile

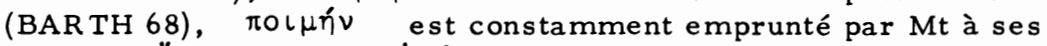
sources, हैं

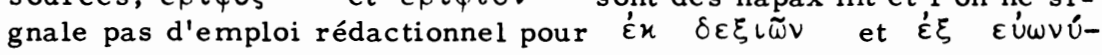
$\mu \omega \nu$.

3 Voir 13,41-42.49-50 et la correction en 24,31.

4 Position adoptée par BROER 278-79; LEGASSE, Enfant 88-89; ROBIN SON, Parable 232, défend la même hypothèse en proposant de l'étendre éventuellement à $32 \mathrm{a}$. 


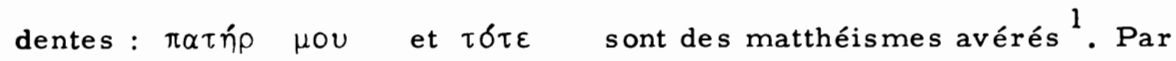

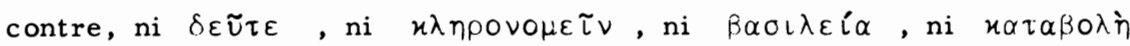

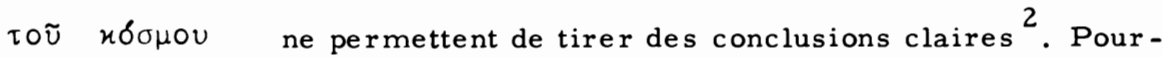
tant la question décisive porte sur $\beta \alpha \sigma \iota \lambda \varepsilon \dot{u} S_{S}$. S'agit-il d'une nouvelle figure introduite par le rédacteur ? Dans ce cas, l'ensemble du v. serait rédactionnel et servirait de transition entre les vv. $31-33$ et $35 \mathrm{ss}^{3}$. Ou bien butons-nous ici sur la suite du morceau traditionnel repris dès le $v$. $32 \mathrm{~b}$, voire sur une nouvelle unité ? Les tenants de l'hypothèse rédactionnelle font remarquer que Mt introduit volontiers la figure du Roi dans les paraboles ${ }^{4}$. Cette constatation - exacte en elle-même - ne résout rien

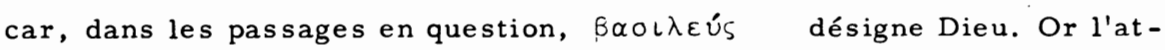
tribution de ce titre au Christ en 25,34 est un fait unique dans le premier évangile ${ }^{5}$. Ainsi puisque ce titre (à l'état absolu) est généralement accor dé à Dieu, puisque notre v. est la seule attestation l'attribuant au ChristJuge, puisque de plus ce prédicat $s$ 'harmonise mal avec la titulature du Fils de l'homme développée par le rédacteur au début de la péricope, il est vraisemblable que Mt compile ici une tradition en l'adaptant à son

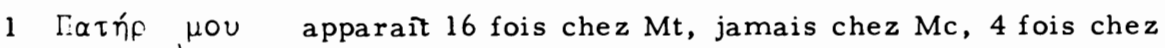
Lc; sur ces 16 emplois mt, 14 sont rédactionnels (ROBINSON, Parable 229); $\tau \delta \tau \varepsilon$ est employé 90 fois par Mt, 6 fois par Mc et 15 fois par Lc.

$2 \Delta \varepsilon \tilde{\cup} \tau \varepsilon$ apparaît 6 fois chez $\mathrm{Mt}$, dont 3 fois en suivant Mc; seul

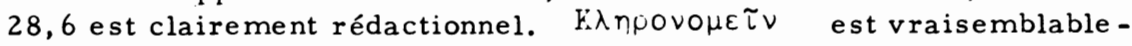
ment rédactionnel en 5, 5 et 19,29; par contre, il n'est pas repris de

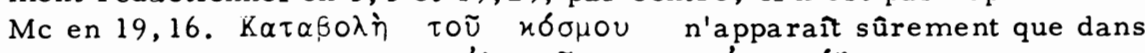

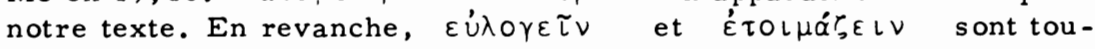
jours traditionnels.

3 Position adoptée notamment par COPE 37 -89; LEGASSE, Enfant 90; ROBINSON, Parable 230.

4 Voir 18,23; 22,2.11. Analyse chez ROBINSON, Parable 230.

5 Il va sans dire qu'il s'agit de $\beta \alpha \sigma \iota \lambda \varepsilon u ́ s$ à l'état absolu. Contraire ment à ce que fait INGELAERE 30 , on ne peut pas identifier sans autre ce titre à ceux de "Roi des Juifs" ou "Roi d'Is raell" qui interviennent dans un contexte différent (voir plus bas). 
propos.

Restent les antithèses des vv. 35-46. La plupart des exégètes pensent que Mt suit une tradition ${ }^{1}$. La statistique terminologique appuie cette appréciation ${ }^{2}$, mais aussi le fait que les oeuvres d'amour évoquées dans notre passage ne jouent aucun rôle dans le reste de l'évangile ${ }^{3}$. La difficulté surgit dès qu' on essaie de délimiter l'ampleur du travail rédactionnel. On s'accorde néanmoins pour attribuer à Mt la paternité du qua-

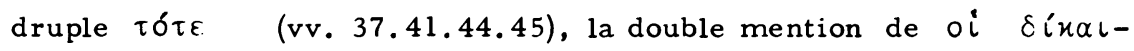

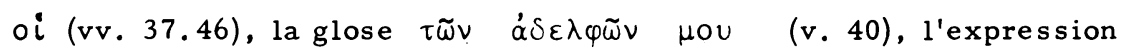

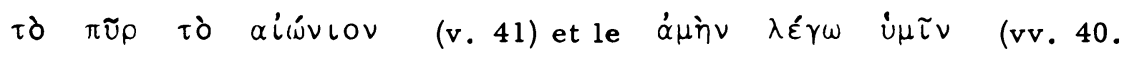
45) ${ }^{4}$. On constate immédiatement que les retouches rédactionnelles ne

1 Voir BROER 281-285; LEGASSE, Enfant 93; ROBINSON, Parable 233. Seul COPE 38-41 considère l'ensemble de ces vv. comme une composition rédactionnelle de $\mathrm{Mt}$.

2 BROER 281-285, a fait une analyse détaillée de la terminologie des vv. 35-40: $\pi \varepsilon \iota \nu \tilde{\alpha} \nu$ -

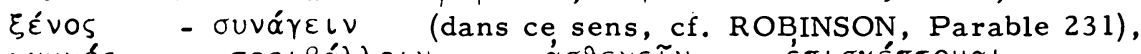

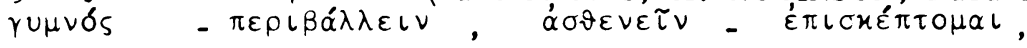
$\varphi \cup \lambda \alpha \varkappa \dot{n} \quad$ sont des termes qui n'appartiennent pas de manière significative à la terminologie $\mathrm{mt}$.

3 Voir p.ex. comment les motifs de la faim et de la soif sont traités de manière rédactionnelle dans les béatitudes (voir notre chap. 11).

4 Pour $\tau o ́ \tau \varepsilon$, voir plus haut. $\Delta i ́ \varkappa \alpha \iota$ เos est un mot cher à Mt : les deux autres fois $(13,43.49)$ où, comme dans notre passage, il désigne les élus lors du jugement, il est rédactionnel. Mt introduit volontiers $\dot{\alpha} \delta \varepsilon \lambda \varphi \delta \delta_{s}$ dans ses sources, cf. $4,18.21 ; 20,24 ; 28,10$. L'expres -

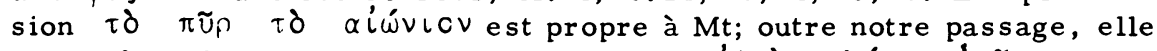

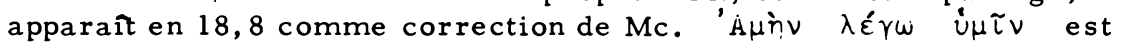
attesté 28 fois chez Mt, 11 fois chez Mc et 5 fois chez Lc. Pour l'ensemble, voir BROER 281-282; à son avis, le double xúpıદ des vv. 37.44 comme désignation du Seigneur eschatologique pourrait égale ment être rédactionnel, cf. 7,21. LEGASSE, Enfant 90-91, partage en gros notre opinion; s'il ne se prononce pas sur la glose du v. 40, il fait

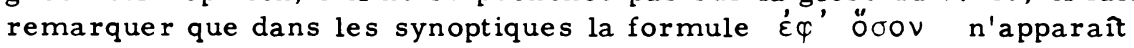
que chez Mt. ROBINSON, Parable $232-233$, tout en se ralliant à l'hy -

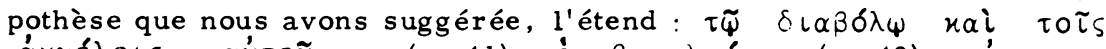

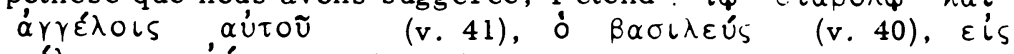

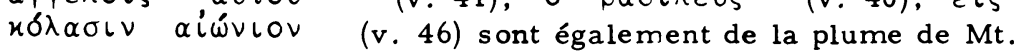


sont pas diffusées dans l'ensemble du texte, mais qu'elles se concentrent surtout dans les parties narratives qui encadrent le dialogue et, dans une plus faible mesure, dans l'énoncé du critère de la sanction. Faut-il alors en déduire avec Robinson ${ }^{1}$ que ces séquences narratives sont l'oeuvre du rédacteur et qu'elles constituent une allégorie de la parabole du berger destinée à introduire les logia des vv. 35-40.42-45 ? Cette hypothèse ne s'impose pas pour la raison suivante. Comme on l'a souvent souligné, $x \delta ́ \lambda \alpha \sigma \iota s$ et $x \alpha \tau \alpha \rho \alpha ́ o \mu \alpha \iota$ (v. 46) sont des hapax dans le cadre du premier évangile. Or les hapax sont l'indice de l'utilisation d'une tradition dans la mesure où l'on peut prouver que, pour exprimer la même idée, le rédacteur préfère recourir à d'autres termes. C'est le cas ici. Pour désigner les ennemis de la volonté de Dieu, Mt n'utilise jamais

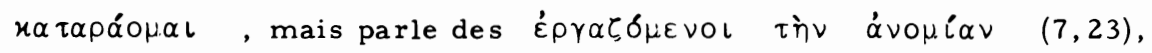

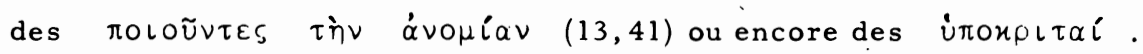
Semblablement, pour décrire le châtiment infernal, Mt préfère l'image

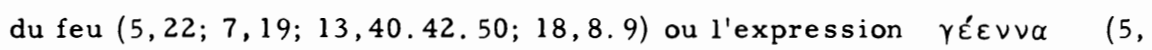

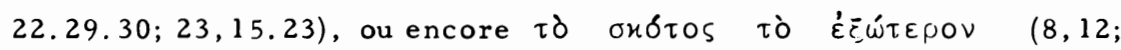
$22,13)$. Cette constatation nous invite à penser que, dans les vv. 35-46, Mt utilise de manière suivie une seule source en lui apportant les retouches que nous avons mentionnées. Une seule difficulté reste à trancher. Nous avons prétendu que Mt s'inspirait d'une tradition dès le v. 32b. La parabole du berger et les antithèses font-elles partie de même ensemble ou s'agit-il d'une combinaison rédactionnelle de deux morceaux indépendants ? Nous pensons avoir affaire à une seule unité car l'expression

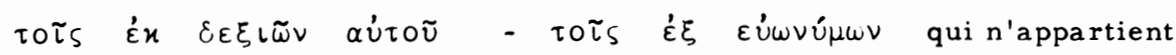
pas à la terminologie spécifiquement $\mathrm{mt}$ surgit aussi bien en $32 \mathrm{~b} .33$ qu'en 34.41 et confère au tout une certaine homogénéité ${ }^{2}$.

1 Parable $233-234$.

2 Avec BROER 284; autrement LEGASSE, Enfant 93, et ROBINSON, Parable 236, qui optent pour deux images primitivement indépendantes. 
En conclusion, il importe de s'interroger sur l'origine de la tradi tion utilisée par Mt dans notre passage. Bultmann a émis l'hypothèse qu'il s'agissait d'une tradition d'origine juive ${ }^{l}$, car si l'on fait abstraction de la figure du Fils de l'homme, ajoutée ultérieurement, le morceau ne contient pas d'argument spécifiquement chrétien. L'assemblée des justiciables est indéterminée. Le titre $\beta \alpha \sigma \iota \lambda \varepsilon u ́ s$ est une métaphore classique pour Dieu dans le judaîsme; semblablement la fonction de juge à la fin des temps est un attribut divin bien connu. Enfin, la matière éthique exposée dans les antithèses est fort proche des oeuvres d'amour chères aux rabbis. Si, pour l'essentiel, cette analyse est convaincante, on doit pourtant lui apporter un correctif qui en modifie le résultat. L'identification du Roi (et donc de Dieu pour le judal'sme) avec l'homme en détresse (vv. 40.45) qui est une partie constitutive des antithèses, n'est attestée ni dans le judaïsme, ni plus largement dans le monde ambiant. Il s'agit d'une affirmation entièrement originale qui confère au texte sa spécificité et en garantit l'origine chrétienne ${ }^{2}$. Pourtant, avec Bultmann, on retiendra la tonalité juive de l'ensemble et l'on attribuera cette tradition à un milieu judéo-chrétien ${ }^{3}$.

Une ultime question mérite d'être posée. La tradition dont s'est servi Mt, parlait-elle de Dieu ou du Christ ? En effet, si l'on fait abstraction de ce que nous avons attribué à l'activité rédactionnelle, à savoir l'intro-

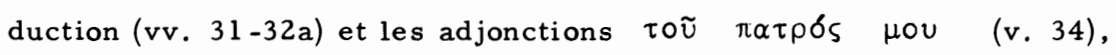
$\tau \tilde{\omega} \nu \alpha \delta \varepsilon \lambda \varphi \tilde{\omega} v$ nov (v. 40), force est d'admettre que le texte est ambigu. Le critère de décision réside dans l'appréciation de la figure du $\beta \alpha \sigma \iota \lambda \varepsilon u ́ s \quad 4$. S'agit-il, à l'origine, d'un titre donné au Christ ou d'une

1 BULTMANN, Trad 130-131.

2 Paradoxalement, BULTMANN, Trad 130, avait déjà souligné ce point sans en tirer les conséquences. Analyse détaillée chez BRAUN, Radikalismus II, 94 (note 2).

3 Avec BROER 286, et HAHN, Hoheitstitel 187.

4 Pour l'analyse du titre $\beta \alpha \sigma \iota \lambda \varepsilon u ́ s$, voir BROER 286-288. 
métaphore désignant Dieu ? Le titre $\beta \alpha \sigma \iota \lambda \varepsilon u ́ s \quad n ' e s t$ jamais conféré au Christ dans les épitres authentiques de Paul; au niveau de la tradition synoptique et dans sa formulation la plus ancienne (Mc), il apparaît exclu sivement dans le récit de la Passion, mais sous forme composée : $\beta \alpha-$

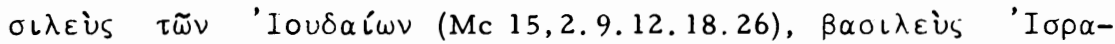
ńl (Mc 15,32). Mt reprend cet usage sans l'étendre ${ }^{1} \cdot 25,31.34$ reste donc le seul passage où $\beta \alpha \sigma \iota \lambda \varepsilon u ́ s$ est utilisé seul, où il est mis en relation avec le Fils de l'homme, où enfin, il désigne le juge eschatologique. Concluons : puisque le titre $\beta \alpha \sigma \iota \lambda \varepsilon u ́ s$ appliqué au Christ n'appartient pas au kérygme primitif ${ }^{2}$ mais que la tradition utilisée par Mt est ancienne, puisque ce prédicat n'apparaît qu'une fois dans le premier é vangile, puisqu'enfin les adjonctions rédactionnelles des vv. $31-32 a .34$ ont pour rôle d'attirer l'attention sur la personne de Jésus, on en déduira qu'à l'origine le texte parlait de Dieu et non du Christ ${ }^{3}$.

Ce dernier résultat n'est pas sans importance car il permet de dégager l'intention directrice de Mt dans notre passage : sa transcription est gouvernée par la volonté d'attribuer au Christ les fonctions du Roi-Juge. Si cette identification n'est pas surprenante et ne fait que mener à chef une ligne déjà présente dans le reste de l'évangile ${ }^{4}$, elle a néanmoins des implications intéressantes. En faisant du Christ à la fois le juge eschatologique et le proclamateur de la Loi qui prévaut à cette occasion, Mt in-

1 Deux exceptions: 2,$2 ; 21,5$.

2 SCHMIDT, art. $\beta \alpha \sigma \iota \lambda \varepsilon \cup ́ s$, ThW 1,578.

3 BROER 287-288; BULTMANN, Trad 131; VIELHAUER, Gottesreich $63-64$.

4 La présentation de Jésus comme Fils de l'homme revenant pour le jugement est largement attestée dans le premier évangile : cf. 10,23; 16,$28 ; 24,27,30-31.37 .39 .44 ; 26,64$. Malgré cette abondance de textes, l'affirmation reste généralement principielle. A part notre péricope, seuls trois passages vont au delà et proclament le critère de la rétri bution. Ce sont 13,41 (rédactionnel), 16,27 (rédactionnel) et 19,28 ( $Q$ fortement réinterprété). 
troduit dans sa tradition une réflexion qui lui était étrangère ${ }^{l}$. Il met en relation la christologie et l'éthique à un double niveau et d'une manière inattendue. D'une part, si le jugement est la prérogative du Fils de l'homme, Jésus est le garant eschatologique de l'éthique. D'autre part, si la sanction eschatologique dépend de la relation entretenue avec le Fils de l'homme par la médiation des "plus petits", l'attitude prise face à Jésus devient le critère de l'éthique. L'étude de l'histoire de la tradition met donc en évidence une problématique qu'il s'agit maintenant d'analyser.

\section{Le champ de l'éthique}

En quoi cette vision à la fois christologique et éthique concerne-t-elle $\underline{\text { le disciple } \mathrm{mt}}$ ? Pour répondre à cette question, il s'agit de résoudre deux problèmes : qui sont les nations qui doivent affronter le jugement (v. 32) ? qui sont "ces plus petits" avec qui le Christ se solidarise et pour lesquels il réclame la miséricorde (vv. 40.45) ? En abordant ces deux questions nous posons le problème du champ de l'éthique. Ce problème est devenu d'autant plus brûlant que, depuis quelques années, un nouveau courant $d^{\prime}$ interprétation ${ }^{2}$ concernant cette péricope a vu le jour. Ce courant, malgré d'assez notables divergences dans l'appréciation du texte, insiste sur deux points : d'une part, l'interprétation globale du passage dépend de la réponse apportée aux deux questions mentionnées ci-dessus; d'autre part, chaque terme utilisé par le rédacteur de manière délibérée

1 La présentation du Christ à la fois comme proclamateur de la Loi et comme juge eschatologique n'est certes pas une création de Mt; elle est bien connue de la christologie judéo-chrétienne (cf. p.ex. Q). Mais, en ce qui concerne 25,31-46, l'apparition de cette conception christologique est l'oeuvre du rédacteur.

2 Voir notamment les art. de BROER, COPE, INGELAERE, LAMBRECHT, LEGASSE, MICHAELS. 
et originale a un sens constant dans l'ensemble de l'évangile ${ }^{l}$. On résout alors les difficultés des vv. 32.40.45 de la manière suivante. Tout d'a bord : qui sont les nations soumises à la Loi du Fils de l'homme et jugées selon elle ? Pour en parler, le v. 32 utilise l'expression $\pi \alpha ́ v \tau \alpha$ $\tau \grave{\alpha}$ E๊ $\vee \eta$. Cette locution n'est pas isolée, elle est chère à l'évangé liste qui, hormis notre v., l'emploie à deux reprises dans le dernier grand discours du Christ $\mathrm{mt}$ : en 24,9 où elle décrit le caractère univer sel de la persécution qui s'abat sur les disciples et en 24,14 où elle évoque la dimension universelle de la proclamation de l'évangile ${ }^{2}$. Elle réapparaît enfin de manière rédactionnelle en 28,19 où elle met en évidence l'universalité de la mission des disciples. Etant donné que tous les passa-

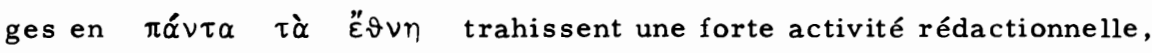
de nombreux exégètes, postulant une homogénéité de sens, interprètent 25,32 a à partir de 24,14 et de 28,19 , en insistant sur la note universalis te et missionnaire de la notion. Dès lors, "toutes les nations" rassemblées devant le Fils de l'homme représentent l'ensemble des hommes qui ont été confrontés à l'évangile et appelés à devenir les disciples de Jésus - qu'ils aient accepté ou non le message ${ }^{-3}$. Cette définition des justicia-

1 Ce double présupposé apparaît très clairement chez BROER 291 -295; INGELAERE $35-38.50$-54; LAMBRECHT 334-339; LEGASSE, Enfant 94-100; MICHAELS $27-29$.

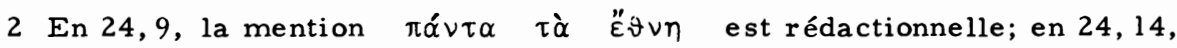
elle est certes empruntée à Mc 13,10, mais le v. est très fortement retravaillé par Mt.

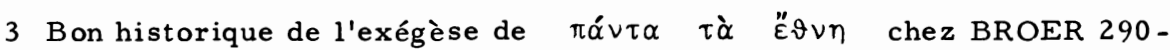
291. Selon BROER 291-292, LEGASSE, Enfant 94-95, MICHAELS 29, "toutes les nations" désignent l'ensemble des hommes qui ont été confrontés à l'évangile grâce à l'accomplissement universel de la tâche missionnaire. LAMBRECHT 334 et WALKER 108-111 soutiennent la même exégèse, tout en excluant Israël de ce rassemblement, car il est déjà jugé. COPE 37.43 opère une autre restriction : les justiciables sont certes les hommes confrontés à l'évangile, mais à l'exclusion de ceux qui l'annoncent - les disciples -. Cette idée d'un jugement diffé rencié est reprise par INGELAERE 35-38 (d'ailleurs à la suite de JEREMIAS, Gleichnisse 206), mais d'une toute autre manière : les hommes qui comparaissent devant le Christ sont les païens non conver- 
bles - et notamment la supposition de leur rencontre avec l'évangile est solidaire de l'interprétation que ces exégètes donnent de l'expres-

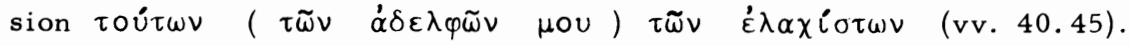
Sur ce point, ils font valoir les deux constatations suivantes : $\mu \iota$ นós , tant en 10,42 que dans le chap. 18, désigne les disciples ${ }^{1}$; semblablement,

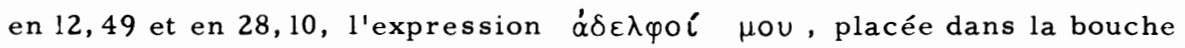
de Jésus, désigne les adhérents du Christ ${ }^{2}$. La conclusion s'impose d'elle-même : "ces plus petits" avec lesquels le Christ-Juge se solidarise, sont ses disciples - et plus particulièrement ses envoyés en mission ${ }^{3}$. Cette exégèse a l'avantage d'éclairer un point difficile du texte, à savoir la nature de la solidarité qui unit le Fils de l'homme au "plus petit". En

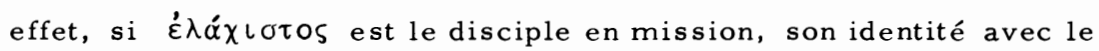

tis et le but du récit est de montrer comment cette catégorie sera jugée. Il faut bien admettre que les arguments décisifs manquent pour retenir une hypothèse plutot qu'une autre, car le texte ne donne aucune indication sur ce point; bien plus, dans ce même v. 32, il passe sans hésitation de la catégorie générique ( $ૈ$ $\vartheta \vee \eta$ ) à la catégorie individuelle ( aúzoús ).

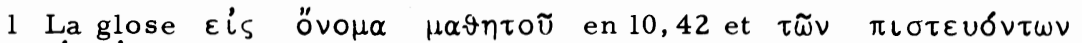
$\varepsilon i \varsigma$ $\varepsilon^{\prime} \mu \varepsilon$ en 18,6 est la démonstration indiscutable de ce point de vue. On remarquera pourtant d'un point de vue philologique que $\mu \iota$ นós et $\dot{\varepsilon} \lambda \alpha ́ \chi \iota \sigma \tau o s$ proviennent de deux racines verbales différentes si bien qu'à proprement parler, Mt n'utilise pas la même notion dans les chap. 10,18 et 25 .

2 En 12,49, l'expression $\dot{\alpha} \delta \varepsilon \lambda \varphi \iota^{\prime} \mu o v$ est certes empruntée à Mc 3, 34, mais l'identification avec le groupe des disciples est une correction rédactionnelle; 28,10 est peut-être une réminiscence de Mc 16,7, mais la mention $\dot{\alpha} \delta \varepsilon \lambda \varphi \iota^{\prime} \mu o v$ est propre à Mt et désigne clairement les disciples (comparer avec 28,16 ).

3 La plupart des auteurs qui identifient "ces plus petits" aux disciples de Jésus rapprochent notre texte de 10,42 et soulignent la vocation mis-

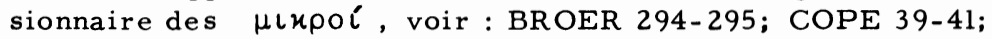

LAMBRECHT 334-339; LEGASSE, Enfant 97-99; MICHAELS 27-29. INGELAERE 50-54 et KLOSTERMANN, Mt 207, insistent plutôt sur la détresse de "ces plus petits" tout en les identifiant également aux croyants. 
Christ se comprend fort bien à partir du droit juif de l'envoi qui affirme une complète identité entre l'envoyé et celui qui l'envoie ${ }^{1}$. Cette explication des expressions-clefs des vv. 38.40.45 ouvre la voie à une interprétation globale de la péricope que nous pouvons schématiser comme suit.

A la fin des temps, lorsque l'évangile aura été proclamé jusqu'aux extrémités de la terre, le Fils de l'homme rassemblera tous les hommes devant lui; il jugera alors chacun selon l'attitude adoptée à l'égard de ses messagers ${ }^{2}$. Ce discours prophétique doit être un sujet de consolation pour les disciples et d'exhortation pour ceux qui les reçoivent.

De prime abord séduisante, cette interprétation soulève pourtant de nombreuses objections au niveau terminologique, littéraire et théologique. Nous nous proposons de le montrer en reconsidérant son fondement, à

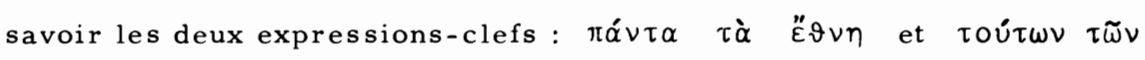
É $\lambda \alpha \chi i \sigma \tau \omega \nu$. Mème les références à 24,9 et 28,19 ne permettent pas de définir avec précision "toutes les nations" dont parle notre texte : s'agit-il des paĩens à l'exclusion des Juifs ? des hommes confrontés à l'évangile ou de ceux qui ne l'ont pas reçu ? Les "petits" font-ils partie

1 Cet argument constamment évoqué par les exégètes est développé par RENGSTORF, art. á $\pi 0 \sigma \tau \varepsilon^{\prime} \lambda \lambda \omega \mu \tau \lambda$, ThW 1, 414-420.426-427. Le locus classicus $\mathrm{mt}$ sur ce point est 10,40 qui fait précisément partie de la même unité littéraire que 10,42. Le recours au droit juif de l'envoi pour expliquer notre passage, se heurte pourtant à une objec-

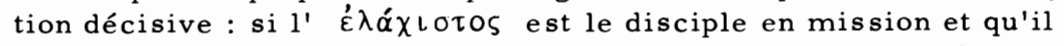
représente totalement son maître, comment faut-il interpréter la surprise des justes (vv. 37-39) et des réprouvés (v. 44) qui n'ont précisément pas reconnu le Christ dans la personne des "plus petits" ?

2 Ainsi BROER 295; COPE 43; LAMBRECHT 339; MICHAELS 28-29. LEGASSE, Enfant 98-100, tout en défendant la même exégèse, voit dans notre passage un avertissement adressé en priorité à l'Eglise : qu'elle ne méprise pas la faiblesse de ses envoyés ! INGELAERE 56.60 s'engage sur une toute autre voie : 25, 31-46 traite du sort des incroyants

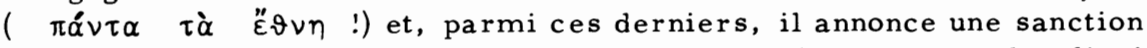
favorable à ceux qui auront fait preuve de bienveillance envers les disciples en difficulté ! 
des "nations" ou s'en distinguent-ils ? Autant de questions qui ouvrent la voie aux hypothèses les plus diverses. Pourtant un fait demeure : le v. 38 ne fournit aucune indication à ce sujet. Et plutôt que d'incriminer l'imprécision de l'auteur, il est préférable d'admettre que son intérêt ne s'est pas porté sur ce point. Le rédacteur n'a pas en vue une quelconque distinction entre Juifs et païens, ou entre croyants et incroyants, il entend simplement souligner que le jugement concerne tous les hommes sans exception 1 .

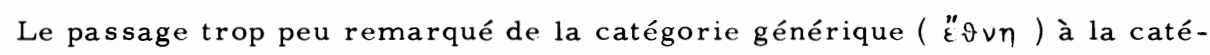
gorie individuelle ( $\alpha$ útoús) dans le seul v. 32 en est la meilleure preuve. On peut faire la même démonstration à propos des $\dot{\varepsilon} \lambda \alpha ́ \chi \iota$ ó de doute qu'en 10,42 et au chap. 18, les "petits" désignent les disciples, mais justement parce que Mt 1 'indique explicitement ${ }^{2}$. Dans notre passage, en revanche, nous ne trouvons aucune allusion directe au statut de disciple ou à un envoi en mission ${ }^{3}$. Le rédacteur veut-il éviter une répétition inutile? Ce n'est pas exclu. Il est pourtant plus raisonnable d'admettre qu'il entend insister sur un autre aspect de la "petitesse". Ce qui est au centre de son propos, c'est la description de la détresse sous toutes ses formes - et il n'est jamais dit dans cette scène qu'elle soit limitée à

1 Point fort opportunément rappelé par BULTMANN, Trad 130 et BORNKAMM, Enderwartung 21. Partagent cet avis : BONNARD, Mt 366; KLOSTERMANN, Mt 205; SCHMID, Mt 352-353; SCHMIDT, art. है จvos, ThW 2, 366-367; SCHNIEWIND, Mt 251; TRILLING, Is rael 26-27; SCHWEIZER, Mt 311; TOEDT 71.

2 Cf. notre p. 337.

3 A la suite de MICHAELS 29-37, LEGASSE, Enfant 92-93, discerne dans les "antithèses" une énumération à la fois classique et caractéristique des épreuves encourues par les premiers missionnaires. Les parallèles les plus frappants de notre passage se trouveraient dans la littérature paulinienne. Si l'existence de telles traditions était prouvée, alors l'identité entre $\mu \iota \varkappa \rho \circ i$ et missionnaires s'imposerait dans notre péricope. Pourtant cette hypothèse n'emporte pas la conviction, car les parallèles avérés aux "antithèses" se situent à un tout autre niveau, voir nos pp. 344-346. 
la condition chrétienne ${ }^{1}$. Dès lors, il est légitime de se demander si les extrapolations auxquelles procèdent plusieurs exégètes pour interpréter les vv. 32.40.45 n'introduisent pas des éléments de signification étrangers au texte. Qu'y a-t-il de commun entre le geste obligé de miséricorde envers le malheureux et l'hospitalité accordée au témoin de l'évangile ? Si Mt avait véritablement voulu parler de l'accueil du Christ dans la personne de ses envoyés, n'aurait-il pas, comme au chap. 10, dépeint l'adhésion à l'évangile ou son refus par des motifs tels que l'écoute de la Parole ${ }^{2}$, l'obéissance de la foi ou la "Nachfolge" plutôt que par l'évocation d'actes de miséricorde ${ }^{3}$ ?

A cette critique de l'analyse des deux notions-clefs s'ajoutent des objections d'ordre littéraire. Les exégètes qui tiennent notre péricope pour une vibrante exhortation à accueillir les disciples - ou encore pour une instruction sur le sort des päiens qui exercent la miséricorde - négli-

1 Voir la bonne remarque de WALKER 110 : "Wäre hier an 'Jünger' zu denken, kämen doch wohl nur die im Namen des Herrn auftretenden, alle Heiden berufenden Boten in Betracht, nicht ganz allgemein die Ohnmächtigen und Menschlich-Bedürftigen, auf die der Text blickt (und zu denen auch 'Jünger' gehören mögen)". Voient également dans

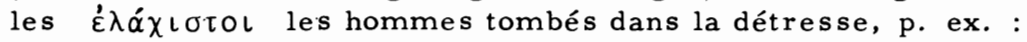
BONNARD, Mt 367; BORNKAMM, Jesus 102; BRAUN, Jesus 127; GRUNDMANN, Mt 528; JEREMIAS, Gleichnisse 205; SCHLATTER, Mt 726; SCHMID, Mt 355; SCHNIEWIND, Mt 253; SCHWEIZER, Mt 312; TOEDT 69. On remarquera par ailleurs que la "petitesse" a une autre tonalité dans les chap. 10 et 18. Dans le chap. 10, il y va de l'envoyé du Christ, dépourvu de tout prestige et exposé à la faiblesse; dans le chap. 18, il s'agit d'un groupe intra-ecclésial sensible au scandale et en danger de s'écarter de la foi.

2 On remarquera qu'en 10,14 , p. ex. il y a équivalence entre l'accueil du missionnaire et l'accueil de sa prédication. Cette équivalence est établie

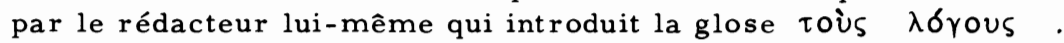

3 Objection signalée, mais pas réfutée par LAMBRECHT 337-338 qui y voit une tension entre tradition et rédaction, et par LEGASSE, Enfant 97-98 qui discerne dans cette terminologie la seule manière de s'adresser à une Eglise qui méprise ses missionnaires. 
gent le contexte rhétorique du passage. Le discours des chap. 24-25 se présente en effet comme une instruction adressée explicitement et exclusivement aux disciples ${ }^{1}$. Or, si 25, 31-46 était une pressante invitation à faire preuve d'amour envers les chrétiens en mission, il faudrait alors admettre que, sans autre indication, Mt s'est tourné vers un autre auditoire que celui signalé en 24,3 . Cela est peu probable si l'on considère le contexte immédiat : les trois paraboles qui précèdent immédiatement notre texte sont au service de la parénèse; elles invitent instamment les disciples à faire preuve de vigilance. Peut-on supposer que la dernière péricope s'éloigne de la thématique du chap. 25 et interrompt la parénèse ecclésiale ? Ce serait contraire au génie du rédacteur qui fait culminer tous ses grands discours dans une exhortation invitant les croyants à la fidélité $^{2}$ : la parabole des deux maisons $(7,24-27)$ clôt le Sermon sur la Montagne, celle du filet (13,47-50) met un point final au discours du chap. 13, celle du débiteur impitoyable (18,23-35) conclut l'instruction du chap. 18. Ainsi, le contexte rhétorique, le contexte immédiat et la manière dont Mt achève ses discours, nous invitent à voir dans 25, 31-46 non pas une parénèse à propos des disciples, mais une parénèse adressée aux disciples.

Enfin, notre point de vue est étayé par une double remarque de théologie mt. Tout d'abord, la conception dont Mt s'inspire pour décrire le jugement, appartient à la tradition vétérotestamentaire-juive ${ }^{3}$. Or, dans l'ensemble, ce courant de pensée n'envisage pas un jugement différencié,

1 Voir la double mention de la locution, $\pi \rho \circ \sigma \tilde{\lambda} \lambda \vartheta \circ v(\alpha \dot{v} \tau \tilde{\Psi})$ oi $\mu \alpha-$ $\vartheta \eta \tau \alpha \hat{\imath} \alpha \dot{u} \tau o \tilde{U}$ en $24,1.3$ et le $n \alpha \tau$ ' $i \delta \ell \alpha \nu$ de 24,3 . Cette formule stéréotypée de $\mathrm{Mt}(\mathrm{cf} .5,1 ; 13,10.36 ; 18,1)$ annonce toujours une instruction ecclésiale du Christ (cf. BONNARD, Mt 367; et BORNKAMM, Enderwartung 19).

2 Voir COPE 33-34.

3 Cf. BOR NKAMM, Enderwartung 21; BULTMANN, Trad 130-131; TRILLING, Is rael 26-27. 
mais un jugement universel où chacun est sanctionné selon le même critère ${ }^{1}$. Ensuite, comme l'a bien montré Bornkamm ${ }^{2}$, chaque fois que, dans son évangile, Mt évoque la venue du jugement - et c'est un thème qui lui est cher -, il ne le fait pas pour consoler les disciples et les renforcer dans la conscience d'un privilege, mais pour les avertir et les mettre en demeure d'obéir, car l'Eglise n'est pas l'assembléc des élus, mais celle des appelés ${ }^{3}$.

Concluons. Sur la base des arguments invoqués et dans l'état actuel de la discussion, nous accordons notre préférence à l'interprétation clas-

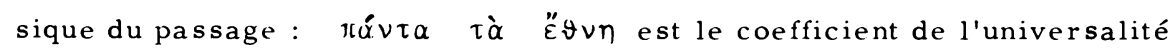
du jugement tandis que le "plus petit" désigne sans autre restriction tout homme tombé dans la détresse. Dans ces conditions, le fil conducteur du passage nous semble ĉtre le suivant : lors de la rétribution finale, le Christ $\mathrm{mt}$, se réclamant de sa solidarité avec "les plus petits", sanctionnera chaque homme selon l'attitude qu'il aura adoptée vis-à-vis du prochain dans la misère. Quelles sont alors les conséquences de cette exégèse pour le problème que nous avons posé, à savoir celui du champ de l'éthique ? Comme dans l'ensemble du premier évangile, mais avec une clarté redoutable, le thème de l'universalité du jugement a ici une fonction parénétique ${ }^{4}$. Chaque homme - et, en premier lieu, le croyant - comparaîtra devant le Fils de l'homme. Cette échéance inéluctable jette une nouvelle

1 Cf. BILL 4, 1199-1212. Même INGALAERE 40-41 qui plaide pour un jugement différencié, concède que cette conception est rare dans le judaisme d'alors.

2 BORNKAMM, Enderwartung 13-21.

3 Voir nos pp. 381-385.

4 Voir BORNKAMM, Enderwartung 19 et SCHMID, Mt 351. BONNARD, Mt 364, écrit sur ce point : ".. Tout au long de ces chapitres, les évocations eschatologiques sur la fin de l'histoire, et même apocalyptiques sur les catastrophes qui signaleront cette fin, sont mises au service de l'éthique ... Or, nous devons faire une remarque analogue au sujet de ces v. $31-46 \ldots$ : leur pointe est dirigée vers l'instruction ou plus exactement vers la prophétie éthique". 
lumière sur son existence présente. L'heure vécue n'est pas indifférente, elle a une importance décisive, car c'est ici et maintenant que le disciple gagne ou perd sa vie. La venue du jugement s'explicite donc, pour Mt, en regard de la vie chrétienne, par une mise en responsabilité radicale de l'homme. Mais - et il faut tout aussitôt l'ajouter -, cette mise en responsabilité n'est pas indéterminée. Elle est clairement axée sur les relations interpersonnelles et plus particulièrement sur la détresse que chacun rencontre dans la personne de son frère. Elle suppose une capacité de discernement et d'ouverture active en faveur de celui dont l'humanité est menacée, voire bafouée. Pourtant cet appel à la miséricorde ne doit pas être compris comme une recommandation éthique valable en elle-même. Il intervient dans un contexte précis qu'il s'agit maintenant d'éclairer.

\section{L'évidence de l'éthique}

Le disciple est mis en situation de responsabilité radicale, mais d'une responsabilité qui se traduit en terme de miséricorde. Quelle est alors la nature de la miséricorde requise de chacun à l'égard du prochain ? Les "antithèses" illustrent cette exigence centrale par six gestes élémentaires : nourrir l'affamé, désaltérer l'assoiffé, recueillir l'étranger, vêtir celui qui est nu, visiter le malade et le prisonnier. Il apparaît immédiatement que, face à des situations de détresse, la miséricorde dont parle le texte, se caractérise à la fois par son évidente simplicité et son impérieuse nécessité. Néanmoins, pour mieux en saisir le relief, il importe de la comparer, d'une part, avec les affirmations similaires du Christ mt dans le reste de l'évangile, d'autre part, avec la conception éthique du monde ambiant.

Pour ce qui est du premier évangile, nous discernons une cohérence essentielle entre la Loi du Juge eschatologique édictée dans notre passage et la prédication du Christ mt dans son ensemble ${ }^{1}$. L'appel à la justice

1 TOEDT 69-71 insiste particulièrement sur ce point. L'appel à la miséricorde des vv. 31-46 ne doit pas être séparé de la conception mt de la 
qui retent it en 5,17 et qui trouve son expression la plus significative dans l'amour de l'ennemi $(5,43-48)$, suppose déjà cette attention illimitée au prochain. La règle d'or $(7,12)$ qui met un point final à l'interprétation de la Loi dans le Sermon sur la Montagne, le commandement d'amour (22, 3440), mais surtout la double citation d'Os 6,6 qui établit la prééminence de la miséricorde $(9,13 ; 12,7)$, confirment que l'amour du prochain est au centre des préoccupations de Mt. Les six gestes exigés par le Fils de l'homme en 25, 31-46 ne sont donc que le prolongement et l'illustration d'une thématique $\mathrm{mt}$ essentielle - l'amour du prochain ${ }^{1}$.

Plus inattendu, mais d'un intérêt capital est le résultat d'une comparaison entre nos antithèses et les parallèles du monde ambiant ${ }^{2}$. Il apparaît en effet que la forme littéraire consistant à énumérer de manière rythmée les oeuvres charitables n'est pas une création du milieu mt; il s'agit d'un procédé connu et pratiqué dans l'ensemble du monde procheoriental $^{3}$. Bien plus, la parenté ne se limite pas à l'utilisation commune d'une forme littéraire. Elle resurgit au niveau du contenu; ici encore, Mt ne fait pas oeuvre originale, il ne fait que reprendre des éléments bien connus du code éthique de l'Orient ancien ". Si les "oeuvres d'amour"

Loi. Elargi'ssant la perspective, cet auteur rappelle à juste titre que la Loi et la christologie vont de pair dans le premier évangile, si bien que les exigences émises par le Fils de l'homme dans notre péricope ne peuvent être assumées que dans la Nachfolge. Cette relation obligée de toute obéissance à la personne du Christ assure la spécificité chrétienne du passage.

1 Sur ce point, voir BROER 289 et nos pp. 309-313.

2 Sur ce point, voir l'excellent art. synthétique de WIKENHAUSER 366-377.

3 INGELAERE 45-46; LEGASSE, Enfant 91; WIKENHAUSER 377. Ce schéma littéraire est utilisé dans l'Orient ancien "soit comme un motif de louer Dieu, soit pour rappeler aux croyants leurs devoirs, soit encore comme arguments à faire valoir devant le juge divin" (LEGASSE, Enfant, ibid.).

3 BULTMANN, Trad 130-131; KLOSTERMANN, Mt 204; LEGASSE, Enfant 91; INGELAERE 45; SCHMID, Mt 353. 
prônées par la tradition rabbinique ${ }^{1}$ paraissent être le parallèle le plus proche, on trouve des analogies frappantes dans l'Ancien Testament ${ }^{2}$, le judaïsme apocalyptique ${ }^{3}$, mais aussi dans le parsisme, le mandéisme et la littérature égyptienne ${ }^{4}$. Ainsi, bien que dans l'économie générale de l'évangile, les antithèses s'intègrent parfaitement à la thématique de l'amour du prochain, du point de vue de l'histoire comparée des religions, elles sont une reprise du code éthique proche-oriental et, en particulier, vétérotestamentaire-juif. La spécificité de la réflexion mt n'apparaît donc ni au niveau de la forme littéraire (des antithèses), ni au niveau du conte-

1 Voir BILL 4,559-579 et WIKENHAUSER 369-371. Même si la liste des oeuvres d'amour et celle de Mt 25,31-46 ne se recouvrent pas exactement (le Christ $m$ t ne parle ni de l'éducation des orphelins, ni de l'ensevelissement des morts; les oeuvres d'amour ne mentionnent pas explicitement la visite des prisonniers), la parenté reste évidente. A titre d'illustration brève et suggestive, nous rappelons le Midrasch Tannaĩm à propos de Dt 15, 9 (cité. par RADEMAKERS, Mt II 317, note 35) où Dieu dit à Israël : "Mes enfants si vous avez donné à manger aux pauvres, je vous le compterai comme si c'était à moi que vous l'aviez fait".

2 Pour les parallèles vétérotestamentaires, voir le matériel rassemblé par WIKENHAUSER 366-367, et, à sa suite, mais avec un classement plus méthodique, INGELAERE 45.

3 Voir WIKENHAUSER 367-369. Le livre des secrets d'Hénoch fournit une analogie particulièrement frappante (cité d'après LEGASSE, Enfant 91): "Hénoch ! Ce lieu est préparé pour les justes ... Ils donnent du pain à ceux qui ont faim et habillent ceux qui sont nus, ils les recouvrent de vêtements, ils relèvent ceux qui sont tombés et ils secourent les malades; ils marchent devant la face de Dieu et le servent, lui seul" (chap. 9); "Hénoch ! Ce lieu est préparé pour les indignes ... Ils ont fait mourir de faim les indigents qu'ils pouvaient rassasier, ils ont dépouillé entièrement de leurs vêtements ceux qui étaient nus alors qu'ils pouvaient les habiller; ils n'ont pas reconnu leur Créateur, mais ont prié des dieux vains. Pour tous ceux-ci ce lieu est préparé en héritage éternel" (chap. 10,4-6).

4 Voir le matériel rassemblé par WIKENHAUSER 373-375 et, en particulier, le chap. 125 du Livre des Morts égyptien. 
nu éthique, mais, comme cela a été souvent souligné, dans la mise en relation de ce contenu avec la personne du Christ ${ }^{1}$. Nous reviendrons en conclusion sur cette particularité décisive, mais il nous paraît préalablement nécessaire de réfléchir sur la signification de cette surprenante convergence entre $\mathrm{Mt}$ et son environnement culturel.

Si l'on renonce à expliquer cette convergence comme une simple coïncidence sans incidence notable, force est de constater que le critère éthique du jugement ne présente aucune originalité, mais constitue un lieu commun admis par l'ensemble du monde ambiant : l'exigence de miséricorde est une évidence qui ne fait l'objet d'aucune discussion ${ }^{2}$. Tant les justes que les réprouvés l'agréent. En conséquence, le problème de l'éthique ne se situe pas au niveau de la norme, qui est évidente. Il surgit bien plutôt dans la découverte de la contradiction suivante : si la miséricorde est une exigence éthique unanimement reconnue, comment se fait-il qu'elle soit accomplie par les uns et violée par les autres ?

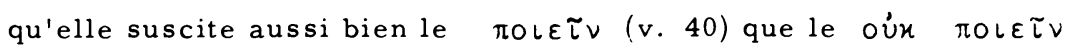
(v. 45) ${ }^{3}$ ? End'autres termes, comment un impératif éthique, évident pour tous, peut-il provoquer aussi bien l'obéissance que la transgression ? Nous butons ici sur le problème central de notre texte, qui est celui de toute éthique. Seule une réflexion sur la spécificité chrétienne de la péricope peut nous permettre de résoudre cette énigme.

1 Tant l'intervention des justes (vv. 37-39) que celle des réprouvés (v. 44) ne porte pas sur la légitimité de la miséricorde comme telle, mais sur la présence mystérieuse du Christ dans la personne des "plus petits".

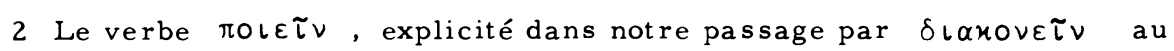
v. 44 (il s'agit donc ici d'un faire au service au prochain), occupe une place centrale dans le premier évangile. Il est utilisé 84 fois (contre 47 fois chez $\mathrm{Mc}$ ). Il traduit un aspect important de l'anthropologie $\mathrm{mt}$ : l'homme se caractérise par ce qu'il fait; il est tout entier dans ses projets, ses soucis, ses décisions, ses engagements et, en définitive, son obéissance. Ainsi, par exemple, le véritable disciple est celui qui fait la volonté de Dieu (12,49-50). Lorsque, dans notre passage, le Fils de l'homme sanctionne le faire des justiciables, il les atteint donc dans leur ultime réalité. 


\section{Le retour au fini}

La spécificité chrétienne du texte ne réside ni dans l'évocation du jugement, ni dans le critère éthique invoqué. Elle se manifeste bien plutôt dans la relation établie entre ces deux éléments et la personne de Jésus. Le Christ mt est désormais le Roi qui opère le tri eschatologique; il ne le fait pas en mesurant chacun à l'aune d'un principe moral, mais sur la base de la solidarité qu'il revendique en faveur de tous les hommes tombés dans la détresse ${ }^{1}$. Cette solidarité du Christ avec ses frères misérables se manifeste sous deux formes dans le passage. D'une part, l'emploi de la première personne dans les antithèses montre que le Fils de l'homme prend fait et cause pour les malheureux; il s'identifie à leur destin au point de le revendiquer comme le sien devant les "nations". D'autre part, les vv. 40.45, en affirmant explicitement cette solidarité, en signalent simultanément la conséquence décisive : toute relation avec le Christ passe désormais par la médiation de "ces plus petits". Cette solidarité du Christ avec les hommes accablés par la détresse est un motif constant du premier évangile même si elle est décrite ici avec une incomparable originalité.

Elle apparaît tout au long de la narration $m$ t dans l'attention que Jésus porte aux malades, aux pécheurs et à ceux que la société rejette ${ }^{2}$. Dans les récits de miracles, par exemple, un des traits dominants du Messie mt est sa douceur ${ }^{3}$, c'est-à-dire sa sollicitude illimitée à l'égard de l'homme malheureux. De même, dans les controverses, le Christ prend parti pour

1 Comme l'indique le genre du morceau qui est une scène de jugement, mais aussi la fonction du Christ qui est celle du juge de la fin des temps, cette solidarité est de nature judiciaire et eschatologique.

2 GRUNDMANN, Mt 528.

3 BORNKAMM, Enderwartung 34 - et, à sa suite, BARTH 117-122 - ont analysé cet aspect de la christologie mt. Les citations d'Es 9, 1-2; 53,$4 ; 42,1-4$ respectivement en Mt 4,15-16; 8,17;12,18-21 en sont des exemples frappants. On peut également y ajouter le sommaire de 9, 35-36. 
le petit peuple rejeté et humilié ${ }^{1}$.

Pourtant - et il convient d'insister sur ce point -, bien que l'exigence de miséricorde soit une évidence pour les justiciables, la révélation de la solidarité du juge eschatologique avec les plus petits les déconcerte; elle est ressentie comme une surprise. Les séries de questions en $\pi \delta-$ $\tau \varepsilon$ posées par les justes comme par les réprouvés l'attestent clairement. Quelle est alors la signification de cette surprise ${ }^{2}$ ? L'étonnement des justiciables interdit tout d'abord une compréhension légaliste du texte : jamais les élus ou les infidèles n'ont imaginé qu'ils aimaient ou haïssaient Dieu en faisant preuve de miséricorde ou d'indifférence envers les plus petits; jamais ils n'ont perçu la détresse d'autrui comme l'occasion de gagner leur salut; jamais ils n'ont subordonné l'exercice de l'amour à l'espérance de la récompense eschatologique. A l'inverse, en insistant sur l'étonnement des justiciables au moment où ces derniers découvrent la solidarité du Christ avec les misérables, le texte entend faire admettre que le champ éthique a une fonction inattendue. Cette fonction cachée, mais capitale, peut se formuler ainsi : à travers l'attention portée à la détresse du prochain se manifeste la relation entre un homme et le Christ - et plus particulièrement entre le disciple et son maître -. Si toute décision prise pour le prochain en détresse est une décision prise pour le Christ, si tout refus opposé a l'homme dans le malheur est un refus signifié à Jésus, c'est que le comportement éthique dévoile en vérité le sérieux des disciples dans leur confession du Fils de l'homme. Nous pouvons dès lors préciser la conception $\mathrm{mt}$ du jugement : il

1 Sans entrer dans le détail, il faut faire état ici de textes tels que 9, 9-13 et 11,19 .

2 La surprise des justiciables est interprétée de diverses manières. BORNKAMM, Jesus 102.131, y voit la preuve que le prochain doit être aimé pour lui-même sans égard à la récompense eschatologique. BONNARD, Mt 366 , pense que cette surprise naft du fait que "le sens plénier de leurs actes ne leur est révélé qu'à la dernière heure". INGELAERE 38 voit dans ce trait le signe que les nations rassemblées sont non-chrétiennes, car les croyants auraient reconnu leur Seigneur. 
$n^{\prime} y$ va pas d'une simple rétribution selon les oeuvres ${ }^{1}$, car ce qui, en définitive, motive la sanction eschatologique, c'est la relation positive ou négative qui se noue et se joue entre l'homme et Jésus. En d'autres termes, le justiciable répond fondamentalement de l'attitude prise envers le Christ, et c'est de cette attitude que ses oeuvres témoignent ${ }^{2}$. Cette interprétation ouvre la voie à une nouvelle appréciation de l'éthique. Le rappel de la solidarité du Christ mt avec chaque homme en détresse dissipe toute illusion quant à la condition du croyant. Dans une église en danger de succomber à un spiritualisme grossier, dans une communauté où les charismes deviennent un alibi $(7,21-23)$ et où la contestation de la Loi (5, 17-20) se double d'un tiédissement de l'amour $(24,12)$, Mt réaffirme avec netteté le lieu de la foi ${ }^{3}$. En liant étroitement la confession du Fils de l'homme à l'exercice de la miséricorde, Mt condamne toute évasion religieuse et récuse les ghettos spirituels. Le Christ n'est véritablement cru et confessé que là où il engage les disciples dans une nouvelle fidélité qui est la fidélité de l'amour. Et cette fidélité n'est qu'un leurre tant qu'elle n'est pas assumée dans le vécu quotidien, dans la rencontre des autres et dans l'ouverture à leur détresse. En définitive, l'exigence de miséricorde montre qu'une authentique relation avec le Christ ne saurait exister sans un retour au fini et que l'éthique est la médiation privilégiée de ce retour. Déclaré heureux $(5,3-12)$, délivré du souci $(6,25-34)$, le

1 16,27 semble défendre la perspective d'un jugement selon les oeuvres

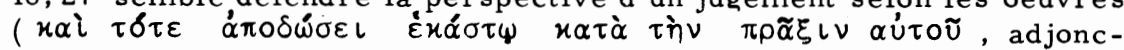
tion rédactionnelle sur la base du Ps 62,13). On fera cependant valoir que ce $v$. intervient dans une instruction exclusivement adressée aux disciples (comparer Mt 16,24 et Mc 8,34!) et qui présente les exigences de la Nachfolge dans la perspective du jugement. La $\pi \rho \tilde{a} \xi \iota \varsigma$ qui décidera du destin eschatologique de l'homme est donc la vie dans la Nachfolge.

2 BARTH 98.

3 Nous renvoyons ici à notre chap. 7. Nous pensons que l'évocation de ce contexte historique n'est pas privée de sens dans notre péricope, car il affleure constamment dans le chap. 24. SCHWEIZER, Mt 314, partage ce point de vue. 
disciple est replongé totalement dans la réalité du monde par le Christ mt et l'éthique proclame à la fois la nécessité et la modalité de cette prise en charge.

En conclusion, il n'est pas inutile de revenir sur la place de notre péricope dans le premier évangile. Notre passage n'est pas seulement la conclusion du dernier grand discours de Jésus, il est également son ultime instruction avant la Passion. Peut-on imaginer que les dernières paroles du Jésus terrestre (selon Mt !) à ses disciples se résument à une digression sur le sort des incroyants ou à une consolation adressée aux missionnaires en péril ${ }^{1}$ ? Toute notre analyse s'oppose à une telle exégèse. Il est préférable de voir dans notre passage l'aboutissement de la réflexion entreprise dans les chap. 24-25: d'abord en se servant du langage apocalyptique $(24,4-36)$, puis en recourant à des paraboles $(24,37-25,30)$, le Christ $\mathrm{mt}$ instruit ses disciples sur le temps de l'Eglise et sur la manière dont il importe de le vivre, à savoir dans une vigilante fidélité. $25,31-46$, loin de rompre l'unité du propos, le reprend dans une scène impressionnante qui doit en marquer le sérieux décisif. Par le biais du discours prophétique, le Maftre met ses disciples en garde contre une illusion qui les menace : il ne saurait y avoir de relation avec lui en dehors du vécu quotidien où s'éprouve et s'édifie toute foi fidèle; il ne saurait y avoir d'adoration sans obéissance dans la profanité du monde; il ne saurait y avoir de confession du Fils de l'homme sans amour "de ces plus petits".

1 Nous trouvons de telles tendances à banaliser le texte chez BROER 295; LAMBRECHT 333-339; JEREMIAS, Gleichnisse 206; INGELAERE 60. Remarquons cependant que notre exégèse se rapporte à la recension $\mathrm{mt}$ de 25, 31-46 et ne préjuge pas du sens pré-mt du passage. 
S I X I E M E P A R T I E

LE CROYANT ET L'EGLISE 
L'instruction finale du Ressuscité $(28,16-20)$ investit les onze d'une mission universelle : faire de toutes les nations les disciples du Christ (v. 19a). Désormais, tout homme quelle que soit sa condition religieuse, sociale ou ethnique est invité à accueillir l'enseignement du Maître et à s'agréger au nouveau peuple que le Christ rassemble par l'intermédiaire de ses envoyés. Cette ouverture inconditionnelle de l'évangile à tout homme - ouverture qui se concrétise dans la mise en oeuvre d'une mission universelle - apparaît avec une clarté incomparable dans la "finale" de Mt. Néanmoins, elle est déjà anticipée dans la généalogie : les quatre femmes citées sont des étrangères. Elle est aussi présente dans les récits d'appel: Pierre et André (4,18-20), Jacques et Jean (4, 21-22), mais surtout Matthieu le collecteur d'impôts $(9,9)$ sont des hommes que rien ne prédispose ou ne qualifie à devenir les disciples de Jésus. Ils appartiennent au petit peuple méprisé par l'élite religieuse et Matthieu n'est qu'un paria aux yeux de la société juive. L'appel de Jésus - complètement inattendu et lié à aucun préalable - transforme et recrée leur existence. Dans sa transgression des interdits sociaux et religieux, dans sa gratuité libératrice, cet appel est exemplaire de la manière dont le Christ s'adresse à tout homme et rassemble un nouveau peuple autour de lui.

Ce thème de l'appel de tout homme à la condition de disciple est l'une des expressions privilégiées de la sotériologie $\mathrm{mt}$. Son implication la plus importante consiste pourtant dans le rassemblement et la vie de cette nouvelle communauté qu'est l'Eglise. Aussi n'est-il pas étonnant que Mt ait retravaillé ce thème de l'appel à un niveau spécifiquement ecclésiologique. Nous en voulons pour preuve trois péricopes que nous nous proposons d'analyser. Il s'agit de la parabole du festin nuptial, de celle des vignerons révoltés, de l'histoire du centurion de Capernaüm. 


\section{L'invitation (Mt 22,1-14)}

Malgré les différences considérables qui existent entre les versions de Mt et de Lc, il n'est pas possible de nier la présence d'une forme primitive de la parabole dans la source $Q^{l}$. La comparaison synoptique nous montre en effet qu'au delà des divergences, le même fil conducteur se retrouve dans les deux recensions et que tout le contenu lc est présent chez $\mathrm{Mt}^{2}$. Pourtant, il est probable que ni Mt, ni Lc n'ont conservé la forme $Q$ de la parabole ${ }^{3}$. Ils l'ont déjà reçue de leur milieu respectif (QLc, QMt) sous des formes quelque peu différentes. A cela s'ajoute le travail de réinterprétation rédactionnelle ${ }^{4}$. Nous avons donc affaire à un processus traditionnel particulièrement complexe. Dans le cadre du présent travail, nous nous limiterons à examiner la constitution de la version mt. Sans prétendre pouvoir en retracer l'histoire complète, nous pensons que sur deux points au moins (vv. 4-7; 10-14), nous disposons d'indices permettant une analyse.

Du point de vue de l'histoire de la tradition, les vv. 11-14 retiennent l'attention, car ils constituent une séquence sans équivalent chez Lc. S'agit-il d'un développement rédactionnel de la parabole ou d'une unité traditionnelle primitivement indépendante ? Et s'il s'agit d'une unité traditionnelle, a-t-elle été fondue avec les vv. 1-10 par Qmt ou par le

1 Ainsi BULTMANN, Trad 189; KLOSTERMANN, Mt 183; LUEHRMANN, Logienquelle 105; SCHULZ, Q 398; STRECKER, Weg 111; TRILLING, Ueberlieferungsgeschichte 265; WALKER 56. Autrement : GRUNDMANN, Mt 465; LINNEMANN 167; SCHNIEWIND, Mt 219; voir aussi BONNARD, Mt 318-319.

2 Relevons la structure suivante : préparation d'un festin; envoi de serviteurs chargés de l'invitation; refus des invités; colère du maître; invitation adressée à d'autres; condamnation des premiers invités. Cf. SCHMID, Mt 309.

3 HAHN, Gleichnis 51-52; LUEHRMANN, Logienquelle 106-106; SCHULZ Q 398.

4 Pour une analyse détaillée, voir les travaux cités de HAHN et de SCHULZ. 
rédacteur de Mt ? L'hypothèse d'une composition purement rédactionnelle des vv. $11 \mathrm{s.13} \mathrm{s}^{\mathrm{l}}$ paraît peu vraisemblable, car on s'expliquerait mal les tensions qui existent entre les vv. 2-10 et 11-13. Le passage des $\delta \circ \tilde{v-}$

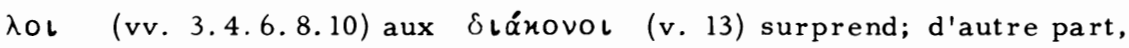
rien dans la parabole du festin nuptial n'indique que les invités insolites et inattendus des vv. 9-10 aient dŭ ou pu se munir d'un vêtement approprié. Le motif de l' $l^{\prime} \vee \delta \cup \mu \alpha$, constitutif des vv. 1l-13a, est totalement étranger aux vv. 2-10. Il faut donc admettre que les vv. 11-13a sont un fragment parabolique pré-mt, dont la provenance reste incertaine ${ }^{2}$. Est-ce le rédacteur qui a placé ce fragment après les vv. 1-10 ou s'y trouvait-il déjà? La majorité des exégètes optent pour une adjonction rédactionnelle ${ }^{3}$. Mais c'est oublier la surprenante tension entre les vv. 11-13 et le v. 14. Les vv. 11-13a évoquent l'indignité d'un seul parmi un grand nombre alors que le

1 Ainsi BARTH 55 (note 7) qui, sur la base de la terminologie utilisée, voit dans les vv. 11-13 une adjonction rédactionnelle calquée sur une parabole rabbinique (BILL 1, 878).

2 Ainsi, p. ex. HAHN, Gleichnis 76; JEREMIAS, Gleichnisse 62; LINNEMANN 102.168; TRILLING, Ueberlieferungsgeschichte 255-258. SCHULZ, Q 398 et SCHWEIZER Mt 271, se rallient avec prudence à cette hypothèse en n'excluant pas une forte empreinte rédactionnelle du fragment. L'introduction primitive de la parabole (v. 2) faisait peut-être partie à l'origine des vv. 11-13 (SCHWEIZER, Mt 271). L'origine du fragment est contestée : si HAHN, JEREMIAS, LINNEMANN y voient le fragment d'une parabole (qu'il est impossible de reconstituer, avec HAHN 76, note 111), TRILLING opte pour un simple motif parabolique. Par ailleurs, HAHN (Gleichnis 76) et TRILLING (Ueberlieferungsgeschichte 256) ont dénoncé à juste titre l'usage abusif que l'exgésèse a fait d'un parallèle rabbinique (BILL 1, 878). Si la parabole juive insiste sur le temps de préparation qui est accordé aux invités, c'est exactement l'inverse qui se produit chez Mt.

3 Ainsi, p. ex. , BONNARD, Mt 319; JEREMIAS, Gleichnisse 62 (?); LINNEMANN 102.168; SCHMID, Mt 310; SCHULZ, Q 398; SCHWEIZER, Mt 271 (avec réserve). 
v. 14 inverse la perspective : parmi le grand nombre, seuls quelques-uns seront sauvés. Même si le v. 14 reprend la problématique des vv. 11-13a 1 , il le fait d'une manière différente, preuve qu'il n'appartient pas à la même couche traditionnelle. Nous en concluons que le v. 14 est un logion isolé dont s'est servi le rédacteur ${ }^{2}$ pour commenter les vv. 11-13a et que ceuxci figuraient déjà dans $Q m t^{3}$. Le v. 13 b est une adjonction rédactionnelle qui, en évoquant le châtiment, introduit l'application mt de la parabole 4.

Les vv. 4-7 sont le second point critique qui permet d'observer la

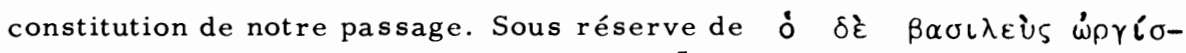
$\vartheta \eta$, les vv. $6 \mathrm{~b}-7$ sont de l'avis unanime ${ }^{5}$ une adjonction secondaire. A l'appui de cette hypothèse, on fait valoir les arguments suivants. On pas-

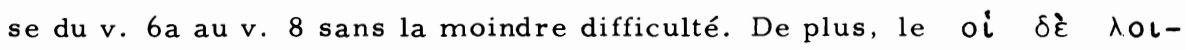
$\pi o i ́$ de $6 \mathrm{~b}$ est une suture maladroite ${ }^{6}$. Mais surtout les vv. $6 \mathrm{~b}-7$ évoquent des événements qui brisent le cadre de la parabole ${ }^{7}$ : on imagine mal que les hôtes choisis assassinent les envoyés du roi pour exprimer leur refus de participer à un repas de noce; on se représente plus mal encore que le roi vexé organise, pendant que le repas attend, une expédition de représailles et tue tous les invités récalcitrants, regroupés par chance dans la même ville; on voit mal enfin le roi regagner tout naturellement son palais pour présider le festin. Comme l'a montré Trilling ${ }^{8}$ il convient pour.

1 L'homme qui ne porte pas le vêtement de noce (vv. 11-13a) est bien évidemment l'image des appelés qui ne sont pas élus (v. 14).

2 Ainsi BULTMANN, Trad 189; HAHN, Gleichnis 77; JEREMIAS, Gleichnisse 105; SCHULZ, Q 398.

3 Ainsi HAHN, Gleichnis 76, à la suite de TRILLING, Ueberlieferungsgeschichte 255-258.

4 Cf. 13,42.50; 24, 51; 25, 30, avec BULTMANN, Trad 352; HAHN, Gleichnis 78; JEREMIAS, Gleichnisse 58 (note 1); LINNEMANN 168.

5 Etat de la discussion, SCHULZ, Q 396 (note 146). Autrement RENGSTORF $126-127$.

6 GRUNDMANN, Mt 468 et SCHULZ, Q 395.

7 JEREMIAS, Gleichnisse 66 et SCHWEIZER, Mt 273.

8 Ueberlieferungsgeschichte 254-255. Voir aussi SCHWEIZER, Gemeinde $117-118$. 
comprendre ce développement allégorique de rapprocher notre passage de la parabole des vignerons révoltés qui précède immédiatement la nôtre. On remarque alors que $22,6 \mathrm{~b}$ est le pendant de 21,$35 ; 22,7$ celui de $21,41 \mathrm{a}$. Mais la convergence va encore plus loin. Dans la version lc de notre parabole, un seul serviteur transmet une seule invitation aux premiers invités alors que Mt évoque deux envois successifs de plusieurs serviteurs. La coïncidence avec $21,34.36$ est frappante et nous amène à penser que dans les vv. 4-7, Mt réinterprète sa source en reprenant le schéma incontestablement rédactionnel qu'il a utilisé dans la parabole précédente (double envoi/sévices/châtiment) ${ }^{1}$. Il existe donc une parenté structurelle entre la parabole des vignerons révoltés et la nôtre. Dans les deux cas, Mt s'est servi du même schéma pour décrire la crise d'Israël et ses conséquences ${ }^{2}$.

Outre ces deux points forts de la rédaction $\mathrm{mt}$, il convient de relever quelques retouches qui assurent une certaine cohérence à l'ensemble. Mis à part les vv. 1-2a qui constituent une introduction typiquement $\mathrm{mt}^{3}$, le thème de la dignité évoqué au v. 8 est probablement rédactionnel 4 , car

1 On peut ajouter que le commentaire de $21,40-42$ se retrouve en $22,3-7$,

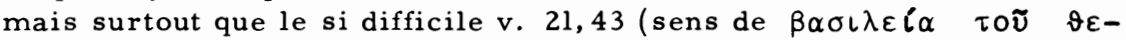
oṽ) se trouve anticipé en 21,31 et développé en 22,8-13.

2 Dans une analyse particulièrement fouillée de la rédaction de Mt 21-25, SCHWEIZER (Gemeinde 116-125) a montré que le thème dominant de cette partie est la crise d'Israël et sa signification pour l'Eglise. L'exposition de ce thème est structurée par le schéma suivant qui revient deux fois $(21,1-22,14$ et $22,15-25,46)$ : comparution d'Israël (21,28-32 et $23,1-32)$, énoncé de la sentence $(21,33-45$ et $23,33-36)$, exécution de la sentence $(22,1-10$ et $23,37-24,2)$, parénèse $(22,11-14$ et $24,3-25,46)$. Dans le cas qui nous occupe, ce schéma permet de comprendre la parenté entre les trois paraboles du jugement des chap. 2122, mais aussi leur spécificité théologique.

3 Cf. HAHN, Gleichnis 53.78; KLOSTERMANN, Mt 174-175; analyse détaillée chez SCHULZ, Q 392.

4 Cf. 3, 8; 10,11.13.37.38. Avec HAHN, Gleichnis 77 et SCHULZ Q 391. 
il n'apparaît que dans le premier évangile. Son intérêt, dans notre péricope, est de fournir le critère théologique qui permet de juger Israël, mais aussi d'annoncer l'idée développée dans les vv. 11-14 - appliquée cette fois-ci à la condition chrétienne -. Semblablement, la clausule

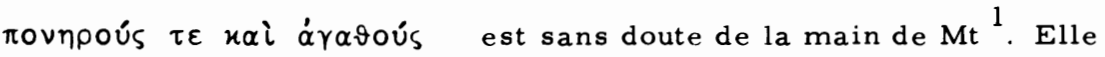
souligne l'ampleur de l'invitation adressée aux seconds invités tout en préparant la dernière scène du morceau.

Les retouches rédactionnelles que nous avons repérées s'inscrivent toutes dans un même projet cohérent : approfondir et systématiser l'allégorie déjà présente en $Q m{ }^{2}$. Dans les vv. 3-6, l'application du schéma "double envoi - persécution - châtiment" vise à décrire la crise d'Israël dans toute sa gravité. L'utilisation de concept $\alpha$ $\varepsilon$, los au v. 8 est une interprétation théologique de l'événement. L'adjonction rovnpoús $\tau \varepsilon$

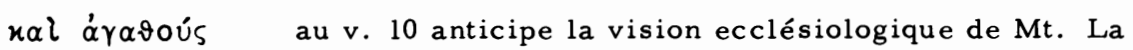
clausule du v. 13 b fait clairement allusion au jugement dernier. Si donc les vv. 2-14 constituent de part en part une allégorie, quel est son objet ?

La plupart des exégètes s'accordent à y voir une illustration de l'histoire du salut $^{3}$. Cette illustration se compose de deux parties ${ }^{4}$ : les vv. 2-7 traitent de l'échec d'Israël, tandis que les vvv. 8-14 décrivent le rassemblement et le destin de l'Eglise. Dans la première scène, l'introduction à la parabole (v. 2) nous fait immédiatement aller à l'essentiel : dans le roi, il faut reconnaître Dieu, dans son fils le Christ, dans les

1 HAHN, Gleichnis ibid.; KLOSTERMANN, Mt 176; SCHULZ, Q 397.

2 Voir p. ex., HAHN, Gleichnis 74; JEREMIAS, Gleichnisse 65-66; LINNEMANN 101; STRECKER, Weg 111.

3 Avant tout JEREMLAS, Gleichnisse 66, approuvé par HAHN, Gleichnis 81; STRECKER, Weg 111-112; WALKER 96-97.

4 Point opportunément relevé par HAHN, Gleichnis 75 : la césure entre 2-10 et 11-14 se justifie du point de vue de 1'histoire de la tradition; au niveau rédactionnel, la péricope doit être considérée dans son ensemble et la répartition qui s'impose est 1-7. 8-14. 
noces l'irruption du Royaume ${ }^{1}$. Si le temps envisagé est bien celui de l'irruption du Royaume, alors le premier envoi fait allusion à la mission pré-pascale auprès d'Israël, tandis que le second (cf. notamment la déclaration du v. 4b) figure la mission post-pascale auprès de ce même Israël ${ }^{2}$. La persécution dont sont victimes les seconds envoyés s'accorde bien avec les données du chap. 10 (cf. aussi 23,34ss). L'expédition punitive du v. 7 , enfin, est un topos représentant la marche des armées romaines sur Jérusalem et sa destruction en $70^{3}$. L'effondrement de la ville sainte est présenté comme le châtiment divin.

La deuxième scène s'attache à révéler la réalité de l'Eglise (vv. 8-14).

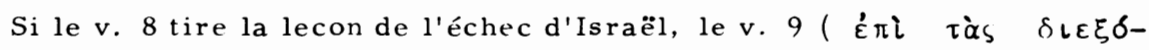

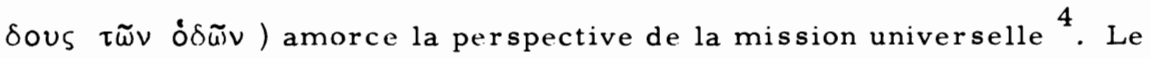

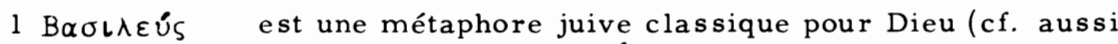
18,23 !) ;elle détermine le sens de viós comme étant le Christ (voir aussi la parenté structurelle avec 21,37-38). Гános désigne le festin messianique (GRUNDMANN, Mt 468; SCHNIEWIND, Mt 218) et, ici le Royaume qui s'approche.

2 Thèse défendue par HAHN, Gleichnis 79-80 et WALKER 91-93. La position classique qui voit dans les premiers envoyés à Israël les prophètes vétérotestamentaires est notamment représentée par JEREMIAS, Gleichnisse 66; LINNEMANN 101; SCHNIEWIND, Mt 221; SCHMID, Mt 311; STRECKER, Weg 33-34; WEISER 66-69 (là, discussion détaillée et état de la recherche). Elle se heurte à trois objections : s'il s'agit des prophètes vétérotestamentaires : quel est l'objet de l'invitation ? pour quand est-elle lancée ? comment leur refus leur vaut-il une nouvelle invitation?

3 RENGSTORF 106-129, a défendu l'hypothèse selon laquelle le v. 7 était un topos connu de l'Orient ancien et décrivant les expéditions guerrières et punitives, topos dans lequel il ne faut chercher aucune allusion aux événements de l'année 70 (art. cit. 125). Ce dernier point est contestable : Mt décrit au contraire la catastrophe de 70 en se servant d'un topos (discussion détaillée chez HAHN, Gleichnis 56-57.80; LINNEMANN 166-167; STRECKER, Weg 35); ce processus d'historicisation est fréquent dans le ler évangile. De plus, il s'insère bien dans un propos allégorique.

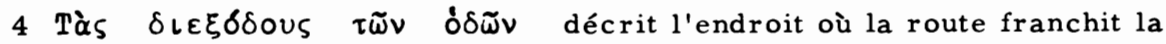
limite de la localité pour déboucher dans la campagne; l'expression devient le symbole du chemin vers le lointain (BONNARD, Mt 450; HAHN, Gleichnis 80; JEREMIAS, Gleichnisse 66; WEISER 68). 
v. 10 nous confronte à une église de masse dont la réalité est ambiguë ${ }^{1}$. Les vv. 11-13 montrent que cette église nouvellement rassemblée est en route vers le jugement et que seule la justice ( $\tilde{\varepsilon} \vee \delta \cup \mu \alpha)$ attestée par chacun prévaudra à cette occasion ${ }^{2}$. Le v. 14 tire l'application parénétique de cette venue du jugement.

Les vv. 2-14 retracent l'histoire du salut à grands traits. Cependant et cet aspect est trop souvent négligé -, cette esquisse est constituée à partir de deux catégories critiques qui président à l'agencement du tout. Ces deux catégories sont l'appel ( $x \alpha \lambda \varepsilon \tau \nu$ vv. 3.4.8.9.14) et la dignité ( $\ddot{\alpha} \xi$ เos v. 8, cf. aussi $\ddot{\varepsilon} \vee \delta \cup \mu \alpha$ vv. 11-12). Cette exposition de l'histoire du salut sous l'angle de l'appel et de la dignité qu'il requiert, ne va pas de soi. En 8,11-12, p. ex., l'histoire du salut est présentée à partir de la foi; en 21, 33-46 à partir de l'obéissance. Dans ce temps qui est celui de l'irruption du Royaume, l'histoire du salut est l'histoire de l'appel que Dieu transmet par ses envoyés. Cet appel met Israël d'abord, l'ensemble des homme ensuite en situation de décision. Par son refus, l'invité se rend indigne de cet appel; par son accord, il s'en révèle digne. Polémique et parénèse sont étroitement liées dans ce morceau ${ }^{3}$. Les vv. 2-7 et 8-14 sont organiquement liés et expriment une seule et même conception théologique qui est celle du rédacteur. Car, d'une part, pour Mt, l'échec d'Israël est la présupposition obligée de la mission auprès des païens - et

1 TRILLING, Ueberlieferungsgeschichte $258 \mathrm{~s}$ qui rapproche notre expression de textes tels que 13,41 .

2 BARTH 55; HAHN, Gleichnis 81; JEREMIAS, Gleichnisse 66. Voir notre analyse détaillée pp. 383-384.

3 Contre TRILLING, Ueberlieferungsgeschichte $263 \mathrm{~s}$, qui attribue la couche parénétique à $Q m$ t et l'accentuation polémique à la rédaction la crise d'Israël deviendrait pour Mt la pointe de l'ensemble -. LINNEMANN 167, et STRECKER, Weg 112 ont à juste titre, contesté ce point de vue. D'une part, l'allégorie de l'histoire du salut donne une homogénéité certaine au tout; d'autre part, parénèse et polémique ne sont pas des critères suffisants pour opérer une distinction entre les couches

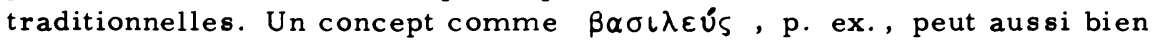
entrer dans la polémique que dans la parénèse. 
donc de la constitution de l'Eglise universelle -. D'autre part, la perdition du peuple élu est le contre-modèle qui met en garde la communauté en route vers le jugement contre toute tentation de légèreté; à ce titre, elle alimente la parénèse.

Si donc il convient de considérer notre passage comme un texte d'un seul tenant, destiné à illustrer le thème de l'appel par l'évocation de l'histoire du salut, la succession des deux parties qui le composent nous permet d'en discerner l'intention directrice : le refus des premiers invités amène le roi à en convier d'autres. En clair : l'échec de la mission des disciples auprès d'Israël conduit Dieu à adresser son appel à d'autres hommes; il provoque et légitime la mission auprès des paíens ${ }^{1}$. Nous touchons ici une affirmation capitale pour l'élaboration de l'ecclésiologie $\mathrm{mt}$, car nous découvrons comment le rédacteur conçoit la constitution de $\underline{1 ' E g l i s e}^{2}$. Nous aimerions, à cet égard, relever deux points essentiels.

Tout d'abord, l'Eglise telle que Mt l'envisage (càd comme le rassemblement de ceux qui acceptent l'invitation divine) n'existe qu'en fonction de l'échec d'Israël et se définit par rapport à lui ${ }^{3}$. Ce n'est que parce qu'Israël a été infidèle à sa vocation et que son incrédulité l'a précipité dans une catastrophe irrémédiable - dont l'année 70 reste le symbole

1 Ce passage de la mission judéo-chrétienne à la mission pagano-chrétienne est retenu comme le point décisif du texte par HAHN, Gleichnis 81; JEREMIAS, Gleichnisse 66; STRECKER, Weg 33-34.

2 WALKER 93-95 conteste que l'on puisse voir dans les invités des vv. 8-10 des chrétiens. Il s'agirait de l'appel des païens que l'on ne saurait sans autre assimiler à l'Eglise qui, elle, serait constituée des seuls

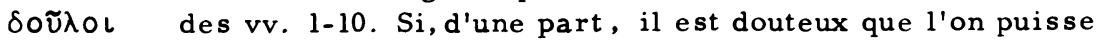
réduire l'Eglise à ses missionnaires, cette exégèse s'accomode mal, d'autre part, des vv. 11-14 (que WALKER ne prend pas en considération !) : sont jugés non pas les hommes en général, mais ceux qui ont accepté la seconde invitation. Cf. le ouvírarov du v. 10.

3 Même argumentation en 8,10-12; 21, 33-46. 
significatif - que l'Eglise se constitue ${ }^{1}$. L'Eglise naft de la crise d'Israël; cette lecture mt de l'histoire confere à la communauté son identité propre et l'assure de son bon droit dans son affrontement avec le judaĩsme.

Pourtant, si l'Eglise résulte du refus que les Juifs ont opposé au Royaume messianique, elle n'est ni la continuation d'Israël, ni un nouvel Israël. Les seconds invités n'ont rien de commun avec les premiers; ils ne sont pas des $x \varepsilon x \lambda$ ńfยvol 2 (v. 3). Si la première invitation était ad personam, la seconde (vv. 8-10), s'adresse à quiquonque veut bien la recevoir. Elle se caractérise par la disparition de toute exclusive, par une ouverture illimitée. Cette profession d'universalisme ${ }^{3}$ dont il s'agira de mesurer les conséquences ecclésiologiques et sotériologiques, apparaft de trois manières dans le texte. D'abord, l'envoi missionnaire ${ }^{4}$ aux $\delta \iota \varepsilon \xi \delta \delta \circ \cup \varsigma$

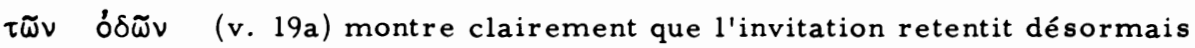
en terre païenne. La barrière séculaire entre Israël et les Gentils est

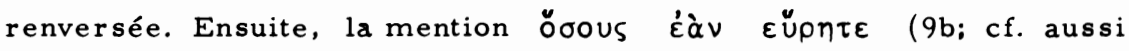

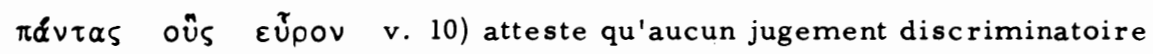
ne saurait être posé dans cette nouvelle aire missionnaire : tout homme rencontré est convié - sans référence à son origine, à son passé, à sa

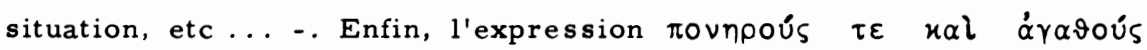

(v. 10) est sans doute la plus surprenante. Comme nous l'avons déjà relevé, elle est élaborée en fonction des vv. 11-14 et ne décrit donc pas un état précédant l'invitation ${ }^{5}$. Mais elle souligne, à sa manière, l'ouvertu-

1 Cf. vv. 6-7. La critique de TRILLING (Ueberlieferungsgeschichte 264) selon laquelle pareille présentation ne correspond pas à la réalité historique n'est juste qu'en apparence : Mt présente sa conception théologique de l'histoire :

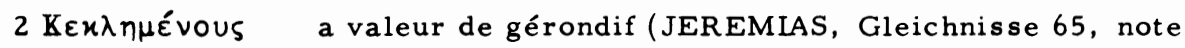
2) et exprime le privilège d'Israël - le fait qu'il était destiné au Royaume - . Cf aussi 8,12 (oi viol $\tau \tilde{n} s \quad \beta \alpha \sigma(\lambda \varepsilon i \alpha \varsigma$ ) et 21,43 .

3 Sur l'universalisme mt, matériel rassemblé par SCHNIEWIND, Mt 219; analyse chez TRILLING, Israel 124-142.

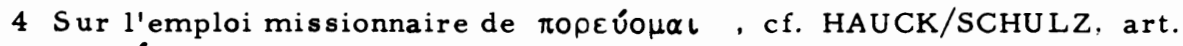

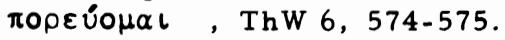

5 BONNARD, Mt 320; KLOSTERMANN, Mt 176; SCHWEIZER, Mt 273-274; WA LKER 93-96. 
re totale et inconditionnelle qui caractérise le rassemblement de l'Eglise.

Concluons. Le thème de l'appel tel qu'il est développé dans notre parabole, a un aspect à la fois sotériologique et ecclésiologique. L'invitation au festin messianique - càd, en définitive, au salut - est désormais adressée en plénitude à tout homme. Aucun préalable n'est exigé pour avoir part à cette initiative divine. Cet universalisme dans l'appel est l'expression de la grầce. Le corrélat ecclésiologique de cette affirmation est double. D'une part, l'Eglise, par le biais de ses missionnaires (

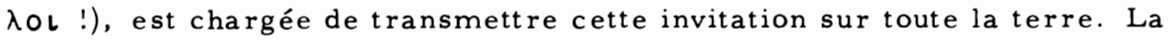
mission mt ne connaît ni limitation, ni restriction. D'autre part, - et c'est l'accent dominant des vv. 8-9-, l'Eglise est conçue comme le lieu d'un vaste rassemblement (v. 10 : ouvńrarov ; $\varepsilon \pi \lambda n ́ \sigma \vartheta \eta \dot{~ v u \mu \varphi w ́ s) ~}$ dont personne n'est exclu. Sa raison d'être est de grouper tous les invités sans égard à leur dignité.

Pareille ouverture dans l'appel n'est pas sans faire problème. Les seconds invités vont-ils éviter l'écueil qui a perdu Israël ? vont-ils se montrer dignes de l'invitation qui leur est adressée ? comment cet appel doit-il être reçu et vécu ? Les vv. 11-14 abordent cette perspective en toute clarté. Nous y reviendrons dans notre paragraphe consacré au corpus mixtum. Préalablement, nous décrirons le statut des invités sous l'angle de la foi et de l'obéissance.

\section{Incrédulité d'Israël, foi de l'Eglise (8,11-12)}

La logion (vv. 11-12) qui va retenir notre attention, a été inséré par Mt (voir plus bas) dans l'histoire du centenier de Capernaüm (8, 5-13). Avant d'en venir à l'analyse de cette parole, il convient d'examiner brièvement le récit qui en constitue le contexte $m$ t et d'en cerner l'intention ${ }^{1}$. Comme en témoigne le par. Lc - et, en particulier, la frappante identité

1 Voir, en particulier, HELD 182-184 et SCHULZ, Q 241-246. 
verbale entre Mt 8,8-10 et Lc 7,6b-9-, le dialogue entre Jésus et le centurion forme le noyau de la péricope. L'élément narratif a presque complètement disparu; la description du miracle est allusive ${ }^{1}$. L'essentiel tient dans la déclaration de confiance de l'officier paĩen et dans la réponse admirative de Jésus. Le thème fondamental de ce débat est la foi ${ }^{2}$. En ce qui concerne le cadre narratif du dialogue central, il est difficile de déterminer qui de Mt ou de Lc est le plus fidèle à $Q$ : peut être même s'agit-il d'une composition rédactionnelle ${ }^{3}$. Quoi qu'il en soit, chez Mt, les vv. 5-7.13 ont un caractère nettement rédactionnel ${ }^{4}$. Ils permettent d'observer comment Mt a compris et utilisé la discussion des vv. 8-10. Considérons tout d'abord la séquence qui ouvre la péricope (vv. 5-7). La venue ( $\pi \rho \circ \sigma \tilde{\eta} \lambda \vartheta \varepsilon v)$ du centurion à Jésus, sa demande implicite ( $\pi \alpha \rho \alpha \varkappa \alpha \Lambda \widetilde{\omega} \nu)$, le titre qu'il accorde au Christ ( $\varkappa u ́ p \iota \varepsilon)$ sont autant de signes de sa confiance ${ }^{5}$. Le v. 7 est fort discuté : a-t-il un sens interrogatif dubitatif, auquel cas il soulignerait l'hésitation du Christ à s'engager en faveur d'un paīen ${ }^{6}$ ? ou est-il affirmatif et exprime-t-il

1 BULTMANN, Trad 39, range de manière significative la péricope dans les apophtegmes. Voir aussi LUEHRMANN, Logienquelle 57 et SCHULZ Q 240-241.

2 L'accord règne sur ce point; cf. p. ex. : HAENCHEN, Probleme 83; HELD 182; LUEHRMANN, Logienquelle 57-58; SCHULZ, Q 241.

3 HELD 183. Etat de la question chez SCHULZ, Q 236-240.

4 HELD $183-184$.

5 Le verbe $\pi \rho \circ \circ \varepsilon \rho \chi \varepsilon \bigcirc \vartheta \alpha \iota$ est un des mots préférés de Mt (52 fois, contre 5 fois chez $\mathrm{Mc}$ et 10 fois chez Lc). Il décrit l'attitude d'un homme venant au Christ avec une prière ou une demande. Le participe $\pi \alpha \rho \alpha \mathcal{K} \alpha \lambda \tilde{\omega} v$ permet de comprendre le $v$. 6 comme une demande d'aide. Chez Mt, le titre $u \cup ́ \rho \iota \varepsilon$ (repris ici de Q) n'est attribué au Christ que par des hommes qui ont une relation positive avec lui - jamais par ses ennemis - (cf. ZUMSTEIN, Maître et disciple 129-132).

6 Cette interprétation est notamment soutenue par HELD 184 à la suite de BULTMANN, Trad 39. En faveur de cette hypothèse, on retient essentiellement trois arguments. D'une part, on invoque la parenté de notre récit avec l'histoire de la Cananéenne où le refus de Jésus est indubitable. Ensuite - et dans la même ligne - on fait valoir qu'en pénétrant dans une maison païenne, le Christ contreviendrait aux règles de pureté rituelle. Enfin, on souligne que la réponse du centurion vise à 
l'acceptation de Jésus ${ }^{1}$ ? Que l'on choisisse l'une ou l'autre hypothèse, il introduit à merveille la réponse du centurion (vv. 8-9) ${ }^{2}$. Ces brèves remarques nous permettent de conclure que l'introduction du récit est composée en vue du dialogue central et à partir de la problématique de la foi. Il n'en va pas autrement de la conclusion rédactionnelle du v. $13^{3}$. La réponse de Jésus qui accorde le secours demandé et clôt le dialogue, résulte de la foi du centurion ( $\dot{\pi} i_{\text {L } \sigma \tau \varepsilon v \sigma \alpha \varsigma}$ ) si clairement manifestée dans les vv. $8-9$.

Que l'on considère le dialogue central ou le cadre narratif dans lequel il est inséré, le récit est de part en part axé sur le thème de la foi. Quelle est la structure de cette foi ? Il ne s'agit évidemment pas du crédit porté par un païen à un thaumaturge particulièrement doué. Le fait que l'histoire soit reprise à l'intention des communautés chrétiennes, signifie que la figure du centurion est saisie dans son exemplarité ${ }^{4}$. De plus, les vv. 11-12 feront de cette foi la condition d'accès au salut eschatologique. L'élément constitutif de la foi du centurion réside dans la confiance

vaincre une résistance. Dans ce sens : KLOSTERMANN, Mt 74; LOHMEYER Mt 157; TRILLING, Israel 105.

1 Ainsi HAENCHEN, Probleme 82-83; SCHLATTER, Mt 274-275;

SCHWEIZER Mt 138. HAENCHEN conteste l'interprétation dubitative en formulant quatre objections : a) l'histoire de la Cananéenne n'est pas un parallèle adéquat car sa progression dramatique ne se retrouve pas dans notre récit; b) le centurion ne demande pas à Jésus de venir chez lui; c) le participe $\dot{\varepsilon} \lambda \vartheta \dot{v} \nu$ est un sémitisme qu'il ne faut pas surinterpréter - l'accent porte sur $\vartheta \varepsilon p a \pi \varepsilon u ́ \sigma \omega ; d)$ si le v. 7 est un refus sous forme de question, la réponse du centurion ne supprime pas la difficulté.

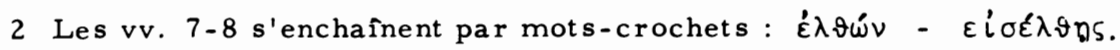

3 Optent pour une formulation rédactionnelle : GRUNDMANN Mt 249; HELD 183; KLOSTERMANN, Mt 75. Analyse terminologique détaillée chez SCHULZ, Q 240 (note 427).

4 HAENCHEN, Probleme 83. 
absolue qu'il met en Jésus ${ }^{1}$ : il n'hésite pas à lui confier la vie gravement menacée de son serviteur. Cette confiance $n^{\prime}$ est pas indéterminée; elle est confiance dans le pouvoir libérateur et transformateur de la parole du Christ (v. $8 \mathrm{~b}: \lambda \delta \gamma \psi)$ ). Mais pourquoi le centurion voue-t-il une foi si totale à la parole de Jésus ? Le v. 9, axé sur le thème de 1 ' $\dot{\varepsilon}-$

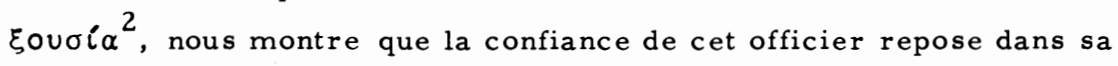
connaissance de la véritable identité du Christ. Le raisonnement qu'il tient sur ce point, mérite d'être rappelé : si lui qui est officier, tient de l'autorité à laquelle il est soumis, un pouvoir qui lui permet d'agir sur le secteur de réalité qui lui est confié, à combien plus forte raison le Christ qui tient son autorité de Dieu, peut-il venir à bout de toutes les forces du mal - et singulièrement de la maladie qui en est l'une des expressions -. En définitive, la foi du centurion consiste en ce qu'il a reconnu dans le dire et le faire de l'homme de Nazareth la présence et l'autorité de Dieu. La parole du Christ est une parole transformatrice et libératrice parce qu'à travers elle c'est Dieu lui-même qui agit. C'est dans le cadre de cette étonnante confession que la déclaration d'indignité du v. $8 \mathrm{~b}$ ( oúx $\varepsilon i \mu i$ ixavós) prend toute sa signification ${ }^{3}$. A un premier niveau, il est incontestable que cette affirmation reflète le problème des relations entre Israël et les nations : en visitant un päien, le Christ contreviendrait aux règles de la pureté rituelle. Mais cette constataiion n'épuise pas l'analyse. Le centurion se déclare indigne non pas devant les Juifs en général, mais devant le Christ en qui il a reconnu la parole et l'agir de Dieu. Son aveu n'est pas seulement l'expression de son respect pour des pratiques religieuses, mais plus fondamentalement la reconnaissance de sa "pauvreté" en face de celui dont il sollicite

1 Bonne formulation chez SCHULZ, Q 244 : "das unerschütterliche Vertrauen gegenüber der Wundermacht des Wortes Jesu".

2 Voir BONNARD, Mt 116, et FOERSTER, art. $\dot{\xi} \xi o v \sigma i \alpha$, ThW 2, 562.

3 Voir SCHNIEWIND, Mt 109, et SCHWEIZER, Mt 138. 
le secours. Le centurion se sait radicalement démuni devant le Christ, n'ayant aucun droit à son aide; et, pourtant, il attend tout de lui. Son attitude rejoint celle du pauvre des béatitudes et figure la juste position de l'homme devant Dieu. En ce sens, l'aveu du v. 8 b est constitutif de la foi.

Toute la thématique que nous venons de développer, Mt l'a trouvée dans sa source $Q$. Il la reprend en la ratifiant totalement. Pourtant il lui donne une application qui lui est propre. Il réoriente la perspective d'ensemble en réinterprétant le v. 10 par l'adjonction des vv. 11-12. Il a certes trouvé les vv. 11-12 dans la source $Q$ (cf. Lc 13,28-29), mais c'est lui qui les a insérés dans l'histoire du centurion de Capernaüm. Au crédit de cette hypothèse généralement admise ${ }^{1}$, on retiendra les arguments suivants. Ces vv. ne figurent pas dans le récit parallèle de Lc. Or si l'on considère que l'appel des païens est une idée chère à Lc, l'omission devient difficilement explicable. Par contre, la propension sans cesse vérifiée de Mt à grouper de manière systématique les matériaux semblables rend l'adjonction vraisemblable. Cette observation générale est soutenue par la présence d'une double suture qui trahit l'insertion secondaire de la sentence dans le récit ${ }^{2}$. Au v. 1l, pour introduire la déclaration de Jésus,

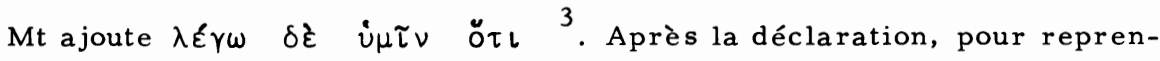
dre le fil de l'histoire, le rédacteur compose une petite transition : $x \alpha i$

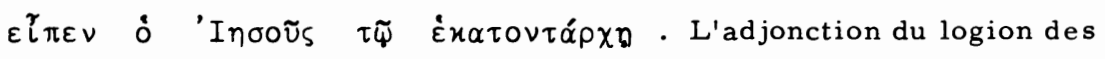
vv. 11-12 est donc le moyen dont se sert Mt pour interpréter le récit.

S'il en est ainsi, qui de Mt ou de Lc a le mieux conservé cette sentence transmise par la source $Q$ ? Il est fort peu probable que ce soit Lc,

1 Voir, p. ex. BOR NKAMM, Enderwartung 25-26; DUPONT, Beaucoup 154; HAENCHEN, Probleme 83; HELD 185; HUMMEL 147; KLOSTERMANN, Mt 75; LUEHRMANN, Logienquelle 57; STRECKER, Weg 100; TRILLING, Israel 88; WALKER 89.

2 Avec DUPONT, Beaucoup 155.

3 Même procédé en $12,6.36 ; 19,9$. 
car on voit mal un logion indépendant débuter par l'adverbe de lieu

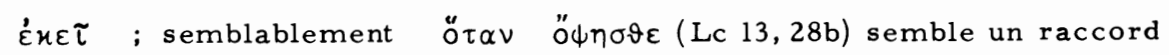
maladroit et postérieur. Par contre, ces éléments s'expliquent fort bien comme adaptation rédactionnelle du logion au contexte ${ }^{1}$. La version $\mathrm{mt}$ qui forme un tout, construit de manière stricte, paraît mieux conserver l'ordonnance primitive de la sentence. Elle lui apporte néanmoins quel-

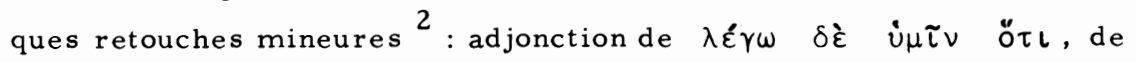

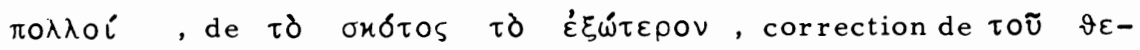

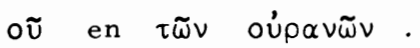

Plus important est l'auditoire que Mt a choisi pour cette déclaration du Christ. Jésus ne répond pas directement au centurion - ce qui correspondrait à une progression normale du dialogue -. Il s'adresse à ceux qui

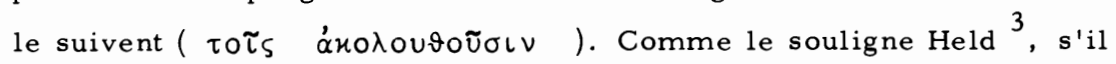
faut voir tout d'abord dans ces auditeurs les accompagnants historiques du Jésus terrestre, il n'en reste pas moins que, comme tout au long de la narration $\mathrm{mt}$, ils sont l'image de l'Eglise. La remarque est d'importance. Elle montre que dans le logion des vv. 11-12, Mt tire à l'intention de l'Eglise la leçon de l'histoire du centurion. Cette leçon est d'ailleurs anticipée dans le v. 10 dans lequel, par une retouche mineure, Mt intro-

1 Le $\dot{\varkappa \varepsilon \tau ~ s u p p o s e ~ u n ~ c o n t e x t e ~ a n t e ́ r i e u r ~ q u e ~ l ' o n ~ t r o u v e ~ e n ~ L c ~ 13, ~} 25$. Le ŏ $\tau \alpha$ ő $\psi \eta \sigma \vartheta \varepsilon$, bien en accord avec la note eschatologique du passage, permet d'introduire l'essentiel de la sentence. La répétition

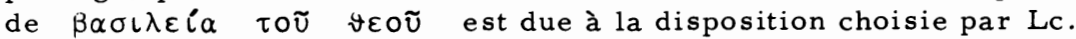
Avec DUPONT, Beaucoup 156; HOFFMANN 207-209; SCHULZ, Q 324 325; ZELLER 223. Contre : STRECKER, Weg 100; TRILLING, Israel 88; WALKER 89.

2 Voir DUPONT, Beaucoup 157; SCHULZ, Q 323-324; HOFFMANN 208

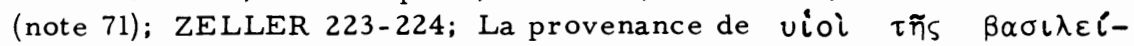
as devrait rester indéterminée, car l'attestation de 13, 38 - incontestablement rédactionnelle - désigne les chrétiens et non les Juifs.

3 Cf. HELD 185-186; voir aussi DUPONT, Beaucoup 163-164. 
duit déjà l'opposition païens/Israël qui domine l'instruction des vv. 11-12. En effet, alors que le par. Lc $(7,9)$ utilise le motif d'Israël pour souligne la grandeur de la foi de l'officier païen - sans pour autant nier celle des

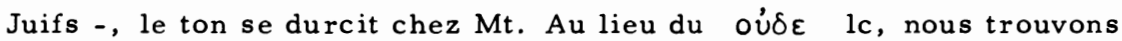

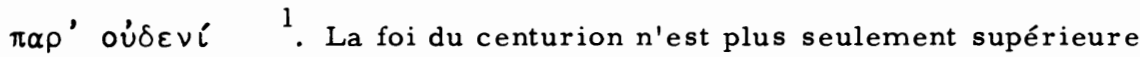
à celle des Juifs, elle n'a pas d'équivalent. Etant donné que dans les vv. 11-12, la foi est la condition d'accès nécessaire au Règne, son manque en Israël devient le signe d'une incrédulité patente ${ }^{2}$. Ainsi, la parole de Jésus sur la foi du centurion (v. 10) est saisie par le communauté mt à la fois comme une promesse faite aux païens et une condamnation d'Israël.

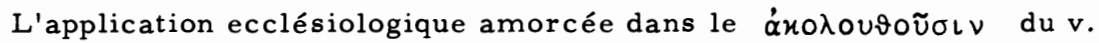
10 et fondée dans l'opposition païens/Israël, est développée dans les vv. 11-12. L'histoire du centurion acquiert une portée inattendue : la foi dont elle rend témoignage, devient la catégorie critique qui éclaire l'histoire du salut et qui conditionne l'accès à la $\beta \alpha \sigma \iota \lambda \varepsilon i ́ \alpha$ eschatologique. Comme en témoigne le parallélisme antithétique des vv. 11-12, la figure de l'officier païen devient le motif d'une promesse pour l'Eglise et d'une parole de jugement pour Israël ${ }^{3}$. Le v. ll développe l'aspect positif du message. Pour l'Eglise,l'histoire de l'officier païen n'est pas seulement

1 Voir STRECKER, Weg 100.

2 La radicalité de la condamnation suppose une situation où les fronts sont déjà durcis, où la mission chrétienne à l'intention d'Israël a essuyé un échec.

3 TRILLING, Israel 89-90 (cf. aussi WALKER 89) ne retient comme pointe du passage que le jugement d'Israël. S'il est vrai que dans les sentences construites de manière parallèle et antithétique, l'accent porte en général sur la deuxième partie (BULTMANN, Trad 207; ZELLER 87-88), il est peu probable que, dans le logion, le v. ll soit simplement le repoussoir rhétorique du v. 12. Pour une église qui mène une mission auprès des paiens, la promesse du v. 11 était inséparable du jugement du v. 12. D'ailleurs, le contexte choisi par Mt pour placer le logion empêche de faire du thème du salut des païens une figure rhétorique. 
l'occasion d'un enseignement sur la foi, mais aussi l'expression d'une promesse. L'enchaînement des vv. 10-11 suggère en effet que le centurion $n^{\prime}$ est pas un cas isolé et idéal ${ }^{l}$. Il est simultanément le premier et le modèle d'une longue lignée de croyants exemplaires que le texte désigne sobrement par $\pi 0 \lambda \lambda \circ \iota^{2}$. Ces derniers - et c'est là une nouveauté décisive - ne se recrutent plus en Israël, mais dans l'ensemble du monde habité ${ }^{3}$. Désormais, l'accès à la $\beta \alpha \sigma \iota \lambda \varepsilon \iota ́ \alpha$ eschatologique ${ }^{4}$ s'effectue sur la seule base de la foi dont le centurion est l'exemple.

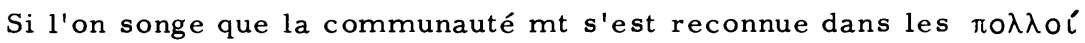
du v. $11^{5}$, l'interprétation que le rédacteur a donnée de l'histoire de l'officier pä̈en est d'une audace et d'une profondeur remarquable. Elle affirme d'abord que le salut est désormais offert à tout homme, et elle justifie ainsi la mission pagano-chrétienne. Elle soutient ensuite que l'accès au Royaume se décide sur la base de la foi dans le Christ ${ }^{6}$ - foi

1 HAENCHEN, Probleme 83-84; cf. aussi DUPONT, Beaucoup 162.

2 Le terme $\pi 0 \lambda \lambda \circ i$ rend bien compte de la perspective mt du corpus mixtum (contre ZELLER 223) : par son indétermination, il préserve la réserve eschatologique. Ce ne sont pas les païens comme tels qui sont sauvés, mais les "nombreux" qui croient, tel le centurion.

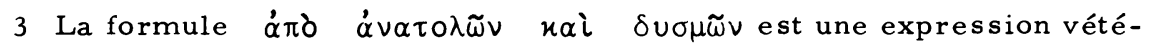
rotestamentaire qui décrit l'universalité (Es 45, 6; 49, 12; 59, 19;

M1 1,11). Cf. DUPONT, Beaucoup 159; KLOSTERMANN, Mt 75; ZELLER 84.

4 De nombreux exégètes - et, en premier lieu, JEREMIAS (Jésus et les païens 49-56) - ont vu dans le v.ll la reprise du motif vétérotestamentaire du pélerinage eschatologique des peuples vers la montagne de Dieu. ZELLER, dans une étude détaillée, a montré les limites de cette hypothèse. S'il est vrai que le v. 11 reprend un motif connu - la venue des païens vers Sion -, il en inverse totalement le sens. Il n'y va plus de la manifestation de la gloire de Dieu en faveur d'Israël, mais de la confusion du peuple élu.

5 BONNARD, Mt 116 (note 1).

6 BOR NKAMM, Enderwartung 26. 
dont le centurion est le paradigme -. Elle déclare enfin que les véritables successeurs des "pères" sont ces nouveaux croyants. La portée ecclésiologique de ces affirmations est évidente : Dieu rassemble un nouveau peuple en fonction d'un nouveau critère : la foi en Christ. Ce nouveau peuple que l'on peut à bon droit appeler le peuple de la foi - est le légitime continuateur des "pères".

Pareille analyse suppose la crise d'Israël ${ }^{l}$. Cette dernière est dénoncée en toute clarté au v. 12. Le titre qui est donné aux Juifs ( vioi $\tau \tilde{n} s$ $\beta \alpha \sigma \iota \lambda \varepsilon i \alpha s$ ) est significatif. Il reconnaît la position particulière d'Israël ${ }^{2}$ : c'est lui qui était destiné au salut. Mais la venue du Christ a démasqué l'incrédulité ( $v$. 10 !) de ceux qui étaient invités au banquet messianique. Cette incrédulité est d'une gravité extrême, car elle conduit au rejet eschatologique. L'élection selon la chair ne sert de rien si elle n'est pas vécue dans la foi $(3,9)$. Abraham, Isaac, Jacob sont les pères des croyants, mais ils n'offrent aucune assurance de salut à des descendants incrédules. Si la figure du centurion est le signe de la promesse pour l'Eglise, elle est le signe de la condamnation pour Israël.

Concluons. Pour Mt, l'histoire du centurion de Capernaüm est un paradigme qui lui permet d'interpréter l'histoire qu'il vit. L'importance eschatologique de la foi l'amène à annoncer le jugement d'Israël perdu par son incrédulité, mais aussi à discerner la naissance d'un nouveau peuple qui met toute sa confiance dans le Christ. De même que la foi est le critère qui condamne Israël, elle est la caractéristique de la communauté des $\pi 0 \lambda \lambda \circ i ́$. L'importance constitutive de la foi pour l'Eglise est à la fois le sujet d'une promesse et d'un avertissement. Promesse tout d'abord, car si le salut dépend exclusivement de la foi, alors il est offert en plénitude à chacun. Avertissement ensuite, car de même que les "pères" ne servent de rien à un Israël incrédule, l'appartenance à l'Eglise ne confère aucune sécurité. Seul le disciple dont la foi est comparable à celle du

1 Analyse détaillée chez HUMMEL 147.

2 Même note dans le Éxßá $\lambda \lambda \varepsilon \sigma \vartheta \alpha \iota$. 
centurion, fera partic de ceux que Dieu accueille ${ }^{1}$.

3. Désobéissance d'Is raël, obéissance de 1'Eglise (Mt 21,33-46)

La parabole des vignerons révoltés a fait l'objet de nombreuses recherches ces dernières années. Notre intention n'est pas de reprendre l'ensemble de cette discussion, mais d'en utiliser les éléments qui nous semblent convaincants pour alimenter notre enquête sur l'ecclésiologie mt. A titre liminaire, une double constatation s'impose. Notre texte n'a pas sa place dans la prédication de Jésus; il émane de la réflexion de la première église. Tant l'interprétation de la mort de Jésus comme le tournant décisif de l'histoire de Dieu avec Israël (vv. 41-42) que la signification messianique du titre viós (vv. 37.38) sont des thèmes postpascaux ${ }^{2}$. En second lieu, il faut admettre que, dès son apparition, notre récit a forme d'allégorie. Il se comprend en effet fort bien comme l'illustration d'un dessein théologique - l'histoire de Dieu avec son peuple -, mais soulève de nombreuses difficultés dès qu'on l'envisage comme une parabole au sens strict ${ }^{3}$. D'ailleurs quoi qu'on puisse penser de la genè-

1 Cf. HELD 186.

2 Démonstration convaincante chez KUEMMEL, Weingärtner 128-131, reprise par HAHN, Hoheitstitel 315-316 et SCHWEIZER, art. viós, ThW 8, 380-381. Même JEREMIAS (Gleichnisse 70-71) qui défend l'authenticité de la parabole admet que la signification messianique de viós n'est pas attestée dans le judaïsme pré-chrétien.

3 KUEMMEL, Weingärtner 121-124 et LOHMEYER, Weingärtner 161-162 ont bien montré les difficultés insurmontables qu'il y a à vouloir reconstruire une parabole. JEREMIAS (Gleichnisse 68-70,72-74), à la suite de DODD, conteste ce point de vue : il pense que notre parabole restitue bien l'état d'esprit des paysans galiléens, travaillés par le mouvement zélote, face aux grands propriétaires terriens résidant à l'étranger (surinterprétation du $\dot{\alpha} \pi \varepsilon \delta \eta ́ n \eta \eta \sigma \nu$ du v. 33 !). Cette hypothèse se heurte à des objections de fond et de forme. Pour le fond qui est supposé restituer une péripétie de la vie quotidienne : comment un propriétaire raisonnable peut-il continuer à envoyer des serviteurs, puis son fils alors qu'il sait ses métayers en révolte ? comment les vignerons peuvent-ils penser s'emparer de l'héritage du fils en le tuant puisque le père est toujours en vie ? Pour la forme : afin de reconstrui- 
se du texte, il demeure que Mt l'a trouvé chez Mc où il a incontestablement la forme d'une allégorie ${ }^{1}$.

La $\underline{\text { rédaction } \mathrm{mt}}$ nous semble avoir visé trois points : une plus grande transparence dans l'allégorie, un développement du motif des "fruits", une transformation de la fin de la parabole ${ }^{2}$. Une plus grande transparence d'abord. Au v. 33b, Mt profile la figure du propriétaire en intro-

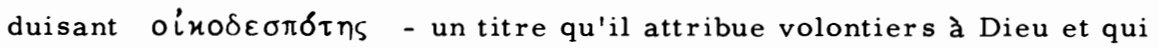
marque bien l'autorité du maître sur la vigne ${ }^{3}$-. Dans les vv. 34-36, il réorganise totalement la présentation de Mc, notamment en l'abrégeant.

On ne compte plus successivement trois envois individuels auxquels vient s'ajouter un envoi massif; on parle de l'envoi de deux groupes compacts. Par ailleurs, alors que Mc fait allusion à des sévices de plus en plus cruels, Mt envisage le même destin tragique pour tous 4 Cette image plus stylisée, mais aussi l'introduction du verbe $\lambda\llcorner\vartheta$ $\beta o \lambda \varepsilon \tau \nu$ ( $v$. 35) montre que le rédacteur pense aux prophètes vétérotesta-

re une parabole, JEREMIAS supprime dans le texte de Mc tous les traits allégoriques (Gleichnisse 71-72); cette procédure n'est possible que si l'on prouve le caractère composite du texte mc - ce qui n'est pas le cas (KUEMMEL, Weingärtner 125-126) -. Voir aussi la contribution récente de BLANK 11-42.

1 Le consensus est réuni sur ce point. Même les exégètes qui défendent l'hypothèse d'un récit purement parabolique à l'origine, admettent que la version de Mc est déjà allégorique (voir p. ex. JEREMIAS, Gleichnisse 68). Même constatation chez TRILLING, Israel 55.

2 Voir GRUNDMANN, Mt 460-61; KLOSTERMANN, Mt 172-73; SCHWEIZER, Mt 270. Tant 1'analyse de TRILLING, Israel 65, qui conteste un approfondissement de l'allégorie chez Mt, que celle de JEREMIAS, Gleichnisse 75, qui discerne dans la recension $\mathrm{mt}$ une allégorie de l'histoire du salut du Sinä̈ au jugement dernier, nous semblent gauchir l'intention $\mathrm{mt}$.

3 GRUNDMANN, Mt 462-63; TRILLING, Israel 56.

4 TRILLING, Israel 56, écrit justement : "Das Handeln der Winzer ist von Anfang an gleich ... So entsteht der Eindruck : Das ist die Regel, das ist die Art dieser Winzer". 
mentaires ${ }^{l}$. Au v. 39s, Mt inverse l'ordre narratif de Mc et crée ainsi une allusion à la Passion : c'est en dehors de Jérusalem que Jésus est mort ${ }^{2}$. La paranomase du v. 41 a est peu claire : pourtant, la parenté de notre parabole avec celle qui suit - et notamment 22,7-plaide pour une allusion à la crise de $70^{3}$.

Outre cet effort de transparence et de concision, il faut relever l'accent porté par le rédacteur sur le motif des "fruits" - motif particulièrement cher à Mt $(3,8.10 ; 7,19 ; 13,26)$-. Si Mc $(12,2)$ dit du propriétaire

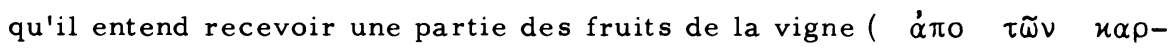
$\pi \tilde{\omega} \nu \tau o \tilde{v} \dot{\alpha} \mu \pi \varepsilon \lambda \tilde{\omega} \nu \circ \not$, Mt signale non seulement la venue du temps des fruits (v. 34a), mais il affirme surtout que le maître réclame, sans par-

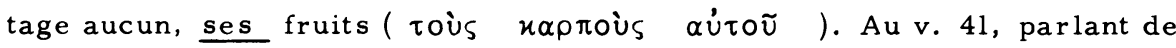
l'affermage de la vigne à d'autres métayers, Mt ajoute une glose, proba-

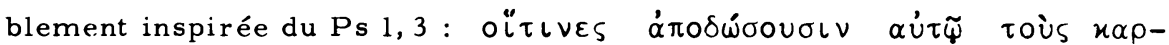

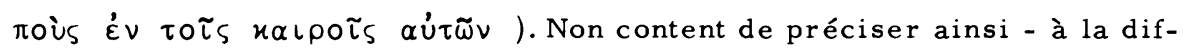
férence de Mc - le comportement des nouveaux vignerons, Mt revient sur ce thème dans le v. 43 qui lui est propre : le Royaume est confié à un peu-

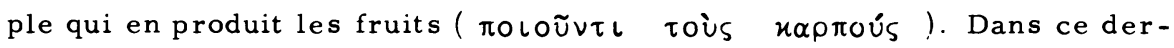
nier v., un glissement significatif s'opère dans l'image : il n'est plus question des fruits de la vigne que les métayers devraient livrer, mais des fruits du peuple ! La connotation éthique du motif, que l'on devinait tout au long du texte, trouve incontestablement son expression ${ }^{4}$.

1 Les commentateurs dans leur ensemble s'accordent à voir dans les

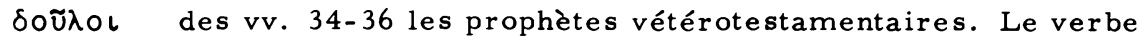
$\lambda \iota \vartheta \circ \beta \circ \lambda \varepsilon \tau \nu$ décrit le destin prophétique (cf. le martyre de Zacharie). Pourtant, JEREMIAS, Gleichnisse 70, KLOSTERMANN, Mt 173, SCHMID, Mt 305, dépassent le contenu du texte en interprétant le double envoi comme un découpage de l'histoire d'Israël. Analyse détaillée de ce point particulier chez WEISER 55-56.

2 JEREMIAS, Gleichnisse 71; TRILLING, Israel 57.

3 HUMMEL 83; JEREMIAS, Gleichnisse 75; STRECKER, Weg 112.

4 TRILLING, Israel 60. 
En troisième lieu, enfin, Mt remanie substantiellement la fin de la parabole. D'une part, ce n'est pas le Christ qui annonce la condamnation des vignerons révoltés, mais les Juifs ${ }^{l}$ : ces derniers annoncent euxmêmes le châtiment qu'ils vont subir. D'autre part - et c'est là le point crucial de l'ensemble - Mt n'achève pas son récit, comme le fait Mc, par la citation du Ps 118,22-23. Il met en quelque sorte cette exaltation christologique entre parcnthèses, et il préfère reprendre dans un v. qui lui est propre (v. 43) le thème annoncé dans le v. 4l. Le châtiment d'Israël et la naissance d'un nouveau peuple sont, en effet, à ses yeux la leçon décisive du texte ${ }^{2}$.

Peut-on alors dégager brièvement la signification de la version $\mathrm{mt}$ de la parabole? Même si la citation d'Es 5,1-2 qui amorce le récit (v. 33) se combine assez mal avec l'allégorie qui suit ${ }^{3}$, elle en donne d'emblée la clef. Le lecteur familier de l'Ecriture sait en effet que la "vigne de Yahvé des armées, c'est la maison d'Israël" (Es 5,7). Il comprend donc que c'est de l'histoire de Yahvé avec son peuple qu'il va s'agir. Aussi n'a-t-il pas grande peine à décrypter les métaphores de l'allégorie proprement dite. Dans le propriétaire de la vigne, il reconnaît Dieu; dans les vignerons : Israël symbolisé par ses chefs; dans les envoyés : les prophètes; dans le fils assassiné : Jésus crucifié; dans la punition : le

1 Conformément à son style, Mt introduit une séquence dialoguée. Le

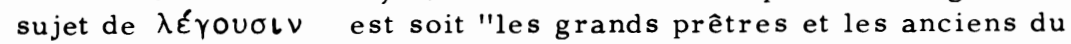
peuple" (v. 23), soit "les grands prêtres et les pharisiens" (v. 45). Dans les deux cas, il s'agit d'une description typiquement mt des adversaires de Jésus (voir notre analyse du chap. 2).

2 Avec TRILLING, Israel 57-58.65.

3 L'agencement de la vigne avec ses divers éléments (clôture, pressoir, tour) ne joue aucun rôle dans la parabole. Le motif de la vigne passe à l'arrière-plan. L'attention se fixe sur le comportement des vignerons et singulièrement sur leur refus de livrer les fruits. Cf. BONNARD, Mt 317; KUEMMEL, Weingärtner 121, 128; TRILLING, Israel 63-64, tout en reconnaissant les tensions existant entre la citation et la parabole, se montre plus réticent. 
rejet d'Israël; dans l'autre peuple : l'Eglise ${ }^{1}$. La nature des relations qui se nouent entre les partenaires est illustrée par le motif des fruits. L'alliance conclue entre Yahvé et son peuple n'est pas respectée. Israël se fermeà l'initiative de Yahvé, il n'honore pas les termes du contrat. Malgré les appels incessants de son Dieu, il se fige dans une désobéissance coupable. Le destin des envoyés permet de mesurer l'étendue de sa révolte ${ }^{2}$. Aussi l'assassinat du Fils à Golgotha n'est-il pas une tragique méprise, mais l'aboutissement fatal d'un endurcissement constant.

Le Christ mt met ses auditeurs en demeure de tirer la lecon du récit. Face à cette histoire qui se voulait salutaire et qui devient perdition, que doit faire le maître de la vigne ? La réponse s'impose d'elle-mème : le châtiment est inéluctable. Il s'exprime de deux manières. Par l'anéantissement d'abord : bien que le texte reste peu clair, le naxoùs nañ̄s

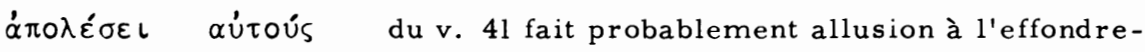
ment de l'année 70 (cf. 22,6). La colère divine commence à se manifester dans l'histoire. Par la dépossession ensuite : Dieu se détourne de son peuple infidèle pour se trouver d'autres vignerons plus fidèles. Si, comme Mc, Mt était exclusivement attaché à dévoiler la culpabilité d'Israël, il conclurait son propos par la citation du Ps 118 qui célèbre la victoire paradoxale du Fils. Sa visée est autre. Déjà au v. 41, il développe le thème du choix de nouveaux vignerons en montrant à quelles conditions ils sont engagés. Mais surtout la citation du v. 42 est suivie d'une importante adjonction (v. 43) ${ }^{3}$ où se trouve énoncé en clair le changement

1 Voir à ce sujet, JEREMIAS, Gleichnisse 68, 74-75.

2 Il est important de noter que, pour Mt, rejet des envoyés et refus de livrer les fruits dévoilent une seule et même réalité : la désobéissance coupable d'Isrä̈l.

3 L'accord règne pour reconnaître que c'est Mt qui a introduit le v, 43 dans la péricope : l'absence du v. chez $\mathrm{Mc}$ et la tension avec le contexte (apparition du thème de la $\beta \alpha \sigma \iota \lambda \varepsilon \mathcal{l} \alpha$ et de $1^{\prime} \varepsilon \varepsilon^{\prime} \vartheta \circ \varsigma_{\text {) }}$ ) en sont la meilleure preuve. S'agit-il d'un logion que Mt a reçu (JEREMIAS, Gleichnisse 75) ou d'une composition rédactionnelle (HUMMEL 148; KILPATRICK 30; TRILLING, Israel 58-60) ? La terminologie utilisée 
qui mène d'Isrä̈l à l'Eglise. C'est dire que le rédacteur reprend toute l'allégorie décrivant la crise d'Israël pour en tirer la leçon ecclésiologique ${ }^{1}$.

Quelle est alors cette leçon ? Elle tient dans deux grandes affirmations déjà présentes au v. 4l. D'une part, l'histoire de Dieu avec les hommes ne s'achève pas sur l'échec irrémédiable d'Israël. Dieu afferme sa vigne à d'autre métayers (v. 4lb); il rassemble un nouveau peuple à son service. Ce peuple est indubitablement l'Eglise. Mais, d'autre part, ce rassemblement s'effectue selon un critère précis : sont engagés des vignerons "qui remettront les fruits en leurs temps" (v. 4lc). Le nouveau peuple dont parle le texte se caractérise par sa fidélité éthique ou, comme on voudra, par son obéissance à la volonté de Dieu.

Le v. 43 qui tire la leçon de la parabole dans son ensemble ${ }^{2}$, reprend cette double thématique. Il le fait en quittant le terrain de l'allégorie ${ }^{3}$ :

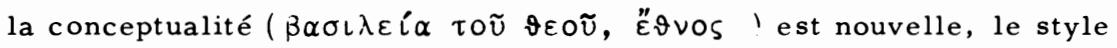
direct ( $\dot{u} \mu \nu)$. C'est le prophète qui parle et qui signifie aux Juifs, re-

ne permet pas de trancher (STRECKER, Weg 169, note 4), car si le

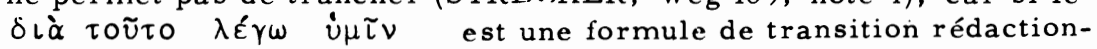
nelle (cf. 12,31), les autres éléments sont tous traditionnels. Néanmoins il faut bien admettre que l'interprétation de la parabole à partir de la notion de $\beta \alpha \sigma \iota \lambda \varepsilon i ́ \alpha$ et la reprise du motif des fruits (accentué par Mt tout au long de la parabole) plaident pour l'hypothèse rédactionnelle.

1 Contre JEREMIAS (Gleichnisse 75), nous ne pensons pas que le but de Mt soit de tracer une image de l'histoire du salut allant du Sinä̈ au jugement dernier. Tout aussi problématique est l'interprétation de WALKER, 82-83 qui conteste la portée ecclésiologique du v. 43 pour

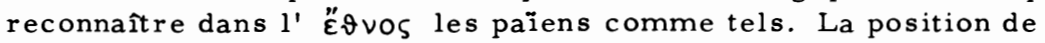
TRILLING, Israel 55-65, est fluctuante : si, d'une part, cet auteur reconnaît dans le v. 43 la pointe $\mathrm{mt}$ du récit et sa portée éminemment ecclésiologique, il voit l'ensemble du texte comme la démonstration de la culpabilité d'Israël.

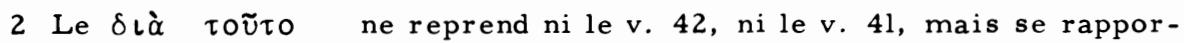
te à la parabole dans son ensemble (TRILLING, Israel 60).

3 TRILLING, Israel 57. 
présentés dans la personne de leurs chefs, le jugement eschatologique. La difficulté d'interprétation soulevée par le v. 43 consiste à décider si l'événement décrit s'est déjà réalisé dans l'histoire ${ }^{1}$, ou s'il s'agit d'une allusion au jugement dernier ${ }^{2}$. Nous penchons pour cette dernière hypothèse pour les arguments que voici. Le couple antithétique $\alpha \rho-$

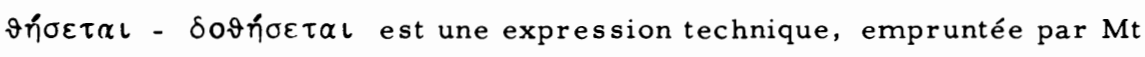
à la tradition synoptique $(13,12 ; 25,29)$ et qui décrit le destin de l'homme lors du jugement ${ }^{3}$; le futur passif a donc un sens eschatologique. Baol-

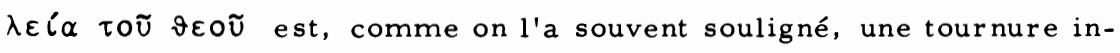
habituelle chez Mt. Notre passage mis à part, elle n'est attestée avec certitude qu'en 12,28 et 21,31 . Si la lecon de 12,28 dépend de $Q$ (cf. Lc $11,20)$ et fait allusion au Royaume qui atteint les hommes en Jésus, 21, 31 provient du Smt et désigne clairement le salut eschatologique. Cette dernière attestation doit retenir notre attention car elle appartient au groupe des trois paraboles qui décrivent la crise d'Israël $(21,28-22,14)$ groupe dont notre texte fait précisément partie -. La similitude thématique entre 21,31 et 21,43 est d'ailleurs frappante. La parabole des deux fils oppose, en effet, polémiçuement les Juifs aux collecteurs d'impôts et aux prostituées. Ces derniers, image des paiens, se caractérisent par leur foi alors qu'Israël se signale par son incrédulité ${ }^{4}$. Le thème de la

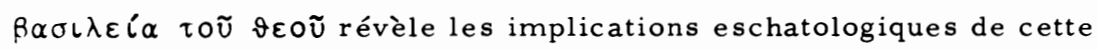
situation : le salut sera accordé à ceux qui en étaient primitivement le plus éloignés tandis que les fils du Royaume (cf. 8,12 ) en seront exclus.

1 SCHMID, Mt 306; STRECKER, Weg 169; TRILLING, Israel 62. Cf. aussi JEREMLAS, Gleichnisse 75.

2 BARTH 56; BORNKAMM, Enderwartung 40; HUMMEL 148-149:

3 BILL 1, 660-662, atteste la provenance juive de la tournure. JEREMIAS, art. $\alpha i \rho \omega$, ThW 1, 185, et STRECKER, Weg 106, montrent sa réinterprétation chrétienne.

4 Voir HUMMEL 23-24 et notre chap. 2. 
Cette parenté thématique et argumentative nous permet de supposer qu'en

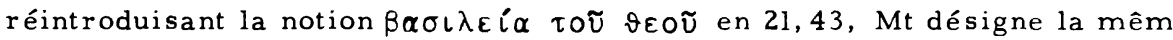

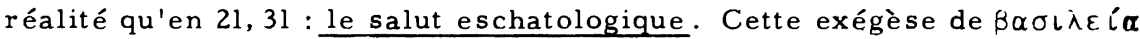

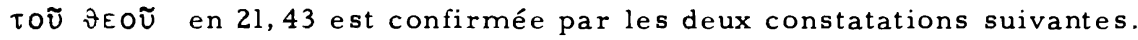
D'une part, le v. 43 - comme c'est souvent le cas chez Mt - lie le don de la $\beta \alpha \sigma \iota \lambda \varepsilon i \alpha$ à l'accomplissement d'une exigence éthique ( $\pi 0 \iota \varepsilon \tau \nu$ toùs naproús); or ce modèle théologique, attesté notamment dans les sentences décrivant les conditions d'entrée dans le Royaume, utilise $\beta \alpha-$

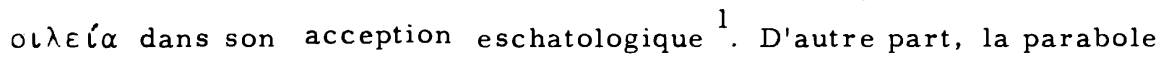
du festin nuptial qui suit la nôtre et s'y apparente de manière structurelle (voir plus haut), est devenue sous la plume de Mt une parabole du Royaume. Or, précisément sur ce point, elle enseigne (vv. 11-12) que les "fruits" des croyants et, partant l'appartenance de ceux-ci à la $\beta \alpha o l-$ $\lambda \varepsilon i \alpha$, ne seront dévoilés que lors du jugement ${ }^{2}$. Remarquons qu'en discer. nant dans la $\beta \alpha \sigma \iota \lambda \varepsilon i ́ \alpha$ donnée ou perdue le destin eschatologique de l'homme, nous n'éliminons en aucune manière la réalité de la $\beta \alpha \sigma \iota \lambda \varepsilon i-$ $\alpha$ sub specie historiae, mais nous lui rendons sa véritable place. Si le v. 43a annonce le jugement d'IBraël sous la forme de la perte de la f $\alpha \sigma \iota \lambda \varepsilon i ́ \alpha$, il présuppose qu'Israēl a eu un lien particulier avec le Royaume. Mais lequel ${ }^{3}$ ? Nous pensons que l'argumentation est analogue

1 Cf. 4,$17 ; 5,19.20 ; 6,33 ; 7,21 ; 8,10-12 ; 13,38.43 ; 18,1-14.23 ; 19,12.14$. $23 ; 21,31.43 ; 22,2-14 ; 23,13 ; 25,13.31-46$ (inventaire de WEISER 99100). Pour l'ensemble de la question, voir WINDISCH, Sprüche vom Eingehen 163-192.

2 Au cas où le v. 44 appartiendrait au texte primitif, il représenterait un argument supplémentaire pour notre exégèse : il annoncerait de manière péremptoire le jugement eschatologique de tout homme qui s'oppose au Fils et développerait la perspective ouverte au v. 43. L'excellente attestation manuscrite plaide pour l'authenticité (JEREMIAS, Gleichnisse 75; STRECKER, Weg 110-111). Par contre, la place occupée par le v. est maladroite et fait penser à une interpolation de

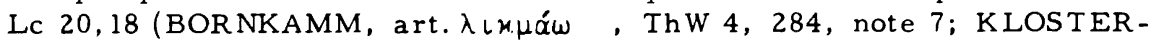
MANN, Mt 173; TRILLING, Israel 57).

3 Inventaire des possibilités d'interprétation chez WALKER 79-80. 
à celle que nous avons trouvée en 8,11-12. Dans l'histoire de Dieu avec les hommes, Israël occupait une place particulière. Choisi de manière inexplicable par Dieu, il était le porteur de son appel et de ses promesses; il était, le premier, destiné à la $\beta \alpha \sigma \iota \lambda_{\ell} \iota \alpha$. Par son endurcissement constant - dont notre parabole est l'illustration -, il a réduit à néant son élection, il a perdu sa vocation au salut.

Mais la désobéissance d'Israël ne met pas un terme à l'action salutaire de Dieu. La $\beta \alpha \sigma \iota \lambda \varepsilon i \alpha$ en tant qu'appel et promesse est le facteur de continuité de l'histoire du salut l. L'accès à la $\beta \alpha \sigma \iota \lambda \varepsilon i ́ \alpha$ est désormais ouvert à tout homme qui en accepte les conditions. Un nouveau peuple se constitue, formé qu'il est de ceux qui reçoivent la $\beta \alpha \sigma \iota \lambda \varepsilon i ́ \alpha$ à la fois en tant que promesse et exigence. Le terme choisi par Mt pour

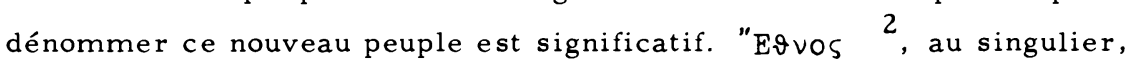
a un sens neutre, indéterminé; il ne comporte pas de nuance restrictive et ne privilégie pas une collectivité historique aux dépens d'une autre. Il ne faut donc pas lire dans ce concept l'appel des päiens par opposition aux Juifs ${ }^{3}$. L'expression a, au contraire, un accent universaliste : tout homme, quelle que soit son origine, peut faire partie de ce nouveau groupe. La spécificité de ce peuple qui se constitue ne réside donc pas dans une quelquonque généalogie historique, mais dans son lien, à la $\beta \alpha \sigma \iota \lambda \varepsilon i \alpha$ prêchée par le Christ mt. En ce sens, nous sommes fondé

\section{WALKER 80.}

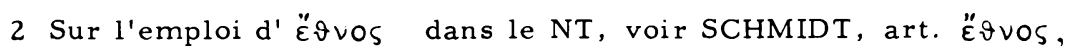
ThW 2, 366-69.

3 L'expression technique pour désigner les pä̈ens par opposition à

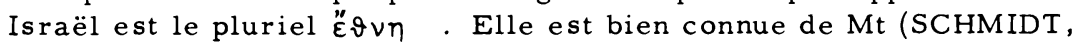
art. cit. 367). Optent néanmoins pour un exégèse soulignant l'appel des paiens : JEREMLAS, Gleichnisse, 75; KILPATRICK il8; WALKER 82. 


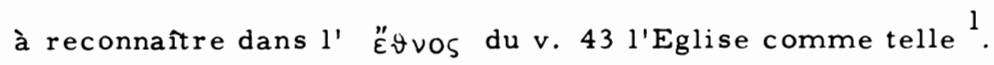

La sobriété et l'originalité de Mt dans le choix du terme ecclésiologique du v. 43, méritent d'être relevées. Ce terme est l'indice d'une rupture frappante avec la terminologie dont se servait Israël pour parler de son élection (p. ex. $\lambda \alpha \delta \varsigma)^{2}$. Cette rupture n'est pas fortuite. Elle montre qu'on ne saurait parler de l'Eglise comme on parlait d'Israël. Pour Mt, l'Eglise n'est pas la simple continuatrice d'Israël déchu dont elle prendrait la place et les privilèges. Dans la perspective $m t$, la caractéristique de l'Eglise ne consiste pas dans la reprise d'un héritage : elle n'est ni un nouvel Israël, ni le véritable Israël ${ }^{3}$. Elle reçoit une vocation propre qui la détermine complètement. Cette voca-

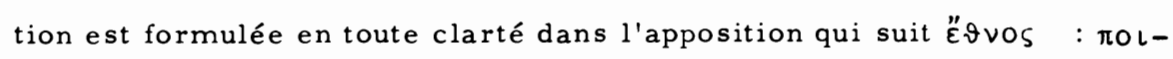

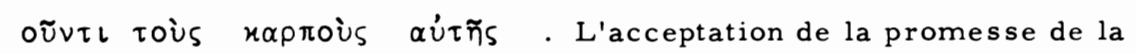
$\beta \alpha \sigma \iota \lambda \varepsilon i \alpha$ implique une fidélité de vie. Cette fidélité consiste dans l'obéissance à la volonté de Dieu, obéissance qui ne saurait être vécue autrement que dans la Nachfolge du Christ. L'Eglise n'est le peuple du Royaume que dans la mesure où elle reste fidele à sa vocation. Le destin d'Israël est un contre-modèle qui a valeur de mise en garde : l'élection ne confere aucune sécurité si elle n'est assumée dans une obéissance sans cesse renouvelée. Le jugement qui vient et qui seul fera connấtre ceux qui ont répondu en vérité à l'appel de la $\beta \alpha \sigma \iota \lambda \varepsilon i ́ \alpha$, donne au

1 BONNARD, Mt 317, écrit à juste titre: "La nation fidèle ( n'est·pas une nation paienne, ni les paīens en général opposés aux Juifs. Ceux qui porteront les fruits du Règne ... seront tous ceux qui, en recevant le Fils, formeront le nouveau peuple de Dieu". Voir aussi KLOSTERMANN, Mt 173; SCHMID, Mt 306; SCHNIEWIND, Mt 217; SCHWEIZER, Mt 270; TRILLING, Israel 61.

2 HUMMEL 154; SCHWEIZER, Gemeinde 34; TRILLING, Israel 61.

3 Contre l'interprétation de TRILLING, Israel 65 : "So ist mit der Kontinuität des Reiches auch die Kontinuität eines Volkes Gottes gegeben ". Cette discontinuité entre Israël et l'Eglise n'empêche pas cette dernière de voir dans l'histoire d'Israël un passé qui est le sien et à travers lequel elle se comprend (HUMMEL 150). 
temps vécu par l'Eglise - et par chaque disciple - son sérieux décisif. Produire des fruits, c'est choisir toujours à nouveau la vie; n'en point porter, c'est succomber à la mort.

Concluons. Comme dans l'histoire du centurion de Capernaüm, la réflexion ecclésiologique de Mt se développe sur l'arrière-fond de la crise d'Israël et de la promesse de la $\beta \alpha \sigma \iota \lambda \varepsilon i \alpha$. Mais alors qu'au chap. 8, la foi était la catégorie qui oriente la lecture de l'histoire du salut, et qui caractérise la communauté des disciples, la parabole des vignerons révoltés met l'accent sur la fidélité éthique. La promesse de la $\beta \alpha \sigma \iota \lambda \varepsilon \hat{l} \alpha$ ne peut être acceptée et vécue que dans un engagement conséquent. S'il fait défaut, elle n'est tout simplement pas reçue. Le nouveau peuple que Dieu rassemble et qui ne sera connu en vérité qu'au jugement, est donc formé de ceux qui portent du fruit, càd de ceux qui placent toute leur existence sous l'autorité de la $\beta \alpha \sigma \iota \lambda \varepsilon i ́ \alpha$ qui vient.

\section{L'Eglise, corpus mixtum}

Ce grand rassemblement qu'est l'Eglise selon Mt, doit se caractériser par la foi et l'obéissance. Notre chap. 7, consacré aux divisions de la communauté $\mathrm{mt}$, nous a fait mesurer l'écart qui existe entre la vocation de l'Eglise et sa réalité historique. $L^{\prime} \dot{\alpha} \nu o \mu \iota_{\alpha} \alpha, l^{\prime}$ égarement ( $\left.\pi \lambda \alpha \nu \widetilde{\alpha} \nu\right)$, le scandale, les faux prophètes ne sont pas l'apanage du monde resté étranger à l'évangile ou du judaĩsme endurci, mais des manifestations qui se développent parmi les croyants. La parénèse du Sermon sur la Montagne (7, 15-23), mais aussi l'apocalypse mt (cf. en particulier 24,914) montrent sans équivoque que, dans la communauté, loups et brebis (cf. 7-15 !) sont mêlés. Le mal n'épargne pas 1'Eglise; non content de dominer le monde, il s'insinue en elle. C'est dire que, pour utiliser une expression remise en honneur par Bornkamm ${ }^{1}$, $1^{\prime}$ Eglise est, aux yeux du premier évangéliste, un corpus mixtum.

1 BORNKAMM, Enderwartung 17. 
Mt n'a pas fait que prendre acte de l'ambiguité fondamentale qui caractérise la réalité ecclésiale. Par le biais de la recension de la tradition synoptique, il lui a consacré une importante réflexion. Ainsi, dans le chap. 13, la parabole de l'ivraie $(13,24-30)$ et son explication $(13,36-43)$, ainsi que la parabole du filet $(13,47-50)$ s'interrogent sur l'énigmatique coexistence du bien et du mal et révèlent par là-même le statut de l'Eglise. Non seulement le monde, mais aussi l'Eglise rassemble indifféremment bons et méchants : l'ivraie se mélange mystérieusement au bon grain et le filet ramène toutes sortes de poissons. L'apport spécifique de Mt est d'avoir mis en relation cette description de l'Eglise avec la thématique du Jugement ${ }^{1}$ (cf. 13,30b. 40-43. 49-50). L'Eglise est, à l'exemple de chaque homme, en route vers le Jugement où parmi les nombreux appelés, Dieu discernera le petit nombre des élus. C'est dire et ici nous retrouvons la parénèse caractéristique de $\mathrm{Mt}$ - que le temps vécu par chaque croyant est le temps de la décision - décision sans cesse renouvelée pour une foi plus fidèle et une obéissance plus authentique - .

Ce bref rappel d'un axe capital de l'ecclésiologie mt doit être vérifié et approfondi. Nous pensons que la parabole de l'invitation au festin nuptial que nous avons déjà analysée sous l'angle de l'appel ${ }^{2}$ s'y prête particulièrement bien. Nous reprenons la deuxième partie du récit (22, 8-14). Les missionnaires chrétiens sont chargés de proclamer la bonne nouvelle du Royaume dans l'ensemble du monde habité et de convier tous les hommes qu'ils rencontrent. Cette universalité de l'appel provoque un

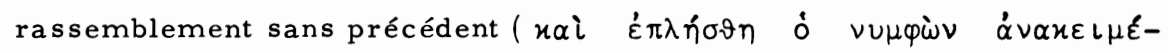
$v(\omega v)$. Le statut de la communauté réunie ne manque pourtant pas d'être problématique, La première partie de la parabole $(22,2-7)$ nous avait en effet montré qu'appel et élection ne vont pas de pair pour Mt : Israël, le premier invité, s'est rendu indigne de l'appel qui lui avait été adressé et son refus a été châtié par le rejet eschatologique dont la catastrophe de 70 est le signe évident.

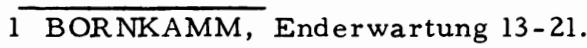

2 Voir nos pp. 353-362. 
Or, de même qu'elle avait été posée à Israël, la question de la dignité est posée à l'Eglise. Et la clausule rédactionnelle de la fin du v. 10

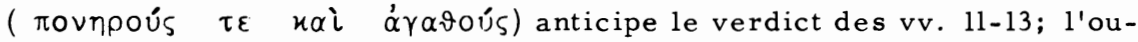
verture illimitée de l'invitation au Royaume a un envers : la réalité ambiguë qu'est l'Eglise. La communauté chrétienne comporte en effet aussi bien des méchants que des bons, càd aussi bien des hommes qui ont répondu de manière conséquente à l'invitation reçue que d'autres qui ont méconnu le sérieux de la parole entendue ${ }^{1}$. L'existence du corpus mixtum est l'envers dramatique - mais inévitable - de la mission universelle.

Les vv. 11-13 confirment notre interprétation des vv. 8-10 et mettent de manière caractéristique en relation Eglise et Jugement. Personne, en effet, ne nie que le fragment parabolique des vv. 1l-13a figure le jugement eschatologique ${ }^{2}$ et personne ne conteste que le justiciable du v. 11 appartienne au cercle des seconds invités, càd à l'Eglise. La question difficile

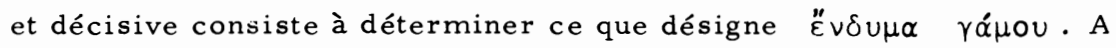
notre avis, la question doit être tranchée à partir du contexte rédactionnel : en ne revêtant pas l'habit de noce, le personnage des vv. 11-13 se rend indigne de l'invitation qu'il avait reçue - comme Israël dans les vv. 2-8 -. Si tel est le cas, $l^{\prime} \varepsilon ̋ \nu \delta u \mu \alpha$ consiste dans les oeuvres requises par le Royaume, et l'absence de vêtement dénote la désobéissance du prévenu. Les oeuvres exigées ne doivent pas être comprises dans un sens

1 Le couple antithétique "bon-méchant", caractéristique du style mt (TRILLING, Ueberlieferungsgeschichte 259, note 21) est à comprendre de manière éthique. Cela ressort très clairement de 13,33-35; cf. aussi 5,45 .

2 La venue en personne du roi, son examen ( $\vartheta \varepsilon \alpha ́ \sigma \alpha \circ \vartheta \alpha \iota)$ des invités sont des éléments caractéristiques du Jugement. La terminologie du v. $13 \mathrm{~b}$ est encore plus claire : elle désigne univoquement le séjour des damnés (voir BARTH 54 et BILL 1, 478). Aussi l'hypothèse de HASLER 30 qui discerne dans le v. 13 une allusion à la discipline ecclésiastique est indéfendable. 
légaliste ou casuistique; elles désignent, comme si souvent chez Mt, "la justice", càd l'adéquation entre la foi et la vie ${ }^{l}$. Si cette adéquation n'est pas réalisée, alors le croyant n'a rien à espérer du juge eschatolologique; il encourt une condamnation sans appel (v. 13).

Mt commente cette scène de jugement par l'adjonction du v. 14. De manière significative, ce logion disjoint appel et élection ${ }^{2}$. Il oppose la masse de ceux qui se réclament apparemment de l'évangile au petit nombre qui le confesse en vérité. Cette disjonction entre appel et élection fait apparaître de manière incisive, la réalité de l'Eglise comme corpus mixtum. Elle signifie notamment que si l'indignité d'Israël est consommée, la dignité de l'Eglise n'est pas pour autant établie. Elle reste une question ouverte et qui sera tranchée lors de l'eschaton; seule prévaudra alors la foi vécue dans l'obéissance. L'avertissement est cinglant : les seconds invités auraient tort de s'enfermer dans de fallacieuses sécurités, prenant prétexte du rejet des premiers pour affirmer leurs

1 Résumé de la discussion sur l'Ë $\vee \delta \cup \mu \alpha$ chez HAHN, Gleichnis 80-81 (note 133). Il ne faut pas penser à un vêtement spécial, mais à un vêtement propre (JEREMIAS, Gleichnisse 186). Dans le langage apocalyptique, le vêtement est le symbole d'une vie digne de participer à l'accomplissement eschatologique (cf. p. ex. Ap. 3,4.5.18; 19,18; matériel rassemblé par JEREMIAS, Gleichnisse 187-188). Même si ce motif apocalyptique est à l'arrière-fond des vv. 1l-13, il ne faut voir dans cet habit ni la justice reçue de Dieu (JEREMIAS, Gleichnisse 188) - le roi de la parabole ne donne rien -, ni l'allégresse eschatologique (SCHNIEWIND, Mt 220). En fait, la problématique est celle de la dignité, posée au v. 8, poursuivie au v. 10 par le couple "bon-mauvais"; l'habit est alors la justice au sens mt (avec BONNARD, Mt 320; GRUNDMANN, Mt 470; HAHN, Gleichnis 80-81; LINNEMANN 102; TRILLING, Ueberlieferungsgeschichte 258-260).

2 Il faut se garder d'aborder ce logion à partir de la théologie paulinienne. Mt ne soulève ni le problème de la prédestination, ni celui de la grâce. Il établit de manière parénétique la corrélation nécessaire entre l'appel salvifique de Dieu et la réponse obéissante de l'homme. 
privilèges. Dieu se tournera contre les croyants infidèles comme il s'est tourné contre Israël. L'appartenance à l'Eglise ne garantit en aucune manière le salut ${ }^{1}$ : seul le présent vécu dans la foi et l'obéissance reçoit l'approbation divine. La mise en relation de l'Eglise comme corpus mixtum et de la venue du Jugement débouche ici encore sur la parénèse si chère à $\mathrm{Mt}$.

1 Conception diamétralement opposée chez TRILLING qui se refuse à intégrer la thématique du corpus mixtum dans sa description tinale de l'ecclésiologie mt. Il écrit (Israel 162-63): "Damit ist ... für die Kirche die Gegenwärtigkeit der Heilswirklichkeit besonders akzentuiert, und zwar nicht nur in der Weise der Proklamation der eschatologischen Botschaft und der Forderung Gottes und des Messias, sondern als wirksame, selbst vor Gott gültige, aber durch Menschen vollzogene $\mathrm{Zu}$ - oder Aberkennung der Gliedschaft in der Heilsgemeinde und damit der Teilnahme oder Nichtteilnahme an dem von Gott geschaffenen Heil überhaupt." 


\section{CHAPITRE QUINZE : L'AMOUR DU FRERE (Mt 18)}

Le chapitre précédent nous a fait découvrir comment Mt aborde le problème de la constitution de l'Eglise. Il y discerne le lieu d'un vaste rassemblement dont personne n'est exclu. La foi et l'obéissance à la parole du Maître sont en effet les seules caractéristiques de ce nouveau peuple en route vers le Royaume. Une telle ouverture est le chiffre même de la grâce, mais elle a son envers : l'Eglise rassemble indifféremment justes et injutes, croyants et incroyants. Elle est la communauté des appelés et non celle des élus. Elle est fondamentalement un "corpus mixtum".

Comme le montre le chap. 13, mais aussi le début du chap. 22, cette situation de corpus mixtum n'est pas une dégradation à laquelle l'Eglise devrait ou pourrait se soustraire. C'est là sa condition historique nécessaire, car le Jugement appartient à Dieu et à lui seul. Dès lors la question qui se pose est la suivante : comment, dans cette situation de tension, l'Eglise va-t-elle rester fidèle à l'enseignement du Maître ? comment va-t-elle vivre et assumer ses conflits dans le quotidien ? comment va-t-elle tolérer le mal qui l'habite sans se renier? Nous pensons que la réponse de Mt à ce problème se trouve dans son chap. 18.

1. La discipline fraternelle $(18,15-20)^{1}$

Cette péricope occupe une place particulière dans le chap. 18 car elle

1 Pour traiter ce chap., nous adoptons le plan proposé par TRILLING (Israel 106-107) qui nous semble refléter fidèlement à la fois le contenu du discours et la démarche de Mt. Ce plan comporte 4 parties : a) la véritable grandeur dans le Royaume des cieux (vv. 1-5); b) la sollicitude envers les petits (vv. 6-14); c) la discipline fraternelle (vv. 15-20); d) le pardon (vv. 21-35). Le plan proposé par PESCH (Gemeindeordnung 232) qui divise le texte en 2 parties (vv. 1-14. 15-35) nous semble moins heureux. Enfin si nous ne prenons pas les péricopes dans l'ordre du texte, c'est pour mieux restituer le mouvement de la réflexion $\mathrm{mt}$. 
seule, à première vuc, mérite d'être appcléc "règle communautaire" (cf. en particulier vv. 15-17) ${ }^{1}$. Le reste du discours contient plutôt des recommandations éthiques : appel à l'humilité (vv. 1-5), à la sollicitude envers les petits (vv. 6-14), au pardon (vv. 21-35) ${ }^{2}$. Cette procédure disciplinaire, destinée à protéger la communauté contre ses membres infidèles, attire d'emblée notre attention sur un point capital. Seule une église qui a la profonde conscience d'être un corpus mixtum, peut avoir conçu pareil instrument; seule une communauté qui se sait habitée par le mal, menacée par l'apostasie, tentée par l'incrédulité, peut envisager de s'amputer pour se préserver. Mais de quelle église s'agit-il ? comment cette règle s'est-elle constituée ?

Les vv. 15-20, dans leur ensemble, sont propres à Mt. Nous analysons tout d'abord les vv. 15-18. Nous constatons que, dans ces vv. qui constituent la règle proprement dite, les vv. 15-17 forment une unité ${ }^{3}$. Le premier membre de cet ensemble (v. 15) a un parallèle en Lc 17,3 . Il est donc légitime d'admettre que le noyau primitif de notre règle se trouve dans la source $Q^{5}$, tout en remarquant que le v. 15 est déjà formulé en fonction des vv. 16-17. Nous ne pensons pas que l'élargissement du logion $\mathrm{Q}$ est imputable au rédacteur $\mathrm{mt}$; il émane plutôt du cercle par lequel la

1 BORNKAMM, Lösegewalt 37.

2 STRECKER, Weg 223.

3 Même si, pris en lui-même, le v. 15 peut constituer une instruction autonome (cf. Lc 17,3), il est formulé en fonction de la procédure d'ensemble qui comprend trois étapes. Il y a correspondance entre les

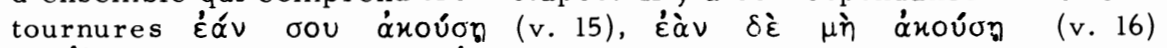

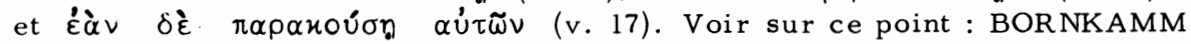
Lösegewalt 39 et TRILLING, Is rael 114.

4 Le logion de Lc 17, 3 contient deux thématiques : celle de la réprimande fraternelle et celle du pardon. Chez Mt, les thèmes sont scindés : 18,15-18 se concentre sur le seul aspect disciplinaire tandis que la discussion sur le pardon commence au v. 21.

5 Cf. l'analyse détaillée de SCHULZ, Q 320-322. 
source $Q$ est parvenue à $M t^{l}$. Trois arguments militent en faveur de cette hypothèse. En premier lieu, la thématique de la règle envisageant l'éventuelle exclusion du pécheur va à l'encontre de celle du contexte rédactionnel défendant la sollicitude (vv. 6-14) et le pardon (vv. 21-35) à l'égard du frère égaré ${ }^{2}$. En deuxième lieu, la citation de Dt 19,15 en 16b, probablement rédactionnelle, s'accorde mal avec le rôle primitif du frère dans le processus disciplinaire ${ }^{3}$. Enfin, la formulation de l'excommunication au v. 17b n'est pas mt, car pour le rédacteur, le païen et le pécheur font partie de l'Eglise ${ }^{4}$.

Si donc Mt a trouvé la règle des vv. 15-17 dans le recueil $Q$ qu'il avait à disposition (=Qmt), qu'en est-il du v. 18 ? A l'origine, ce v. est un logion indépendant qui peut être considéré comme une variante secondaire de $16,19^{5}$. Cette variante se distingue sur deux points de 16,19: le pouvoir de lier et de délier n'est plus confié à Pierre, mais à l'église locale; il ne s'agit plus d'autorité doctrinale, mais de pouvoir disciplinaire (vv. 15-17). Avant d'entrer plus avant dans l'analyse de ce logion, il convient de se demander si ce v. 18 faisait déjà partie de la règle reçue par Mt ou si il y va d'une adjonction rédactionnelle. Nous préférons la première hypothèse pour les raisons suivantes. En premier lieu, com-

1 Défendent ce point de vue : BARTH 78; BOR NKAMM, Lösegewalt 38; BULTMANN, Trad 151; LUEHRMANN, Logienquelle 112-113; PESCH, Seelsorger 38; STRECKER, Weg 203; TRILLING, Israel 114 (113 : "ein Stück alter Kirchendisziplin").

2 Nous parlons de contexte rédactionnel, car, dans les vv. 6-35, c'est Mt qui a déterminé l'ordre des péricopes (voir plus bas).

3 Selon le contexte de la règle, la présence du frère vise à rendre plus pressante l'exhortation alors que la citation de Dt 19,5 transforme le frère en témoin juridique (avec BARTH 78; BULTMANN, Trad 151; KLOSTERMANN, Mt 150-151; STRECKER, Weg 223). Une telle tension atteste que la règle est antérieure à la citation.

4 Cf. LUEHRMANN, Logienquelle 112 et notre analyse de ces deux termes, pp. $76-80$.

5 BOR NKAMM, Lösegewalt 39; BULTMANN, Trad 150; LUEHRMANN, Logienquelle 113. STRECKER, Weg 223. 


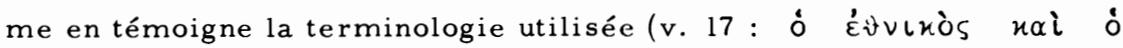

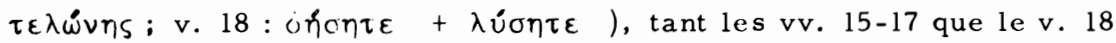
proviennent de la même tradition judéo-chrétienne palestinienne. En second lieu, du point de vue de la forme littéraire, les vv. 15-18 forment une unité; ils sont construits selon le schéma halachique : une série de conditionnelles de type casuistique (vv. 15-17) suivie d'une proposition en $\dot{\alpha} \mu \grave{n} v \quad \lambda \varepsilon \gamma \omega \dot{u} \mu \tilde{\tau} \nu$ qui sert de fondement eschatologique au tout (v. 18). Enfin, une suture rédactionnelle particulièrement perceptible apparaît au

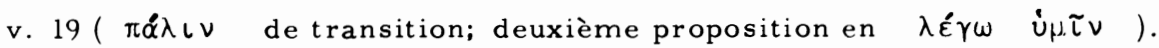
Cet enchaînement abrupt nous montre que $M t$ a reçu une règle qui compor tait déjà les vv. 15-18, et qu'il a jugé nécessaire de la commenter par l'adjonction d'un logion supplémentaire (vv. 19-20).

Quel est alors le contenu de cette règle (vv. 15-18) reprise par Mt ? Cette instruction ecclésiale entend réglementer la procédure à suivre lorsqu'un conflit éclate entre la communauté et l'un de ses membres ${ }^{1}$. La communauté ne doit faire preuve ni d'amertume, ni de haine à l'égard du fautif (cf. Lv 19,17-18); elle doit, au contraire, s'engager pleinement pour tenter de le sauver. La nature du conflit n'est pas précisée; le verbe $\dot{\alpha} \mu \alpha \rho \tau \alpha \dot{\alpha} v \omega$ laisse entendre qu'il s'agit d'une transgression de l'enseignement du Maître ${ }^{2}$. Le premier échelon de la procédure (v. 15) est la réprimande fraternelle : un membre de la communauté reprend ( $\dot{\varepsilon} \lambda \varepsilon \varepsilon_{\gamma}$ $\chi \in \iota)$ en privé le fautif pour l'exhorter à la repentance ${ }^{3}$. En cas de

1 'A $\delta \varepsilon \lambda \varphi \delta \varsigma$ désigne ici le membre de la communauté religieuse plus

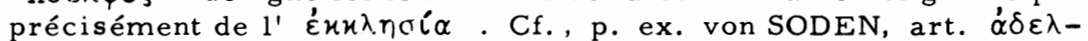
фơs, ThW 1, 145.

2 La lecon $\varepsilon i_{\zeta}$ бé qui figure dans quelques témoins importants est secondaire. Elle est une harmonisation avec le v. 21 (Lc 17,4). Cf. KLOSTERMANN, Mt 150; STRECKER, Weg 224; TRILLING, Israel 114. La règle n'aborde pas le problème spécial de l'offense personnelle, mais celui de la transgression comme telle.

3 Le verbe $\dot{\varepsilon} \lambda \varepsilon ́ \gamma \chi \varepsilon \iota \vee$ a un fort accent disciplinaire et juridique; il signifie "reprendre", "réprimander". Cf. BONNARD, Mt 274; MICHEL, art. $\dot{\varepsilon} \lambda \varepsilon \varepsilon_{\chi \omega}$, ThW 2, 470-71; TRILLING, Israel 114; voir aussi BILL 1, 787 . 


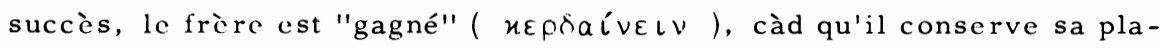
ce dans l'Eglisc. En cas d'échec, une deuxième tentative est entreprise en présence de frères dont le rôle est de rendre plus pressante l'exhortation (v. 16). Enfin, en cas de nouvel échec, c'est la communauté locale ${ }^{1}$ qui est instruite de l'affairc par les frères devenus témoins $(D t 19,15)$ et qui prononce un ultime appel. Si l'intéressé persiste dans son refus, l'excommunication est prononcée $(v .17)^{2}$. On ne peut certes nier que cette règle soit animée d'une volonté de fraternité : la communauté ne se désintéresse pas du pécheur, elle en est au contraire responsable et son premier souci est de le regagner; d'ailleurs, la triple répétition de l'exhortation présuppose de la patience vis-à-vis de l'égaré. Mais l'aboutissement de la procédure est inéluctable pour le fautif endurci : l'exclusion le frappe et le prive du salut.

Le v. 18 fonde ${ }^{3}$ cette réglementation disciplinaire (cf. $\left.\alpha \dot{\alpha} \hat{\eta} \nu x^{\prime} \tau\right)$.

1 Le mouvement même de la règle (réprimande entre quat re yeux, puis

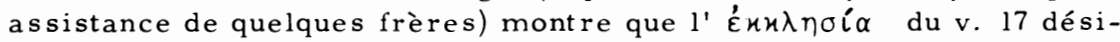
gne la communauté locale assemblée. Voir p. ex. : BOR NKAMM, Lösegewalt 39 (versammelte Gemeinde); LUEHRMANN, Logienquelle 112 (die ganze Gerneindeversammlung): PESCH, Seelsorger 41 (eine judenchristliche Ortskirche); STRECKER, Weg 224 (die Versammlung der Ortsgemeinde).

2 Pour exprimer l'exclusion de l'Eglise, nous avons la formule :

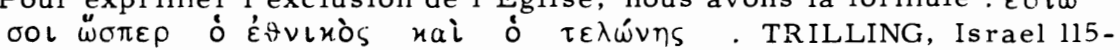
116 a consacré une longue analyse à cette expression. Il en relève à la fois l'originalité dans le cadre des synoptiques et l'orientation strictement juive-exclusiviste. Le païen est celui qui n'appartient pas à la communauté nationale et religieuse, celui qui n'est pas Israélite (BER-

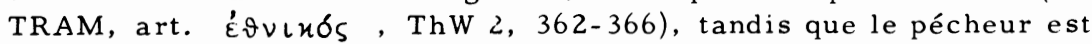
celui qui est extérieur à la communauté religieuse juive - l'Israélite

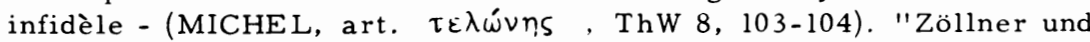
Heide vertreten die zwei Gruppen der nicht zur Gemeinde gehörenden Menschheit (MICHEL, art. cit. 103). "Cette manière de décrire celui qui est exclu de la communauté ne peut être que le fait d'une église qui ne s'est pas encore clairement séparée de la communauté juive, plus précisément de la synagogue.

3 BORNKAMM, Lösegewalt 39; LUEHRMANN, Logienquelle 113. 
Il le fait en attribuant à l'église locale ${ }^{1}$ le pouvoir de lier et de délier.

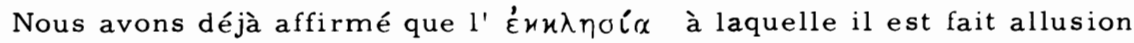
est la communauté rassemblée. Concernant le pouvoir de lier et de délier la cohérence avec le contexte immédiat qui traite de réprimande et d'exclusion, recommande l'acception disciplinaire ${ }^{2}$. Le sens du v. 18 est alors le suivant : la discipline fraternelle n'est pas un acte de simple opportunité ou l'exercice d'une juridiction toute humaine ${ }^{3}$; elle est l'expression de la vocation de l'Eglise qui se voit dotée d'un pouvoir eschatologique : par l'exclusion ou le pardon, l'Eglise consacre l'attitude du disciple égaré, elle anticipe son destin ${ }^{4}$. En effet, en acceptant ou en refusant le

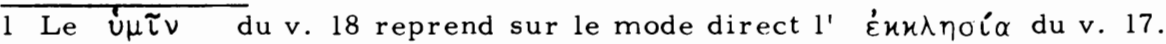
On peut aussi le référer plus largement aux disciples du v. l (voir aussi vv. 2.10.12.13) qui sont la figure de l'Eglise. Quoi qu'il en soit, on remarquera que l'exercice de la discipline n'est remis ni à un groupe spécial de la communauté (p. ex. les ministres), ni à un individu particulier, mais à $1^{\prime} \dot{\varepsilon} x \varkappa \lambda \eta i^{\prime} \alpha$ dans son ensemble, comme le démontre l'usage de l'expression indéterminée $\delta$ úo $\hat{n} \tau \rho \varepsilon \tau \varsigma$ au v. 20

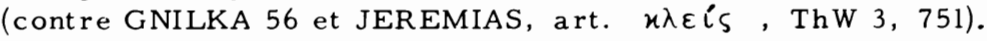

2 Les verbes $\delta \varepsilon \varepsilon \iota \nu$ et $\lambda u ́ \varepsilon \iota \nu$ proviennent de la terminologie rabbinique dans laquelle ils désignent l'autorité à la fois disciplinaire et doctrinale des rabbis; ces derniers peuvent exclure quelqu'un de la synagogue, puis le réintégrer; en matière halachique, ils peuvent déclarer quelque chose permis ou défendu (BILL 1, 738-747; BUECHSEL, art. $\delta \varepsilon \omega$, ThW 2, 59-60; BULTMANN, Trad 147). On ne saurait sans autre reprendre cette signification pour la reporter sur notre texte. Il faut à la fois se souvenir que Mt reçoit une tradition chrétienne et surtout il convient de tenir compte du contexte immédiat (vv. 15-17). Or ce dernier est clair : il parle de restaurer ou de rompre la communion avec le pécheur. L'accent disciplinaire est donc irréfutable (avec BORNKAMM, Lösegewalt 39.46-47; GRUNDMANN, Mt 420; HUMMEL 62; SCHWEIZER, Mt 242; STRECKER, Weg 224).

3 La différence avec la pratique synagogale doit être soulignée : le ban y est un moyen limité dans le temps et graduel; il est un instrument de discipline intra-synagogal sans rapport avec l'excommunication $m$ à valeur eschatologique (STRECKER, Weg 225).

4 Par le pouvoir de lier ou de délier, l'Eglise anticipe la sanction eschatologique rendue par Dieu au Jugement (GNILKA, Kirche 56; JEREMIAS, art. $x \lambda \varepsilon i ́ s$, ThW 3, 753); autre exégèse de TRILLING, Israel 116, qui insiste sur la valeur immédiate de la juridiction ecclésiale. 
pardon, le membre incriminé se révèle fondamentalement dans sa foi ou dans son incrédulité.

Peut-on alors situer avec quelque précision l'église qui se dote d'une telle discipline? Les verbes "lier" et "délier" au sens d'exercer l'autorité disciplinaire et doctrinale, sont des tournures empruntées au milieu juif rabbinique. Semblablement, l'expression "païen" et "pécheur" fait allusion à ceux qui sont exclus de la communauté juive dans sa dimension nationale et religieuse. De plus, Trilling ${ }^{1}$ n'a pas manqué de rapprocher notre règle des écrits de Qumrân et il a mis en évidence des parallèles frappants en l QS 5, 25-6,1 et en CD 9,2-4, passages auxquels il faut ajouter CD 13,9-10 2. Dans l'état actuel de la recherche, il n'est pas possible d'utiliser ces parallèles pour en déduire des rapports de dépendance historique. Il n'en reste pas moins qu'ils attestent une parenté certaine et témoignent de leur appartenance à un même courant traditionnel situé dans l'aire syro-palestienne ${ }^{3}$. Enfin, c'est encore à l'univers juif-rabbinique que nous renvoie le schéma "halachique" que nous avons relevé dans les vv. 15-18. La conclusion s'impose d'elle-même : la tradition dont sont issus les vv. 15-18 est judéo-chrétienne et syro-palestinienne. Elle nous fait découvrir une église fort proche de la synagogue, lui empruntant son langage, mais attachée à définir son identité propre ${ }^{4}$.

La double sentence des vv. 19-20 fait partie du Smt dans lequel elle constitue déjà une unité : la promesse d'exaucement de la prière (v. 19) a

1 Israel $117-121$.

2 Israel 117-120; voir aussi BRAUN, Qumran I, 38-40 et GNILKA, Kirche 54-56. La parallèle avec 1 QS en particulier est frappant : même accent sur la fraternité réciproque, même point de départ dans la réprimande fraternelle, même procédure en trois étapes.

3 Cf. BRAUN, Qumran I, 40; TRILLING, Israel 120. Il se peut, p. ex. , que ce schéma disciplinaire ait été repris par la communauté palestinienne, dépositaire de Qmt.

4 BOR NKAMM, Lösegewalt 44-45; TRILLING, Israel 121. 
son fondement dans la présence du xúplos (v. 20) ${ }^{1}$. Pour bien apprécier la fonction de ces deux vv. dans l'économie du discours, il convient de déterminer si leur contexte est pré-mt ${ }^{2}$ ou rédactionnel ${ }^{3}$. Nous penchons pour la seconde hypothèse car, d'une part, le $\pi \alpha{ }^{\prime} \lambda \iota \nu$ (v. 19) est une transition rédactionnelle, d'autre part, la succession de deux paroles en ( $\dot{\alpha}-$

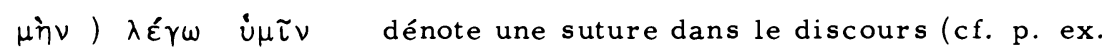
8,10-11). Si donc le rédacteur fait intervenir directement les vv. 19-20 après la règle disciplinaire, par quelle intention est-il animé ? ou, en d'autres termes, quelle relation existe-t-il entre la discipline fraternelle et les vv. 19-20 ? Etant donné la stricte ordonnance dont Mt fait preuve dans le chap. 18, on ne peut guère songer à un enchaînement fortuit ou à un simple changement de thème ${ }^{4}$. Selon toute vraisemblance, Mt ajoute à la discipline fraternelle un commentaire destiné à l'éclairer dans la perspective rédactionnelle ${ }^{5}$. De toute évidence, comme l'a bien montré ${ }^{6}$ Trilling, le thème de $l^{\prime} \dot{\varepsilon}\langle\varkappa \lambda \eta \sigma i ́ \alpha$ qui était au centre des vv. 15-18 est repris avec force. Mais comment?

1 BORNKAMM, Lösegewalt 40; LUEHRMANN, Logienquelle 114; STRECKER Weg 223; voir aussi BULTMANN, Trad 151-52 qui aborde les vv. 19-20 comme un tout. Formellement, $\delta$ úo est le mot-crochet qui lie les deux vv.

2 Ainsi, p. ex., PESCH, Seelsorger 43; SCHWEIZER, Mt 244.

3 Ainsi, p. ex., BONNARD, Mt 275; BORNKAMM, Lösegewalt 40; LUEHRMANN, Logienquelle 114; STRECKER, Weg 223. La liaison se fait par

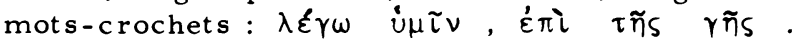

4 Contre KLOSTERMANN, Mt 151 et STRECKER, Weg 223. Mt n'est pas contraint à cet enchaînement par le contexte préétabli d'une source (BOR NKAMM, Lösegewalt 43).

5 BONNARD, Mt 275 et Composition 135-136; BORNKAMM, Lösegewalt 43; LUEHRMANN, Logienquelle 114; SCHWEIZER, Mt 244-45; TRILLING Israel 121. Voir aussi STRECKER, Weg 225.

6 TRILLING, Israel 121, parle à juste titre de la "Kirchenbewusstsein" qui sous-tend le logion. 
Tout d'abord, le v. 19 n'est pas une leçon sur la prière en général et sur la promesse de son exaucement (cf. p. ex. Mc 11, 24 par.). Il parle d'une prière prononcée par une communauté rassemblée au nom de Jésus ${ }^{1}$ et de son unité dans l'intercession. C'est à cette éxxhncía locale unie dans une même intention de prière qu'est promis l'exaucement du Père. On ne peut alors manquer de rapprocher cette intention de prière communautaire $(\pi \varepsilon \rho i \text { } \pi \alpha \nu \tau o ̀ s ~ \pi \rho \alpha ́ \gamma \mu \alpha \tau \sigma \zeta)^{2}$ de la règle qui précède et singulièrement du pouvoir de lier et de délier qui est précisément attribué à $1^{\prime} \dot{\varepsilon} \varkappa x \lambda$ noía locale ${ }^{3}$. Le différend avec le frère ne se tranche pas de manière administrative ou juridique, mais dans la prière. Et cette prière est garantie d'exaucement en vertu de la présence du Christ dans l' $\sigma i \alpha$.

Comment le Christ est-il présent dans l'Eglise ? Le fait que le Christ soit présent au milieu de ceux qui se réunissent pour invoquer son nom (v. 20a) ${ }^{4}$ montre bien que nous sommes en situation post-pascale et que la présence dont il est parlé ici est celle du Seigneur élevé ${ }^{5}$. Nous retrouvons donc une perspective analogue à celle qu'évoque la conclusion de l'évangile (voir notamment 28,20) : 1'assistance active que le Ressuscité promet à tous les disciples est accordée ici à la communauté locale ${ }^{6}$.

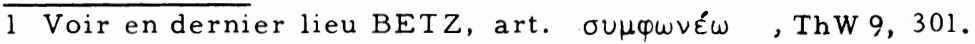

2 BONNARD, Mt 275 et SCHWEIZER, Mt 244.

3 BONNARD, Mt 275; BORNKAMM, Lösegewalt 243; PESCH, Seelsorger

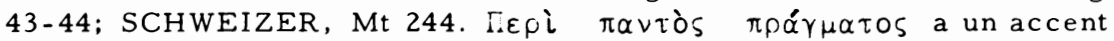
juridique et désigne le différend. STRECKER, Weg 225, hésite entre la prière pour l'apostat et celle subsumant le pouvoir des clefs.

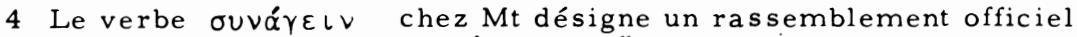

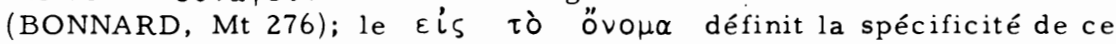
rassemblement qui est rassemblement au nom de Jésus et donc assemblée ecclésiale (TRILLING, Israel 41-42).

5 Avec p. ex. : BARTH 127; BONNARD, Mt 275; BORNKAMM, Lösegewalt 43-44; GRUNDMANN, Mt 420-421; KLOSTERMANN, Mt 151. PESCH, Seelsorger 44; SCHWEIZER, Mt 244; STRECKER, Weg 225-26; TRILLING, Is rael 42.

6 Voir notre analyse de Mt 28,20, pp 103s. Comparent 28, 20 et 18, 20 : BORNKAMM (Lösegewalt 44) qui relève la parenté, et IRILLING (Israel 41-42) qui se montre plus hésitant. 
Comme le note à juste titre BORNKAMM ${ }^{1}$, cette expérience de la présence de l'Elevé est représentative de la foi au Kyrios telle qu'elle apparaît dans le christianisme hellénistique. L'étonnant est que cette foi à la présence du Kyrios - typiquement hellénistique - est exprimée dans les termes mêmes dont se sert la tradition juive rabbinique pour parler du lien entre l'étude de la Tora et la présence de la Schechina. Le traité Abot $(3,2)$ déclare p. ex. : "... Si deux se réunissent pour étudier les paroles de la Tora, la Schechina est parmi eux..." 2 . Le parallélisme entre Mt 18, 20 et Ab 3,2 est frappant même si - comme le constate BARTH ${ }^{3}$ - le nom de Jésus remplace la Tora et sa personne prend la place de la Schechina. Comment faut-il alors expliquer que, pour exprimer la présence du Kyrios dans l'Eglise, Mt reprenne un logion si fortement apparenté à une sentence juive sur la Tora ? On pourrait imaginer une intention polémique vis-à-vis du judäisme rabbinique. Le nom et la présence du Kyrios remplacent désormais la Tora. Mais pareille interprétation s'accorde mal avec la conception $\mathrm{mt}$ de la Loi et sa lutte contre les antinomistes (cf. 5,17-20 !). Bornkamm nous semble mieux résoudre la difficulté lorsqu'il écrit : "Pour l'évangéliste et la communauté qu'il représente, la présence de l'Elevé en son sein peut seulement signifier et impliquer que le Seigneur présent ... est le même que celui qui a valablement interprété et accompli la Loi par son enseignement, qui l'a rendue obligatoire pour sa communauté". Ainsi l'utilisation d'un theologoumenon juif sur la Tora s'oppose à toute vision spiritualiste et charismatique de la présence du Kyrios : de même que l'Elevé et le terrestre sont inséparables, semblablement la présence du Christ dans son Eglise ne saurait être dé-

1 Lösegewalt 43-44.

2 Autre matériel chez BILL, 794-795.

3 Gesetzesverständnis 127.

4 Lösegewalt 44 . 
tachée de son enseignement ${ }^{1}$.

Il s'agit maintenant de préciser en quoi les vv. 19-20 réorientent la discipline fraternelle que Mt a trouvée dans sa communauté. L'Eglise qui lie et qui délie, ne peut le faire que dans la prière et en présence de son Seigneur. Cette relation obligée avec le Maître ressuscité implique que la communauté ne saurait appliquer la discipline sans autre, qu'ici comme en toute circonstance elle est tenue d'être fidèle à l'enseignement du terrestre. Or que dit l'enseignement du terrestre ? Le chap. 18 nous en offre un aperçu suggestif : il est appel à l'humilité (vv. 1-5), à l'amour (vv. 614), au pardon (vv. 21-35).

La règle des vv. 15-18 n'est donc pas un corps étranger dans le chap. 18. Néanmoins, par le biais de la christologie qui renvoie en définitive à l'enseignement du terrestre (vv. 19-20), elle est replacée dans un contexte qui l'empêche de devenir une grotesque anticipation du Jugement ou de tourner à la tentation sectaire. Elle doit rester une possibilité extrême sans cesse replacée dans le cadre de la prédication du Maître. Que dit alors ce Maître à une église qui a reçu le pouvoir de s'amputer pour ne pas se renier?

\section{La sollicitude envers les petits $(18,6-14)$}

Avant la discipline fraternelle - et pour bien en préciser le sens -, Mt a placé un enseignement de Jésus sur la sollicitude particulière dont la communauté doit faire preuve à l'égard des "petits" (vv. 6.10.14). Cette unité thématique ${ }^{2}$ se compose de deux péricopes : la première (vv. 6-9) est une mise en garde contre le scandale, la seconde (vv. 10-14) rapporte la parabole de la brebis perdue.

1 La similitude avec 28,16-20 est frappante : de même que dans le manifeste, devenir disciple c'est observer les commandements, ici accueillir la présence du Christ, c'est accueillir son enseignement.

2 BORNKAMM, Lösegewalt 41 et TRILLING, Israel 110 . 
a) Le scandale (vv. 6-9)

La péricope sur le scandale (vv. 6-9) est étroitement liée à l'instruction sur la véritable grandeur (vv. 1-5). Comme le remarque à juste titre Lührmann ${ }^{1}$, les vv. 5.6 sont des propositions antithétiques : à l'accueil d'un enfant par les disciples (v. 5) - accueil qui est le signe de la foi - est opposé le scandale qui égare les petits et qui est le signe de la perdition (v. 5 : ős $\varepsilon^{\alpha} \alpha$; v. 6 : ôs $\delta^{\prime \prime \alpha \nu ~) . ~ P o u r ~ c r e ́ e r ~ c e t ~ e n c h a i ̂ n e m e n t ~ d e ~}$ caractère systématique entre $1-5$ et $6-9$, Mt a éliminé l'apophtegme de l'exorciste étranger (Mc 9,38-41) qui ne s'intégrait pas à son discours ${ }^{2}$. Les vv. 6-9 sont une reprise avec de multiples retouches de Mc 9, 42-47 3 . La dépendance littéraire par rapport à $M c$ est attestée par le fait que $M t$ reprend les logia sur la main et le pied, logia qui conviennent médiocrement à son propos ${ }^{4}$. La retouche la plus importante consiste dans l'introduction du logion du v. 7 qui est emprunté à la source $Q$ (cf. Lc 17, ${ }^{5}$ ).

Avec le v. 6 apparaît un groupe qui occupe l'avant-scène dans les vv.

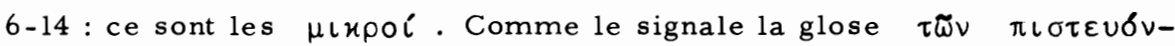
$\tau \omega \nu \varepsilon \iota_{\varsigma} \varepsilon \varepsilon^{\prime}{ }^{6}$, il s'agit de croyants. On notera d'emblée la connotation

1 Logienquelle 110.

2 L'apophtegme de l'exorciste étranger briserait certes l'unité thématique du discours, mais surtout il défend une conception des charismes que Mt ne partage pas (BONNARD, Composition 134 et SCHWEIZER, Mt 237); pour Mt, il n'y a pas de charisme authentique en dehors de la condition du disciple. Le logion de $\mathrm{Mc} 9,40 \mathrm{~s}$ 'oppose également à la vision $\mathrm{mt}$, cf. $12,30:$ Mc 9,41, enfin, est repris en 10,42 .

3 BOR NKAMM, Lösegewalt 38; BULTMANN, Trad 155; LUEHRMANN, Logienquelle 110; PESCH, Gemeindeordnung 222-223.

4 Cf. BONNARD, Composition 134. A son habitude, Mt simplifie et clarifie les vv. 8-9, voir l'analyse de KLOSTERMANN, Mt 149.

5 Cette insertion s'explique d'autant mieux que Lc 17, 2 est un parallèle de Mt 18,6. Il est fort possible que ce soit Mt qui ait composé le premier membre du v. 7 (PESCH, Gemeindeordnung 223; TRILLING, Israel 111).

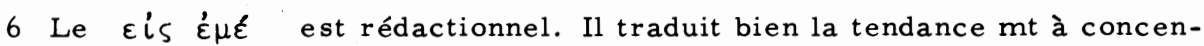
trer la foi sur la personne du Christ (matériel chez TRILLING, Israel $110)$. 
sémantique du terme choisi : ce groupe de chrétiens est vu sous l'angle de sa petitesse, de sa fragilité. Et de fait, ces disciples sont dangereusement menacés dans leur foi au Christ par d'autres membres de la communauté $^{1}$ qui sont devenus pour eux une occasion de chute ( $\left.\sigma \kappa \alpha \nu \delta \alpha \lambda \zeta \zeta \omega\right)$. Le problème qui dominait la règle - celui du disciple égaré et, partant, du corpus mixtum - réapparaît ici, mais par un tout autre biais : il s'agit bien d'éviter la perte du "petit", mais en combattant ce qui est la cause de sa chute - le fauteur de scandale -. La mise en garde est tranchante : celui qui amène un frère à renier sa foi ${ }^{2}$ et le condamne ainsi au rejet eschatologique, subira le même sort. Car à quoi la mort par noyade estelle préférable sinon à la condamnation lors du Jugement ${ }^{3}$ ?

La double lamentation prophétique du v. 7 reprend le v. 6 en l'approfondissant. Elle situe le scandale dans la perspective de l'apocalyptique 4. Le scandale n'est pas un accident isolé, ni une défaillance individuelle, mais une réalité constitutive et inéluctable ( $\alpha$ $\nu \alpha ́ \gamma \not \eta n)$ du temps de l'Eglise. Dans un monde dominé par le mal, le Christ mt ouvre la grande crise $(11,6)$ qui précède la fin des temps. Le temps de l'Eglise est donc par exellence le temps de la tentation, le temps de l'épreuve, le temps qui se caractérise par une recrudescence des occasions de chute. Cette lecture du

1 Le cadre communautaire peut être établi comme suit : le ôs $\delta^{\prime \prime} \alpha \nu$ qui ouvre le v. 6 reprend de manière antithétique le ŏs du v. 5, res-

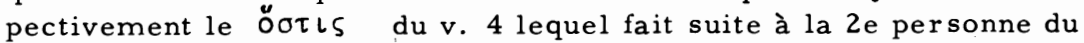
pluriel du v. 3 qui figure le groupe des disciples.

$2 \sigma n \alpha \nu \delta \alpha \lambda i \zeta \omega$ au v. 6 signifie détruire la foi qu'un frère a placée dans le Christ et donc le priver de la "vie" (cf. vv. 8.9). Voir sur ce point STAEHLIN, art. cit, ThW 7, 345, 351-52, mais aussi notre analyse de la terminologie du scandale (pp. 175-178).

3 Mt a grossi l'image du v. 6b pour rendre l'avertissement plus frappant :

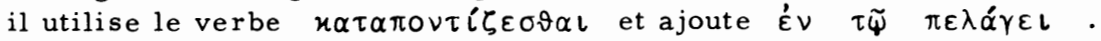
Le fauteur de scandale est, à proprement parler, jeté au fond de l'abîme. Cf. TRILLING, Israel 111; STAEHLIN, art. cit., ThW 7, 351-352.

4 GRUNDMANN, Mt 416; SCHWEIZER, Mt 237; STAEHLIN, art. cit., ThW 7, 346-47. 
temps de l'Eglise n'est pas isolée dans le premier évangile. Elle réapparaît notamment dans les chap. 10 et 24. Cependant, Mt lui a consacré une réflexion particulière dans l'explication de la parabole de l'ivraie (13, 36 $43)^{l}$ : le mal est une réalité constitutive de l'histoire, une réalité qui s'insinue partout, même dans l'Eglise.

La lamentation du v. 7c est une exhortation indirecte. Le mal est une réalité et non une fatalité. Le scandale surgit dès l'instant où un disciple devient le médiateur du mal dans la communauté et qu'il risque de détruire la foi de ses frères les plus faibles. Il ne faut à aucun prix que cela se produise.

Le rapport des vv. 8-9 avec les vv. 6-7 fait difficulté. Pour surmonter ce problème, Pesch ${ }^{2}$ propose une compréhension allégorique de ces deux vv. L'Eglise, corps du Christ, se préserverait de la ruine en excommuniant ceux de ses membres qui sont fauteurs de scandale. Cette explication est peu probable, car elle projette dans le premier évangile une conception ecclésiologique - celle de l'Eglise comme corps - qui lui est étrangère. Il est préférable d'admettre que les vv. 8-9 renforce l'exhortation qui précède : le disciple ne doit à aucun prix donner prise au scandale 3 sous peine de perdre son accès à la "vie". Si, dans la 2e antithèse (5,2930), les logia sur la main, le pied et l'oeil éclairent la radicalité de la volonté de Dieu, dans notre passage, ils servent de manière hyperbolique, à souligner la responsabilité illimitée de chaque disciple vis-à-vis de ses frères.

Au terme de cette brève analyse des vv. 6-9, une double remarque s'impose. D'une part, la problématique du scandale n'est pas posée en fonction de l'existence chrétienne individuelle. Elle est abordée dans le

1 Voir notre analyse de ce passage, pp. 187-195.

2 Gemeindeordnung 223-24 et Seelsorger 27.

3 Il ne s'agit pas du scandale que le disciple pourrait se donner à luimême, mais de celui qu'il donne aux autres. Même si ce sens contrevient à la perspective primitive de ces logia, il est commandé par le contexte. 
contexte de la relation que chaque disciple entretient avec ses frères - et singulièrement ses frères les plus faibles -. La gravité du scandale ne consiste pas d'abord dans les répercussions personnelles qu'il peut avoir, mais dans le danger mortel qu'il représente pour le compagnon dont la foi est fragile. La vie communautaire du disciple doit donc avoir pour critèr $\epsilon$ le souci des petits. Elle doit être animée par l'amour des plus faibles, amour qui ne peut avoir d'autre désir que leur maintien dans la foi. Cet appel pressant à l'amour vigilant de chaque croyant afin qu'aucun petit ne défaille, amène notre seconde remarque. Le scandale dont parle le texte est d'une gravité irréparable puisqu'il débouche sur l'apostasie. Il ne pro-

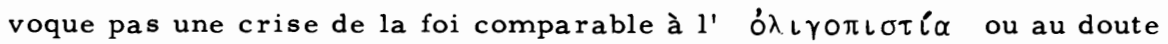
mt, mais la destruction de la foi. Quels sont alors le geste ou la parole qui menacent à ce point les $\mu \iota x p o l$ et les induisent à un reniement fatal ? Nos vv. ne nous disent rien sur ce point. Mais la parabole qui suit, nous livre un faible indice, notamment à cause de la terminologie utilisée.

\section{b) La brebis perdue (vv. 10-14)}

Attaché à développer le thème de l'amour dû aux "petits", Mt abandonne la source Mc qui lui avait servi de support jusqu'ici. Pour poursuivre sa réflextion, il puise la parabole de la brebis perdue dans la source $Q$ (cf. Lc 15,3-7) ${ }^{1}$. La récurrence du terme $\mu \iota$ นós (vv. 10.14) établit la continuité avec les vv. 6-9 et constitue une inclusion qui fait ressortir le thème dominant du morceau. La recension mt de cette parabole est particulièrement caractéristique. Elle apparaît tout d'abord dans l'adjonction de deux logia (vv. 10.14) qui encadrent l'image et qui en livrent l'in-

1 Le v. 11 manque dans la plupart des manuscrits les plus anciens et doit être considéré comme une interpolation tardive de Lc 19, 10 opérée à cause de la similitude des contextes. 
terprétation ${ }^{l}$. Le premier (v. 10) -d'origine inconnue, mais proche de l'angélologie juive ${ }^{2}$ - assure la transition avec les vv. 6-9 et indique la perspective dans laquelle doit être lue la parabole. Le second (v. 14) en tire l'application pratique ${ }^{3}$. La parabole proprement dite (vv. 12-13), in-

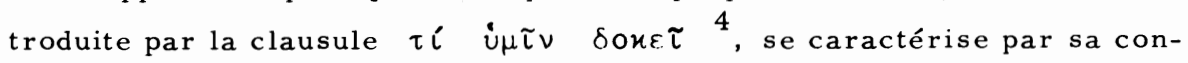
cision. L'élément narratif est réduit à sa plus simple expression. La description de la joie des retrouvailles - capitale chez Lc - occupe une place restreinte chez Mt si bien que l'accent porte sur la recherche entreprise par le berger. Le fait que le rédacteur préfère le verbe $\pi \lambda \alpha \vee \alpha u(\omega)$ (cf. vv. 12.13) au verbe $\alpha \dot{\alpha} \delta \lambda \lambda u \mu \iota$ atteste que le fond prend le pas sur l'image, car, au niveau du récit parabolique, une pareille distinction est sans importance ${ }^{5}$. Enfin, comme l'a relevé Lührmann ${ }^{6}$, nous retrouvons

1 Sur cette interprétation de la parabole par la mise en place d'un nouveau contexte, voir : BONNARD, Mt 271; BORNKAMM, Lösegewalt 38; JEREMIAS, Gleichnisse 36; LUEHRMANN, Logienquelle 114; PESCH, Gemeindeordnung 224-25; TRILLING, Israel 112.

2 BULTMANN, Trad 155; LEGASSE, Enfant 62-63; PESCH, Gemeindeordnung 224.

3 Avec BARTH 114; KILPATRICK 29; PESCH, Gemeindeordnung 231 et Seelsorger 30-31; STRECKER, Weg 149, nous pensons que ce v. est rédactionnel. A l'appui de cette hypothèse, nous relevons la terminologie typiquement $\mathrm{mt}$ et la propension du rédacteur à conclure les paraboles par une application pratique. Autrement : LEGASSE, Enfant 6162.

4 Formule de provenance rédactionnelle en 21, 28 ?, 22, 42, 26,66 (avec LEGASSE, Enfant 60 et SCHULZ, Q 387).

5 Il n'en va pas de même pour une communauté : $\pi \lambda \alpha \nu \alpha ́ \omega$ y décrit l'errance doctrinale, $\dot{\alpha} \pi \delta\langle\lambda \cup \mu \iota$ le rejet eschatologique. Voir LEGASSE, Enfant 60; TRILLING, Israel 112 .

6 Logienquelle 115. La concision de la version mt a souvent été invoquée pour démontrer sa plus grande ancienneté (p. ex. BULTMANN, Trad 184; KLOSTERMANN, Mt 149). L'argument ne convainc guère car la concision est une technique constante de la rédaction mt et dans le cas présent, elle paraît dépendre du schéma halachique utilisé. Cf. aussi TRILLING, Israel 112. 
le schéma "halachique" si fréquent chez Mt : deux séquences introduites

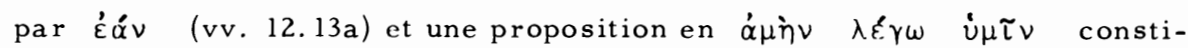
tuent la structure du récit. L'emploi de cette forme montre que, pour $\mathrm{Mt}$, cette parabole est devenue une règle communautaire.

Si les vv. 6-9 invitaient les disciples à faire preuve d'amour à l'égard des "petits" en évitant de devenir pour eux une occasion de scandale, la perspective se déplace dans les vv. 10-14. Il s'agit non plus d'éviter de scandaliser les "petits", mais de se consacrer à ceux d'entre eux qui se sont égarés. L'exhortation du v. 10 est une claire mise en garde : l'égarement des "petits" n'autorise aucune forme de mépris. Le verbe $\varkappa \alpha \tau \alpha \varphi \rho \circ-$ $\nu \varepsilon \tilde{\iota} \nu$ décrit une attitude pratique qui consiste à négliger son frère, à le laisser à son sort ${ }^{1}$. Cet appel à ne pas se réfugier dans l'indifférence

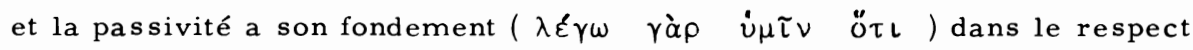
indiscutable dans lequel Dieu tient les "petits". Comme le montre le motif des anges (v. 10b $)^{2}$, Dieu attache un prix tout particulier à ceux-là même que la communauté ignore. S'occuper d'eux équivaut à s'occuper de ceux que Dieu gratifie d'un amour tout particulier.

Qui sont donc ces $\mu \iota x p o i^{3}$ ? Le v. 6 nous montrait en eux ceux qui sont vulnérables au scandale. La parabole de la brebis perdue par son

1 Voir l'analyse du sens de $\chi \alpha \tau \alpha \varphi p o v \varepsilon \tau \nu$ dans le milieu biblique chez LEGASSE, Enfant 68.

2 Le motif des anges qui accompagnent tout homme durant sa destinée terrestre est assez flou dans le milieu juif (voir BILL 1, 781-783). S'agit-il d'anges gardiens? ou d'anges intercesseurs ? Quoi qu'il en soit, en attribuant un médiateur céleste individuel aux petits, notre logion entend les valoriser. Cet effort de valorisation est particulièrement rehaussé par le fait que les anges des petits voient la face de Dieu. Il s'agit là d'un priviliège inouï généralement refusé aux anges, selon le

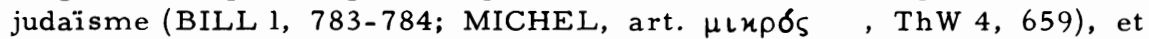
qui montre bien l'intimité et l'amour dans lesquels Dieu tient les petits. Cf. GNILKA, Kirche 53 et LEGASSE, Enfant 71-72.

3 Nous tentons de définir le sens de ce terme au niveau de la rédaction $m$ et dans le cadre du chap. 18. 
emploi délibéré du verbe $\pi \lambda \alpha \vee \alpha ́ \omega$ nous fait découvrir, dans la personne des "petits", des croyants qui s'égarent. Cet égarement n'est ni fortuit, ni involontaire : il est de nature doctrinale et consiste à abandonner la vraie foi pour l'apostasie ${ }^{1}$. Le v. 14 souligne la gravité de cet égarement en montrant qu'il peut conduire au rejet eschatologique ( $\left.\dot{\alpha} \pi \lambda_{\lambda} \lambda u \mu \iota\right)$ si le disciple infidèle n'est pas regagné. La faiblesse des $\mu \iota$ upó consiste donc dans la vulnérabilité de leur foi - et singulièrement dans le gauchis sement possible de leurs convictions -. Le "corpus mixtum" a son origine non seulement dans la question de la fidélité éthique, mais aussi dans celle de la rectitude doctrinale.

Face à ces croyants dont la foi est falsifiée, Mt répudie tout geste de rejet ou de jugement. Comme le montre la parabole qui est l'illustration didactique du v. 10, la sollicitude infinie est la seule attitude évangélique. De même que le berger n'abandonne pas à son sort la brebis perdue, mais se met à sa recherche jusqu'à ce qu'il la trouve, ainsi chaque membre de la communauté est-il obligé à cette miséricorde envers le frère égaré. Dès lors la pointe de la parabole n'est plus la joie de Dieu à l'égard du pécheur qui se repent (ainsi Lc); l'histoire devient une instruction à l'adresse de l'Eglise - instruction dans laquelle se trouve décrite l'attitude requise vis-à-vis du disciple infidèle ${ }^{2}$-. Le v. 14 qui tire la leçon de la parabole ${ }^{3}$ confirme notre interprétation. Ce que Dieu veut, c'est qu'au-

1 Autre hypothèse chez BONNARD, Composition 134, qui voit dans les $\mu \iota$ น $\rho \circ$ des petites gens sans instruction et candidats au baptême.

2 Cette interprétation rallie un large consensus. Voir p. ex. : BONNARD, Composition 134; BORNKAMM, Lösegewalt 41; GRUNDMANN, Mt 417; JEREMIAS, Gleichnisse 35-36; LEGASSE, Enfant 70; PESCH, Gemeindeordnung 225; SCHNIEWIND, Mt 228; SCHWEIZER, Mt 281; TRILLING Is rael 112-113.

3 Le v. 14 tire la lecon de la parabole, mais en décalage par rapport à l'image. En effet, la conclusion attendue qui s'appuie sur le tertium comparationis, serait la suivante : de même que le berger a recherché la brebis perdue, vous aussi vous devez retrouver le frère égaré. Mt ne s'en tient pas à cela; il préfère immédiatement parler de la volonté de Dieu à l'égard des "petits". 
cun croyant - aussi fragile soit-il - ne renie sa foi : c'est dire que le berger a agi comme la situation l'exigeait; c'est dire que chaque disciple est impérativement responsable de ramener au Christ le frère égaré.

L'instruction des vv. 10-14 développe de manière exemplaire les rapports entre la vérité et l'amour. Le gauchissement de la foi est une réalité que l'Eglise prend au sérieux : il y a des frères qui s'égarent au point d'encourir le rejet eschatologique. Face à cette situation de crise, il n'est nullement demandé à la communauté de faire taire son exigence de vérité. Elle doit, au contraire, la maintenir et l'affirmer clairement. L'image du berger qui défend l'intégrité du troupeau en est la meilleure illustration. Cependant la fidélité à la foi authentique n'autorise en aucune manière des attitudes de rejet ou de jugement à l'égard des disciples apostats; elle exige au contraire un amour infini sous peine de renier ce qu'elle prétend défendre. Cet amour n'est évidemment pas une marque de complaisance vis-à-vis du croyant déchu. Il consiste à vouloir inlassablement son salut. Le comportement du berger est exemplaire en ceci, qu'il recherche patiemment et attentivement la brebis perdue afin de lui permettre de réintégrer le troupeau qu'elle n'aurait jamais dù quitter ! Ainsi, en situation d'apostasie, fidélité à la vérité et fidélité à l'amour se conjuguent de la façon suivante : aimer le frère infidèle, c'est chercher sans relâche et avec une sollicitude infinie à le faire quitter le chemin de l'erreur et à le ramener à la foi authentique.

Pareille instruction nous fait découvrir une église en proie à des difficultés internes extrêmement sérieuses. Les conflits que cette règle communautaire essaie de résoudre, sont de nature doctrinale. La situation de corpus mixtum est créée, dans ce cas, par l'apparition de l'hérésie. Peut-on en préciser les contours ? Nous avons déjà émis notre hypothèse sur ce point ${ }^{l}$. A notre avis, les $\mu \iota x \rho \iota^{\prime}$ qui succombent au scandale sont les victimes des faux prophètes. Leur égarement consiste dans leur adhésion à une foi enthousiaste qui ne connaît plus l'autorité de la Loi. L'amour, du point de vue de $\mathrm{Mt}$, vise à les ramener à une compré1 Voir nos pp. 171-200. 
hension fidèle de l'enseignement du Christ. Mais, fait capital - et qui nous avait déjà frappé dans l'exégèse des vv. 15-20-, la communauté ne saurait se prévaloir de l'enseignement du terrestre sans le pratiquer. Aussi, pour Mt, dans une situation de conflit doctrinal, il n'y a pas de défense authentique de la vérité sans amour vécu vis-à-vis du frère égaré. C'est dans cette difficile dialectique de la vérité et de l'amour que l'Eglise est invitée à assumer son statut de corpus mixtum.

\section{Le pardon $(18,21-35)$}

La mise en perspective théologique de la discipline communautaire à partir de l'enseignement du terrestre est effectuée par l'ordonnance du discours. Les vv. 15-20 sont précédés d'un enseignement sur l'amour dû aux petits (vv. 6-14); ils sont suivis par une instruction sur le pardon (vv. 21-35). Cette dernière se compose d'un entretien d'école (vv. 21-22) et d'une parabole (vv. 23-35).

\section{a) l'entretien sur le pardon (vv. 21-22)}

Mt tire son instruction sur le pardon de la source Q (cf. Lc 17,4). Cette tradition prend d'autant mieux sa place ici que dans $Q$ déjà, les thèmes de la réprimande fraternelle et du pardon sont liés (cf. Lc 17, 3 par. Mt 18, 15; Lc 17, 4 par. Mt 18,22). Le mérite essentiel du rédacteur est d'avoir donné à ces matériaux traditionnels une forme claire et systématique.

Par rapport au logion lc, la structure dialoguée des vv. 21-22 est secondaire et doit être attribuée à la rédaction ${ }^{1}$. Tant la terminologie utilisée $^{2}$ que l'apparition de Pierre ${ }^{3}$, que la prédilection de Mt pour le dia-

1 Voir p. ex. BOR NKAMM, Lösegewalt 40, BULTMANN, Trad 151; GRUNDMANN, Mt 21; KLOSTERMANN, Mt 151; PESCH, Gemeindeord nung 229 et Seelsorger 45; SCHMID, Mt 274; SCHULZ, Q 321; STRECKER, Weg 203.

$2 \tau \delta \tau \varepsilon, \pi \rho \circ \sigma \varepsilon \rho \chi \varepsilon \sigma \hat{\vartheta} \alpha \iota$ et $\chi u ́ \rho \iota \varepsilon$ sont des éléments classiques du vocabulaire $\mathrm{mt}$.

3 Autres apparitions rédactionnelles : 15,15;17, 24-25. 
logue ${ }^{l}$, étayent cette hypothèse. L'essentiel de la terminologie utilisée dans la question de Pierre est puisé dans le logion emprunté à $Q{ }^{2}$.

La réponse de Jésus contient un élément supplémentaire, inconnu de

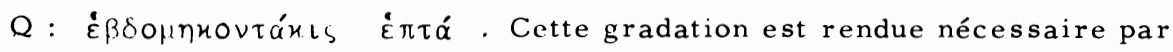
la structure dialoguée. Elle rappelle Gn 4, 24 et correspond bien à l'utilisation intensive de l'Ecriture par $\mathrm{Mt}^{3}$.

La figure de Pierre ${ }^{4}$, introduite par le rédacteur au v. 21, a une double signification. En tant que porte-parole des disciples, Pierre représente l'Eglise qui se fait instruire par son maître. Le titre que Pierre attribue à Jésus, montre bien que l'instruction reçue du Jésus terrestre est la parole normative dont l'Eglise vit jusqu'à la fin des temps. En tant que figure individuelle, Pierre est le garant traditionnel, pour le milieu $\mathrm{mt}$, de l'authenticité de l'enseignement reçu ${ }^{5}$. La question posée par Pierre s'accorde bien avec la thématique d'ensemble du chap. 18; quelle attitude la communauté doit-elle adopter vis-à-vis du disciple infidèle ${ }^{6}$ ? La nouveauté réside en ce que la question va être traitée à partir du thème du pardon. La manière dont Pierre envisage le problème est typiquement juive ${ }^{7}$. Même si le pardon est l'attitude requise vis-à-

1 Voir l'analyse de HELD $221-224$.

$2 \dot{\alpha} \mu \alpha \rho \tau \alpha \dot{\alpha} \nu \omega \varepsilon_{\zeta}, \dot{\alpha} \delta \varepsilon \lambda \varphi \sigma o s$ (par. Lc 17,3), $\dot{\alpha} \varphi i n \mu \iota, \dot{\varepsilon} \pi \tau \alpha \dot{\alpha} \iota \varsigma$.

3 La parenté avec Gn 4, 24 LXX est dans la structure formelle, mais non dans le thème; en effet, Gn 4,24 est un chant de vengeance :

4 Sur ce point, voir nos pp. 24-25.

5 Cf. HUMMEL 60.62 et PESCH, Seelsorger 46.

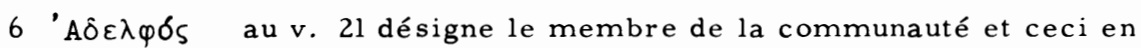
continuité avec la règle qui précède (v. 15), cf. PESCH, Seelsorger 46 et TRILLING, Israel 121. La transgression est précisée : il s'agit d'une faute $\varepsilon i_{S} \sigma \varepsilon$, càd qui affecte les relations intracommunautaires. Cette précision est cohérente avec la thématique du pardon.

7 Le judaïsme réfléchit sur la fréquence du pardon (GRUNDMANN, Mt 421; PESCH, Seelsorger 46; SCHWEIZER, Mt 246). Il affirme que Dieu pardonne deux à trois fois la même offense; or le fidèle est invité à imiter Yahvé (BILL 1, 795-97). 
vis du pécheur, pour Pierre, ce mode d'agir reste expressément limité. La réponse de Jésusconteste radicalement cette perspective : le chiffre

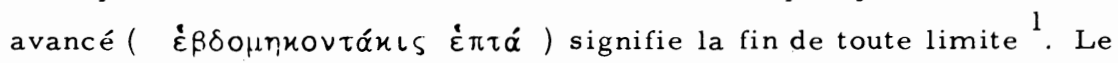
pardon requis à l'égard du pécheur est illimité; il n'est lié à aucune condition, aucun préalable. Il est la seule voie et la possibilité toujours renouvelée de dépasser une relation perturbée. Il s'oppose à toute entreprise casuistique (tension avec les vv. 15-18 !).

b) Le serviteur impitoyable (vv. 23-35)

A l'entretien sur-le pardon (vv. 21-22), Mt joint la parabole du serviteur impitoyable ${ }^{2}$ qui illustre et approfondit le thème abordé. Si le débat entre Jésus et Pierre porte sur la fréquence du pardon, la parabole traite du pardon comme tel ${ }^{3}$. La conviction commune des exégètes est que cette parabole est empruntée au Smt ${ }^{4}$. Néanmoins le rédacteur apporte quelques retouches significatives à la tradition reçue. Il convient donc de localiser ces interventions et d'en mesurer l'importance.

$L^{\prime}$ introduction ( $v$. 23a) et la conclusion ( $v$. 35) sont des adjonctions rédactionnelles qui intègrent la parabole dans le chap. 18 et l'adaptent à sa thématique ${ }^{5}$. Cette appréciation est soutenue par les arguments sui-

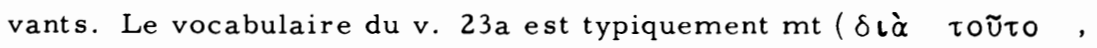

1 Voir en particulier RENGSTORF, art. $\dot{\varepsilon} \pi \tau \dot{\alpha} \quad x \tau \lambda$, ThW 2, 627-28.

2 Pour l'ensemble de cette parabole, nous nous inspirons largement de l'analyse détaillée de WEISER 75-104.

3 Voir p. ex. DIETZFELBINGER 438; JEREMIAS, Gleichnisse 96; LINNEMANN 111-113; WEISER, 99.

4 JEREMIAS, Gleichnisse 78; PESCH, Gemeindeordnung 230; WEISER 92.

5 Défendent l'origine rédactionnelle de 23a : BULTMANN, Trad 195; LINNEMANN 113; SCHMID, Mt 271; STRECKER, Weg 214; WEISER 99; autrement : SCHWEIZER, Gemeinde 98-105. Celle du v. 35 : DIETZFELBINGER 438; JEREMIAS, Gleichnisse 108; LINNEMANN 113; SCHMID, Mt 276; WEISER 100. 


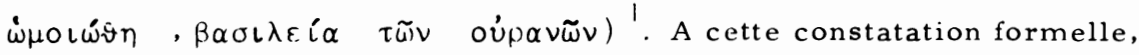
il faut ajouter deux remarques de fond : d'une part, le v. 23a opère la transition entre l'entretien et la parabole; or, dans la mesure où il y a décalage de problématique entre les deux morceaux - le premier traitant de la fréquence du pardon, le second du pardon comme tel -, la transition est secondairc. D'autre part, le fait d'associer les paraboles à la thématique du Royaume et notamment de lier une exigence éthique (ici le pardon) à l'accès au Royaume est un trait constitutif de la théologie mt. Le v. 35 est une application parénétique qui correspond bien au génie du ler évangile. Comme éléments terminologiques typiquement $\mathrm{mt}$, nous re-

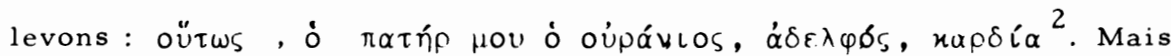
plus que ces particularités de vocabulaire, il faut souligner trois faits. D'une part, le v. 35 qui livre l'application de la parabole n'en restitue que très partiellement la pointe; il n'en retient que l'aspect négatif et menaçant, sans plus faire allusion au miracle libérateur qu'est le pardon du Père ${ }^{3}$; la parénèse finale est donc secondaire et elle est conçue en fonction de l'ensemble de la problématique du chap. 18. Ensuite, Weiser 4

$1 \delta \iota \grave{\alpha}$ toũ̃o est utilisé 10 fois par Mt $(12,31 ; 13,13.52 ; 21,43 ; 24,44$

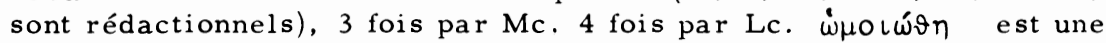
forme verbale propre à quelques paraboles chez Mt $(13,24 ; 18,23$;

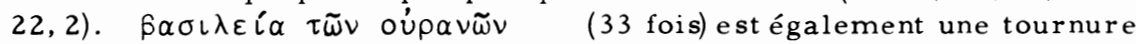
spécifiquement $\mathrm{mt}$.

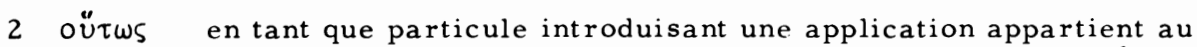
style $\mathrm{mt}$ (Mt : 15 fois; $\mathrm{Mc}: 3$ fois; Lc 10 fois). Même constat pour ó $\pi \alpha \tau$ ńp pou (Mt : 14 fois, Mc jamais; Lc : 4 fois) ainsi que pour le

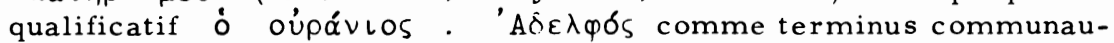
taire apparaît 17 fois chez Mt, 2 fois chez Mc et 7 fois chez Lc. Enfin

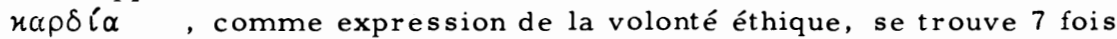
chez Mt, 3 fois chez Mc et 2 fois chez Lc.

3 Bonne formulation de SCHWEIZER, Gemeinde 114 : "Wiederum unterstreicht er nicht etwa die unendliche Grösse der Vergebung Gottes, (...) sondern allein die Konsequenzen, die Gottes vergebendes Handeln für das Handeln des Bruders in der Gemeinde gegenüber seinen Mitchristen aufdrängt".

4 Knechtsgleichnisse 101-102. 
a relevé que Mt utilise ici l'expression "mon père" alors qu'on attendrait plus volontiers "votre père"; cet emploi est pourtant cohérent avec la théologie mt qui réserve cette locution à des contextes en relation avec la volonté de Dieu et le jugement; or, au v. 35, nous sommes précisément en contexte de parénèse. Enfin, alors que la parabole traite de la situation de l'homme devant Dieu et des hommes entre eux (du moins à son niveau pré-mt), le v. 35, par l'emploi du concept $\alpha \delta \varepsilon \lambda \varphi \sigma \zeta s$, en donne une application ecclésiale qui, pour être bien adaptée au chap. 18, n'en est pas moins secondaire.

Dans le corps même de la parabole, nous relevons trois retouches légères et deux interventions majeures. Les corrections mineures concernent les métaphores de la parabole qu'elles explicitent ou infléchissent. Ainsi, au v. 23, la formulation $\ddot{\alpha} \nu \vartheta \rho \omega \pi \circ \varsigma$ $\beta \alpha \sigma l \lambda \varepsilon u ́ s$ est probablement rédactionnelle ${ }^{1}$ car seul $\mathrm{Mt}$, dans les paraboles, emploie de manière pléonastique $\ddot{\alpha} \nu \vartheta \rho \omega \pi$ os lié à un autre substantif (cf. 13,45.52; 20,1; 22, 2). Cette adjonction ne transforme pas notre histoire en une "parabole royale" : elle l'était dans la source ${ }^{2}$. Mais, par ce biais, Mt élucide le xúplos du récit (vv. 25.27.31.32.34) qui devient plus nettement encore

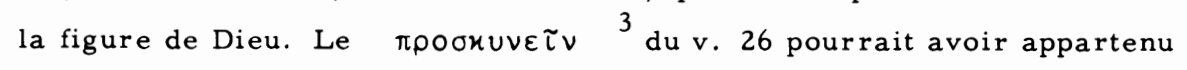
à la parabole dès l'origine, car il s'intègre fort bien au cérémonial des cours orientales. Cependant, dans le premier évangile, ce verbe exprime constamment l'adoration de l'homme pour Dieu ou pour le Christ. Or le roi devant lequel le débiteur s'incline est en vérité Dieu. Nous voyons donc ici une retouche qui exploite l'ambivalence du discours. Enfin, pour désigner les collègues de travail du débiteur, le texte utilise le terme

1 JEREMIAS, Gleichnisse 24 (note 1); TRILLING, Israel 56 (note 6); WEISER 75.

2 La grandeur de la dette, l'autorité avec laquelle le xúplos grâcie, puis condamne, sont autant d'éléments du récit qui renvoient à un personnage royal, cf. JEREMIAS, Gleichnisse 24; LINNEMANN 174 (note 8); SCHMID, Mt 275; WEISER 75.

3 HELD 217; WEISER 80-81. 


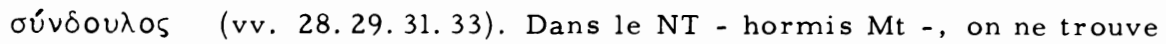
.ce terme que dans Col et Ap où il désigne le frère dans la foi. Il est incontestable que oúvôoùos l's'adapte parfaitement à l'imagerie de la parabole et dénomme dans ce cadre le fonctionnaire royal. Mais il est tout de même frappant que, dans ce chap. 18 où la problématique communautaire est essentielle, notre texte utilise une métaphore classique de la terminologie ecclésiale. Il serait étonnant que cette ambivalence ne soit pas délibérée surtout si l'on songe que, dans l'application du v. 35 (rédaction !), c'est le correspondant $\alpha \dot{\delta} \varepsilon \lambda \varphi \delta_{s}$ qui apparaît.

Comme modification majeure, il faut retenir l'adjonction rédactionelle du v. $31^{2}$. Ce v. survient en effet comme un élément adventice dans la narration et son exégèse s'intègre mal au mouvement de la parabole íquelle est la portée de cette tristesse ? pourquoi rapporter l'événement au roi ?). Comme le suggère Bonnard ${ }^{3}$, ce v. exprime "l'étonnement des disciples à la vue des conflits qui surgissent parmi eux". Il s'agirait alors d'une glose actualisante de la main de l'évangéliste qui inscrirait son vécu ecclésial dans le récit. Quatre arguments soutiennent ce point de vue. Tout d'abord, l'enchaînement du v. 30 au v. 32, se fait très harmonieusement. Ensuite, la péripétie évoquée est sans importance pơr la progression et la pointe du récit. De plus, la seule explication valable de la tristesse du v. 31 est l'étonnement ressenti par la communauté devant le mal qui la ravage. Enfin, la terminologie est strictement $\mathrm{mt}^{4}$.

Si l'on considère l'ensemble des retouches apportées par Mt à la tradition qu'il a reçue, une ligne directrice se dégage. L'inscription du récit

1 BONNARD, Mt 277; BUECHSEL, art. $\lambda u ́ \tau \rho O \nu$, ThW 4, 349; HELD 217; PESCH, Seelsorger 47; WEISER 83.

2 WEISER 85-86 est le premier à défendre cette idée de manière conséquente.

3 BONNARD, Mt 279.

4 Le verbe $\lambda \cup \pi \varepsilon \tau \nu$, appliqué aux disciples, est également rédactionnel en 17,23. Analyse détaillée chez WEISER 85-86. 
dans la perspective du Royaume et de l'ordre qu'il suppose (v. 23a), la claire mise en demeure contenue dans l'application finale (v. 35), le souci de faire transparaître dans la narration les relations des chrétiens

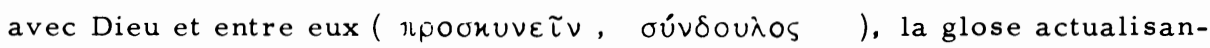
te du v. 31 , tous ces éléments constituent un tissu d'indices univoques. La parabole est résolument inscrite dans le contexte ecclésial : elle est orientée de manière à dire comment doivent être vécus les conflits communautaires. Elle devient une leçon à l'usage du corpus mixtum qu'est l'église mt, l'instruction que le Maître donne à ses disciples divisés.

Venons-en au contenu de la parabole. Cette dernière se divise en trois scènes : a) la miséricorde du roi (vv. 24-27); b) la dureté du débiteur (vv. 28-31); c) le jugement (vv. 32-34). Nous les analysons rapidement. L'imagerie nous introduit dans la cour d'un despote oriental. Ce souverain décide de régler ses comptes avec son administration. A cet effet, il fait comparaître devant lui un haut fonctionnaire (un satrape !) qui se trouve être son plus grand débiteur ${ }^{1}$. La dette évoquée est colossale ${ }^{2}$. Comme le justiciable n'est pas en mesure d'honorer sa dette, le roi prend une mesure qui n'est nullement l'expression de sa colère ${ }^{3}$, mais qui correspond au droit oriental : pour recouvrer une partie de son dû, il se propose

1 L'exégèse récente (p. ex. JEREMIAS, Gleichnisse 208; LINNEMANN 113-114.175; WEISER 76) est d'accord pour reconnaître que l'histoire se joue entre un despote oriental et un de ses hauts fonctionnaires. Il n'est pas nécessaire de supposer (ainsi JEREMIAS 208) que le haut fonctionnaire est incarcéré et que sa comparution a lieu durant sa détention.

2 La dette évoquée correspond à près de 100 millions de francs suisses (BILL 1, 290-294; JEREMIAS, Gleinisse 208; LINNEMANN 114; WEISER 78). On peut certes penser à l'encaissement des impôts de provinces entières par des satrapes, mais, même dans ce cas, on reste loin du compte. La somme mentionnée sort du cadre du récit et renvoie au fond (avec JEREMIAS et WEISER contre LINNEMANN).

3 Contre JEREMIAS, Gleichnisse 209. 
de vendre le coupable, sa famille et ses biens '. L'accusé, à toute extrémité, tente une ultime manoeuvre. Il fait une promesse qu'il ne pourra évidemment pas tenir, mais qui lui assurerait un délai ${ }^{2}$. Et l'inattendu se produit : le roi se laisse fléchir; non seulement, il renonce à sa menace ${ }^{3}$, mais encore il efface cette dette colossale. Il va bien au delà de ce que le fonctionnaire osait espérer.

L'histoire peut sembler extravagante ${ }^{4}$. Pourtant l'auditeur comprend immédiatement de quoi il s'agit. Dans le milieu juif dont notre parabole

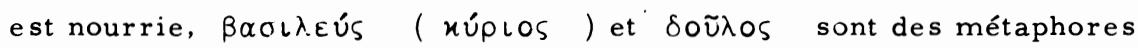
classiques qui désignent respectivement Dieu et l'homme ${ }^{5}$. La réinterprétation $m$ t du récit (voir plus haut) nous invite à voir dans le $\delta \circ \tilde{\lambda}$ os non pas l'homme en général, mais le croyant. Cette double métaphore ne transforme pourtant pas la narration en une allégorie dans la mesure où tout ce qui se passe reste dans les limites possibles des relations entre un roi oriental et ses serviteurs. Les rapports entre Dieu et le croyant sont grevés d'une dette colossale, impossible à honorer. Le milieu juif connaît cette image; dans ce passif, il discerne la culpabilité infinie de l'homme devant Dieu ${ }^{6}$. Cette culpabilité radicale met le croyant dans une

1 Il s'agit d'une mesure connue en Palestine, mais qui n'était pas pratiquée par les Juifs. En cas de vol, la Tora autorisait, en effet, la vente du coupable - s'il n'était pas en mesure de rembourser - ainsi que de ses enfants, mais pas de sa femme (JEREMIAS, Gleichnisse 208-209; BILL 1, 797-798; WEISER 79).

2 Depuis JUELICHER, à propos du v. 26, les exégètes parlent à juste titre de "Angst - und Notversprechen" (cf. WEISER 80).

3 Le verbe $\alpha$ ádúw (v. 27) se rapporte non pas à une libération de prison du justiciable, mais à la levée de la menace du v. 25.

4 BONNARD, Mt 277 : "Tout est énorme et invraisemblable dans ce récit".

5 BILL 1, 797; RENGSTORF, art. EO $\tilde{\lambda}$.OS, ThW 2, 270-71; SCHMIDT,

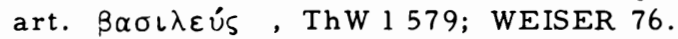

6 JEREMIAS, Gleichnisse 26; SCHMID, Mt 275; SCHNIEWIND, Mt 201; WEISER 77. Autrement LINNEMANN 175 (note 12). 
situation sans issue (v. 25) où il ne peut dire que son désespoir et chercher des faux-fuyants (v. 26). Alors même que la comparution de l'homme devant Dieu a révélé sa perdition irrémédiable, l'inattendu se produit : le roi se laisse émouvoir, il suspend le châtiment et renonce à son dû. Tout en restant dans le cadre de l'image, les verbes utilisés au v. 27 ont une connotation théologique extrêmement forte : $\sigma \pi \lambda \alpha \gamma \chi \nu \iota \zeta \varepsilon \sigma \vartheta \alpha \iota$ décrit, dans le premier évangile, la compassion du Messie pour le petit

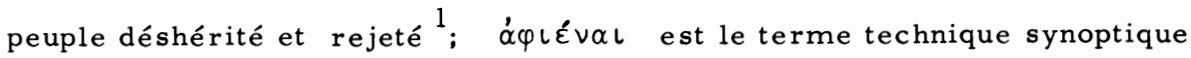
du pardon ${ }^{2}$. Dans le geste du roi se manifeste le contenu central de l'évangile : Dieu prend une initiative totalement inattendue et libératrice qui permet au croyant de passer d'une culpabilité devenue fatalité, à une vie entiè rement nouvelle.

La seconde scène (vv. 28-31) témoigne simultanément d'une similitude formelle voulue et d'un contraste frappant par rapport aux vv. 24-27. A peine libéré d'une situation sans issue et rendu à une vie toute nouvelle, le haut fonctionnaire tombe sur l'un de ses subordonnés - peut-être un percepteur d'impôts ${ }^{3}$ - qui lui doit une somme dérisoire ${ }^{4}$ en regard de la dette dont il vient d'être délivré. Il réclame son dû comme le droit le lui autorise ${ }^{5}$. Son employé, en difficulté, lui demande de surseoir à son exigence dans les termes mêmes que notre homme avait utilisés au v. 26 . Le lecteur pense évidemment que le débiteur grâcié va céder eu égard à

1 Cf. 9, 36; 14, 14; 15, 32; 30,34. Sur la signification du verbe, voir KOESTER, art. $\sigma \pi \lambda \alpha ́ \gamma \chi \operatorname{OO\nu } x \tau \lambda$, ThW 7, 553-555, et WEISER 81.

2 BULTMANN, art. '́㇒ínul, ThW 1, 506-509.

3 JEREMIAS, Gleichnisse 209; LINNEMANN 115; WEISER 83.

4100 deniers (BILL 1, 291) valent un peu moins de 100 francs suisses. A titre de comparaison 1 talent vaut entre 5'000 et 10'000 deniers.

5 Les verbes $x \rho \alpha \tau \varepsilon \tau \nu$ et $\pi \nu i \gamma \varepsilon \iota \nu$ ne dépeignent pas ici une violence mal contenue. Dans l'Antiquité et le judaïsme (BILL 1, 799; JEREMIAS, Gleichnisse 209; WEISER 82), ils décrivent les gestes classiques du créancier vis-à-vis de son débiteur pour lui empêcher tout échappatoire. 
la mansuétude royale dont il a bénéficié.Pourtant, l'incroyable se produit le haut fonctionnaire reste délibéremment inflexible ( oúx envoie son subordonné en prison afin de recouvrer son argent ${ }^{1}$.

Cette deuxième scène - si elle est lue en référence au contexte ecclésial - aborde un point capital de la réflexion mt. Comment le disciple vat-il répercuter dans ses relations avec ses frères la grâce dont il a été l'objet ? L'image que trace la parabole est particulièrement pénible. Le croyant grâcié ${ }^{2}$ mesure ses frères dans la foi et, particulièrement, ceux qui lui sont redevables, à l'aulne d'une justice tout humaine, celle précisément dont il a été exempté. Il y a une contradiction évidente et scandaleuse entre le régime dont il bénéficie et celui qu'il impose à son prochain. C'est une distorsion qui suscite la profonde tristesse de la communauté : comment un chrétien au bénéfice du pardon divin peut-il agir de la sorte? cette contradiction entre le pardon reçu et le pardon refusé n'est-elle pas significative de l'irruption du mal parmi les croyants ? n'est-elle pas le signe d'un gauchissement de l'évangile?

La troisième scène (vv. 32-34) est celle du jugement. Le roi, informé du comportement de son serviteur, le fait comparaître devant lui. Il lui donne à comprendre que l'acquittement dont il a bénéficié (v. 32b), devait l'amener à agir de même à l'égard de ses collègues (v. 33). Son ingrati-

1 Le judaïsme ne connaît pas la prison pour dette. Par contre, les pays environnants pratiquaient la contrainte par corps pour recouvrer de l'argent : soit le débiteur incarcéré remboursait sa dette par son travail, soit les parents désintéressaient les créanciers (JEREMIAS, Gleichnisse 209). La vente en esclavage est ici exclue, car la somme obtenue serait bien supérieure à la créance.

2 Le biais par lequel la parabole aborde le problème du corpus mixtum est le même que dans les vv. 10-14 et 15-20: il n'y va pas du problème du mal comme tel, mais de l'attitude du disciple dans une situation de conflit dont le prochain est responsable. 
tude est châtiée : il est livré aux tortionnaires ${ }^{1}$.

L'intervention finale du roi (vv. $32 b-33$ ) mérite une attention particulière. Elle pose avec une vigueur remarquable ce qu'il est convenu d'appeler la dialectique de l'indicatif et de l'impératif. Le me la relation nécessaire et infrangible entre le pardon reçu et le pardon à donner. La grâce appelle et permet une nouvelle fidélité. Cette correspondance essentielle entre libération et obéissance est soulignée dans le texte par l'emploi du même verbe ( $\dot{\lambda} \lambda \varepsilon \varepsilon \tau َ \nu)$ pour exprimer ce double événement. La miséricorde ${ }^{2}$ décrit tout à la fois l'initiative divine qui ouvre une vie nouvelle au disciple et la norme sous laquelle est placée cette vie nouvelle. La liberté créée gratuitement par la miséricorde divine est une liberté pour la miséricorde.

Le v. 34 radicalise la prédication : recevoir et accorder le pardon vont de pair. Toute rupture entre indicatif et impératif est dramatique; elle précipite dans la situation désespérée et sans issue qui pointait au début de la parabole : la perdition éternelle ${ }^{3}$.

1 La torture pénale n'existait pas en Israël. Par contre, dans les pays voisins, elle était utilisée contre les hauts fonctionnaires indélicats, soit pour les forcer à révéler où ils avaient dissimulé l'argent, soit pour exercer un chantage sur les parents et amis en vue de les amener à honorer la créance (JEREMIAS, Gleichnisse 210).

2 Parmi les synoptiques, Mt est celui qui utilise le plus fréquemment la

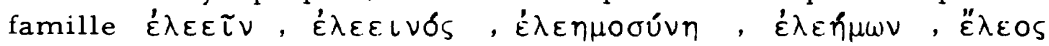
(Mt 15 fois, Mc 3 fois, Lc 12 fois). 9 emplois sont rédactionnels : $5,7.7 ; 9,13 ; 12,7 ; 15,22 ; 17,15 ; 20,30.31 ; 23,23$. Il s'agit donc d'une particularité de la terminologie théologique $\mathrm{mt}$.

3 Etant donné l'importance de la dette, il est clair que le débiteur impitoyable n'arrivera pas à sortir de sa situation dramatique si bien que la torture qu'il subit, sera sans fin (JEREMIAS, Gleichnisse 210; LINNEMANN 116; WEISER 88). Ce châtiment sans fin est pour Mt l'image de la condamnation eschatologique. WEISER (ibid) fait remarquer à juste titre que, dans la parabole elle-même, la peine ne s'applique pas à la dette, mais à la dureté. 
L'application du v. 35 semble en retrait par rapport au message central de la parabole. Elle paraît situer la pointe au v, 34 alors que cette dernière se trouve au v. $33^{1}$. Elle met l'accent sur le jugement qui menace tout chrétien inconséquent quand bien même le récit culmine dans l'affirmation de la réciprocité nécessaire entre la grâce et l'obéissance. Dans la perspective du chap. 18, ce décalage s'explique fort bien : ce qu'il convient en premier lieu de rappeler à une communauté divisée par de graves querelles où chacun entend faire prévaloir son bon droit sur l'autre, c'est que le pardon mutuel et véridique est la seule fidélité possible pour des hommes qui se réclament du Christ. Le mérite de Mt est d'avoir non seulement souligné cette exigence, mais de l'avoir enracinée dans son fondement évangélique : la grâce divine.

\section{La véritable grandeur $(18,1-5)$}

Pour achever notre analyse du chap. 18, nous en venons à la péricope qui erı constitue le début (vv. 1-5) et qui place l'ensemble du discours dans une perspective théologique fondamentale (voir plus bas). Mt a trouvé ce débat d'école dans sa source Mc (9,33-37), mais il le retravaille profondément pour en faire une instruction cohérente et traitant le seul thème de la véritable grandeur ${ }^{2}$. Ainsi il recompose entièrement l'introduction (v. 1) ${ }^{3}$. En supprimant l'indication topographique (Mc 9, 33) 4 , la dispute des disciples (Mc 9,34), la mention du cercle des douze (Mc 9, 35), Mt dépouille le récit de tout trait anecdotique. De ce fait, les $\mu \alpha \vartheta \eta \tau \alpha i ́$

\section{LINNEMANN 113.}

2 Voir avant tout, BORNKAMM, Lösegewalt 43; LEGASSE, Enfant 32-33; TRILLING, Israel 109.

3 Voir notamment BORNKAMM, Lösegewalt, ibid.; LEGASSE, Enfant ibid.; PESCH, Gemeindeordnung 221; TRILLING, Israel 106-107.

4 Cette indication topographique est probablement rétrocédée en 17, 24 25. 
deviennent les représentants de la communauté se faisant instruire par leur maitre ${ }^{l}$. La question posée (v. lb) ne se limite plus au cercle des douze; elle soulève le problème de la grandeur eschatologique comme

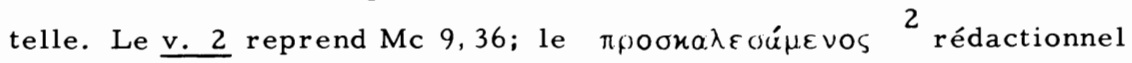
nous permet déjà de deviner le rôle que Mt attribue à l'enfant : il ne s'agira pas d'abord de le considérer comme une personne digne d'accueil (ainsi Mc), mais comme un exemple pour la vie dans la foi. Le $\underline{v} 3$ est une adjonction de Mt. Pour formuler l'instruction du Christ, il reprend le logion de Mc 10,15 en le réinterprétant totalement ${ }^{3}$. Si l'usage de la 2 e personne résulte de l'adaptation au contexte, si le pluriel généralisant $\pi \alpha \iota \delta i ́ \alpha$ correspond bien au ton catéchétique du passage, la modification la plus importante consiste évidemment dans la correction

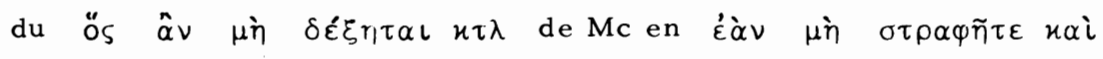

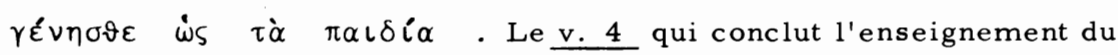
Christ, est également une adjonction de Mt. Inspiré d'un logion indépendant bien attesté dans la tradition (cf. Mt 23,12; Lc 14,11;18,14 4), il est rédigé en fonction du contexte; ainsi le rédacteur introduit les motifs de

1 BORNKAMM, Lösegewalt 41; LEGASSE, Enfant 32; PESCH, Gemeindeordnung 221; TRILLING, Israel 109.

$2 \pi \rho \circ \sigma \chi \alpha \lambda \varepsilon \sigma \alpha ́ \mu \varepsilon v o s \quad \varkappa \tau \lambda$ est une formule classique dans les synoptiques pour introduire une instruction du Christ à l'intention des auditeurs présents (LEGASSE, Enfant 33).

3 On a souvent évoqué la présence des deux parallèles de Mt 18, 3 , à savoir Mc 10,15 et Jn 3,5. On s'est demandé si ce logion n'avait pas été transmis de manière indépendante et si Mt n'en avait pas gardé la forme primitive (état de la discussion: LEGASSE, Enfant 33-35). Quoi qu'il en soit, la critique des sources nous confronte à un fait patent : dans la scène de l'accueil des enfants (19,13-15 par. Mc 10,1316), Mt omet consciemment Mc 10,15; il a donc probablement déplacé ce logion pour l'insérer dans un contexte jugé plus favorable (ainsi p. ex. TRILLING, Is rael 108).

4 Le futur $\tau \alpha \pi \varepsilon \iota \nu \omega ́ \sigma \varepsilon \iota$, la parenté de contenu et de terminologie atteste la parenté entre 18,4 et Mt 23,12, respectivement Lc 14,11; 18,14 . 
l'enfant et du Royaume des cieux, il remplace le verbe $\dot{\psi} \psi \delta \omega$ par $\varepsilon \sigma-$ $\tau \iota \nu$ o $\mu \varepsilon \iota \zeta \omega \nu^{1}$. Par ces différentes retouches il reprend les termes du v. lb, mais sous la forme d'une déclaration conclusive. Avec le v. 5 Mt en revient à sa source Mc $(9,37)$; il ne retient que la première partie du logion, car il a déjà utilisé la seconde en $10,40^{2}$.

Du récit quelque peu cahotique de Mc, nous passons à une scène bien ordonnée et abordant le seul thème de la véritable grandeur. Schweizer ${ }^{3}$ a fait remarquer que ce débat d'école se rapproche de la forme $\mathrm{mt} d u$ récit de miracle. L'introduction est constituée par la question d'un homme venu à Jésus pour lui demander quelque chose (cf. v. lb!). La réponse du Christ consiste dans un acte significatif (cf. v. 2) et dans une instruction répondant à la question posée (cf. vv. 3-5 !).

La question posée par les disciples rejoint un thème fort discuté dans le monde antique - et dans le judaïsme palestinien ${ }^{4}$ en particulier -. Pourtant en liant le débat sur la grandeur à la réalité du Royaume, Mt se place d'emblée dans une perspective spécifiquement chrétienne ${ }^{5}$. La difficulté majeure du v. l consiste à savoir si le Royaume dont il est fait état, est une grandeur présente ou future. Mt fait-il allusion à un "ordre divin" prévalant hic et nunc ${ }^{6}$ ? ou pense-t-il au Royaume eschatologique ${ }^{7}$ ? A notre avis, l'argument décisif se trouve au v. 3 et consiste dans l'utilisa-

1 LEGASSE, Enfant 35.

2 Mt a utilisé Mc 9,37b dans le discours d'envoi $(10,40)$, car le contexte théologique s'y prêtait, ce qui n'est pas le cas ici.

3 SCHWEIZER, Mt 235.

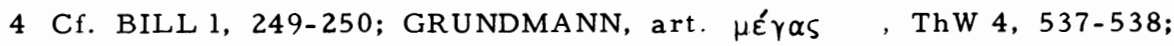
SCHLATTER, Mt 543-544.

5 Le fait que des parallèles rabbiniques posent le problème de la grandeur en fonction du monde à venir (GRUNDMANN, art. $\mu \varepsilon \gamma \alpha \varsigma$, ThW 4, 538) ne met pas en cause notre affirmation, si l'on se souvient de l'étroite relation qui existe entre le Christ et le Royaume chez Mt.

6 Ainsi, p. ex., BONNARD, Mt 268; LEGASSE, Enfant 33; PESCH, Seelsorger 19; TRILLING, Israel 107.

7 Ainsi p. ex., GRUNDMANN, Mt 413; KLOSTERMANN, Mt 147; SCHMID Mt 267; SCHWEIZER, Mt 236. 


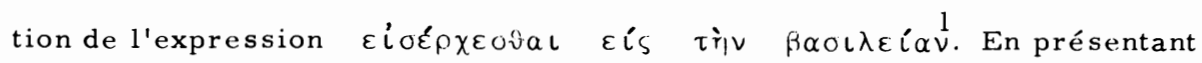
la grandeur comme une condition d'accès au Royaume, Mt entend $\beta \alpha \sigma \iota-$ $\lambda \varepsilon i \alpha$ au sens eschatologique. La question de la véritable grandeur est donc placée sous le signe de l'à-venir de Dieu. Cet à-venir éclaire le temps vécu par le disciple et confère à la thématique de la grandeur son véritable sérieux ${ }^{2}$.

A la question des disciples répond l'instruction du Christ (vv. 2-5). Celle-ci se développe en deux temps : elle s'ouvre par un geste symbolique (v. 2) qui est élucidé ensuite par une brève explication (vv. 3-4). Une application (v. 5) conclut le passage. Le geste symbolique accompli par le Christ est hautement paradoxal : pour illustrer la grandeur approuvée par Dieu, il fait appel à un enfant, càd à un représentant d'une catégorie sociale dépourvue de tout prestige dans le monde juif ${ }^{3}$. En quoi l'enfant est-il exemplaire de la véritable grandeur ? Les notions utilisées dans l'explication des vv. 3-4 nous montrent que la condition enfantine est saisie comme l'image d'une attitude intérieure ${ }^{4}$. Devenir comme un enfant consiste à changer globalement l'orientation de sa vie ( $\sigma \tau \rho \varepsilon ́ \varphi \varepsilon \iota \nu)$ et à

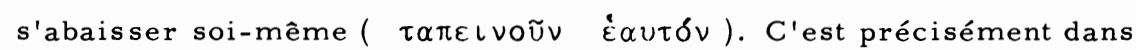
l'évocation de la $\tau \alpha \pi \varepsilon i ́ v \omega \sigma \iota s$ que se trouve la clef de l'image ${ }^{5}$. L'ex-

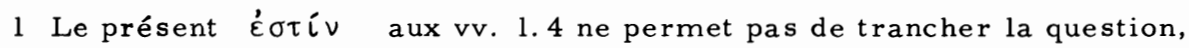
car ce verbe au présent peut également avoir un sens futur (cf. les béatitudes). L'argument de TRILLING (Israei 107) selon lequel le v. 3 est emprunté à Mc, d'où Mt tiendrait la sentence d'entrée dans le Royaume, ne porte pas, car Mt a spécialement placé ce logion ici.

2 L'alternative entre la réalité présente du Royaume et sa venue est superficielle, car l'à-venir du Royaume détermine entièrement le présent.

3 Cf. OEPKE, art. $\pi \alpha \tau \varsigma$, ThW 5, 644-45.

4 Cf. TRILLING, Israel 108. Dans les vv. 3-4, le raisonnement procède par élucidations successives : se convertir consiste à devenir comme les enfants ( $x \alpha i ́$ explicatif); devenir comme des enfants équivaut à s'abaisser (v. 4).

5 JEREMIAS, Gleichnisse 190. 
emplarité de l'enfant ne réside ni dans sa naïveté, ni dans son innoncence, ni dans sa prétendue pureté morale ${ }^{1}$. Elle consiste dans le fait qu'il est petit, qu'il le sait et qu'il se conduit en conséquence. En d'autres termes : de même que l'enfant est petit par rapport à l'adulte, le croyant est invité à se reconnaître petit par rapport à Dieu; de même que l'enfant s'abandonne avec confiance à l'adulte et attend de lui tout ce qui est nécessaire à sa vie, ainsi le disciple doit-il renoncer à toute prétention et s'en remettre totalement à Dieu ${ }^{2}$. La véritable grandeur dont l'enfant est l'image, consiste donc dans la juste attitude du disciple devant Dieu.

La solennelle mise en demeure du v. 3 ( $\dot{\alpha} \mu \grave{\eta} \nu \quad \lambda \varepsilon \gamma \omega \dot{v} \mu \tau \nu)$ nous montre que cette juste attitude de l'homme devant Dieu qui seule ouvre l'accès du Royaume, est contraire à ce que l'homme pense et croit - et notamment à sa manière d'envisager la grandeur -. Pour l'atteindre, il doit complètement changer l'orientation de sa vie $(\sigma \tau \rho \varepsilon \varphi \varepsilon \iota \nu)^{3}$. Et il n'est pas inutile de souligner que, conformément à la théologie mt, c'est la parole du Christ qui lui en ouvre la possibilité en lui en montrant le chemin.

Le v. 5 fait difficulté. Est-il une invite adressée à la communauté et la mettant en demeure d'accueillir ces croyants qui ont précisément re-

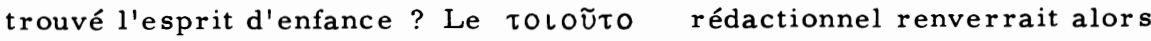
à la condition chrétienne telle qu'elle est décrite dans les vv. 3-4 et $\pi \alpha \iota-$ Síov aurait un sens imagé ${ }^{4}$. Pour séduisante qu'elle soit, nous ne retenons pas cette hypothèse. Le v. 5 doit être compris en continuité avec le

1 BONNARD, Mt 268.

2 GRUNDMANN, art. $\tau \alpha \pi \varepsilon \iota \nu \delta s \quad x \tau \lambda$, ThW 8,17; SCHNIEWIND, Mt 196; TRILLING, Israel 108.

3 Le verbe $\sigma \tau p \varepsilon ́ \varphi \varepsilon \iota \nu$ ne signifie pas revenir à un point de départ (ainsi JEREMIAS, Gleichnisse 189, note 2; LEGASSE, Enfant 217), mais changer de conduite, se convertir (ainsi BARTH 110; BONNARD, Mt 268; KLOSTERMANN, Mt 148; SCHMID Mt 268; SCHNIEWIND, Mt 197; STRECKER, Weg 227; TRILLING, Israel 108).

4 Ainsi notamment KLOSTERMANN, Mt 148; LEGASSE, Enfant 35-36; PESCH, Gemeindeordnung 222. 
geste symbolique du v. 2 et la comparaison du v. 4 ( $\dot{\omega} s$ $\tau \dot{\delta} \pi \alpha \iota \delta$ íov

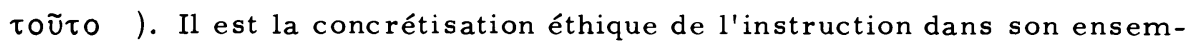
ble $^{1}$ : le disciple qui adopte la juste attitude devant Dieu est en mesure d'accueillir celui pour lequel la société n'éprouve aucun intérêt. Mais, sans doute, la portée de cette attitude ne se limite pas à cet acte d'accueil. Elle s'étend à tout le chap. $18^{2}$ : seul celui qui se sait petit devant Dieu, qui s'abandonne à lui et attend tout de lui, peut ne pas être une occasion de scandale pour ses frères (vv. 6-9), peut leur vouer une sollicitude sans limite (vv. 10-14), peut veiller à leur salut (vv. 15-20) et leur offrir un pardon inépuisable (vv. 21-35). Le débat sur la véritable grandeur, en resituant l'homme devant Dieu, lui apprend à rencontrer ses frères avec amour. Toutes les recommandations communautaires qui suivent s'enracinent et trouvent leur condition de possibilité dans l'authentique relation qui s'institue entre le disciple et son Dieu et qui consiste dans la conversion à la véritable grandeur prêchée par le Christ.

1 Avec BONNARD, Mt 268-269; GRUNDMANN, Mt 414; SCHWEIZER, Mt 336.

2 Point bien souligné par TRILLING, Is rael 109. 
Après avoir traité le rassemblement de l'Eglise, sa vocation à la foi et à l'obéissance - mais aussi sa condition de corpus mixtum -, après avoir montré comment cette assemblée pleine de contradiction était appelée à vivre communautairement l'enseignement d'amour de son Seigneur. il nous faut nous tourner maintenant vers un thème ecclésiologique qui, ces dernières années, est devenu un slogan : l'Eglise et le monde.

La première rencontre entre Jésus et ses compagnons culmine dans le "je vous ferai pêcheur d'hommes" $(4,19)$ et la dernière instruction que le Ressuscité adresse aux siens, déclare notamment : "Allez donc : de toutes les nations faites des disciples" $(28,19)$. L'Eglise est responsable des hommes qui vivent en dehors d'elle et chaque croyant est simultanément un témoin sous peine de faillir à sa condition. La petite péricope de Mt 5,13-16 nous permettra de développer cet aspect. Par le biais de $\mathrm{Mt} \mathrm{10,1.7-8}$, nous aborderons le problème du contenu de ce témoignage rendu devant le monde; nous constaterons alors qu'il s'inscrit dans une continuité essentielle avec celui du Jésus terrestre : il s'agit de la même proclamation et des mêmes actes charismatiques. Cette prédication en actes qui authentifie la parole prêchée a fait problème pour la communauté mt. Mt 17,14-20 nous permettra de mesurer l'ampleur de la crise ouverte, mais aussi de découvrir la solution qui lui est donnée par le rédacteur.

Comment les hommes vont-ils répondre à cette prétention folle de l'Eglise d'être le sel de la terre et la lumière du monde ? Comment vontils accueillir les disciples en chemin pour propager l'évangile du Royaume et en ériger les signes ? Le constat de Mt est clair et net : pas plus qu'Israël, le monde n'est disposé à recevoir son Seigneur. La souffrance et l'échec seront le lot des disciples en mission (Mt 10,17-25).

\section{Devenez ce que vous êtes (Mt 5,13-16)}

Entre les béatitudes et la déclaration programmatique du Christ $\mathrm{mt}$ sur 
le statut de sa prédication, Mt a inséré une petite péricope qui traite de la vocation des disciples à l'égard du monde. Il s'agit d'une instruction absolument originale. L'activité rédactionnelle ne réside pas simplement dans le choix d'un contexte paradoxal - les "pauvres" des béatitudes deviennent la lumière du monde ! - mais dans une composition minutieuse de l'ensemble. Leux logia de la source Q (Mt 5, 13 bc par. Lc 14, 34-35 ${ }^{1}$; Mt 5, 15 par. Lc 11, $33^{2}$ ) sont introduits à chaque fois par deux affirmations thétiques du rédacteur $(5,13 a \cdot 14 a)^{3}$. De plus, $14 a$ est commenté en 14b par un logion du Smt ${ }^{4}$. L'application parénétique du tout (v. 16), enfin, est également de la main du rédacteur ${ }^{5}$. Le morceau, dans son en-

1 La sentence sur le sel est connue des deux sources de Mt (cf. Mc 9, 50; Lc 14,34-35). Le premier évangile a retenu ici la leçon de Q. Etant donné que Lc combine les leçons de $M c$ et de $Q$, il est malaisé de reconstruire la version primitive de $Q$. Il n'y a néanmoins aucune raison

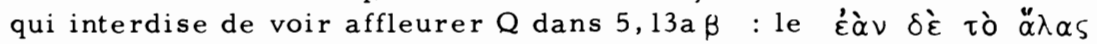
$\mu \omega \rho \alpha \nu \vartheta \tilde{\text { ñ }}$ (identité verbale avec Lc 14,34 ) est bien distinct du

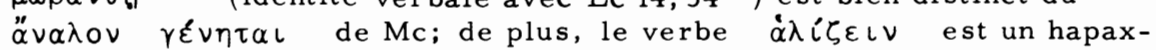
legomenon $\mathrm{mt}$. La conclusion de la sentence transmise par $Q$ - bien qu'elle soit formulée de manière fort différente par Mt et par Lc - est inconnue de Mc. L'idée essentielle en est que le sel devenu fade doit

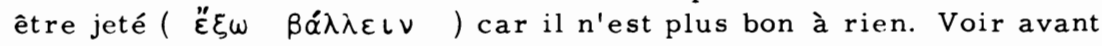
tout : SCHNACKENBURG 179-182; SCHULZ, Q 470-471.

2 La sentence sur la lampe est connue des deux sources de Mt (cf. Mc $4,21 ;$ Lc 8,$16 ; 11,33)$. Le premier évangile a retenu ici la leçon de $Q$ (par. Lc 11,33); la version mc comporte le motif du lit,inconnu de $\mathrm{Mt}$ 5,15 , et elie omet le motif de l'illumination de la pièce. La version $\mathrm{mt}$ est plus ancienne que celle de Lc : la 3 e personne du pluriel pour exprimer le "on", la forme parataxique, l'allusion à la demeure palestinienne sont autant d'arguments. Voir : SCHNACKENBURG 183-184; SCHULZ, Q 474-475.

3 Le consensus règne sur ce point, voir : BULTMANN, Trad 95-96. 100; CONZELMANN, art. $\varphi \tilde{\omega} \varsigma$, ThW 9, 335; DUPONT, Béatitudes I 85. 91; GRUNDMANN, Mt 136; KILPATRICK 17; SCHNACKENBURG 180. 183-84; SCHWEIZER, Mt 58; SCHULZ Q 470.

4 BULTMANN, Trad 100; DUPONT, Béatitudes I 83; SCHNACKENBURG, 183-184: ce logion est attesté par l'évangile de Thomas $(1,32)$.

5 Le v. est d'ailleurs composé à partir de termes présents dans les vv. 13-15. Soutiennent la provenance rédactionnelle: BULTMANN, Trad 100; CONZELMANN, art. $\varphi \tilde{\omega} \varsigma$, ThW 9, 335; DUPONT, Béatitudes I 85; EICHHOLZ 56; GRUNDMANN, Mt 140; SCHWEIZER, Mt 59. 
semble, est donc caractéristique de la rédaction mt.

La structure formelle de notre passage mérite attention, car, comme l'a relevé Suggs ', elle est analogue à celle de 16,17-19: pour présenter la mission des disciples et celle de Pierre, Mt use du même modèle ce qui suppose une similitude de condition -. Tout d'abord, un macarisme célèbre le bonheur des destinataires $\left(5,11-12^{2} ; 16,17\right)$; ensuite la nouvelle fonction attribuée par une déclaration en "tu es/vous êtes" $(5,13$ 15; 16, 18) est décrite: enfin, l'ordre de mission est donné $(5,16 ; 16,19)$.

La difficulté principale du passage consiste à donner une interprétation cohérente des images composites qu'il rassembie. La lumière (v. 14a), la lampe (v. 15), mais surtout la ville (v. 14b) et le sel (v. 13) ont suscité des débats considérables quant à leur signification dans le milieu vétérotestamentaire et juif palestinien ${ }^{3}$. Sans vouloir contester la valeur de ces études, il nous semble opportun, au niveau rédactionnel, de rappeler une règle établie notamment par Jeremias ${ }^{4}$. Le sel et la lumière, la ville et la lampe sont des images couplées. Dès lors, elles portent le même accent, elles expriment la même idée. Il n'est donc pas indiqué d'insister sur telle ou telle propriété du sel; il faut plutot se demander: quel sens y a-til à décrire conjointement la condition du disciple par les images de la lumière et du sel ? Semblablement, il n'est pas recommandé d'insister

1 Wisdom 120-127; voir aussi SCHWEIZER, Gemeinde 43.

2 L'hypothèse de SUGGS n'est pertinente qu'à condition de considérer la béatitude des persécutés $(5,11-12)$ et la péricope qui suit $(5,13-16)$ comme un tout.

3 Pour le sel, voir, p. ex. : BILL 1, 232-236; GRUNDMANN, Mt 137-138; HAUCK, art. "̋ $\lambda \alpha \varsigma$, ThW 1, 229; JEREMLAS, Gleichnisse 168-169. Pour la lumière: BILL 1, 236-238; CONZELMANN, art. $\varphi \tilde{\omega} s$, ThW 9, 335; SCHNACKENBURG 190-191. Pour la ville: JEREMIAS, Gleichnisse 215; von RAD, Die Stadt auf dem Berg, EvTh 8, 1948-49, 439447; SCHNACKENBURG, 191-192. Pour la lampe : JEREMIAS, Gleichnisse $120-121$.

4 Gleichnisse 89. Idée bien exploitée par EICHHOLZ 56. 
sur la valeur typologique de la ville située sur la montagne ${ }^{1}$, il faut comprendre cette image en continuité avec celle de la lampe.

Un deuxième point nous semble décisif pour l'exégèse. Tant l'image du sel que de la lumière sont présentées, sous l'angle de contrastes qui frisent parfois le grotesque ${ }^{2}$. Cette argumentation sert, de manière négative, à mettre en évidence la fonction spécifique de la lumière et du sel $^{3}$. Est-il pensable que le sel cesse de saler ? Est-il imaginable de construire une ville sur une montagne pour la faire passer inaperçue ? Est-il concevable d'allumer une lampe pour l'éteindre aussitôt ? La réponse qui s'impose est évidemment négative. Si - ce qui est chimiquement impossible - le sel devient fade ${ }^{4}$, il doit être jeté à la rue. Une ville située sur une montagne s'impose aux regards des voyageurs. Une lampe allumée illumine l'ensemble de la pièce ${ }^{5}$. La conclusion s'impose d'ellemême : le propre du sel est de saler, le propre de la lumière est d'éclai-

1 A la suite de l'art. de von RAD (Stadt), de nombreux exégètes (p. ex. JEREMIAS et SCHNACKENBURG) voient dans le v. $14 \mathrm{~b}$ une allusion au pélerinage eschatologique des peuples vers Sion. Cet arrière-fond est peu probable au niveau rédactionnel, car la ville et la lampe sont des images couplées; de plus, tant "ville" que "montagne" sont employées de manière indéterminée dans notre logion; enfin, la construction d'une. ville sur une colline est une affaire courante dans la Palestine du ler s. (dans le même sens : GRUNDMANN, Mt 139; SCHWEIZER, Mt 58).

2 Cf. EICHHOLZ 57-58; JEREMIAS, Gleichnisse 120-121, relève pour le v. 13 le contraste saler/jeter, pour le v. 15 allumer/éteindre.

3 SCHLATTER, Mt 146.

4 L'hypothèse d'un sel fade - et donc jeté - est tout-à-fait défendable. On sait que des barres de sel servant de catalyseur dans les fours étaient usées et donc jetées après environ quinze ans. Par ailleurs le sel domestique qui provenait des côtes maritimes était grevé d'impuretés et parfois impropre à la consommation (JEREMIAS, Gleichnisse 168-169). Le contexte (cf. vv. 14b. 15) recommande plutôt de voir dans le v. 14 une image provocante - comme si souvent dans la tradition synoptique -

5 Il faut penser à l'humble ferme palestinienne, composée d'une seule pièce, sans fenêtre, ni cheminée (JEREMIAS, Gleichnisse 120). 
rer. Quel est alors le propre du disciple pour qu'il soit disciple - puisqu' il est déclaré tout à la fois sel et lumière ${ }^{1}$ - ?

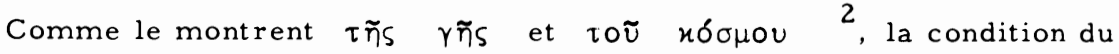
disciple est invisagée par rapport au monde. Qu'est-ce donc qui authentifie le disciple dans sa condition vis-à-vis du monde? Quelle fonction doitil assumer sous peine de déchoir ? Le sel qui assaisonne et conserve, la lumière qui est indispensable à la vie sont des éléments absolument nécessaires à l'existence humaine. "Nihil esse utilius sale et sole" (Pline l'Ancien : Hist. nat. 31.102) ${ }^{3}$. Aussi ces notions ont-elles été utilisées comme des métaphores privilégiées dans la tradition vétérotestamentaire-juive pour exprimer les réalités essentielles de la foi. Le sel est non seulement associé à l'Alliance et aux sacrifices, mais il désigne également la Tora ${ }^{4}$. Semblablement, l'expression "lumière du monde" peut dénommer aussi bien Dieu, que la Tora, le Temple ou Israël ${ }^{5}$. Sans vouloir presser le texte ou solliciter abusivement cet arrière-fond, l'interprétation qui s'impose nous semble être la suivante : les notions de sel de la terre et de lumière du monde se subsument dans la catégorie du témoignage. De même qu'Isräël avait reçu la vocation d'être le témoin de Yahvé parmi les hommes ${ }^{6}$, ainsi le disciple est sel de la terre et lumiè-

1 La tradition vétérotestamentaire-juive n'applique jamais l'image du sel à la condition du croyant (voir p. ex. GRUNDMANN, Mt 137; aporie relevée par BILL 1, 236). Par contre, l'application de la métaphore de la lumière à de grands personnages est connue (BILL 1, 237; CONZEL MANN, art. $\varphi \tilde{\omega}$, ThW 9, 335).

$2 \gamma \tilde{n}$ et $x \delta o \mu o s$ sont des expressions équivalentes qui désignent l'ensemble du monde habité, $\gamma \tilde{n}$ étant le pendant sémitique de xóopos (voir, p. ex. CONZELMANN, art. $\varphi \tilde{\omega} s$, ThW 9, 335; SCHWEIZER, Mt 59).

3 Cité par BONNARD, Mt 58.

4 Cf. HAUCK, art. $\ddot{\alpha} \lambda \alpha_{5}$, ThW 1, 229.

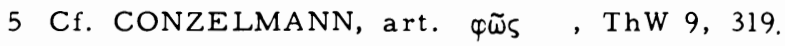

6 SCHNACKENBURG 190-194 interprète l'ensemble du passage à partir de l'opposition : véritable Israël - judaïsme incroyant. Sans nier que la communauté assume, dans notre passage, la vocation qui était celle d'Israël, il faut relever l'absence de toute note polémique explicite. 
re du monde dans la mesure où il est le porteur de la révélation divine parmi les hommes. Le v. 16 qui dit que "les belles oeuvres" des croyants permettent au monde de reconnaître et de confesser Dieu, authentifie de manière irrécusable cette exégèse.

Cette concentration de la condition du disciple dans le témoignage appelle deux précisions. Tout d'abord, comme l'illustrent les violents contrastes des vv. 13b. 14b.15, le témoignage n'est pas une fonction réservée à un groupe particulier de la communauté. Il appartient constitutivement à la condition du croyant ${ }^{l}$. Le nier équivaudrait à un gauchissement irrémédiable de la vie dans la foi. Le croyant qui refuse de témoigner est le sel fou ${ }^{2}$ qui mérite d'être piétiné, la ville placée sur une montagne qui se croit invisible, la lumière qui s'allume pour s'éteindre aussitôt. Le deuxième point qui mérite d'être souligné est que cette condition de témoin est présentée à la fois sur le mode indicatif ( $\dot{u \mu \varepsilon} \tau_{\varsigma} \quad \dot{\sigma} \sigma \varepsilon \quad \varkappa \tau \lambda$ ) et impératif ( $\lambda \alpha \mu \psi \alpha \dot{\tau} \omega)$. ${ }^{3}$ Nous retrouvons ici le schéma qui sous tend les béatitudes et qui établit une parfaite correspondance entre la grâce et l'exigence. Appelé par la seule grâce du Christ à une nouvelle condition - et singulièrement au bonheur eschatologique (vv. 3-12 !) -, le disciple est mis en situation de responsabilité radicale. Semblablement ici, la déclaration immotivée et gracieuse du Christ installant ses disciples comme témoins (vv. 13-15), précède l'exhortation du v. 16. Le croyant n'est pas mis en demeure d'accomplir une tâche qui le mènerait à l'état souhaité, mais de devenir ce qu'il est déjà.

1 Point bien souligné par EICHHOLz 59, à la suite de SCHLATTER, Mt 147.

2 On s'est souvent demandé si le $\mu \omega \rho \alpha \nu \vartheta \tilde{n}$ du v. 13 était une erreur de traduction car la racine araméenne "taphel" peut aussi bien signifier "devenir fade" qu" être fou". Il pourrait aussis'agir d'une incursion du fond dans l'image : le disciple qui n'est pas fidèle est un dis-

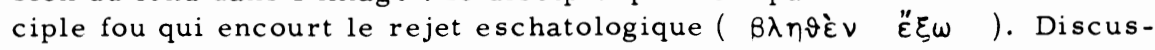
sion détaillée chez JEREMIAS, Gleichnisse 168.

3 Point développé par BONNARD, Mt 58 ("un être fondé sur la présence et l'activité du Christ"); EICHHOLZ 57; SCHWEIZER, Mt 59-61. 
Le problème majeur du v. 16 réside dans l'interprétation des "belles

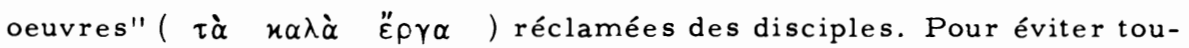
te erreur d'appréciation, il convient de souligner que la thématique est axée sur le témoignage - et non sur l'éthique -. A ceci, il faut ajouter deux remarques. D'une part, bien que l'image de la lumière au v. 14 s'applique à la personne du disciple et qu'au v. 16 elle décrive les oeuvres, il ne faut pas en déduire une distinction subtile car, pour l'anthropologie biblique, la personne est inséparable de son faire ${ }^{1}$. D'autre part - et ici la catégorie du témoignage trouve son application -, les "belles oeuvres" évoquées n'attirent pas l'attention sur le croyant qui les accomplit, elles ne sont pas le gage de sa perfection, mais elles renvoient à Dieu. Leur seule raison d'être et leuir finalité est d'amener les hommes à confesser leur Seigneur ${ }^{2}$.

Demandons-nous pour terminer comment le croyant fera ces "belles oeuvres" ou - ce qui revient au même - assumera sa condition de témoin. Nous avons souligné plus haut que le disciple était constitutivement un témoin sous peine de se renier. Il nous faut insister maintenant sur l'aspect inverse: est témoin, celui qui reste fidèle à sa condition de disciple telle qu'elle vient d'être développée dans les béatitudes. C'est le pauvre quant à son esprit, celui qui pleure, qui est doux, miséricordieux, pur de coeur, faiseur de paix, persécuté à cause de la justice, c'est ce disciplelà qui produit les belles oeuvres propres à interpeller le monde ${ }^{3}$. Il s'agit donc d'un témoignage profondément paradoxal où - pour employer un vocabulaire paulinien- la faiblesse est appelée à confondre la puissance, la folie à détruire toute sagesse.

1 Cf.CONZELMANN, art. $\varphi \tilde{\omega} 5$, ThW 9, 335. La même constatation peut déjà être faite au niveau de l'image : la lumière ne peut être séparée de sa source.

2 SCHWEIZER, Mt 59.61 souligne fortement cet aspect. Le verbe $\delta 0-$ $\xi \alpha \dot{\alpha} \zeta \omega$ a un sens profilé "reconnaître comme seul viai Dieu" (BONNARD, Mt 60).

3 Avec EICHHOLZ 59; GRUNDMANN, Mt 136. 


\section{Un témoignage en paroles et en actes}

Mt 5,13-16 a établi la vocation et la responsabilité de chaque disciple comme témoin, devant le monde. Nous aimerions commenter cette affirmation fondamentale, d'une part, en analysant les traditions d'envoi (chap. 10), d'autre part, en insistant sur la dimension charismatique de ce témoignage (Mt 17,14-20), car c'est là un trait original et trop peu remarqué de la théologie $\mathrm{mt}$.

\section{a) L'envoi en mission $(10,1.7-8)$}

Les vv. 1. 7-8 appartiennent à la première partie du discours du chap. 10. Pour composer les vv. 1-16, Mt s'est servi d'un matériel traditionnel extrêmement diversifié ${ }^{1}$ (Mc 6,7-13; Lc 9,1-15, respectivement 10, 3 11). Le commun dénominateur des traditions rassemblées est le thème de l'envoi, mais il est traité sous des aspects si variés que la structure des vv. 1-16 est composite ${ }^{2}$. L'exégèse des vv. 1.7-8 pris isolément est donc méthodologiquement possible. Avant de l'entreprendre, il convient de s'interroger sur le statut du chap. 10 dans son ensemble : s'agit-il d'instructions limitées à la seule mission pré-pascale ou sont-elles également valables pour les disciples des générations ultérieures ${ }^{3}$ ? Dans son état actuel, la recherche $\mathrm{mt}^{4}$ admet que le discours d'envoi dépasse le cadre

1 Pour le cataloguedes "douze", cf. encore Mc 3,13-19, Lc 6,13-16. Voir le tableau d'ensemble dressé par KLOSTERMANN, Mt 83.

2 HAHN, Mission 34. Le plan des vv. 1-16 peut être élaboré comme suit : v. 1 : habilitation des douze au miracle; vv. 2-4 : catalogue des douze; vv. 5-6: aller vers les brebis perdues d'Israël; vv. 7-8: habilitation à la prédication et aux miracles; vv. 9-10: le bagage des envoyés; vv. 11-14: le comportement dans les maisons et dans les villes; $v$. $15: 1$ le thème du jugement; v. 16 : le logion de l'envoi parmi les loups.

3 Voir sur ce point déjà nos pp. 33-34.

4 BARTH 93; BORNKAMM, Enderwartung 16; BULTMANN, Trad 155; GRUNDMANN, Mt 284; KLOSTERMANN, Mt 84; LUZ, Jünger 143146; SCHMID, Mt 176; SCHWEIZER, Gesetz und Enthusiasmus 57-58. 
de l'activité galiléenne de Jésus pourtant encore bien visible en 10,5-10. Selon Schlatter ${ }^{1}$, le chap. 10 donne à Mt "l'occasion de décrire toute l'activité apostolique jusqu'à la parousie" et Grundmann ${ }^{2}$ renchérit dans ce sens en écrivant : "Il y va ... purement et simplement de l'envoi et de la destinée des messagers de la communauté". Cependant, cet avis n'est pas entièrement partagé par les exégètes qui discernent dans le premier évangile une tendance historicisante se traduisant par la mise en place d'une histoire du salut; pour eux ${ }^{3}$, la valeur exemplaire du discours d'envoi n'apparaît que dès le v. 17 alors que les 16 premiers vv. - d'ailleurs en opposition avec 28,16-20 - ne concernent que l'époque pré-pascale. A cette interprétation (étayée par les difficiles vv. 5 et $6^{4}$ ) qui voit dans Mt 10,1-16 le reflet d'une époque passée et sans rapport immédiat avec le temps de l'Eglise, il faut opposer deux arguments. Premièrement, dès le début du discours d'envoi, Mt est avant tout animé par un projet thématique; le fait qu'il fond en une seule composition les traditions de Mc $(6,6 b-13)$ et de $Q(L c 10,2-12)$, montre bien que son intérêt n'est pas historique "au sens moderne" et que la distinction entre les traditions pré et post-pascales ne lui tient pas fondamentalement à coeur. De plus, le rédacteur n'introduit aucune césure explicite entre les vv. 16.17 qui justifierait une telle distinction entre les vv. 1-16 et 17-42 ${ }^{5}$. Deuxièmement, il est intéressant d'inventorier les éléments que Mt a retranchés de ses

1 SCHLATTER, Mt 337.

2 GRUNDMANN, Mt 284.

3 Cf. notamment STRECKER, Weg 134.196; WALKER, 114-118.

4 Il est clair qu'en continuité avec 9,35, l'identité de la mission des disciples avec celle du Christ, exige qu'ils soient envoyés d'abord au seul Israël (vv. 5-6). Mais cette note historisante rend-elle l'ensemble des instructions missionnaires caduques pour l'époque post-pascale ? Nous ne le pensons pas, car cette tendance est fortement contrebalancée par les autres arguments que nous évoquons.

5 Dans le même sens, LUZ, Jünger 146. 
deux sources. Le rédacteur a supprimé, d'une part, le récit de l'exécution de l'ordre d'envoi (Mc 6,12-13 par. Lc 9,6), d'autre part, la description du retour des envoyés (Mc 6,30 et Lc 9,10;10,17-20). La tendance de l'évangéliste est donc d'effacer les circonstances concrètes liées aux traditions de l'envoi. Bien loin de faire oeuvre "historicisante", Mt - par cette double suppression - "brise la situation d'un alors historique situé durant l'activité terrestre de Jésus $1^{\prime \prime}$. Le discours d'envoi mt n'est donc pas clos sur lui-même, mais ouvert vers l'avenir, et il décrit une possibilité présente de la communauté mt. La grâce dont les douze furent l'objet, et l'exigence sous laquelle ils furent placés, demeurent valables pour tout disciple dépositaire de la Parole ${ }^{2}$. Ce présupposé contextuel est indispensable à l'exégèse de 10,1.7-8.

Le v. 1 a son parallèle en Mc 6,7. Mis à part trois retouches sans

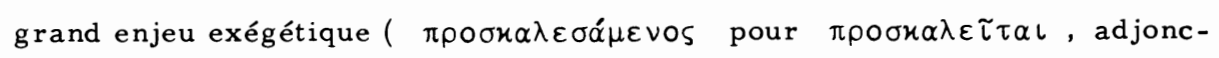

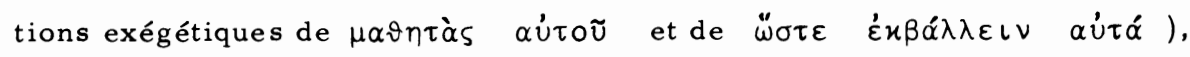
Mt opère deux changements significatifs. Premièrement, il supprime la

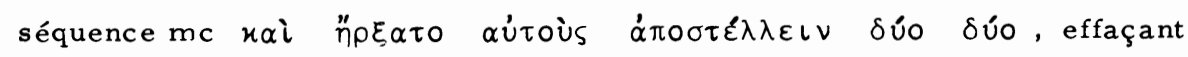
ainsi une circonstance marquant la réalisation historique du discours $d^{\prime}$

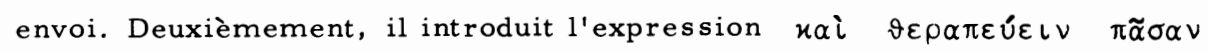

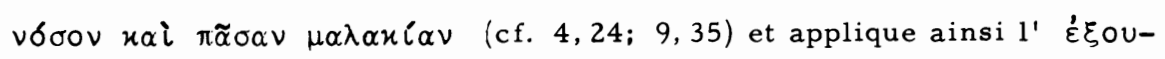
oí $\alpha$ conférée par Jésus à toutes les maladies alors que Mc ne parle que

1 BARTH 93-94; LUZ, Jünger 146, déclare "Die Gebote Jesu sind aber grundsätzlich gültig"; il applique ce jugement également à 10, 1.7-8 (voir Jünger 146, note 23 a).

2 BARTH 94 : "... Die Aussendungsrede spricht nun von Aussendung der Jünger überhaupt. Mehr noch, es geht nicht um Aussendung besonderer Missionare, sondern Aussendung und Verfolgung sind für das Jüngersein überhaupt wesentlich $(10,24 \mathrm{f}$.). Christ sein heisst ausgesendet sein, unterwegs mit dem Wort, das Jesu eigenes Wort ist, unter einem zerschundenen und zerschlagenen Volk, das den Boten hasst und verfolgt". Thèse inverse chez STRECKER, Weg 196. 
d'exorcismes. Les vv. 7-8a sont vraisemblablement d'origine rédactionnelle et n'ont pas de parallèles immédiats. Ils contiennent cependant deux motifs également présents en Lc 9,2 et 10,9. A son habitude, Mt comment la double mission des disciples par un logion (8b) dont on ne connaît pas l'origine ${ }^{1}$.

9, 35-38 avait posé le problème de la mission dans son urgence et sa nécessité. En 10,1s, Jésus appelle ${ }^{2}$ ses douze disciples ${ }^{3}$ pour leur donner les instructions nécessaires à l'accomplissement de cette tầche. A travers, eux, ce sont les chrétiens de l'église mt qui sont enseignés. Le discours du Christ $\mathrm{mt}$ commence par une habilitation. La comparaison avec Mc montre d'ailleurs que tout l'accent de notre v. porte sur ce point. Concrètement, Jésus habilite les douze à la guérison des malades. Le verbe

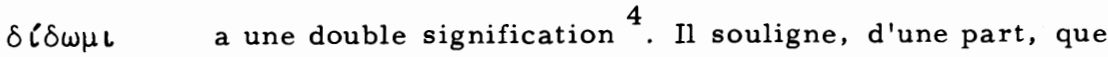
l'autorité des disciples est fondée en lui seul; leur relation avec Jésus est la source exclusive de leur $\dot{\varepsilon} \xi 0 v \sigma i ́ \alpha$. Mais, d'autre part, cette autorité n'a de sens que dans le cadre de la mission décrite, elle est une autorité au service de la prédication missionnaire et non pas une qualité enrichissant quelques privilégiés (cf. la parénèse du Sermon sur la Montagne!).

Alors que Mc limite l'ȩ̧́ovoía des disciples à l'exorcisme, Mt l'étend

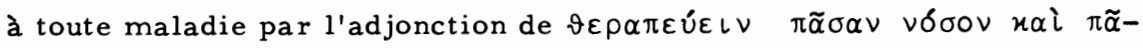
$\sigma \alpha \nu \mu \alpha \lambda \alpha \varkappa\lceil\alpha \nu$, expression rédactionnelle déjà utilisée en 4, 23 et 9,35,

1 Analyse détaillée chez SCHULZ, Q 406-407.

2 Le verbe $\pi p \circ \sigma k \alpha \lambda \varepsilon \tau \nu$ ( SCHMIDT, ThW 3, 502) n'est pas un terminus technicus de l'élection. Ici, comme en 15,32 et 20,25, le Christ fait venir ses disciples pour les instruire (cf. aussi 18,2 et 15,10). Mt présuppose l'existence du cercle des douze sans jamais relater son institution.

$3 \Delta \omega ́ \delta \varepsilon x \alpha$ (appliqué aux $\mu \alpha \vartheta \eta \tau \alpha i$ ) apparaft en 10,1 pour la première fois dans l'évangile selon $M t$. Pour lui, l'identification des douze avec les disciples va de soi et n'a pas d'accent particulier (LUZ, Jünger 142-143).

4 BONNARD, Mt 143. 
mais à propos de Jésus ${ }^{1}$. En utilisant les mêmes mots pour décrire l'oeuvre du Christ et celle des disciples, l'évangélistes veut souligner que

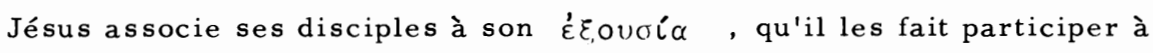
sa toute-puissance pour qu'ils puissent assumer la même tầche que lui ou - si l'on préferre - la développer et la perpétuer. Il élève donc ses compagnons au rang de collaborateurs jouissant des mêmes pouvoirs et astreints au même travail ${ }^{2}$. Bien que soumis au Christ et dépendant de lui, le disciple est son égal sitôt qu'il annonce et manifeste le Royaume qui s'approche.

Les vv. 7-8 confirment la thèse défendue au v. 1 : par l'envoi en mission, le disciple devient le collaborateur de Jésus et partage sa toute-puissance. A l'exemple du Christ mt qui est le "messie de la parole et des actes", le disciple en mission proclame le Royaume qui s'approche et en érige les signes.

Le v. 7 définit le contenu de la prédication des disciples en parfaite similitude avec celle de Jésus : de même que Jésus annonce la proximité

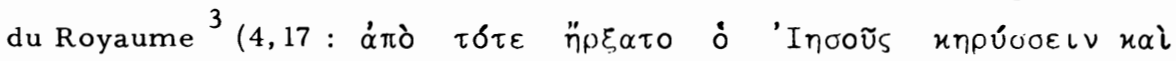

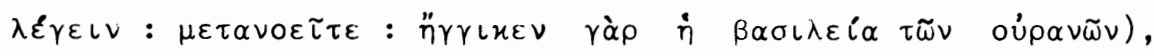

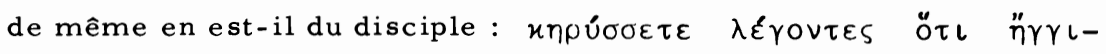

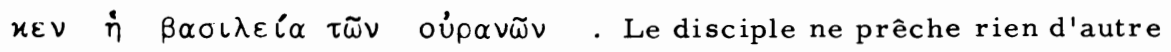

1 La répétition des formules rédactionnelles stéréotypées est une technique de mise en évidence propre à Mt et précieuse pour son interprétation.

2 HELD 237-238, suivi par LUZ, Jünger 156, et SCHWEIZER, Gesetz und Enthusiasmus 58.

3 Nous n'entreprenons pas ici le détail de l'exégèse de l'expression

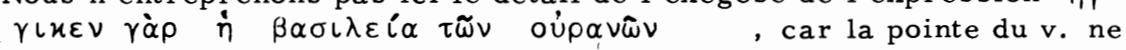
consiste pas dans le contenu de ces termes, mais dans la similitude du message de Jésus et de ses disciples. Néanmoins, deux remarques :

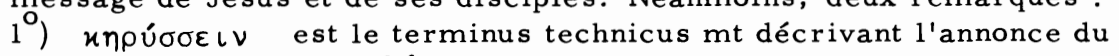
Royaume alors que $\delta \iota \hat{\alpha} \sigma x \varepsilon \iota v$ est utilisé pour désigner la proclamation de la volonté de Dieu (cf. BORNKAMM, Enderwartung 35). $2^{\circ}$ ) La seule différence notable entre 4,17 et 10,7 est l'absence de l'appel à la repentance en 10,7 : nous ne pensons pourtant pas qu'il y ait là matière à discussion, car la proclamation de la proximité du Royaume est par 
que ce que le maître a dit; il est habilité à annoncer ce que Jésus a proclamé, et cela seulement. Sa prédication, si elle est fidèle, se confond avec celle du maître. Cette identité de la prédication du Christ et de celle de ses disciples rejoint le grand thème de la normativité de l'enseignement du Jésus terrestre pour l'Eglise, particulièrement mis en évidence en 5,17-20 et $28,16-20^{\mathrm{l}}$. Cette convergence souligne la profonde cohérence de l'évangile sur ce point.

L'énumération du v. 8a, propre à $\mathrm{Mt}$, a ceci d'intéressant qu'elle reprend toute la gamme des miracles décrits dans les chapitres 8-9 à l'exception de la guérison des aveugles. Mais cette omission est sans doute

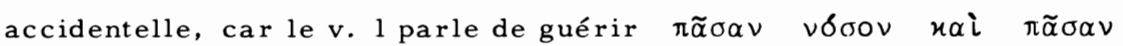
$\mu \alpha \lambda \alpha \varkappa \iota_{\alpha} \nu^{2}$. Ici encore la similitude entre l'activité de Jésus et celle des disciples est frappante, puisque ces derniers sont habilités et invités à faire précisément ce que Jésus a accompli dans les chap. 8-9 ${ }^{3}$. Le souci de Mt dans les v. 1.7-8 apparaît clairement : établir une identité complète entre la mission de Jésus et celle des disciples aussi bien au niveau

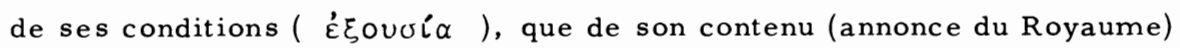
et de ses modalités (prédication et miracle).

Mt - comme si souvent dans son évangile - introduit un logion $(10,8 \mathrm{~b})$ qui commente ce qui précède. Le thème en est la gratuité ( 2 fois $\delta \omega p \varepsilon-$ $\left.\alpha_{\alpha} \nu\right)$ : puisque les disciples ont reçu gratuitement la bonne nouvelle du Royaume d'abord, l'ordre et l'habilitation à la mission ensuite, ils ont aussi à donner gratuitement. Ce topos ${ }^{4}$ de la gratuité de la Parole de Dieu, de sa non-utilisation à des fins pécuniaires ou personnelles, est bien connu du monde judéo-chrétien. Ici il nous semble - de par le contexte - revêtir le sens suivant : s'il est vrai que la mission du maître et du disciple

elle-même une invite à la repentance. Son omission n'introduit pas une différence de contenu (ainsi STRECKER, Weg 195-96; autrement BORNKAMM, Enderwartung 15 et GRUNDMANN, Mt 290).

1 Voir nos chap. 3 et 4.

2 SCHWEIZER, Gesetz und Enthusiasmus 54-55.

3 Voir BILL 1, 563 et SCHULZ, Nachfolgen und Nachahmen 30. 
est identique, elle l'est par grâce, et non à cause des mérites ou des qualités des disciples. Leur rappeler la gratuité de l'annonce du Royaume, c'est leur rappeler qu'eux aussi l'ont reçu gratuitement et gracieusement de celui qui les élève maintenant au rang de collaborateurs. L'identité maître-disciple prend alors son véritable sens.

\section{b) La crise des charismes (Mt 17,14-20)}

Institué témoin devant le monde par son Seigneur, le croyant est habilité à une prédication en paroles et en actes. Nous avons déjà abordé le problème de la rectitude de l'enseignement chrétien en relevant, d'une part, l'accent mis par la christologie mt sur la valeur normative de la prédication du Jésus terrestre 1 , en analysant, d'autre part, les "disputes confessionnelles" qui ont agité l'église $\mathrm{mt}^{2}$. Nous ne reprenons donc pas la question de la $\delta \iota \delta \alpha \chi \eta^{\prime}$ mt, mais nous nous concentrons sur la dimension charismatique du témoignage chrétien. A cet égard, deux faits méritent d'être relevés. Mt connaît tout aussi bien une distorsion des charismes (cf. 7, 21-23) que leur possible disparition ${ }^{3}$. Face à cette crise vécue par la communauté $\mathrm{mt}$, Mt prend clairement position. L'épisode de l'enfant épileptique (17, 14-20) lui permet de souligner la nécessité des charismes, et d'en discerner théologiquement la condition de possibilité.

La source de Mt 17,14-20 ${ }^{4}$ est Mc 9,14-29 ${ }^{5}$, mais les versions s'avèrent si différentes qu'il n'est pas inutile de faire un inventaire des diver-

1 Voir nos chap. 3-5.

2 Voir notre chap. 7.

3 Voir l'état de la question chez LUZ, Jünger 155.

4 La recension de Mc 9, 29 en Mt 17, 21 par plusieurs manuscrits importants est probablement secondaire. Elle est due à la volonté d'harmoniser $\mathrm{Mt}$ et Mc, mais s'oppose au dessein théologique de Mt.

5 Mc 9,14-29 est une combinaison de deux histoires de miracles (BULTMANN, Trad 225-226) : la première décrit l'incapacité des disciples à guérir un malade et rappelle la plainte de Jésus sur l'incrédulité de sa génération (Mc 9,14-19); la seconde - axée sur le dialogue entre Jésus 
gences. Mt résume dans le seul v. 14 Mc 9,14-17a. Il élimine la querelle des disciples avec les scribes, l'étonnement de la foule et la question de Jésus. Toute l'attention est fixée sur la venue implorante d'un homme à

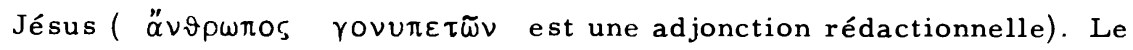
v. 15 a reproduit dans une terminologie entièrement originale Mc 9,17b. 1

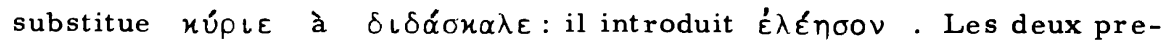
mières modifications ont une signification théologique. Le v. $15 \mathrm{~b}$ fait une brève description de la maladie sur la base de Mc 9,22a. Mc 9, 18. 20 qui sont consacrés à la peinture de la maladie sont supprimés. Mt n'entend donc pas s'attarder sur ce point. Le v. 16 reprend dans d'autres termes

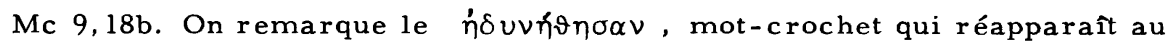

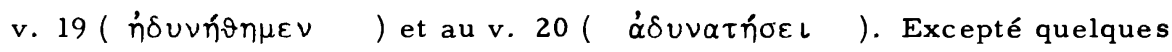
améliorations de style, le v. 17 est le parallèle de Mc 9,19. Fait beaucoup plus important, Mt ne retient pas du tout le dialogue entre Jésus et le père - entretien axé sur la foi (vv. 21-24) -. Il passe immédiatement à la guérison. Le v. 18 conte l'acte d'exorcisme avec une extrême sobriété. Le luxe de détails donnés par Mc 9,25-27 est évité. Le miracle comme tel n'est pas mis en valeur. La conclusion $m t$ du récit ( $v$. 20) frappe par son originalité; elle est vraisemblablement rédactionnelle. Le v. 20a fait

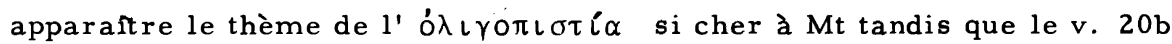
reprend un logion de la source $Q$ (par. Lc 17,6), célébrant la puissance de la foi ${ }^{l}$.

Cette rapide comparaison montre que la recension de Mt se signale par sa brièveté. L'abréviation est devenue un moyen d'interprétation ou, si l'on préfère, une technique permettant de "fixer l'attention sur l'essentiel" ${ }^{2}$. L'absence de tout développement sur la maladie de l'épileptique,

et le père du malade - dévoile le paradoxe de la foi incroyante (Mc 9, 20 27). A ces deux miracles s'ajoute un débat d'école (Mc 9, 28-29) qui explique l'impuissance des disciples et qui constitue à ce titre le point culminant de la péricope.

1 Analyse littéraire détaillée chez SCHULZ, Q 465-467.

2 BONNARD, Mt 278. 
puis sur sa guérison, la suppression du dialogue entre Jésus et le père de l'enfant font que le récit se concentre principalement sur l'échec des disciples et son explication ${ }^{1}$. Cette hypothèse est soutenue par la double série de mots-crochets qui scelle la cohérence du texte et trahit son inten-

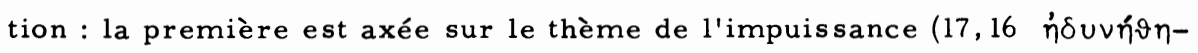

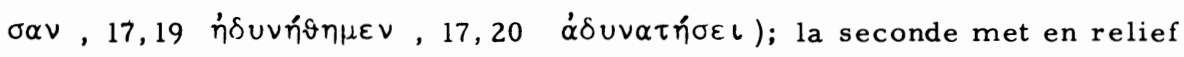

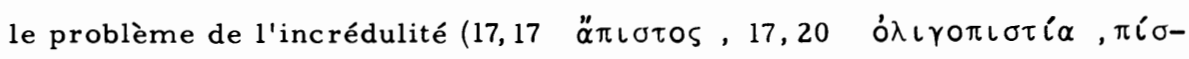
$\tau \iota \varsigma$ ). Ainsi l'histoire du miracle passe à l'arrière-plan; le récit a son point de départ dans l'échec des disciples et il est tout entier orienté vers le dialogue final qui illustre comment cette défaillance peut être dépassée dans la foi.

On ne s'étonnera dès lors pas que la place prépondérante du dialogue final dans l'économie du récit influe sur la détermination de sa forme littéraire. Il s'agit moins d'un miracle et plus d'un enseignement $\overline{2}$. L'auditeur doit moins s'émerveiller de l'extraordinaire puissance de Jésus et plus se reconnaître dans le disciple acculé à l'échec, mais appelé à la foi qui, seule, permet de dépasser son impuissance ${ }^{3}$. Si telle est l'intention fondamentale de la recension mt, comment cette thématique est-elle développée au fil de la péricope?

Mt a considérablement allégé la mise en scène du récit (v. 14). L'attitude de l'homme qui vient à Jésus est décrite par une expression stéréo-

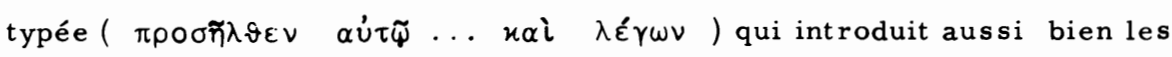
récits de miracles que les paradigmes mt. En placant le titre $\chi u ́ \rho \iota$ dans la bouche du père, Mt le décrit d'emblée comme un croyant qui voue une confiance absolue au Christ, et rend ainsi inutile le dialogue de Mc

1 HELD 178.

2 HELD ibid. Voir aussi BULTMANN ( Trad 65) qui classe ce récit dans les apophtegmes et DIBELIUS (Formgeschichte 77 ) qui le range dans les paradigmes.

3 SCHLA T TER (Mt 534) écrit : "Das Ziel dieser Erzählung liegt ... in der Aufrichtung der Jünger zum Glauben". 
9, 22-24 ${ }^{1}$. Lc motif rédactionnel de la proskynèse appuie cette hypothèse.

La demande adressée par le père à Jésus (v. 15) a la forme d'une priè-

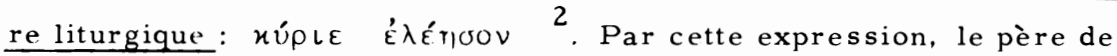
l'épileptique confesse son impuissance et demande un geste concret de secours; en donnant à cette requète une forme liturgique, Mt veut faire comprendre à son église que cette prière est encore une possibilité actuelle pour elle ${ }^{3}$, possibilité par laquelle elle rencontre son Seigneur élevé.

Mt caractérise la maladie de l'enfant par un terme technique de la mé-

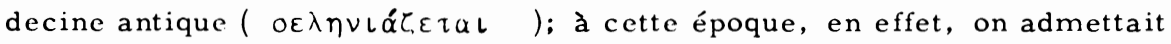
que la fréquence des crises d'épilepsie était fonction des phases lunaires ${ }^{4}$. La description de l'épilepsie qui suit, est extrêmement sobre et évite toute motivation démoniaque.

L'élimination que Mt a exercée sur sa source, ne touche pas le v. 16 qui revêt ainsi une importance certaine aux yeux du rédacteur. Il y est question - sous forme d'accusation - de l'impuissance des disciples à guérir l'enfant qui est présenté maintenant à Jésus. Cette impuissance n'est pas normale, car, selon 10,1, Jésus a conféré à ses disciples le

1 Avec GRUNDMANN, Mt 406; HELD 180; LOHMEYER, Mt 270. On remarquera non sans intérêt qu'une fois encore, les modèles de la foi dans l'évangile ne sont pas empruntés au cercle des disciples, mais à des personnages périphériques. Il ne peut d'ailleurs en être autrement si le disciple est à la fois le compagnon historique de Jésus et la figure du croyant. Car, comme tel, il n'est pas modèle de foi, mais appelé à la foi. Le choix des exemples de la foi à l'extérieur du cercle des adhérents, a donc un rôle parénétique.

2 Ceci est un trait constant des requêtes dans les récits de miracles $\mathrm{mt}$ : ainsi on retrouve le titre $x u ́ p \iota \varepsilon$ en $8,2.6 ; 15,22 ; 17,15 ; 20,30.31$ alors que Mc ne l'utilise jamais dans ce contexte et Lc une seule fois $(5,12)$; $\dot{\lambda} \hat{n} \eta$ ov comme appel au secours miraculeux de Jésus apparaît en 9,$27 ; 15,22 ; 17,15 ; 20,30.31(15,22$ et 17,15 sont rédactionnels). Cf. HELD 253.

3 HELD ibid.

4 L'amulette des épileptiques s'appelait d'ailleurs $\sum \varepsilon \lambda \eta \nu i ́ s$ (GRUNDMANN, Mt 406. 
pouvoir de guérison et d'exorcisme. Comme le confirme le v. suivant,

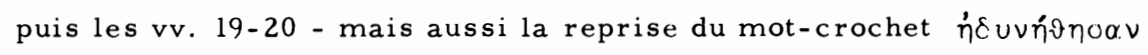
aux vv. 19-20-, cet échec est le véritable problème auquel est consacrée notre péricope alors que l'activité miraculeuse du Christ comme telle a une fonction secondaire.

Cette défaillance des compagnons historiques du Christ n'est pas seulement un accident isolé et passé. Elle évoque sans doute une incapacité douloureusement ressentie par l'église $m t^{l}$. Une fois encore, les disciples sont l'image de la communauté croyante ${ }^{2}$. A cet égard, on remarquera - et ce n'est pas qu'une coïncidence - que l'échec des disciples se produit en l'absence de Jésus, absence qui figure la situation post-pascale (même phénomène en $8,23-28 ; 14,22-33$ ).

A l'accusation du père formulée au v. 16, répond la plainte de Jésus (v. 17). Les termes de cette plainte rappelle le lyrisme vétéro-testamentaire $(\stackrel{\boldsymbol{\omega}}{\omega}+2$ fois $\check{\varepsilon} \omega \varsigma$ ) exprimant l'indignation prophétique, càd la douleur du juste voyant ses semblables refuser le salut divin ${ }^{3}$.

Dans l'analyse de cette plainte, la difficulté principale consiste à savoir à qui s'applique le terme $\gamma \varepsilon v \varepsilon \alpha ́ \alpha$. Il ne peuts'agir du père, décrit comme étant croyant, ni de la foule qui n'est pas en cause ici (juste mentionnée au v. 14). C'est l'impuissance des disciples qui provoque la plainte de Jésus; d'ailleurs au v. 20, cette impuissance est expliquée par

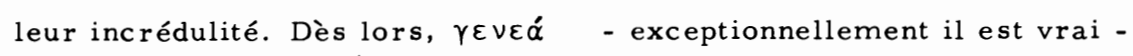
désigne les disciples 4 . Notre v. s'intègre donc au débat ouvert au v. 16 et conclu au v. 20.

1 LUZ, Jünger 155; SCHWEIZER, Gesetz und Enthusiasmus 58.

2 BONNARD, Mt 259.

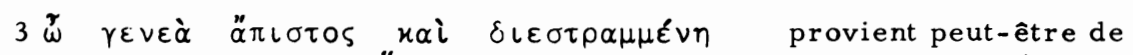
Dt $32,5 \mathrm{t}$ andis que $\check{\varepsilon} \omega S \pi \delta \tau \varepsilon$ évoque le fameux מת (cf.

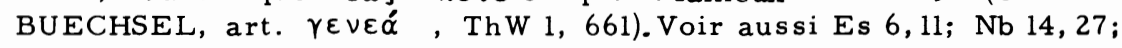
Es 65,2 .

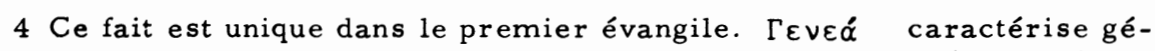
néralement, chez Mt, les Juifs dans un sens dépréciatif (cf. 11, 16; $12,39 ; 16,4 ; 12,41.42 .45 ; 23,26 ; 24,34)$. Semblablement, le $\mu \alpha \vartheta \eta \tau \hat{n} \varsigma$ 
Notre option, en défendant la cohérence du passage, ouvre la voie à une exégèse homogène au contexte. Par sa plainte, Jésus fournit une première explication de l'échec des disciples : leur incrédulité ( ä

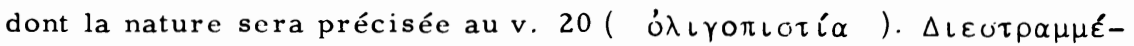
$\eta$ appartient à la terminologie biblique du péché et désigne une distorsion, une perversion du coeur de l'homme.

Le $\tau \delta \nu$ du v. 16 : ce que les disciples n'ont su faire par incrédulité, Jésus va l'accomplir. Le miracle en lui-même (v. 18) est raconté avec une sobriété proche de la stylisation ${ }^{1}$ : un ordre de Jésus, son exécution, la constatation de la guérison. Cette manière de procéder démontre - si besoin est que le narrateur passe rapidement sur les points de l'histoire qui ne servent pas directement son propos, qu'"'il a hâte d'en revenir à son enseignement sur la foi ${ }^{2}$ ".

Le v. 19 introduit le dialogue vers lequel tend toute la péricope. La formulation stéréotypée du v. 19a se retrouve en effet au début des débats d'école et des récits de controverses; elle nous indique qu'ici commence l'instruction proprement dite. Le succès de Jésus permet aux disciples qui réapparaissent en pleine lumière de s'interroger fondamentalement sur la signification de leur défaillance.

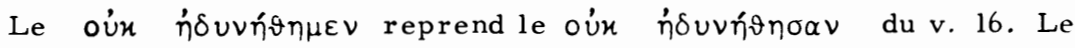
$\delta \iota \grave{\alpha} \tau \grave{l}$ montre que l'échec subi par les disciples, n'est pas ressenti comme normal. Les traditions de l'envoi (chap. 10) indiquent en effet que la mission des disciples est semblable à celle du Christ et que le Christ leur donne le moyen de l'accomplir. Si donc les disciples ${ }^{3}$ échouent dans

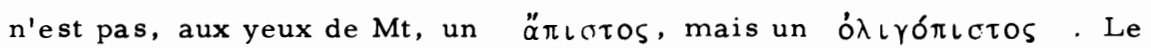
contexte cependant impose cette interprétation (avec HELD 180-181).

1 LOHMEYER, Mt 271.

2 BONNARD, Mt 260.

3 Le $\dot{n} \mu \varepsilon \tau \varsigma$ est un "nous" communautaire dans lequel se reconnaît la communauté mt (BONNARD, Mt 261; SCHLATTER, Mt 534). 
ce à quoi ils sont habilités par leur maître, c'est que leur relation avec le Christ n'est plus correctement comprise, ni vécue. Leur impuissance face à l'épileptique est l'indice d'une crise plus grave qui affecte leur rapport avec Jésus. Leur question adressée à Jésus montre leur désir de surmonter leur défaillance.

La réponse de Jésus ( $v$. 20) - rédactionnelle - se divise en deux parties. Le maître explique tout d'abord la raison de l'échec des disciples, puis il ajoute une parole paradoxale sur la grandeur de la foi.

L'échec des disciples s'explique par leur ó $\lambda \iota \gamma o \pi \iota \sigma \tau \iota \alpha$. Ce terme, typiquement $\mathrm{mt}$ (cf. 6,$30 ; 8,26 ; 14,31 ; 16,8$ ), atteste que 1 'impuissance des disciples n'est pas due à une maladresse ou à une ignorance, mais à une crise fondamentale de leur relation avec Jésus 1 . Concrètement,

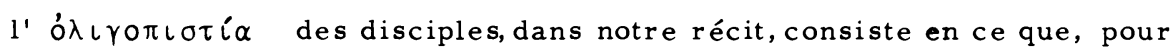
eux, l'évidence de la maladie de l'enfant est plus forte que leur pouvoir de guérison. La réalité dernière n'est plus la foi, mais la maladie qui se transforme ainsi en une fatalité aliénante.

A cette élucidation critique de l'échec des disciples, le Christ mt ajoute une lecon sur la puissance merveilleuse de la foi. Ce logion apparaît quatre fois dans la tradition synoptique ${ }^{2}$ (Mc 11, 23 par. Mt 21, 21; Mt 17, 20 par. Lc 17,6). Mt reprend ici la forme $Q$ du logion, mais il lui apporte deux importantes modifications si bien que sa version est secondai-

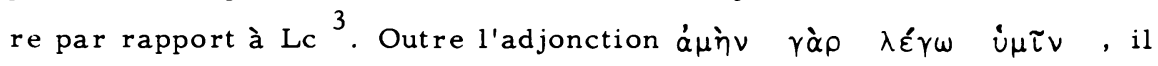
substitue - probablement sous l'influence de Mc 11,23 - au motif du mûrier planté dans la mer celui du déplacement des montagnes. L'image rappelle une tournure proverbiale courante dans le monde juif ${ }^{4}$ et ren-

1 Voir supra nos pp. 239 ss.

2 Pour l'analyse, voir HUNZINGER, art. oíva $\pi$, ThW 7, 287-289; SCHULZ, Q 465-467.

3 Ainsi, p. ex., LUEHRMANN, Logienquelle 108-109; SCHULZ Q ibid.

4 BILL 1, 759. 
force le paradoxe ${ }^{1}$.

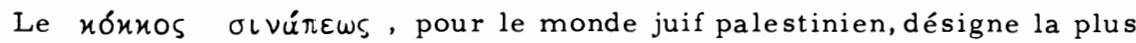

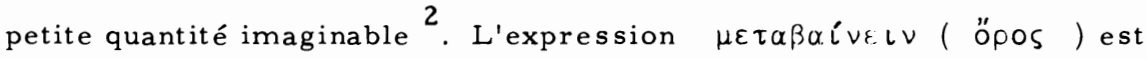
également enracinée dans le monde juif, où l'image signifie : rendre possible quelque chose d'impossible ${ }^{3}$. Le sens du logion est alors le suivant : à la plus petite foi imaginable est déjà accordée la plus grande promesse. L'aide prodiguée par Dieu ne dépend pas de la grandeur de la foi. La foi, aussi petite soit-elle, remet toutes choses à Dieu et le laisse agir. Elle est dès lors capable de l'impossible ${ }^{4}$, car ce qui est inaccessible à l'homme est soumis à Dieu. Cette foi trouve son expression achevée dans la prière.

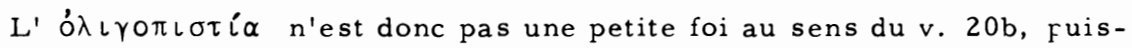
que la plus petite foi réussit l'impossible alors que précisément les disciples ont échoué. Elle est incrédulité puisque les disciples dans les situations critiques cèdent aux évidences du monde, mais aussi fidélité puisque, dans le reniement même, les disciples continuent de suivre le Christ. La réponse de Jésus a dès lors un double but : dénoncer l'erreur coupable de ses adhérents et leur montrer le moyen de la surmonter en leur proposant

1 L'opposition $m t$ grain de sénevé/montagne est plus frappante que le couple grain de sénevé/mûrier bien que le sens final soit le même.

2 Cf. Nid 5, 2; Nazir 1,5; BILL 1, 669. Même interprétation chez BONNARD, Mt 261; KLOSTERMANN, Mt 145; HUNZINGER, art. cit. 289; SCHLATTER, Mt 534. L'accent n'est donc pas mis sur la fragilité et la faiblesse du grain de sénevé (ainsi GRUNDMANN, Mt 407, et Michel art. nóxnos, ThW 3, 811), ni sur le fait qu'il ait été semé par Dieu (GRUNDMANN, Mt ibid), ni enfin sur l'opposition entre son inapparence actuelle et son épanouissement eschatologique (LOHMEYER, Mt 273).

3 Cf. BILL 1, 759. Le "déplacement des montagnes" est également un topos eschatologique (cf. Es 40,4ss; 49,11) décrivant la suppression de tous les obstacles dressés devant le peuple de Dieu (cf. BONNARD, Mt 261; SCHLATTER, Mt 535). Cette signification est étrangère à notre contexte.

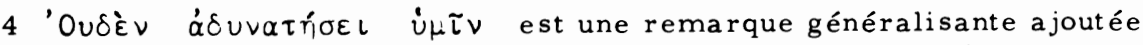
par le rédacteur (BULTMANN, Trad 98; BONNARD, Mt 26l; GRUNDMANN, Mt 407; SCHULZ, Q 467). 
l'exemple d'une foi comme un grain de sénevé.

Concluons : l'épisode de l'enfant épileptique a donné au rédacteur du premier évangile l'occasion d'interpréter la crise que traverse sa communauté. En élucidant la carence charismatique vécue par les croyants à l'aide de la notion d'incrédulité, il révèle la gravité du mal, mais aussi sa racine. A ses yeux, la question fondamentale est celle de la foi. Le disciple qui voue une confiance illimitée à son maître, partage sa toutepuissance; confession authentique du Kyrios et don des charismes vont de pair. Le disciple qui échoue, est, au contraire, celui qui doute de son Seigneur. En définitive, ce qui est en cause, ce n'est pas une habilitation particulière du croyant pour la mission, mais sa relation avec le Christ. L'Eglise $m$ t est ainsi appelée à s'interroger sur le fondement de son existence.

\section{Souffrance et échec (Mt 10,17-25)}

Si Mt décrit en toute clarté la mission de chaque croyant dans le monde, il ne reste pas muet sur l'attitude que le monde va prendre à l'égard de ce témoignage. Déjà les deux dernières béatitudes $(5,10-12)$, mais aussi la parabole du festin nuptial $(22,6)$ ne laissent aucun doute : les envoyés post-pascaux soulèveront une opposition violente qui culminera dans la persécution et le martyre. Semblablement, le discours contre les scribes et les pharisiens (voir en particulier 23, 24) et l'apocalypse mt (voir en particulier 24 ,9) détruisent toute illusion : la propagation de l'évangile va susciter une crise d'une gravité extrême. Le chap. 10, axé sur le thème de l'envoi, ne pouvait se désintéresser de ce problème. Il le traite de manière approfondie dans les vv. 17-25. Il convient donc d'analyser cette péricope.

Mt 10,17-25 est une composition originale et théologiquement cohérente du premier évangéliste. Mt rassemble dans notre passage - et c'est là son originalité - des traditions d'origines diverses à l'aide desquelles il décrit la destinée de souffrance des disciples. Le travail rédactionnel 
consiste donc ici plus dans la constitution d'une péricope à partir d'un matériel diversifié que dans les retouches de détail apportées aux unités traditionnelles utilisées ${ }^{1}$. Les traditions recueillies par Mt et groupées dans les vv. 17-25 peuvent schématiquement se diviser en trois groupes. D'une part, dans les vv. 17-22, Mt reprend des unités traditionnelles que Mc (13, 9-13), et, à sa suite, Lc (21,12-19) placent dans leurs "apocalypses". Ce changement de contexte est important; il signifie que, pour Mt, la persécution n'est pas seulement un signe de la fin des temps, mais appartient constitutivement à la condition du disciple ${ }^{2}$. En second lieu, le v. 23 est une sentence isolée appartenant au Smt ${ }^{3}$. Son insertion dans la tradition de l'envoi est secondaire. Les deux motifs qui en constituent l'armature la fuite eschatologique ${ }^{4}$ et l'attente de la fin - sont bien connus de l'apoca lyptique juive. Leur combinaison fait de notre v. une parole de consolation dans le temps de la persécution ${ }^{5}$. Enfin, les vv. 24-25 sont empruntés

1 Nous renonçons en conséquence à présenter une comparaison synoptique détaillée, nous bornant à relever ce qui suit. Pour les vv. 17-22, hormis les différences dues à des motifs stylistiques, Mt introduit $x \alpha i$

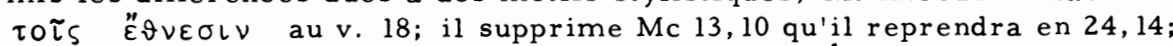

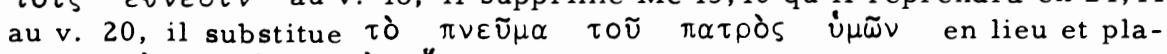

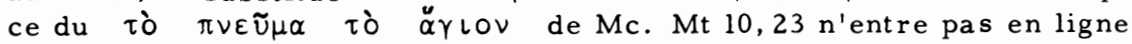
de compte (Smt !); Mt 10, 24-25 sera analysé spécialement.

2 SCHWEIZER, Mt 158.

3 Pour l'analyse de ce logion, nous renvoyons avant tout à VIELHAUER, Gottesreich 64-67. Voir aussi : BARTH 94; COLPE, art. ¿́ viós toṽ $\dot{\alpha} \vee \vartheta \rho \omega ́ \pi 0 v \quad$, ThW 8, 464; STRECKER, Weg 41-42.

4 Attestations dans le bas-judaïsme rabbinique : BILL 1, 951-52. Ce topos joue un rôle central à Qumrân; voir les références rassemblées par BRAUN, Radikalismus II 104 (note 2).

5 Un tel logion est avant tout concevable dans le monde palestinien. Il présuppose l'existence d'une synagogue juive persécutant le christianisme naissant et d'une communauté chrétienne qui non seulement attend intensément la venue du Fils de l'homme, mais encore limite sa mission à Israël. A ce titre, ce logion devrait provenir de la Palestine d'avant 70 et avoir été prononcé par un prophète inspiré parlant au nom du Christ élevé (BRAUN, Radikalismus II 46, note 1; BULTMANN, Trad 129; HAHN, Hoheitstitel 38; VIELHAUER, Gottesreich 66). 
par Mt à la source $Q\left(\right.$ par. Lc 6,40) ${ }^{1}$. Le motif constitutif de ce logion est bien attesté dans le bas-judaĩsme rabbinique ${ }^{2}$. Du point de vue de l'histoire de la tradition, il semble que la version lc - plus brève - est plus ancienne, d'autant plus que l'empreinte rédactionnelle du premier évangéliste est très nette. On remarquera en effet que le double parallélisme de notre logion (v. 24 a par. v. 24b; v. $25 a \alpha$ par. v. 25a $\beta$ ) appartient au

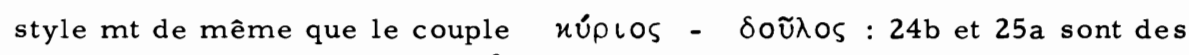
élargissements rédactionnels ${ }^{3}$. Il en va de même de $25 \mathrm{~b}$ qui contient une

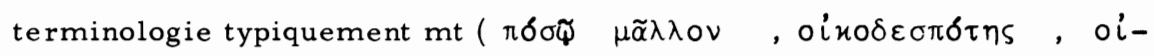

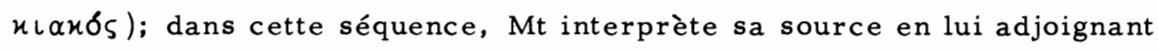
une très ancienne tradition que l'on retrouve en 9, 32 - 4 .

La triple adjonction que Mt apporte à sa source $(24 b, 25 a \alpha \beta, 25 b)$ est ici un véritable moyen d'interprétation. D'une part, dans cette parole qui évoque la relation du maître et du disciple, Mt élucide le titre $\delta \iota \delta \alpha ́ \sigma-$

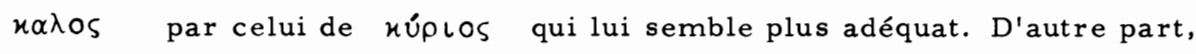
par l'adjonction de $25 \mathrm{~b}$, il interprète $Q$ autrement que $L c$ : pour Lc, le logion marque les limites de la compétence du disciple; pour Mt, il souligne que l'identité du maître et du disciple réside dans leur commune destinée de souffrance.

Quel est alors le thème central de cette composition liant des traditions si disparates et quel auditoire vise-t-elle ? Les exégètes ${ }^{5}$ sont unanimes

1 Voir les analyses détaillées, mais divergentes dans leurs résultats, de SCHULZ, Q 449-450; TRILLING, Israel 82-83.

2 bBer 58b Bar (cité par GRUNDMANN, Mt 295) : "Il suffit au serviteur d'être comme son maître"; cf. aussi BILL 1, 577-78. On se souviendra enfin que le disciple rabbinique, pour apprendre la Tora, s'efforce de modeler sa vie d'après l'image de son maître.

3 SCHULZ, Q 449, tient au contraire l'ensemble de la version $\mathrm{mt}$ pour primitive.

4 Ainsi aussi BRAUN, Radikalismus II 106 (note 1).

5 Voir, p. ex., BONNARD, Mt 147; GRUNDMANN, Mt 292-293; TRILLING, Israel 83-84; SCHWEIZER, Mt 156-158. 
à voir dans la destinée souffrante des disciples la problématique de la péricope et dans les vv. 24-25 la pointe du passage. Le v. 23 qui décrit le sort des envoyés de Jésus entre Pâques et la parousie ${ }^{1}$ de même que l'emploi typologique du couple $\mu \alpha \vartheta \eta \tau n ́ s ~-~ \delta o \tilde{\lambda} o s \quad 2$ dans les vv. 24 25 montrent clairement que cette règle communautaire ${ }^{3}$ s'attache à présenter la condition chrétienne comme telle ${ }^{4}$. Si la condition chrétienne est une condition souffrante, quelle est la nature de cette souffrance et comment doit-elle être assumée ?

Le v. $17 \mathrm{a}$, composé par $\mathrm{Mt}$, reprend le thème de la prudence introduit au v. 16. Il formule un avertissement : il s'agit d'être constamment sur ses gardes et de ne pas se nourrir d'illusions ${ }^{5}$. Les hommes dont il faut

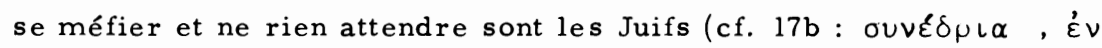

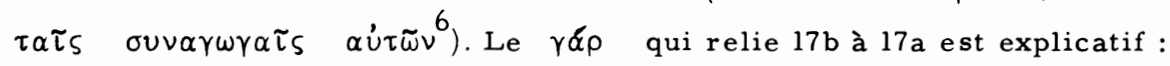
il convient de ne rien attendre des Juifs, car ces derniers s'acharneront à persécuter les croyants. Selon le v. 17, cette persécution revêt

1 BARTH 93-94.

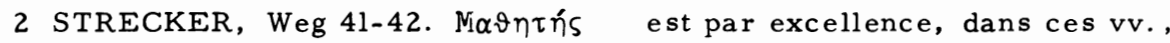
une notion paradigmatique dans laquelle le croyant est appelé à se reconnaiftre.

3 Cf. GRUNDMANN, Mt 292-293 et STRECKER, Weg 134.

4 L'analyse minutieuse de HARE 96-114 soutient ce point de vue : la mission est décrite dans des termes qui font penser à la situation de la diaspora (cf. comparution des croyants devant les instances politiques paiennes, séparation entre la synagogue et l'Eglise, etc ...).

5 Le verbe $\pi \rho \circ \sigma \varepsilon ́ \chi \varepsilon \iota \nu$ ámò $\tau$ ívos est typiquement $\mathrm{mt}$ (6 fois chez $\mathrm{Mt}$, jamais chez $\mathrm{Mc}$ ); il décrit la vigilance chrétienne (BAUER, WB 1417; BONNARD, Mt 148).

6 Le $\alpha \dot{\tau} \tilde{\omega} \nu$ qui qualifie les synagogues juives (cf. 12, 9; 13, 54; 23, 34), montre que l'église $\mathrm{mt}$ a conquis une nette conscience de son identité par rapport à Israël quels que soient ses rapports sociologiques avec le judaĩsme. Cf. sur ce point particulier HARE 104-106; SCHWEIZER, Mt 157. 
deux formes : la citation devant les tribunaux juifs ${ }^{1}$ et la flagellation ${ }^{2}$.

Pour décrire la comparution des disciples devant les tribunaux juifs, $\mathrm{Mt}$, à la suite de $\mathrm{Mc}$, utilise un terme appartenant à la terminologie chrétienne de la souffrance, à savoir le verbe $\pi \alpha \rho \alpha \delta i \delta \omega \mu \iota$. П $\alpha \rho \alpha \delta i \delta \delta \omega \mu \iota$ est l'expression classique utilisée pour décrire la passion du Christ ${ }^{3}$ et, par analogie, les tribulations des disciples en butte aux persécutions 4. Le sens du v. nous semble alors être le suivant : à l'image de leur mấtre et à cause de leur foi en lui, les disciples vont eux aussi déclencher l'hostilité violente de la synagogue et subir sa persécution active; leur Nachfolge les introduit dans une destinée de souffrance.

Mais la persécution n'émane pas des seuls sanhédrins de Palestine et de la diaspora. Elle est générale même si elle est provoquée par les Juifs (v. 18). Les disciples comparaîtront également devant les autorités païen-

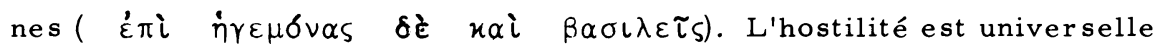
et inexorable. Mais les croyants connaissent la raison de cette haine criminelle : c'est parce qu'ils se réclament sans détour du Christ qu'ils sont

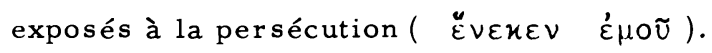

1 Les instances devant lesquelles les croyants sont appelés à comparaître

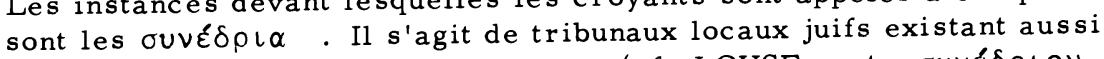

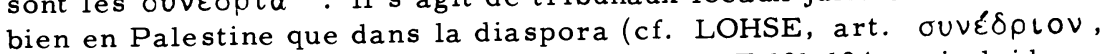
ThW 7, 864-865). Analyse détaillée chez HARE 101-104, qui plaide pour

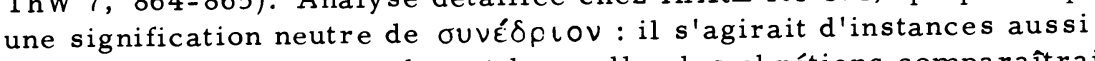
bien juives que paiennes devant lesquelles les chrétiens comparaîtraient pour avoir troublé la paix civile.

2 Le verbe $\mu \alpha \sigma \tau \iota \gamma \delta \omega$ (SCHNEIDER, ThW 4, 521-525) évoque le châtiment de la flagellation qui était effectivement subi dans la synagogue et consistait dans l'application de 39 coups de fouet. Il était appliqué à tout Juif convaincu de manquements divers à la Loi.

310,$4 ; 17,22 ; 20,18.19 ; 26,2.15 .16 .21 .33 ; 26,24.25 .45 .46 .48 ; 27,2.3 .4$. 18. 26 .

$410,17.19 .21 ; 24,9$. Voir aussi BUECHSEL, art. $\pi \alpha \rho \alpha \delta i \delta \omega \mu \iota$, ThW 2, 172. 
Les violences endurées par les disciples ne sont pourtant pas radicalement infructueuses. Elles sont l'occasion d'un témoignage public ( $\mu \alpha \beta-$ túpıov) que les persécutés doivent rendre à leur Seigneur. Ce témoigna ge s'adresse à tous les hommes sans exception: dans l'expression $\alpha \dot{U}-$

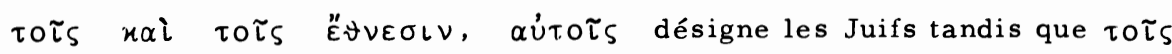

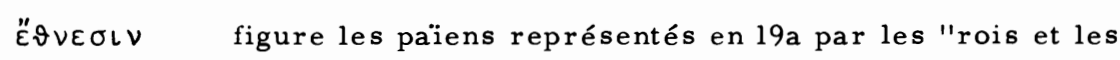
gouverneurs "l. L'idée qui domine les vv. 17-18 peut alors s'énoncer ainsi : les disciples - parce qu'ils sont les disciples de Jésus - vont devenir l'objet de la haine des hommes; ils ne devront ni la fuir, ni s'y dérober, mais y trouver l'occasion de témoigner.

Le témoignage au sein de la persécution peut pourtant devenir un objet de souci parmi les disciples (v. 19). Ce souci concerne aussi bien la forme $(\pi \tilde{\omega} S)$ que le contenu $(\tau \hat{\imath})$ du témoignage à rendre au Seigneur ${ }^{2}$. Dans notre cas - et comme le montre le v. 19b -, le souci du croyant est inutile, car Dieu le prend en charge ( $\delta \circ \vartheta n ́ \sigma \varepsilon \tau \alpha \iota$ est un passif sémitique dont l'agent sous-entendu est Dieu, cf. v. 20). Dieu ne libère pourtant pas le croyant du souci en le soustrayant à l'épreuve - ici la per sécution -, mais en lui apprenant à ne pas craindre l'insécurité dans laquelle il est plongé ${ }^{3}$, car, dans cette insécurité même ${ }^{4}$, il n'est pas abandonné à lui-même.

Le v. 20 précise la nature de l'aide que le croyant persécuté est en droit d'attendre de son Dieu. Cette aide consiste dans le don "de l'Esprit

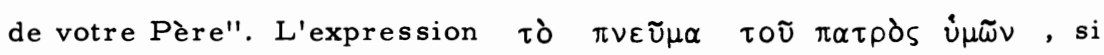
typiquement $\mathrm{mt}$, appelle deux remarques. $1^{\circ}$ ) Les affirmations en $\pi \alpha-$

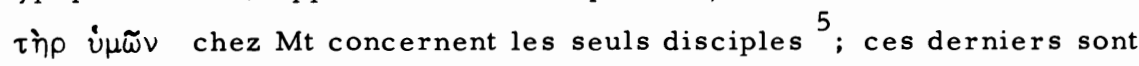

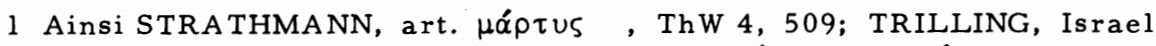

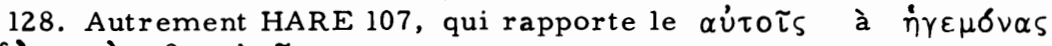
$\delta \varepsilon \quad$ น

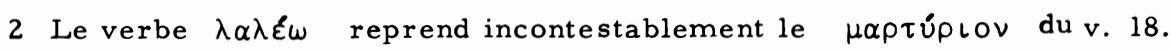

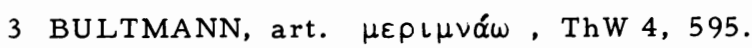

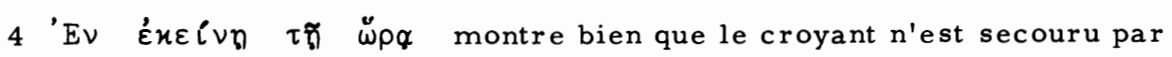
Dieu nulle part ailleurs qu'à l'heure critique de la persécution vécue.

5 SCHRENK, art. $\pi \alpha \tau \hat{n} \rho$, ThW 5, 987. 
donc l'objet d'une attention particulière du Père. Cette sollicitude qui s'oppose au $\mu \varepsilon \rho \iota \mu \nu \alpha ́ \omega$ (cf. Mt 6,26.32), ne fléchit pas dans la souffrance ou dans la persécution; elle culmine au contraire dans le don de l'Esprit ${ }^{1} .2^{\circ}$ ) Qu'est-ce qu'alors que cet Esprit qui est donné par le Père aux croyants ? Chez Mt, $\pi \nu \varepsilon \tilde{v} \mu \alpha$ a encore une consonnance vétérotestamentaire, il est la force donnée par Dieu aux croyants pour les habiliter à certains actes $^{2}$ - ici le témoignage dans la persécution -. La force que Dieu donne au fidèle persécuté le libère de tout souci quant au témoignage à rendre.

En résumé, la condition du disciple telle qu'elle est décrite dans les vv. 17-20, est paradoxale : d'une part, le croyant est placé devant la nécessité inexorable de l'épreuve, mais, d'autre part, son Seigneur lui donne les moyens de l'affronter.

Le v. 21 est en rupture avec le v. 20. Il reprend néanmoins le thème d'ensemble qui est celui de la persécution. Le verbe $\pi \alpha \rho \alpha \delta i \delta \omega \mu \iota$, déjà présent aux vv. 17-20, joue le rôle de mot-crochet, et atteste la cohérence thématique de la péricope.

Pour décrire la destinée souffrante des disciples, Mt recourt ici, par le biais d'une libre réminiscence de Mi 7,6, à un motif bien connu de 1'AT et du bas-judaisme ${ }^{3}$, celui de la division mortelle des familles dans les derniers temps. Mais il le réinterprètechristologiquement : la prédication et l'oeuvre de Jésus provoquent la crise finale attendue par le basjudaĩsme et la seule confession de Jésus par les disciples suffit à déclencher les affres apocalyptiques ${ }^{4}$. Parmi ceux-ci, figure le déchirement dramatique des familles. Ce déchirement a lieu maintenant, car la confession de Jésus suffit à diviser les parents naturels.

1 SCHRENK, art. cit., ThW 5, 991-992.

2 SCHWEIZER, art. $\pi \nu \varepsilon \tilde{U} \mu \alpha$, ThW 6, 394.

$34^{\mathrm{e}}$ Esd 6,24, p. ex., déclare : "En ce temps-là, les amis se battront les uns contre les autres comme des ennemis si bien que la terre avec tous ses habitants en sera épouvantée" (cf. KAUT ZSCH, Apokryphen 2, 366).

4 SCHRENK, art. $\pi \alpha \tau n \dot{p}$, ThW 5, 983. 
Les verbes $\pi \alpha p \alpha \delta i \delta \omega \mu \iota$ et $\varepsilon \pi \alpha \nu i \sigma \tau \alpha \mu \alpha \iota$ l'évoquent l'activité des accusateurs qui se lèvent contre les disciples pour les faire comparaître devant les tribunaux et les condamner à mort ( $\varepsilon i \varsigma_{\text {S }} \vartheta \alpha \alpha$ tov, $\vartheta \alpha \nu \alpha \tau \delta \omega)$. Et ces accusateurs ne sont autres que les familiers des

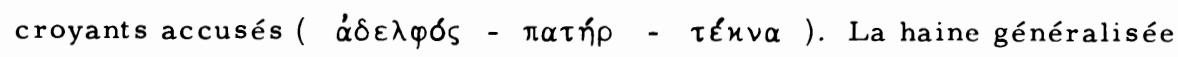
qui s'abat sur les disciples, contamine même leurs proches. Ils ne sont désormais sûrs de personne.

Le v. 22a reprend et résume le thème de la haine universelle qui sous -

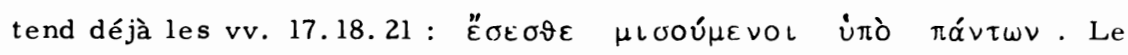
verbe $\mu\llcorner\sigma \varepsilon ́ \omega$ a un sens "théologique" : plutôt qu'à l'agressivité implacable et féroce des ennemis, il fait allusion à la situation d'épreuve et de tentation qu'affronte le disciple dans les "derniers temps" ${ }^{2}$. Cette situation dans laquelle le disciple est appelé à vivre et à témoigner fidèlement, n'est pourtant pas une fatalité absurde ou accidentelle. Elle est provoquée par la confession que les disciples rendent publiquement à leur Seigneur

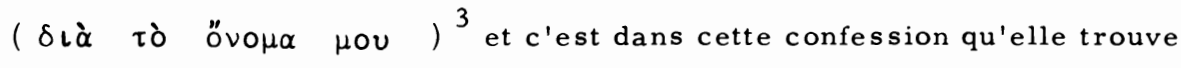
aussi son sens. La confession du nom de Jésus est inséparable de la souffrance qu'elle provoque.

A l'annonce de cette destinée souffrante sont liés à la fois un avertissement et une promesse ${ }^{4}$ : c'est l'objet du v. 22b. La promesse du "salut" n'est en effet valable que pour celui qui "persévérera jusqu'à la fin". Le verbe $\dot{\pi}{ } \mu \varepsilon^{\nu} \omega$ fait partie de la terminologie de la souffrance des disciples; il signifie supporter, persévérer dans l'épreuve, sans renier sa fidélité au Christ ${ }^{5}$. La persévérance fidèle dans l'épreuve est une

1 BONNARD, Mt 148-149.

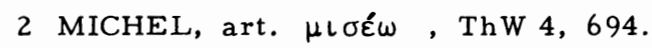

3 BIETENHARD, art. ővo $\mu \alpha$, ThW 5, 278.

4 Nous retrouvons le même mode d'argumentation qu'aux vv. 17-20; les vv. 17-18 annoncent la comparution devant les tribunaux, les vv. 18-19 promettent aux accusés la force de témoigner.

5 HAUCK, art. U் $\pi$ Hé $\nu \omega$, ThW 4, 590. 
persévérance "jusqu'à la fin". L'expression $\varepsilon$ is $\tau$ ť̉os 1 a ici un sens adverbial. Elle peut soit revêtir une nuance temporelle et désigner alors celui qui aura résisté jusqu'à la dernière extrémité, càd la mort martyre ${ }^{2}$; mais elle peut aussi avoir un sens quantitatif et décrire alors celui qui aura résisté totalement, sans défaillance. Le contexte immédiat recommande le sens temporel (cf. v. 21), sans d'ailleurs exclure le sens quantitatif. Le oữos qui introduit l'énoncé de la promesse, a un sens restrictif : "celui-là et celui-là seulement qui aura résisté jusqu'à la dernière extrémité". Le contenu de la promesse est le don du salut; $\sigma \omega \vartheta \hat{\eta} \circlearrowleft \varepsilon \tau \alpha \iota$ ne signifie pas que Dieu délivrera le disciple de la persécution, mais qu'il lui fera surmonter le jugement eschatologique. Celui qui suit le Christ en acceptant une destinée souffrante, sera sauvé.

Le v. 23a fait difficulté, car il semble en contradiction avec le v. 22; il paraît, en effet, inviter à la dérobade, alors que le v. 22 exigeait une fidélité allant jusqu'au martyre. La solution de cette contradiction apparente se trouve peut-être dans le fait que 23 a est, comme le v. 21, inspiré d'un motif apocalyptique juif, celui de la fuite eschatologique: L'auteur voudrait alors souligner, par le biais de ce topos, l'insécurité dans laquelle le disciple est appelé à vivre. De plus, ce topos traduirait bien l'expérience des missionnaires chrétiens dans la diaspora juive - chas sés qu'ils étaient de synagogue en synagogue sous prétexte d'agitation 3.

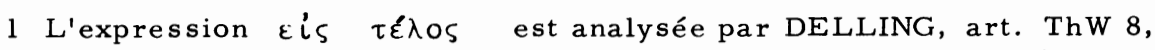
56. Remarquons qu'elle a toujours une valeur adverbiale et qu'à ce titre, elle n'est jamais le terminus technicus de la parousie dans les synoptiques.

2 Le thème de la haine universelle, mais aussi du martyre, confirme notre hypothèse : notre péricope dépasse nettement le cadre de la seule mission galiléenne pour mieux s'intégrer à l'ère apostolique (avec GRUNDMANN, Mt 294, mais aussi HAENCHEN, Weg 231-234).

3 Voir BONNARD, Mt 149; BRAUN, Radikalismus II 102 (note 4); HAENCHEN, Weg 231-234; HARE 111-112. 
A cette nouvelle prédiction de souffrance est ajouté le v. 23 b. Il a soulevé de vives discussions. Nous essaierons simplement de le comprendre dans son contexte rédactionnel, en laissant en suspens les autres questions. Le verbe $\tau \varepsilon \hat{\lambda} \varepsilon ́ \omega$ signifie ici "venir à bout, terminer". Mais en quel sens les disciples n'en auront-ils pas fini avec les villes d'Israël L'ensemble du passage évoque la souffrance encourue par le croyant, membre d'une église évangélisant juifs et païens et qui ne s'attend plus à un proche retour de son Seigneur. Dès lors, cette "absence de fin" qualifie de manière paradoxale le temps de la mission : l'hostilité rencontrée ne s'apaisera qu'au terme de l'économie présente. La propagation de la Parole se heurtera constamment à l'opposition et à la violence - voire à l'échec - .

Les vv. 17-23 ont mis en évidence la condition souffrante des disciples, en expliquant et en justifiant cette souffrance par l'appartenance à Jésus (cf. notamment les vv. 18-22). Sette thématique culmine avec netteté dans les vv. 24-25 qui forment la pointe de la péricope.

Nos deux vv. sont construits à l'aide de trois couples de notions : $\mu \alpha-$

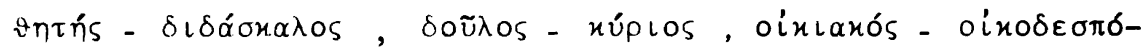
Ins. Or, fait capital, ces trois couples qui, pris séparément, ont une signification générale et non-typique, s'élucident les uns les autres terme à

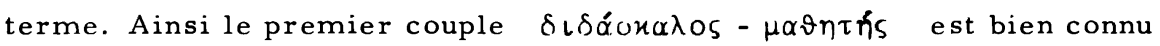
dans le monde juif et n'a rien de spécifiquement chrétien; il établit, dans des termes classiques, la relation du maître et du disciple. Mais en ver-

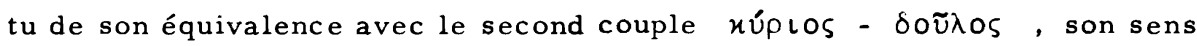
se précise : le $\delta \iota \delta \alpha ́ \sigma K \alpha \lambda o s$ Jésus n'est personne d'autre que le Kúplos, de même que le $\mu \alpha \vartheta \eta \tau$ ńs n'est personne d'autre que le serviteur de son Seigneur. A cette première élucidation, le troisième couple oíxo-

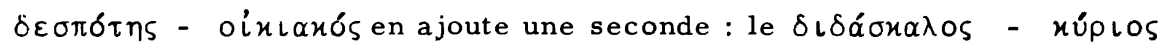
est le chef d'une maison - la familia Dei - et les disciples sont les membres de cette maison. Ainsi Jésus, en tant que xúplos et oíxo$\delta \varepsilon \sigma \pi \delta$ ins, cesse d'être un $\delta \iota \delta \alpha ́ \sigma x \alpha \lambda o s$ au sens juif ${ }^{1}$ et, semblablement

1 BORNKAMM, Enderwartung 38. 


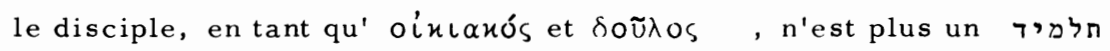
au sens rabbinique. Le didascale Jésus est le xúplos qui règne avec autorité sur ses disciples. Les $\mu \alpha \vartheta \eta \tau \alpha i ́$ ont conscience d'appartenir à leur maître et de le servir.

Par ces trois couples, Mt met en relation la condition du maître et celle du disciple pour affirmer leur similitude. Tant le double oún $\dot{u}-$ $\pi \varepsilon ́ \rho$ du v. 24 que le double $\dot{\omega}$ de v. 25 a visent à établir cette identité.

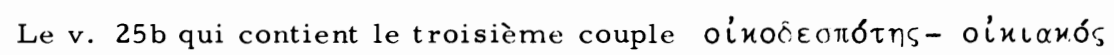
s'éclaire à partir du droit juif de l'envoi pour lequel l'envoyé représente totalement celui qui l'envoie : c'est encore l'idée d'une commune destinée qui domine.

Il convient alors de se demander sur quel point la destinée du disciple est identique à celle du Jésus terrestre. La réponse de l'évangéliste se trouve au v. 25b qui est une adjonction de sa main. De façon analogue à 9,34 et 12,24 , elle montre un Christ en butte à l'hostilité mortelle des pharisiens allant jusqu'à l'accuser d'être un démoniaque. C'est donc le Christ souffrant, méprisé et rejeté, et enfin crucifié qui apparaît. La condition du maître et celle des disciples sont identiques en ceci que l'hostilité des hommes vaut à l'un et aux autres la même dérision et la même souffrance ${ }^{2}$. La Nachfolge est une Nachfolge dans la souffrance.

1 HAHN, Hoheitstitel 78.

2 Ainsi, p. ex., BONNARD, Mt 150, et BORNKAMM, Jesus 133. SCHWEIZER (Gesetz und Enthusiasmus 58) et, à sa suite, LUZ (Jünger 156), interprètent, sur la base de 9,34, la similitude de condition entre Jésus et ses disciples comme le partage par les croyants de la toute-puissance de leur Seigneur. Si cette note est présente dans le chap. 10 (cf. vv. 1 et 8 ), elle n'est pas recommandée par le contexte immédiat. 


\section{Abréviations}

Les abréviations utilisées dans notre travail sont, en règle générale, empruntées au registre d'abréviations établi par l'ouvrage "DIE RELIGION IN GESCHICHTE UND GEGENWART", 3e éd. , édité par Kurt GALLING, tome 1, 1957, XVI - XXIX. A cette liste devenue classique, nous avons ajouté deux compléments. D'une part, les abréviations utilisées pour citer les livres bibliques sont celles de la "Traduction oecuménique de la Bible" (TOB), Nouveau Testament, 1972, 824; d'autre part, les sigles utilisés pour citer les textes de Qumrân sont ceux établis par E. LOHSE, Die Texte aus Qumran, hebräisch und deutsch, 1964, IX-XI. Dérogent ou s'ajoutent aux recueils de références indiqués :

a) littérature rabbinique :

$\mathrm{Ab}$

Abot

Nid

Nidda

b) abréviations techniques:

lc

lucanien

$\mathrm{mc}$

marcinien

$\mathrm{mt}$

matthéen

Q

source des logia

Q1c source des logia telle qu'elle est transmise dans le milieu lucanien

Qmt source des logia telle qu'elle est transmise dans le milieu matthéen

SM Sermon sur la Montagne

Smt traditions propres à l'évangile selon Matthieu

c) collections de monographies, revues, ouvrages:

$\mathrm{B} 1-\mathrm{D}$ F. BLASS - A. DEBRUNNER, Grammatik des neutestamentlichen Griechisch, 12 e éd., 1965, avec un "Ergänzungsheft" de D. TAB ACHOWIT $Z$.

Bibl. Ephemer. Theologic. Lovan.

Bibliotheca Ephemeridum Theologicarum Lovaniensium.

CNT Commentaire du Nouveau Testament.

CTh Cahier théologique. 
ET L

HTR

NSTh

RHPR

RNT

RTP

SBS

ScE

StANT

Trad

WB

WMANT

Ephemerides Theologicae Lovianienses.

The Harward Theological Review.

Nouvelle série théologique.

Revue d'Histoire et de Philosophie religieuses.

Regensburger Neues Testament.

Revue de Théologie et de Philosophie.

Stuttgarter Bibelstudien.

Sciences ecclésiastiques.

Studien zum Alten und Neuen Testament.

(R. BULTMANN), Die Geschichte der synoptischen Tradition, FRLANT 29, 6e éd., 1964.

W. BAUER, Griechisch-deutsches Wörterbuch zu den Schriften des Neuen Testaments und der übrigen urchristlichen Literatur, 5e éd., 1963.

Wissenschaftliche Monographien zum Alten und Neuen Testament. 


\section{Bibliographie}

1. Textes et sources

Die Apokryphen und Pseudepigraphen des Alten Testaments, 2 tomes, traduits et édités par E. KAUTZSCH, 2e réimpression, 1962.

J. BONSIRVEN, Textes rabbiniques des deux premiers siècles chrétiens, Rome, 1955.

Die Mischna IV/9, Abot. Texte, Uebersetzung, Erklärung von K. MARTI-G. BEER, 1927.

Novum Testamentum Graece, édité par E. NESTLE et K. A LAND, 25e éd., 1963.

Septuaginta, id est Vetus Testamentum Graece juxta LXX Interpretes, éditée par A. RAHLFS, 7e éd., 1962.

Les Stoiciens, textes traduits par Emile BREHIER, édités sous la direction de Pierre-Maxime SCHUHL, Bibliothèque de la Pléiade, 1964.

Synopsis Quattuor Evangeliorum, édité par K. ALAND, ze éd., 1964.

Die Texte aus Qumran, hebräisch und deutsch, édités par E. LOHSE, 1964.

Les textes de Qumrân, traduits et annotés par :

Tome 1, J. CARMIGNAC et P. GUILBERT, Paris 1961

Tome 2, J. CARMIGNAC, E. COTHENET et H. LIGNEE, Paris, 1963.

2. Instruments de travail

W. BAUER, Griechisch-deutsches Wörterbuch zu den Schriften des Neuen Testaments und der übrigen urchristlichen Literatur, 5e éd., 1963.

F. BLASS - A. DEBRUNNER, Grammatik des neutestamentlichen Griechisch, 12e éd., 1965, avec un "Ergänzungsheft" de D. TABACHOWITZ.

L. KOEHLER - W. BAUMGARTNER, Lexikon in Veteris Testamenti Libros, 2e éd., 1958.

W. F. MOULTON - A.S. GEDEN, A Concordance to the Greek Testament, 3e éd., 1953.

Die Religion in Geschichte und Gegenwart, 3e éd., éditée par K. GALLING, 6 tomes, 1957-1962 et un registre, 1965. 
A. SCHMOLLER, Handkonkordanz zum griechischen Neuen Testament, 12 e éd., 1960.

Theologisches Wörterbuch zum Neuen Testament, 9 tomes; tomes 1 - 4 édités par G. KITTEL, tomes 5 - 9 édités par G. FRIEDRICH; paraît depuis 1933.

N. B. : les articles de la $\mathrm{RGG}^{3}$ et du ThW qui ont été consultés, n'ont pas été reportés dans la bibliographie afin de ne pas gonfler abusivement cette dernière. Ils sont cités en note avec indication de l'auteur, du titre de l'article, du tome et de la page. Nous prions les auteurs incriminés de nous excuser de cette procédure, car leurs contributions méritaient d'être mentionnées au même titre que les autres.

3. Commentaires consacrés à l'évangile selon Matthieu

W. C. ALlEN, A Critical and Exegetical Commentary on the Gospel according to St. Matthew, 3e éd., 1947.

P. BENOIT, L'Evangile selon Saint Matthieu, dans : La Sainte Bible, 3e éd. , 1961.

P. BONNARD, L'Evangile selon Saint Matthieu, CN'T 1, 2e éd., revue et augmentée, 1970.

J. C. FENTON, The Gospel of St. Matthew, The Pelican Gospel Commentaries, 1963.

F. V. FILSON, A Commentary on the Gospel according to St. Matthew, dans : Black's New Testament Commentaries, 1960.

P. GAECHTER, Das Matthäusevangelium, 1964.

W. GRUNDMANN, Das Evangelium nach Matthäus, ThHK 1, 1968.

E. KLOSTERMANN, Das Matthäusevangelium, HNT 4, 4e éd., 1971.

M. - J. LAGRANGE, Evangile selon Saint Matthieu, 1923.

E. LOHMEYER, Das Evangelium des Matthäus, MeyerK Sonderband, édité par W.SCHMAIICH, 3e éd., revue, 1962.

J. RADEMAKERS, Au fil de l'évangile selon saint Matthieu, 2 tomes, 1972.

A. SCHLATTER, Der Evangelist Matthäus. Seine Sprache, sein Ziel, seine Selbständigkeit. Ein Kommentar zum ersten Evangelium, 6e éd., 1963.

J. SCHMID, Das Evangelium nach Matthäus, RNT 1, 5e éd. revue, 1965.

J. SCHNIEWIND, Das Evangelium nach Matthäus, NTD 2, 10 éd., 1965. 
E. SCHWEIZER, Das Evangelium nach Matthäus, NTD 2, 13 éd., 1973.

(H. L. STRACK) P. BILLERBECK, Kommentar zum Neuen Testament aus Talmud und Midrasch, 4 tomes, 1922-1928 (4 éd., 1965).

W. TRILLING, L'évangile selon Matthieu, Collection "Parole et Prière", 3 tomes, 1971.

B. WEISS, Das Matthäus-Evangelium, MeyerK I, 1, 4e éd., 1898.

J. WEISS, Dic drei älterenEvangelien, dans : Die Schriften des Neuen Testaments I, 3e éd., 1917.

J. WELlHAUSEN, Das Evangelium Matthaei, 1904.

4. Articles, autres commentaires, monographies

S. AGOURIDES, La tradition des Béatitudes chez Matthieu et Luc, dans : Mélanges bibliques offerts au R. P. Béda Rigaux, 1970, 9-27.

T. ARVEDSON, Das Mysterium Christi. Eine Studie zu Mr 11, 25-30, 1937.

G. BARTH, Das Gesetzesverständnis des Evangelisten Matthäus, dans : G. BOR NKAMM - G. BARTH - H. J. HELD, Ueberlieferung und Auslegung im Matthäusevangelium, WMANT 1, 5e éd., 1968, $54-154$.

H. W. BARTSCH, Parusieerwartung und Osterbotschaft, dans : Entmythologisierende Auslegung, ThF 26, 1962, 61-69.

- Die Passions - und Ostergeschichten bei Matthäus, dans ouvr, cit., 80-92.

J. B. BAUER, Das milde Joch und die Ruhe, Mt 11, 28-30, ThZ 17, 1961, 99-106.

G. BAUMBACH, Das Verständnis des Bösen in den synoptischen Evangelien, ThA 19, 1963.

- Die Mission im Matthäusevangelium, ThLZ 92, 1967, 889893.

K. BERGER, Die Amen-Worte Jesu, BZNW 39, 1970.

J. BLANK, Die Sendung des Sohnes. Zur christologischen Bedeutung des Gleichnisses von den bösen Winzern Mk 12,1-12, dans : Neues Testament und Kirche (Festschrift Schnackenburg), $1974,11-41$.

J. BIENECK, Sohn Gottes als Christusbezeichnung der Synoptiker, A ThANT 21, 1951.

D. BONHOEFFER, Le prix de la grâce - Sermon sur la Montagne -, 1962. 
P. BONNARD, "Composition et signification historique de Matthieu 18. Règle ecclésiastique ou éthique du Royaume ?", dans : De Jésus aux Evangiles. Tradition et rédaction dans les évangiles synoptiques, Bibl. Ephemer. Theologic. Lovan. 25, $1967,130-140$.

- Matthieu, éducateur du peuple chrétien, dans : Mélanges Rigaux 1-7.

G. BORNKAMM, Jesus von Nazareth, Urban-Bücher 19, 7e éd., 1966.

- Enderwartung und Kirche im Matthäusevangelium, dans :

G. BORNKAMM - G. BARTH - H. J. HELD, Ueberlieferung und Auslegung im Matthäusevangelium, 13-47.

- Die Sturmstillung im Matthäusevangelium, dans ouvr. cit., 48-53.

- Der Auferstandene und der Irdische, Mt 28,16-20, dans ouvr. cit., 289-310.

- Die Verzögerung der Parusie dans : Geschichte und Glaube I (Gesammelte Aufsätze III), BEvTh 48, 1968, 4655.

- Die Binde - und Lösegewalt in der Kirche des Matthäus, dans : Geschichte und Glaube II (Gesammelte Aufsätze IV), BEvTh 53, 1971, 37-50.

- Wandlungen im alt - und neutestamentlichen Gesetzesver ständnis, dans ouvr. cit., 73-119.

W. BOUSSE'T, Kyrios Christos, FRLANT 21, 2e éd. , 1921.

G. BRAUMANN, Zum traditionsgeschichtlichen Problem der Seligpreisungen, Mt 5,3-12, NovTest 14, 1960, 253-260.

- Der sinkende Petrus, ThZ 22, 1966, 403-414.

H. BRAUN, Spätjüdisch-häretischer und frühchristlicher Radikalismus, tome 1: Das Spätjudentum, tome 2: Die Synoptiker, BHTh $24,1957$.

- Qumran und das Neue Testament, 2 tomes, 1966.

- Jesus. Der Mann aus Nazareth und seine Zeit, Themen der Theologie l, 1969.

I. BROER, Das Gericht des Menschensohnes über die Völker, Mt 25, 3146, Bibel und Leben 11, 1970, 273-295.

L. BRUN, Die Auferstehung in der urchristlichen Ueberlieferung, 1925.

R. BULTMANN, Die Geschichte der synoptischen Tradition, FRLANT 29, 6e éd., 1964; G. THEISSEN et P. VIELHAUER, Ergänzungsheft, $4 \mathrm{e}$ éd., 1971).

- Jesus, Siebenstern - Taschenbuch 17, 2e éd., 1965.

H. Fr. von CAMPENHAUSEN, Der Ablauf der Osterereignisse und das leere Grab, SHA, 3e éd., 1966. 
- Kirchliches Amt und geistliche Vollmacht in den drei ersten Jahrhunderten, BHTh 14, 2e éd., 1963.

C. E. CARLSTON, The Things that Defils (Mark 7,14) and the Law in Matthew and Mark, NTS 15, 1968-69, 75-96.

F. CHRIST, Jesus Sophia. Die Sophia-Christologie bei den Synoptikern, A ThANT 57, 1970.

L. CERFAUX, Les sources scripturaires de Mt 11, 25-30, ETL 30, $1954,740-746 ; 31,1955,331-342$.

H. CONZELMANN, Die Mitte der Zeit, Studien zur Theologie des Lukas, BHTh 17, 3e éd., 1960.

- Die Apostelgeschichte, HNT 7, 1963.

L. COPE, Matthew 25,31-46, "The Sheep and the Goats" Reinterpreted, NovTest 11, 1969, 32-44.

E. COTHENET, Les prophètes chrétiens, dans : L'Evangile selon Matthieu. Rédaction et Théologie, Bibl. Ephemer. Theologic. Lovan. 29, 1972, 281-308.

W. D. DAVIES, Knowledge in the Dead See Scrolls and Matthew 11, 25-30, HTR 46, 1953, 133-140.

- Matthew 5,17-18, dans : Mélanges bibliques offerts à

A. Robert, 1957, 428-456.

- The Setting of the Sermon on the Mount, 2e éd., 1966.

G. DELLING, Die Zueignung des Heils in der Taufe. Eine Untersuchung zum neutestamentlichen "taufen auf den Namen", 1961.

A. DESCAMPS, Les justes et la justice dans les évangiles et dans le christianisme primitif, 1950.

- Essai d'interprétation de Mt 5,17-48, dans : Studia Evangelica I (TU 73), 1959, 156-173.

F. DIBELIUS, Die kleinsten Gebote, ZNW 11, 1910, 188-190.

M. DIBELIUS, Die Formgeschichte des Evangliums, 3e éd., 1959.

- Die Pastoralbriefe, HNT 13, 4e éd., 1966.

- Die Bergpredigt, dans : Botschaft und Geschichte I, 1953, 79-174.

C. DIETZFELBINGER, Das Gleichnis von der erlassenen Schuld. Eine theologische Untersuchung von Matthäus 18, 23-35, EvTh 37, $1972,437-451$.

E. DINKLER, Jesu Wort vom Kreuztragen, dans : Signum Crucis (Aufsätze zum Neuen Testament und zur christlichen Archäologie), 1967, 77-98.

E. von DOBSCHUETZ, Matthäus als Rabbi und Katechet, ZNW 27, $1928,338-348$. 
J. DUPONT, L'interprétation des béatitudes, Foi et Vie, Cahier biblique $4,1966,17-39$.

- "Beaucoup viendront du levant et du couchant ..." (Matthieu 8,11-12; Lc 13, 28-29), ScE 19, 1967, 153-168.

- Nova et Vetera (Matthieu 13,52), dans : Mélanges Leenhardt, 1968, 338-348.

- Les Béatitudes I. Le problème littéraire, 2e éd., 1969.

- Le point de vue de Matthieu dans le chapitre des parabo-

les, dans : l'Evangile selon Matthieu. Rédaction et théologie, 221-259.

G. EICHHOLZ, Auslegung der Bergpredigt, BSt 46, 1965.

A. FEUillet, Jésus et la Sagesse divine d'après les évangiles synoptiques, RB 62, 1955, 161-196.

- Morale ancienne et morale chrétienne d'après Mt 5, 17-20, NTS 17, 1970-71, 123-137.

H. FRANKEMOELLE, Die Makarismen (Mt 5,1-12; Lk 6, 20-23). Motive und Umfang der redaktionnellen Komposition, BZ NF 15, $1971,52-75$.

E. FUCHS, Die vollkommene Gewissheit, zur Auslegung von Matthäus 5,48 , dans : Neutestamentliche Studien für Rudolf Bultmann, BZNW 21, 2e éd., 1957, 130-136.

T.F. GLASSON, Anti-Pharisaism in St. Matthew, JQR 51, 1961-62, 316320.

J. GNILKA, Die Kirche des Matthäus und die Gemeinde von Qumran, BZ NF 7, 1963, 43-63.

H. GRASS, Ostergeschehen und Osterberichte, 2 éd., 1962.

E. GRAESSER, Das Problem der Parusieverzögerung in den synoptischen Evangelien und in der Apostelgeschichte, BZNW 22, 2e éd., 1960 .

H. GROLLINGER, "Ihr wisst nicht, an welchem Tag euer Herr kommt", Auslegung von Mt 24,37-51, Bibel und Leben 11, 1970, 238-247.

G. GROSS, Die "geringsten Brüder" Jesu in Mt 25, 40 in Auseinandersetzung mit der neueren Exegese, Bibel und Leben 5, 1964 , $172-180$.

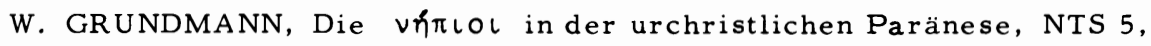
1958-59, 180-205.

- Matth. 11, 27 und die johanneischen "Der Vater - Der Sohn" Stellen, NTS 12, 1965-66, 42-49.

- Das Evangelium nach Markus, ThHK II, 3e éd., 1968.

E. HAENCHEN, Matthäus 23, ZThK 48, 1951, 38-63. 
- Die Apostelgeschichte, MeyerK III, 14 éd., 1965.

- Johanneische Probleme, dans : Gott und Mensch (Gesammelte Aufsätze), 1965, 78-113.

- Der Weg Jesu, ST, Zweite Reihe, Band 6, 1966.

Th. HAERING, Mt 11,28-30, dans: Aus Schrift und Geschichte (Festschrift Schlatter), 1922, 3-15.

Ferd. HAHN, Das Verständnis der Mission im Neuen Testament, WMANT 13, 1963.

- Christologische Hoheitstitel. Ihre Geschichte im frühen Christentum, FRLANT 83, 3e éd., 1966.

- Das Gleichnis von der Einladung zum Festmahl, dans : Verborum Veritas (Festschrift Stählin), 1970, 51-82.

D. R. A. HARE, The Theme of Jewish Persecution of Christians in the Gospel according to St Matthew, Society for New Testament Studies, Monograph Series 6, 1967.

V. HASLER, Die königliche Hochzeit, Matth 22, 1-14, ThZ 18, 1962, 25 35.

G. HAUFE, "Soviel ihr getan habt einem dieser geringsten Brüder", dans : Ruf und Antwort (Festschrift Fuchs), 1964, 484-493.

H. J. HELD, Matthäus als Interpret der Wundergeschichten, dans :

G. BORNKAMM - G. BARTH - H. J. HELD, Ueberlieferung und Auslegung im Matthäusevangelium 155-287.

M. HENGEL, Nachfolge und Charisma, BZNW 34, 1968.

- Das Gleichnis von den bösen Weingärtnern Mk 12,1-12 im Licht der Zenonpapyri und der rabbinischen Gleichnisse, ZNW 59, 1968, 1-39.

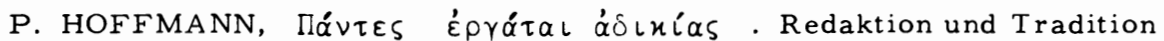
in Lc 13, 22-30, ZNW 58, 1967, 188-214.

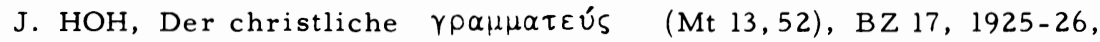
256-269.

R. HUMMEL, Die Auseinandersetzung zwischen Kirche und Judentum im Matthäusevangelium, BEvTh 33, 2e éd., revue et augmentée, 1966.

A. M. HUNTER, Crux Criticorum - Matth 11, 25-30 - A Reappraisal, NTS 8, 1961-62, 241-249.

B. M.F. van IERSEL, "Der Sohn" in den synoptischen Jesuworten. Christusbezeichnung oder Selbstbezeichnung Jesu ? (Supplements to Novum Testamentum, Vol. 3), 1961.

J. C. INGElaERE, La "Parabole" du Jugement dernier (Matthieu 25, 31-46), RHPR 50, 1970, 23-60. 
J. JEREMIAS, Zöllner und Sünder, ZNW 30, 1931, 293-300.

- Jésus et les païens, CTh 39, 1956.

- Die Gleichnisse Jesu, 7e éd., 1965.

- Jérusalem au temps de Jésus, 1967.

E. KAESEMANN, Sätze heiligen Rechtes im Neuen Testament, dans : Exegetische Versuche und Besinnungen II, 5e éd., 1967, 69-82.

- Die Anfänge christlicher Theologie, dans ouvr. cit., 83-104.

G. D. KILPATRICK, The Origins of the Gospel according to St. Matthew, 2e éd., 1950.

H.G. KLEMM, Das Wort von der Selbstbestattung der Toten, NTS 16, $1969,60-75$.

K. KOCH, Was ist Formgeschichte? Neue Wege der Bibelexegese, 1964.

G. KRETSCHMAR, Ein Beitrag zur Frage nach dem Ursprung frühchristlicher Askese, ZThK 61, 1964, 27-67.

Th. KRUIJF, Der Sohn des lebendigen Gottes. Ein Beitrag zur Christologie des Matthäusevangeliums, Analecta Biblica 16, 1962.

W. G. KUEMMEL, Das Gleichnis von den bösen Weingärtnern, dans : Aux sources de la tradition chrétienne (Mélanges Goguel), $1950,120-131$.

- (P. Feine, J. Behm), Einleitung in das Neue Testament, $13 \mathrm{e}$ éd. , 1964.

- Jesus und der jüdische Traditionsgedanke, dans : Heilsgeschehen und Geschichte (Gesammelte Aufsätze 1933-1964), Marburger Theol. Studien 3, 1965, 15-35.

J. LAMBRECHT, The Parousia Discourse. Composition and Content in Mt., XXIV-XXV, dans : L'Evangile selon Matthieu. Rédaction et théologie 309-342.

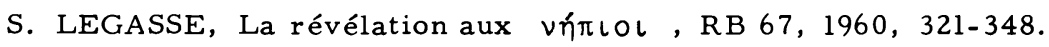

- Jésus et l'enfant. "Enfants", "petits" et "simples" dans

la tradition synoptique, Etudes bibliques, 1969.

- L'Evangile selon Matthieu, dans : Le ministère et les ministères dans le Nouveau Testament, Collection "Parole de Dieu", 1974, 182-206.

X. LEON-DUFOUR, La tempête apaisée, dans : Etudes d'Evangile, Collection "Parole de Dieu", 1965, 150-182.

- L'épisode de l'enfant épileptique, dans ouvr. cit., 184227.

- La parabole des vignerons homicides, dans ouvr. cit., 304-344. 
E. LINNEMANN, Gleichnisse Jesu. Einführung und Auslegung, 4e éd. , 1966.

E. LOHMEYER, "Mir ist gegeben alle Gewalt ! "Eine Exegese von Mt 28,16-20, dans : In Memoriam Ernst Lohmeyer, 1951, 22- 49 .

- Das Gleichnis von den bösen Weingärtnern (Mark 12,1-12), dans : Urchristliche Mystik, 1955, 159-181.

- Das Evangelium des Markus, MeyerK I, 2, 16 éd., 1963.

E. LOEVESTAM, Wunder und Symbolhandlung, KuD 8, 1962, 124-135.

D. LUEHRMANN, Das Offenbarungsverständnis bei Paulus und in paulinischen Gemeinden, WMANT 16, 1965.

- Die Redaktion der Logienquelle, WMANT 33, 1969.

U. LUZ, Die Jünger im Matthäusevangelium, ZNW 62, 1971, 141-171.

- Die wiederendeckte Logienquelle, EvTh 33, 1973, 527 533.

I. MAISCH, Das Gleichnis von den klugen und törichten Jungfrauen, Auslegung von Mt 25,1-13, Bibel und Leben 11, 1970, 247259.

W. MANSON, Bist du, der da kommen soll ?, 1952.

H. MERTENS, L'Hymne de jubilation dans les synoptiques, 1957.

E. MEYER, Ursprung und Anfänge des Christentums, Band I : Die Evangelien, 1921.

J.R. MICHAELS, Apostolic Hardships and Righteous Gentiles, Mt 25, 31-46, JBL 84, 1965, 27-37.

O. MICHEL, Der Abschluss des Matthäusevangeliums, EvTh 10, 1950 $51,16-26$.

P. S. MINEAR, False Prophecy and Hypocrisy in the Gospel of Matthew, dans : Neues Testament und Kirche (Festschrift Schnackenburg), 76-93.

J. MUNCK, Discours d'adieux dans le Nouveau Testament et dans la littérature biblique, dans : Aux sources de la tradition chrétienne 155-170.

Neues Testament (Das Buch der Bücher), Einführungen, Texte, Kommentare, in Verbindung mit Hermann TIMM, herausgegeben von Gerhard IBER, 1972.

E. NORDEN, Agnostos Theos. Untersuchungen zur Formgeschichte religiöser Rede, 1913.

E. PERCY, Die Botschaft Jesu. Eine traditionskritische und exegetische Untersuchung, 1953. 
W. PESCH, Die sogenannte Gemeindeordnung Mt 18, BZ NF 7, 1963, $220-235$.

- Matthäus der Seelsorger, SBS 2, 1966.

- Eschatologie und Ethik, Auslegung von Mt 24,1-36, Bibel und Leben 11, 1970, 223-238.

E. PETERSON, Die Einholung des Kurios, ZsTh 7, 1929-30, 682-702.

G. von RAD, Die Stadt auf dem Berg, EvTh 8, 1948-49, 439-447.

K. H. RENGSTORF, Die Stadt der Mörder (Mt 22,7), dans : Judentum Urchristentum, Kirche (Festschrift Jeremias), BZNW 26, $1960,106-129$.

B. RIGAUX, Témoignage de l'évangile de Matthieu. Pour une histoire de Jésus II, 1967.

J.A. ROBINSON, The "Parable" of the Sheep and the Goats, NTS 2, $1955-56,225-237$.

- Le kérygme de l'Eglise et le Jésus de l'histoire, NSTh $11,1960$.

A. SAND, Die Polemik gegen "Gesetzeslosigkeit" im Evangelium nach Matthäus und bei Paulus, BZ NF 14, 1970, 112-125.

A. SATAKE, Die Gemeindeordnung in der Johannesapokalypse, WMANT 21, 1966, 171-191.

W. SCHMAUCH, Orte der Offenbarung und der Offenbarungsort im Neuen Testament, 1956.

K. L. SCHMIDT, Der Rahmen der Geschichte Jesu, 2e éd., 1969.

R. SCHNACKENBURG, "Ihr seid das Salz der Erde, das Licht der Welt" zu Mt 5,13-16, dans : Schriften zum Neuen Testament, 1971, $177-200$.

A. SCHULZ, Nachfolgen und Nachahmen, StANT 6, 1962.

S. SCHULZ, Q. Die Spruchquelle der Evangelisten, 1972.

E. SCHWEIZER, Matth, 5,17-20. Anmerkungen zum Gesetzesverständnis des Matthäus, ThLZ 77, 1952, 479-485.

- Erniedrigung und Erhöhung bei Jesus und seinen Nachfolgern, AThANT 28, 2e éd., revue, 1962.

- Gesetz und Enthusiasmus bei Matthäus, dans : Beiträge zur Theologie des Neuen Testaments (Neutestamentliche Aufsätze 1955-1970), 1970, 49-70.

- Matthäus und seine Gemeinde, SBS 71, 1974.

D. J.F. SEITZ, Love your Enemies, NTS 16, 1969-70, 39-54.

O. H. STECK, Israel und das gewaltsame Geschick der Propheten, WMANT 23, 1967. 
K. STENDAHL, The School of St. Matthew and its Use of the Old Testament, 2e éd., 1968.

G. STRECKER, Der Weg der Gerechtigkeit. Untersuchung zur Theologie des Matthäus, FRLANT 82, 2e éd., 1966.

- Das Geschichtsverständnis des Matthäus, EvTh 26, 1966, 57-74.

- Les macarismes du discours sur la montagne, dans :

L'Evangile selon Matthieu. Rédaction et théologie 185-208.

A. STROBEL, Zum Verständnis von Mt XXV 1-13, NovTest 2, 1958, 199-227.

P. STUHLMACHER, Das paulinische Evangelium. I. Vorgeschichte, FRLANT 95, 1968.

M. J. SUGGS, Wisdom, Christology and Law in Matthew's Gospel, 1970.

W. G. THOMPSON, Matthew's Advice to a Divided Community, Mt 17, 22-18, 35, Analecta Biblica 44, 1970.

R. THYSMANN, Communauté et directives éthiques (la catéchèse de Matthieu), 1974.

S. van TILBORG, The Jewish Leaders in Matthew, 1972.

H. E. TOEDT, Der Menschensohn in der synoptischen Ueberlieferung, 2e éd., 1963.

W. TRILLING, Zur Ueberlieferungsgeschichte des Gleichnisses vom Hochzeitsmahl, Mt 22,1-14, BZ NF 4, 1960, 251-265.

- Das wahre Israel. Studien zur Theologie des MatthäusEvangeliums, StANT 10, 3e éd., 1964.

- Christusverkündigung in den synoptischen Evangelien. Beispiele gattunsgemässer Auslegung, Biblische Handbibliothek 4, 1969.

- Amt und Amtverständnis bei Matthäus, dans : Mélanges Rigaux 29-44.

P. VIElHAUER, Die Prophetie, dans : E. Hennecke - W. Schneemelcher, Neutestamentliche Apokryphen, tome 2 (Apostolisches, Apokalypsen und Verwandtes), 3e éd., 1964, 422-427.

- Gottesreich und Menschensohn in der Verkündigung Jesu, dans : Aufsätze zum Neuen Testament, ThB 31, 1965, 55-91.

A. VOEGTLE, Das christologische und ekklesiologische Anliegen von Mt 28,16-20, dans : Studia Evangelica II (TU 87), 1964, $266-294$.

R. WALKER, Die Heilsgeschichte im ersten Evangelium, FRLANT 91, 1967. 
N. WALTER, Die Bearbeitung der Seligpreisungen durch Matthäus, dans : Studia Evangelica IV (TU 102), 1968, 246-258.

A. WEISER, Die Knechtsgleichnisse der synoptischen Evangelien, StANT 29, 1971.

A. WIKENHAUSER, Die Liebeswerke in dem Gerichtsgemälde, Mt 25, 31-46, BZ 20, 1932, 366-377.

U. WILCKENS, Auferstehung, Themen der Theologie 4, 1970.

W. WILKENS, Die Redaktion des Gleichniskapitels Markus 4 durch Matthäus, ThZ 20, 1964, 305-327.

H. WINDISCH, Die Sprüche vom Eingehen in das Reich Gottes, ZNW 27 , $1928,163-192$.

- Der Sinn der Bergpredigt. Ein Beitrag zum geschichtlichen Verständnis der Evangelien und zum Problem der richtigen Exegese, 2e éd., 1937.

H. T. WREGE, Die Ueberlieferungsgeschichte der Bergpredigt, WUNT 9, 1968.

D. ZELLER, Das Logion Mt 8, 11f, Lk 13, 29f und das Motiv der Völkerwallfahrt, BZ NF 15, 1971, 222-237; 16, 1972, 84-93.

J. ZUMSTEIN, La relation du maître et du disciple dans le bas-judaîsme palestinien et dans l'évangile selon Matthieu, Mémoire de l'Institut des sciences bibliques de l'Université de Lausanne, 1971 (ronéographié).

- Matthieu 28,16-20, RTP 22, 1972, 14-33. 